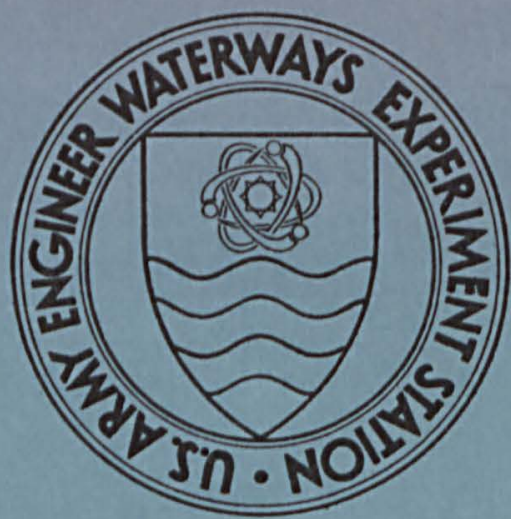

TECHNICAL REPORT N-70-7

\title{
AN EXPERIMENTAL INVESTIGATION OF SOIL-STRUCTURE INTERACTION IN A COHESIVE SOIL \\ Volume II \\ by
}

G. E. Jester
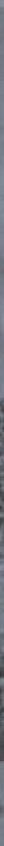

March 1970

Sponsored by Defense Atomic Support Agency

Conducted by U. S. Army Engineer Waterways Experiment Station, Vicksburg, Mississippi 


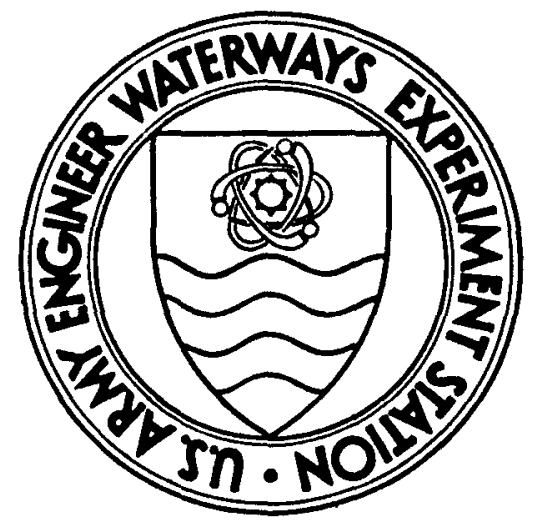

TECHNICAL REPORT N-70-7

\section{AN EXPERIMENTAL INVESTIGATION OF SOIL-STRUCTURE INTERACTION IN A COHESIVE SOIL \\ Volume II \\ by}

G. E. Jester

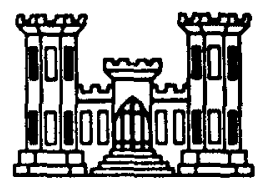

Morch 1970

Sponsored by Defense Atomic Support Agency NWER Subtask SC210

Conducted by U. S. Army Engineer Waterways Experiment Station, Vicksburg, Mississippi 
W34

No. $N-70-7$

v. 2

$\operatorname{cog} 3$

CONTENTS

$\underline{\text { Page }}$

BIBLIOGRAPHY -

336

TABLES 1 THROUGH 7-_. 350

FIGURES 1 THROUGH 99-1 365

APPENDIX A PROPERTIES OF COMPACTED BUCKSHOT CLAY; WALL

FRICTION REDUCTION; SOIL, GAGE, AND TEST DEVICE

PLACEMENT-.......... 460

APPENDIX B DESIGN OF SPRING-RING TEST DEVICE-_._. 515

APPENDIX C OSCILIOGRAPH RECORDS FROM THE DYNAMIC TESTS-.....-.- 528 


\section{BIBLIOGRAPHY}

Abbott, P. A. (1966), Nonlinear Static Arching for Vertically Buried Prismatic Structures, AFWL TR 65-160, University of New Mexico for Air Force Weapons Laboratory, Kirtland AFB, New Mexico.

Adine, A., and Dai, P. (1963), "An Approximate Study of Arching in Soil," Computation of Underground Structural Response, Chapter 5, p. 123-139; DASA 1386, edited by A. Ang and N. M. Newmark for Defense Atomic Support Agency.

Aggarwal, H. R., Soldate, A. M., and Hook, J. F. (1963), A Theoretical Analysis of Stress Wave Interaction in a Model Soil, final report. AFSWC-TDR-63-43, National Engineering Science Company for Air Force Special Weapons Center, Kirtland AFB, New Mexico.

Ahlers, E. B. (1961), Experimental Methods of Determining the Behavior of Underground Structures under Dynamic Loads, Armour Research Foundation, Chicago, Illinois.

Airy, W. (1897), "The Pressure of Grain," Minutes of Proceedings of the Institute of Civil Engineers, Vol. 81, Paper No. 3049.

Allgood, J. R. (1965), The Behavior of Shallow-Buried Cylinders, Technical Report R344, U. S. Naval Civil Engineering Laboratory, Port Hueneme, California.

Allgood, J. R., and Seabold, R. H. (1965), Shallow Buried Model Arches Subjected to a Traveling-Wave Ioad, Technical Report R375, U. S. Naval Civil Engineering Laboratory, Port Hueneme, California.

American Society of Civil Engineers (1961), Design of Structures to Resist Nuclear Weapons Effects, Manuals of Engineering Practice No. 42, New York, New York.

Ang, A., and Newmark, N. M. (1963), Computation of Underground Structural Response, DASA 1386, Department of Civil Engineering, University of Illinois, Urbana, Illinois.

Bakex, W. J., and Janza, F. J. (1966), Investigation of Flash X-ray Techniques in Soil Dynamics and Interaction Problems, 
AFWL-TR-66-50, U. S. Air Force Weapons Laboratory, Kirtland AFB, New Mexico.

Baron, M. I., and Parnes, R. (1962), Further Studies on the Diffraction of a Pressure Wave by an Elastically Lined Cylindrical Cavity in an Elastic Medium, SR-72, Paul Weidlinger for the Mitre Corporation.

Bedesem, W. B. (1964), A Continuum Theory of Soil-Structural Interaction in Granular Media, Ph. D. Thesis, University of Illinois, Urbana, Illinois.

Bedesem, W. B., Das, Y. C., and Robinson, A. R. (1964), Quasi-Static Theory of Soil-Structure Interaction, AFWL-TDR-64-91, prepared by the University of Illinois for Air Force Weapons Laboratory, Kirtland AFB, New Mexico.

Berdan, D., and Bernhard, R. K. (1950), "Pilot Studies of Soil Density Measurements by Means of X-Rays," Proceedings of the American Society for Testing and Materials, Vol. 50, pp 13281342 .

Bloedow, F. H. (1962), Radiographic Instrumentation Study, final report AFSWC-TDR-62-44, Air Force Special Weapons Center, Kirtland AFB, New Mexico.

Boynton Associates (1960), Operation Manual for 250 PSI 4-Foot Diameter Dynamic Load Generator, La Canada, California.

Brecht, W. A., and Wahl, A. M. (1952), The Radially Tapered Disk Spring, Paper APM-52-4, American Society of Mechanical Engineers, New York, New York.

Caquot, A. I. (1934), Equilibre des Massifs a Frottement Interne, Gauthier-Villars, Paris.

Caquot, A. I., and Kérisel, J. (1949), Traité De Mécanique Des Sols, Gauthier-Villars, Paris.

Caquot, A. I. (1957), "Pressure in Elevators," ("La Pression Dans Les Silos, ") Proceedings of the Fourth International Conference on Soil Mechanics and Foundation Engineering, Vol. II, pp 191-195 (Translation No. 64-11, U. S. Army Engineer Waterways Experiment Station, by Jan C. Van Tienhoven, December 1964). 
Carlson, R. W. (1936), Development and Analysis of a Device for Measuring Compressive Stress in Concrete, Sc. D. Thesis, Massachusetts Institute of Technology, Cambridge, Massachusetts.

Carlson, R. W., and Pirtz, D. (1953), "Development of a Device for the Direct Measurement of Compressive Stress," Proceedings, American Concrete Institute, Title No. 49-15, Vol. 49, pp 201-215, Detroit, Michigan.

Carroll, W. F. (1963), Dynamic Bearing Capacity of Soils, Report 5; Vertical Displacements of Spread Footings on Clay: Static and Impul sive Loadings, Technical Report No, 3-599, U. S. Army Engineer Waterways Experiment Station, Vicksburg, Mississippi.

Chelapati, C. V. (1964), Arching in Soil Due to the Deflection of a Rigid Horizontal Strip, Technical Note N-591, U. S. Naval Civil Engineering Laboratory, Port Hueneme, California.

Chiapetta, R. L., and Constantino, C. J. (1967), A Bibliography on the Response of Earth Media and Buried Structures to Ground Shock Loading, IIT Research Institute Project Report M 6177, also published as U. S. Army Engineer Waterways Experiment Station, Vicksburg, Mississippi, Contract Report No. 3-168.

Christensen, R. W., and Tien Hsing Wu (1964), "Analysis of Clay Deformation as a Rate Process," Journal of the Soil Mechanics and Foundation Division, American Society of Civil Engineers, Proceedings, Vol. 90, SM 6, pp 125-157.

Costantino, C. J., Robinson, R. R., and Salmon, M. A. (1964), "A Simplified Soil Structure Interaction Model to Investigate the Response of Buried Silos and Cylinders," Proceedings of the Symposium on Soil Structure Interaction, University of Arizona, Tucson, Arizona, pp 303-314.

Costantino, C. J. (1966), Response of Crushable Foam Encased Buried Cylinders, Ph. D. Thesis, Illinois Institute of Technology, Chicago, Illinois.

Crandall, S. H., and DahI, N. C. (1959), An Introduction to the Mechanics of Solids, McGraw-Hill, Inc., New York, New York.

Davis, H. E., and Woodward, J. R. (1949), "Some Laboratory Studies 
of Factors Pertaining to the Bearing Capacity of Soils," Highway Research Board Proceedings, Vol 29, pp 467-476.

Dorris, A. F. (1965), Response of Horizontally Oriented Buried CyIinders to Static and Dynamic Loading, Technical Report 1-682, Vicksburg, Mississippi, U. S. Army Engineer Waterways Experiment Station.

Drake, J. L., Considerations for Determining Airblast-Induced Soil Stress, U. S. Army Engineer Waterways Experiment Station, Vicksburg, Mississippi (in preparation).

Engesser, Fr. (1882), "Ueber den Erddruck Gegen Innere StützWände (Tunnelwände)," Deutsche Bauzeitung, No. 16, pp 91-93.

Finn, W. D. (1960), Stresses in Soil Masses Under Various Boundary Conditions, $\mathrm{Ph}$. D. Thesis, University of Washington.

Gardner, G. C. (1963), "The Best Hopper Profile for Cohesive Material," Chemical Engineering Science, Vol. 18, No. 1, pp 35-39.

Gravesen, S. (1959), "Elastic Half-Space Covered by a Stiff Plate with a Circular Piston," Bulletin No. 10, Laboratory for Building Research, Technical University of Denmark, Copenhagen.

Haase, M. C. (1967), X-Radiography of Unopened Soil Cores, Miscellaneous Paper No. 3-918, U. S. Army Engineer Waterways Experiment Station, Vicksburg, Mississippi.

Hadala, P. F. (1965), Dynamic Bearing Capacity of Soils, Report 4, Investigation of a Dimensionless Load-Displacement Relation for Footings on Clay, Technical Report No. 3-599, U. S. Army Engineer Waterways Experiment Station, Vicksburg, Mississippi.

Hadala, P. F. (1967a), The Effects of Placement Method on the Response of Soil Stress Gages, Technical Report No. 3-803, U. S. Army Engineer Waterways Experiment Station, Vicksburg, Mississippi.

Hadala, P. F. (1967b), Sidewall Friction Reduction in Static and Dynamic Small Blast Load Generator Tests, Technical Report, U. S. Army Engineer Waterways Experiment Station, Vicksburg, Mississippi (in preparation).

Hadala, P. F. (1967c), Spectral Analysis of Fundamental Studies 
Tests, Memorandum on DASA's Program, 20 June 1967, U. S. Army Engineer Waterways Experiment Station, Vicksburg, Mississippi (not publicly distributed).

Havers, J. A. (1963), Structural Materials for Hardened Personnel Shelters, IIT Research Institute for Office of Civil Defense, Department of Defense, Washington, D. C.

Hendron, A. J. (1963), The Behavior of Sand in One-Dimensional Compression, Ph. D. dissertation, Dept. of Civil Engineering, University of Illinois, Urbana, Illinois.

Hendron, A. J. (1968), An Experimental Study of Arching In Clay, Technical Report, U. S. Army Engineer Waterways Experiment Station, Vicksburg, Mississippi (in preparation).

Höeg, Kaare (1966), Pressure Distribution on Underground Structural Cylinders, AFWL TR-65-98, Massachusetts Institute of Technology for Air Force Weapons Laboratory, Kirtland AFB, New Mexico.

Hughes, G. T., Locker, J. G., and Stewart, W. D. (1965), Bibliography of Soil Dynamics and Soil Structure Interaction During Dynamic or Similar Loadings, DR-170, Department of Civil Engineering, Royal Military College of Canada, Kingston, Ontario.

Hvorslev, M. J. (1949), Subsurface Exploration and Sampling of Soils for Civil Engineering Purposes, report on research project of ASCE, edited and printed by U. S. Army Engineer Waterways Experiment Station, Vicksburg, Mississippi.

Hvorslev, M. Juul (1966), Comments on Research Proposal, private communications with author, U. S. Army Engineer Waterways Experiment Station, Vicksburg, Mississippi.

Ingram, J. K. (1965), "The Development of a Free-Field Soil Stress Gage for Static and Dynamic Measurements," Instruments and Apparatus for Soil and Rock Mechanics, ASTM STP 392, also issued as U. S. Army Engineer Waterways Experiment Station, Vicksburg, Mississippi, Technical Report No. 1-814.

Ingram, L. F. (1967), Instrumentation for Earth Stresses and Motions Produced by Explosions, Miscellaneous Paper No. 1-885, U. S. Army Engineer Waterways Experiment Station, Vicksburg, Mississippi. 
Jackson, J. G., Jr., and Hadala, P. F. (1964), Dynamic Bearing Capacity of Soils, Report 3; The Application of Similitude to Small-Scale Footing Tests, Technical Report No. 3-599, U. S. Army Engineer Waterways Experiment Station, Vicksburg, Mississippi.

Jaky, J. (1948), "Pressure in Silos," Second International Conference on Soil Mechanics and Foundation Engineering, Proceedings, Vol. 1, pp 103-107.

Jamieson, J. A. (1904), "Grain Pressures in Deep Bins," Engineexing News, Vol. 5I, pp 236-239.

Janssen, H. A. (1895), "Versuche über Getreidedruchs in Silozellen," Verein Deutscher Ingenieure, Zeitschrift, Vol. 39.

Jenike, A. W., Elsey, P. J., and Woolley, R. H. (1958), "Flow of Bulk Solids Progress Report," Bulletin No. 96, Utah Engineering Experiment Station, (Revised Reprint July, 1959), Salt Lake City, Utah.

Jenike, A. W (1964), "Storage and Flow of Solids," Bulletin No. 123, Utah Engineering Experiment Station, University of Utah, Salt Lake City, Utah.

Johnson, S. J. (1961), An Alternative Method for Determining the Constrained Modulus and the Coefficient of Elastic Vertical Compression, report by Moron, Proctor, Mueser, and Rutledge for Ballistic Systems Division of U. S. Air Force.

Kallstenius, Torsten (1959), "A Study of the Flow Pattern When Emptying a Vessel," Proceedings No. 17, Royal Swedish Geotechnical Institute, pp 67-70, Appendix, to "Measurements in Grain Silos During Filling and Emptying," by Werner Bergau, Stockholm.

Kane, H., Davisson, M. T., Oleson, R. E., and Sinnamon, G. K. (1964), A Study of the Behavior of a Clay Under Rapid and Dynamic Ioading in the One-Dimensional and Triaxial Tests, final report, RTD TDR 63-3II6, University of Illinois for Air Force Weapons Laboratory, Kirtland AFB, New Mexico.

Kennedy, T. E., Dynamic Response of Buried Concrete Arches, Project 3.2 , OPERATION SNOWBALL, Technicrl Report, U. S. Army Engineer Waterways Experiment Station, Vicksburg, Mississippi (in preparation). 
Kogler, F., and Scheidig, A. (1927), "Druckverteilung im Baugrunde-I. Die Ergebnisse neuerer Versuche," Die Bautechnik, 5. Jahrgang, Heft 29, pp 418-42l, Berlin, Germany.

Kvapil, Rudolf (1965), "Gravity Flow of Granular Materials in Hoppers and Bins," International Journal of Rock Mechanics and Mining Science, Vol. 2, pp 25-41, Pergamon Press.

Lenczner, D. (1966), "Cohesive Arching of Bulk Materials in Bunkers and Silos," Civil Engineering and Public Works Review, Vo1. 61, No. 724, pp 1393, 1395-1396.

Mason, H. G., Criner, O. H., Waissar, R., and Wallace, N. R. (1963), A Study of the Dynamic Soil-Structure Interaction Characteristics of Real Soil Media, RTD TDR-63-3075, United Research Services for Air Force Weapons Laboratory, Kirtland AFB, New Mexico.

Mason, H. G. (1965), Effects of Structural Compressibility on Active and Passive Arching in Soil-structure Interaction, final report, DASA 1718, URS Corporation for Defense Atomic Support Agency, Washington, D. C.

McNuIty, J.W. (1965), An Experimental Study of Arching in Sand, Technical Report No. 1-674, U. S. Army Engineer Waterways Experiment Station, Vicksburg, Mississippi.

Melin, J.W., and Sutcliffe, S. (1959), Development of Procedures for Rapid Computation of Dynamic Structural Response, SRS 171, Department of Civil Engineering, University of Illinois, Urbana, IlIinois.

Merritt, J. I., and Newmark, N. M. (1964), "Effects on Underground Structures and Equipment," Nuclear Geoplosics, Part V, DASA $1285(\mathrm{~V})$, Stanford Research Institute, Menlo Park, California.

Monfore, G. E. (1950), "An Analysis of the Stress Distributions in and Near Stress Gages Embedded in Elastic Solids," Structural Research Iaboratory Report No. SP 26, U. S. Bureau of Reclamation, Denver, Colorado.

Mosborg, R. J., and Talda, P. M. (1966), An Experimental Investigation of the Arching Phenomenon Occurring Over a Buried Rectangular Plate, AFWI TR-65-78, prepared by University of 
Illinois for Air Force Weapons Laboratory, Kirtland AFB, New Mexico.

Mow, C. C. (1964), Dynamic Response of Lined and Unlined Underground Openings, Memorandum RM-3962-PR, The Rand Corporation, Santa Monica, California.

Newmark, N. M. (1949), Methods of Analysis for Structures Subjected to Dynamic Loading, report to Physical Vulnerability Division. Directorate of Intelligence, U. S. Air Force.

Newmark, N. M., Briscoe, J. W., and Merritt, J. I. (1960), Analysis and Design of Flexible Underground Structures, Volume I, prepared for U. S. Army Engineer Waterways Experiment Station, Vicksburg, Mississippi.

Newmark, Hansen and Associates (1961), Protective Construction Review Guide-Hardening, Vol. 1, prepared for the Office of the Assistant Secretary of Defense.

Newmark, N. M., and Hall, W. J. (1962), Preliminary Design Methods for Underground Protective Structures, AFSWC-TDR-62-6, prepared by University of Illinois for Air Force Special Weapons Center, Kirtland AFB, New Mexico.

Newmark, N. M., and Haltiwanger, J. D. (1962), Principles and Practices for Design of Hardened structures, AFSWC-TDR-62-138, prepared by University of Illinois for Air Force Special Weapons Center, Kirtland AFB, New Mexico.

Newmark, N. M. (1964), "The Basis of Current Criteria for the Design of Underground Protective Construction," Proceedings of the Symposium on Soil-Structure Interaction, University of Arizona, Tucson, Arizona, pp 1-24.

Neuber, Heinz (1946), Theory of Notch Stresses, Edwards Bros., Ann Arbor, Michigan.

Peck, R. B., Hanson, W. E., and Thornburn, T. H. (1953), Foundation Engineering; John Wiley \& Sons, Inc., New York, New York.

Peck, R. B. (1960), "Soft Ground Tunneling," From Theory to Practice in Soil Mechanics, selections from the writings of Karl Terzaghi, John Wiley and Sons, Inc., New York, New York. 
Perry, Edward B. (1968), The Effect of Shallow Burial on the LoadDisplacement-Time Response of Square Footings in Clay Under Impulsive Loading, M.S. Thesis, Department of Civil Engineering, Mississippi State University, State College, Mississippi.

Richardson, A. M., Jr. (1965), The Response of Soils to Dynamic Loading, Report 16; Effective Stress Versus Strength, Saturated Fat Clay, Massachusetts Institute of Technology Research Project 63-20, also published as U. S. Army Engineer Waterways Experiment Station, Vicksburg, Mississippi, Contract Report No. 3-26.

Richardson, A. M., Jr., and Whitman, R. V. (1963), Effect of Strain Rate upon Undrained Shear Resistance of a saturated Fat Clay, P63-15, Massachusetts Institute of Technology.

Richart, F. E., Jr. (1959), Arching in Granular Elastic Media, final report on Project 5810, Engineering and Industrial Experiment Station, University of Florida.

Richmond, O. (1963), "Gravity Hopper Design," Mechanical Engineering, Vol. 85, No. I, pp 46-49.

Robinsky, E. I., and Morrison, C. F. (1964), "Sand Dispiacement and Compaction Around Model Friction Piles," Canadian Geotechnical Journal, Vol. I, No. 2, pp 81-93.

Roscoe, K. H., Arthur, J. R. F., and James, R. G. (1963), "The Determination of Strains in Soils by an X-Ray Method," (Part 2), Civil Engineering and Public Works Review, Vol. 58, No. 685, pp 1009-1012.

Schindler, I. (1967), An Improved Facility for Testing Soils in OneDimensional Compression, paper submitted to the Symposium on Wave Propagation and Dynamic Properties of Earth Materials, Albuquerque, New Mexico.

Seaman, Lynn (1966), One-Dimensional Stress Wave Propagation in Soils, DASA Report 1757, prepared by Stanford Research Institute, Menlo Park, California.

Seely, F. B., and Smith, J. O. (1952), Advanced Mechanics of Materials, and ed., John Wiley \& Sons, Inc., New York, New York.

Selig, E. T. (1960), "An Analytical Method for the Design of 
Underground Structures," Shock, Vibration and Associated Environments, Bulletin No. 28, Part III, pp 37-47, Office of the Secretary of Defense, Research and Engineering, Washington, D. C.

Selig, E. T. (1961a), "A Technique for Observing Structure-Soil Interaction," Materials Research and Standards, Vol. 1, No. 9, pp 717-719.

Selig, E. T., McKee, K. E., and Vey, Eben (1961b), "Underground Structures Subject to Air Overpressure," Transactions of the American Society of Civil Engineers, Vol. 126, Part I, pp 1627-1649.

Sirieys, P. M. (1964), "Champs de Contraintes Autour des Tunnels Circulaires en Elastoplasticite," Rock Mechanics and Engineering Geology, Vol. II, No. I, pp 68-75.

Spangler, M. G. (1948), "Underground Conduits - An Appraisal of Modern Research," Transactions of the American Society of Civil Engineers, Vol. 113, No. 2337, New York.

Spangler, M. G. (1956), "Stresses in Pressure Pipelines and Protective Casing Pipes," Journal of the Structural Division, Proceedings of the American Society of Civil Engineers, Vol. 82, No. 1054, Ann Arbor, Michigan.

Steen, Leo L. (1966a), "Revised Procedure for Lining Small Blast Load Generator Specimens to Reduce Sidewall Friction," Memorandum for Blast Load Generator Soils Laboratory Files, U. S. Army Engineer Waterways Experiment Station, Vicksburg, Mississippi.

Steen, Leo L. (1966b), "Compaction Methods for Various Degrees of Saturation of Buckshot Clay," Memorandum for Blast Load Generator Soils Laboratory Files, U. S. Army Engineer Waterways Experiment Station, Vicksburg, Mississippi.

Sylwestrowicz, W. (1953), "Experimental Investigation of the Behavior of Soil Under a Punch or Footing," Journal of the Mechanics and Physics of Solids, Vol. I, pp 258-264.

Taylor, D. W. (1945), Review of Pressure Distribution Theories, Pressure Cell Investigation and Pressure Distribution Data, Massachusetts Institute of Technology, Cambridge, Massachusetts. 
Teng, Wayne C. (1962), Foundation Design, Prentice-Hall, Inc., Englewood Cliffs, New Jersey.

Terzaghi, Karl (1919), "Die Erddruckerscheinungen in örtlich beanspruchten Schüttungen und die Entstehung Von

'Tragkörpern'," Osterreichische Wochenschrift fur offentlichen Baudienst, Nos. 17-19, pp 194-199, 206-210, and 218-223.

Terzaghi, Karl (1936a), "A Fundamental Fallacy in Earth Pressure Computations," Journal of the Boston Society of Civil Engineers, Vol. 23, No. 2, pp 71-88.

Terzaghi, Karl (1936b), "Arching in Sands," Engineer News-Record, Vol. 116, pp 690-693.

Terzaghi, Karl (1936c), "Stress Distribution in Dry and in Saturated Sand Above a Yielding Trap-Door," First International Conference on Soil Mechanics and Foundation Engineering, Vol. I, pp 307-311, Cambridge, Massachusetts.

Terzaghi, Karl (1943), Theoretical Soil Mechanics, John Wiley \& Sons, Inc., New York, New York.

Terzaghi, Karl, and Peck, R. B. (1948), Soil Mechanics in Engineering Practice, John Wiley \& Sons, Inc., New York, New York.

Terzaghi, Karl, and Richart, F. E., Jr. (1952), "Stresses in Rock About Cavities," Geotechnique, Vol. 3, No. 2, pp 57-90.

Triandafilidis, G. E., Hampton, D., and Spanovich, N. (1964), Experimental Study of Arching Stresses on Buried Vertical Cylinders, RTD TDR-63-3106, University of New Mexico for Air Force Weapons Laboratory, Kirtland AFB, New Mexico.

Tschebotarioff, G. P. (1951), Soil Mechanics, Foundations, and Earth Structures, McGraw-Hill, Inc., New York, New York.

Tschebotarioff, G. P. (1954), Discussion of Field Study of SheetPile Bulkhead (ASCE Proceedings Separate No. 155) by Duke, C. M., presented at ASCE Meeting, 1950, Los Angeles, ASCE Proceedings Separate No. 379.

Turnbull, W. J., and Foster, C. R. (1958), "Stabilization of Materials 
by Compaction," Transactions, American Society of Civil Engineers, Vol. 123, pp 1-26.

U. S. Army Engineer Waterways Experiment Station (1944), Soil Pressure Cell Investigation, interim report, Technical Memorandum No. 210-1, Vicksburg, Mississippi.

U. S. Army Engineer Waterways Experiment Station (1958), Geological Investigation of the Yazoo Basin, Technical Report No. 3-480, Vicksburg, Mississippi, revised March 1968.

U. S. Army Engineer Waterways Experiment Station (1963), Status Report for Blast Load Generator Facility, Vicksburg, Mississippi.

U. S. Corps of Engineers (1965), Laboratory Soils Testing, EM 1110-2-1906.

Van Horn, D. A., and Tener, B. K. (1963a), A Study of Loads on Underground Structures, Project 434-S, Iowa Engineering Experiment Station, Ames, Iowa.

Van Horn, D. A. (1963b), A Study of Loads on Underground Structures, Part I, an annotated bibliography, DASA 1406, Iowa Engineering Experiment Station, Iowa State University, Ames, Iowa.

Van Horn, D. A. (1964), "A. Study of Loads on Underground Structures," Proceedings of the Symposium on Soil-structure Interaction, University of Arizona, Tucson, Arizona, pp 256-282.

Voellmy, Adolph (1937), Imbedded Pipes, Static Analysis of Buried Conduits with Consideration of the Elasticity (English translation by Professor and Mrs. Edward Allen, 1961-1962, Ames, Iowa).

Walker, D. M. (1966), "An Approximate Theory for Pressures and Arching in Hoppers, "Chemical Engineering Science, Vol. 2l, Oxford, England, pp 975-997.

Watkins, R. K. (1957), Characteristics of the Modulus of Passive Resistance of Soil, Ph. D. Thesis, Iowa State College, Ames, Iowa.

Watkins, R. K. (1964), "Structural Design Trends in Buried Flexible Conduits," Proceedings of the Symposium on Soil-structure 
Interaction, University of Arizona, Tucson, Arizona, pp 246255.

Watkins, R. K. (1966), Structural Design of Buried Circular Conduits, U. S. Army Engineer Waterways Experiment Station, Vicksburg, Mississippi.

Whiffin, A. C., and Morris, S. A. H. (1962), "Piezoelectric Gauge for Measuring Dynamic Stresses Under Roads," The Engineer, Vol. 213, No. 5544, pp 741-746.

Whipple, C. R. (1961), The Dynamic Response of Shallow-Buried Arches Subjected to Blast Ioading, Ph. D. Thesis, Department of Civil Engineering, University of Illinois, Urbana, Illinois.

Whitman, R. V., Roberts, J. E., and Shieh-Wen Mao (1960), The Response of Soils to Dynamic Ioadings, Report 4; One-Dimensional Compression and Wave Velocity Tests, prepared by Massachusetts Institute of Technology for U. S. Army Engineer Waterways Experiment Station, Vicksburg, Mississippi, and Defense Atomic Support Agency, Contract Report No. 3-26, DASA 1199.

Whitman, R. V., Richardson, A. M., Jr., and Nasim, N. M. (1962a), The Response of Soils to Dynamic Loadings, Report 10; Strength of Saturated Fat Clay, Massachusetts Institute of Technology Research Project R62-22, also published as U. S. Army Engineer Waterways Experiment Station, Vicksburg, Mississippi, Contract Report No. 3-26.

Whitman, R. V., Getzler, Zvi, and Höeg, Kaare (1962b), The Response of Soils to Dynamic Loadings, Report 12; Static Tests Upon Thin Domes Buried in Sand, Massachusetts Institute of Technology Research Project R62-41, also published as U. S. Army Engineer Waterways Experiment Station, Vicksburg, Mississippi, Contract Report No. 3-26.

Whitman, R. V. (1963), The Response of Soils to Dynamic Loadings, Report 17; Stress-Strain-Time Behavior of Soil in OneDimensional Compression, Massachusetts Institute of Technology Research Report R63-25, also published as U.S. Army Engineer Waterways Experiment Station, Vicksburg, Mississippi, Contract Report No. 3-26.

Wiedermann, A. H. (1960), Concepts of Preliminary Design of Structure Projects for Underground Nuclear Detonations, Appendix B; The 
Interaction of Buried Structures with Ground Shock, AFSWCTR-60-3, Armour Research Foundation for Air Force Special Weapons Center, Kirtland AFB, New Mexico.

Wiehle, C. K. (1965), Soil-Structure Interaction Under Dynamic Load, final report, DASA 1711, URS 645-7, URS Corporation, Burlingame, California.

Yoshiharo, T., Robinson, A. R., and Merritt, J. L. (1963), Interaction of Plane Elastic Waves with an Elastic Cylindrical Shell, University of Illinois, Structural Research Series No. 261 , for the Office of Naval Research. 
Tabie 1

Surmary of Static Tests

\begin{tabular}{|c|c|c|c|c|c|c|c|c|c|c|c|c|c|c|c|c|c|c|}
\hline \multirow[b]{2}{*}{$\begin{array}{l}\text { Test } \\
\text { Mo. }\end{array}$} & \multirow{2}{*}{\multicolumn{2}{|c|}{$\begin{array}{l}\text { Pressure } \\
\quad \mathrm{psi} \\
\end{array}$}} & \multirow{2}{*}{\multicolumn{2}{|c|}{$\begin{array}{l}\text { Depth of } \\
\text { Cover }\end{array}$}} & \multirow[b]{2}{*}{$\begin{array}{c}\mathrm{K}_{\mathrm{T}} \\
\mathrm{psi} / \mathrm{in} .\end{array}$} & \multirow[b]{2}{*}{$\begin{array}{r}M_{S} \\
\text { psi } \\
\end{array}$} & \multirow[b]{2}{*}{$\begin{array}{c}K_{S} \\
p s i / i n .\end{array}$} & \multirow{2}{*}{\multicolumn{2}{|c|}{$\frac{\mathrm{K}_{\mathrm{T}}}{\mathrm{K}_{\mathrm{S}}}$}} & \multirow[b]{2}{*}{$\begin{array}{r}\Delta \mathrm{P} \\
\mathrm{psi} \\
\end{array}$} & \multirow[b]{2}{*}{$\frac{\frac{\partial \Delta P}{q_{u}}}{}$} & \multicolumn{3}{|c|}{ Maximum Arching } & \multirow[b]{2}{*}{$\frac{d_{t}}{B} \times 1000$} & \multirow{2}{*}{\multicolumn{2}{|c|}{$\frac{\text { Avg Soils Data }}{q_{u}}$}} & \multirow[b]{2}{*}{ Remarks } \\
\hline & & & & & & & & & & & & $\frac{P_{T}}{P_{S}}$ & $\frac{\Delta D}{B} \times 1000$ & $\frac{D_{T}}{B} \times 100$ & & & & \\
\hline 1 & 37.8 & 38.0 & 2 & $1 / 3$ & NA & 4700 & 940 & NA & IIA & -24.5 & -1.67 & 0.35 & -73.01 & 8.64 & -78.33 & 26.0 & $\sim 14.70$ & Top of test device lowered \\
\hline \multirow[t]{3}{*}{2} & 37.5 & 28.3 & 6 & 1 & NA & 1700 & 340 & NA & $\mathrm{NA}$ & $-37.0+$ & -2.32 & 0.02 & -21.17 & 2.72 & -26.67 & 24.3 & 16.97 & Top of test device lowered \\
\hline & 37.5 & 26.5 & - & - & NA & 2040 & 404 & NA & $\mathrm{NA}$ & +8.7 & +0.54 & 1.24 & +74.17 & 7.29 & +82.67 & -- & $\cdots$ & Top of test device raised \\
\hline & 34.8 & 27.1 & -- & -- & NA & 1740 & 348 & NA & $\mathrm{NA}$ & -33.5 & -2.10 & 0.04 & -78.00 & 8.80 & -81.67 & - & $\cdots$ & Lowering top of device $2 \mathrm{~d}$ time \\
\hline \multirow[t]{3}{*}{3} & 37.5 & 27.7 & 18 & 3 & NA & 1870 & 375 & NA & NA & -36.5 & -2.02 & 0.03 & -11.84 & 2.13 & -16.00 & 24.7 & 18.06 & Top of test device lowered \\
\hline & 37.5 & 30.0 & - & -- & NA & 1130 & 226 & NA & NA & +7.8 & +0.43 & 1.21 & +15.17 & 1.47 & +25.83 & -- & -- & Raising top of device \\
\hline & 50.0 & 40.6 & 18 & 3 & INA & 1640 & 328 & NA & NA & -49.3 & -2.73 & 0.01 & -26.51 & 10.24 & -24.17 & -- & $-\cdot$ & Top of test device lowered $2 \mathrm{~d}$ time \\
\hline \multirow[t]{3}{*}{4} & 75.0 & 67.2 & 6 & 1 & NA & 1700 & 340 & NA & $\mathrm{NA}$ & -45.2 & -3.32 & 0.40 & -77.17 & 7.85 & -81.83 & 25.2 & 13.61 & Top of test device lowered \\
\hline & 75.0 & 66.2 & -- & -- & NA & 1650 & 330 & RA & NA & +36.1 & +2.65 & 1.48 & +71.67 & 6.97 & +82.67 & -- & -- & Raising top of device \\
\hline & 75.0 & 67.8 & 6 & 1 & $\mathrm{NA}$ & 1690 & 338 & IIA & NA & -50.6 & -3.72 & 0.33 & -78.83 & 8.35 & -82.17 & - & -- & Lowering top of device $2 \mathrm{a}$ time \\
\hline \multirow[t]{2}{*}{5} & 37.6 & -- & 6 & 1 & INA & UNK & UTiK & $\mathrm{NA}$ & ra & +38.8 & +2.79 & 2.03 & +61.18 & 6.06 & +72.28 & 25.5 & 13.89 & Top of device raised \\
\hline & 37.5 & -- & -- & -- & NA & UNK & UNK & MA & IAA & -36.4 & -2.62 & 0.03 & -58.51 & 4.85 & -24.63 & - & -- & Top of device lowered \\
\hline \multirow[t]{5}{*}{6} & 37.5 & 33.2 & 6 & 1 & 189 & 1570 & 314 & 0.5 & 0.60 & -14.1 & -1.04 & 0.62 & -2.17 & 13.72 & -20.67 & 24.7 & 13.61 & Spring system used, pressure raised \\
\hline & 38.5 & 36.2 & -- & -- & 189 & 1550 & 310 & 0.5 & 0.61 & -15.9 & -1.17 & 0.59 & -2.17 & 15.32 & -21.67 & -- & -- & Raised pressure 2d time \\
\hline & 38.0 & 39.0 & -- & -- & 189 & 1525 & 305 & 0.5 & 0.62 & -11.9 & -0.87 & 0.69 & -0.67 & 16.67 & $-23 . \infty$ & -- & - & Max load after $15.5 \mathrm{hr}$ creep allowed \\
\hline & 38.7 & 39.0 & -- & -- & 189 & 1565 & 313 & 0.5 & 0.60 & -12.1 & -0.89 & 0.69 & -1.17 & 17.19 & -23.50 & -- & -- & Max load after $51.8 \mathrm{hr}$ creep allowed \\
\hline & 38.5 & 39.0 & -- & -- & 189 & 1565 & 313 & 0.5 & 0.60 & -11.9 & -0.87 & 0.69 & -1.50 & 17.22 & -23.50 & -- & -- & Max load after $62.6 \mathrm{hr}$ creep allowed \\
\hline 7 & 37.5 & 46.2 & 6 & 1 & 958 & 3830 & 766 & 3.0 & 1.25 & -5.6 & -0.42 & 0.85 & -4.00 & 14.68 & -6.00 & 25.7 & 13.47 & Spring system used, pressure raised \\
\hline \multirow[t]{3}{*}{8} & 175.0 & -- & 18 & 3 & 522 & 6150 & 1230 & 0.7 & 0.42 & -54.0 & -4.9 & 0.74 & -27.67 & 31.26 & -41.33 & 27.3 & 11.11 & $\begin{array}{l}\text { Spring system used, first reached } \\
\text { max load on top of device }\end{array}$ \\
\hline & 240.0 & -- & -- & -- & 532 & 7950 & 1590 & 0.7 & 0.33 & -58.0 & -5.2 & 0.76 & -40.17 & 33.16 & -57.50 & -- & -- & Max load on top of device at $\max P_{S}$ \\
\hline & 240.0 & $\cdots$ & -- & -- & 532 & 7450 & 1490 & 0.7 & 0.36 & -35.0 & 3.2 & 0.85 & -45.01 & 33.94 & -60.33 & -- & -- & $\begin{array}{l}\text { Max load of top of device after } \\
\text { creep allowed }\end{array}$ \\
\hline
\end{tabular}

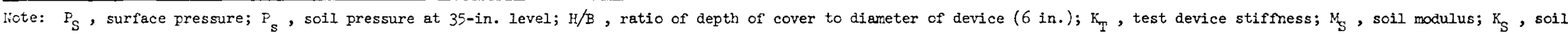
stiffness; $\Delta F$, differential pressure $\left(P_{T}-P_{S}\right) ; q_{2}$, unconfined compressive strength of soil; $P_{T}$, average force acting on top of test device; $\Delta 0$, differential deflection $\left(D_{T}-D_{S}\right) ; D_{T}$, total deflection of test device $\left(d_{b}+d_{t}\right) ; a_{t}$, deflection of top with respect to base of test device; wC, water content of soil in percent of dry weight. $+(-)$ pressure on top of device higher (lower) than surface pressure or deflection of top of device is less (more) than the soil in the "free field" at the same level. 
Tabie 2

Summary of Dynamic Tests

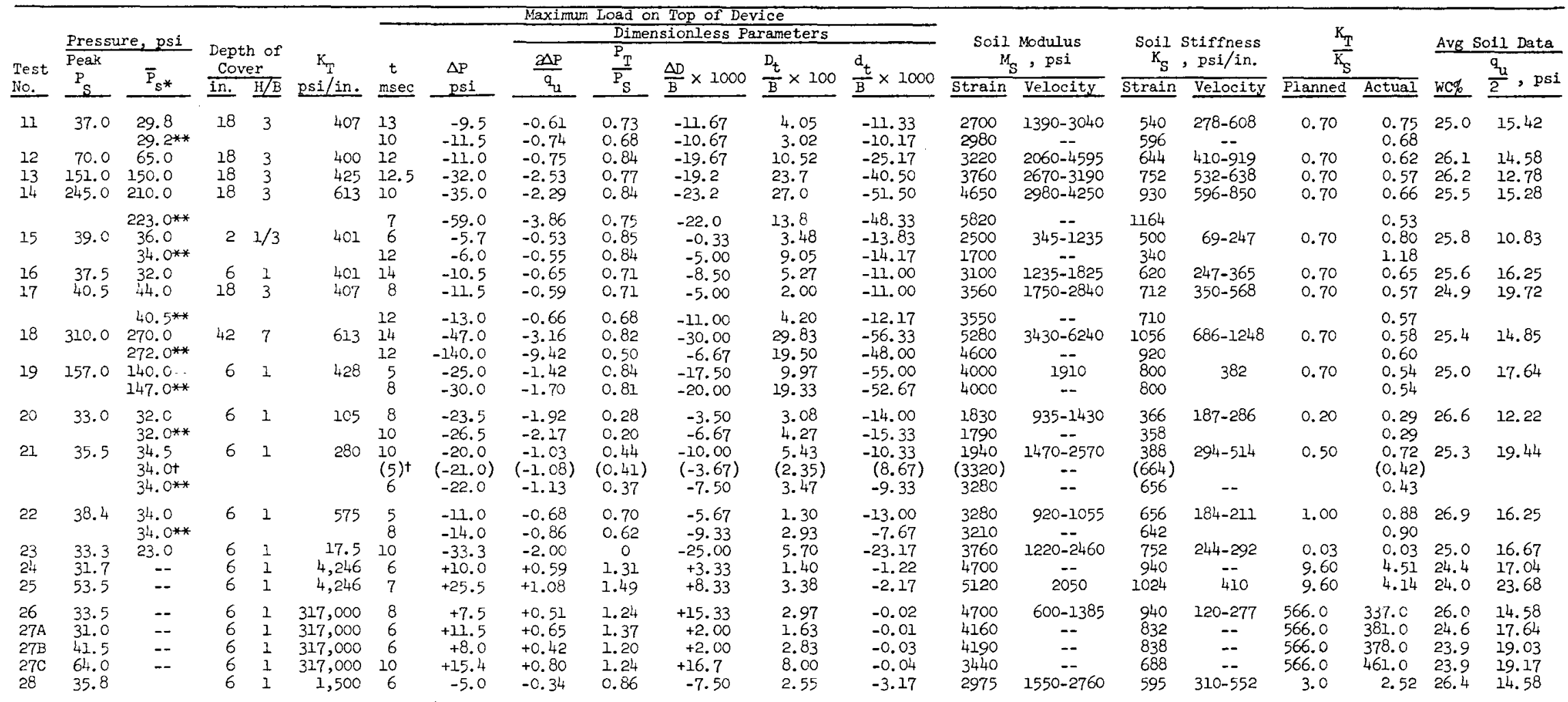

* $\overline{\mathrm{P}}_{\mathrm{S}}$, approximate soil pressure at 35 -in. level at time indicated; other symbols are defined in table 1.

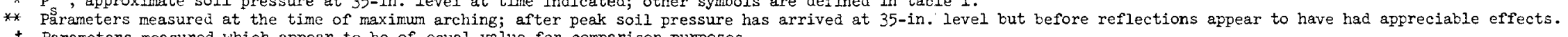
+ Parameters measured which appear to be of equal value for comparison purposes. 
Table 3

Characteristics of Test Device

\begin{tabular}{|c|c|c|c|c|c|c|c|c|c|c|c|c|c|c|c|c|c|c|c|c|}
\hline \multirow[b]{2}{*}{$\begin{array}{l}\text { Test } \\
\text { No. } \\
\end{array}$} & \multicolumn{3}{|c|}{ Spring stiffness } & \multicolumn{5}{|c|}{$\begin{array}{l}\text { Components of Spring } \\
\text { Rings }\end{array}$} & \multirow[b]{2}{*}{$\begin{array}{l}\text { Total } \\
\text { Wt } \\
\text { Ib } \\
\end{array}$} & \multirow[b]{2}{*}{$\begin{array}{l}\text { Wt of } \\
\text { Top } \\
\text { 1b } \\
\end{array}$} & \multirow{2}{*}{$\begin{array}{l}\text { wt of Top } \\
\text { +50\% of } \\
\text { Active Rings } \\
\text { 1b } \\
\end{array}$} & \multicolumn{3}{|c|}{$\begin{array}{l}\text { Calculated Properties without } \\
\text { Damping }\end{array}$} & \multicolumn{6}{|c|}{ Observed Results of Damping Test in Air } \\
\hline & $\begin{array}{c}k \\
1 b / \text { in. } \\
\end{array}$ & $\begin{array}{c}K_{T} \\
\mathrm{psi} / \mathrm{in} .\end{array}$ & $\begin{array}{c}M_{T} \\
\text { psi } \times 10^{3} \\
\end{array}$ & №. & $\begin{array}{l}\text { Rings } \\
\text { No. } \\
\text { Active }\end{array}$ & $\overline{\text { Size }}$ & $\frac{\text { Spac }}{\text { No.- }}$ & $\frac{\mathrm{ers}}{\mathrm{size}}$ & & & & $\begin{array}{l}\text { Period } \\
\underline{\text { T, msec }}\end{array}$ & $\begin{array}{c}\text { Damping } \\
\text { Mass } \\
\mathrm{m}, \mathrm{lb} \text { in. } / \mathrm{sec}^{2}\end{array}$ & $\begin{array}{l}\text { Frequency } \\
\text { f, Hertz }\end{array}$ & $\begin{array}{l}\text { Wt in } \\
\text { Motion } \\
\text { Ib } \\
\end{array}$ & $\begin{array}{l}\text { Damping, } c \\
2 \mathrm{~b} / \mathrm{in} . \mathrm{sec} \\
\end{array}$ & $\begin{array}{l}\text { Critical Damping } \\
\mathrm{c}_{c}, \mathrm{lb} / \mathrm{in} . / \mathrm{sec} \\
\end{array}$ & $\begin{array}{l}\text { Period } \\
\text { T, msec }\end{array}$ & $\begin{array}{c}\text { Damped } \\
\text { Frequency } \\
\text { Hertz } \\
\end{array}$ & $\begin{array}{c}\text { Natural } \\
\text { Frequency } \\
\text { Hertz } \\
\end{array}$ \\
\hline 1 & $\mathrm{NA}$ & -- & -- & $\mathrm{NA}$ & $\mathrm{NA}$ & IXA & NA & $M$ & 13.36 & 3.96 & -. & NA & -- & -- & NA & $\mathrm{NA}$ & $\mathrm{NA}$ & $\mathrm{NA}$ & IIA & NAA \\
\hline 2 & $\mathrm{NA}$ & .. & -- & $\mathrm{M}$ & $\mathrm{NA}$ & IAA & $\mathrm{MA}$ & $\mathrm{NA}$ & 13.36 & 3.96 & -- & NA & -- &.- & $\mathrm{NA}$ & $\mathrm{NA}$ & $\mathrm{NA}$ & $\mathrm{NA}$ & NTA & $\mathrm{MA}$ \\
\hline 3 & NA. & -- & - & HA & IA & NA & NA & IKA & 13.36 & 3.96 & -- & MA & -- & -- & $\mathrm{NA}$ & NA & NA & NA & $\mathrm{MA}$ & NA \\
\hline 4 & WA & -- & -- & $\mathrm{NA}$ & NA & $\mathrm{NA}$ & IXA & $\mathrm{NA}$ & 13.36 & 3.96 & -- & NAA & -- & -- & $\mathrm{NA}$ & $\mathrm{NA}$ & IA & $\mathrm{NA}$ & NA & $\mathrm{NA}$ \\
\hline 5 & NA & -- & -- & NA & NA & NA & $\mathrm{NA}$ & MA & 13.36 & 3.96 & -- & $\mathrm{NA}$ & -- & -- & NA & NA & NA & $\mathrm{NA}$ & $\mathrm{NA}$ & $\mathrm{NA}$ \\
\hline 6 & 5,340 & 190 & 0.950 & 20 & 13 & $1 / 8$ & 5 & $1 / 4$ & 11.72 & 3.60 & 5.51 & INA & -- & -. & NA & IAA & $\mathrm{NA}$ & INA & $\mathrm{NA}$ & NA \\
\hline 7 & 31,750 & 1,124 & 5.620 & 12 & 5 & $1 / 4$ & 4 & $1 / 4$ & 9.34 & 4.51 & 6.00 & IAA & -- & -- & $\mathrm{NA}$ & NAA & $\mathrm{NA}$ & IAA & $\mathrm{NA}$ & $\mathrm{NA}$ \\
\hline 8 & 14,700 & 522 & 2.621 & 20 & 14 & $1 / 8$ & 7 & $1 / 4$ & 11.65 & 3.62 & 5.72 & NA & -- &.- & $\mathrm{NA}$ & NA & $\mathrm{NA}$ & NA & NA & NA \\
\hline 11 & 11,500 & 407 & 2.035 & 20 & 28 & $1 / 8$ & 7 & $1 / 4$ & 11.88 & 3.02 & 5.71 & 7.09 & 0.01473 & 140.9 & 4.50 & 2.12 & 23.2 & 6.35 & 157.0 & 158 \\
\hline 12 & 11,330 & 401 & 2.005 & 20 & 28 & $1 / 8$ & 7 & $1 / 4$ & 11.91 & 3.02 & 5.71 & 7.17 & 0.01473 & 139.4 & 4.50 & 2.11 & 23.0 & 6.40 & 156.0 & 157 \\
\hline 13 & 11,950 & 4,25 & 2.125 & 20 & 17 & $1 / 8$ & 7 & $1 / 4$ & 11.91 & 3.02 & 5.56 & 6.85 & 0.01434 & 145.8 & -- & $* 3.0$ & $* 24.1$ & -- & -- & -- \\
\hline 14 & 17,300 & 613 & 3.065 & 20 & 18 & $1 / 8$ & 8 & $1 / 8$ & 11.73 & 3.01 & 5.62 & 5.72 & 0.01450 & 174.8 & 5.56 & 7.63 & 32.8 & 5.96 & 168.0 & 173 \\
\hline 15 & 11,330 & 401 & 2.005 & 20 & 18 & $1 / 8$ & 7 & $1 / 4$ & 12.45 & 3.51 & 6.20 & 7.46 & 0.01600 & 134.0 & 5.27 & 2.50 & 27.4 & 6.50 & 154.0 & 155 \\
\hline 16 & 11,330 & 402 & 2.005 & 20 & 18 & $1 / 8$ & 7 & $1 / 4$ & 12.45 & 3.51 & 6.20 & 7.46 & 0.01600 & 134.0 & 5.27 & 2.50 & 27.4 & 6.50 & 154.0 & 155 \\
\hline 17 & 11,500 & 407 & 2.035 & 20 & 18 & $1 / 8$ & 7 & $1 / 4$ & 11.88 & 3.02 & 5.71 & 7.09 & 0.01473 & 140.9 & 4.47 & 2.12 & 23.2 & 6.35 & 157.0 & 158 \\
\hline 18 & 17,300 & 613 & 3.065 & 20 & 18 & $1 / 8$ & 8 & $1 / 8$ & 11.73 & 3.01 & 5.62 & 5.72 & 0.01450 & 174.8 & 5.56 & 7.63 & 32.8 & 5.95 & 168.0 & 173 \\
\hline 19 & 12,075 & 428 & 2.14 & 20 & 17 & $1 / 8$ & 7 & $3 / 4$ & 11.91 & 3.02 & 5.56 & 6.84 & 0.01434 & 146.0 & -- & $* 3.1$ & $* 24.3$ & -. & -- & .. \\
\hline 20 & 2,960 & 105 & 0.525 & 42 & 40 & $1 / 16$ & 11 & $1 / 8$ & 11.57 & 2.86 & 5.54 & 33.79 & 0.01429 & 72.5 & 5.66 & 1.91 & 13.3 & 14.12 & 70.8 & 72 \\
\hline 21 & 7,900 & 280 & 1.400 & 20 & 13 & $1 / 8$ & 6 & $1 / 4$ & 11.82 & 3.61 & 5.54 & 8.41 & 0.01429 & 118.8 & -- & $* 1.95$ & $* 17.4$ & -- & -- & -. \\
\hline 22 & 16,250 & 575 & 2.875 & 12 & 10 & $1 / 4$ & 4 & $1 / 4$ & 13.05 & 3.32 & 6.30 & 6.28 & 0.01625 & 159.2 & 6.14 & 6.38 & 32.1 & 6.39 & 156.0 & 161 \\
\hline 23 & 495 & 27.5 & 0.0875 & 42 & 40 & $1 / 16$ & 7 & $1 / 8$ & 11.46 & 2.86 & 5.49 & 33.67 & 0.01420 & 29.7 & 4.73 & 0.62 & 4.9 & 33.0 & 30.3 & 32 \\
\hline 24 & 120,000 & 4,246 & 22.330 & 12 & 8 & $1 / 4$ & 7 & $1 / 4$ & 13.18 & 3.93 & 6.35 & 2.14 & 0.01638 & 466.0 & 4.00 & 9.6 & 66.6 & 1.65 & 606.0 & 614 \\
\hline 25 & 120,000 & 4,246 & 22.330 & 12 & 8 & $1 / 4$ & 7 & $1 / 4$ & 13.18 & 3.93 & 6.35 & 2.14 & 0.01638 & 466.0 & 4.00 & 9.6 & 66.6 & 1.65 & 606.0 & 614 \\
\hline 26 & $8,950,000$ & 317,000 & $1,585.0$ & & Machined & Cylin & & & 11.30 & 2.61 & 5.41 & 0.24 & 0.01396 & 4040.0 & -- & -- & -- & -- & -- & -- \\
\hline 27 & $8,950,000$ & 317,000 & $1,585.0$ & & Macinined & Cylin & & & 11.30 & 2.61 & 5.41 & 0.24 & 0.01396 & 4040.0 & - & -- & -- & -- & $\cdots$ & -- \\
\hline 28 & 42,390 & 1,500 & 7,500 & 12 & 20 & $1 / 4$ & 5 & $1 / 4$ & 13.08 & 3.32 & 6.32 & 3.89 & 0.01631 & 257.0 & -- & $* 8.4$ & $* 41.0$ & -- & -- & -- \\
\hline
\end{tabular}


Table 4

Summary of Buckshot Clay Properties

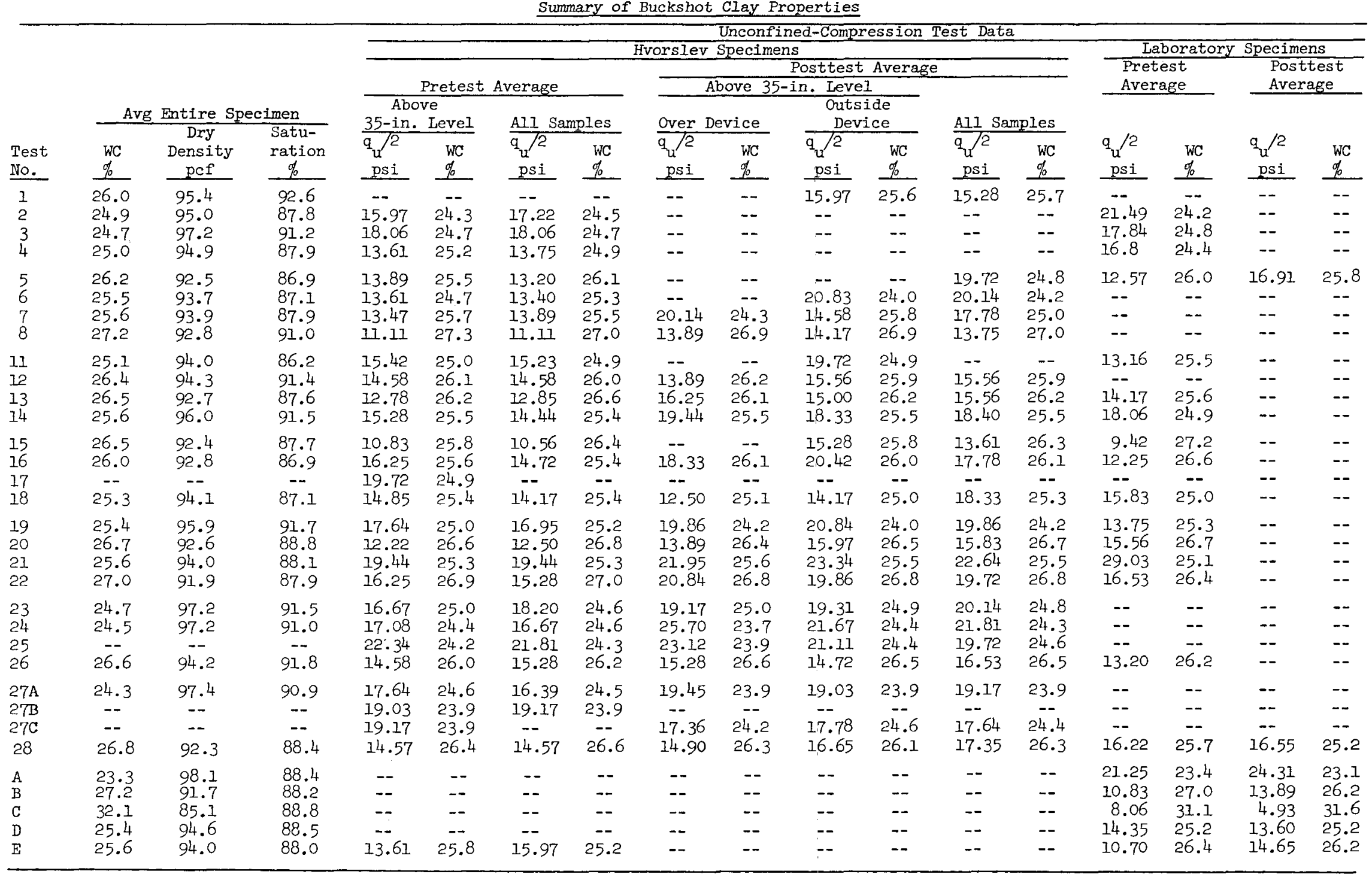

Note: $W C$, water content; $q_{u}$, unconfined compressive strength. 


\begin{tabular}{|c|c|c|c|c|c|c|c|c|c|c|c|c|c|c|}
\hline \multirow{4}{*}{$\begin{array}{c}t \\
\text { hr:min }\end{array}$} & \multirow{3}{*}{\multicolumn{2}{|c|}{$\begin{array}{c}\text { Pressure } \\
\text { ps1 }\end{array}$}} & \multirow{3}{*}{\multicolumn{5}{|c|}{ Deflections, in. }} & \multirow{3}{*}{\multicolumn{3}{|c|}{$\begin{array}{l}\text { Force Per Unit } \\
\text { Area Acting on } \\
\text { Top of Device, psi } \\
\end{array}$}} & \multirow{2}{*}{\multicolumn{4}{|c|}{ 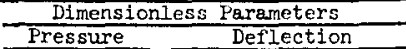 }} \\
\hline & & & & & & & & & & & & & & \\
\hline & & & $\mathrm{D}_{\mathrm{S}}$ & & & & & & & & \multirow{2}{*}{$\frac{a \Delta P}{q_{u}}$} & \multirow{2}{*}{$\frac{P_{T}}{P_{S}}$} & \multirow{2}{*}{$\frac{\Delta D}{B} \times 1000$} & \multirow{2}{*}{$\frac{D_{\mathrm{T}}}{\mathrm{B}} \times 100$} \\
\hline & $P_{S}$ & ${ }_{s}$ & ${ }_{S}$ & $a$ & ${ }_{t}$ & $\mathrm{D}_{\mathrm{T}}$ & $\Delta D$ & $\mathrm{P}_{\mathrm{C}}^{\mathrm{P}}$ & $P_{\text {T }}$ & $\Delta \mathrm{P}$ & & & & \\
\hline & & & & & & & est 1 & & & & & & & \\
\hline $0-3: 53$ & $0-37.5$ & $0-37.4$ & 0.997 & 1.072 & 0 & 1.072 & -0.075 & -- & -- & -- & -- & -- & -12.50 & 17.87 \\
\hline \multicolumn{15}{|c|}{ Lowering top of test device } \\
\hline $\begin{array}{l}0: 41 \\
0: 54 \\
1: 08 \\
1: 20 \\
1: 31\end{array}$ & $\begin{array}{l}37.5 \\
37.8 \\
37.7 \\
37.8 \\
37.7\end{array}$ & $\begin{array}{l}37.6 \\
37.6 \\
37.9 \\
37.9 \\
37.9\end{array}$ & $\begin{array}{l}0.016 \\
0.021 \\
0.029 \\
0.033 \\
0.035\end{array}$ & $\begin{array}{l}0.016 \\
0.020 \\
0.021 \\
0.026 \\
0.028\end{array}$ & $\begin{array}{l}0.001 \\
0.005 \\
0.008 \\
0.016 \\
0.025\end{array}$ & $\begin{array}{l}0.017 \\
0.025 \\
0.032 \\
0.042 \\
0.053\end{array}$ & $\begin{array}{l}-0.001 \\
-0.004 \\
-0.003 \\
-0.009 \\
-0.018\end{array}$ & $\begin{array}{l}83.5 \\
79.5 \\
75.5 \\
67.5 \\
62.0\end{array}$ & $\begin{array}{l}37.1 \\
35.3 \\
33.6 \\
30.0 \\
27.6\end{array}$ & $\begin{array}{r}-0.4 \\
-2.5 \\
-4.1 \\
-7.8 \\
-10.1\end{array}$ & $\begin{array}{l}-0.03 \\
-0.17 \\
-0.28 \\
-0.53 \\
-0.69\end{array}$ & $\begin{array}{l}0.99 \\
0.93 \\
0.89 \\
0.79 \\
0.73\end{array}$ & $\begin{array}{l}-0.17 \\
-0.67 \\
-0.50 \\
-1.50 \\
-3.00\end{array}$ & $\begin{array}{l}0.28 \\
0.42 \\
0.53 \\
0.70 \\
0.88\end{array}$ \\
\hline $\begin{array}{l}1: 48 \\
2: 02 \\
2: 15 \\
2: 38 \\
2: 51\end{array}$ & $\begin{array}{l}37.7 \\
37.7 \\
37.7 \\
37.8 \\
37.8\end{array}$ & $\begin{array}{l}37.9 \\
37.9 \\
37.9 \\
38.2 \\
38.2\end{array}$ & $\begin{array}{l}0.042 \\
0.044 \\
0.049 \\
0.052 \\
0.056\end{array}$ & $\begin{array}{l}0.031 \\
0.033 \\
0.034 \\
0.037 \\
0.037\end{array}$ & $\begin{array}{l}0.041 \\
0.055 \\
0.081 \\
0.091 \\
0.145\end{array}$ & $\begin{array}{l}0.072 \\
0.088 \\
0.115 \\
0.131 \\
0.182\end{array}$ & $\begin{array}{l}-0.030 \\
-0.044 \\
-0.066 \\
-0.079 \\
-0.126\end{array}$ & $\begin{array}{l}58.5 \\
54.0 \\
50.0 \\
48.5 \\
40.5\end{array}$ & $\begin{array}{l}26.0 \\
24.0 \\
22.2 \\
21.6 \\
18.0\end{array}$ & $\begin{array}{l}-11.7 \\
-13.7 \\
-15.5 \\
-16.2 \\
-19.8\end{array}$ & $\begin{array}{l}-0.80 \\
-0.93 \\
-1.05 \\
-1.10 \\
-1.35\end{array}$ & $\begin{array}{l}0.69 \\
0.64 \\
0.59 \\
0.57 \\
0.48\end{array}$ & $\begin{array}{r}-5.00 \\
-7.33 \\
-11.00 \\
-13.17 \\
-21.00\end{array}$ & $\begin{array}{l}1.20 \\
1.47 \\
1.92 \\
2.18 \\
3.03\end{array}$ \\
\hline $\begin{array}{l}3: 06 \\
3: 30 \\
4: 00 \\
4: 21 \\
4: 52\end{array}$ & $\begin{array}{l}37.9 \\
38.0 \\
37.9 \\
37.9 \\
37.8\end{array}$ & $\begin{array}{l}38.2 \\
38.2 \\
38.0 \\
38.0 \\
38.0\end{array}$ & $\begin{array}{l}0.058 \\
0.067 \\
0.072 \\
0.076 \\
0.080\end{array}$ & $\begin{array}{l}0.038 \\
0.042 \\
0.045 \\
0.047 \\
0.048\end{array}$ & $\begin{array}{l}0.189 \\
0.269 \\
0.347 \\
0.410 \\
0.470\end{array}$ & $\begin{array}{l}0.227 \\
0.311 \\
0.392 \\
0.457 \\
0.518\end{array}$ & $\begin{array}{l}-0.169 \\
-0.244 \\
-0.320 \\
-0.381 \\
-0.438\end{array}$ & $\begin{array}{l}37.5 \\
36.5 \\
34.0 \\
32.5 \\
30.0\end{array}$ & $\begin{array}{l}16.7 \\
16.2 \\
15.1 \\
14.4 \\
13.3\end{array}$ & $\begin{array}{l}-21.2 \\
-21.8 \\
-22.8 \\
-23.5 \\
-24.5\end{array}$ & $\begin{array}{l}-1.44 \\
-1.48 \\
-1.55 \\
-1.60 \\
-1.67\end{array}$ & $\begin{array}{l}0.44 \\
0.43 \\
0.40 \\
0.38 \\
0.35\end{array}$ & $\begin{array}{l}-28.17 \\
-40.67 \\
-53.34 \\
-63.51 \\
-73.01\end{array}$ & $\begin{array}{l}3.78 \\
5.18 \\
6.53 \\
7.62 \\
8.64\end{array}$ \\
\hline $\begin{array}{l}19: 13 \\
19: 39 \\
19: 49 \\
20: 08 \\
21: 00\end{array}$ & $\begin{array}{r}37.8 \\
29.2 \\
19.5 \\
10.2 \\
0.0\end{array}$ & $\begin{array}{r}38.4 \\
30.5 \\
21.6 \\
12.9 \\
0.5\end{array}$ & $\begin{array}{r}0.134 \\
0.128 \\
0.104 \\
0.042 \\
-0.159\end{array}$ & $\begin{array}{r}0.093 \\
0.082 \\
0.051 \\
-0.0214 \\
-0.251\end{array}$ & $\begin{array}{l}0.474 \\
0.473 \\
0.464 \\
0.442 \\
0.364\end{array}$ & $\begin{array}{l}0.567 \\
0.555 \\
0.515 \\
0.418 \\
0.113\end{array}$ & $\begin{array}{l}-0.433 \\
-0.427 \\
-0.411 \\
-0.376 \\
-0.272\end{array}$ & $\begin{array}{r}35.0 \\
33.0 \\
26.5 \\
16.0 \\
0.5\end{array}$ & $\begin{array}{r}15.6 \\
14.7 \\
11.8 \\
7.1 \\
0.2\end{array}$ & $\begin{array}{r}-22.2 \\
-14.5 \\
-7.7 \\
-3.1 \\
+0.2\end{array}$ & $\begin{array}{l}-1.51 \\
-0.99 \\
-0.52 \\
-0.21 \\
+0.01\end{array}$ & $\begin{array}{l}0.41 \\
0.50 \\
0.60 \\
0.70 \\
--\end{array}$ & $\begin{array}{l}-72.18 \\
-71.18 \\
-68.51 \\
-62.68 \\
-45.34\end{array}$ & $\begin{array}{l}9.32 \\
9.25 \\
8.59 \\
6.97 \\
1.88\end{array}$ \\
\hline \multicolumn{15}{|c|}{ Test 2} \\
\hline $0-0: 1+1$ & $0-37.5$ & 0.28 .0 & 0.440 & 0.430 & 0 & 0.430 & -0.010 & -- & -- & -- & - & -- & -1.67 & 7.17 \\
\hline \multicolumn{15}{|c|}{ Lowering top of test device } \\
\hline $\begin{array}{l}0: 21 \\
0: 35 \\
0: 48 \\
1: 00 \\
1: 12\end{array}$ & $\begin{array}{l}37.5 \\
37.5 \\
37.5 \\
37.5 \\
37.5\end{array}$ & $\begin{array}{l}28.1 \\
28.1 \\
28.1 \\
28.4 \\
28.1\end{array}$ & $\begin{array}{l}0.001 \\
0.002 \\
0.004 \\
0.006 \\
0.011\end{array}$ & $\begin{array}{l}0.005 \\
0.006 \\
0.007 \\
0.008 \\
0.012\end{array}$ & $\begin{array}{l}0.001 \\
0.004 \\
0.006 \\
0.010 \\
0.014\end{array}$ & $\begin{array}{l}0.006 \\
0.008 \\
0.013 \\
0.018 \\
0.026\end{array}$ & $\begin{array}{l}-0.005 \\
-0.008 \\
-0.009 \\
-0.012 \\
-0.015\end{array}$ & $\begin{array}{r}9.0 \\
81.2 \\
74.2 \\
61.8 \\
52.7\end{array}$ & $\begin{array}{l}40.0 \\
36.1 \\
33.0 \\
27.5 \\
23.4\end{array}$ & $\begin{array}{r}+2.5 \\
-1.4 \\
-4.5 \\
-10.0 \\
-14.1\end{array}$ & $\begin{array}{l}+0.16 \\
-0.09 \\
-0.28 \\
-0.63 \\
-0.88\end{array}$ & $\begin{array}{l}1.07 \\
0.96 \\
0.88 \\
0.73 \\
0.62\end{array}$ & $\begin{array}{l}-0.83 \\
-1.33 \\
-1.50 \\
-2.00 \\
-2.50\end{array}$ & $\begin{array}{l}0.10 \\
0.13 \\
0.22 \\
0.30 \\
0.43\end{array}$ \\
\hline $\begin{array}{l}1: 25 \\
1: 39 \\
1: 52 \\
2: 12 \\
2: 36\end{array}$ & $\begin{array}{l}37.5 \\
37.5 \\
37.5 \\
37.5 \\
37.5\end{array}$ & $\begin{array}{l}28.2 \\
28.2 \\
28.5 \\
28.4 \\
28.4\end{array}$ & $\begin{array}{l}0.020 \\
0.024 \\
0.033 \\
0.036 \\
0.037\end{array}$ & $\begin{array}{l}0.014 \\
0.015 \\
0.016 \\
0.013 \\
0.009\end{array}$ & $\begin{array}{l}0.022 \\
0.030 \\
0.039 \\
0.060 \\
0.079\end{array}$ & $\begin{array}{l}0.036 \\
0.045 \\
0.055 \\
0.073 \\
0.088\end{array}$ & $\begin{array}{l}-0.016 \\
-0.021 \\
-0.022 \\
-0.037 \\
-0.051\end{array}$ & $\begin{array}{r}40.0 \\
28.2 \\
20.2 \\
13.0 \\
6.6\end{array}$ & $\begin{array}{r}17.8 \\
12.5 \\
9.0 \\
5.8 \\
2.9\end{array}$ & $\begin{array}{l}-19.7 \\
-25.0 \\
-28.5 \\
-31.7 \\
-34.6\end{array}$ & $\begin{array}{l}-1.23 \\
-1.57 \\
-1.78 \\
-1.98 \\
-2.17\end{array}$ & $\begin{array}{l}0.47 \\
0.33 \\
0.24 \\
0.15 \\
0.08\end{array}$ & $\begin{array}{l}-2.67 \\
-3.50 \\
-3.67 \\
-6.17 \\
-8.50\end{array}$ & $\begin{array}{l}0.60 \\
0.75 \\
0.92 \\
1.22 \\
1.47\end{array}$ \\
\hline $\begin{array}{l}2: 52 \\
3: 08 \\
3: 20 \\
3: 33 \\
3: 47\end{array}$ & $\begin{array}{l}37.5 \\
37.5 \\
37.5 \\
37.5 \\
37.5\end{array}$ & $\begin{array}{l}28.4 \\
28.2 \\
28.2 \\
28.1 \\
28.1\end{array}$ & $\begin{array}{l}0.036 \\
0.036 \\
0.039 \\
0.040 \\
0.043\end{array}$ & $\begin{array}{l}0.005 \\
0.003 \\
0.003 \\
0.002 \\
0.000\end{array}$ & $\begin{array}{l}0.099 \\
0.160 \\
0.220 \\
0.310 \\
0.400\end{array}$ & $\begin{array}{l}0.104 \\
0.163 \\
0.223 \\
0.312 \\
0.400\end{array}$ & $\begin{array}{l}-0.068 \\
-0.127 \\
-0.184 \\
-0.272 \\
-0.357\end{array}$ & $\begin{array}{l}2.0 \\
1.5 \\
2.4 \\
3.0 \\
1.5\end{array}$ & $\begin{array}{l}0.9 \\
0.7 \\
1.1 \\
1.3 \\
0.7\end{array}$ & $\begin{array}{l}-36.6 \\
-37.0 \\
-36.4 \\
-36.2 \\
-37.0\end{array}$ & $\begin{array}{l}-2.29 \\
-2.32 \\
-2.28 \\
-2.27 \\
-2.32\end{array}$ & $\begin{array}{l}0.02 \\
0.02 \\
0.03 \\
0.03 \\
0.02\end{array}$ & $\begin{array}{l}-11.34 \\
-21.17 \\
-30.67 \\
-45.34 \\
-59.51\end{array}$ & $\begin{array}{l}1.73 \\
2.72 \\
3.72 \\
5.20 \\
6.67\end{array}$ \\
\hline $\begin{array}{l}4: 00 \\
4: 09 \\
4: 18 \\
4: 27 \\
4: 55\end{array}$ & $\begin{array}{l}37.5 \\
37.5 \\
37.5 \\
37.5 \\
37.5\end{array}$ & $\begin{array}{l}28.2 \\
28.5 \\
28.5 \\
28.4 \\
28.4\end{array}$ & $\begin{array}{l}0.043 \\
0.044 \\
0.046 \\
0.054 \\
0.053\end{array}$ & $\begin{array}{l}0.001 \\
0.001 \\
0.002 \\
0.003 \\
0.004\end{array}$ & $\begin{array}{l}0.438 \\
0.456 \\
0.474 \\
0.483 \\
0.499\end{array}$ & $\begin{array}{l}0.439 \\
0.457 \\
0.476 \\
0.486 \\
0.503\end{array}$ & $\begin{array}{l}-0.396 \\
-0.413 \\
-0.430 \\
-0.432 \\
-0.450\end{array}$ & $\begin{array}{l}0.5 \\
0.8 \\
1.0 \\
1.0 \\
0\end{array}$ & $\begin{array}{l}0.2 \\
0.4 \\
0.4 \\
0.4 \\
0\end{array}$ & $\begin{array}{l}-37.3 \\
-37.1 \\
-37.1 \\
-37.1 \\
-37.5\end{array}$ & $\begin{array}{l}-2.33 \\
-2.32 \\
-2.32 \\
-2.32 \\
-2.35\end{array}$ & $\begin{array}{l}0.01 \\
0.01 \\
0.01 \\
0.01 \\
--\end{array}$ & $\begin{array}{l}-66.01 \\
-68.85 \\
-71.68 \\
-72.01 \\
-75.00\end{array}$ & $\begin{array}{l}7.32 \\
7.62 \\
7.93 \\
8.10 \\
8.39\end{array}$ \\
\hline \multicolumn{15}{|c|}{ Raizing top of test dcrice } \\
\hline $\begin{array}{l}5: 17 \\
5: 26 \\
5: 34 \\
5: 42 \\
5: 57\end{array}$ & $\begin{array}{l}34.5 \\
35.0 \\
37.0 \\
37.5 \\
34.0\end{array}$ & $\begin{array}{l}23.3 \\
25.6 \\
29.0 \\
29.0 \\
24.8\end{array}$ & $\begin{array}{l}0.054 \\
0.063 \\
0.069 \\
0.068 \\
0.067\end{array}$ & $\begin{array}{l}0.003 \\
0.003 \\
0.007 \\
0.009 \\
0.010\end{array}$ & $\begin{array}{l}0.496 \\
0.486 \\
0.471 \\
0.437 \\
0.211\end{array}$ & $\begin{array}{l}0.499 \\
0.489 \\
0.478 \\
0.446 \\
0.221\end{array}$ & $\begin{array}{l}-0.445 \\
-0.426 \\
-0.409 \\
-0.378 \\
-0.154\end{array}$ & $\begin{array}{l}17.4 \\
21.5 \\
32.0 \\
38.0 \\
60.0\end{array}$ & $\begin{array}{r}7.7 \\
9.6 \\
14.2 \\
16.9 \\
26.7\end{array}$ & $\begin{array}{l}-26.8 \\
-25.4 \\
-22.8 \\
-20.6 \\
-7.3\end{array}$ & $\begin{array}{l}-1.68 \\
-1.59 \\
-1.43 \\
-1.29 \\
-0.46\end{array}$ & $\begin{array}{l}0.22 \\
0.27 \\
0.38 \\
0.45 \\
0.79\end{array}$ & $\begin{array}{l}-74.17 \\
-71.00 \\
-68.17 \\
-63.00 \\
-25.67\end{array}$ & $\begin{array}{l}8.32 \\
8.15 \\
7.97 \\
7.43 \\
3.68\end{array}$ \\
\hline $\begin{array}{l}6: 05 \\
6: 13 \\
6: 19 \\
6: 30 \\
6: 35\end{array}$ & $\begin{array}{l}37.0 \\
37.5 \\
37.5 \\
36.7 \\
36.6\end{array}$ & $\begin{array}{l}25.3 \\
27.2 \\
27.2 \\
26.5 \\
26.5\end{array}$ & $\begin{array}{l}0.068 \\
0.062 \\
0.062 \\
0.062 \\
0.062\end{array}$ & $\begin{array}{l}0.023 \\
0.040 \\
0.050 \\
0.061 \\
0.062\end{array}$ & $\begin{array}{l}0.073 \\
0.037 \\
0.020 \\
0 \\
0\end{array}$ & $\begin{array}{l}0.096 \\
0.077 \\
0.070 \\
0.061 \\
0.062\end{array}$ & $\begin{array}{l}-0.028 \\
-0.015 \\
-0.008 \\
+0.001 \\
0\end{array}$ & $\begin{array}{r}80.0 \\
96.0 \\
102.5 \\
11.6 \\
10.2\end{array}$ & $\begin{array}{l}35.6 \\
42.7 \\
45.6 \\
51.6 \\
45.3\end{array}$ & $\begin{array}{r}-1.4 \\
+5.2 \\
+8.1 \\
+14.9 \\
+8.7\end{array}$ & $\begin{array}{l}-0.09 \\
+0.33 \\
+0.51 \\
+0.93 \\
+0.54\end{array}$ & $\begin{array}{l}0.96 \\
1.14 \\
1.22 \\
1.41 \\
1.24\end{array}$ & $\begin{array}{l}-4.67 \\
-2.50 \\
-1.33 \\
+0.17 \\
0\end{array}$ & $\begin{array}{l}1.60 \\
1.28 \\
1.17 \\
1.02 \\
1.03\end{array}$ \\
\hline \multicolumn{15}{|c|}{ Lowering top of test device } \\
\hline $\begin{array}{l}6: 37 \\
6: 44 \\
6: 56 \\
7: 05 \\
7: 15\end{array}$ & $\begin{array}{r}36.5 \\
35.8 \\
35.2 \\
34.8 \\
0.0\end{array}$ & $\begin{array}{l}27.1 \\
29.2 \\
29.6 \\
27.1\end{array}$ & $\begin{array}{r}0.062 \\
0.067 \\
0.063 \\
0.060 \\
-0.074\end{array}$ & $\begin{array}{r}0.062 \\
0.047 \\
0.04+1 \\
0.038 \\
-0.122\end{array}$ & $\begin{array}{l}0.002 \\
0.141 \\
0.3^{4} 99 \\
0.490 \\
0.492\end{array}$ & $\begin{array}{l}0.064 \\
0.188 \\
0.390 \\
0.528 \\
0.370\end{array}$ & $\begin{array}{l}-0.002 \\
-0.321 \\
-0.327 \\
-0.468 \\
-0.444\end{array}$ & $\begin{array}{r}93.2 \\
14.5 \\
7.0 \\
3.0 \\
0.0\end{array}$ & $\begin{array}{r}41.4 \\
6.4 \\
3.1 \\
1.3 \\
0.0\end{array}$ & $\begin{array}{r}+4.9 \\
-29.4 \\
-32.1 \\
-33.5 \\
0.0\end{array}$ & $\begin{array}{c}+0.31 \\
-1.84 \\
-2.01 \\
-2.10 \\
0.0\end{array}$ & $\begin{array}{l}1.13 \\
0.18 \\
0.09 \\
0.04 \\
--\end{array}$ & $\begin{array}{r}-0.33 \\
-20.17 \\
-54.50 \\
-78.00 \\
-74.00\end{array}$ & $\begin{array}{l}1.07 \\
3.13 \\
6.50 \\
8.80 \\
6.17\end{array}$ \\
\hline \multicolumn{15}{|c|}{ (Continued) } \\
\hline
\end{tabular}

ivcte: $t$, time after zero; $P_{S}$, surface pressure at time $t ; P_{S}$, soil pressure at $35-i n$. level; $D_{S}$, soil deflection at 3r-in. level; $d_{0}$, deflection of base of test device; $d_{t}$, deflection of top with respect to base of test device; $D_{T}$, total deflection of test device $\left(d_{\mathrm{b}}+d_{t}\right) ; \Delta D$, differential deflection $\left(D_{T}-D_{S}\right) ; P_{C}$, pressure acting on inside of test device; $P_{T}$, average force actinc on top of test device; $\Delta P$, differential pressure $\left(P_{T}-P_{S}\right) ; q_{u}$, unconfined compressive strength of soil; $B$, diameter of test device.

( 1 of 5 sheets) 


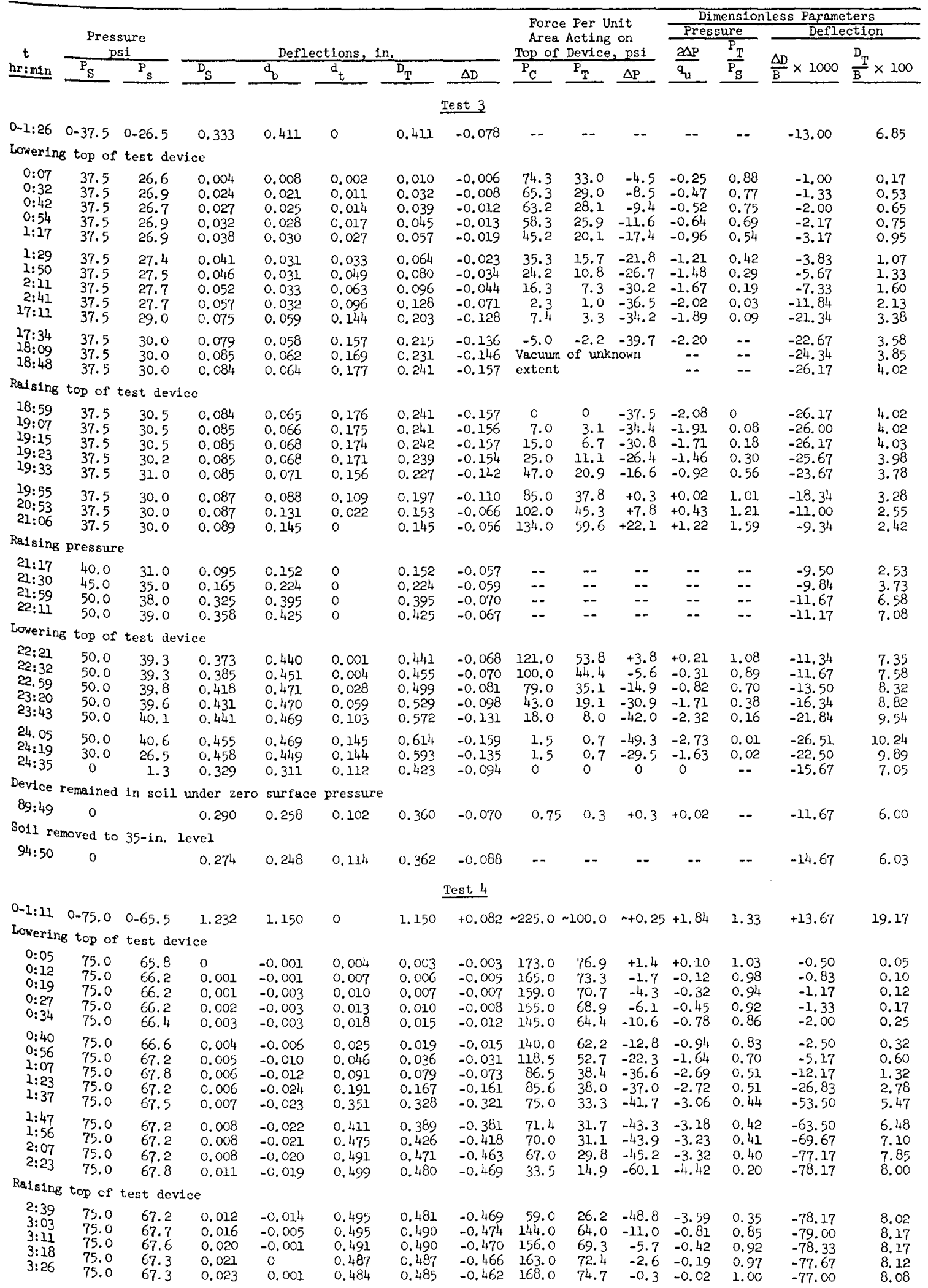




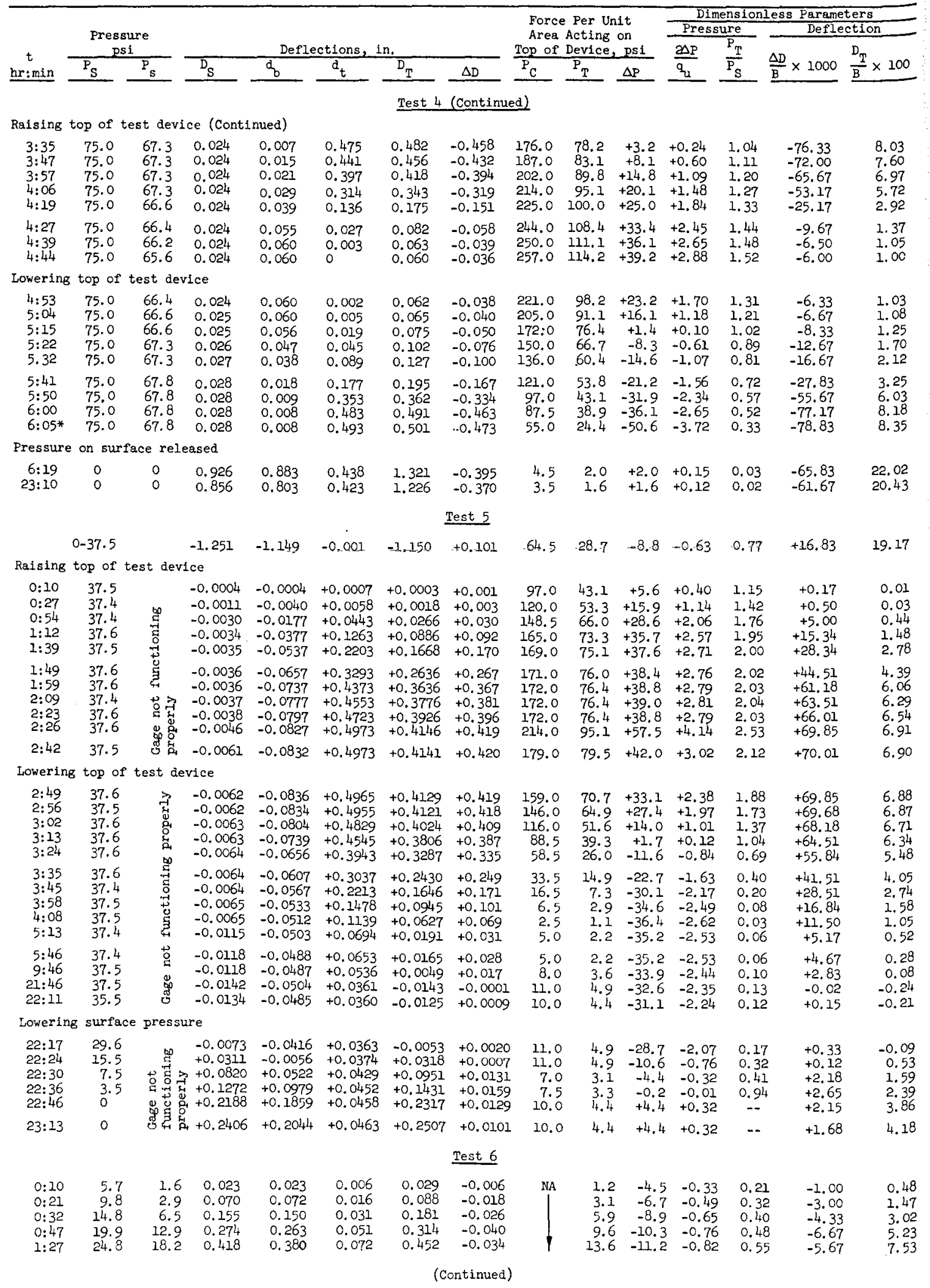

Note: NA, not applicable.

* Force on top not reliable, top into stop. 


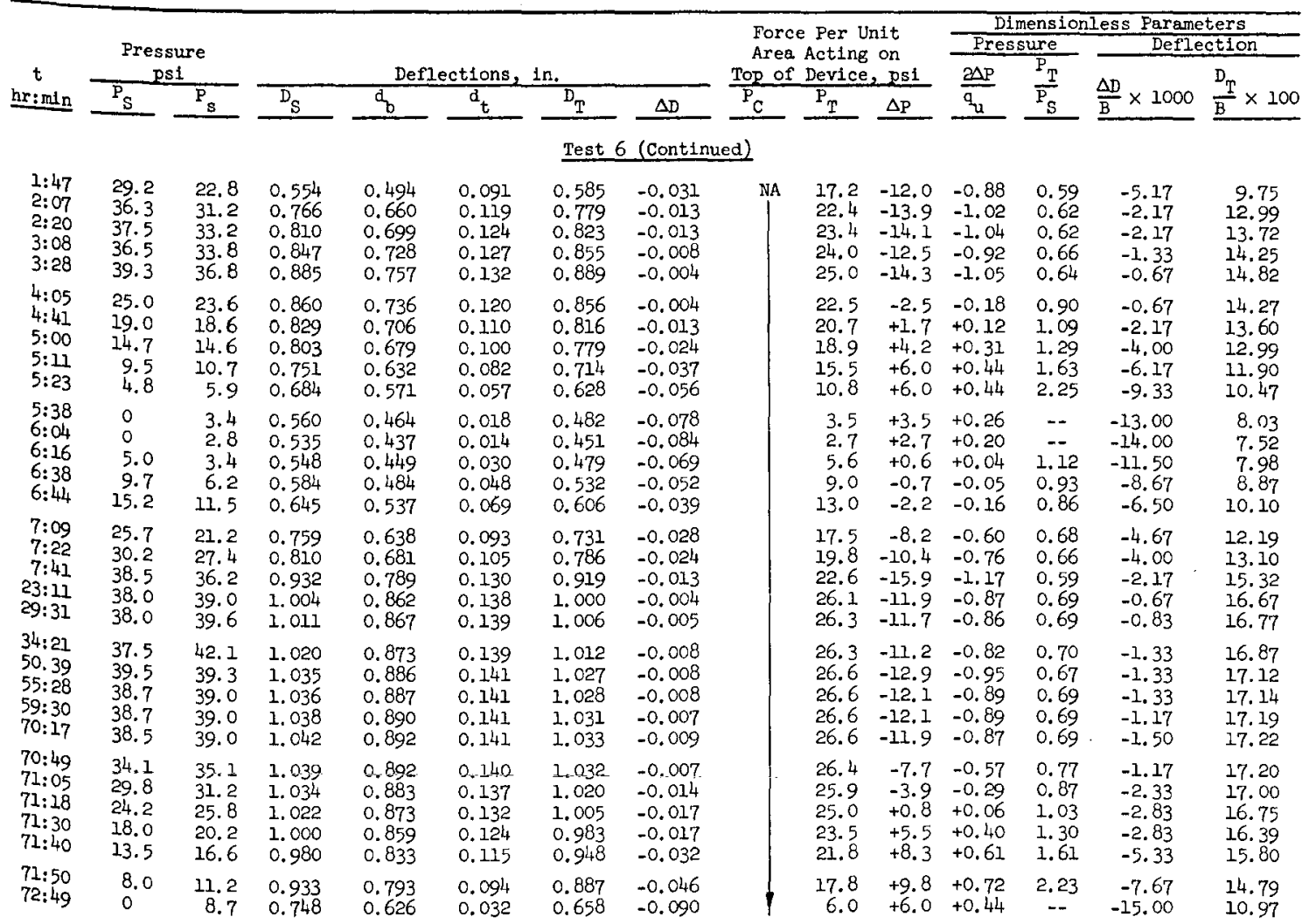

$\begin{array}{lccccc}0: 18 & 5.5 & 5.2 & 0.016 & 0.019 * * & 0.005 \\ 0: 29 & 10.0 & 10.7 & 0.063 & 0.069 * * & 0.011 \\ 0: 39 & 15.0 & 16.5 & 0.150 & 0.160 * * & 0.016 \\ 0: 54 & 20.2 & 22.3 & 0.277 & 0.296 * * & 0.021 \\ 1: 08 & 24.9 & 28.2 & 0.411 & 0.430 * * & 0.025 \\ 1: 16 t & 29.7 & -- & -- & -- & -. \\ 1: 33 & 0 & 0 & 0.292 & 0.287 * * & -- \\ 0 & 0 & 0 & 0.275 & 0.262 * * & -- \\ 0: 22 & 10.0 & 12.1 & 0.302 & 0.296 * * & 0.008 \\ 0: 36 & 19.9 & 21.0 & 0.398 & 0.401 * * & 0.019 \\ 0: 57 & 30.0 & 36.1 & 0.579 & 0.584 * * & 0.029 \\ 1: 16 & 35.2 & 43.4 & 0.755 & 0.752 * * & 0.034 \\ 1: 37 & 37.5 & 46.2 & 0.857 & 0.845 * * & 0.036 \\ 2: 06 & 37.4 & 46.1 & 0.894 & 0.879 * * & 0.036 \\ 2: 21 & 29.8 & 37.1 & 0.884 & 0.873 * * & 0.031 \\ 2: 31 & 20.0 & 25.1 & 0.856 & 0.839 * * & 0.023 \\ 2: 42 & 10.1 & 1.0 & 0.789 & 0.763 * * & 0.011 \\ 2: 57 & 0 & 0 & 0.636 & 0.603 * * & 0 \\ 4: 06 & 0 & 0 & 0.605 & 0.566 * * & 0\end{array}$

Test 7

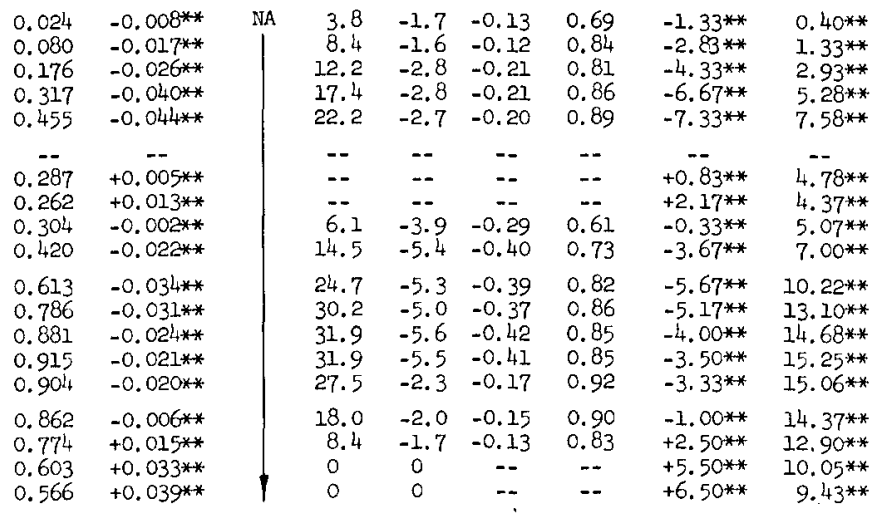

$\begin{array}{lccccccc}0: 08 & 5.0 & 1.7 & 0.031 & 0.029 & 0.004 & 0.033 & -0.002 \\ 0: 18 & 10.0 & 5.4 & 0.127 & 0.118 & 0.013 & 0.131 & -0.004 \\ 0: 43 & 20.0 & -- & 0.399 & 0.373 & 0.031 & 0.404 & -0.005 \\ 1: 30 & 30.0 & -- & 0.727 & 0.685 & 0.048 & 0.733 & -0.006 \\ 1: 58 & 40.0 & -. & 0.980 & 0.922 & 0.066 & 0.988 & -0.007 \\ 2: 09 & 45.0 & 50.9 & 1.086 & 1.020 & 0.074 & 1.094 & -0.008 \\ 2: 21 & 50.0 & 56.3 & 1.184 & 1.112 & 0.082 & 1.194 & -0.010 \\ 2: 32 & 55.0 & 61.4 & 1.263 & 1.184 & 0.090 & 1.274 & -0.011 \\ 2: 46 & 60.0 & 66.3 & 1.344 & 1.259 & 0.097 & 1.356 & -0.012 \\ 3: 04 & 65.0 & 72.0 & 1.408 & 1.324 & 0.106 & 1.430 & -0.022 \\ 3: 19 & 70.0 & 76.7 & 1.445 & 1.365 & 0.113 & 1.478 & -0.033 \\ 3: 34 & 75.0 & 79.9 & 1.480 & 1.403 & 0.120 & 1.523 & -0.043 \\ 3: 57 & 100.0 & 100.7 & 1.596 & 1.524 & 0.155 & 1.679 & -0.083 \\ 4: 22 & 125.0 & -- & 1.661 & 1.584 & 0.186 & 1.770 & -0.109 \\ 4: 48 & 150.0 & -- & 1.690 & 1.622 & 0.216 & 1.838 & -0.148\end{array}$

NA $\begin{array}{rrrrrr}2.0 & -3.0 & -0.03 & 0.40 & -0.33 & 0.55 \\ 7.0 & -3.0 & -0.3 & 0.70 & -0.67 & 2.18 \\ 16.0 & -4.0 & -0.4 & 0.80 & -0.83 & 6.73 \\ 25.0 & -5.0 & -0.4 & 0.83 & -1.00 & 12.22 \\ 34.0 & -6.0 & -0.5 & 0.85 & -1.17 & 16.47 \\ 39.0 & -6.0 & -0.5 & 0.87 & -1.33 & 18.24 \\ 43.0 & -7.0 & -0.6 & 0.86 & -1.67 & 19.90 \\ 47.0 & -8.0 & -0.7 & 0.85 & -1.83 & 21.23 \\ 51.0 & -9.0 & -0.8 & 0.85 & -2.00 & 22.60 \\ 55.0 & -10.0 & -0.9 & 0.85 & -3.67 & 23.84 \\ 59.0 & -11.0 & -1.0 & 0.84 & -5.50 & 24.64 \\ 63.0 & -12.0 & -1.1 & 0.84 & -7.17 & 25.39 \\ 81.0 & -19.0 & -1.6 & 0.81 & -13.84 & 27.99 \\ 97.0 & -28.0 & -2.5 & 0.78 & -18.17 & 29.51 \\ 13.0 & -37.0 & -3.2 & 0.75 & -24.67 & 30.64 \\ & & & & & \end{array}$

(Continued)

* Questionable values due to mal unction in one of base gages.

( 4 of 5 sheets) 
Table 5 (Concluded)

\begin{tabular}{|c|c|c|c|c|c|c|c|c|c|c|c|c|c|c|}
\hline \multirow{3}{*}{$\begin{array}{c}\mathrm{t} \\
\mathrm{hr}: \min \end{array}$} & \multirow{2}{*}{\multicolumn{2}{|c|}{$\begin{array}{c}\text { Pressure } \\
\text { psi }\end{array}$}} & \multirow{2}{*}{\multicolumn{5}{|c|}{ Deflections, in. }} & \multirow{2}{*}{\multicolumn{3}{|c|}{$\begin{array}{l}\text { Force Per Unit } \\
\text { Area Acting on } \\
\text { Top of Device, psi }\end{array}$}} & \multicolumn{4}{|c|}{ Dimensionless Parameters } \\
\hline & & & & & & & & & & & $2 \mathrm{AP}$ & $\mathrm{P}_{\mathrm{T}}$ & & \\
\hline & $\mathrm{P}_{\mathrm{S}}$ & $\mathrm{P}_{\mathrm{s}}$ & $\mathrm{D}_{\mathrm{S}}$ & $a_{b}$ & $a_{t}$ & $\mathrm{D}_{\mathrm{T}}$ & $\Delta \mathrm{D}$ & $\bar{P}_{C}$ & $\mathrm{P}_{\mathrm{T}}$ & $\Delta P$ & $q_{u}$ & $\overline{\mathrm{P}_{\mathrm{S}}}$ & $\frac{\mathrm{DD}}{\mathrm{B}} \times 1000$ & $\frac{T}{B} \times 100$ \\
\hline & & & & & & Test & at. & & & & & & & \\
\hline $\begin{array}{l}5: 18 \\
5: 41 \\
5: 58 \\
6: 54 \\
7: 18\end{array}$ & $\begin{array}{l}175.0 \\
200.0 \\
215.0 \\
216.0 \\
240.0\end{array}$ & & $\begin{array}{l}1.709 \\
1.722 \\
1.733 \\
1.737 \\
1.748\end{array}$ & $\begin{array}{l}1.627 \\
1.635 \\
1.640 \\
1.641 \\
1.644\end{array}$ & $\begin{array}{l}0.248 \\
0.280 \\
0.303 \\
0.317 \\
0.345\end{array}$ & $\begin{array}{l}1.875 \\
1.915 \\
1.943 \\
1.958 \\
1.989\end{array}$ & $\begin{array}{l}-0.166 \\
-0.193 \\
-0.210 \\
-0.221 \\
-0.241\end{array}$ & NA & $\begin{array}{l}129.0 \\
146.0 \\
158.0 \\
163.0 \\
182.0\end{array}$ & $\begin{array}{l}-54.0 \\
-54.0 \\
-57.0 \\
-53.0 \\
-58.0\end{array}$ & $\begin{array}{l}-4.9 \\
-4.9 \\
-5.1 \\
-4.8 \\
-5.2\end{array}$ & $\begin{array}{l}0.74 \\
0.73 \\
0.73 \\
0.75 \\
0.76\end{array}$ & $\begin{array}{l}-27.67 \\
-32.17 \\
-35.01 \\
-36.84 \\
-40.17\end{array}$ & $\begin{array}{l}31.26 \\
31.92 \\
32.39 \\
32.64 \\
33.16\end{array}$ \\
\hline $\begin{array}{l}22: 00 \\
22: 08 \\
22: 23 \\
22: 36 \\
22: 45\end{array}$ & $\begin{array}{l}240.0 \\
235.0 \\
230.0 \\
225.0 \\
220.0\end{array}$ & $\begin{array}{l}\overrightarrow{\mid} \\
\vec{a} \\
\mathscr{a}_{1} \\
0_{1}\end{array}$ & $\begin{array}{l}1.766 \\
1.764 \\
1.764 \\
1.764 \\
1.767\end{array}$ & $\begin{array}{l}1.674 \\
1.671 \\
1.671 \\
1.670 \\
1.673\end{array}$ & $\begin{array}{l}0.362 \\
0.361 \\
0.361 \\
0.360 \\
0.359\end{array}$ & $\begin{array}{l}2.036 \\
2.032 \\
2.032 \\
2.030 \\
2.032\end{array}$ & $\begin{array}{l}-0.270 \\
-0.268 \\
-0.268 \\
-0.266 \\
-0.265\end{array}$ & & $\begin{array}{l}205.0 \\
204.0 \\
204.0 \\
202.0 \\
201\end{array}$ & $\begin{array}{l}-35.0 \\
-31.0 \\
-26.0 \\
-23.0 \\
-19.0\end{array}$ & $\begin{array}{l}-3.2 \\
-2.8 \\
-2.3 \\
-2.1 \\
-1.7\end{array}$ & $\begin{array}{l}0.85 \\
0.87 \\
0.89 \\
0.90 \\
0.91\end{array}$ & $\begin{array}{l}-45.01 \\
-44.68 \\
-44.68 \\
-44.34 \\
-44.18\end{array}$ & $\begin{array}{l}33.94 \\
33.87 \\
33.87 \\
33.84 \\
33.87\end{array}$ \\
\hline $\begin{array}{l}22: 58 \\
23: 13 \\
23: 26 \\
23: 40 \\
23: 58\end{array}$ & $\begin{array}{l}215.0 \\
210.0 \\
205.0 \\
200.0 \\
195.0\end{array}$ & 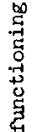 & $\begin{array}{l}1.767 \\
1.766 \\
1.766 \\
1.764 \\
1.764\end{array}$ & $\begin{array}{l}1.673 \\
1.672 \\
1.672 \\
1.672 \\
1.671\end{array}$ & $\begin{array}{l}0.356 \\
0.354 \\
0.352 \\
0.350 \\
0.347\end{array}$ & $\begin{array}{l}2.029 \\
2.026 \\
2.024 \\
2.022 \\
2.018\end{array}$ & $\begin{array}{l}-0.262 \\
-0.260 \\
-0.258 \\
-0.258 \\
-0.254\end{array}$ & & $\begin{array}{l}195.0 \\
191.0 \\
189.0 \\
186.0 \\
183.0\end{array}$ & $\begin{array}{l}-20.0 \\
-19.0 \\
-16.0 \\
-14.0 \\
-12.0\end{array}$ & $\begin{array}{l}-1.8 \\
-1.7 \\
-1.4 \\
-1.3 \\
-1.1\end{array}$ & $\begin{array}{l}0.91 \\
0.91 \\
0.92 \\
0.93 \\
0.94\end{array}$ & $\begin{array}{l}-43.68 \\
-43.34 \\
-43.01 \\
-43.01 \\
-42.34\end{array}$ & $\begin{array}{l}33.82 \\
33.77 \\
33.74 \\
33.71 \\
33.64\end{array}$ \\
\hline $\begin{array}{l}24: 06 \\
24: 27 \\
24: 39 \\
24: 56 \\
25: 11\end{array}$ & $\begin{array}{r}190.0 \\
165.0 \\
140.0 \\
115.0 \\
90.0\end{array}$ & 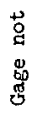 & $\begin{array}{l}1.764 \\
1.765 \\
1.764 \\
1.764 \\
1.767\end{array}$ & $\begin{array}{l}1.671 \\
1.671 \\
1.672 \\
1.672 \\
1.673\end{array}$ & $\begin{array}{l}0.344 \\
0.328 \\
0.305 \\
0.274 \\
0.243\end{array}$ & $\begin{array}{l}2.015 \\
1.999 \\
1.977 \\
1.946 \\
1.916\end{array}$ & $\begin{array}{l}-0.251 \\
-0.234 \\
-0.213 \\
-0.182 \\
-0.149\end{array}$ & & $\begin{array}{l}181.0 \\
171.0 \\
159.0 \\
143.0 \\
127.0\end{array}$ & $\begin{array}{r}-9.0 \\
+6.0 \\
+19.0 \\
+28.0 \\
+37.0\end{array}$ & $\begin{array}{l}-0.8 \\
+0.5 \\
+1.7 \\
+2.5 \\
+3.3\end{array}$ & $\begin{array}{l}0.95 \\
1.04 \\
1.14 \\
1.24 \\
1.41\end{array}$ & $\begin{array}{l}-41.84 \\
-39.01 \\
-35.51 \\
-30.34 \\
-24.84\end{array}$ & $\begin{array}{l}33.59 \\
33.32 \\
32.96 \\
32.44 \\
31.94\end{array}$ \\
\hline $\begin{array}{l}25: 28 \\
25: 48 \\
26: 03 \\
26: 34 \\
27: 03\end{array}$ & $\begin{array}{c}65.0 \\
40.0 \\
15.0 \\
0 \\
0\end{array}$ & & $\begin{array}{l}1.767 \\
1.766 \\
1.752 \\
1.034 \\
0.973\end{array}$ & $\begin{array}{l}1.674 \\
1.645 \\
1.513 \\
0.984 \\
0.943\end{array}$ & $\begin{array}{l}0.202 \\
0.145 \\
0.075 \\
0.008 \\
0.005\end{array}$ & $\begin{array}{l}1.876 \\
1.790 \\
1.588 \\
0.992 \\
0.948\end{array}$ & $\begin{array}{l}-0.109 \\
-0.024 \\
+0.164 \\
+0.042 \\
+0.025\end{array}$ & 1 & $\begin{array}{r}105.0 \\
76.0 \\
39.0 \\
4.0 \\
3.0\end{array}$ & $\begin{array}{r}+40.0 \\
+36.0 \\
+24.0 \\
+4.0 \\
+3.0\end{array}$ & $\begin{array}{l}+3.6 \\
+3.2 \\
+2.2 \\
+0.4 \\
+0.3\end{array}$ & $\begin{array}{c}1.62 \\
1.90 \\
2.60 \\
-- \\
--\end{array}$ & $\begin{array}{r}-18.17 \\
-4.00 \\
+27.33 \\
+7.00 \\
+4.17\end{array}$ & $\begin{array}{l}31.27 \\
29.84 \\
26.47 \\
16.54 \\
15.80\end{array}$ \\
\hline
\end{tabular}


Table 6

Surmary of Dynamic Arching Data

\begin{tabular}{|c|c|c|c|c|c|c|c|c|c|c|c|c|c|c|c|c|}
\hline \multirow[b]{3}{*}{$\begin{array}{c}t \\
\text { msec }\end{array}$} & \multirow{2}{*}{\multicolumn{2}{|c|}{$\begin{array}{c}\text { Pressure } \\
\text { psi }\end{array}$}} & \multirow{2}{*}{\multicolumn{5}{|c|}{ Deflections, in. }} & \multirow{2}{*}{\multicolumn{5}{|c|}{$\begin{array}{l}\text { Force per Unit Area } \\
\text { Auting on Top of Device, psi }\end{array}$}} & \multicolumn{4}{|c|}{ Dimensionless Parameters } \\
\hline & & & & & & & & & & & & & \multicolumn{2}{|c|}{ Pressure } & \multicolumn{2}{|c|}{ Deflection } \\
\hline & $\mathrm{P}_{\mathrm{S}}$ & $\overline{\mathrm{P}}_{\mathrm{s}}$ & $\mathrm{D}_{\mathrm{S}}$ & Def & $\frac{\text { ections }}{d_{t}}$ & $\frac{\mathrm{in} .}{\mathrm{D}}$ & $\Delta D$ & $\frac{\text { Ant }}{\text { Dampling }}$ & $\begin{array}{l}\text { ing on } \mathrm{T} \\
\text { Inertia }\end{array}$ & $\begin{array}{l}\mathrm{p} \text { of Dey } \\
\text { Spring }\end{array}$ & $\frac{c e, p s}{\mathrm{P}_{\mathrm{T}}}$ & $\Delta P$ & $\frac{2 \Delta \vec{p}}{q_{\mu}}$ & $\begin{array}{r}\frac{P_{T}}{P_{S}} \\
\end{array}$ & $\frac{\Delta D}{B} \times 1000$ & $\frac{\mathrm{D}_{\mathrm{T}}}{\mathrm{B}} \times 100$ \\
\hline \multicolumn{17}{|c|}{ Test 11} \\
\hline $\begin{array}{c}\text { nitial } \\
2 \\
3 \\
4 \\
5\end{array}$ & $\begin{array}{c}0 \\
25.0 \\
34.0 \\
36.1 \\
37.0\end{array}$ & $\begin{array}{c}3.5 \\
0 \\
2.4 \\
7.0 \\
12.7\end{array}$ & $\begin{array}{l}0.011 \\
0 \\
0 \\
0.008 \\
0.016\end{array}$ & $\begin{array}{l}0.007 \\
0 \\
0 \\
0 \\
0.006\end{array}$ & $\begin{array}{l}0.013 \\
0 \\
0 \\
0.009 \\
0.013\end{array}$ & $\begin{array}{l}0.020 \\
0 \\
0 \\
0.009 \\
0.019\end{array}$ & $\begin{array}{l}-0.009 \\
0 \\
0 \\
-0.001 \\
-0.003\end{array}$ & $\begin{array}{l}0 \\
0 \\
+0.1 \\
+0.3 \\
+0.8\end{array}$ & $\begin{array}{c}0 \\
0 \\
+1.3 \\
+1.9 \\
+3.8\end{array}$ & $\begin{array}{l}1.3 \\
0 \\
0 \\
3.7 \\
5.3\end{array}$ & $\begin{array}{l}1.30 \\
0 \\
1.40 \\
5.90 \\
9.90\end{array}$ & $\begin{array}{l}-2.2 \\
-25.0 \\
-32.5 \\
-30.0 \\
-27.0\end{array}$ & $\begin{array}{l}-0.14 \\
-1.61 \\
-2.09 \\
-1.94 \\
-2.74\end{array}$ & $\begin{array}{c}-- \\
0.04_{4} \\
0.16 \\
0.27\end{array}$ & $\begin{array}{l}-1.50 \\
0 \\
0 \\
-0.17 \\
-0.50\end{array}$ & $\begin{array}{l}0.33 \\
0 \\
0 \\
0.15 \\
0.32\end{array}$ \\
\hline $\begin{array}{l}6 \\
7 \\
8.5 \\
10 \\
12\end{array}$ & $\begin{array}{l}36.8 \\
36.6 \\
36.3 \\
36.2 \\
36.0\end{array}$ & $\begin{array}{l}18.2 \\
21.2 \\
25.2 \\
29.2 \\
30.4\end{array}$ & $\begin{array}{l}0.028 \\
0.048 \\
0.088 \\
0.117 \\
0.1 .53\end{array}$ & $\begin{array}{l}0.012 \\
0.031 \\
0.080 \\
0.120 \\
0.155\end{array}$ & $\begin{array}{l}0.023 \\
0.030 \\
0.048 \\
0.061 \\
0.066\end{array}$ & $\begin{array}{l}0.035 \\
0.061 \\
0.128 \\
0.181 \\
0.221\end{array}$ & $\begin{array}{l}-0.007 \\
-0.023 \\
-0.040 \\
-0.064 \\
-0.068\end{array}$ & $\begin{array}{l}+1.3 \\
+1.3 \\
+0.9 \\
+0.6 \\
+0.3\end{array}$ & $\begin{array}{c}+3.8 \\
+2.3 \\
0 \\
-0.8 \\
-1.9\end{array}$ & $\begin{array}{r}9.4 \\
12.2 \\
19.5 \\
24.8 \\
26.9\end{array}$ & $\begin{array}{l}14.50 \\
15.80 \\
20.40 \\
24.60 \\
25.30\end{array}$ & $\begin{array}{l}-22.5 \\
-21.0 \\
-16.0 \\
-11.5 \\
-10.5\end{array}$ & $\begin{array}{l}-1.45 \\
-1.35 \\
-1.02 \\
-0.74 \\
-0.68\end{array}$ & $\begin{array}{l}0.39 \\
0.43 \\
0.56 \\
0.68 \\
0.70\end{array}$ & $\begin{array}{r}-1.17 \\
-2.17 \\
-6.67 \\
-10.67 \\
-11.33\end{array}$ & $\begin{array}{l}0.58 \\
1.02 \\
2.14 \\
3.02 \\
3.68\end{array}$ \\
\hline $\begin{array}{l}13 \\
15^{*} \\
17^{*} \\
20 * \\
50 * *\end{array}$ & $\begin{array}{l}36.0 \\
35.5 \\
35.0 \\
35.0 \\
34.0\end{array}$ & $\begin{array}{l}29.8 \\
29.2 \\
27.9 \\
24.8 \\
21.0\end{array}$ & $\begin{array}{l}0.173 \\
0.197 \\
0.210 \\
0.218 \\
0.234\end{array}$ & $\begin{array}{l}0.175 \\
0.212 \\
0.219 \\
0.225 \\
0.237\end{array}$ & $\begin{array}{l}0.068 \\
0.068 \\
0.066 \\
0.064 \\
0.059\end{array}$ & $\begin{array}{l}0.243 \\
0.280 \\
0.285 \\
0.289 \\
0.296\end{array}$ & $\begin{array}{l}-0.070 \\
-0.083 \\
-0.075 \\
-0.071 \\
-0.062\end{array}$ & $\begin{array}{c}+0.20 \\
0 \\
+0.10 \\
0 \\
0\end{array}$ & $\begin{array}{l}-1.6 \\
-1.5 \\
-0.8 \\
0 \\
0\end{array}$ & $\begin{array}{l}27.7 \\
27.7 \\
26.9 \\
26.0 \\
24.0\end{array}$ & $\begin{array}{l}26.30 \\
26.20 \\
26.20 \\
26.00 \\
24.00\end{array}$ & $\begin{array}{r}-9.5 \\
-9.5 \\
-9.0 \\
-9.0 \\
-10.0\end{array}$ & $\begin{array}{l}-0.62 \\
-0.61 \\
-0.58 \\
-0.58 \\
-0.64\end{array}$ & $\begin{array}{l}0.73 \\
0.74 \\
0.75 \\
0.74 \\
0.71\end{array}$ & $\begin{array}{l}-11.67 \\
-13.84 \\
-12.50 \\
-11.84 \\
-10.35\end{array}$ & $\begin{array}{l}4.05 \\
4.67 \\
4.75 \\
4.82 \\
4.93\end{array}$ \\
\hline $\begin{array}{l}100 * * \\
500 * *\end{array}$ & $\begin{array}{r}28.0 \\
8.6\end{array}$ & 20.0 & $\begin{array}{l}0.234 \\
0.160\end{array}$ & $\begin{array}{l}0.237 \\
0.164\end{array}$ & $\begin{array}{l}0.058 \\
0.018\end{array}$ & $\begin{array}{l}0.295 \\
0.182\end{array}$ & $\begin{array}{l}-0.061 \\
-0.022\end{array}$ & $\begin{array}{l}0 \\
0\end{array}$ & $\stackrel{0}{0}$ & $\begin{array}{r}23.6 \\
7 \cdot 3\end{array}$ & $\begin{array}{r}23.60 \\
7.30\end{array}$ & $\begin{array}{l}-4.5 \\
-1.3\end{array}$ & $\begin{array}{l}-0.29 \\
-0.08\end{array}$ & $\begin{array}{l}0.84 \\
0.85\end{array}$ & $\begin{array}{r}-10.16 \\
-5.67\end{array}$ & $\begin{array}{l}4.92 \\
3.23\end{array}$ \\
\hline
\end{tabular}

$\begin{array}{cccccccc}\text { Initial } & 0 & 1.1 & 0.089 & 0.050 & 0.004 & 0.054 & +0.035 \\ 3 & 66.0 & 7.0 & 0 & 0 & 0 & 0 & 0 \\ 4 & 70.0 & 14.0 & 0.002 & 0 & 0.008 & 0.008 & -0.006 \\ 5 & 70.0 & 32.0 & 0.035 & 0.015 & 0.029 & 0.044 & -0.009 \\ 6 & 69.0 & 47.0 & 0.082 & 0.025 & 0.070 & 0.095 & -0.013 \\ 7 & 69.0 & 57.0 & 0.140 & 0.047 & 0.110 & 0.157 & -0.017 \\ 10 & 69.0 & 62.0 & 0.373 & 0.295 & 0.139 & 0.434 & -0.061 \\ 11 & 69.0 & 63.0 & 0.443 & 0.392 & 0.145 & 0.537 & -0.094 \\ 12 & 68.0 & 65.0 & 0.520 & 0.480 & 0.151 & 0.631 & -0.119 \\ 12 & 68.0 & 67.0 & 0.594 & 0.579 & 0.154 & 0.733 & -0.139 \\ 14 & 68.0 & 67.0 & 0.650 & 0.670 & 0.154 & 0.824 & -0.174 \\ 15 & 67.0 & 67.0 & 0.700 & 0.770 & 0.153 & 0.923 & -0.223 \\ 16 & 67.0 & -- & -- & -- & 0.152 & -- & -- \\ 18 * & 66.0 & 69.0 & 0.870 & 0.960 & 0.153 & 1.113 & -0.243 \\ 24 * & 66.0 & 77.0 & 1.050 & 1.140 & 0.161 & 1.301 & -0.251 \\ 50 * * & 59.0 & 57.0 & 1.040 & 1.130 & 0.148 & 1.278 & -0.238 \\ 100 * * & 44.0 & 52.0 & 1.060 & 1.130 & 0.144 & 1.274 & -0.214 \\ 500 * * & 0 & 18.0 & 1.025 & 1.071 & 0.096 & 1.167 & -0.142 \\ \text { F1na1 } & 0 & 11.0 & 0.627 & 0.603 & 0.002 & 0.605 & +0.022\end{array}$

Test 12

\begin{tabular}{|c|c|c|c|c|c|c|c|c|}
\hline 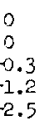 & $\begin{array}{r}0 \\
0 \\
4.0 \\
+20.2 \\
+9.1\end{array}$ & $\begin{array}{c}1.5 \\
0 \\
3.0 \\
11.0 \\
26.6\end{array}$ & $\begin{array}{c}1.5 \\
0 \\
7.3 \\
32.4 \\
38.2\end{array}$ & $\begin{array}{r}+0.5 \\
-66.0 \\
-62.5 \\
-37.5 \\
-31.0\end{array}$ & $\begin{array}{l}+0.03 \\
-4.53 \\
-4.29 \\
-2.57 \\
-2.13\end{array}$ & $\begin{array}{l}1.36 \\
-- \\
0.10 \\
0.46 \\
0.55\end{array}$ & $\begin{array}{l}+5.83 \\
0 \\
-1.00 \\
-1.50 \\
-2.17\end{array}$ & $\begin{array}{l}0.90 \\
0 \\
0.13 \\
0.73 \\
1.58\end{array}$ \\
\hline & $\begin{array}{c}+2.6 \\
+3.0 \\
0 \\
-1.1 \\
=2 .-3 . \\
-2.4 \\
-3.6 \\
-3.6 \\
-3.6 \\
-3.0\end{array}$ & $\begin{array}{l}41.8 \\
52.8 \\
55.1 \\
57.4 \\
58.5 \\
58.5 \\
58.1 \\
57.8 \\
58.1 \\
61.2\end{array}$ & $\begin{array}{l}46.2 \\
55.6 \\
55.7 \\
56.9 \\
56.5 \\
56.7 \\
54.4 \\
54.2 \\
54.2 \\
58.2\end{array}$ & $\begin{array}{l}-23.0 \\
-13.5 \\
-1.3 .5 \\
-11.0 \\
-11.5 \\
-11.5 \\
-12.5 \\
-13.0 \\
-12.0 \\
-8.0\end{array}$ & $\begin{array}{l}-1.58 \\
-0.93 \\
-0.93 \\
-0.75 \\
-0.79 \\
-0.79 \\
-0.86 \\
-0.89 \\
-0.82 \\
-0.55\end{array}$ & $\begin{array}{l}0.67 \\
0.81 \\
0.81 \\
0.834 \\
0.84 \\
0.83 \\
0.81 \\
0.81 \\
0.82 \\
0.88\end{array}$ & $\begin{array}{c}-2.83 \\
-10.17 \\
-15.67 \\
-19.67 \\
-23.17 \\
-29.00 \\
-37.17 \\
-- \\
-40.50 \\
-42.80\end{array}$ & $\begin{array}{r}2.62 \\
7.23 \\
8.95 \\
10.52 \\
12.22 \\
13.73 \\
15.39 \\
1 \overline{18.55} \\
21.68\end{array}$ \\
\hline & $\begin{array}{l}0 \\
0 \\
0 \\
0\end{array}$ & $\begin{array}{r}56.2 \\
54.7 \\
36.5 \\
0.8\end{array}$ & $\begin{array}{r}56.2 \\
54.7 \\
36.5 \\
0.8\end{array}$ & $\begin{array}{r}-3.0 \\
+10.5 \\
+36.5 \\
+1.0\end{array}$ & $\begin{array}{l}-0.21 \\
+0.72 \\
+2.50 \\
+0.07\end{array}$ & $\begin{array}{l}0.95 \\
1.24 \\
=- \\
=-\end{array}$ & $\begin{array}{r}-39.70 \\
-35.67 \\
-23.67 \\
+3.67\end{array}$ & $\begin{array}{l}21.30 \\
21.23 \\
19.45 \\
10.08\end{array}$ \\
\hline \multicolumn{9}{|c|}{13} \\
\hline & $\begin{array}{r}+1.8 \\
+1.8 \\
+19.0 \\
+39.5 \\
+0.5\end{array}$ & $\begin{array}{r}1.3 \\
2.8 \\
17.7 \\
25.5 \\
67.5\end{array}$ & $\begin{array}{r}3.10 \\
5.00 \\
39.60 \\
72.20 \\
82.90\end{array}$ & $\begin{array}{r}-137.0 \\
-146.0 \\
-109.0 \\
-77.0 \\
-66.0\end{array}$ & $\begin{array}{r}-10.72 \\
-11.42 \\
-8.56 \\
-6.01 \\
-5.17\end{array}$ & $\begin{array}{l}0.02 \\
0.03 \\
0.27 \\
0.48 \\
0.56\end{array}$ & $\begin{array}{l}-0.50 \\
-1.00 \\
-0.83 \\
-0.83 \\
-1.50\end{array}$ & $\begin{array}{l}0.05 \\
0.10 \\
0.68 \\
1.30 \\
4.30\end{array}$ \\
\hline & $\begin{array}{l}+7.1 \\
+6.5 \\
+1.4 \\
-0.7 \\
-2.8\end{array}$ & $\begin{array}{r}81.8 \\
87.2 \\
93.5 \\
104.3 \\
109.0\end{array}$ & $\begin{array}{r}92.90 \\
96.00 \\
96.20 \\
105.70 \\
106.00\end{array}$ & $\begin{array}{l}-54.0 \\
-49.0 \\
-45.0 \\
-32.3 \\
-32.0\end{array}$ & $\begin{array}{l}-4.23 \\
-3.83 \\
-3.51 \\
-2.53 \\
-2.53\end{array}$ & $\begin{array}{l}0.63 \\
0.66 \\
0.68 \\
0.77 \\
0.77\end{array}$ & $\begin{array}{r}-3.33 \\
-4.33 \\
-8.17 \\
-19.20 \\
-30.00\end{array}$ & $\begin{array}{r}7.00 \\
9.80 \\
16.30 \\
23.70 \\
28.20\end{array}$ \\
\hline & $\begin{array}{c}-5.0 \\
-7.0 \\
0 \\
0 \\
0\end{array}$ & $\begin{array}{r}106.3 \\
107.2 \\
105.7 \\
100.9 \\
33.0\end{array}$ & $\begin{array}{r}101.20 \\
101.50 \\
105.70 \\
100.90 \\
33.00\end{array}$ & $\begin{array}{r}-35.8 \\
-35.5 \\
-15.3 \\
-8.1 \\
+33.0\end{array}$ & $\begin{array}{l}-2.80 \\
-2.78 \\
-1.20 \\
-0.63 \\
+2.58\end{array}$ & $\begin{array}{l}0.74 \\
0.74 \\
0.87 \\
0.93 \\
-.\end{array}$ & $\begin{array}{l}-43.30 \\
-50.00 \\
-55.00 \\
-60.00 \\
-26.70\end{array}$ & $\begin{array}{l}32.20 \\
37.70 \\
38.20 \\
37.80 \\
31.20\end{array}$ \\
\hline
\end{tabular}

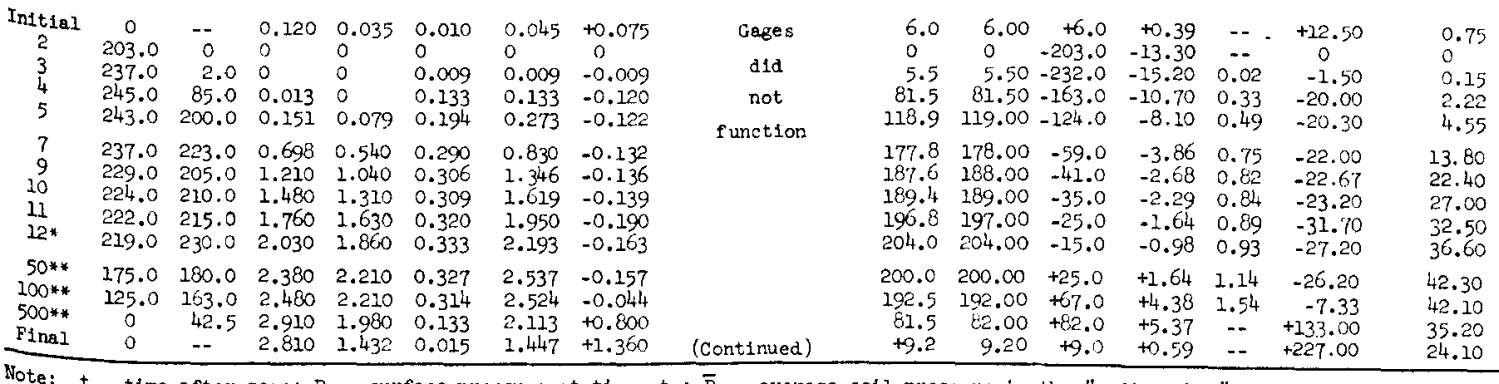

Test 14

$\begin{array}{lrrllllll}2 & 140.0 & 2.5 & 0 & 0 & 0.003 & +0.003 & -0.003 \\ 3 & 151.0 & 8.0 & 0 & 0 & 0.006 & +0.006 & -0.006 \\ 4 & 149.0 & 30.0 & 0.036 & 0 & 0.041 & 0.041 & -0.005 & + \\ 4.5 & 149.0 & 57.0 & 0.075 & 0.020 & 0.060 & 0.080 & -0.005 & + \\ 6 & 149.0 & 112.0 & 0.250 & 0.102 & 0.157 & 0.259 & -0.009 & + \\ 7 & 147.0 & 132.0 & 0.402 & 0.232 & 0.190 & 0.422 & -0.020 & + \\ 8 & 145.0 & 147.0 & 0.564 & 0.387 & 0.203 & 0.590 & -0.026 & + \\ 10 & 141.0 & 143.0 & 0.929 & 0.760 & 0.218 & 0.978 & -0.049 & +1 \\ 12.5 & 138.0 & 150.0 & 1.305 & 1.180 & 0.243 & 1.420 & -0.115 & +2 \\ 13.5 & 138.0 & 146.0 & 1.510 & 1.440 & 0.254 & 1.590 & -0.180 & -0 \\ 15.0 * & 137.0 & 137.0 & 1.670 & 1.680 & 0.248 & 1.930 & -0.260 & - \\ 17.0 * & 137.0 & 145.0 & 1.260 & 2.010 & 0.250 & 2.260 & -0.300 & +1 \\ 50.0 * * & 121.0 & 110.0 & 1.960 & 2.040 & 0.248 & 2.290 & -0.330 & \\ 100 * * & 109.0 & 95.0 & 1.910 & 2.030 & 0.237 & 2.270 & -0.360 & 0 \\ 100 * * & 0 & 23.5 & 1.710 & 1.790 & 0.078 & 1.870 & -0.160\end{array}$

Note: $t$, time after zero; $P_{S}$, surface pressure at time $t ; \bar{P}_{s}$, average soll pressure in the "undisturbed" region at the $35-i n$.

0.90

13

.58

2.62
7.23

8.95

12.22

3.73
5.39

$-5$

1.68

10.08

.05

.10

.30

.00

9.80

3.70

2.20

7.70

37.80

31.20 of test device; $D_{T}$, total deflection of test device $\left(d_{b}+d_{t}\right) ; \infty$, differential deflection $\left(D_{T}-D_{S}\right) ; P_{T}$, average force acting on top of test device; $\angle P$, differentlal pressure $\left(P_{T}-P_{S}\right) ; q_{q}$, unconfined compressive strength of soll; $B$, diameter of test device.

* Results measurably affected by reflections.

* Affected by reflections and/or reduced surface pressure. 


\begin{tabular}{|c|c|c|c|c|c|c|c|c|c|c|c|c|c|c|c|c|}
\hline \multirow[b]{3}{*}{$\begin{array}{c}t \\
\text { msec } \\
\end{array}$} & \multirow{2}{*}{\multicolumn{2}{|c|}{$\begin{array}{c}\text { Pressure } \\
\text { psi }\end{array}$}} & & \multirow{2}{*}{\multicolumn{5}{|c|}{$\begin{array}{r}\text { Force per Unit Area } \\
\text { Acting on Top of Device, psi }\end{array}$}} & \multicolumn{4}{|c|}{ Dimensionless Paraneters } \\
\hline & & & & & & & & & & & & & \multicolumn{2}{|c|}{ Pressure } & \multicolumn{2}{|c|}{ Deflection } \\
\hline & $\mathrm{P}_{\mathrm{S}}$ & $\overline{\mathrm{I}}_{\mathrm{S}}$ & $\mathrm{D}_{\mathrm{S}}$ & $d_{b}$ & $\frac{\operatorname{ect} 3.0 n s}{d_{t}}$ & $\mathrm{D}_{\mathrm{T}}$ & $\Delta \mathrm{D}$ & Damping & Inertia & $p$ or Dev1 & $\frac{\mathrm{ce}, \mathrm{psi}}{\mathrm{P}_{\mathrm{T}}}$ & $\Delta^{P}$ & $\frac{2 \Delta P}{q_{u}}$ & $\frac{\mathrm{P}_{\mathrm{T}}}{\mathrm{P}_{\mathrm{S}}}$ & $\frac{\mathrm{DD}}{\mathrm{B}} \times 2000$ & $\frac{\mathrm{D}_{\mathrm{T}}}{\mathrm{B}} \times 100$ \\
\hline \multicolumn{17}{|c|}{ Test 15} \\
\hline $\begin{array}{c}\text { Initial } \\
1 \\
2 \\
4 \\
5\end{array}$ & $\begin{array}{c}0 \\
28.0 \\
31.0 \\
38.5 \\
39.0\end{array}$ & $\begin{array}{r}0.5 \\
17.0 \\
23.0 \\
36.0 \\
36.0\end{array}$ & $\begin{array}{l}0.060 \\
0.005 \\
0.030 \\
0.105 \\
0.149\end{array}$ & $\begin{array}{l}0.060 \\
0 \\
0.001 \\
0.042 \\
0.080\end{array}$ & $\begin{array}{l}0 \\
0.011 \\
0.036 \\
0.071 \\
0.081\end{array}$ & $\begin{array}{l}0.060 \\
0.011 \\
0.037 \\
0.113 \\
0.161\end{array}$ & $\begin{array}{l}0 \\
-0.006 \\
-0.007 \\
-0.008 \\
-0.072\end{array}$ & $\begin{array}{c}0 \\
+0.5 \\
+2.1 \\
+0.4 \\
-0.5\end{array}$ & $\begin{array}{r}0 \\
+13.3 \\
+4.0 \\
+0.7 \\
+0.7\end{array}$ & $\begin{array}{c}0 \\
4.4 \\
14.4 \\
28.5 \\
32.5\end{array}$ & $\begin{array}{c}0 \\
18.20 \\
20.50 \\
29.60 \\
32.70\end{array}$ & $\begin{array}{r}-0.5 \\
-10.0 \\
-10.5 \\
-9.0 \\
-6.5\end{array}$ & $\begin{array}{l}-0.05 \\
-0.92 \\
-0.97 \\
-0.83 \\
-0.59\end{array}$ & $\begin{array}{l}-. \\
0.65 \\
0.66 \\
0.77 \\
0.84\end{array}$ & $\begin{array}{l}0 \\
-1.00 \\
-1.17 \\
-1.33 \\
-2.00\end{array}$ & $\begin{array}{l}1.00 \\
0.18 \\
0.62 \\
1.88 \\
2.68\end{array}$ \\
\hline $\begin{array}{c}6 \\
8 \\
10 \\
12 \\
14 *\end{array}$ & $\begin{array}{l}39.0 \\
38.5 \\
38.5 \\
38.0 \\
37.5\end{array}$ & $\begin{array}{l}36.0 \\
36.0 \\
36.0 \\
34.0 \\
35.0\end{array}$ & $\begin{array}{l}0.207 \\
0.312 \\
0.416 \\
0.513 \\
0.602\end{array}$ & $\begin{array}{l}0.126 \\
0.233 \\
0.357 \\
0.458 \\
0.561\end{array}$ & $\begin{array}{l}0.083 \\
0.084 \\
0.085 \\
0.085 \\
0.085\end{array}$ & $\begin{array}{l}0.209 \\
0.317 \\
0.442 \\
0.543 \\
0.646\end{array}$ & $\begin{array}{l}-0.002 \\
-0.005 \\
-0.026 \\
-0.030 \\
-0.044\end{array}$ & $\begin{array}{l}-0.7 \\
-0.8 \\
-1.0 \\
-0.4 \\
+0.3\end{array}$ & $\begin{array}{l}+0.7 \\
0 \\
-0.4 \\
-1.7 \\
-2.5\end{array}$ & $\begin{array}{l}33.3 \\
33.7 \\
34.1 \\
34.1 \\
34.1\end{array}$ & $\begin{array}{l}33.30 \\
32.80 \\
32.70 \\
32.00 \\
31.90\end{array}$ & $\begin{array}{l}-5.7 \\
-5.6 \\
-5.8 \\
-6.0 \\
-5.5\end{array}$ & $\begin{array}{l}-0.53 \\
-0.52 \\
-0.54 \\
-0.55 \\
-0.51\end{array}$ & $\begin{array}{l}0.85 \\
0.85 \\
0.85 \\
0.84 \\
0.85\end{array}$ & $\begin{array}{l}-0.33 \\
-0.83 \\
-4.33 \\
-5.00 \\
-7.33\end{array}$ & $\begin{array}{r}3.48 \\
5.28 \\
7.37 \\
9.05 \\
10.77\end{array}$ \\
\hline $\begin{array}{l}16^{*} \\
18^{*} \\
20^{*} \\
22^{*} \\
50^{*}\end{array}$ & $\begin{array}{l}37.0 \\
37.0 \\
37.0 \\
36.5 \\
34.0\end{array}$ & $\begin{array}{l}35.0 \\
34.0 \\
33.0 \\
31.0 \\
29.0\end{array}$ & $\begin{array}{l}0.654 \\
0.684 \\
0.690 \\
0.680 \\
0.669\end{array}$ & $\begin{array}{l}0.633 \\
0.670 \\
0.684 \\
0.670 \\
0.641\end{array}$ & $\begin{array}{l}0.085 \\
0.086 \\
0.084 \\
0.082 \\
0.079\end{array}$ & $\begin{array}{l}0.718 \\
0.756 \\
0.768 \\
0.752 \\
0.720\end{array}$ & $\begin{array}{l}-0.064 \\
-0.072 \\
-0.078 \\
-0.072 \\
-0.051\end{array}$ & $\begin{array}{c}+0.2 \\
+0.4 \\
0 \\
-1.2 \\
0\end{array}$ & $\begin{array}{l}-2.8 \\
-2.4 \\
-1.7 \\
-0.6 \\
0\end{array}$ & $\begin{array}{l}34.1 \\
34.5 \\
33.7 \\
32.9 \\
31.7\end{array}$ & $\begin{array}{l}31.50 \\
32.50 \\
32.00 \\
31.20 \\
31.70\end{array}$ & $\begin{array}{l}-5.5 \\
-4.5 \\
-5.0 \\
-5.5 \\
-2.3\end{array}$ & $\begin{array}{l}-0.51 \\
-0.42 \\
-0.46 \\
-0.51 \\
-0.21\end{array}$ & $\begin{array}{l}0.85 \\
0.88 \\
0.86 \\
0.85 \\
0.93\end{array}$ & $\begin{array}{r}-10.67 \\
-12.00 \\
-13.00 \\
-12.00 \\
-8.50\end{array}$ & $\begin{array}{l}11.97 \\
12.60 \\
12.80 \\
12.53 \\
12.00\end{array}$ \\
\hline $\begin{array}{l}100^{* *} \\
500^{* *}\end{array}$ & $\begin{array}{c}31.5 \\
0\end{array}$ & $\begin{array}{r}24.0 \\
3.2\end{array}$ & $\begin{array}{l}0.654 \\
0.379\end{array}$ & $\begin{array}{l}0.573 \\
0.357\end{array}$ & $\begin{array}{l}0.073 \\
0.008\end{array}$ & $\begin{array}{l}0.646 \\
0.365\end{array}$ & $\begin{array}{l}+0.008 \\
+0.014\end{array}$ & $\begin{array}{l}0 \\
0\end{array}$ & $\begin{array}{l}0 \\
0\end{array}$ & $\begin{array}{r}29.3 \\
3.2\end{array}$ & $\begin{array}{r}29.30 \\
3.20\end{array}$ & $\begin{array}{l}-2.2 \\
+3.2\end{array}$ & $\begin{array}{l}-0.20 \\
+0.30\end{array}$ & $\begin{array}{c}0.93 \\
\ldots\end{array}$ & $\begin{array}{l}+1.30 \\
+2.30\end{array}$ & $\begin{array}{r}10.77 \\
6.08\end{array}$ \\
\hline \multicolumn{17}{|c|}{ Test 16} \\
\hline $\begin{array}{c}\text { Initial } \\
3 \\
4 \\
5 \\
6\end{array}$ & $\begin{array}{c}0 \\
33.5 \\
37.0 \\
37.5 \\
37.3\end{array}$ & $\begin{array}{r}2.8 \\
20.5 \\
23.0 \\
28.0 \\
28.0\end{array}$ & $\begin{array}{l}0.037 \\
0.017 \\
0.046 \\
0.064 \\
0.096\end{array}$ & $\begin{array}{l}0.075 \\
0 \\
0.029 \\
0.033 \\
0.067\end{array}$ & $\begin{array}{l}0.001 \\
0.022 \\
0.030 \\
0.048 \\
0.058\end{array}$ & $\begin{array}{l}0.076 \\
0.012 \\
0.049 \\
0.081 \\
0.125\end{array}$ & $\begin{array}{l}-0.039 \\
-0.001 \\
-0.003 \\
-0.017 \\
-0.029\end{array}$ & $\begin{array}{c}0 \\
+1.2 \\
+1.5 \\
+0.9 \\
+0.5\end{array}$ & $\begin{array}{c}0 \\
+4.8 \\
0 \\
0 \\
0\end{array}$ & $\begin{array}{r}0.4 \\
4.4 \\
12.0 \\
19.2 \\
23.3\end{array}$ & $\begin{array}{r}0.40 \\
10.40 \\
13.50 \\
20.10 \\
23.80\end{array}$ & $\begin{array}{r}-2.4 \\
-23.0 \\
-23.5 \\
-17.5 \\
-13.5\end{array}$ & $\begin{array}{l}-0.15 \\
-1.41 \\
-1.44 \\
-1.08 \\
-0.83\end{array}$ & $\begin{array}{l}-.- \\
0.31 \\
0.36 \\
0.54 \\
0.64\end{array}$ & $\begin{array}{l}-6.5 \\
-0.17 \\
-0.50 \\
-2.83 \\
-4.83\end{array}$ & $\begin{array}{l}1.27 \\
0.20 \\
0.82 \\
1.35 \\
2.08\end{array}$ \\
\hline $\begin{array}{l}8 \\
10 \\
12 \\
14 \\
16 *\end{array}$ & $\begin{array}{l}37.2 \\
37.0 \\
37.0 \\
36.5 \\
36.5\end{array}$ & $\begin{array}{l}29.0 \\
30.0 \\
30.0 \\
32.0 \\
31.0\end{array}$ & $\begin{array}{l}0.147 \\
0.186 \\
0.231 \\
0.265 \\
0.279\end{array}$ & $\begin{array}{l}0.129 \\
0.190 \\
0.226 \\
0.250 \\
0.265\end{array}$ & $\begin{array}{l}0.064 \\
0.064 \\
0.065 \\
0.066 \\
0.066\end{array}$ & $\begin{array}{l}0.193 \\
0.254 \\
0.291 \\
0.316 \\
0.331\end{array}$ & $\begin{array}{l}-0.046 \\
-0.068 \\
-0.060 \\
-0.051 \\
-0.052\end{array}$ & $\begin{array}{l}+0.6 \\
+0.8 \\
+0.7 \\
+0.4 \\
+0.4\end{array}$ & $\begin{array}{l}-1.8 \\
-1.7 \\
-1.0 \\
-1.1 \\
-0.7\end{array}$ & $\begin{array}{l}25.7 \\
25.7 \\
26.1 \\
26.5 \\
26.5\end{array}$ & $\begin{array}{l}24.50 \\
24.80 \\
25.80 \\
25.80 \\
26.20\end{array}$ & $\begin{array}{l}-12.5 \\
-12.0 \\
-11.0 \\
-10.5 \\
-10.0\end{array}$ & $\begin{array}{l}-0.77 \\
-0.74 \\
-0.68 \\
-0.65 \\
-0.62\end{array}$ & $\begin{array}{l}0.66 \\
0.67 \\
0.70 \\
0.71 \\
0.72\end{array}$ & $\begin{array}{r}-7.67 \\
-11.33 \\
-10.00 \\
-8.50 \\
-8.67\end{array}$ & $\begin{array}{l}3.22 \\
4.23 \\
4.85 \\
5.27 \\
5.52\end{array}$ \\
\hline $\begin{array}{c}18^{*} \\
20 * \\
50^{* *} \\
100^{*} \\
500^{* *} \\
\text { FinaI }\end{array}$ & $\begin{array}{c}36.5 \\
-36.0 \\
34.0 \\
30.5 \\
0 \\
0\end{array}$ & $\begin{array}{l}31.0 \\
29.0 \\
27.0 \\
24.0 \\
0 \\
0\end{array}$ & $\begin{array}{r}0.279 \\
0.279 \\
0.293 \\
0.293 \\
0.143 \\
0.011\end{array}$ & $\begin{array}{l}0.273 \\
0.271 \\
0.269 \\
0.269 \\
0.152 \\
0.003\end{array}$ & $\begin{array}{l}0.067 \\
0.067 \\
0.065 \\
0.064 \\
0.008 \\
0.002\end{array}$ & $\begin{array}{r}0.340 \\
.0 .338 \\
0.334 \\
0.333 \\
0.160 \\
0.005\end{array}$ & $\begin{array}{l}-0.061 \\
-0.059 \\
-0.041 \\
-0.040 \\
-0.017 \\
+0.006\end{array}$ & $\begin{array}{c}+0.4 \\
+0.3 \\
0 \\
0 \\
0 \\
0\end{array}$ & $\begin{array}{c}-0.3 \\
0 \\
0 \\
0 \\
0 \\
0\end{array}$ & $\begin{array}{r}26.9 \\
26.9 \\
26.1 \\
25.7 \\
3.2 \\
0.8\end{array}$ & $\begin{array}{r}27.00 \\
27.20 \\
26.10 \\
25.70 \\
3.20 \\
+0.80\end{array}$ & $\begin{array}{l}-9.5 \\
-9.0 \\
-8.0 \\
-5.0 \\
+3.2 \\
+0.8\end{array}$ & $\begin{array}{l}-0.58 \\
-0.55 \\
-0.49 \\
-0.31 \\
+0.20 \\
+0.05\end{array}$ & $\begin{array}{l}0.74 \\
0.76 \\
0.77 \\
0.84 \\
-- \\
--\end{array}$ & $\begin{array}{r}-10.17 \\
-9.83 \\
-6.83 \\
-6.67 \\
-2.83 \\
+1.00\end{array}$ & $\begin{array}{l}5.67 \\
5.63 \\
5.57 \\
5.55 \\
2.67 \\
0.08\end{array}$ \\
\hline \multicolumn{17}{|c|}{ Test 17} \\
\hline $\begin{array}{l}2 \\
3 \\
4 \\
5 \\
6\end{array}$ & $\begin{array}{l}39.0 \\
40.5 \\
40.0 \\
40.0 \\
40.0\end{array}$ & $\begin{array}{r}0.5 \\
4.0 \\
5.5 \\
14.0 \\
31.0\end{array}$ & $\begin{array}{l}0 \\
0 \\
0.006 \\
0.016 \\
0.034\end{array}$ & $\begin{array}{l}0 \\
0 \\
0 \\
0.004 \\
0.012\end{array}$ & $\begin{array}{l}0 \\
0.003 \\
0.009 \\
0.018 \\
0.048\end{array}$ & $\begin{array}{l}0 \\
0.003 \\
0.009 \\
0.022 \\
0.060\end{array}$ & $\begin{array}{l}0 \\
-0.003 \\
-0.003 \\
-0.006 \\
-0.026\end{array}$ & $\begin{array}{l}+0.05 \\
+0.2 \\
+0.4 \\
+1.0 \\
+1.4\end{array}$ & $\begin{array}{l}+0.8 \\
+1.3 \\
+2.5 \\
+5.1 \\
+3.4\end{array}$ & $\begin{array}{r}0 \\
1.2 \\
3.8 \\
6.9 \\
19.5\end{array}$ & $\begin{array}{r}0.85 \\
2.70 \\
6.70 \\
13.00 \\
24.30\end{array}$ & $\begin{array}{l}-38.0 \\
-38.0 \\
-33.5 \\
-27.0 \\
-16.0\end{array}$ & $\begin{array}{l}-1.94 \\
-1.94 \\
-1.71 \\
-1.38 \\
-0.82\end{array}$ & $\begin{array}{l}0.02 \\
0.07 \\
0.17 \\
0.32 \\
0.61\end{array}$ & $\begin{array}{l}0 \\
-0.50 \\
-0.50 \\
-1.00 \\
-4.33\end{array}$ & $\begin{array}{l}0 \\
0.05 \\
0.15 \\
0.37 \\
1.00\end{array}$ \\
\hline $\begin{array}{c}8 \\
10 \\
12 \\
14 \\
16 *\end{array}$ & $\begin{array}{l}40.0 \\
40.0 \\
40.0 \\
40.0 \\
39.5\end{array}$ & $\begin{array}{l}44.0 \\
40.0 \\
40.5 \\
37.3 \\
37.0\end{array}$ & $\begin{array}{l}0.090 \\
0.141 \\
0.186 \\
0.224 \\
0.243\end{array}$ & $\begin{array}{l}0.054 \\
0.115 \\
0.179 \\
0.217 \\
0.233\end{array}$ & $\begin{array}{l}0.066 \\
0.072 \\
0.073 \\
0.073 \\
0.074\end{array}$ & $\begin{array}{l}0.120 \\
0.187 \\
0.252 \\
0.290 \\
0.307\end{array}$ & $\begin{array}{l}-0.030 \\
-0.046 \\
-0.066 \\
-0.066 \\
-0.064\end{array}$ & $\begin{array}{l}+0.8 \\
+0.3 \\
+0.3 \\
-0.1 \\
+0.2\end{array}$ & $\begin{array}{l}+0.7 \\
-2.0 \\
-2.7 \\
-2.3 \\
-1.3\end{array}$ & $\begin{array}{l}26.9 \\
29.3 \\
29.7 \\
29.7 \\
30.1\end{array}$ & $\begin{array}{l}28.40 \\
27.60 \\
27.30 \\
27.30 \\
29.00\end{array}$ & $\begin{array}{l}-11.5 \\
-12.5 \\
-13.0 \\
-13.0 \\
-10.5\end{array}$ & $\begin{array}{l}-0.59 \\
-0.64 \\
-0.66 \\
-0.66 \\
-0.54\end{array}$ & $\begin{array}{l}0.71 \\
0.69 \\
0.68 \\
0.68 \\
0.73\end{array}$ & $\begin{array}{r}-5.00 \\
-7.67 \\
-11.00 \\
-11.00 \\
-10.67\end{array}$ & $\begin{array}{l}2.00 \\
3.12 \\
4.20 \\
4.83 \\
5.12\end{array}$ \\
\hline $\begin{array}{c}17^{*} \\
20^{*} \\
50^{*} \\
100^{* *} \\
500^{* *} \\
\text { Pinal }\end{array}$ & $\begin{array}{c}39.5 \\
39.5 \\
38.0 \\
36.5 \\
18.0 \\
0\end{array}$ & $\begin{array}{c}36.7 \\
35.0 \\
31.5 \\
30.5 \\
0.5 \\
-.\end{array}$ & $\begin{array}{l}0.253 \\
0.250 \\
0.255 \\
0.275 \\
0.260 \\
0.064\end{array}$ & $\begin{array}{l}0.236 \\
0.231 \\
0.236 \\
0.242 \\
0.230 \\
0.013\end{array}$ & $\begin{array}{l}0.074 \\
0.073 \\
0.069 \\
0.069 \\
0.049 \\
0.003\end{array}$ & $\begin{array}{l}0.310 \\
0.304 \\
0.305 \\
0.311 \\
0.279 \\
0.016\end{array}$ & $\begin{array}{r}0.057 \\
-0.054 \\
-0.050 \\
-0.036 \\
-0.019 \\
+0.048\end{array}$ & $\begin{array}{c}0 \\
+0.1 \\
0 \\
0 \\
0 \\
0\end{array}$ & $\begin{array}{c}-1.0 \\
0 \\
0 \\
0 \\
0 \\
0\end{array}$ & $\begin{array}{r}30.1 \\
29.7 \\
28.1 \\
28.1 \\
19.9 \\
1.2\end{array}$ & $\begin{array}{r}29.10 \\
29.80 \\
28.10 \\
28.10 \\
20.00 \\
1.20\end{array}$ & $\begin{array}{r}-10.4 \\
-9.5 \\
-10.0 \\
-8.5 \\
+2.0 \\
+1.0\end{array}$ & $\begin{array}{l}-0.53 \\
-0.48 \\
-0.51 \\
-0.43 \\
+0.10 \\
+0.05\end{array}$ & $\begin{array}{l}0.74 \\
0.75 \\
0.74 \\
0.77 \\
1.11 \\
--\end{array}$ & $\begin{array}{l}-9.50 \\
-9.00 \\
-8.33 \\
-6.00 \\
-3.17 \\
+8.00\end{array}$ & $\begin{array}{l}5.17 \\
5.07 \\
5.08 \\
5.18 \\
4.65 \\
0.27\end{array}$ \\
\hline \multicolumn{17}{|c|}{ Test 18} \\
\hline $\begin{array}{c}\text { Initial } \\
7 \\
8 \\
9 \\
10\end{array}$ & $\begin{array}{c}0 \\
300.0 \\
298.0 \\
294.0 \\
290.0\end{array}$ & $\begin{array}{r}6.0 \\
25.0 \\
247.0 \\
305.0 \\
285.0\end{array}$ & $\begin{array}{l}0.137 \\
0 \\
0.030 \\
0.301 \\
0.527\end{array}$ & $\begin{array}{l}0.060 \\
0 \\
0 \\
0.133 \\
0.299\end{array}$ & $\begin{array}{l}0.007 \\
0.005 \\
0.109 \\
0.248 \\
0.358\end{array}$ & $\begin{array}{l}0.067 \\
0.005 \\
0.109 \\
0.381 \\
0.657\end{array}$ & $\begin{array}{l}+0.070 \\
-0.005 \\
-0.079 \\
-0.080 \\
-0.130\end{array}$ & $\begin{array}{c}0 \\
0 \\
+6.9 \\
+19.3 \\
+4.1\end{array}$ & $\begin{array}{c}0 \\
0 \\
+151.0 \\
+35.0 \\
-22.0\end{array}$ & $\begin{array}{r}4.3 \\
3.1 \\
67.2 \\
153.0 \\
220.8\end{array}$ & $\begin{array}{r}4.30 \\
3.10 \\
245.10 \\
207.30 \\
202.90\end{array}$ & $\begin{array}{r}+2.0 \\
-297.0 \\
-53.0 \\
-87.0 \\
-87.0\end{array}$ & $\begin{array}{r}+0.13 \\
-19.98 \\
-3.57 \\
-5.85 \\
-5.85\end{array}$ & $\begin{array}{l}-.- \\
0.01 \\
0.82 \\
0.71 \\
0.70\end{array}$ & $\begin{array}{r}+11.67 \\
-0.83 \\
-13.17 \\
-13.34 \\
-21.67\end{array}$ & $\begin{array}{r}1.12 \\
0.08 \\
1.82 \\
6.35 \\
10.95\end{array}$ \\
\hline $\begin{array}{l}11 \\
12 \\
13 \\
14 \\
15\end{array}$ & $\begin{array}{l}283.0 \\
273.0 \\
274.0 \\
270.0 \\
267.0\end{array}$ & $\begin{array}{l}267.0 \\
272.0 \\
290.0 \\
270.0 \\
267.0\end{array}$ & $\begin{array}{l}0.803 \\
1.130 \\
1.380 \\
1.610 \\
1.880\end{array}$ & $\begin{array}{l}0.575 \\
0.801 \\
1.180 \\
1.450 \\
1.720\end{array}$ & $\begin{array}{l}0.298 \\
0.288 \\
0.318 \\
0.338 \\
0.368\end{array}$ & $\begin{array}{l}0.873 \\
1.170 \\
1.500 \\
1.790 \\
2.090\end{array}$ & $\begin{array}{l}-0.070 \\
-0.040 \\
-0.120 \\
-0.180 \\
-0.210\end{array}$ & $\begin{array}{l}-8.2 \\
-28.3 \\
-30.2 \\
-21.2 \\
-33.2\end{array}$ & $\begin{array}{r}-8.7 \\
-11.0 \\
+11.0 \\
+35.0 \\
+26.4\end{array}$ & $\begin{array}{l}183.9 \\
177.8 \\
196.5 \\
208.9 \\
227.2\end{array}$ & $\begin{array}{l}167.00 \\
138.50 \\
177.30 \\
222.70 \\
220.40\end{array}$ & $\begin{array}{r}-116.0 \\
-140.0 \\
-97.0 \\
-47.0 \\
-47.0\end{array}$ & $\begin{array}{l}-7.81 \\
-9.42 \\
-6.53 \\
-3.16 \\
-3.16\end{array}$ & $\begin{array}{l}0.59 \\
0.50 \\
0.65 \\
0.82 \\
0.83\end{array}$ & $\begin{array}{r}-11.67 \\
-6.67 \\
-20.00 \\
-30.00 \\
-35.00\end{array}$ & $\begin{array}{l}14.55 \\
19.50 \\
25.00 \\
29.83 \\
34.83\end{array}$ \\
\hline $\begin{array}{l}50 * \\
200 * * \\
500^{* *} \\
\text { Final }\end{array}$ & $\begin{array}{c}233.0 \\
202.0 \\
0 \\
0\end{array}$ & $\begin{array}{r}250.0 \\
200.0 \\
68.0 \\
11.0\end{array}$ & $\begin{array}{l}2.380 \\
2.480 \\
2.260 \\
1.860\end{array}$ & $\begin{array}{l}2.130 \\
2.150 \\
2.140 \\
1.460\end{array}$ & $\begin{array}{l}0.350 \\
0.417 \\
0.412 \\
0.079\end{array}$ & $\begin{array}{l}2.480 \\
2.570 \\
2.550 \\
1.540\end{array}$ & $\begin{array}{l}-0.100 \\
-0.090 \\
-0.290 \\
+0.320\end{array}$ & $\begin{array}{l}0 \\
0 \\
0 \\
0\end{array}$ & $\begin{array}{l}0 \\
0 \\
0 \\
0\end{array}$ & $\begin{array}{r}214.6 \\
255.6 \\
252.6 \\
48.4\end{array}$ & $\begin{array}{r}214.60 \\
255.60 \\
252.60 \\
48.40\end{array}$ & $\begin{array}{r}-18.0 \\
+54.0 \\
+253.0 \\
+48.0\end{array}$ & $\begin{array}{r}-1.21 \\
+3.63 \\
+17.02 \\
+3.23\end{array}$ & $\begin{array}{c}0.92 \\
1.27 \\
\cdots \\
\cdots\end{array}$ & $\begin{array}{l}-43.34 \\
-15.00 \\
-48.33 \\
+53.33\end{array}$ & $\begin{array}{l}41.33 \\
42.83 \\
42.50 \\
25.67\end{array}$ \\
\hline
\end{tabular}

* Results measurably affected by reflections.

* Results affected by reflections and/or reduced surface pressure.

( 2 of 5 sheets) 


\begin{tabular}{|c|c|c|c|c|c|c|c|c|c|c|c|c|c|c|c|c|}
\hline \multirow{4}{*}{$\begin{array}{c}t \\
\text { msec }\end{array}$} & \multirow{3}{*}{\multicolumn{2}{|c|}{$\begin{array}{c}\text { Pressure } \\
\text { psi }\end{array}$}} & \multirow{3}{*}{\multicolumn{5}{|c|}{ Deflections, in. }} & \multirow{3}{*}{\multicolumn{5}{|c|}{$\begin{array}{c}\text { Force per Unit Area } \\
\text { Acting on Top of Device, psi }\end{array}$}} & \multicolumn{4}{|c|}{ Dimensionless Parameters } \\
\hline & & & & & & & & & & & & & \multicolumn{2}{|c|}{ Pressure } & \multicolumn{2}{|c|}{ Deflection } \\
\hline & & & & & & & & & & & & & \multirow{2}{*}{$\frac{2 \Delta P}{q_{u}}$} & \multirow{2}{*}{$\begin{array}{l}\mathrm{P}_{\mathrm{T}} \\
\overline{\mathrm{P}_{\mathrm{S}}} \\
\end{array}$} & \multirow{2}{*}{$\frac{\Delta D}{B} \times 1000$} & \multirow{2}{*}{$\frac{\mathrm{D}_{\mathrm{T}}}{\mathrm{B}} \times 100$} \\
\hline & $\mathrm{P}_{\mathrm{S}}$ & $\overline{\mathrm{F}}_{\mathrm{S}}$ & $\bar{D}_{S}$ & $d_{0}$ & $d_{t}$ & $\mathrm{D}_{\mathrm{T}}$ & $\Delta D$ & Damping & Intertia & Spring & $\mathrm{P}_{\mathrm{T}}$ & $\Delta \mathrm{P}$ & & & & \\
\hline \multicolumn{17}{|c|}{ Test 19} \\
\hline $\begin{array}{l}2 \\
3 \\
4 \\
5 \\
6\end{array}$ & $\begin{array}{l}123.0 \\
145.0 \\
157.0 \\
157.0 \\
156.0\end{array}$ & $\begin{array}{c}0 \\
88.0 \\
136.0 \\
155.0 \\
157.0\end{array}$ & $\begin{array}{l}0 \\
0.109 \\
0.301 \\
0.493 \\
0.661\end{array}$ & $\begin{array}{l}0 \\
0 \\
0.108 \\
0.268 \\
0.451\end{array}$ & $\begin{array}{l}0.039 \\
0.175 \\
0.273 \\
0.330 \\
0.327\end{array}$ & $\begin{array}{l}0.039 \\
0.175 \\
0.381 \\
0.598 \\
0.778\end{array}$ & $\begin{array}{l}-0.039 \\
-0.066 \\
-0.080 \\
-0.105 \\
-0.117\end{array}$ & $\begin{array}{r}+5.4 \\
+10.4 \\
-4.2 \\
-10.0 \\
-12.6\end{array}$ & $\begin{array}{r}+58.3 \\
+5.0 \\
0 \\
+1.0 \\
+3.5\end{array}$ & $\begin{array}{r}16.7 \\
74.9 \\
116.0 \\
141.2 \\
140.0\end{array}$ & $\begin{array}{r}80.40 \\
90.40 \\
112.00 \\
132.00 \\
131.00\end{array}$ & $\begin{array}{l}-42.5 \\
-55.0 \\
-45.0 \\
-25.0 \\
-25.0\end{array}$ & $\begin{array}{l}-2.41 \\
-3.12 \\
-2.55 \\
-1.42 \\
-1.42\end{array}$ & $\begin{array}{l}0.65 \\
0.62 \\
0.71 \\
0.84 \\
0.84\end{array}$ & $\begin{array}{r}-6.50 \\
-11.00 \\
-13.34 \\
-17.50 \\
-19.20\end{array}$ & $\begin{array}{r}0.65 \\
2.95 \\
6.35 \\
9.97 \\
12.97\end{array}$ \\
\hline $\begin{array}{c}8 \\
10 * \\
12 * \\
50 * \\
100 \\
500\end{array}$ & $\begin{array}{c}155.0 \\
153.0 \\
153.0 \\
142.0 \\
112.0 \\
0\end{array}$ & $\begin{array}{r}148.0 \\
149.0 \\
152.0 \\
121.0 \\
108.0 \\
19.0\end{array}$ & $\begin{array}{l}1.040 \\
1.370 \\
1.620 \\
1.750 \\
1.790 \\
1.450\end{array}$ & $\begin{array}{l}0.845 \\
1.260 \\
1.660 \\
1.800 \\
1.870 \\
1.470\end{array}$ & $\begin{array}{l}0.316 \\
0.324 \\
0.346 \\
0.377 \\
0.365 \\
0.104\end{array}$ & $\begin{array}{l}1.160 \\
1.580 \\
2.010 \\
2.180 \\
2.240 \\
1.570\end{array}$ & $\begin{array}{l}-0.120 \\
-0.210 \\
-0.390 \\
-0.430 \\
-0.450 \\
-0.120\end{array}$ & $\begin{array}{c}-11.9 \\
-15.6 \\
-14.3 \\
0 \\
0 \\
0\end{array}$ & $\begin{array}{c}+2.8 \\
0 \\
-6.4 \\
0 \\
0 \\
0\end{array}$ & $\begin{array}{r}134.3 \\
138.7 \\
148.1 \\
160.2 \\
156.0 \\
44.2\end{array}$ & $\begin{array}{r}125.00 \\
123.00 \\
127.40 \\
160.00 \\
156.00 \\
44.00\end{array}$ & $\begin{array}{l}-30.0 \\
-30.0 \\
-26.0 \\
+18.0 \\
+44.0 \\
+44.0\end{array}$ & $\begin{array}{l}-1.70 \\
-1.70 \\
-1.47 \\
+1.02 \\
+2.49 \\
+2.49\end{array}$ & $\begin{array}{l}0.81 \\
0.80 \\
0.83 \\
1.13 \\
1.39 \\
-.-\end{array}$ & $\begin{array}{l}-20.00 \\
-35.00 \\
-65.00 \\
-71.67 \\
-75.00 \\
-20.00\end{array}$ & $\begin{array}{l}19.34 \\
26.33 \\
33.50 \\
36.33 \\
37.33 \\
26.17\end{array}$ \\
\hline
\end{tabular}

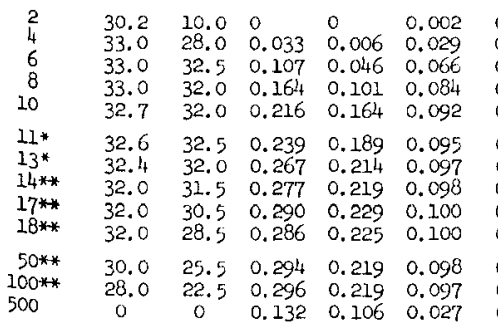

$\begin{array}{ll}0.002 & -0.002 \\ 0.035 & -0.002 \\ 0.112 & -0.005 \\ 0.185 & -0.021 \\ 0.256 & -0.040 \\ 0.284 & -0.045 \\ 0.311 & -0.044 \\ 0.317 & -0.040 \\ 0.329 & -0.039 \\ 0.325 & -0.039 \\ 0.317 & -0.023 \\ 0.316 & -0.020 \\ 0.133 & -0.001\end{array}$

\section{Test 20}

$\begin{array}{cc}+0.7 & +5.3 \\ +1.7 & +1.9 \\ +1.0 & 0 \\ +0.7 & -0.2 \\ +0.5 & -3.8 \\ +0.4 & -4.0 \\ +0.1 & -3.6 \\ +0.1 & -2 . \\ -0.1 & -1 . \\ -0.2 & -0.6 \\ 0 & 0 \\ 0 & 0\end{array}$

\section{Test 21}

$\begin{array}{lllll}28.6 & 8.5 & 0.005 & 0\end{array}$

$\begin{array}{lllll}31.0 & 11.0 & 0.011 & 0 & 0.006 \\ 33.0 & 25.0 & 0.081 & 0.046 & 0.044\end{array}$

$\begin{array}{llllll}33.0 & 25.0 & 0.084 & 0.046 & 0.044\end{array}$

$\begin{array}{lllll}35.5 & 31.0 & 0.119 & 0.089 & 0.052\end{array}$

$\begin{array}{lllll}35.5 & 34.0 & 0.163 & 0.152 & 0.056 \\ 35.5 & 35.5 & 0.228 & 0.224 & 0.061\end{array}$

$\begin{array}{lllll}35.5 & 34.5 & 0.266 & 0.264 & 0.062\end{array}$

$\begin{array}{llllll}35.5 & 32.5 & 0.282 & 0.264 & 0.062\end{array}$

$\begin{array}{llllll}35.0 & 31.0 & 0.279 & 0.248 & 0.061\end{array}$

$\begin{array}{lllll}34.0 & 30.5 & 0.260 & 0.246 & 0.060\end{array}$

$\begin{array}{llllll}32.0 & 30.0 & 0.279 & 0.246 & 0.061\end{array}$

$\begin{array}{llllll}100 * * & 29.0 & 30.5 & 0.279 & 0.216 & 0.061 \\ 29.0 & 0.274 & 0.216 & 0.060\end{array}$

$\begin{array}{rrrrrr}0 & 2.0 & 0.119 & 0.102 & 0.011\end{array}$
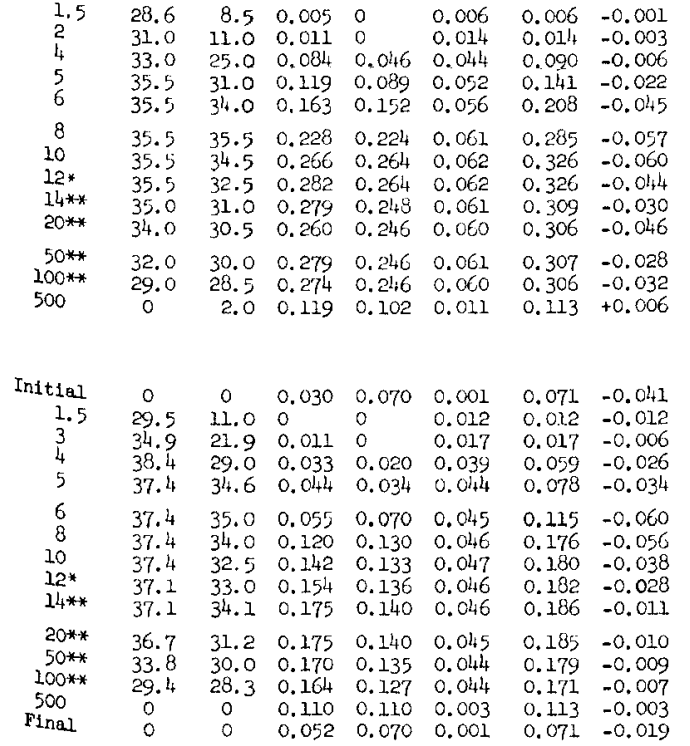

$+1,0$

+0.6
+1.0
+0.8
+0.3
+0.2
0
0
+0.1
0
-0.1
0
0
0

$+6.1$

+6.5
-1.3

-1.3
-0.5

$-2.6$

$-2.8$

$-1.9$

$+0.8$

$+0.6$

0
0
0

\section{Test 22}

$0.071 \quad-0.041$ $\begin{array}{ll}0.0 .22 & -0.012 \\ 0.017 & -0.006\end{array}$ $\begin{array}{ll}0.017 & -0.006 \\ 0.059 & -0.026\end{array}$ $\begin{array}{ll}0.059 & -0.026 \\ 0.078 & -0.034\end{array}$ $0.115-0.060$ $0.176-0.056$ $0.180-0.038$ $0.182-0.028$ $\begin{array}{lll}0.186 & -0.011\end{array}$ $0.185-0.010$ $0.179-0.009$ $0.171-0.007$ $\begin{array}{ll}0.113 & -0.003 \\ 0.071 & -0.019\end{array}$ $\begin{array}{lllllll}0 & 0.6 & 0.60 & +0.6 & +0.04 & --\end{array}$ $\begin{array}{rrrrrr}+3.9 & 6.9 & 12.10 & -17.5 & -1.08 & 0.41 \\ +1.5 & 9.8 & 17.90 & -17.0 & -1.05 & 0.51\end{array}$ $\begin{array}{rrrrrr}+1.5 & 9.8 & 17.90 & -17.0 & -1.05 & 0.51 \\ +1.4 & 22.4 & 26.30 & -12.0 & -0.74 & 0.68\end{array}$ $\begin{array}{llllll}+0.5 & 25.3 & 26.30 & -11.0 & -0.68 & 0.70\end{array}$

$\begin{array}{llllll}0 & 25.9 & 26.30 & -11.0 & -0.68 & 0.70\end{array}$ $\begin{array}{llllll}-2.5 & 26.5 & 23.20 & -14.0 & -0.86 & 0.62\end{array}$ $\begin{array}{llllll}-2.1 & 27.0 & 23.90 & -12.5 & -0.77 & 0.67\end{array}$

$\begin{array}{llllll}-1.6 & 26.5 & 2 t_{4} .90 & -12.0 & -0.7^{4} & 0.67\end{array}$

$\begin{array}{lllll}26.5 & 26.00 & -11.0 & -0.68 & 0.70\end{array}$

$\begin{array}{lllll}25.9 & 26.50 & -10.5 & -0.64 & 0.72\end{array}$

$\begin{array}{lllll}25.3 & 25.30 & -8.5 & -0.52 & 0.75\end{array}$

$\begin{array}{lllll}25.3 & 25.30 & -4.0 & -0.25 & 0.86\end{array}$ $1.7 \quad 1.70$
$+1.7+0.10=$ $\begin{array}{ll}0.6 & 0.60\end{array}$

$\begin{array}{ll}-0.33 & -0.03 \\ -0.33 & 0.58 \\ -0.83 & 1.87 \\ -3.50 & 3.08 \\ -6.67 & 4.27 \\ -7.50 & 4.73 \\ -7.33 & 5.18 \\ -6.67 & 5.28 \\ -6.50 & 5.48 \\ -6.50 & 5.42 \\ -3.83 & 5.28 \\ -3.33 & 5.27 \\ +0.17 & 2.22\end{array}$

$-0.16$

0.10 0.23 1.50 2.35
3.47 4.75 5.43 5.43 5.15 5.20 5.12 5.10 1.88

$-6.83$

1.18

0.20

0.28

0.98
1.30

1.92

1.92
2.93

3.00

3.00
3.03
3.10

3.08

2.98

2.85

1.88
1.18

\section{Test 23}

$\begin{array}{ll}+0.5 & +11.9 \\ +0.8 & +0.7 \\ +0.6 & -0.2 \\ +0.4 & -1.0 \\ +0.4 & -3.6 \\ +0.2 & -3.5 \\ +0.1 & -2.3 \\ 0 & -1.8 \\ 0 & -1.3 \\ 0 & +0.8 \\ +0.2 & +0.3 \\ 0 & 0 \\ 0 & 0 \\ 0 & 0\end{array}$

$\begin{array}{lllll}0.2 & 12.60 & -13.5 & -0.81 & 0.48\end{array}$ $\begin{array}{llllll}1.2 & 2.70 & -30.0 & -1.80 & 0.08\end{array}$ $2.2 \quad 1.60$ $2.4 \quad-0.80$

$\begin{array}{ll}2.6 & -0.70\end{array}$

$\begin{array}{ll}2.7 & 0.50 \\ 2.7 & 0.90\end{array}$

$2.7 \quad 1.40$

$\begin{array}{ll}2.7 & 3.50 \\ 2.7 & 3.20\end{array}$

2.62 .60

$2.6 \quad 2.60$ $1.9 \quad 2.30$ $\begin{array}{llll}-31.0 & -1.86 & 0.08\end{array}$

$\begin{array}{lll}-31.5 & -1.89 & 0.05\end{array}$ $-33.3-2.00 \quad 0$

$\begin{array}{llll}-33.0 & -1.98 & 0\end{array}$

$\begin{array}{lll}-31.5 & -1.89 & 0.02 \\ -31.0 & -1.86 & 0.03\end{array}$

$\begin{array}{llll}30.5 & -1.83 & 0.04\end{array}$

$\begin{array}{rrr}-28.5 & -1.71 & 0.11\end{array}$

$\begin{array}{llll}-28.0 & -1.68 & 0.10\end{array}$

$\begin{array}{lll}-25.5 & -1.53 & 0.09\end{array}$

$\begin{array}{llll}-18.0 & -1.08 & 0.13\end{array}$
$-1.50$

$-1.17$

$-0.50$

$-3.20$

$-1.00$

$-6.50$

-16.50
-19.20

$-25.00$

$-27.80$

-27.50
-27.20

$-25.80$

$-24.50$

$-24.20$

$-22.00$

-23.20
-8.17
0.20

1.43

2.82
4.43

5.70

6.50

6.88

6.95

6.58

6.58

6.50

6.55
2.90 


\begin{tabular}{|c|c|c|c|c|c|c|c|c|c|c|c|c|c|c|c|c|}
\hline \multirow[b]{3}{*}{$\begin{array}{c}t \\
\text { msec }\end{array}$} & \multirow{2}{*}{\multicolumn{2}{|c|}{$\begin{array}{c}\text { Pressure } \\
\text { psi }\end{array}$}} & \multirow[b]{3}{*}{$\overline{\mathrm{D}_{\mathrm{s}}}$} & \multirow{2}{*}{\multicolumn{4}{|c|}{ Deflections, in. }} & \multirow{2}{*}{\multicolumn{5}{|c|}{$\begin{array}{l}\text { Force per Unit Area } \\
\text { Acting on Top of Device, psi }\end{array}$}} & \multicolumn{4}{|c|}{ Dimensionless Parameters } \\
\hline & & & & & & & & & & & & & \multicolumn{2}{|c|}{ Pressure } & \multicolumn{2}{|c|}{ Deflection } \\
\hline & $P_{S}$ & $\overline{\mathrm{P}}_{\mathrm{s}}$ & & $\frac{\text { Defled }}{\mathrm{d}_{0}}$ & $\frac{d_{t}}{d_{1}}$ & $\overline{\mathrm{D}}_{\mathrm{T}}$ & $\Delta D$ & Act & ing on To & $\frac{\text { of Dev }}{\text { Spring }}$ & $\frac{\mathrm{ee}_{2}, \mathrm{psi}}{\mathrm{P}_{\mathrm{T}}}$ & $\Delta p$ & $\frac{\partial \Delta p}{q_{u}}$ & $\frac{\mathrm{P}_{\mathrm{T}}}{\mathrm{P}_{\mathrm{S}}}$ & $\frac{\Delta D}{B} \times 1000$ & $\frac{\mathrm{T}}{\mathrm{B}} \times 100$ \\
\hline \multicolumn{17}{|c|}{ Test 24} \\
\hline $\begin{array}{l}1.5 \\
2 \\
3 \\
4 \\
5\end{array}$ & $\begin{array}{l}26.5 \\
28.0 \\
30.6 \\
31.2 \\
31.5\end{array}$ & $\begin{array}{l}0 \\
25.0\end{array}$ & $\begin{array}{l}0 \\
0.006 \\
0.021 \\
0.045 \\
0.075\end{array}$ & $\begin{array}{l}0 \\
0.005 \\
0.014 \\
0.023 \\
0.046\end{array}$ & $\begin{array}{l}0.003 \\
0.004 \\
0.005 \\
0.006 \\
0.007\end{array}$ & $\begin{array}{l}0.003 \\
0.009 \\
0.019 \\
0.029 \\
0.053\end{array}$ & $\begin{array}{l}-0.003 \\
-0.003 \\
+0.002 \\
+0.016 \\
+0.022\end{array}$ & $\begin{array}{l}+1.7 \\
+0.7 \\
+0.7 \\
+1.1 \\
+1.0\end{array}$ & $\begin{array}{l}+2.7 \\
+2.3 \\
+0.7 \\
+0.5 \\
+1.1\end{array}$ & $\begin{array}{l}11.2 \\
15.5 \\
25.8 \\
35.6 \\
38.7\end{array}$ & $\begin{array}{l}15.60 \\
18.50 \\
27.20 \\
37.20 \\
40.80\end{array}$ & $\begin{array}{r}-11.0 \\
-9.5 \\
-3.4 \\
+6.0 \\
+9.5\end{array}$ & $\begin{array}{l}-0.64 \\
-0.56 \\
-0.20 \\
+0.35 \\
+0.56\end{array}$ & $\begin{array}{l}0.59 \\
0.66 \\
0.89 \\
1.19 \\
1.30\end{array}$ & $\begin{array}{l}-0.50 \\
-0.50 \\
+0.33 \\
+2.67 \\
+3.67\end{array}$ & $\begin{array}{l}0.05 \\
0.15 \\
0.32 \\
0.48 \\
0.88\end{array}$ \\
\hline $\begin{array}{c}6 \\
7 \\
8 * \\
10 * \\
12^{*}\end{array}$ & $\begin{array}{l}31.7 \\
31.7 \\
31.7 \\
31.7 \\
31.7\end{array}$ & 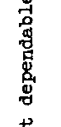 & $\begin{array}{l}0.107 \\
0.130 \\
0.155 \\
0.187 \\
0.205\end{array}$ & $\begin{array}{l}0.077 \\
0.102 \\
0.129 \\
0.165 \\
0.179\end{array}$ & $\begin{array}{l}0.0073 \\
0.0070 \\
0.0070 \\
0.0070 \\
0.0074\end{array}$ & $\begin{array}{l}0.084 \\
0.109 \\
0.136 \\
0.172 \\
0.186\end{array}$ & $\begin{array}{l}+0.023 \\
+0.021 \\
+0.019 \\
+0.015 \\
+0.019\end{array}$ & $\begin{array}{l}+0.5 \\
+0.2 \\
-0.6 \\
-0.1 \\
-0.3\end{array}$ & $\begin{array}{l}+0.3 \\
-0.2 \\
-1.0 \\
-1.5 \\
-0.9\end{array}$ & $\begin{array}{l}40.8 \\
37.7 \\
39.3 \\
39.3 \\
41.3\end{array}$ & $\begin{array}{l}41.60 \\
37.70 \\
37.70 \\
37.70 \\
40.10\end{array}$ & $\begin{array}{r}+10.0 \\
+6.0 \\
+6.0 \\
+6.0 \\
+8.5\end{array}$ & $\begin{array}{l}+0.59 \\
+0.35 \\
+0.35 \\
+0.35 \\
+0.50\end{array}$ & $\begin{array}{l}1.31 \\
1.19 \\
1.19 \\
1.19 \\
1.26\end{array}$ & $\begin{array}{l}+3.83 \\
+3.50 \\
+3.17 \\
+2.50 \\
+3.17\end{array}$ & $\begin{array}{l}1.40 \\
1.82 \\
2.27 \\
2.87 \\
3.10\end{array}$ \\
\hline $\begin{array}{l}14^{*} \\
16^{*} \\
18^{*} \\
20^{* *} \\
50^{* *}\end{array}$ & $\begin{array}{l}31.7 \\
31.4 \\
31.4 \\
31.2 \\
29.0\end{array}$ & 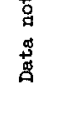 & $\begin{array}{l}0.211 \\
0.204 \\
0.198 \\
0.197 \\
0.204\end{array}$ & $\begin{array}{l}0.175 \\
0.172 \\
0.172 \\
0.172 \\
0.179\end{array}$ & $\begin{array}{l}0.0070 \\
0.0070 \\
0.0065 \\
0.0064 \\
0.0065\end{array}$ & $\begin{array}{l}0.182 \\
0.179 \\
0.179 \\
0.178 \\
0.186\end{array}$ & $\begin{array}{l}+0.029 \\
+0.025 \\
+0.019 \\
+0.019 \\
+0.018\end{array}$ & $\begin{array}{l}-0.3 \\
-1.0 \\
0 \\
+0.4 \\
0\end{array}$ & $\begin{array}{c}-0.3 \\
+0.3 \\
+0.2 \\
+0.1 \\
0\end{array}$ & $\begin{array}{l}38.7 \\
37.7 \\
36.2 \\
35.6 \\
36.2\end{array}$ & $\begin{array}{l}38.10 \\
37.00 \\
36.40 \\
36.10 \\
36.20\end{array}$ & $\begin{array}{l}+6.5 \\
+5.5 \\
+5.0 \\
+5.0 \\
+7.0\end{array}$ & $\begin{array}{l}+0.38 \\
+0.32 \\
+0.29 \\
+0.29 \\
+0.41\end{array}$ & $\begin{array}{l}1.20 \\
1.18 \\
1.16 \\
1.16 \\
1.25\end{array}$ & $\begin{array}{l}+4.83 \\
+4.17 \\
+3.17 \\
+3.17 \\
+3.00\end{array}$ & $\begin{array}{l}3.03 \\
2.98 \\
2.98 \\
2.97 \\
3.10\end{array}$ \\
\hline $\begin{array}{l}100 * * \\
500\end{array}$ & $\begin{array}{c}25.0 \\
0\end{array}$ & 8.6 & $\begin{array}{l}0.205 \\
0.098\end{array}$ & $\begin{array}{l}0.179 \\
0.083\end{array}$ & 0.006 & $\begin{array}{l}0.185 \\
0.083\end{array}$ & $\begin{array}{l}+0.020 \\
+0.015\end{array}$ & $\begin{array}{l}0 \\
0\end{array}$ & $\begin{array}{l}0 \\
0\end{array}$ & $\begin{array}{r}34.1 \\
8.8\end{array}$ & $\begin{array}{r}34.10 \\
8.80\end{array}$ & $\begin{array}{l}+9.0 \\
+0.2\end{array}$ & $\begin{array}{l}+0.53 \\
+0.01\end{array}$ & $\begin{array}{l}1.36 \\
-.\end{array}$ & $\begin{array}{l}+3.33 \\
+2.50\end{array}$ & $\begin{array}{l}3.08 \\
1.38\end{array}$ \\
\hline
\end{tabular}

\begin{tabular}{|c|c|c|c|c|c|c|c|}
\hline $\begin{array}{l}1.5 \\
2 \\
2.5 \\
3 \\
4\end{array}$ & $\begin{array}{l}37.8 \\
41.9 \\
45.5 \\
47.7 \\
51.3\end{array}$ & $\begin{array}{l}\frac{2}{4} \\
\frac{2}{6}\end{array}$ & $\begin{array}{l}0 \\
0.009 \\
0.022 \\
0.042 \\
0.087\end{array}$ & $\begin{array}{l}0 \\
0.003 \\
0.007 \\
0.014 \\
0.045\end{array}$ & $\begin{array}{l}0.004 \\
0.006 \\
0.008 \\
0.009 \\
0.011\end{array}$ & $\begin{array}{l}0.004 \\
0.009 \\
0.015 \\
0.023 \\
0.056\end{array}$ & $\begin{array}{c}-0.004 \\
0 \\
+0.007 \\
+0.019 \\
+0.031\end{array}$ \\
\hline $\begin{array}{c}5 \\
6 \\
7 \\
8 * \\
10^{*}\end{array}$ & $\begin{array}{l}53.5 \\
52.5 \\
52.0 \\
52.0 \\
52.0\end{array}$ & 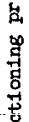 & $\begin{array}{l}0.145 \\
0.200 \\
0.253 \\
0.290 \\
0.365\end{array}$ & $\begin{array}{l}0.090 \\
0.142 \\
0.190 \\
0.243 \\
0.330\end{array}$ & $\begin{array}{l}0.012 \\
0.012 \\
0.013 \\
0.013 \\
0.013\end{array}$ & $\begin{array}{l}0.102 \\
0.154 \\
0.203 \\
0.256 \\
0.343\end{array}$ & $\begin{array}{l}+0.043 \\
+0.046 \\
+0.050 \\
+0.034 \\
+0.022\end{array}$ \\
\hline $\begin{array}{c}12^{*} \\
16^{*} \\
20^{*} \\
50^{*} \\
100^{* *}\end{array}$ & $\begin{array}{l}51.7 \\
51.9 \\
51.7 \\
49.0 \\
40.0\end{array}$ & $\begin{array}{l}0 \\
3 \\
30\end{array}$ & $\begin{array}{l}0.427 \\
0.478 \\
0.475 \\
0.485 \\
0.485\end{array}$ & $\begin{array}{l}0.390 \\
0.438 \\
0.428 \\
0.440 \\
0.438\end{array}$ & $\begin{array}{l}0.012 \\
0.013 \\
0.012 \\
0.012 \\
0.011\end{array}$ & $\begin{array}{l}0.402 \\
0.451 \\
0.440 \\
0.452 \\
0.449\end{array}$ & $\begin{array}{l}+0.025 \\
+0.027 \\
+0.035 \\
+0.033 \\
+0.036\end{array}$ \\
\hline $\begin{array}{l}500 \\
\text { Final }\end{array}$ & $\begin{array}{l}0 \\
--\end{array}$ & & $\begin{array}{l}0.321 \\
0.232\end{array}$ & $\begin{array}{l}0.311 \\
0.208\end{array}$ & $\begin{array}{l}0.001 \\
0\end{array}$ & $\begin{array}{l}0.312 \\
0.208\end{array}$ & $\begin{array}{l}+0.009 \\
+0.024\end{array}$ \\
\hline
\end{tabular}

Test 25

$\begin{array}{lcccccccc}+1.2 & +4.8 & 21.8 & 27.80 & -10.0 & -0.42 & 0.74 & -0.67 & 0.07 \\ +3.0 & +4.2 & 33.3 & 40.50 & -1.5 & -0.06 & 0.97 & 0 & 0.15 \\ +3.7 & +6.3 & 43.2 & 53.20 & +7.5 & +0.32 & 1.17 & +1.17 & 0.25 \\ +4.7 & +2.9 & 51.7 & 59.30 & +11.5 & +0.49 & 1.24 & +3.17 & 0.38 \\ +5.6 & +1.5 & 62.3 & 69.40 & +18.0 & +0.76 & 1.35 & +5.17 & 0.93 \\ +6.1 & +1.4 & 65.8 & 73.30 & +20.0 & +0.84 & 1.37 & +7.17 & 1.70 \\ +7.0 & +0.8 & 67.9 & 75.70 & +23.0 & +0.97 & 1.44 & +7.67 & 2.57 \\ +6.8 & +0.2 & 70.4 & 77.40 & +25.5+1.08 & 1.49 & +8.33 & 3.38 \\ +6.6 & -0.5 & 70.1 & 76.20 & +24.0 & +1.01 & 1.47 & +5.67 & 4.27 \\ +5.2 & -2.4 & 69.7 & 72.50 & +20.5+0.87 & 1.39 & +3.67 & 5.72 \\ +3.1 & -1.9 & 68.6 & 69.80 & +18.0+0.76 & 1.35 & +4.17 & 6.70 \\ +0.6 & -1.0 & 69.6 & 69.20 & +17.5+0.74 & 1.33 & +4.50 & 7.52 \\ 0 & -0.1 & 64.7 & 64.60 & +13.0 & +0.55 & 1.25 & +5.83 & 7.33 \\ 0 & 0 & 66.5 & 66.50 & +17.5 & +0.74 & 1.36 & +5.50 & 7.53 \\ 0 & 0 & 61.6 & 61.60 & +21.5 & +0.91 & 1.54 & +6.00 & 7.48 \\ 0 & 0 & 0.9 & 0.90 & +0.9 & +0.04 & -- & +1.50 & 5.20 \\ -- & -- & 0 & 0 & 0 & 0 & -- & +4.00 & 3.47\end{array}$

Test 26

\begin{tabular}{|c|c|c|c|c|c|c|c|}
\hline Initial & 0 & & 0.090 & 0.040 & $1.000+$ & 0.040 & +0.050 \\
\hline $\begin{array}{l}4 \\
6 \\
8\end{array}$ & $\begin{array}{l}33.5 \\
33.0 \\
32.5\end{array}$ & 号 & $\begin{array}{l}0.070 \\
0.150 \\
0.270\end{array}$ & $\begin{array}{l}0.020 \\
0.095 \\
0.178\end{array}$ & $\begin{array}{l}1.200 \\
1.200 \\
1.200\end{array}$ & $\begin{array}{l}0.020 \\
0.095 \\
0.178\end{array}$ & $\begin{array}{l}\text { Results } \\
+0.050 \\
+0.055 \\
+0.092\end{array}$ \\
\hline $\begin{array}{l}10 \\
12^{*} \\
14^{*} \\
20^{*} \\
50^{* *}\end{array}$ & $\begin{array}{l}32.0 \\
32.0 \\
31.7 \\
30.5 \\
28.5\end{array}$ & 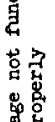 & $\begin{array}{l}0.350 \\
0.410 \\
0.450 \\
0.500 \\
0.470\end{array}$ & $\begin{array}{l}0.275 \\
0.355 \\
0.410 \\
0.448 \\
0.443\end{array}$ & $\begin{array}{l}1.300 \\
1.300 \\
1.000 \\
1.100 \\
1.000\end{array}$ & $\begin{array}{l}0.275 \\
0.355 \\
0.410 \\
0.448 \\
0.443\end{array}$ & $\begin{array}{l}+0.075 \\
+0.055 \\
+0.040 \\
+0.052 \\
+0.027\end{array}$ \\
\hline $\begin{array}{l}100 * * \\
500\end{array}$ & $\begin{array}{c}22.0 \\
0\end{array}$ & & $\begin{array}{l}0.475 \\
0.265\end{array}$ & $\begin{array}{l}0.443 \\
0.241\end{array}$ & $\begin{array}{l}0.900 \\
0\end{array}$ & $\begin{array}{l}0.443 \\
0.241\end{array}$ & $\begin{array}{l}+0.032 \\
+0.024\end{array}$ \\
\hline $\begin{array}{l}2 \\
3 \\
4 \\
6 \\
7\end{array}$ & $\begin{array}{l}27.5 \\
30.5 \\
31.0 \\
31.0 \\
30.0\end{array}$ & 范 & $\begin{array}{l}0.002 \\
0.016 \\
0.045 \\
0.110 \\
0.155\end{array}$ & $\begin{array}{l}0.004 \\
0.015 \\
0.039 \\
0.098 \\
0.134\end{array}$ & $\begin{array}{l}0.070 t \\
0.360 \\
0.650 \\
0.750 \\
0.800\end{array}$ & $\begin{array}{l}0.004 \\
0.015 \\
0.039 \\
0.098 \\
0.134\end{array}$ & $\begin{array}{l}-0.002 \\
+0.001 \\
+0.006 \\
+0.012 \\
+0.021\end{array}$ \\
\hline $\begin{array}{l}8 \\
10 \\
12^{*} \\
14 * \\
16 * *\end{array}$ & $\begin{array}{l}30.0 \\
29.0 \\
29.0 \\
29.0 \\
28.0\end{array}$ & 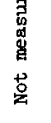 & $\begin{array}{l}0.191 \\
0.250 \\
0.285 \\
0.300 \\
0.300\end{array}$ & $\begin{array}{l}0.169 \\
0.221 \\
0.261 \\
0.276 \\
0.275\end{array}$ & $\begin{array}{l}0.850 \\
1.100 \\
1.000 \\
1.050 \\
1.050\end{array}$ & $\begin{array}{l}0.169 \\
0.221 \\
0.261 \\
0.276 \\
0.275\end{array}$ & $\begin{array}{l}+0.022 \\
+0.029 \\
+0.024 \\
+0.024 \\
+0.025\end{array}$ \\
\hline $\begin{array}{l}50 * * * \\
100 * * \\
500\end{array}$ & $\begin{array}{l}26.0 \\
22.0 \\
0\end{array}$ & & $\begin{array}{l}0.290 \\
0.290 \\
0.169\end{array}$ & $\begin{array}{l}0.270 \\
0.270 \\
0.160\end{array}$ & $\begin{array}{l}0.900 \\
0.850 \\
0\end{array}$ & $\begin{array}{l}0.270 \\
0.270 \\
0.160\end{array}$ & $\begin{array}{l}+0.020 \\
+0.020 \\
+0.009\end{array}$ \\
\hline
\end{tabular}

\begin{tabular}{|c|c|c|c|}
\hline \multirow{3}{*}{ 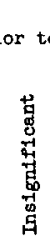 } & 0 & 22.2 & 22.20 \\
\hline & $\begin{array}{l}+5.3 \\
+2.6 \\
+1.3\end{array}$ & $\begin{array}{l}35.4 \\
37.2 \\
38.9\end{array}$ & $\begin{array}{l}40.70 \\
39.80 \\
40.20\end{array}$ \\
\hline & $\begin{array}{l}-2.2 \\
-3.4 \\
-2.8 \\
-0.8 \\
0\end{array}$ & $\begin{array}{l}40.2 \\
40.2 \\
37.5 \\
34.0 \\
31.3\end{array}$ & $\begin{array}{l}38.00 \\
36.80 \\
34.70 \\
33.20 \\
31.30\end{array}$ \\
\hline & $\begin{array}{l}0 \\
0\end{array}$ & $\underset{0}{29.5}$ & $\begin{array}{c}29.50 \\
0\end{array}$ \\
\hline
\end{tabular}

$\begin{array}{rccr}+22.2+1.52 & -- & +8.33 \\ +7.2+0.49 & 1.21 & +8.33 \\ +7.0+0.48 & 1.21 & +9.17 \\ +7.5+0.51 & 1.24 & +15.33 \\ +6.0+0.41 & 1.19 & +12.50 \\ +5.0+0.34 & 1.15 & +9.17 \\ +3.0+0.21 & 1.09 & +6.67 \\ +2.5+0.17 & 1.09 & +8.67 \\ +3.0+0.21 & 1.10 & +4.50 \\ +7.5 & +0.51 & 1.34 & +5.33 \\ 0 & 0 & -- & +4.00\end{array}$

0.67

0.33

2. 97

4.58

5.92
6.83

6.83
7.47
7.38

7.38

Test $27 \mathrm{~A}$

\begin{tabular}{|c|c|c|c|c|c|c|c|}
\hline $\begin{array}{r}+3.8 \\
+4.8 \\
+3.8 \\
+1.1 \\
+0.2\end{array}$ & $\begin{array}{l}12.2 \\
26.8 \\
37.4 \\
41.4 \\
41.4\end{array}$ & $\begin{array}{l}16.00 \\
31.60 \\
41.20 \\
42.50 \\
41.60\end{array}$ & $\begin{array}{r}-11.5 \\
+1.0 \\
+10.0 \\
+11.5 \\
+11.5\end{array}$ & $\begin{array}{l}-0.65 \\
+0.06 \\
+0.57 \\
+0.65 \\
+0.65\end{array}$ & $\begin{array}{l}0.58 \\
1.04 \\
1.33 \\
1.37 \\
1.39\end{array}$ & $\begin{array}{l}-0.33 \\
+0.17 \\
+1.00 \\
+2.00 \\
+3.50\end{array}$ & $\begin{array}{l}0.07 \\
0.25 \\
0.65 \\
1.63 \\
2.23\end{array}$ \\
\hline $\begin{array}{l}-1.0 \\
-2.5 \\
-2.5 \\
-3.1 \\
-0.8\end{array}$ & $\begin{array}{l}42.2 \\
41.4 \\
41.4 \\
40.6 \\
37.4\end{array}$ & $\begin{array}{l}41.20 \\
38.90 \\
38.90 \\
37.50 \\
36.60\end{array}$ & $\begin{array}{r}+11.0 \\
+10.0 \\
+10.0 \\
+8.5 \\
+8.5\end{array}$ & $\begin{array}{l}+0.62 \\
+0.57 \\
+0.57 \\
+0.48 \\
+0.48\end{array}$ & $\begin{array}{l}1.37 \\
1.34 \\
1.34 \\
1.29 \\
1.31\end{array}$ & $\begin{array}{r}+3.67 \\
+4.83 \\
+4.00 \\
+4.00 \\
+4.17\end{array}$ & $\begin{array}{l}2.82 \\
3.68 \\
4.35 \\
4.60 \\
4.58\end{array}$ \\
\hline $\begin{array}{l}0 \\
0 \\
0\end{array}$ & $\begin{array}{c}31.7 \\
28.4 \\
0\end{array}$ & $\begin{array}{c}31.70 \\
28.40 \\
0\end{array}$ & $\begin{array}{c}+5.5 \\
+6.5 \\
0\end{array}$ & $\begin{array}{c}+0.31 \\
+0.37 \\
0\end{array}$ & $\begin{array}{l}1.22 \\
1.29 \\
0\end{array}$ & $\begin{array}{r}+3.33 \\
+3.33 \\
+1.50\end{array}$ & $\begin{array}{l}4.50 \\
4.50 \\
2.67\end{array}$ \\
\hline
\end{tabular}

\section{Test 27B}

\begin{tabular}{|c|c|c|c|c|c|c|c|}
\hline $\begin{array}{l}2 \\
3 \\
4 \\
6 \\
8\end{array}$ & $\begin{array}{l}35.7 \\
39.5 \\
40.7 \\
41.5 \\
40.7\end{array}$ & & $\begin{array}{l}0.007 \\
0.038 \\
0.083 \\
0.182 \\
0.287\end{array}$ & $\begin{array}{l}0.012 \\
0.042 \\
0.083 \\
0.170 \\
0.252\end{array}$ & $\begin{array}{l}0.100 t \\
1.100 \\
1.300 \\
1.500 \\
1.600\end{array}$ & $\begin{array}{l}0.012 \\
0.042 \\
0.083 \\
0.170 \\
0.252\end{array}$ & $\begin{array}{l}-0.005 \\
-0.004 \\
0 \\
+0.012 \\
+0.035\end{array}$ \\
\hline $\begin{array}{l}10 \\
12 * \\
14^{*} \\
16^{*} \\
50^{*}\end{array}$ & $\begin{array}{l}40.7 \\
40.5 \\
40.5 \\
40.5 \\
38.0\end{array}$ & 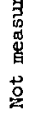 & $\begin{array}{l}0.375 \\
0.423 \\
0.456 \\
0.453 \\
0.440\end{array}$ & $\begin{array}{l}0.334 \\
0.390 \\
0.405 \\
0.410 \\
0.397\end{array}$ & $\begin{array}{l}1.600 \\
1.600 \\
1.600 \\
1.500 \\
1.200\end{array}$ & $\begin{array}{l}0.334 \\
0.390 \\
0.405 \\
0.410 \\
0.397\end{array}$ & $\begin{array}{l}+0.041 \\
+0.033 \\
+0.051 \\
+0.043 \\
+0.043\end{array}$ \\
\hline $\begin{array}{l}100 * * \\
500\end{array}$ & $\begin{array}{c}30.0 \\
0\end{array}$ & & $\begin{array}{l}0.431 \\
0.326\end{array}$ & $\begin{array}{l}0.395 \\
0.297\end{array}$ & $\begin{array}{c}1.100 \\
-\end{array}$ & $\begin{array}{l}0.395 \\
0.297\end{array}$ & $\begin{array}{r}0.036 \\
+0.029\end{array}$ \\
\hline
\end{tabular}

* Results measurably affected by reflections.

* Affected by reflections and/or reduced surface pressure.

$+7.5 \quad 21.8 \quad 29.30$

$\begin{array}{rrr}+5.8 & 36.5 & 42.30 \\ +3.3 & 42.4 & 45.70\end{array}$

$+1.4 \quad 48.2 \quad 49.60$

$-1.4 \quad 49.6 \quad 48.20$

$\begin{array}{lll}-3.1 & 51.1 & 48.00\end{array}$

$\begin{array}{lll}-3.1 & 51.5 & 48.40 \\ -2.8 & 50.0 & 47.20\end{array}$

$-1.0 \quad 46.0 \quad 45.00$

39.439 .40

$\begin{array}{rr}35.0 & 35.00 \\ 4.6 & 4.60\end{array}$ $\begin{array}{llll}-6.4 & -0.34 & 0.82 & -0.83\end{array}$

$\begin{array}{llll}+3.0 & +0.16 & 1.07 & -0.67\end{array}$

$+8.0+0.26-1.12$

$+8.0+0.421 .20$
$+7.5+0.39$

$+7.5+0.39 \quad 1.18$

$+8.0+0.42 \quad 1.19$

$+6.5+0.34 \quad 1.17$

$+4.5+0.24 \quad 1.11+7.17$

$+1.5+0.08 \quad 1.04 \quad+7.17$

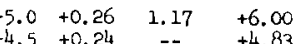

.07

.25

.93

.57

.72

70

.33

.20

67

33




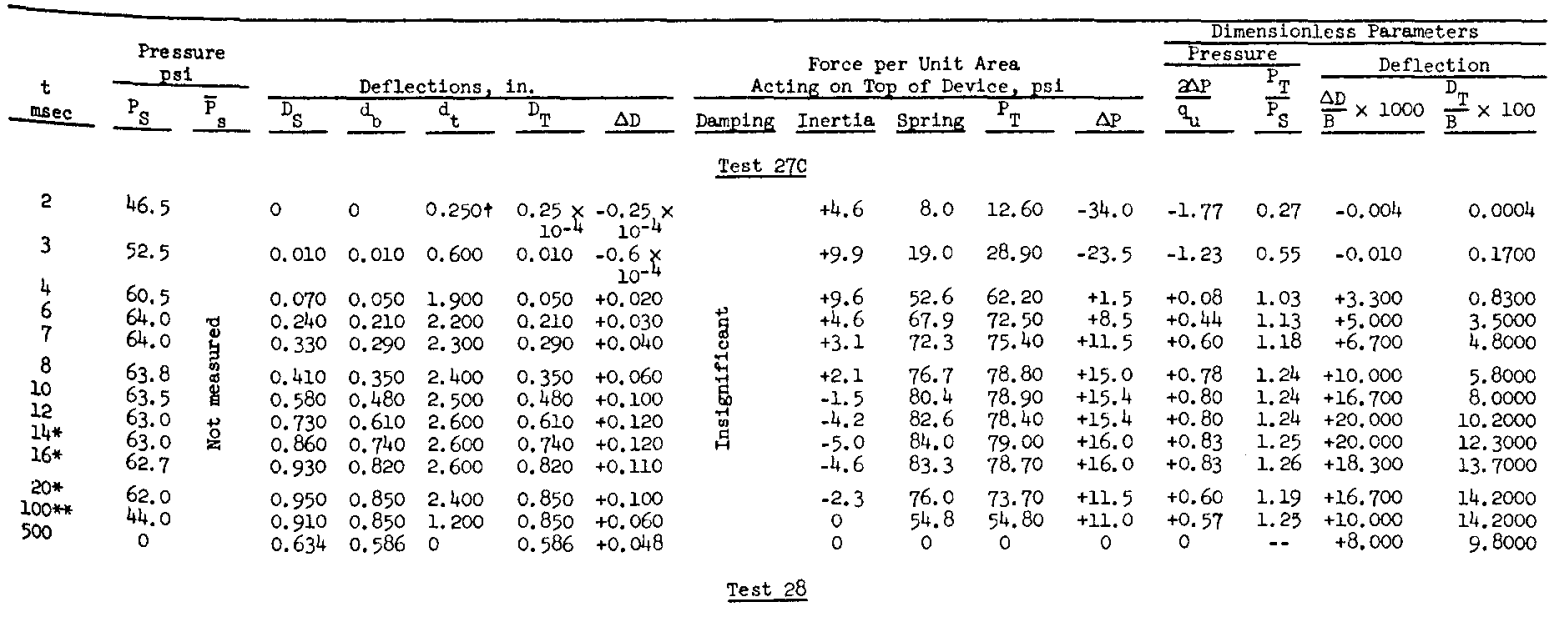

Most of instrumentation lost during test 
Table 7

Rate of Pressure Rise and Rise Time at 35-in. Level

\begin{tabular}{|c|c|c|c|c|c|c|c|c|c|c|c|c|c|c|}
\hline \multirow[b]{2}{*}{$\begin{array}{l}\text { Test } \\
\text { No. }\end{array}$} & \multirow{2}{*}{\multicolumn{2}{|c|}{ 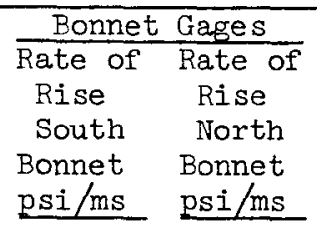 }} & \multicolumn{4}{|c|}{ Soil Near Device } & \multirow{2}{*}{$\begin{array}{c}\text { Period } \\
\text { of } \\
\text { Test } \\
\text { Device } \\
\underline{T}, \mathrm{~ms} \\
\end{array}$} & \multirow[b]{2}{*}{$\mathrm{t}_{\mathrm{r}} / \mathrm{T}$} & \multirow[b]{2}{*}{$\mathrm{t}_{\mathrm{O}} / \mathrm{T}$} & \multicolumn{2}{|c|}{$\begin{array}{c}\text { Damped } \\
\text { Amplification } \\
\text { Factor for }\end{array}$} & \multicolumn{2}{|c|}{$\mathrm{P}_{\mathrm{T}} / \mathrm{P}_{\mathrm{S}}^{*} \div \times \mathrm{AF}$} & \multirow[b]{2}{*}{$\mathrm{K}_{\mathrm{T}} / \mathrm{K}_{\mathrm{S}}{ }^{*}$} \\
\hline & & & $\begin{array}{c}\text { Channel } \\
\text { No. } \\
\end{array}$ & $\begin{array}{c}\text { Rise } \\
\mathrm{psi} / \mathrm{ms} \\
\end{array}$ & ${ }^{t_{r}}, \mathrm{~ms}$ & $\mathrm{t}_{0}, \mathrm{~ms}$ & & & & $\mathrm{t}_{r} / T$ & $t_{0} / T$ & $t_{r}$ & $t_{0}$ & \\
\hline 11 & 41 & -- & SII & 5.82 & 6.80 & 4.75 & 6.35 & 1.07 & 0.75 & 1.06 & 1.21 & 0.69 & 0.60 & 0.75 \\
\hline 12 & 55 & 31 & SII & 11.4 & 6.08 & 3.58 & 6.40 & 0.95 & 0.56 & 1.03 & 1.42 & 0.81 & 0.59 & 0.62 \\
\hline 13 & 94 & 188 & $\mathrm{~S} 11$ & 28.8 & 5.48 & 2.78 & 6.85 & 0.80 & 0.41 & $1.14 t$ & $1.49+$ & 0.67 & 0.52 & 0.57 \\
\hline 14 & 224 & 222 & $\mathrm{SII}$ & 91.5 & 2.24 & 0.70 & 5.96 & 0.38 & 0.12 & 1.36 & 1.45 & 0.62 & 0.58 & 0.66 \\
\hline 15 & 40 & 41 & $\mathrm{~S} 11$ & 24.6 & 1.25 & 0.75 & 6.50 & 0.19 & 0.12 & 1.71 & 1.73 & 0.50 & 0.49 & 0.80 \\
\hline 16 & 32 & 35 & SII & 8.3 & 3.05 & 2.90 & 6.50 & 0.47 & 0.45 & 1.52 & 1.53 & 0.47 & 0.46 & 0.65 \\
\hline 17 & 40 & 36 & Sll & 10.0 & 5.65 & 3.90 & 6.35 & 0.89 & 0.61 & 1.08 & 1.36 & 0.66 & 0.52 & 0.57 \\
\hline 18 & 268 & 343 & SII & 123.0 & 3.00 & 0.75 & 5.95 & 0.50 & 0.13 & 1.30 & 1.44 & 0.63 & 0.57 & 0.58 \\
\hline 19 & 146 & 92 & Sll & 58.5 & 2.50 & 1.25 & 6.84 & 0.37 & 0.18 & $1.52 t$ & $1.61 t$ & 0.55 & 0.52 & 0.54 \\
\hline 20 & 39 & 28 & S1O & 10.1 & 3.25 & 1.75 & 14.12 & 0.23 & 0.12 & 1.56 & 1.60 & 0.18 & 0.17 & 0.29 \\
\hline 21 & 54 & 54 & S1O & 18.6 & 2.50 & 1.60 & 8.41 & 0.30 & 0.19 & $1.59+$ & $1.66+$ & 0.28 & 0.26 & 0.72 \\
\hline 22 & 51 & 53 & Slo & 11.9 & 3.44 & 1.70 & 6.39 & 0.54 & 0.27 & 1.32 & 1.47 & 0.53 & 0.48 & 0.88 \\
\hline 23 & -- & 25 & S1I & 5.46 & 3.60 & 1.80 & 33.0 & 0.11 & 0.05 & 1.63 & 1.64 & 0.00 & 0.00 & 0.03 \\
\hline 24 & 30 & 27 & S8** & 13.8 & 4.30 & 4.00 & 1.65 & 2.62 & 2.42 & 1.06 & 1.08 & 1.39 & 1.42 & 4.51 \\
\hline 25 & 44 & 41 & S5 & 19.4 & 2.35 & 1.65 & 1.65 & 1.42 & 1.00 & 1.14 & 1.00 & 1.55 & 1.36 & 4.14 \\
\hline 26 & 30 & 29 & $\mathrm{~s} 18$ & 10.4 & 2.90 & 2.00 & 0.24 & 12.1 & 8.34 & 1.00 & 1.00 & 1.24 & 1.24 & 337.0 \\
\hline 27 & + & & & & & — No & Record- & & & & & & & \\
\hline 28 & 30 & 51 & SIO & 12.3 & 3.60 & 2.00 & 3.89 & 0.92 & 0.51 & 1.04 & 1.31 & 0.89 & 1.13 & 2.52 \\
\hline
\end{tabular}

Note: $t_{r}=$ time to maximum pressure (no reflection). $t_{0}=$ time to first pressure peak (steep part of pressure trace).

* Extracted from table 2.

* Close-in gage lost.

+ No damping tests--estimated value. 


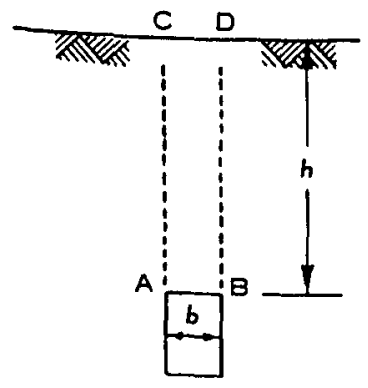

(a)

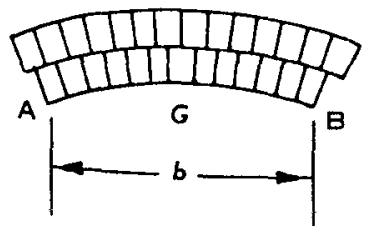

(b)
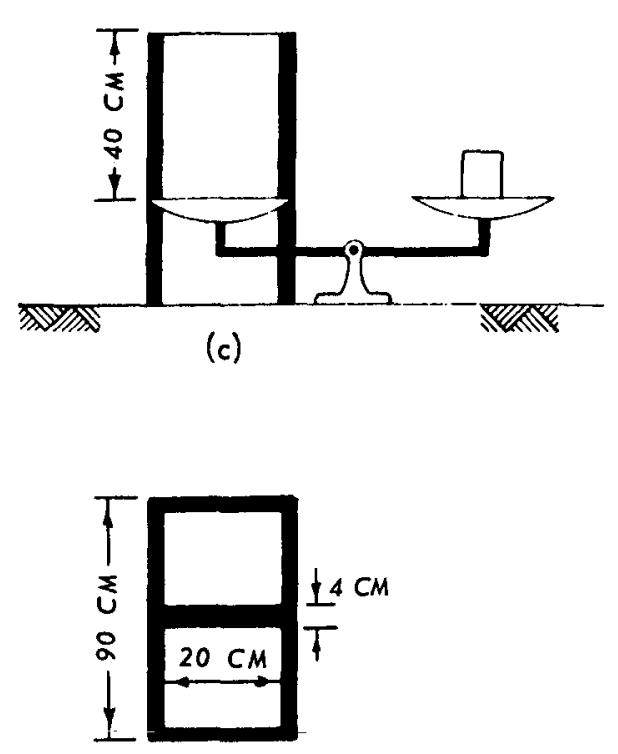

(d)

Fig. 1. Analytical and experimental study by Engesser

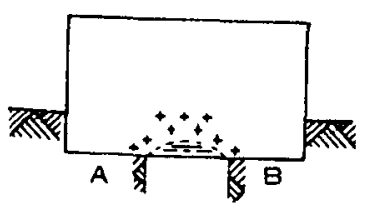

(a)

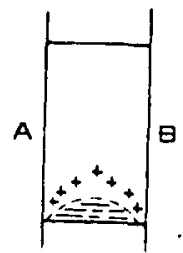

(b)

Fig. 2. Earth pressure phenomena in locally stressed fills by Terzaghi (1919) 


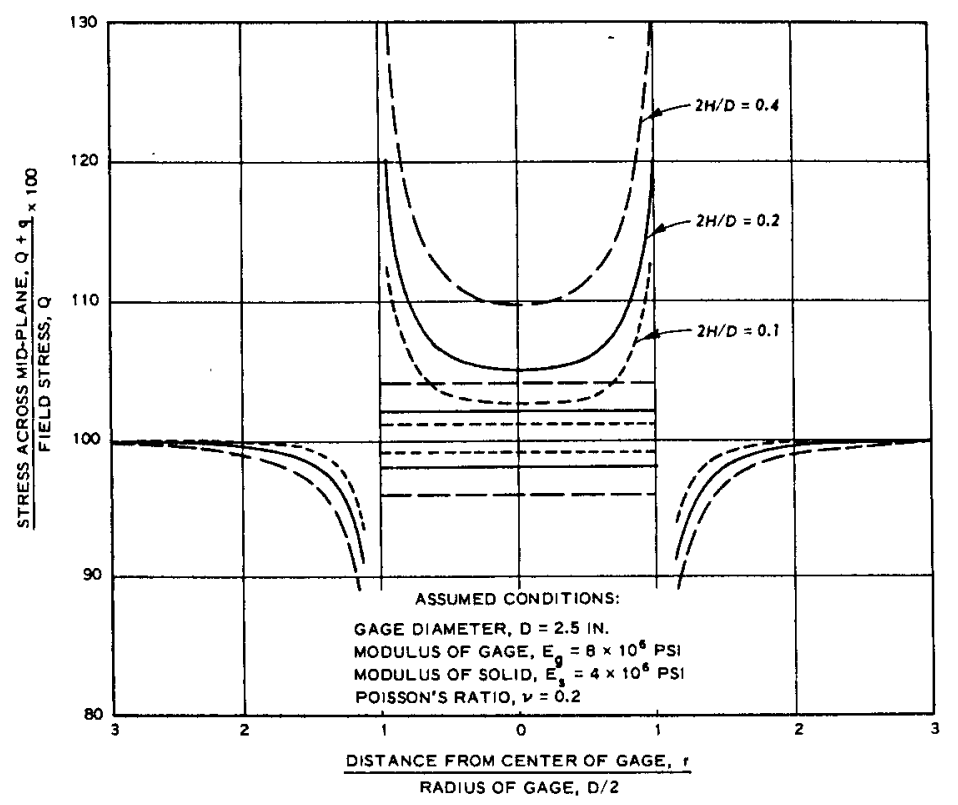

a. EFFECTS OF GEOMETRY

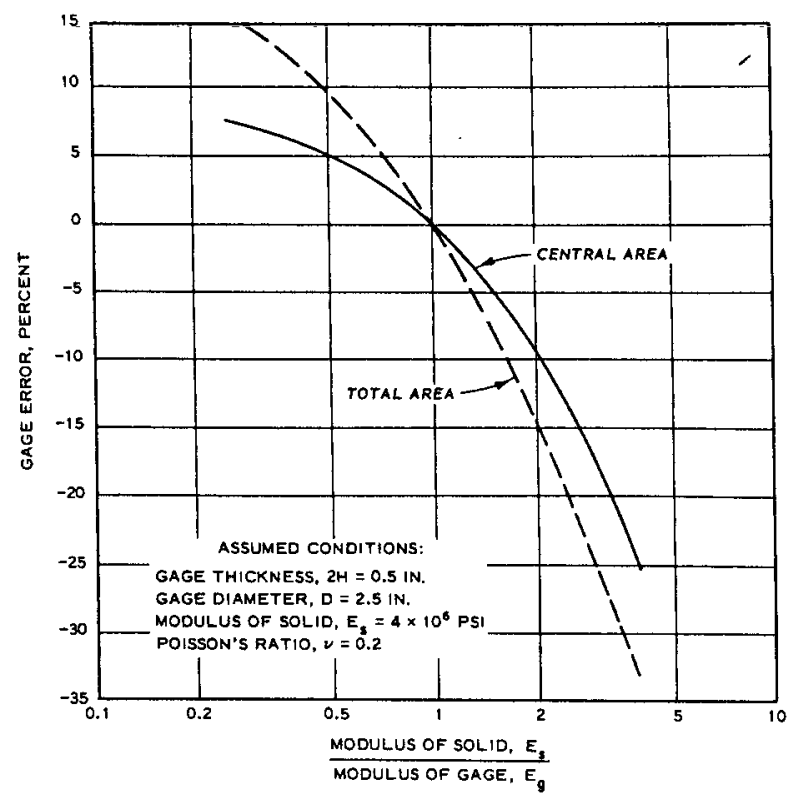

b. EFFECTS OF MODULUS

Fig. 3. Monfore's distribution of pressure as determined by elastic analysis 


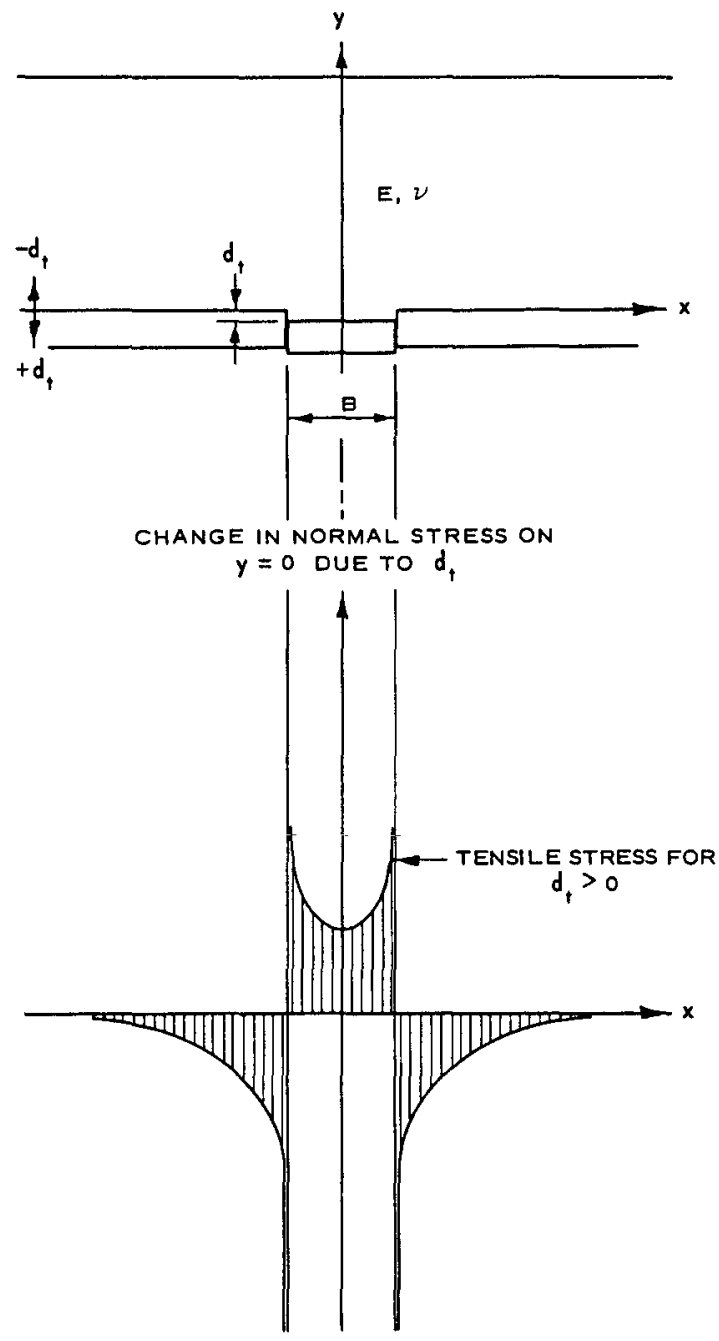

Fig. 4. Distribution of arching stresses from an elastic solution by Finn 


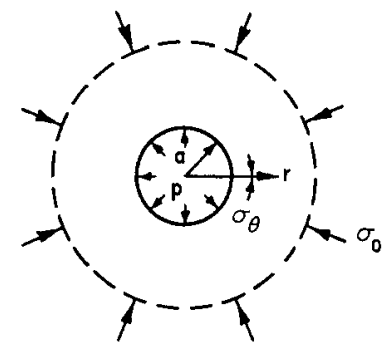

(a) GEOMETRY AND BOUNDARY CONDITIONS

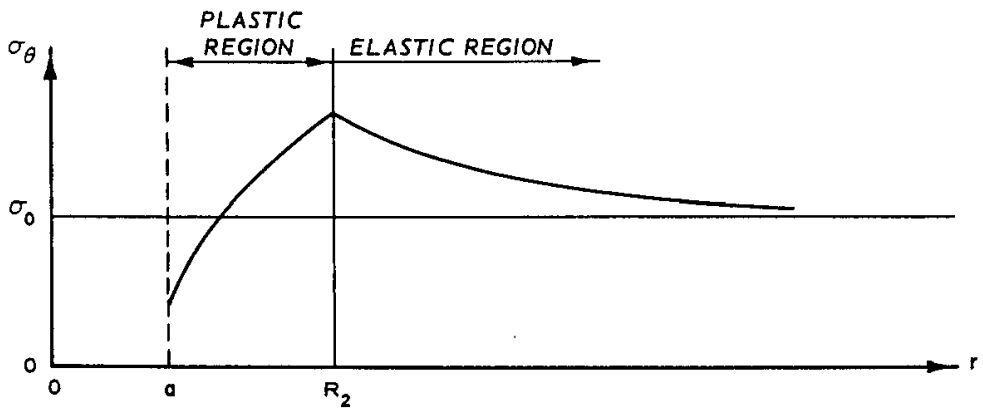

(b) ACTIVE ARCHING $-p<<\sigma_{0}$

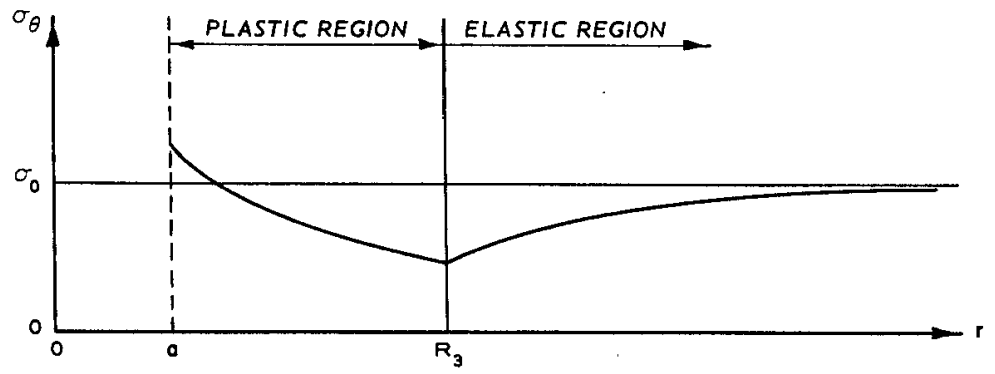

(c) PASSIVE ARCHING $-p>>\sigma_{0}$

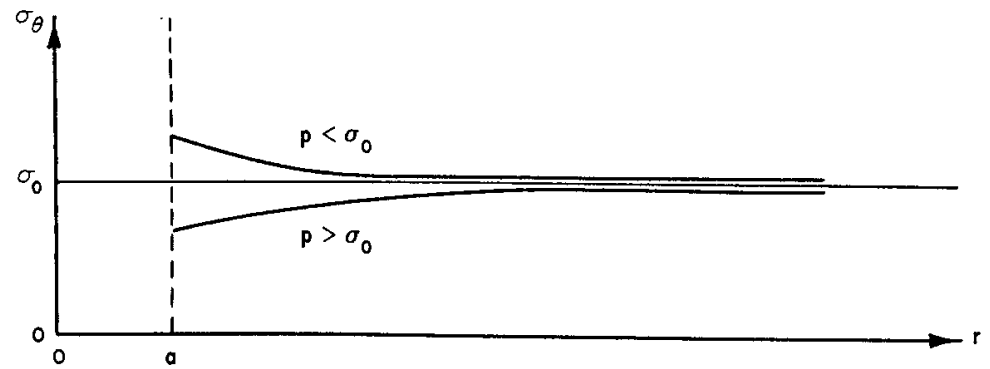

(d) ELASTIC SITUATION $-p \approx \sigma_{0}$

Fig. 5. Circumferential stress distribution from an elastoplastic solution by Sirieys as modified by Hendron (1968) 


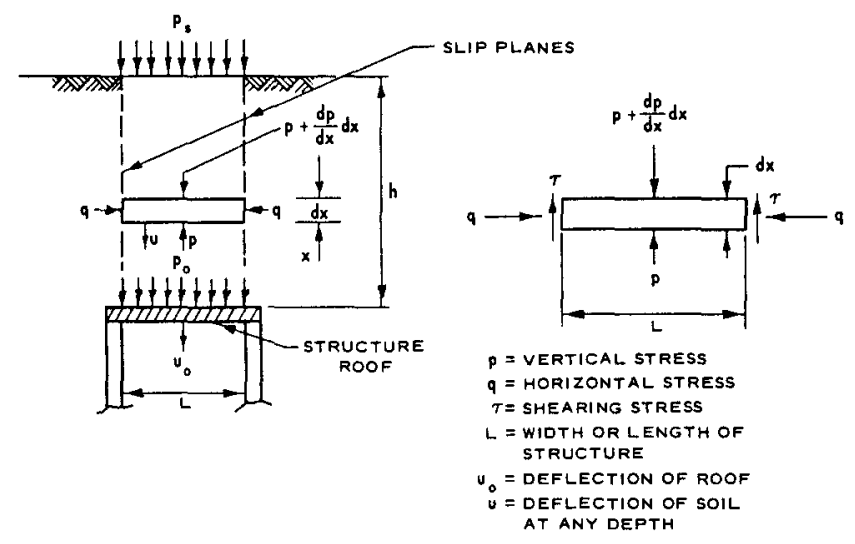

a. ASSUMED FORCE FIELD

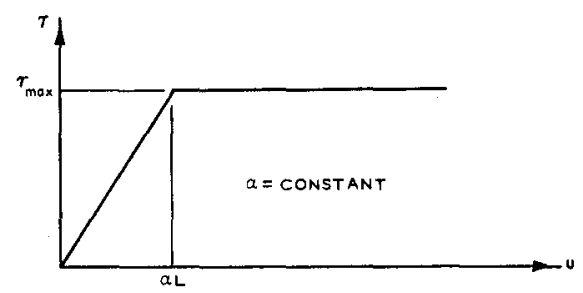

b. ASSUMED VARIATION OF SHEARING STRESS VERSUS DISPLACEMENT

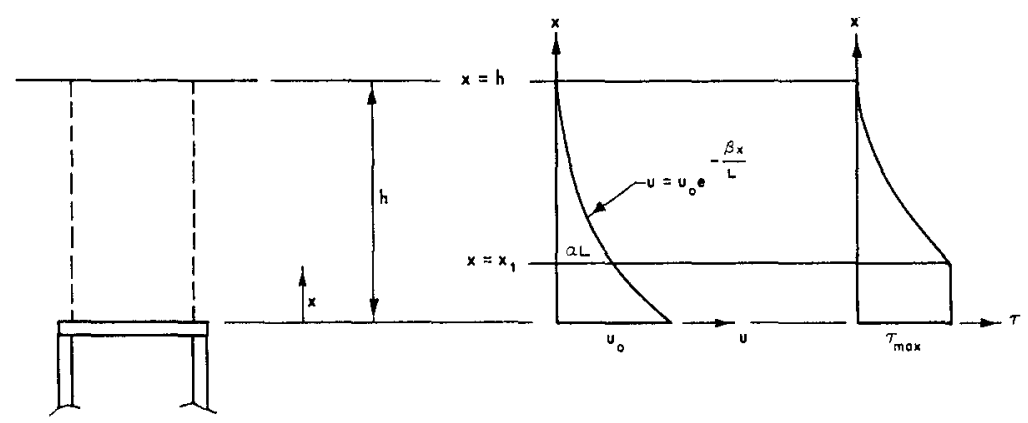

c. VARIATION OF DISPLACEMENT AND SHEARING STRESS WITH DEPTH

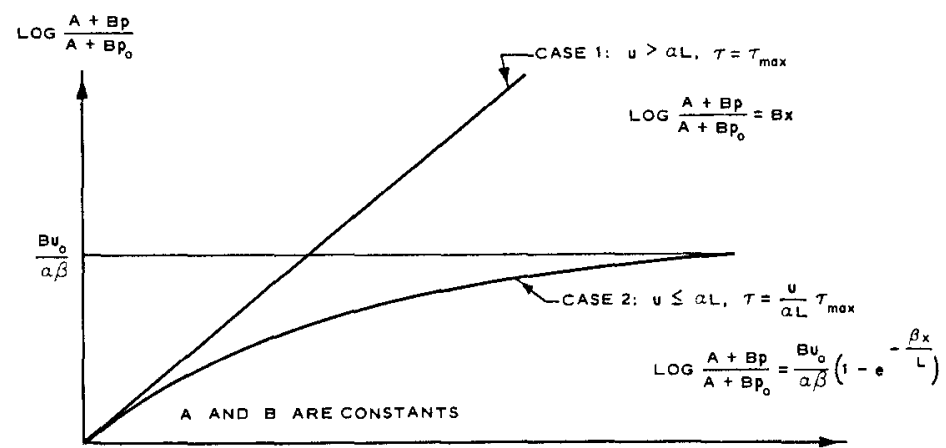

d. VARIATION OF STRESS WITH DEPTH

Fig. 6. Calculation of arching loads by Newmark and Haltiwanger 


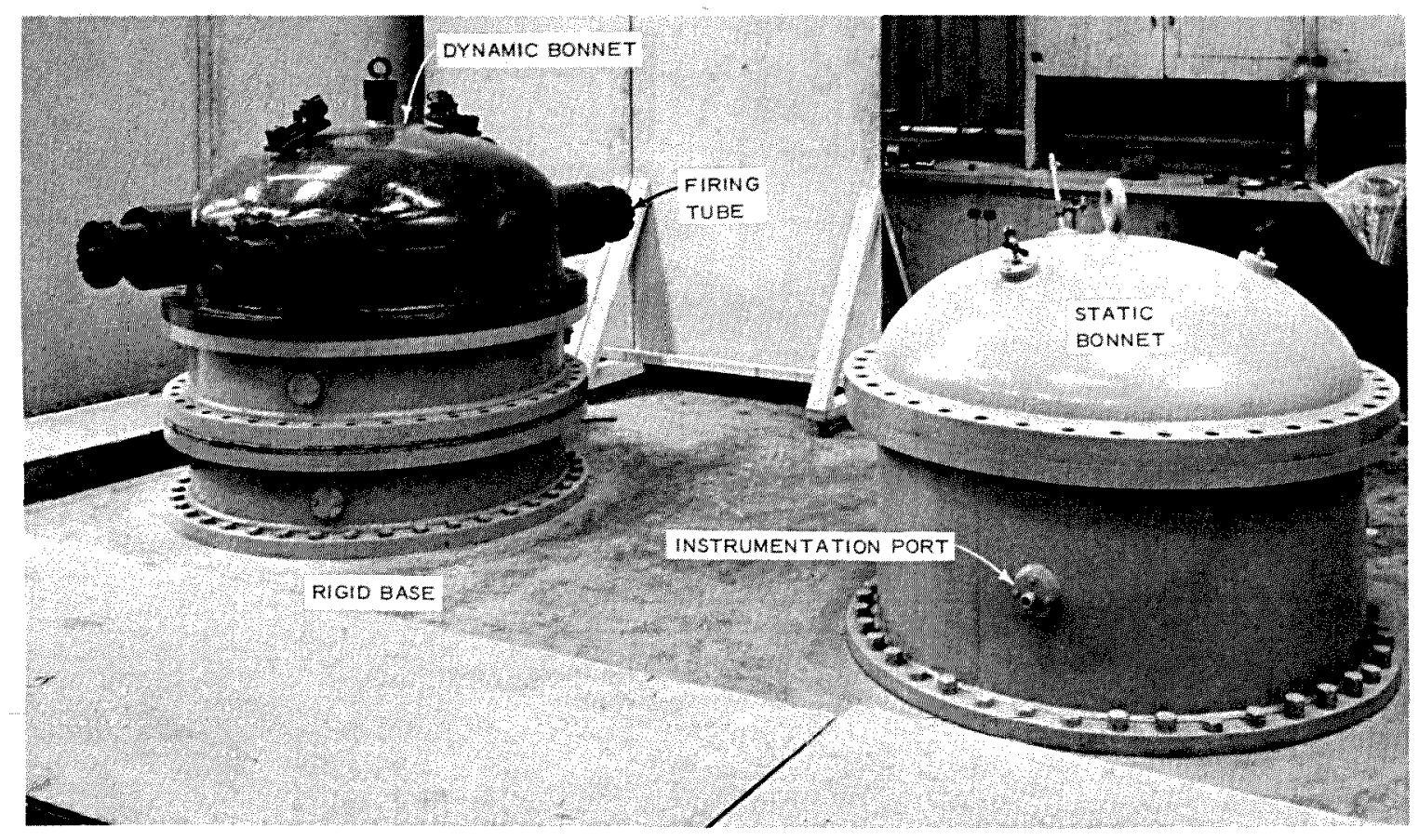

Fig. 7. Small Blast Load Generator facilities (SBIG)

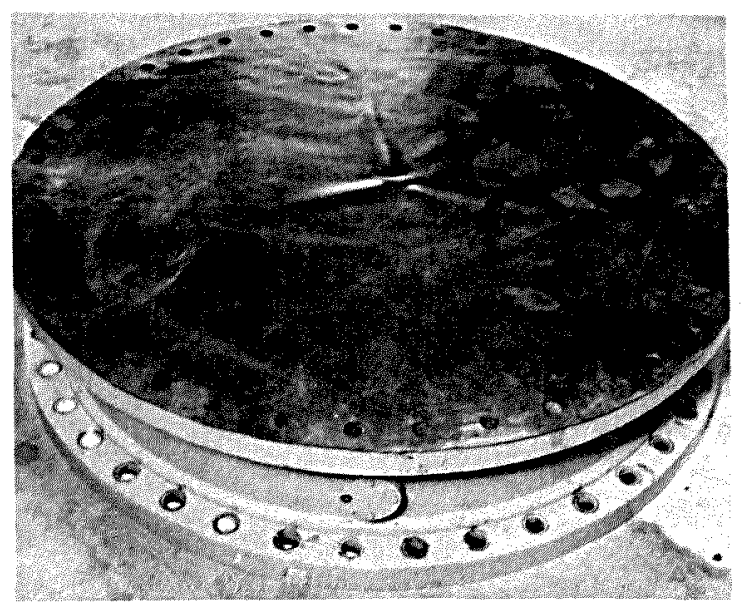

a. Normal diaphragm

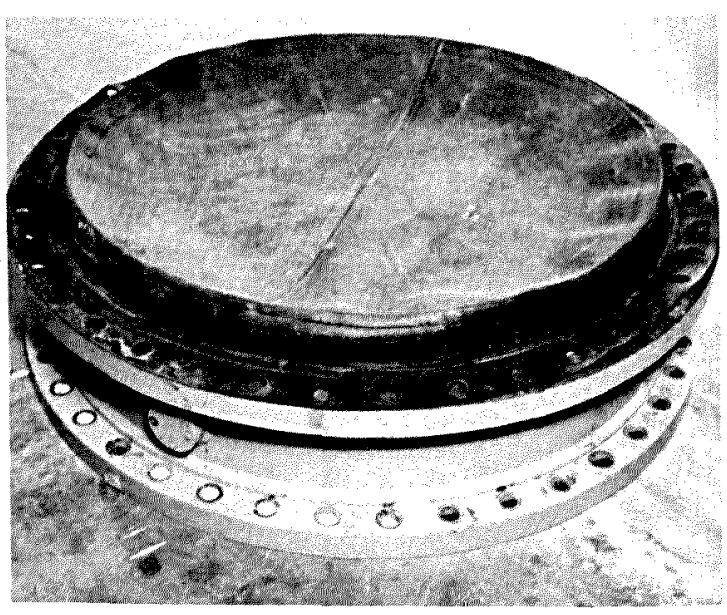

b. "Rolled top" diaphragm

Fig. 8. Protective diaphragms 

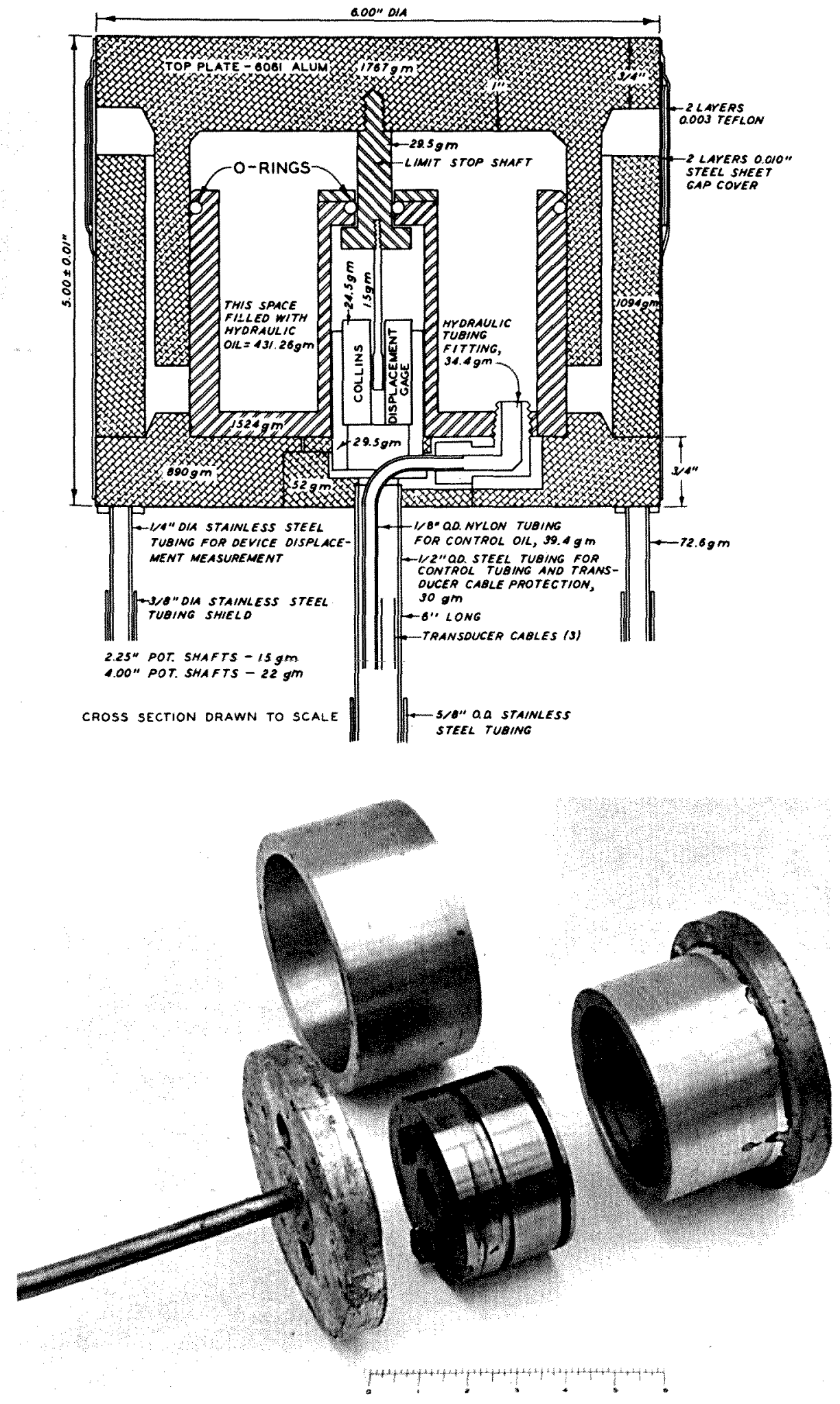

Fig. 9. Hydraulically controlled test device 


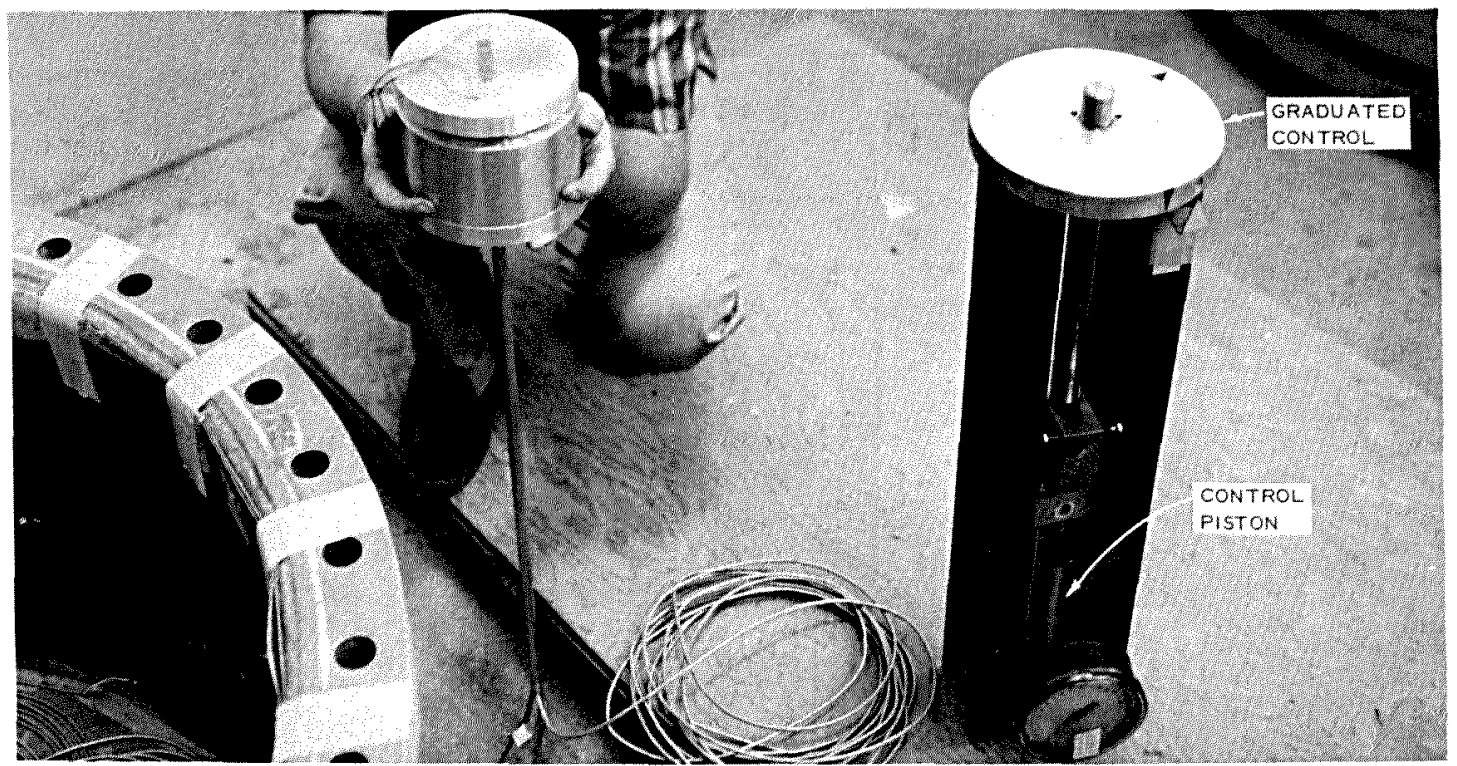

Fig. 10. Hydraulically controlled test device and control piston

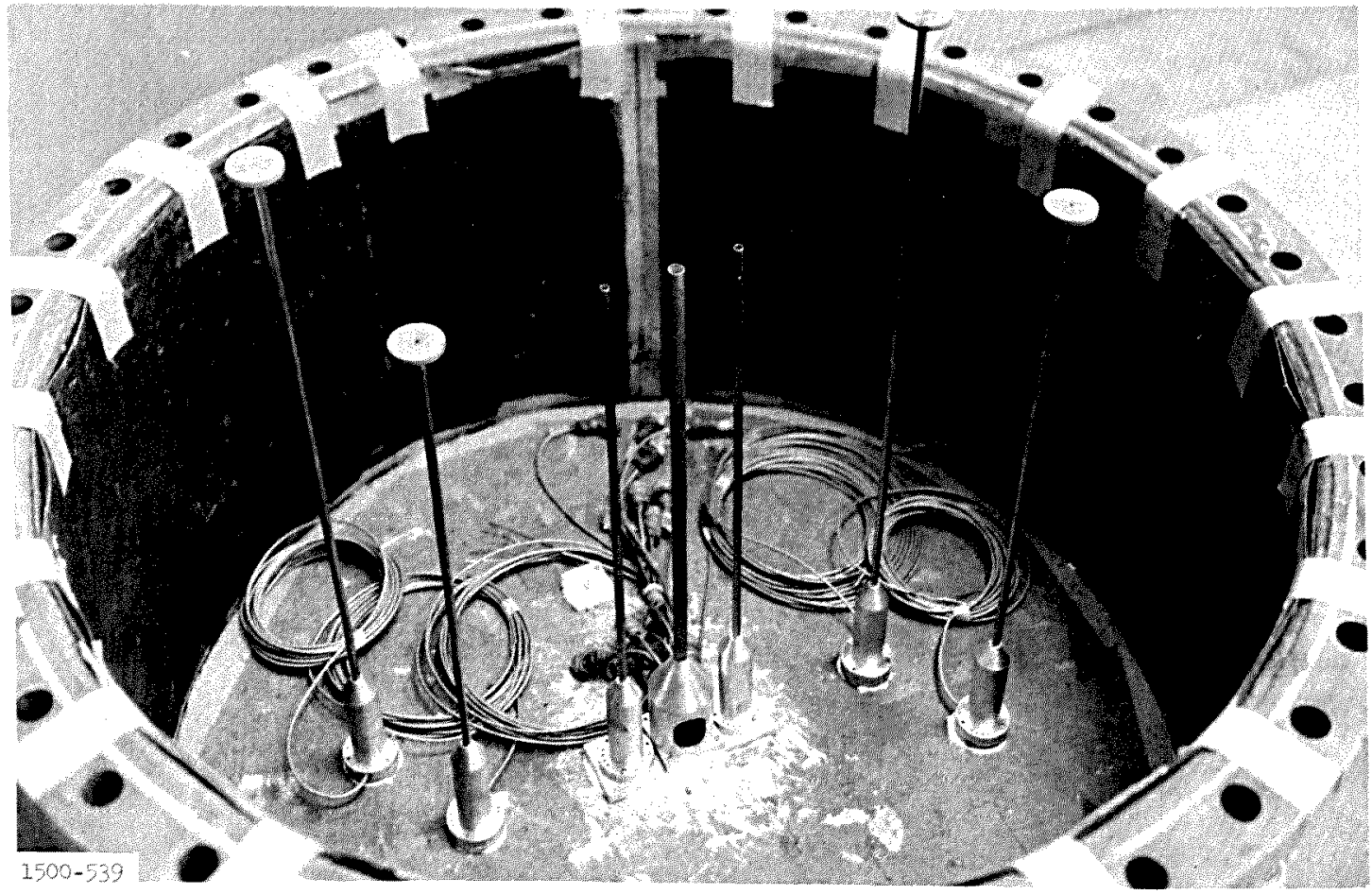

Fig. 11. Base of test chamber with deflection control rods and conduits installed 

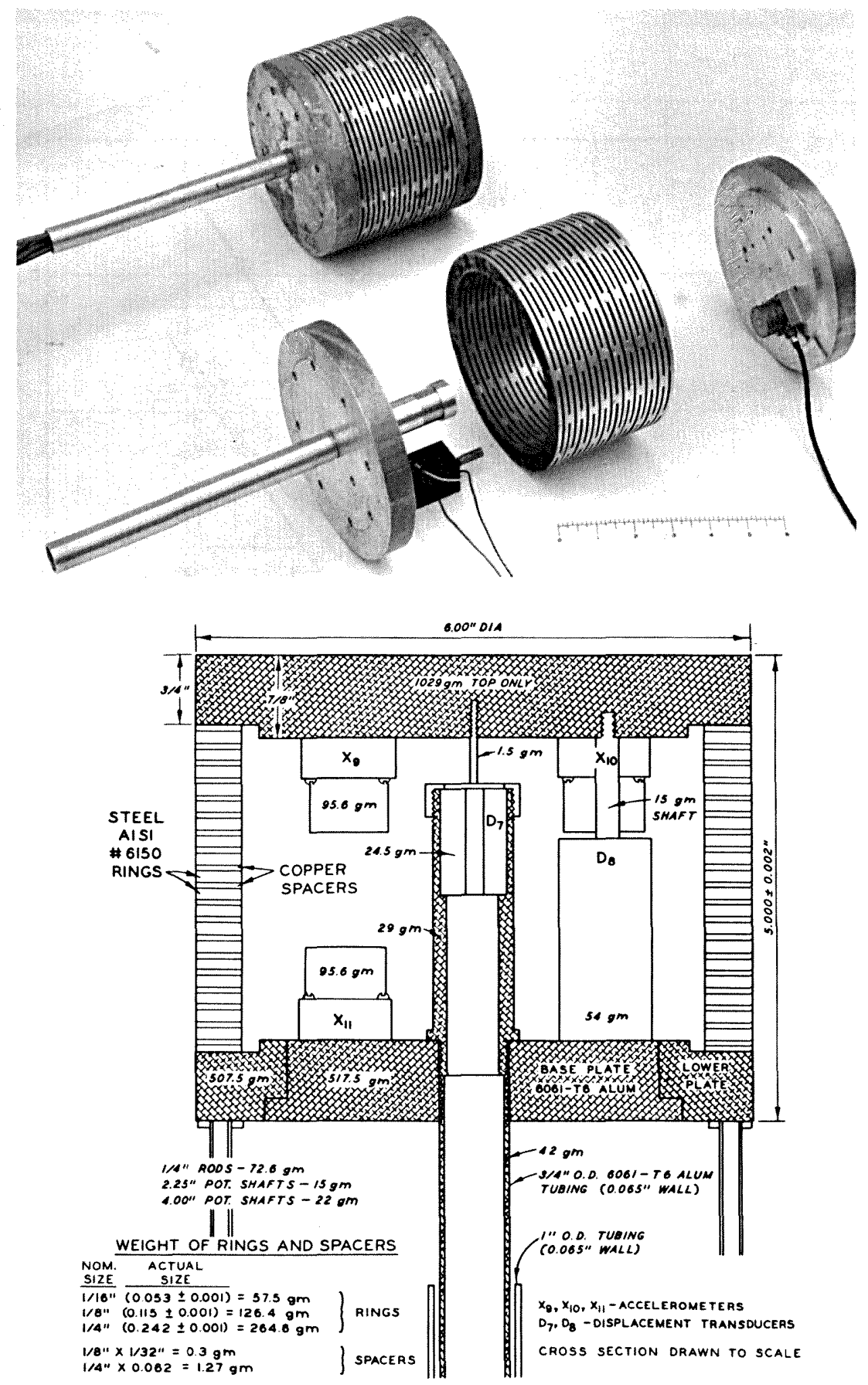

Fig. 12. Spring-ring test device 


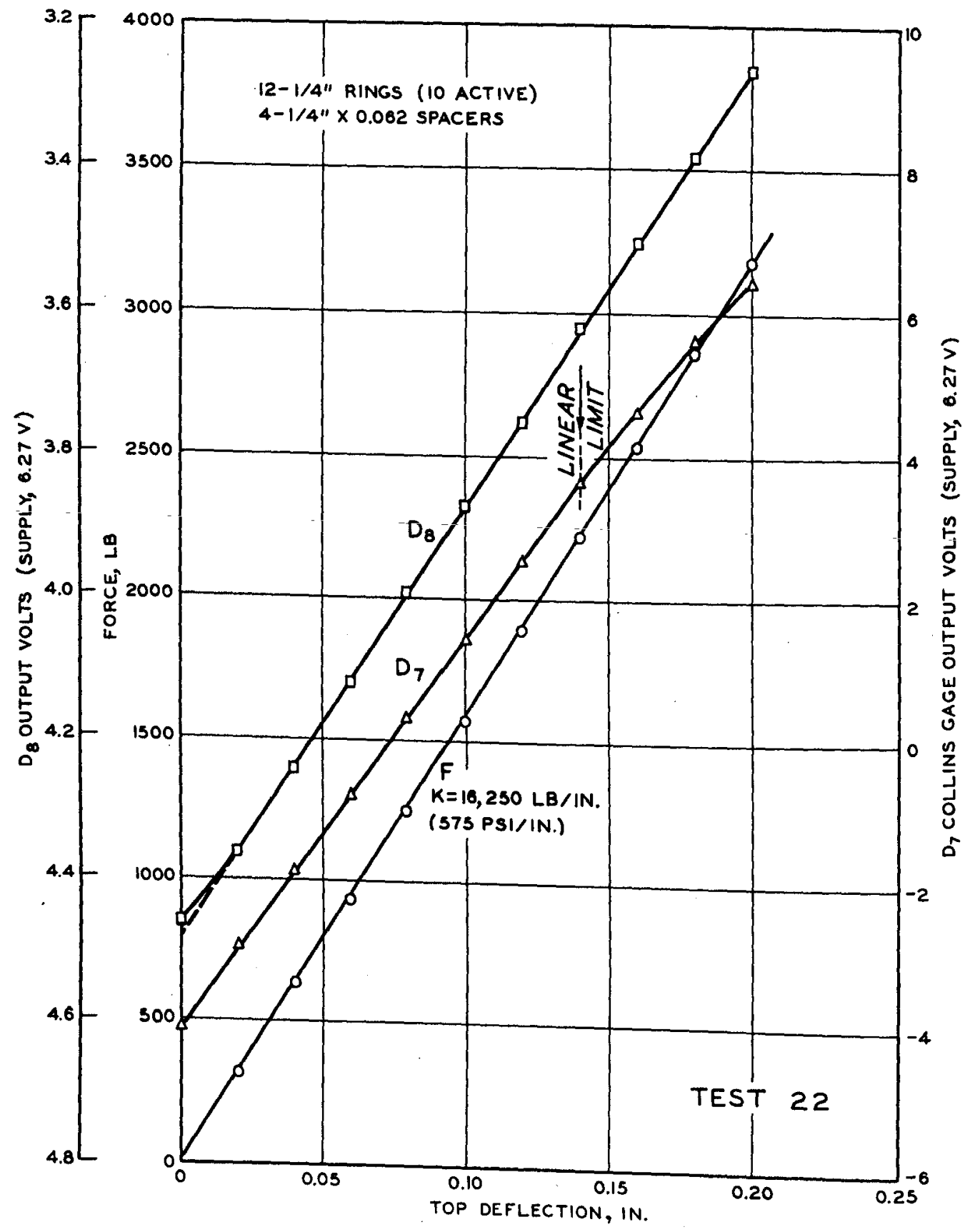

Fig. 13. Typical static calibration curve for spring-ring test device 


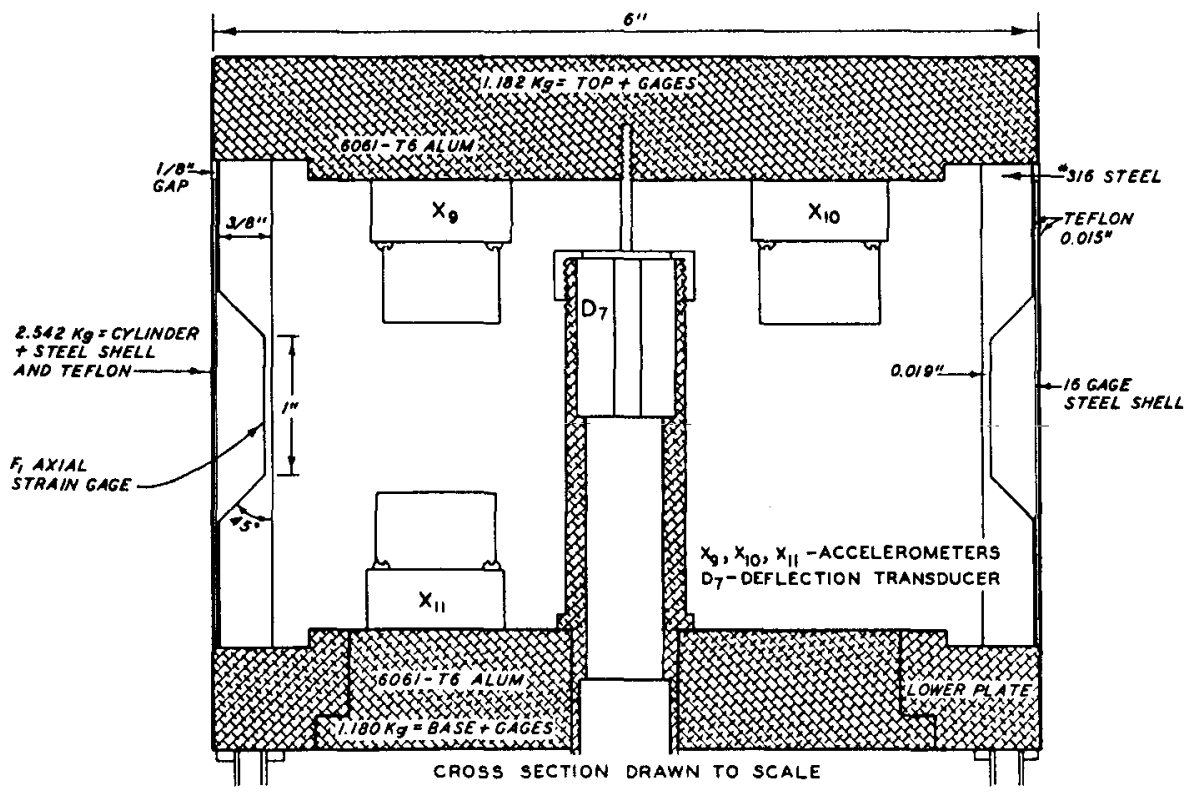

Fig. 14. "Rigid" test device 


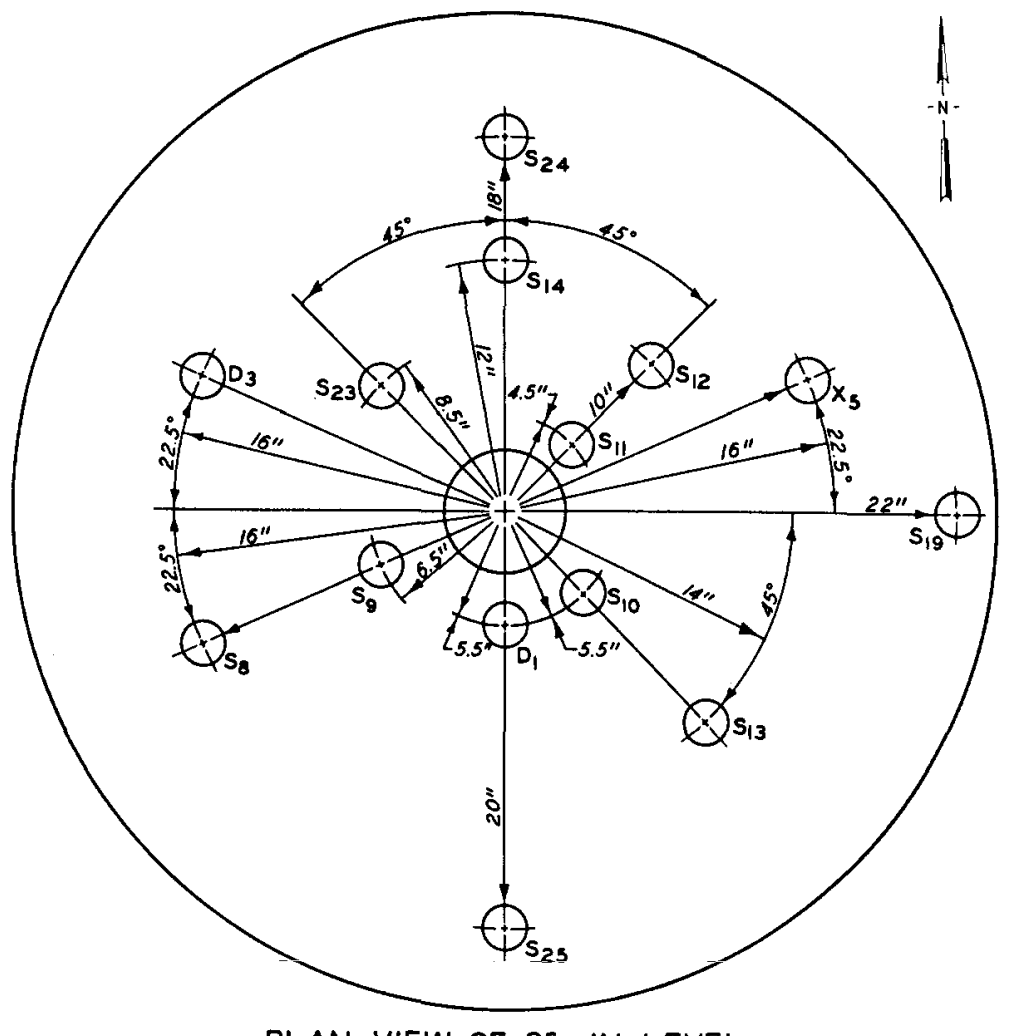

PLAN VIEW OF $35-1 N$. LEVEL

$77 "$ SURFACE LEVEL

D= DEFLECTION GAGE

$S=S O I L$ PRESSURE GAGE

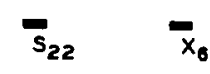

$71^{\prime \prime}$

$X=A C C E L E R O M E T E R$

$P_{3}=B O N N E T$ PRESSURE GAGE

$P_{I}=$ PRESSURE INSIDE TEST DEVICE

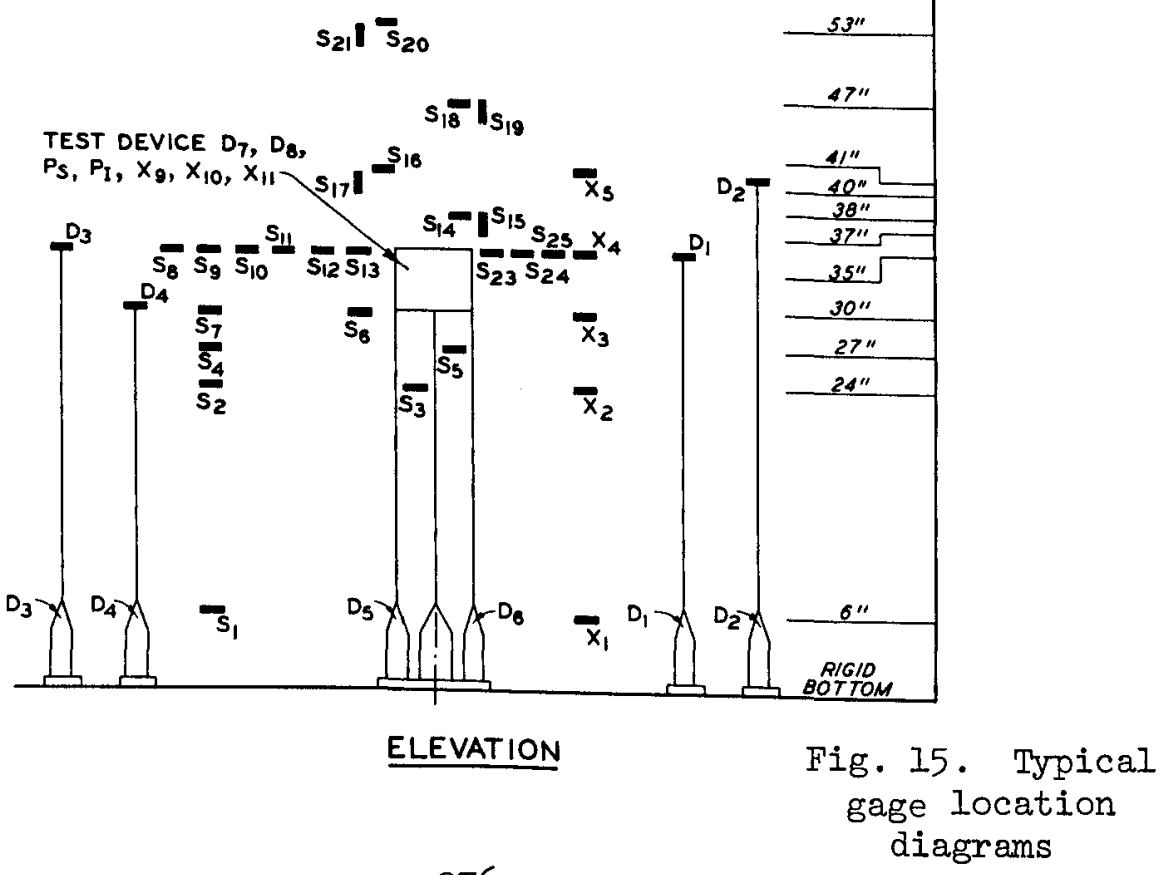




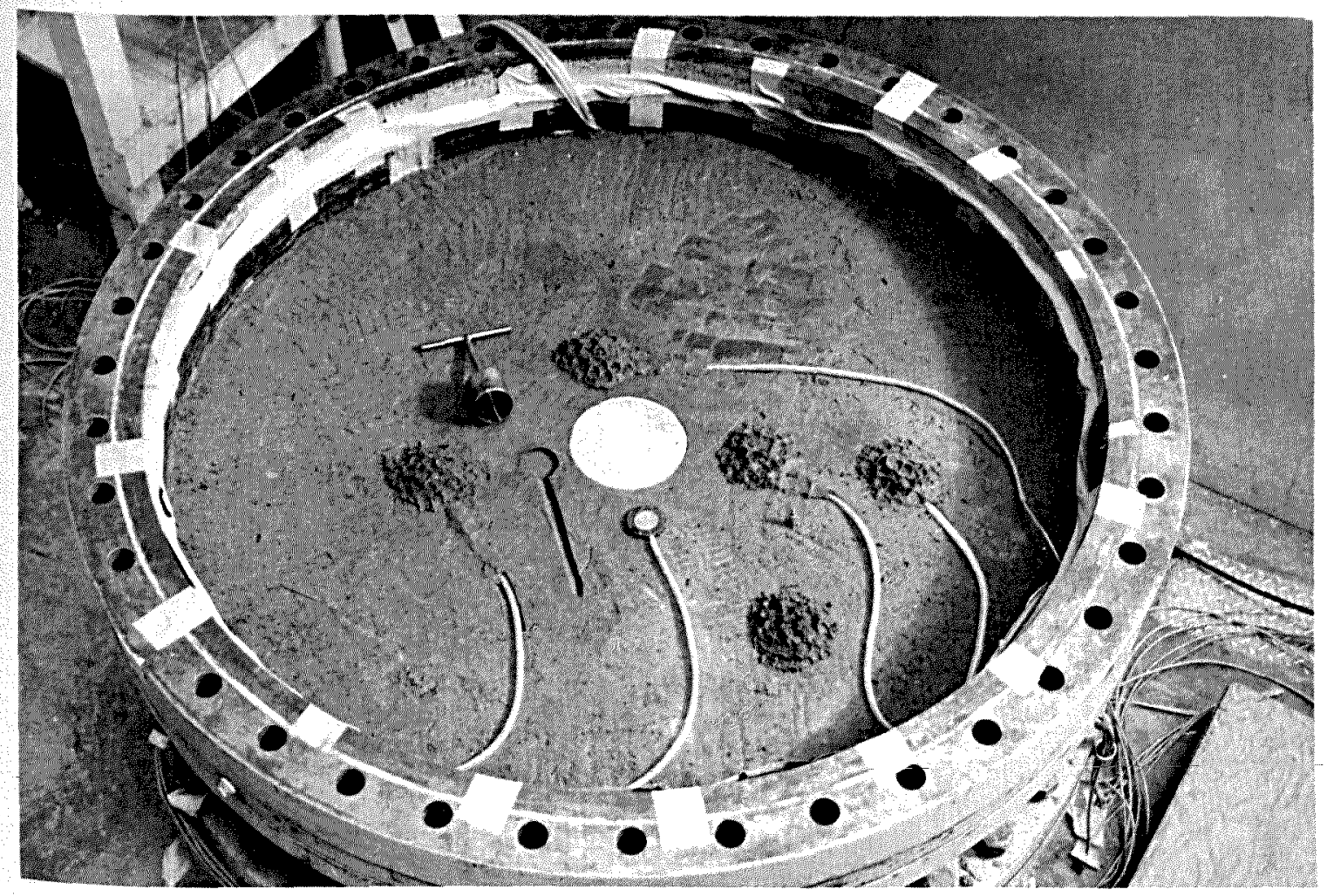

Fig. 16. Soil pressure gage placement at the 35-in. level

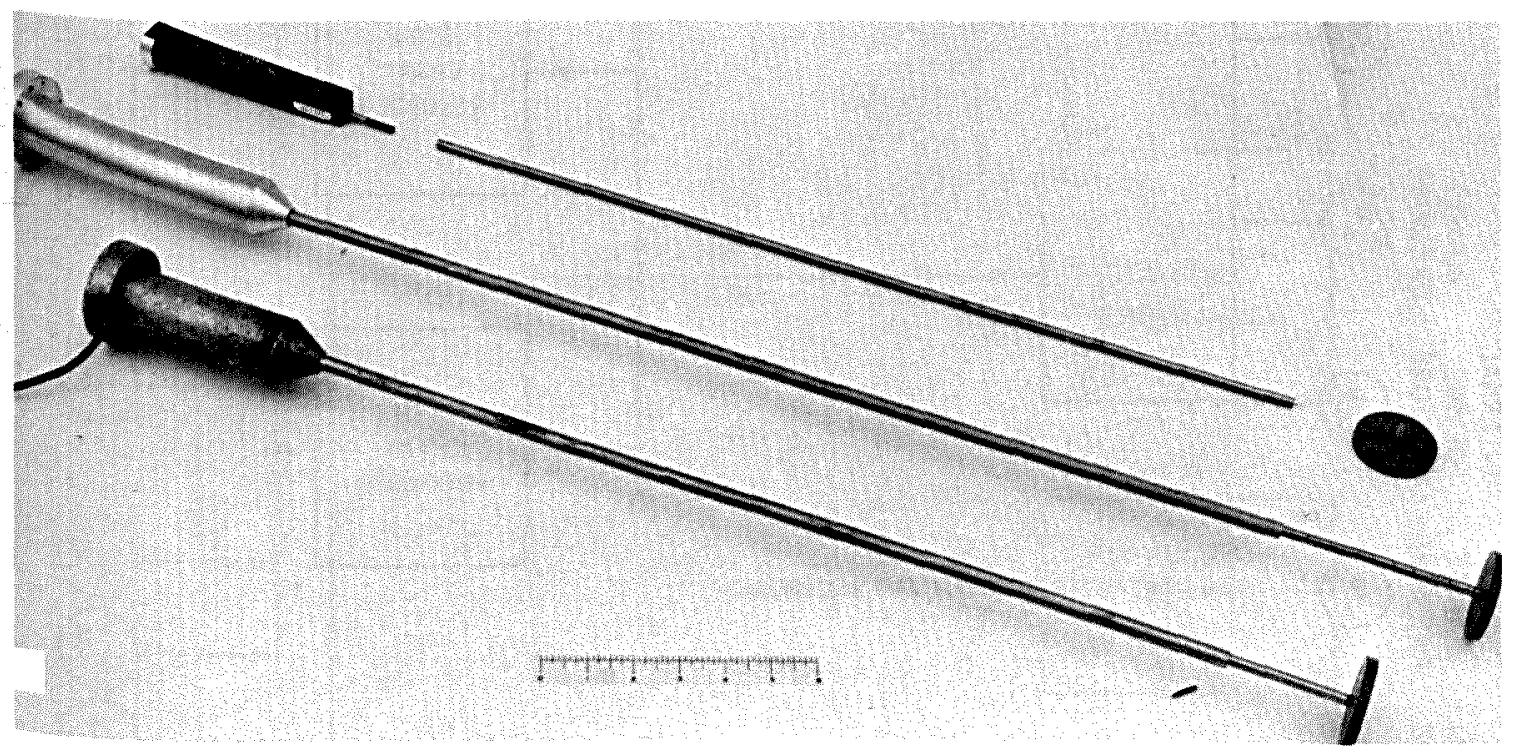

Fig. 17. WES soil deflection measuring device 

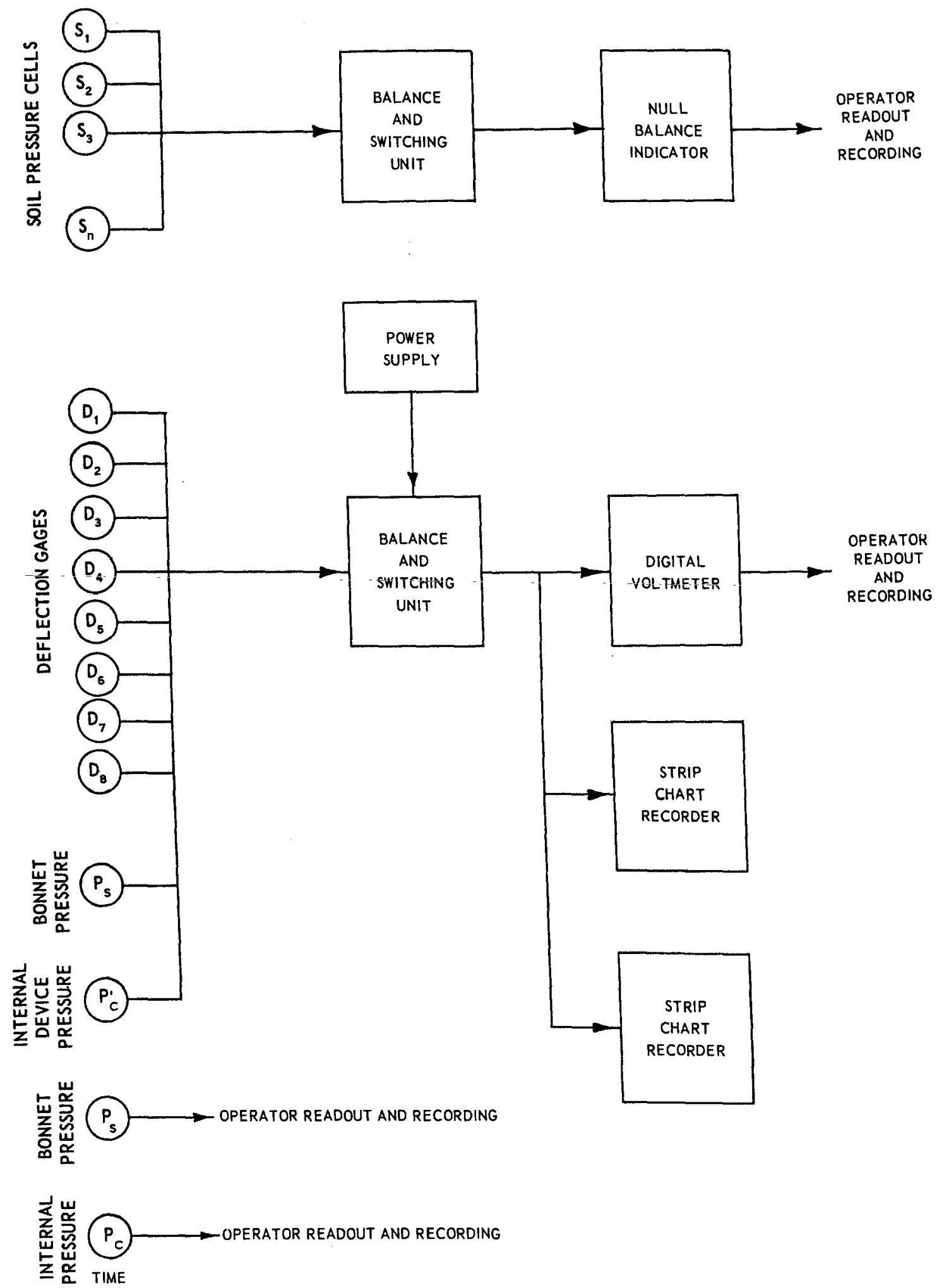

Fig. 18. Block diagram of measurement and control system used for static tests 


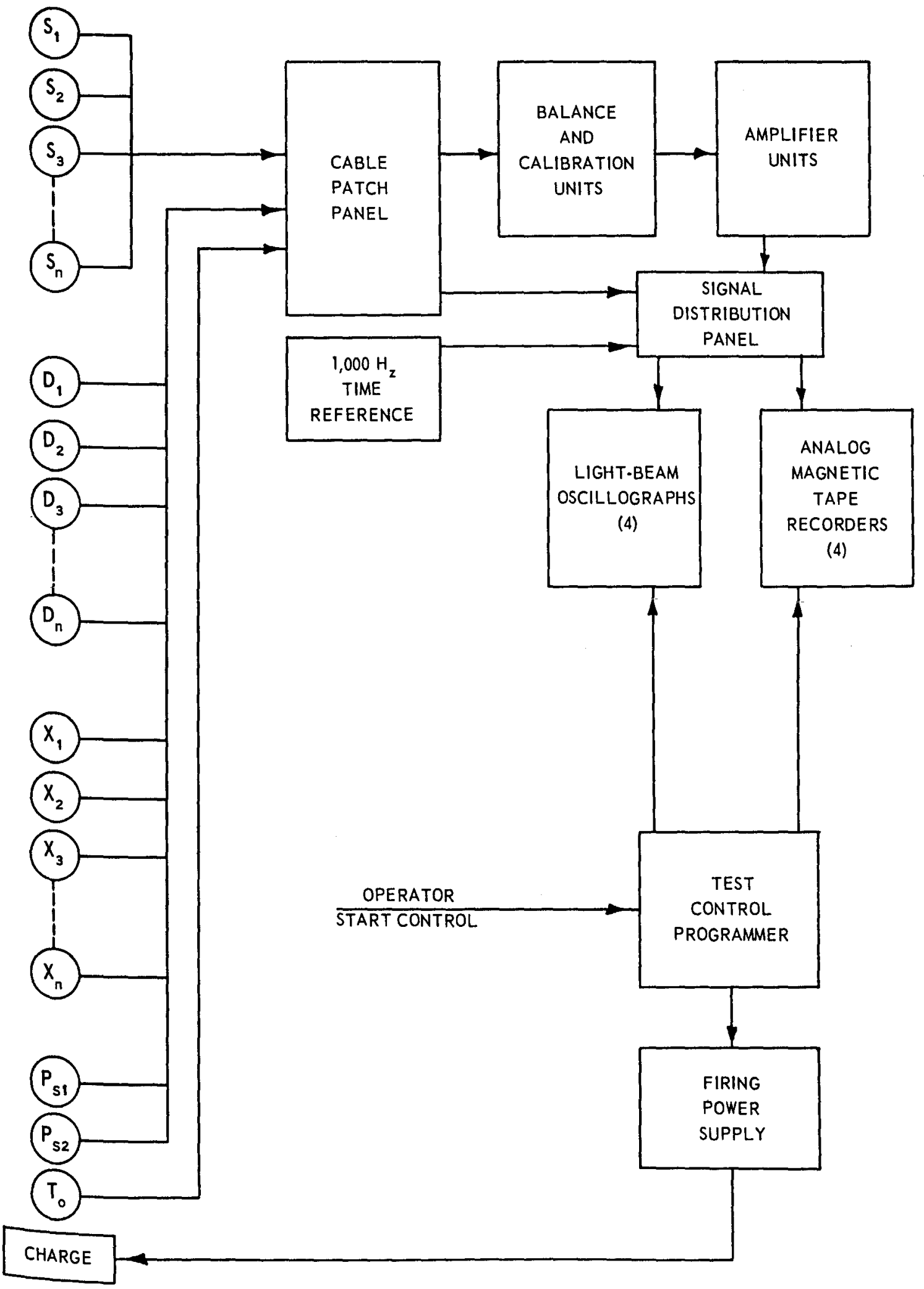

Fig. 19. Block diagram of measurement and control system used for dynamic tests 

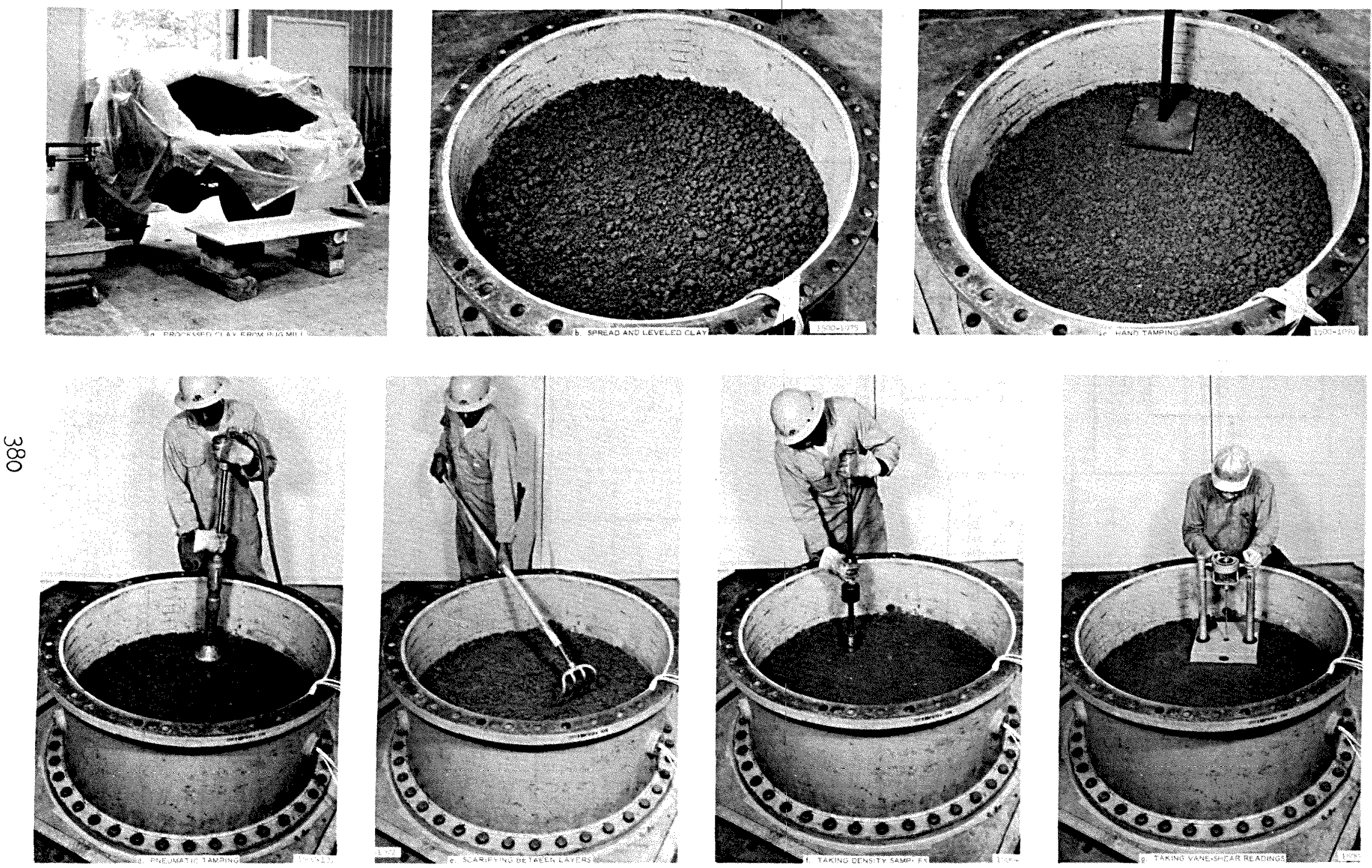

Fig. 20. Placement of buckshot clay in WES Small Blast Load Generator 

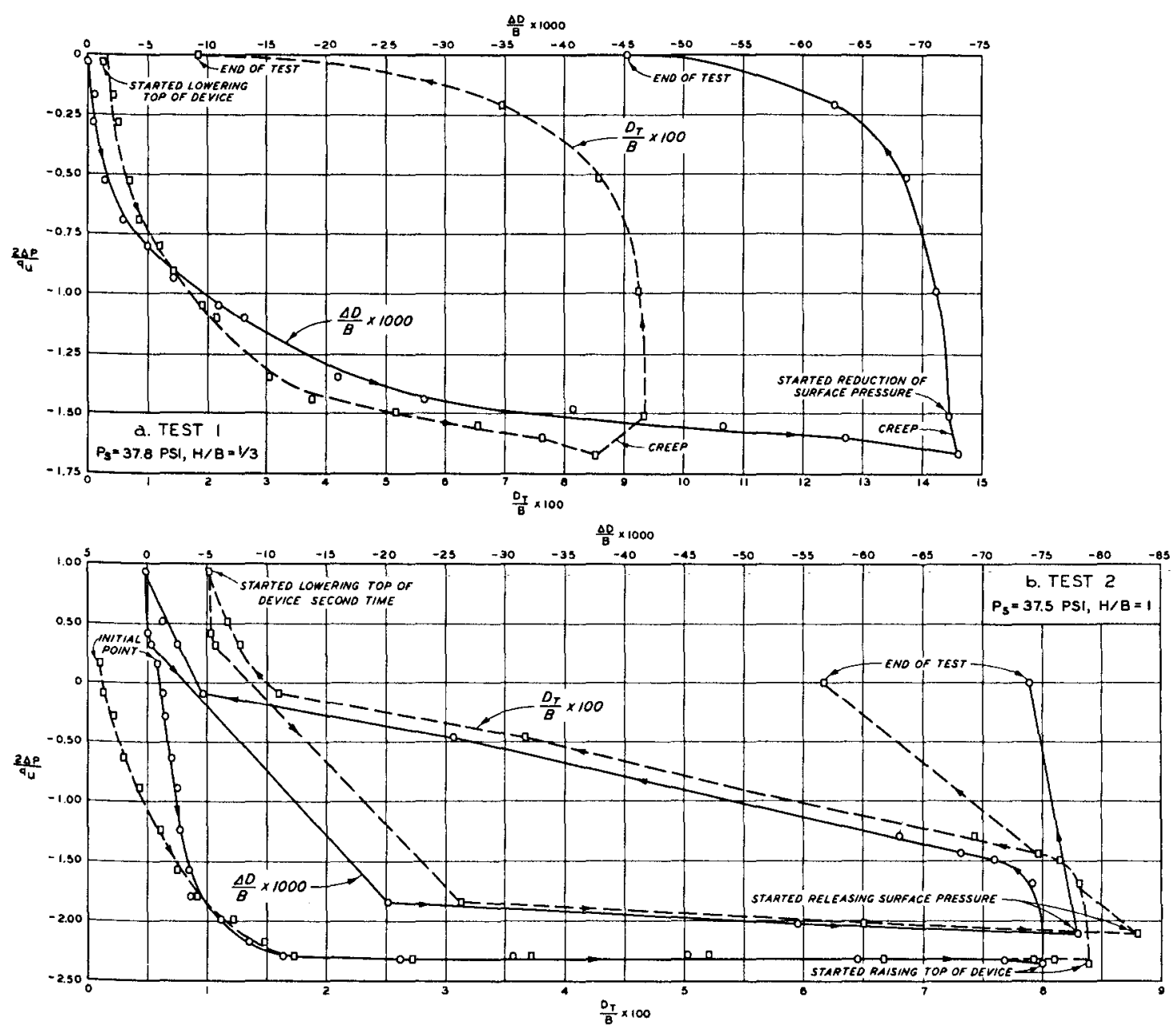

Fig. 2l. Dimensionless plot of pressure versus deflection for static Tests 1 and 2 

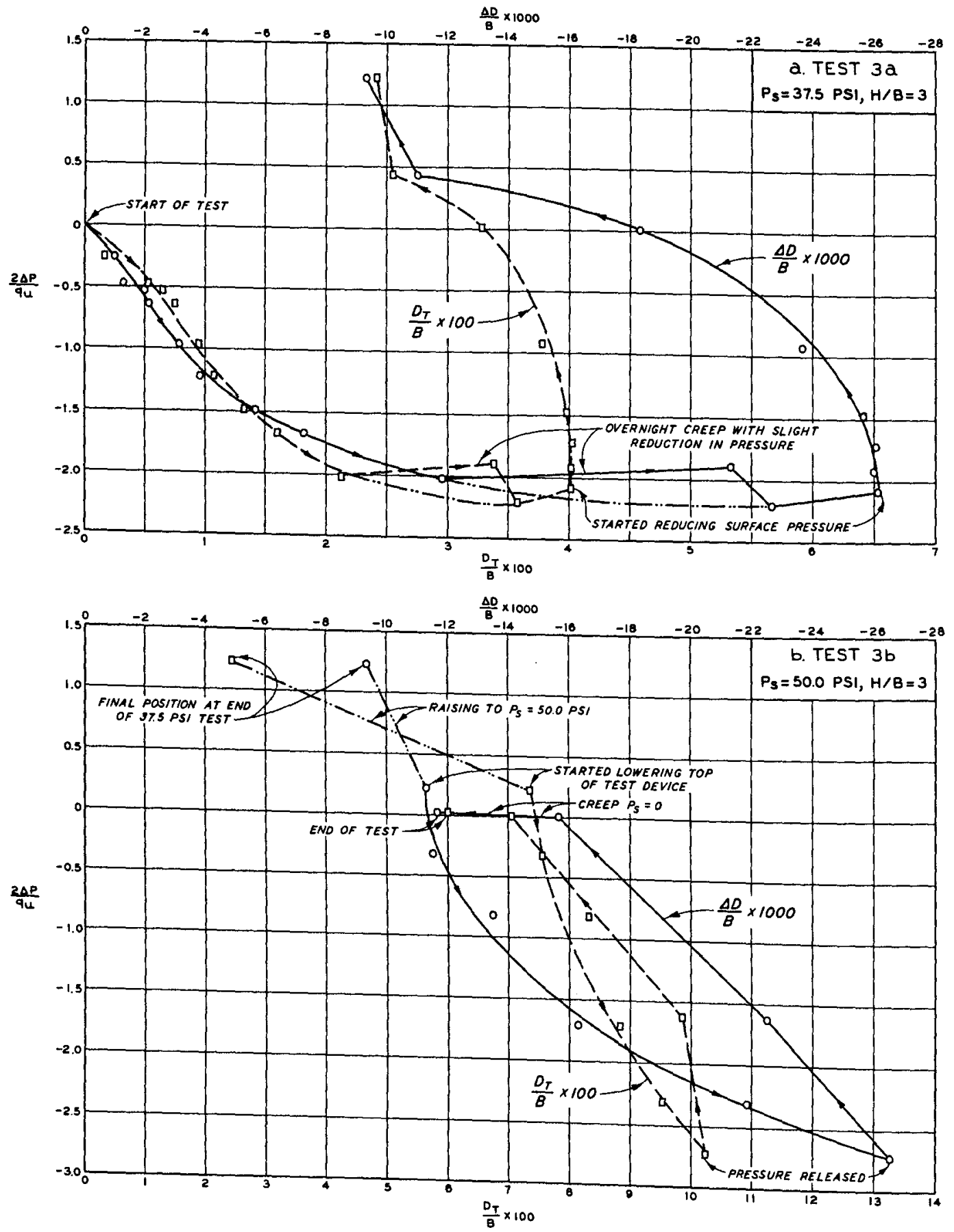

Fig. 22. Dimensionless plot of pressure versus deflection for static Test 3 


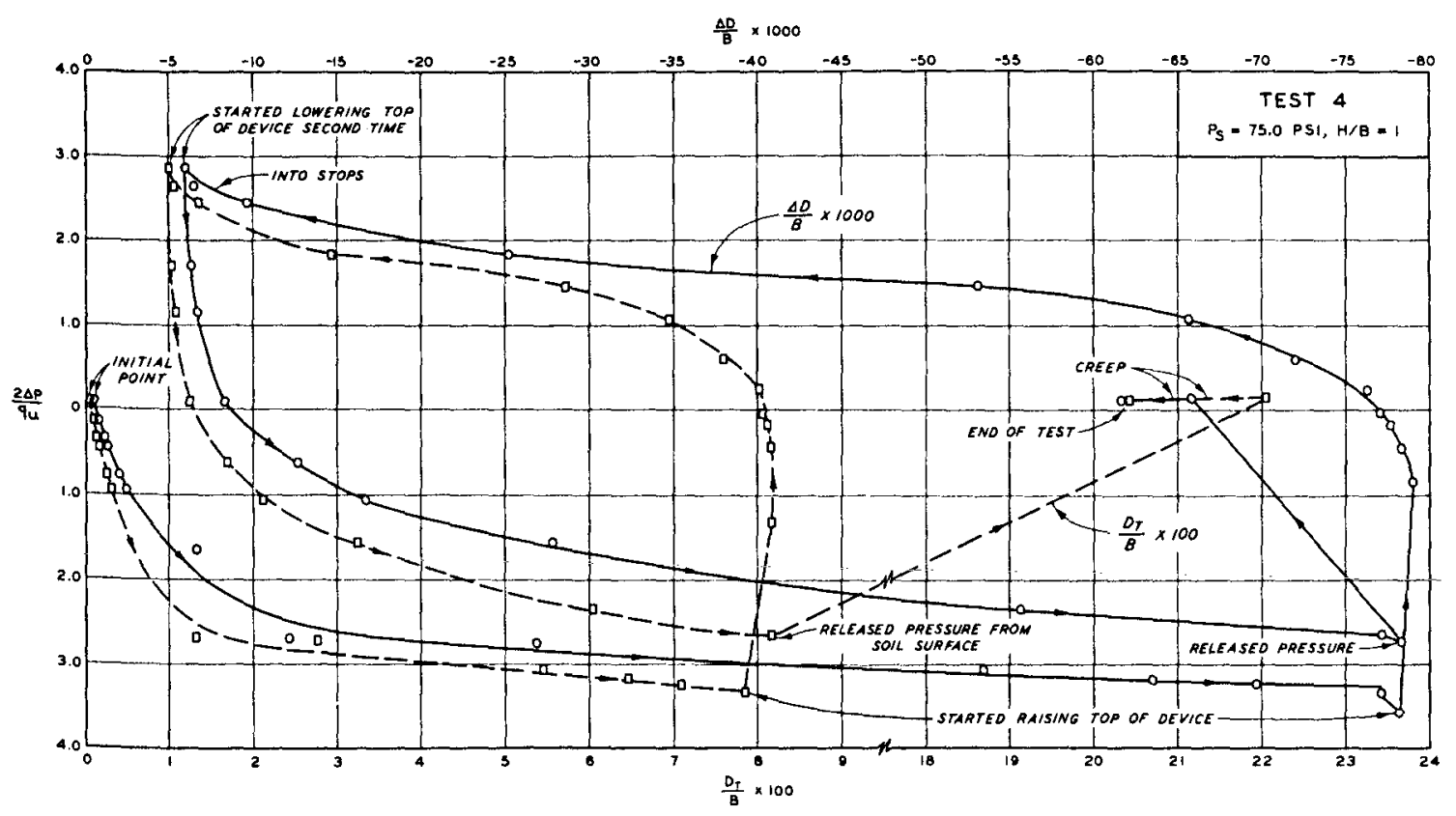

Fig. 23. Dimensionless plot of pressure versus deflection for static Test 4 

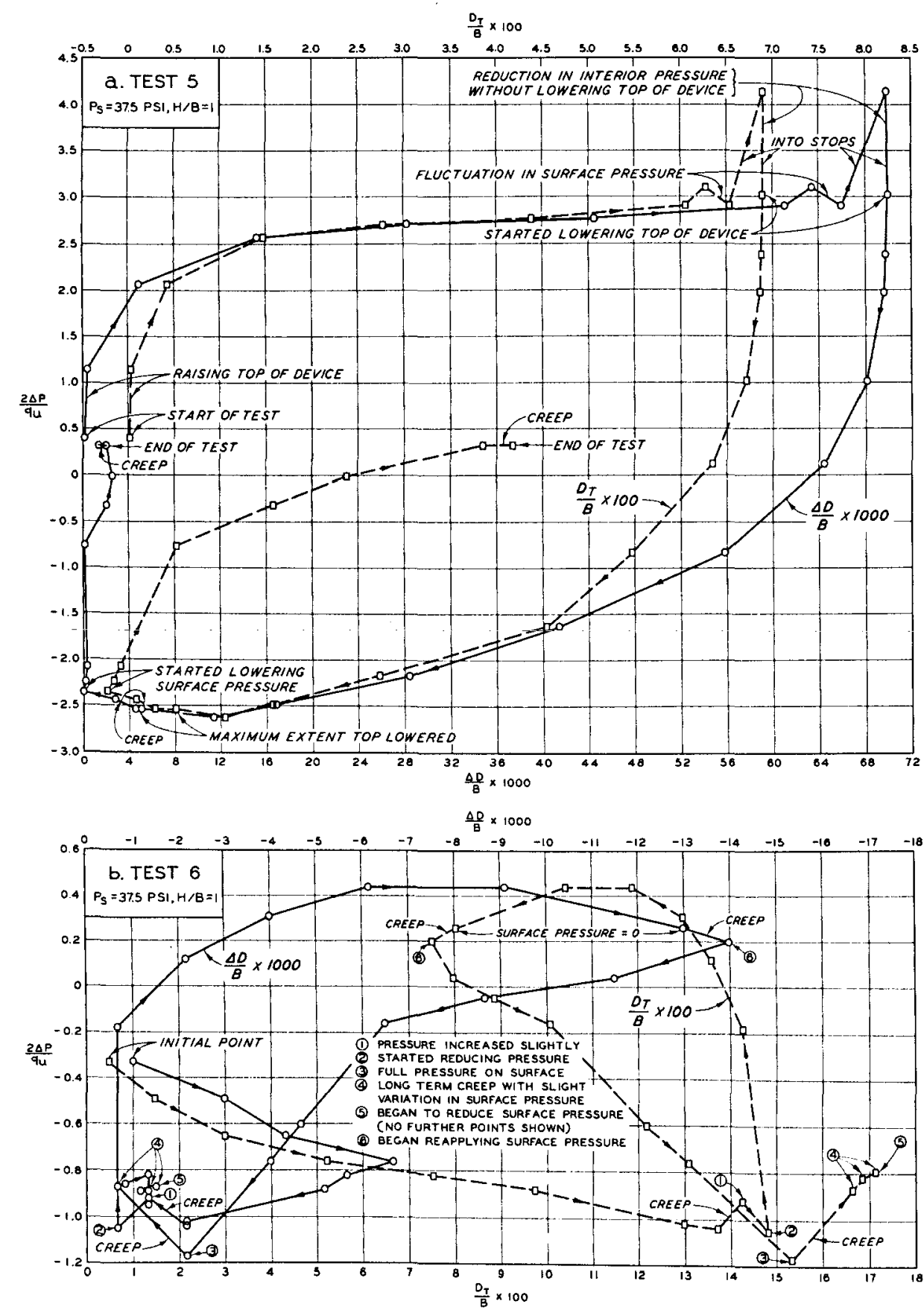

Fig. 24. Dimensionless plot of pressure versus deflection for static Tests 5 and 6 

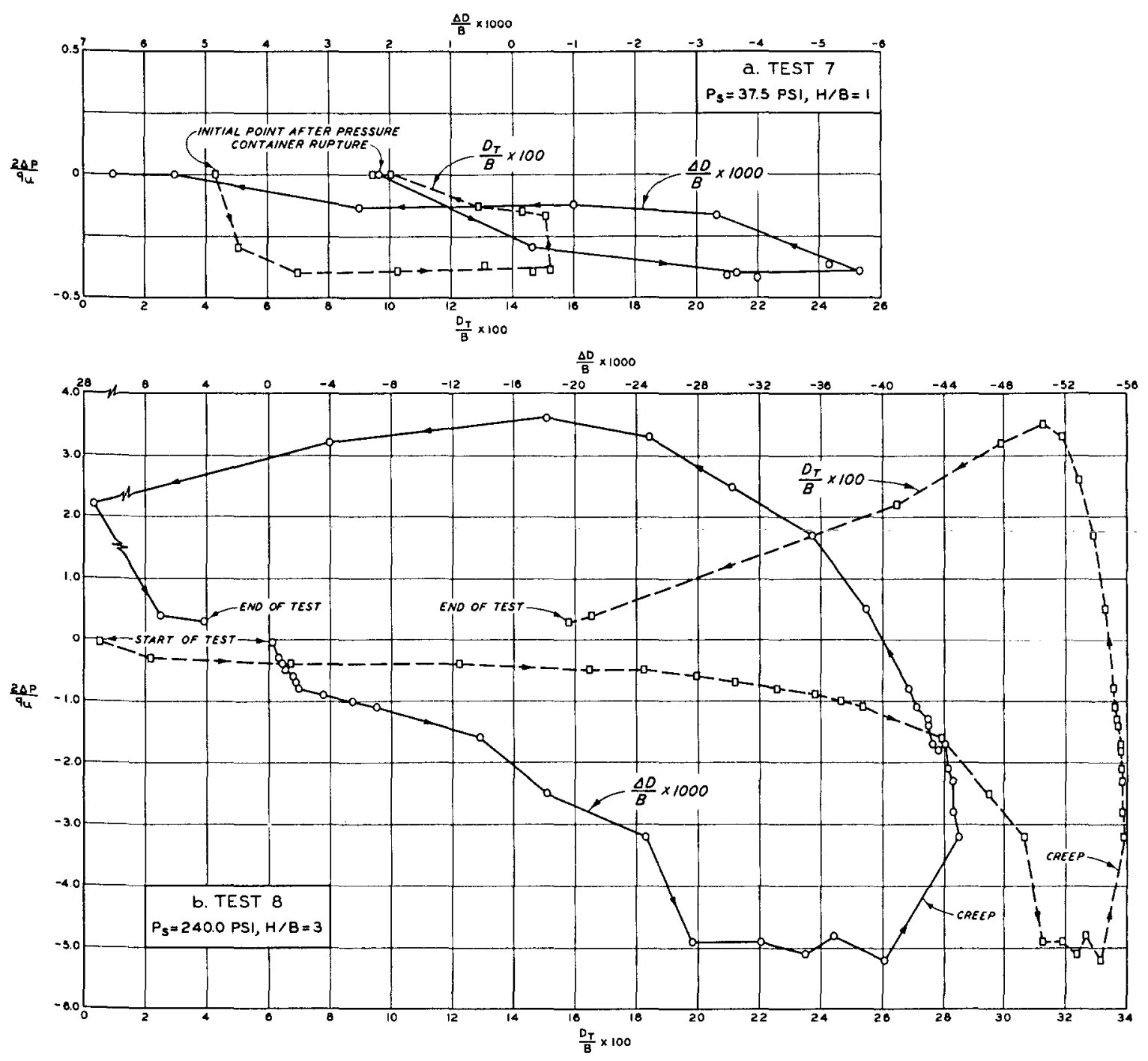

Fig. 25. Dimensionless plot of pressure versus deflection for static Tests 7 and 8 
a. TEST 1 - ACTIVE, $H / B=1 / 3$

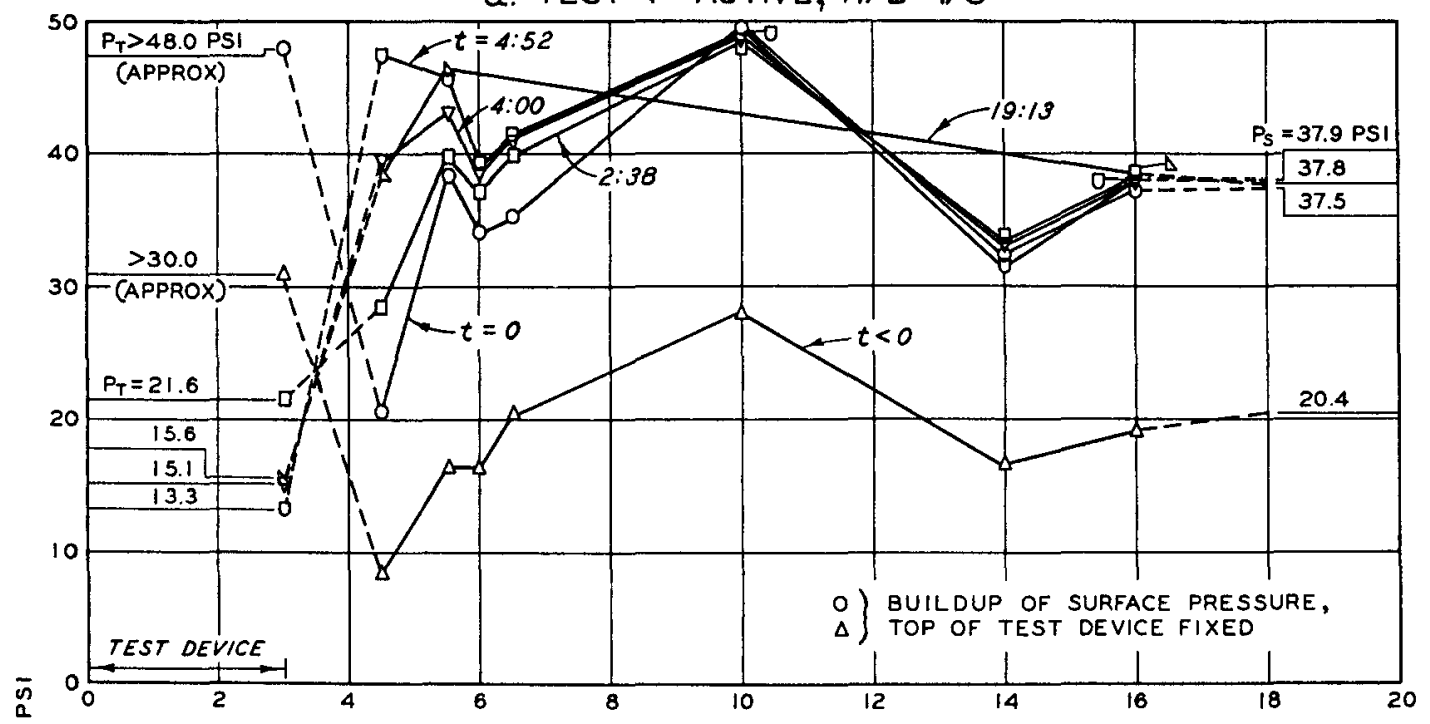

b. TEST 2 - ACTIVE, $H / B=1$

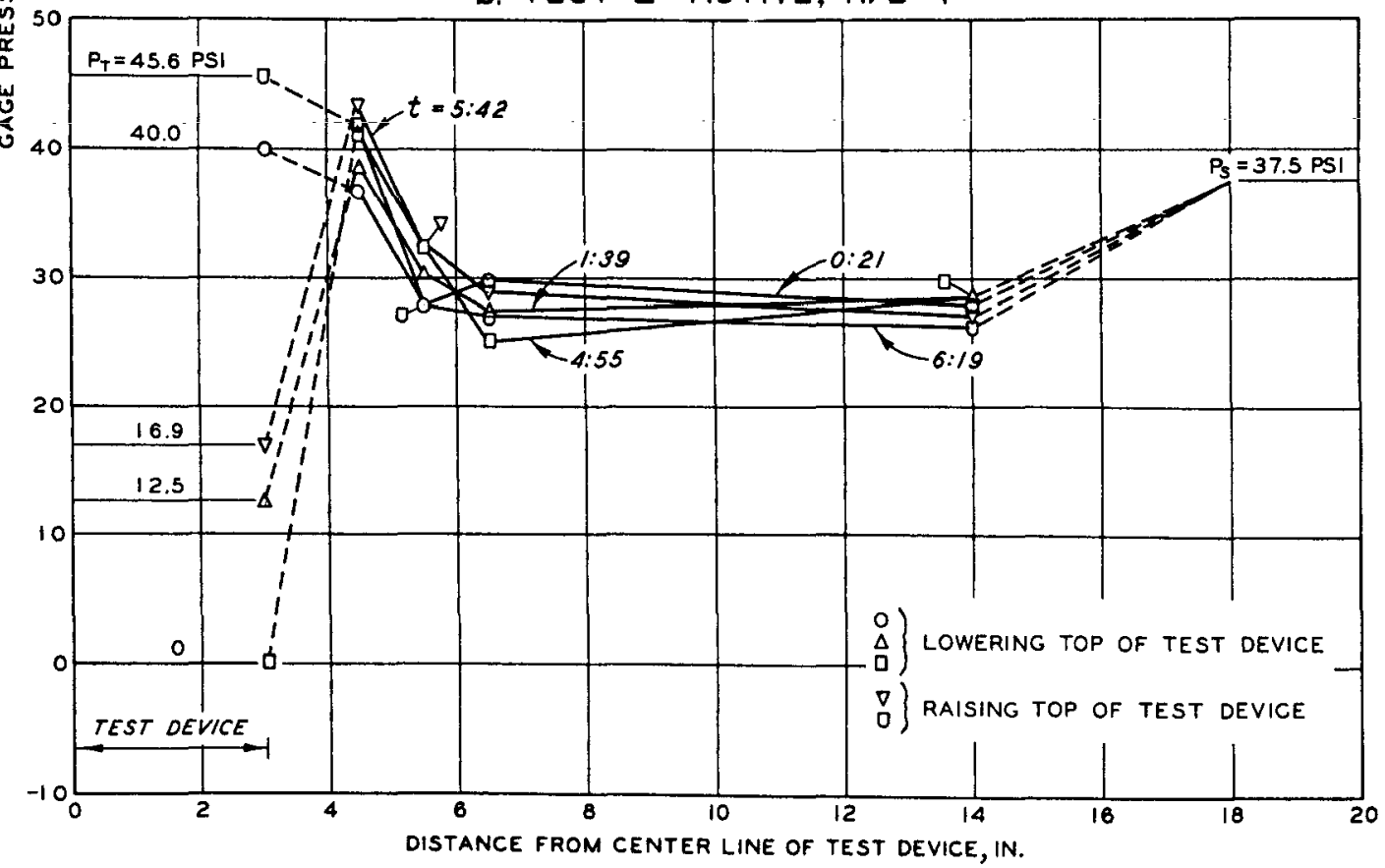

Fig. 26. Change in vertical soil stress at the 35-inch level due to deflection of the top of test device, Tests 1 and 2 
a. TEST 3 - ACTIVE, H/B $=3$

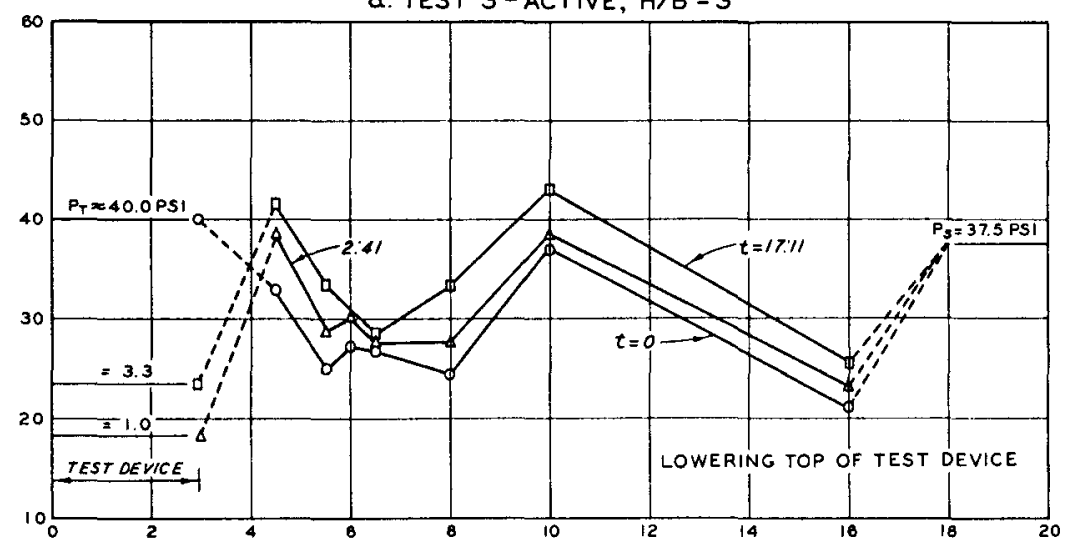

b. TEST 3 -PASSIVE, $H / B=3$

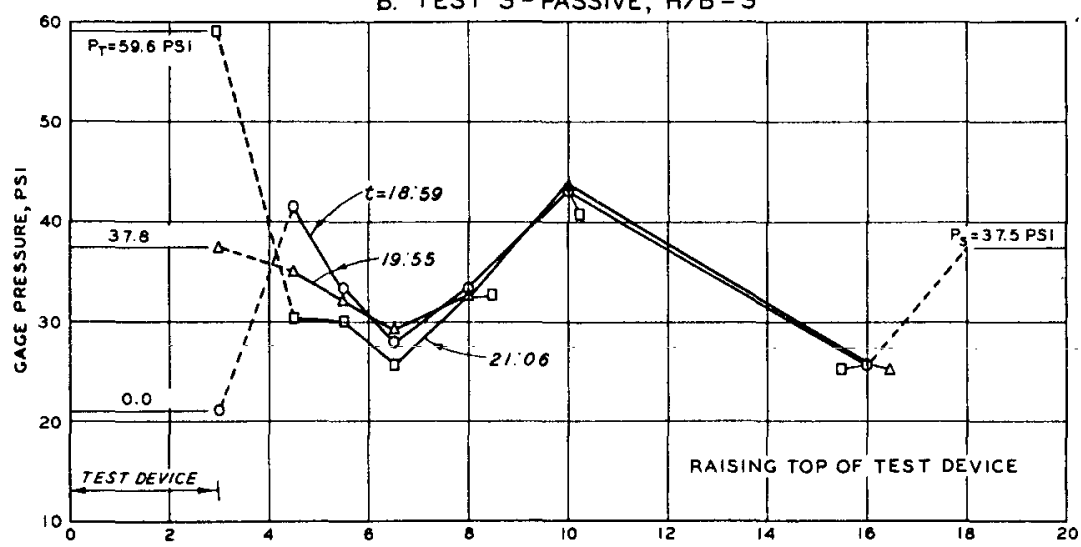

c. TEST 4-ACTIVE, $H / B=1$

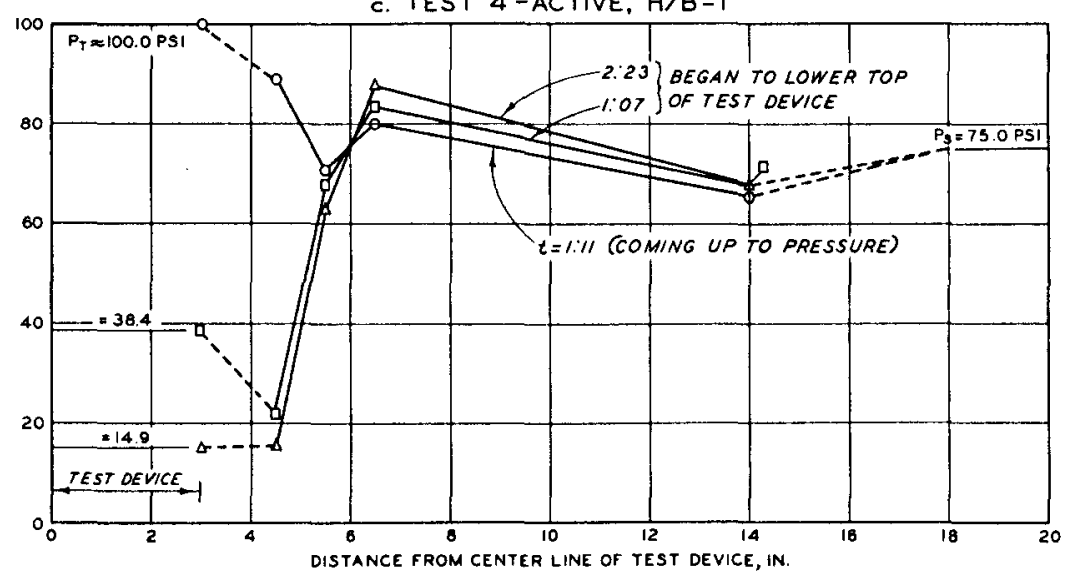

Fig. 27. Change in vertical soil stress at the 35-inch level due to deflection of the top of test device, Tests 3 and 4 
a. TEST 6 -ACTIVE, $H / B=1$

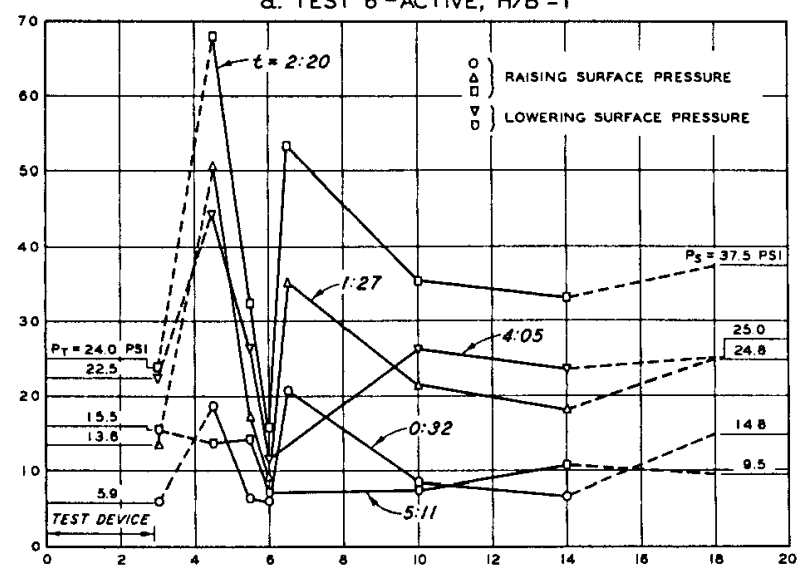

b. TEST 6 - ACTIVE, $H / B=1$

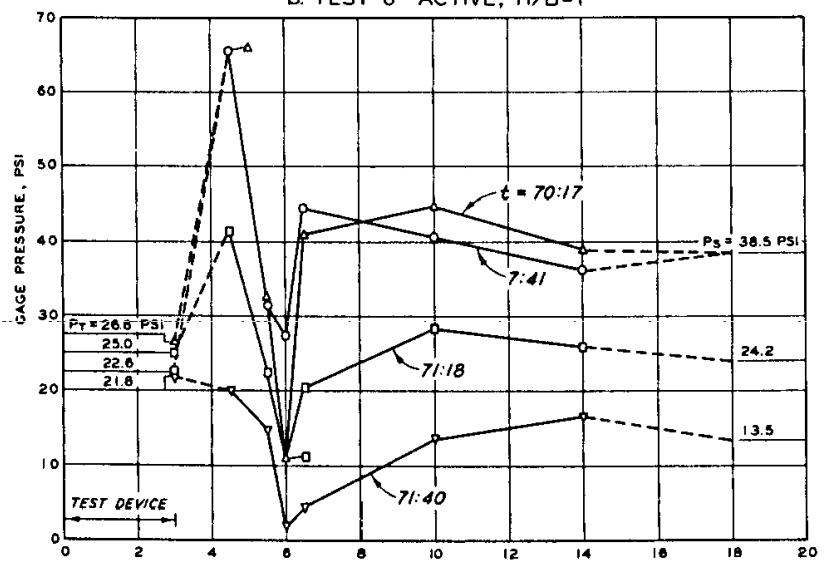

c. TEST 7-ACTIVE-PASSIVE, $H / B=1$

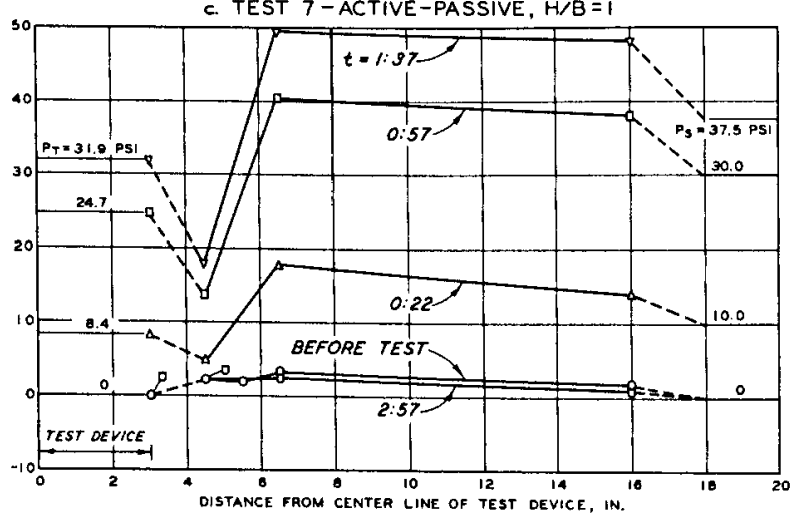

Fig. 28. Change in vertical soil stress at the 35-inch level due to deflection of top of test device, Tests 6 and 7 
a.

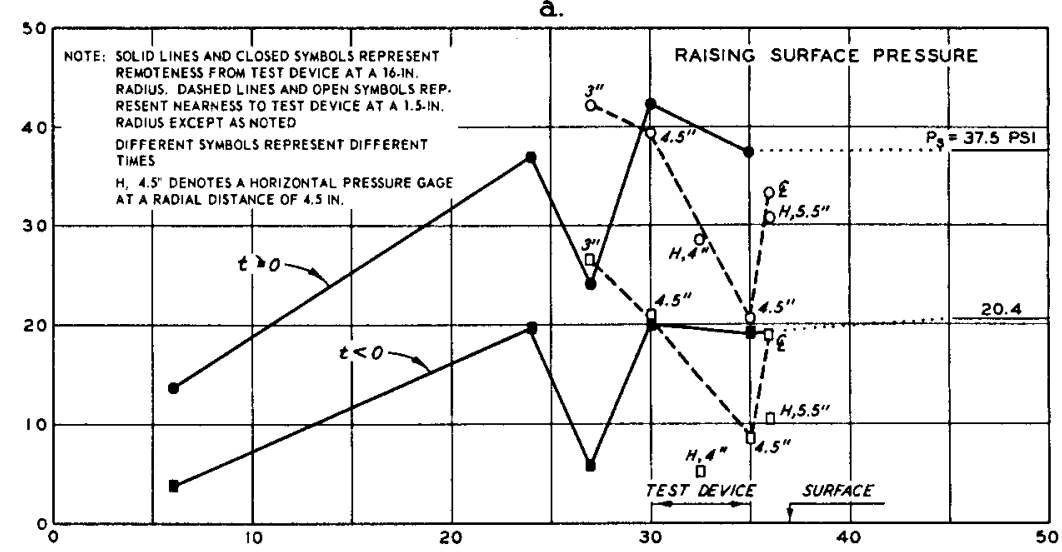

b.

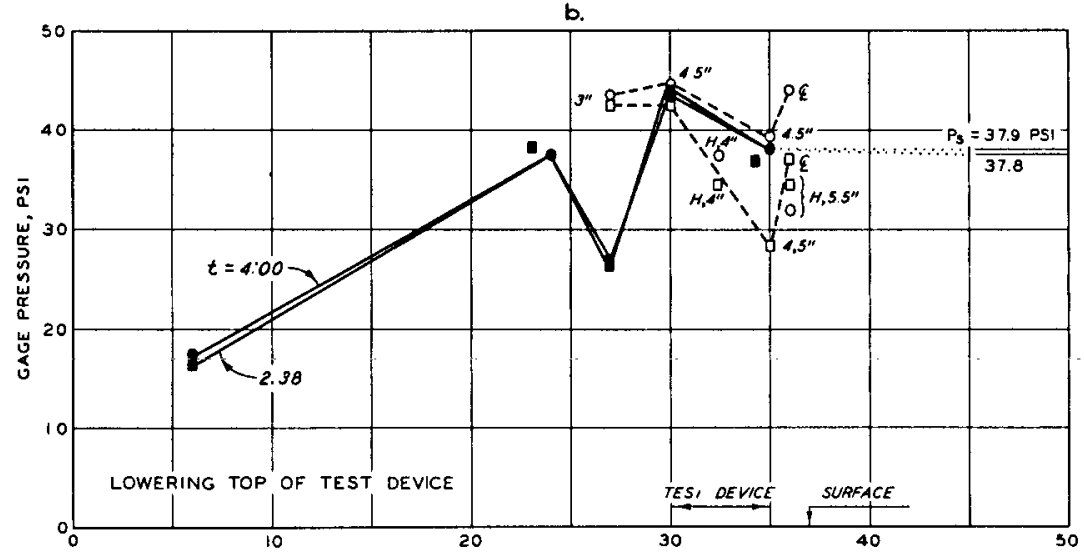

c.

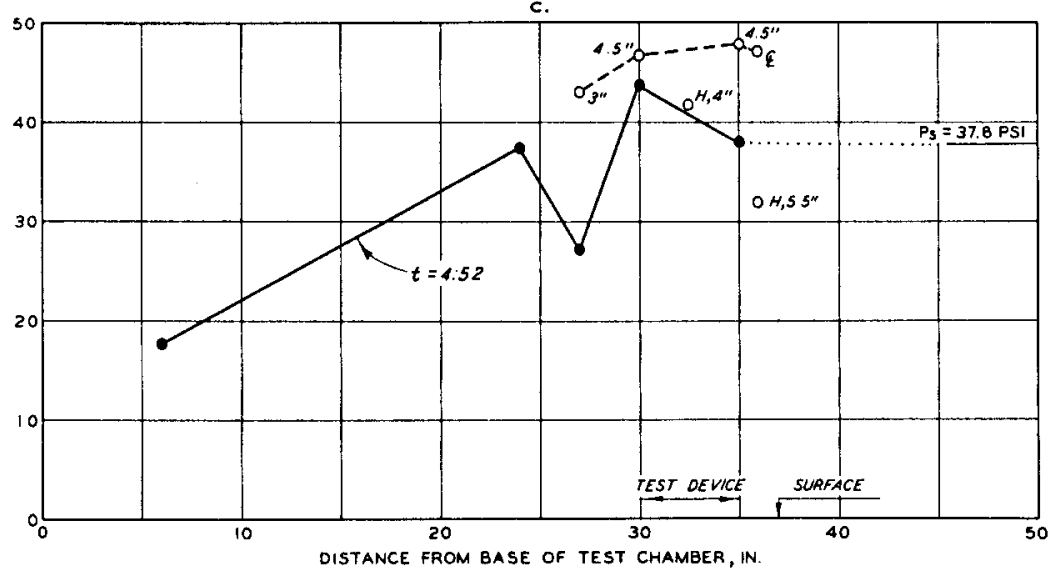

Fig. 29. Change in distribution of vertical soil stress with depth, Test $1 ; \mathrm{H} / \mathrm{B}=1 / 3$ 
a.
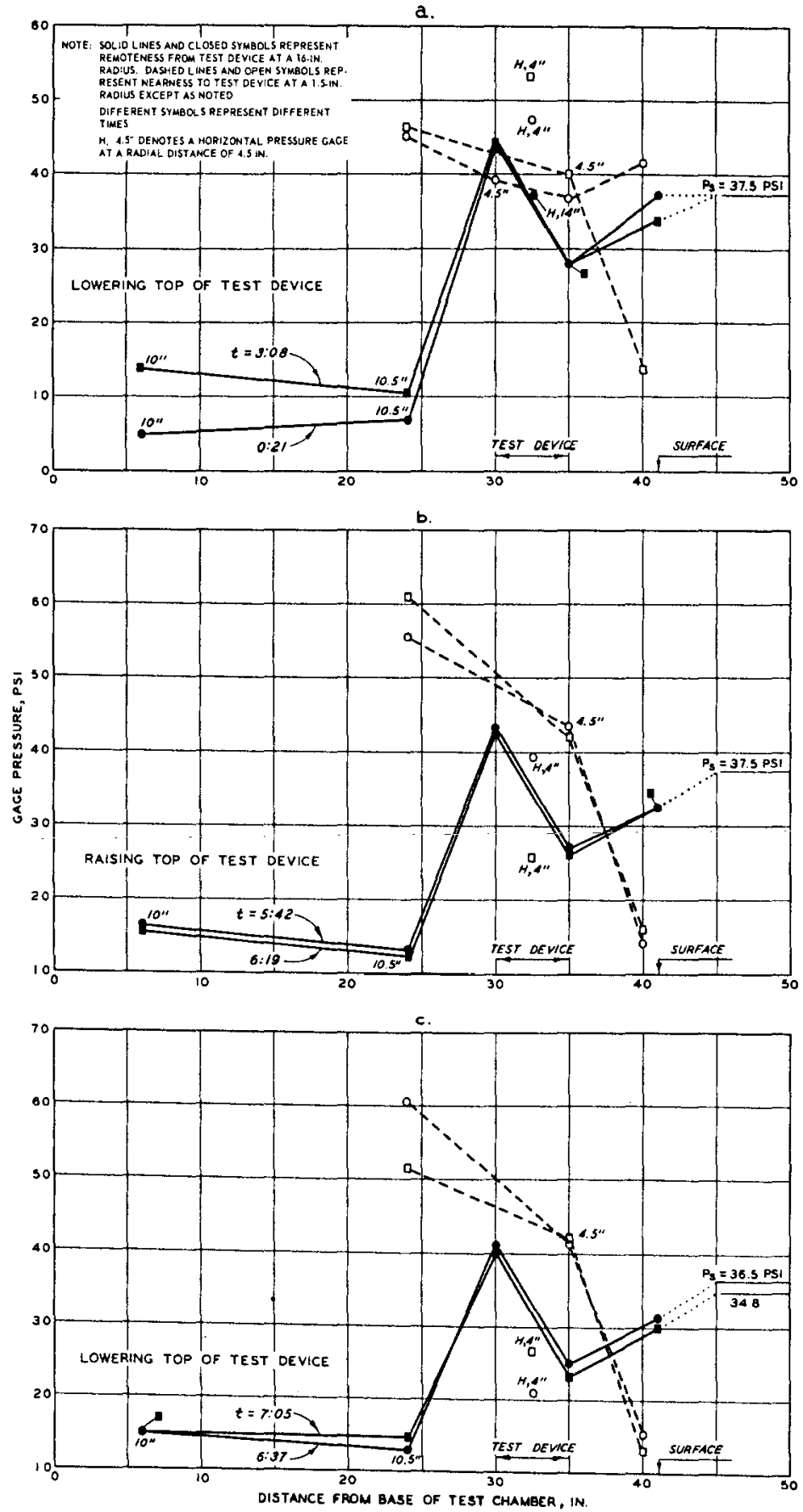

Fig. 30. Change in distribution of vertical soil stress with depth, Test $2 ; \mathrm{H} / \mathrm{B}=1$ 

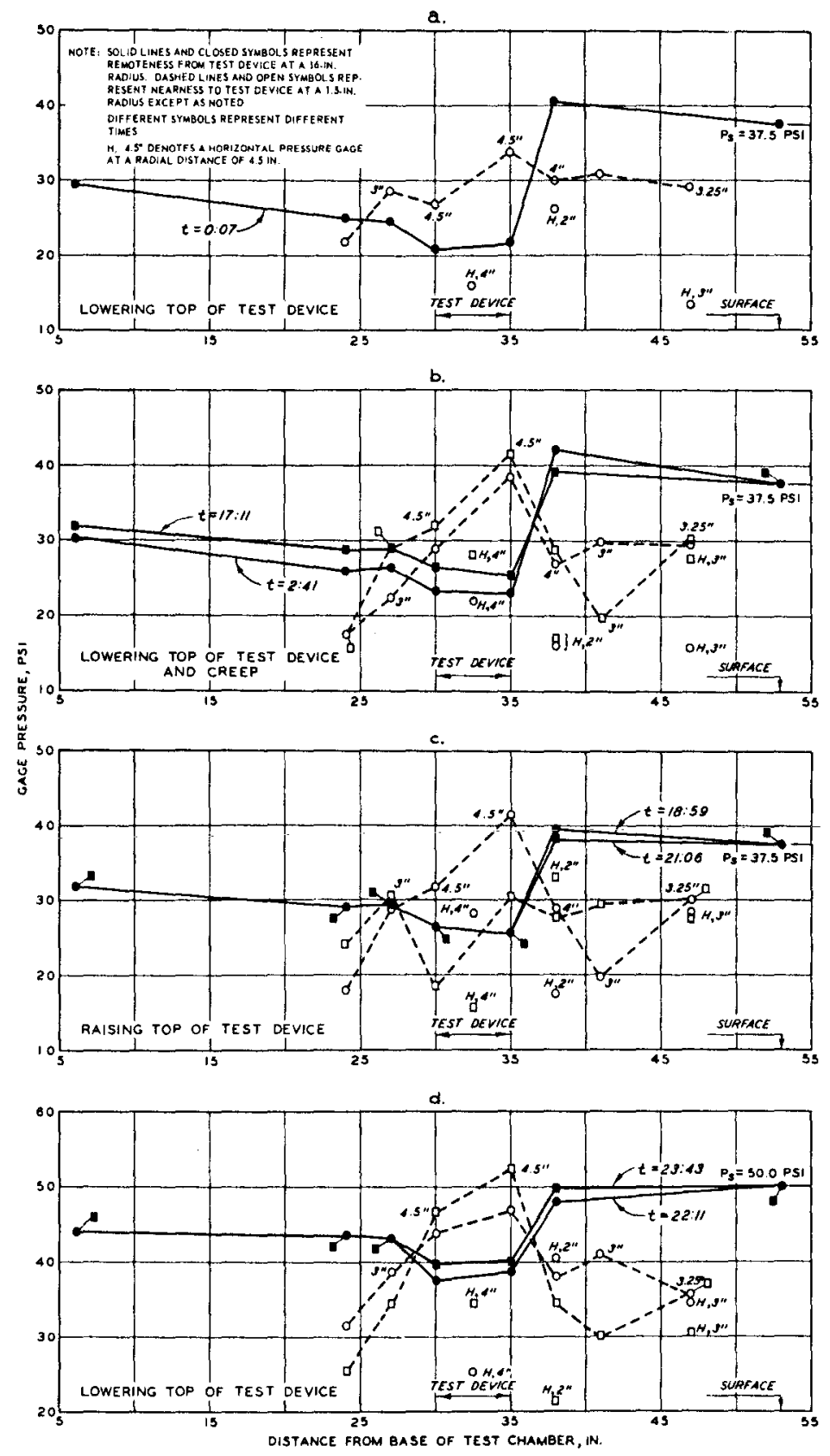

Fig. 31. Change in distribution of vertical soil stress with depth, Test $3 ; \mathrm{H} / \mathrm{B}=3$ 


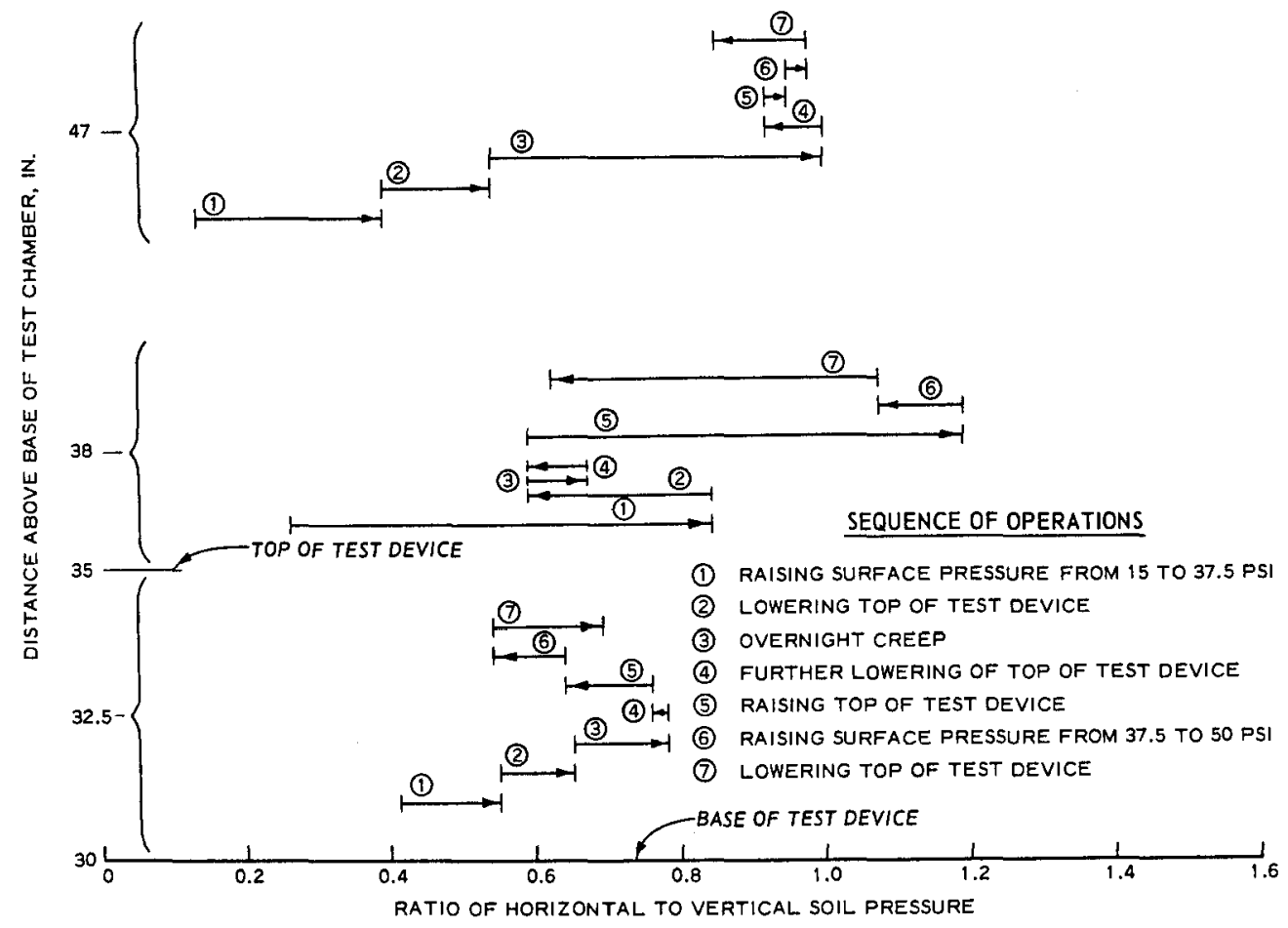

a. TEST 3

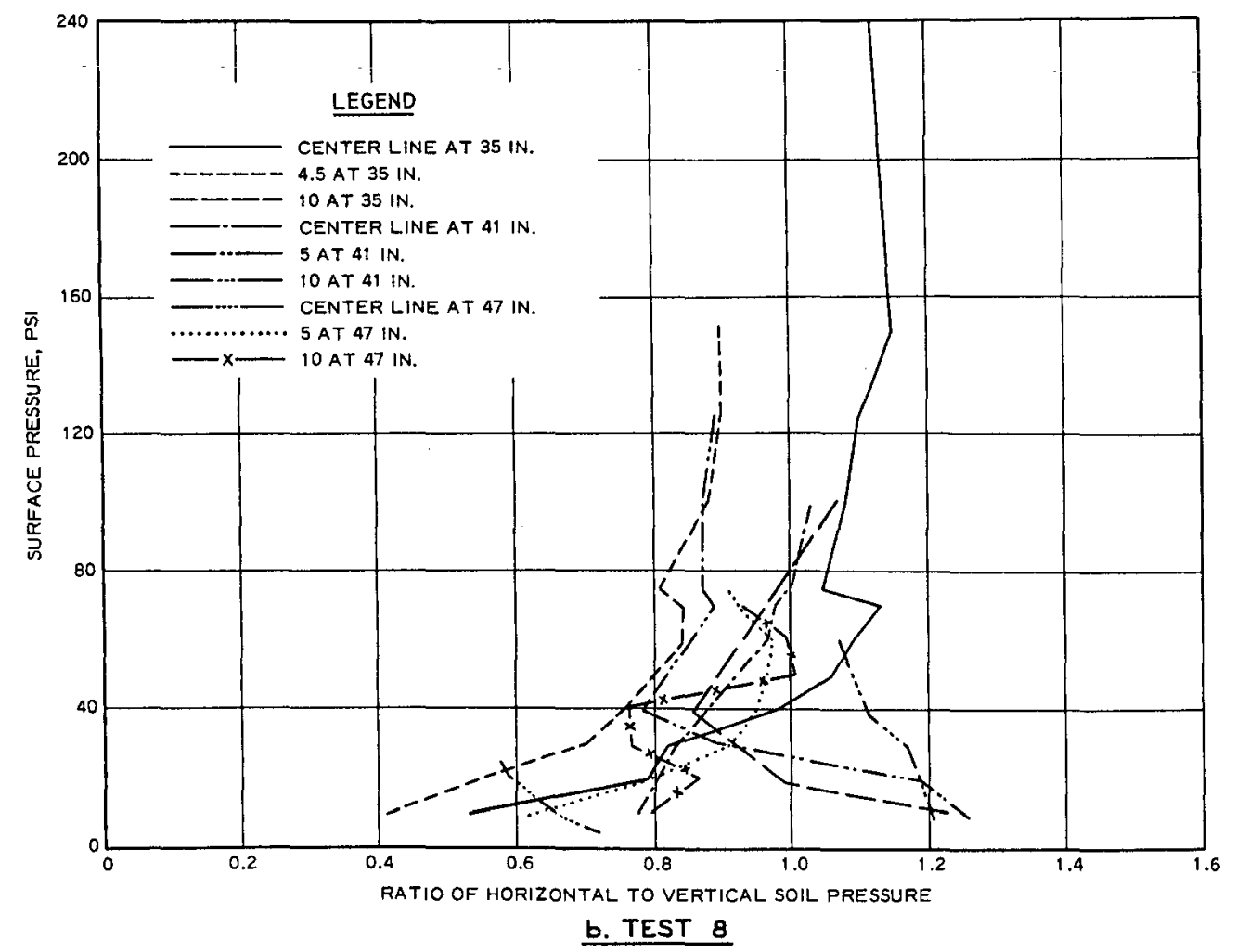

Fig. 32. Variation of horizontal to vertical soil pressure ratio with structural deflection and surface pressure 


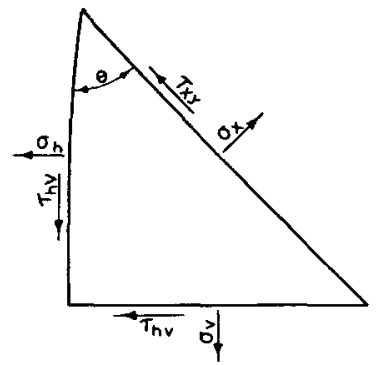

a. STRESS COMPONENTS ACTING ON FACES OF A SMALL WEDGE CUT FROM A BODY WHICH IS IN A STATE OF PLANE STRESS

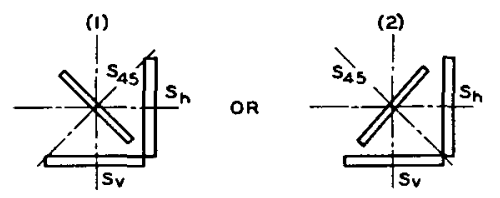

b. PLACEMENT OF SOIL STRESS GAGES
35* LEVEL
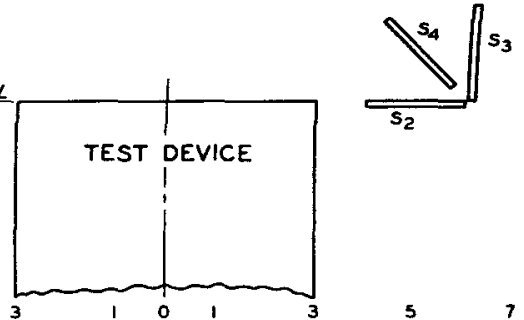

$\mathbf{s}_{2}$ DISTANCE FROM CENTER LINE OF TEST CHAMBER INCHES c. TEST 8-CONFIGURATION OF GAGE ARRAY WITH RESPECT TO TEST DEVICE

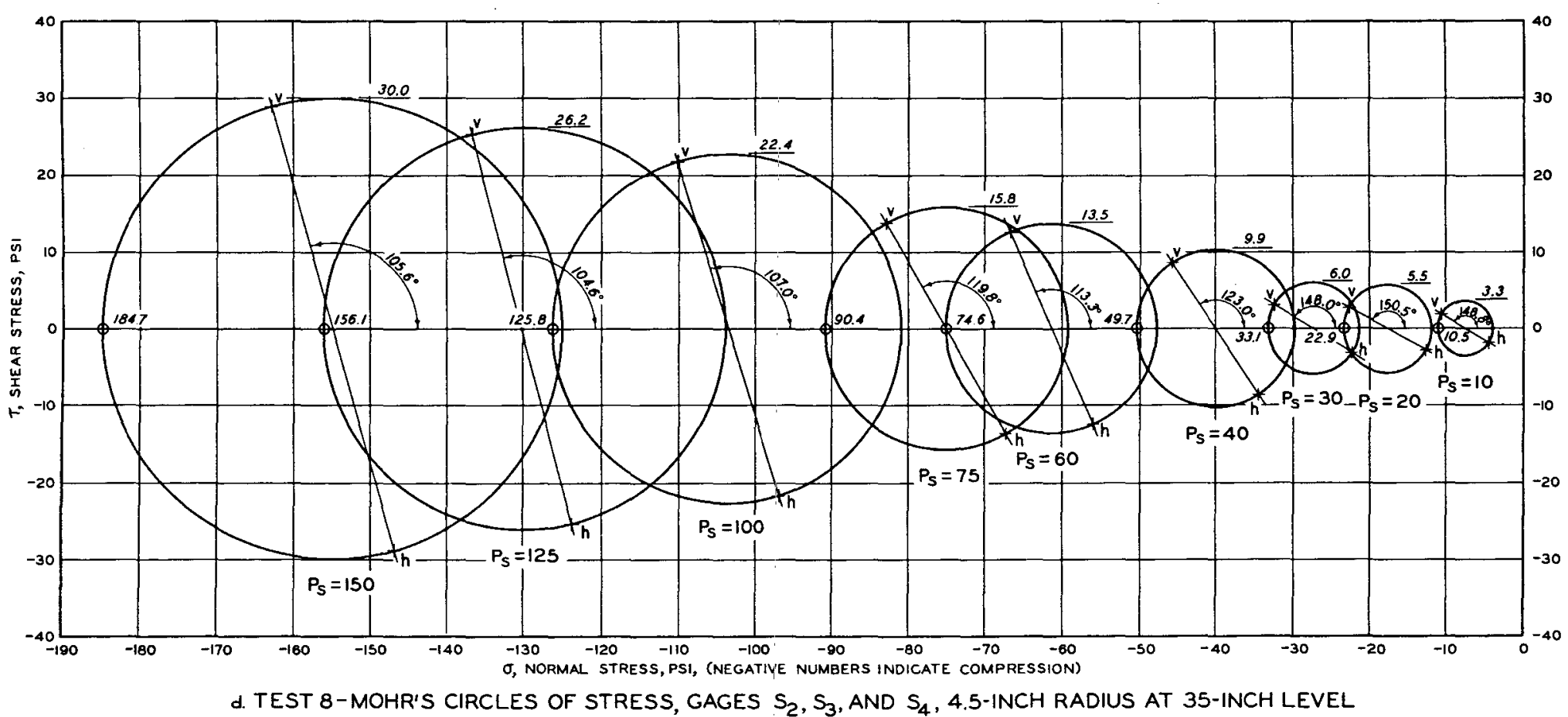

Fig. 33. Determination of stress components 


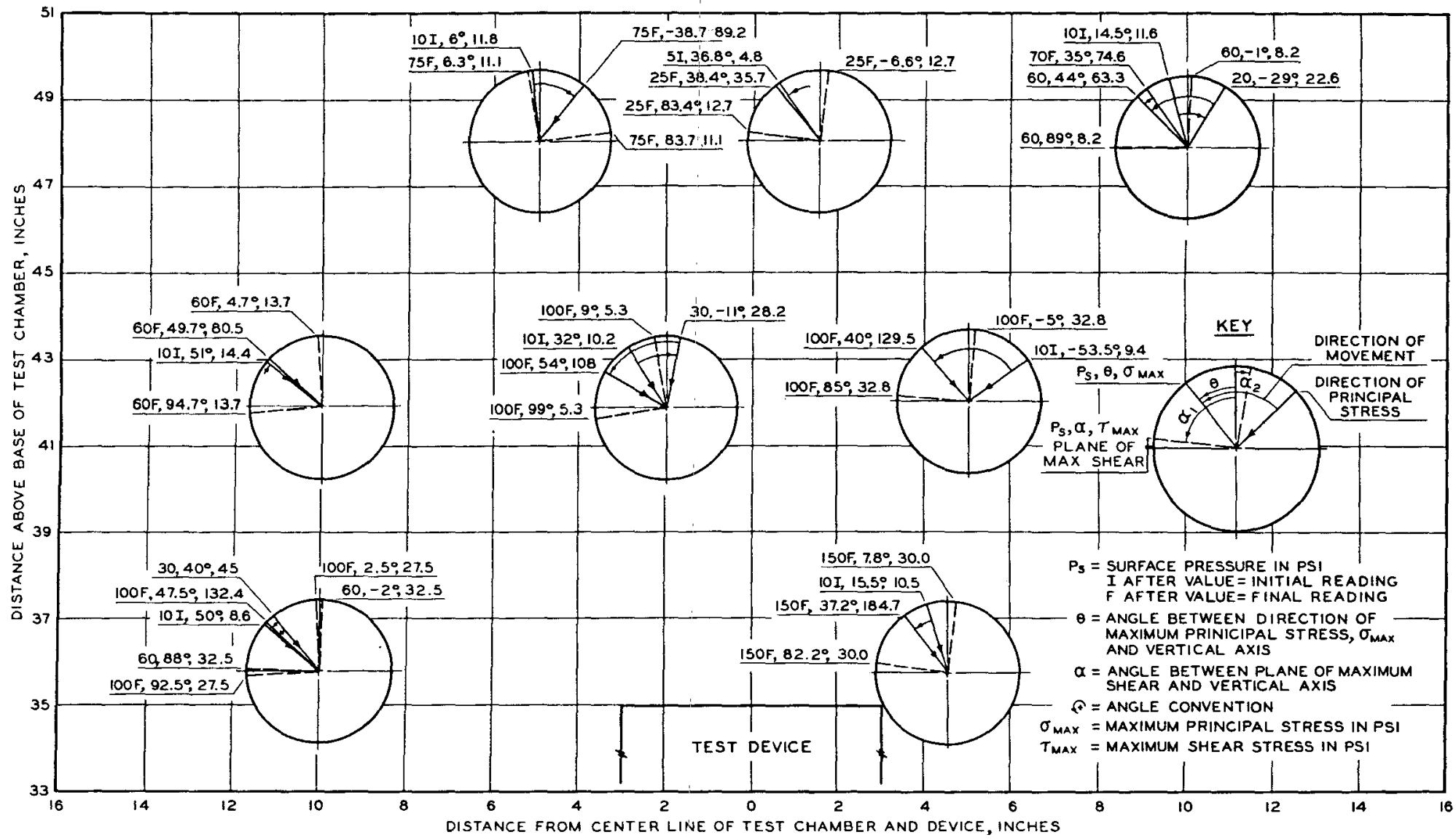

Fig. 34. Directions of principal stresses and location of planes of maximum shear, Test 8 


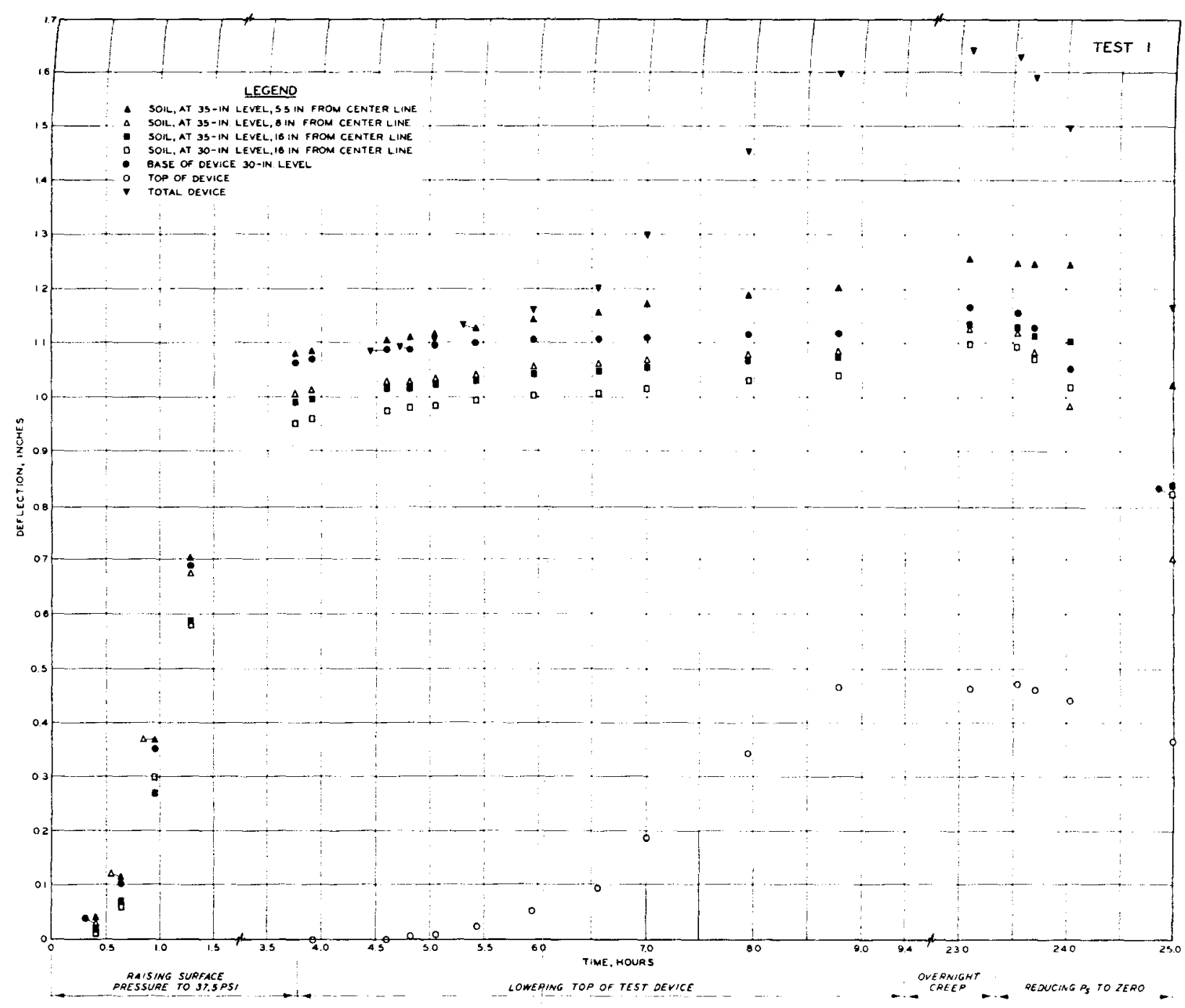

Fig. 35. Soil and structure deflections versus time; Test $1, H / B=1 / 3$, $\mathrm{P}_{\mathrm{S}}=37.5 \mathrm{psi}$ 


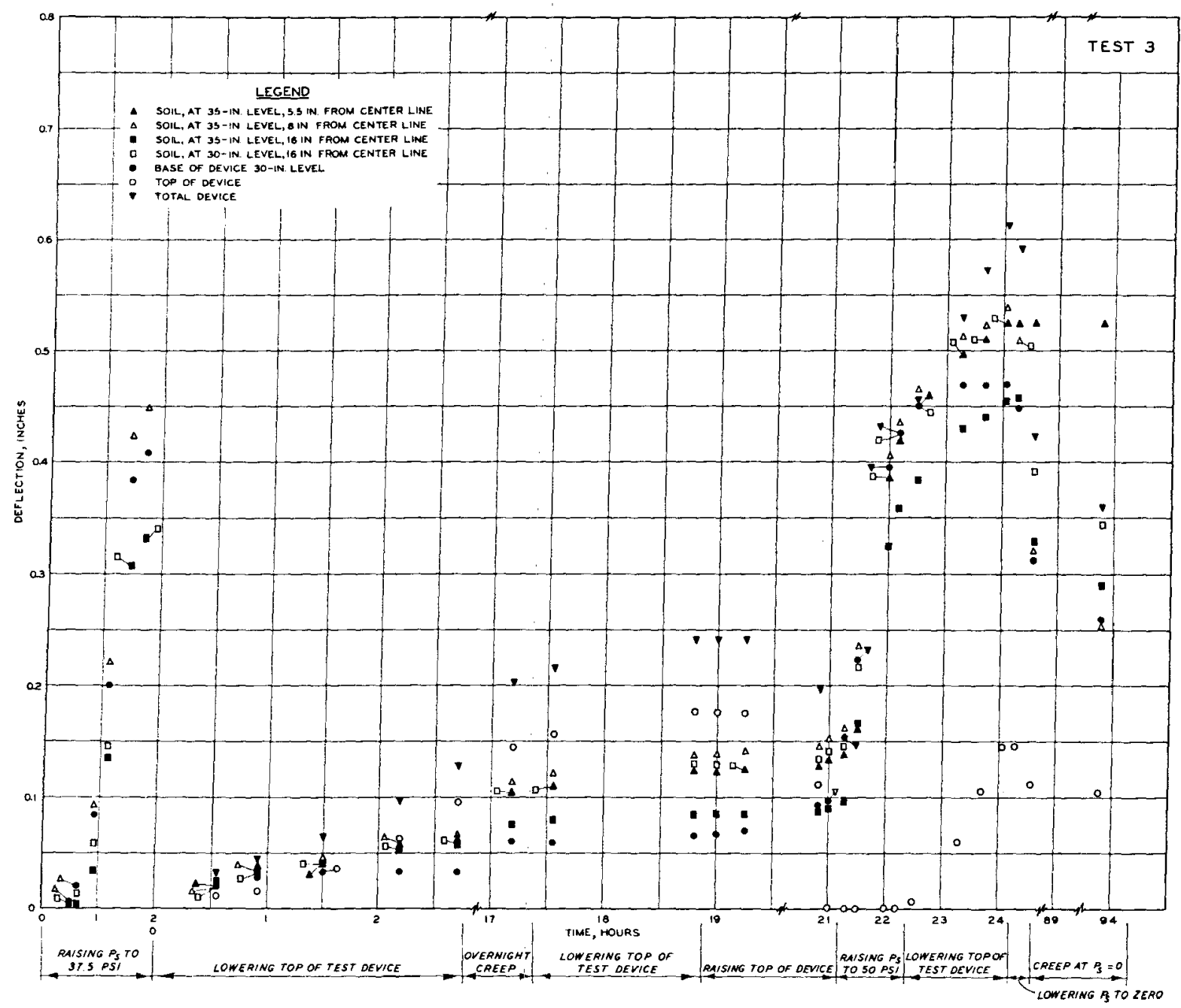

Fig. 36. Soil and structure deflections versus time; Test $3, \mathrm{H} / \mathrm{B}=3$, $\mathrm{P}_{\mathrm{S}}=37.5$ psi 


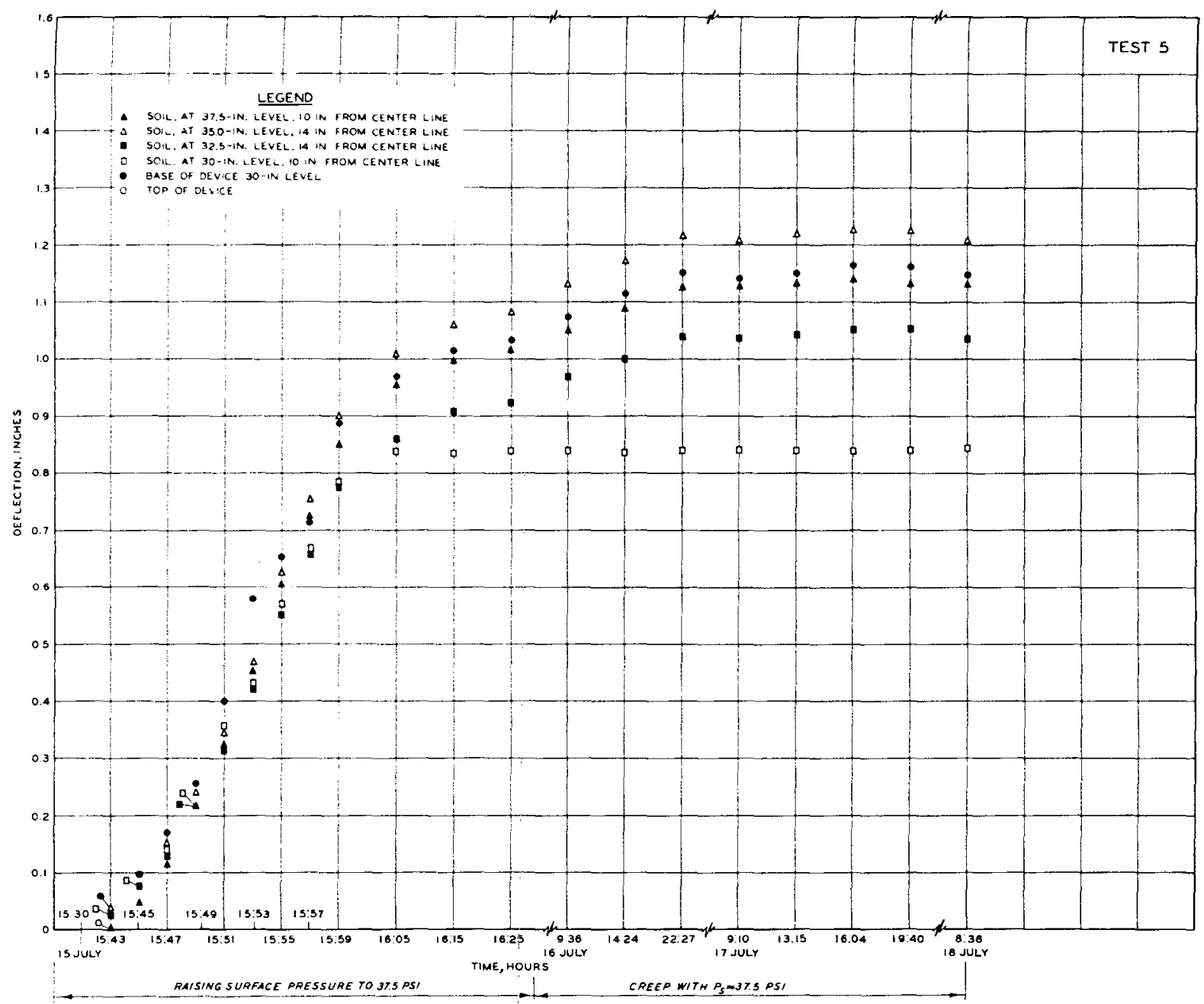

Fig. 37. Soil and structure deflections versus time; Test 5, H/B $=1$, $\mathrm{P}_{\mathrm{S}}=37.5 \mathrm{psi}$ 


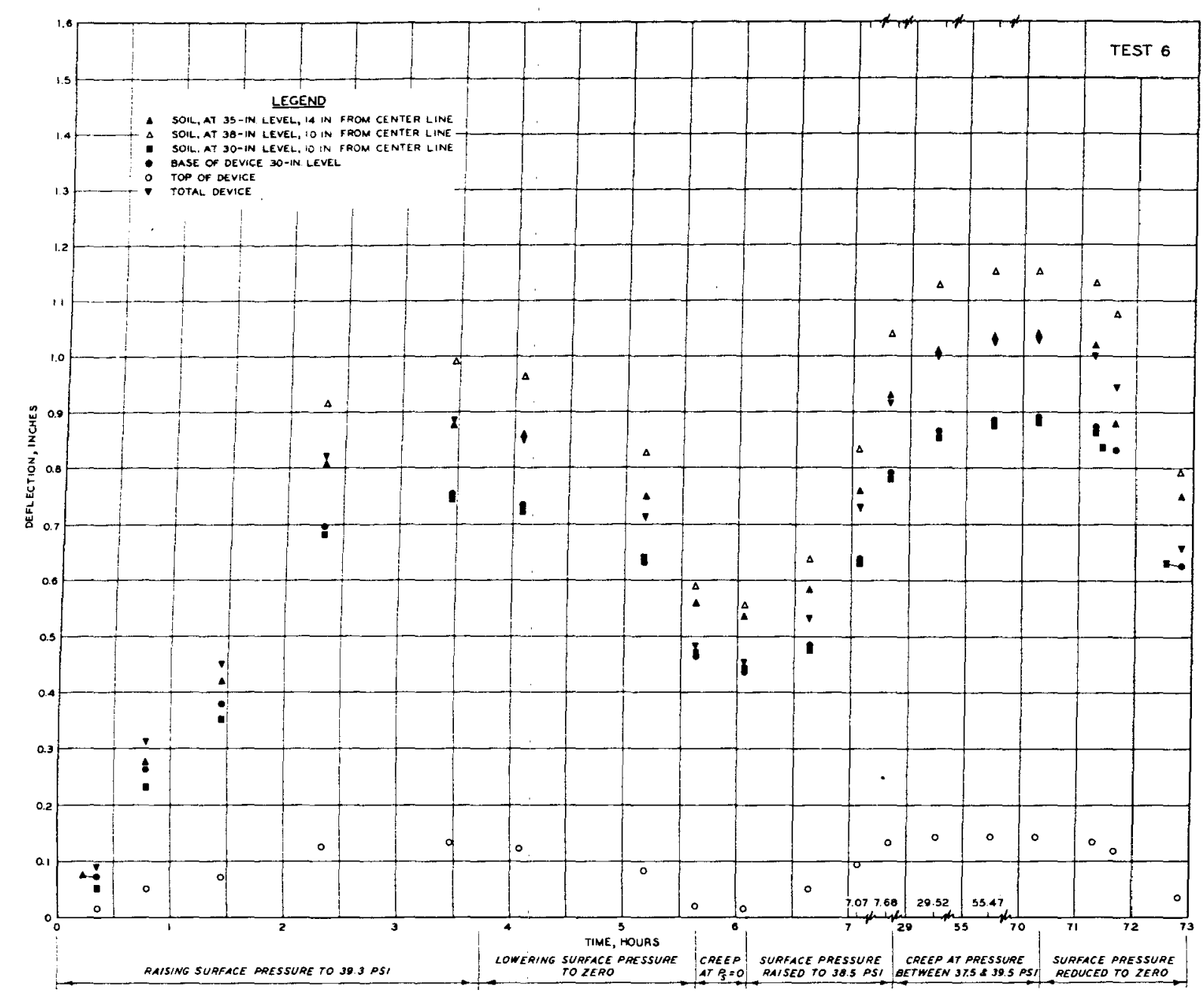

Fig. 38. Soil and structure deflections versus time; Test $6, \mathrm{H} / \mathrm{B}=1$, $\mathrm{P}_{\mathrm{S}}=39.3 \mathrm{psi}$ 


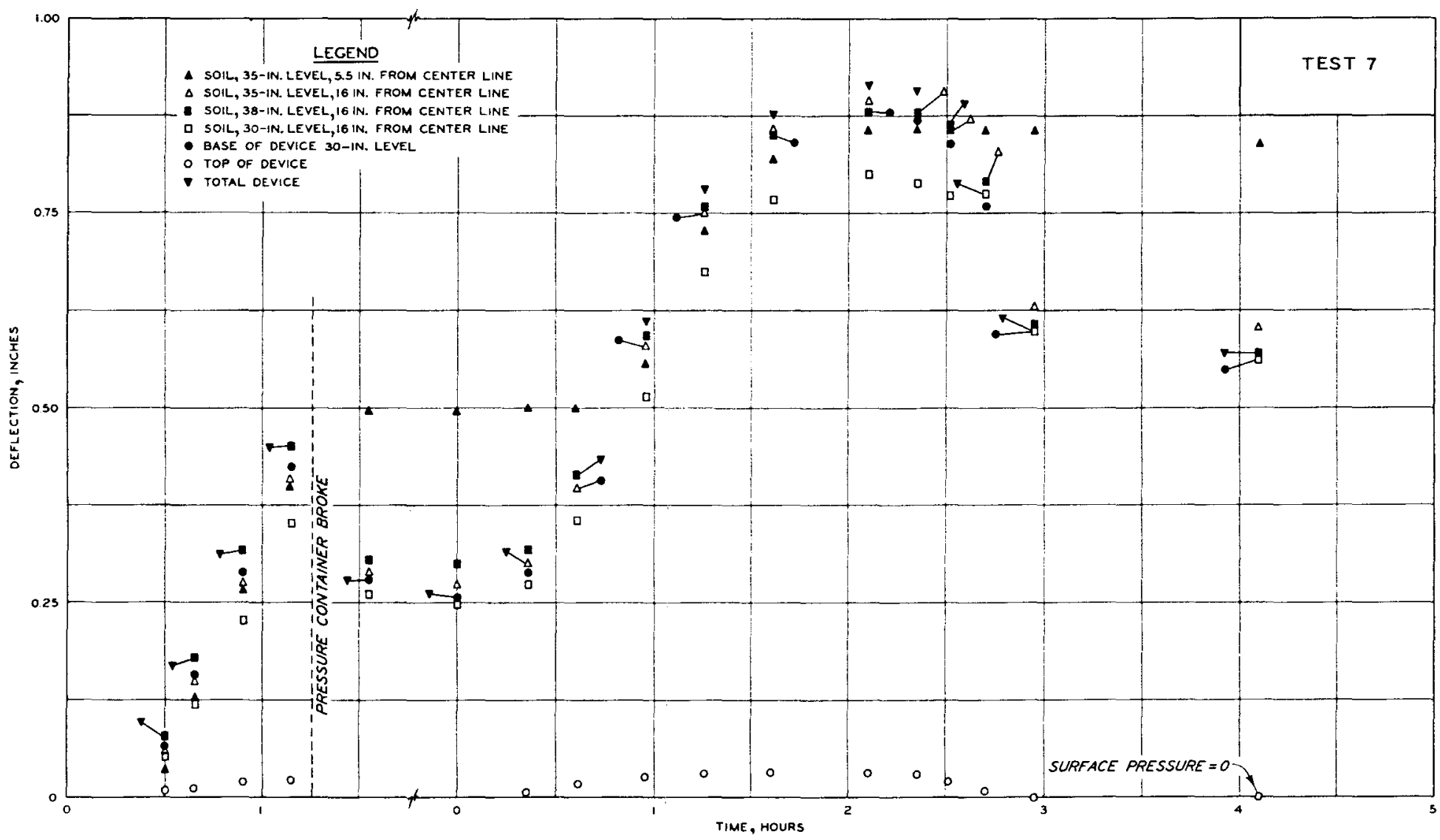

Fig. 39. Soil and structure deflections versus time; Test 7, H/B = I, $\mathrm{P}_{\mathrm{S}}=37.5$ psi 


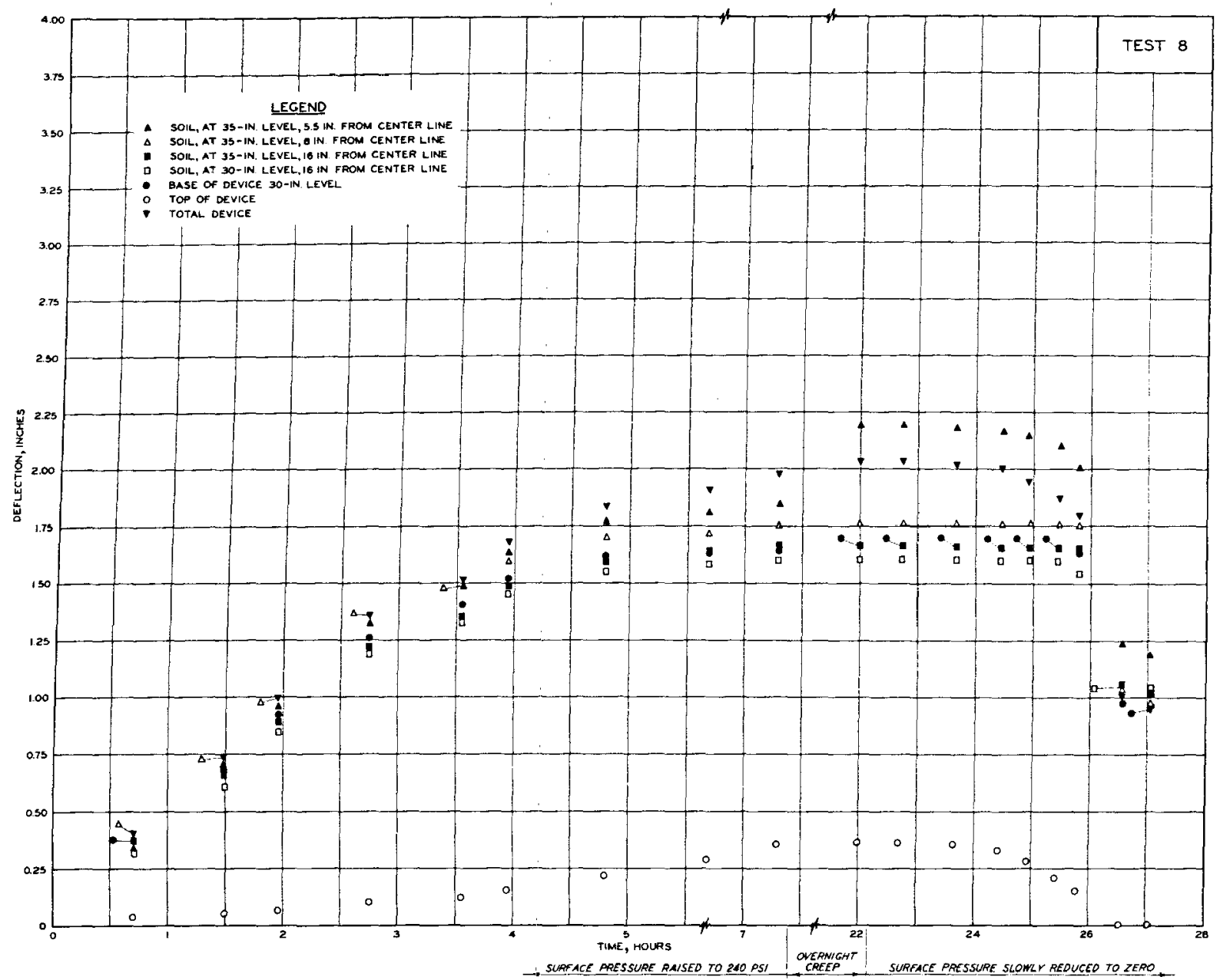

Fig. 40. Soil and structure deflections versus time; Test $8, \mathrm{H} / \mathrm{B}=3$, $\mathrm{P}_{\mathrm{S}}=240 \mathrm{psi}$ 


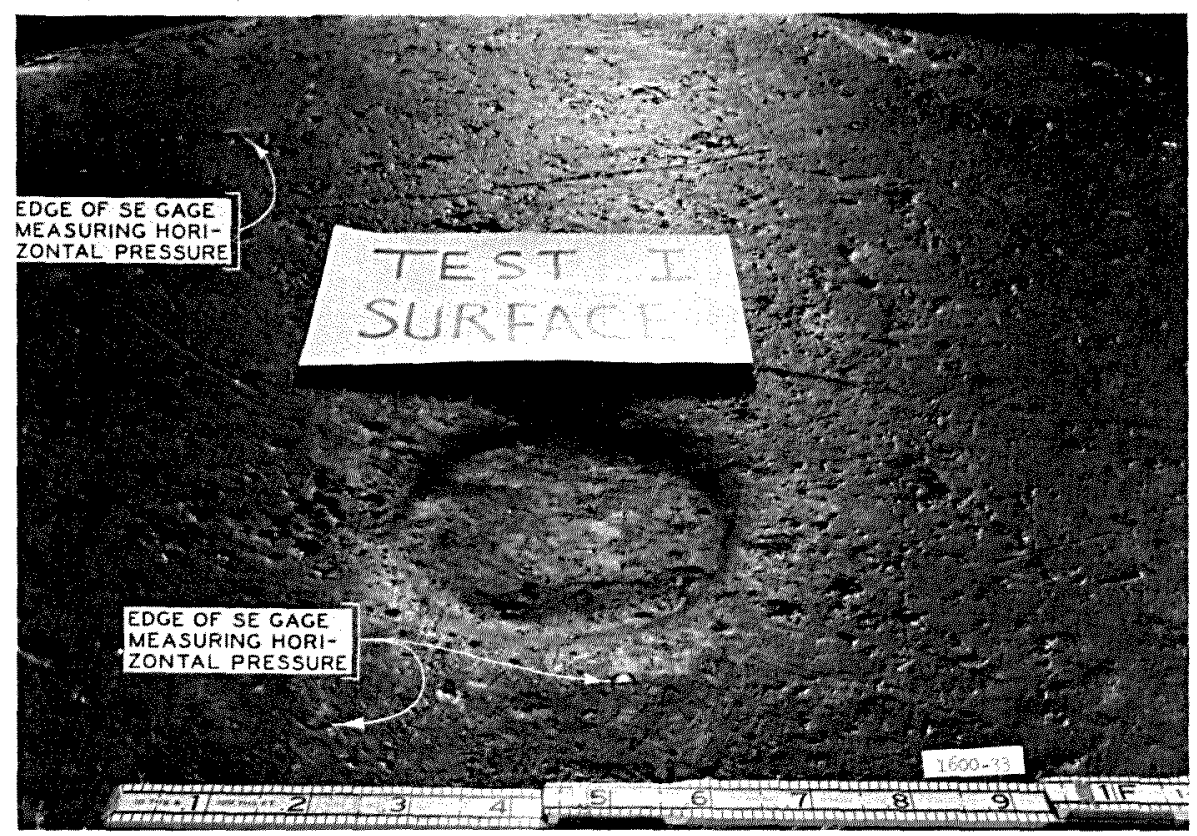

a. Oblique view of soil surface

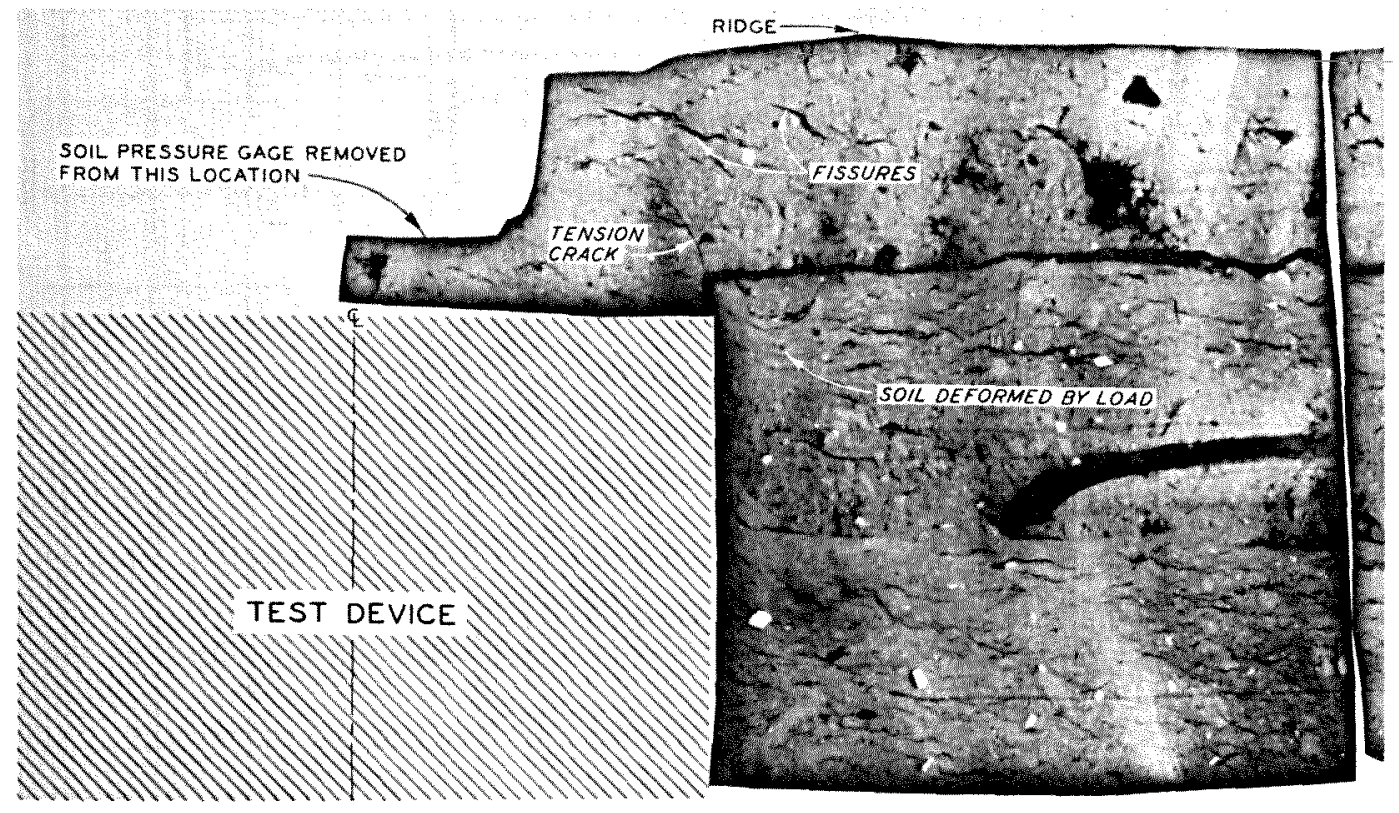

b. Cross-section radiograph of depression

Fig. 41. Depression in the soil surface above test device, Test $1 ; \mathrm{H} / \mathrm{B}=1 / 3, \mathrm{P}_{\mathrm{S}}=37.8 \mathrm{psi}$ 


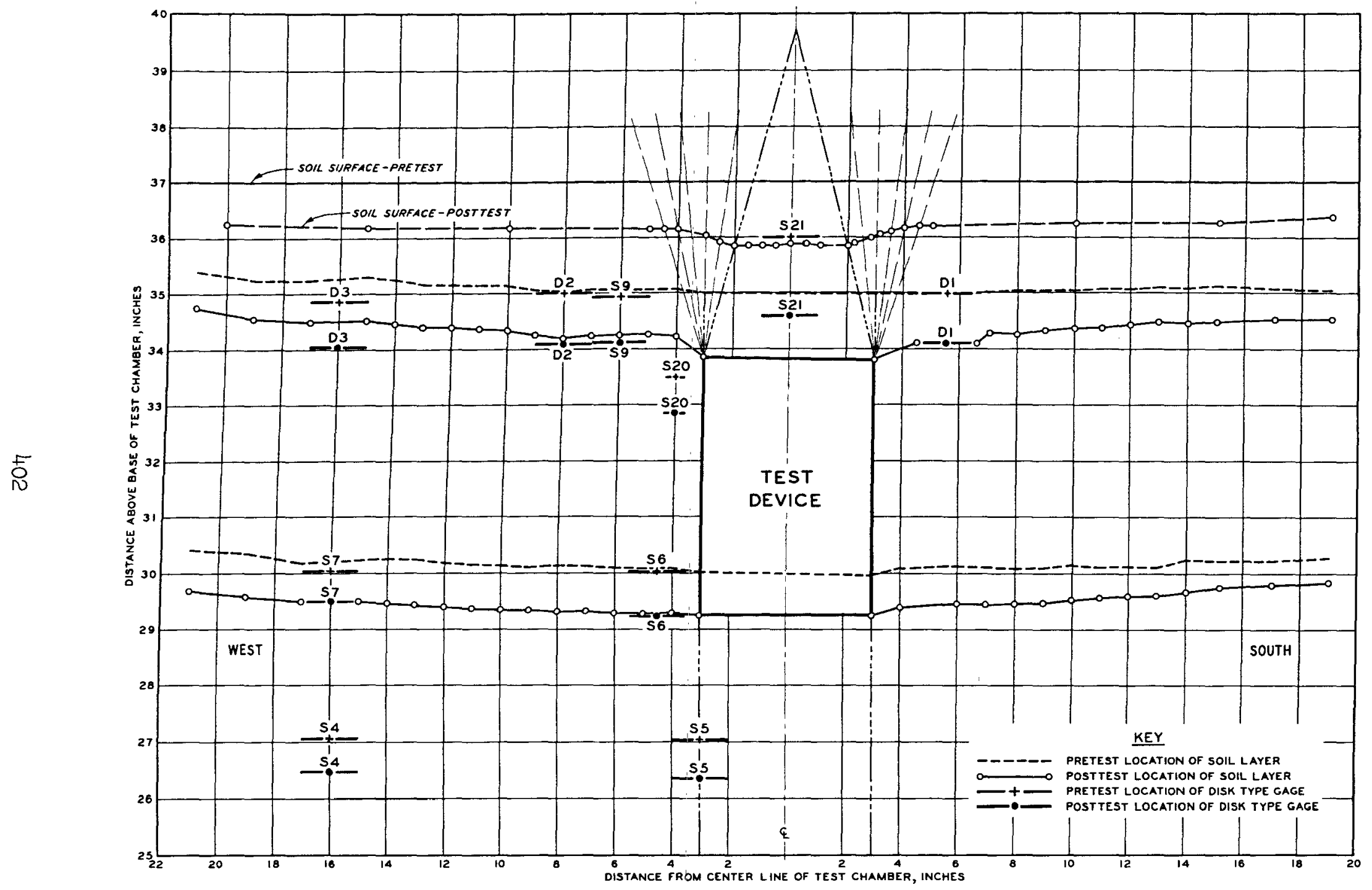

Fig. 42. Cross-section view of soil deformation, Test $1 ; \mathrm{H} / \mathrm{B}=1 / 3, \mathrm{P}_{\mathrm{S}}=\$ 7.8 \mathrm{psi}$ 


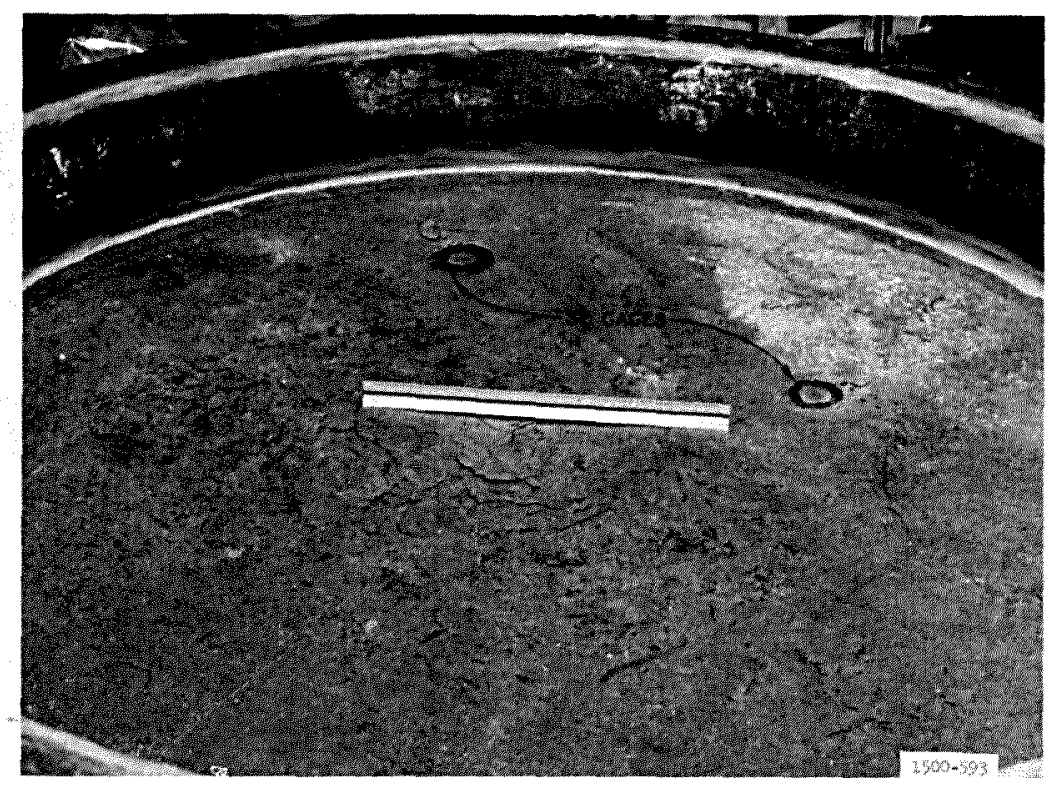

a. Oblique view of soil surface

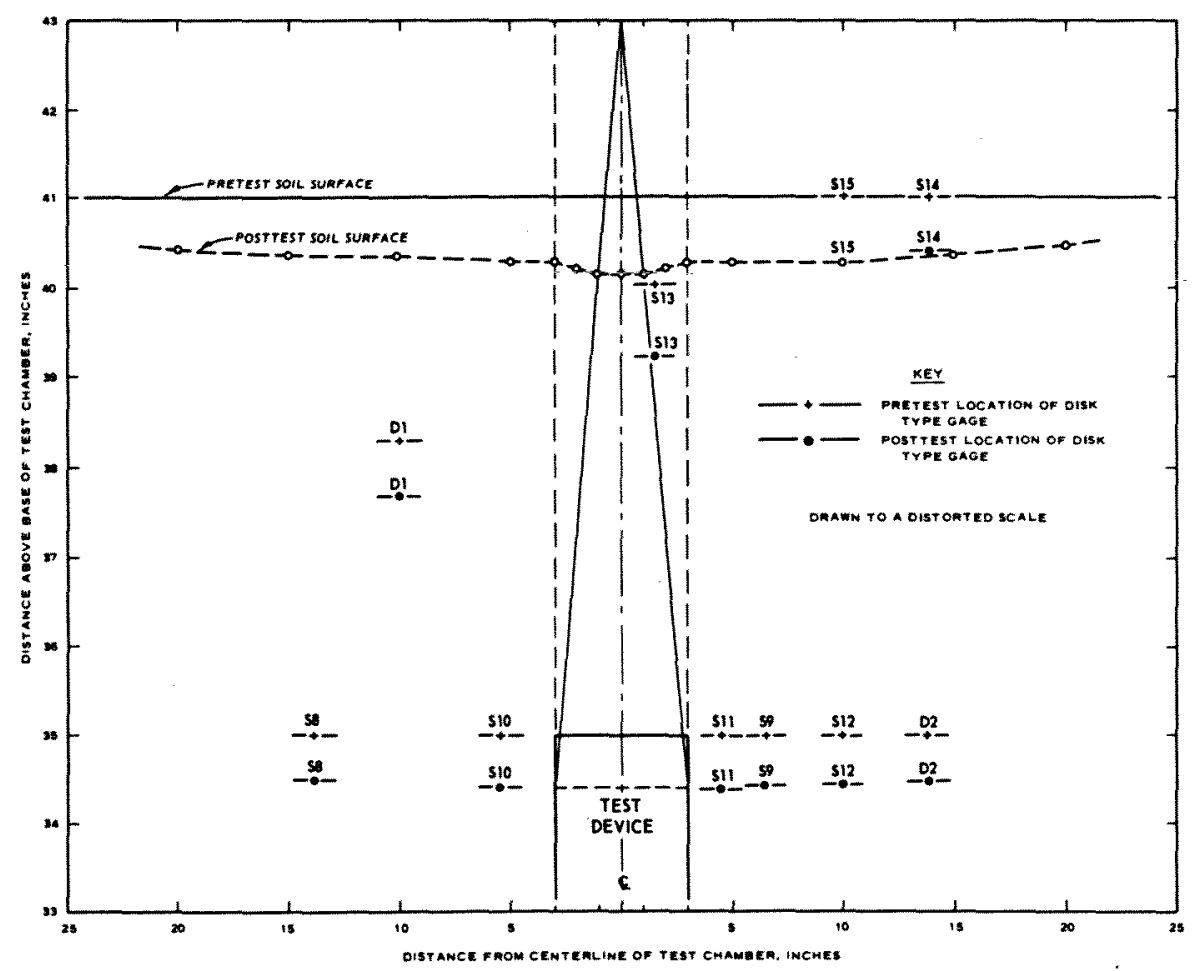

b. Cross-section view of soil deformation

Fig. 43. Depression in soil surface above test device, Test 2; $\mathrm{H} / \mathrm{B}=1, \mathrm{P}_{\mathrm{S}}=37.5 \mathrm{psi}$ 


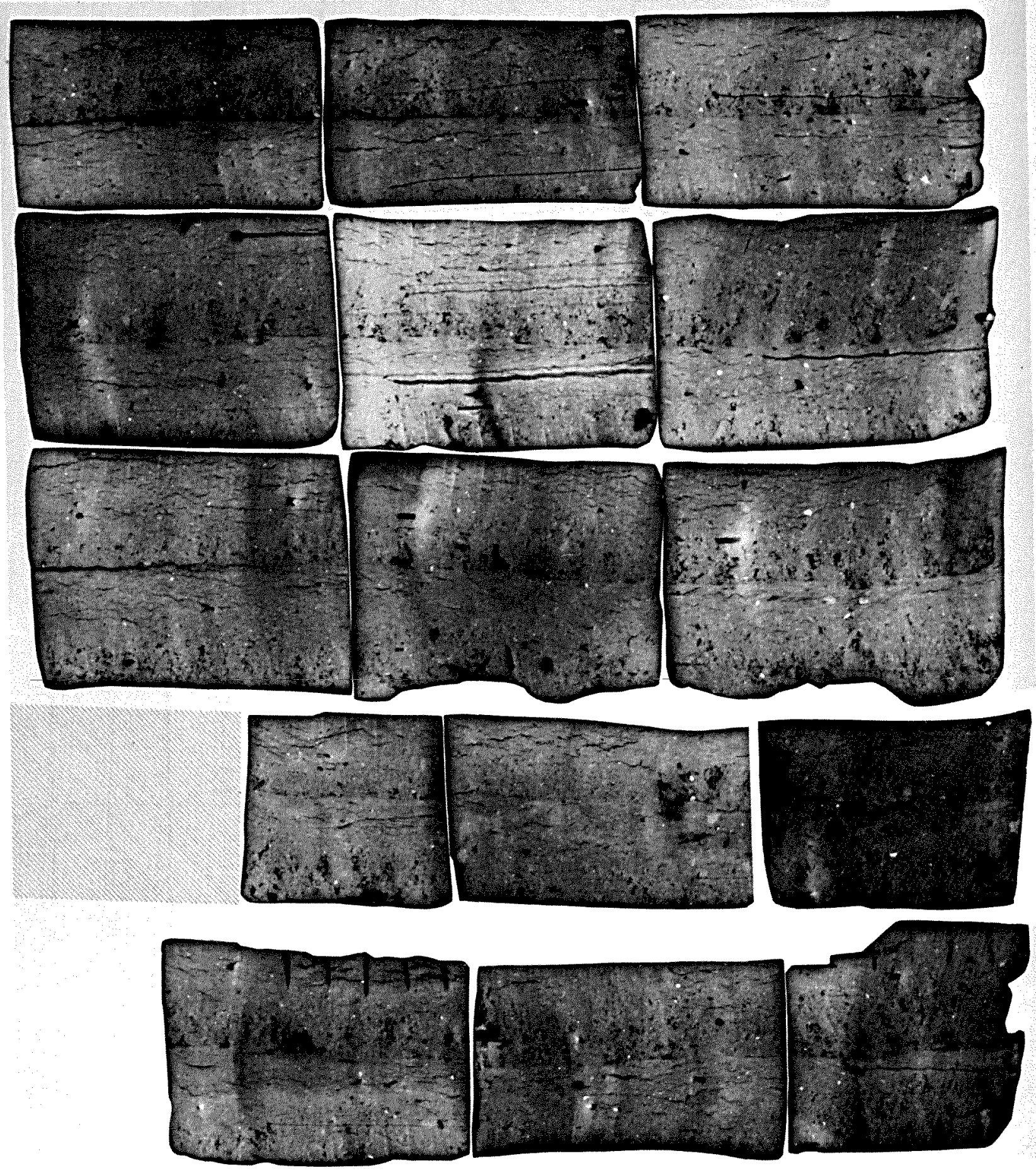

Fig. 44. Radiograph of soil deformation pattern, Test 3; $\mathrm{H} / \mathrm{B}=3, \mathrm{P}_{\mathrm{S}}=37.5 \mathrm{psi}$ 


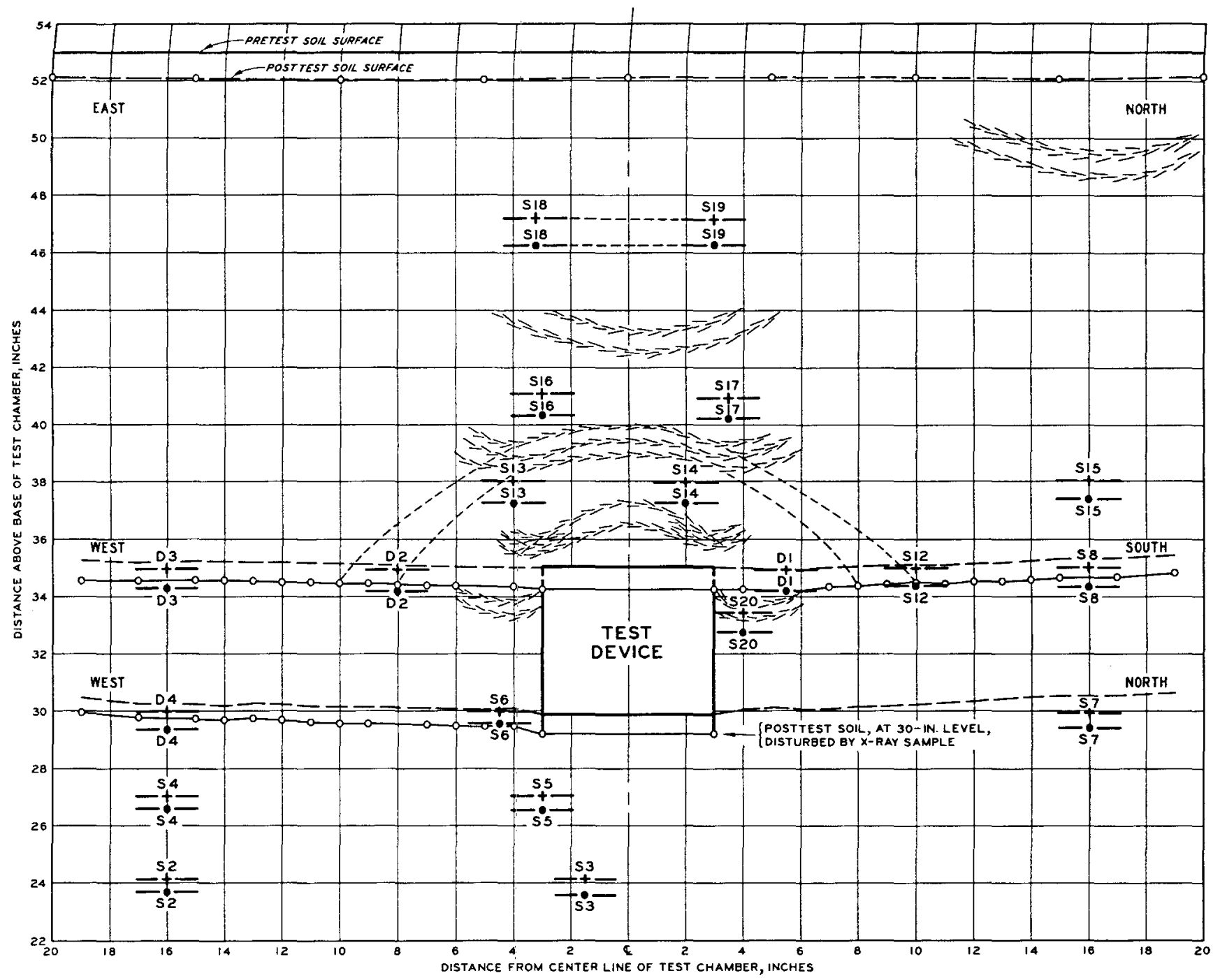

Fig. 45. Sketch of soil deformation patterns found in radiograph and visual inspection, Test $3 ; \mathrm{H} / \mathrm{B}=3, \mathrm{P}_{\mathrm{S}}=37.5 \mathrm{psi}$ 


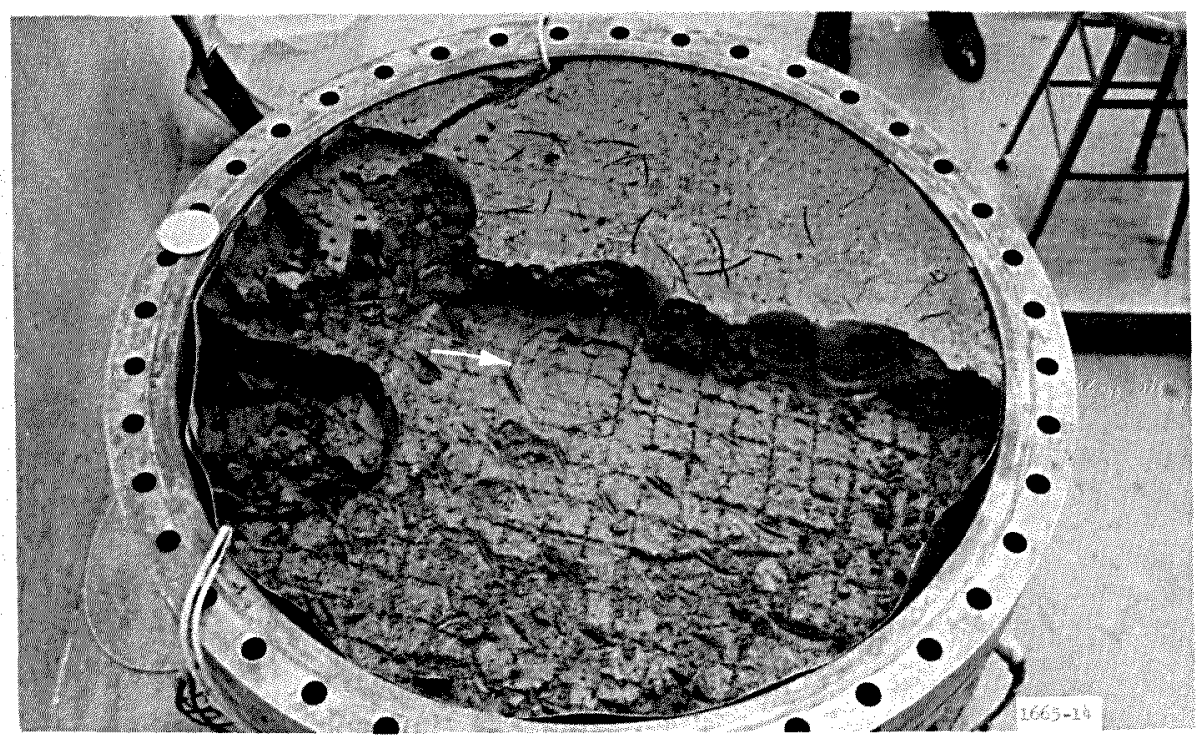

a. Oblique view of 38 -inch soil level
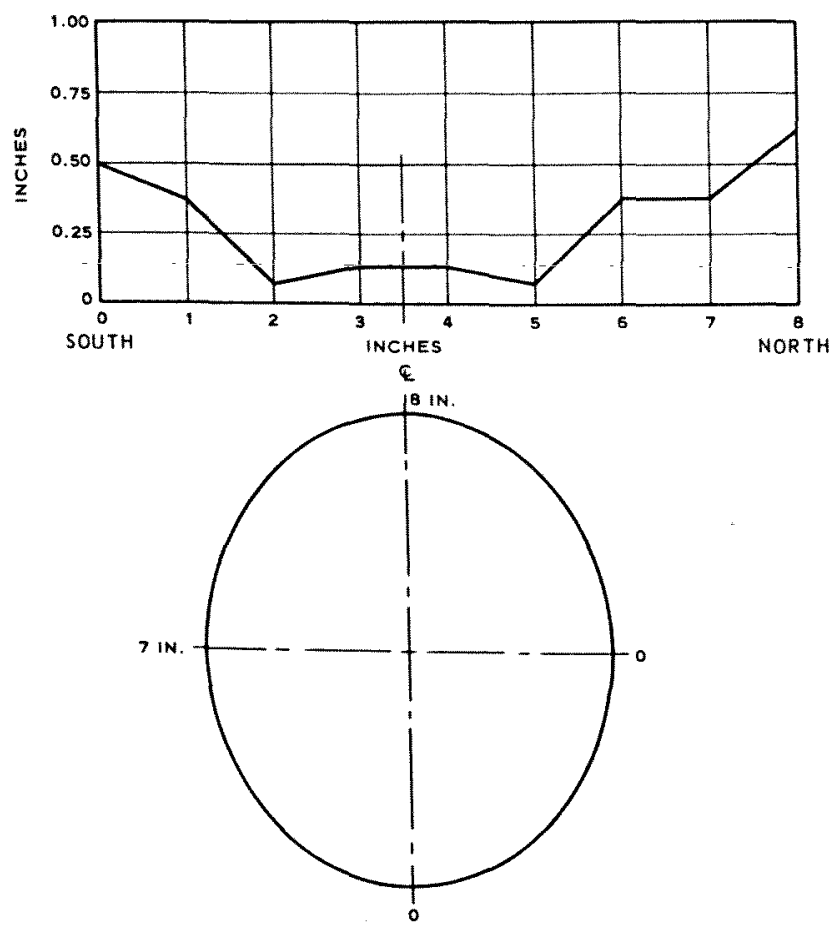

b. Profile of depression at 38-inch level

Fig. 46. Depression above test device, Test 4A; $\mathrm{H} / \mathrm{B}=1, \mathrm{P}_{\mathrm{S}}=75 \mathrm{psi}$ 


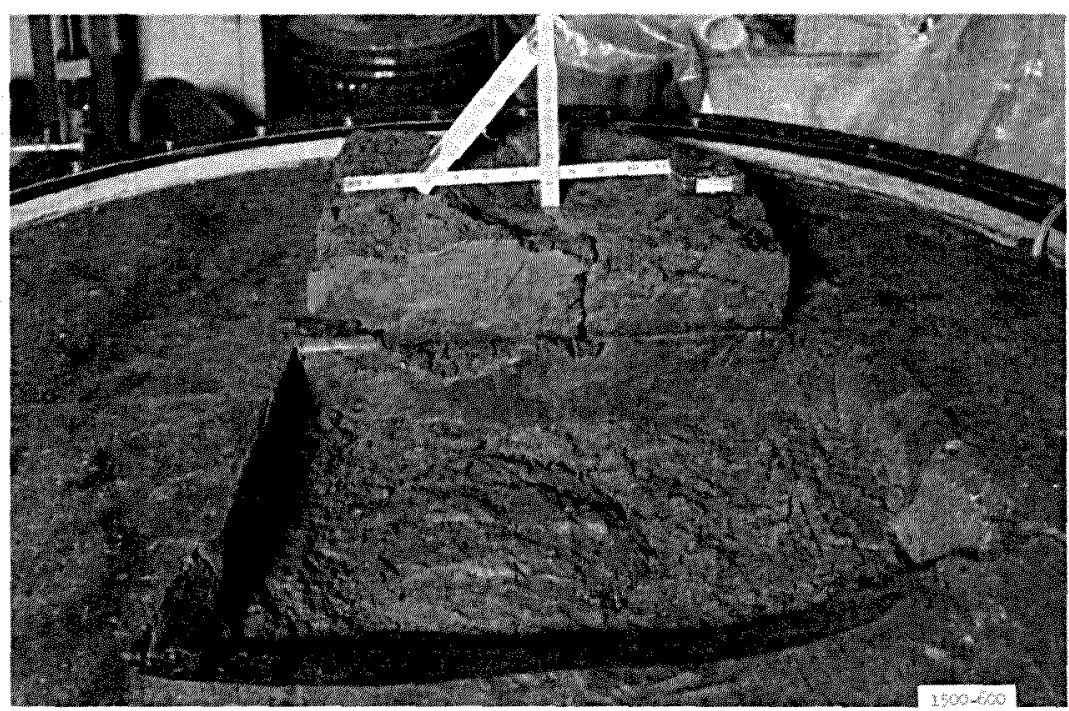

a. Oblique view of 38-inch soil level

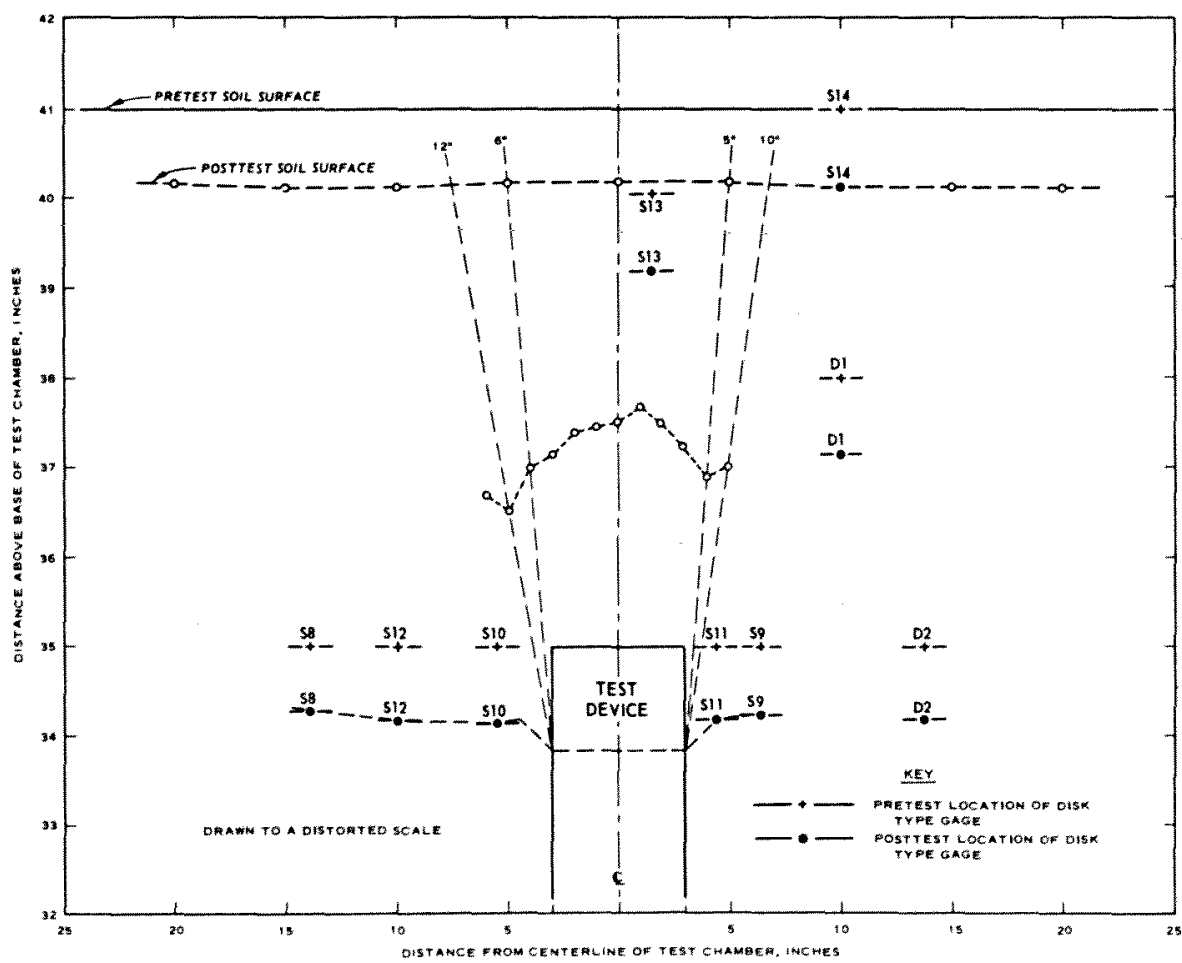

b. Profile of soil deformations

Fig. 47. Hump above test device, Test 4B; H/B $=1, P_{\mathrm{S}}=75$ psi 


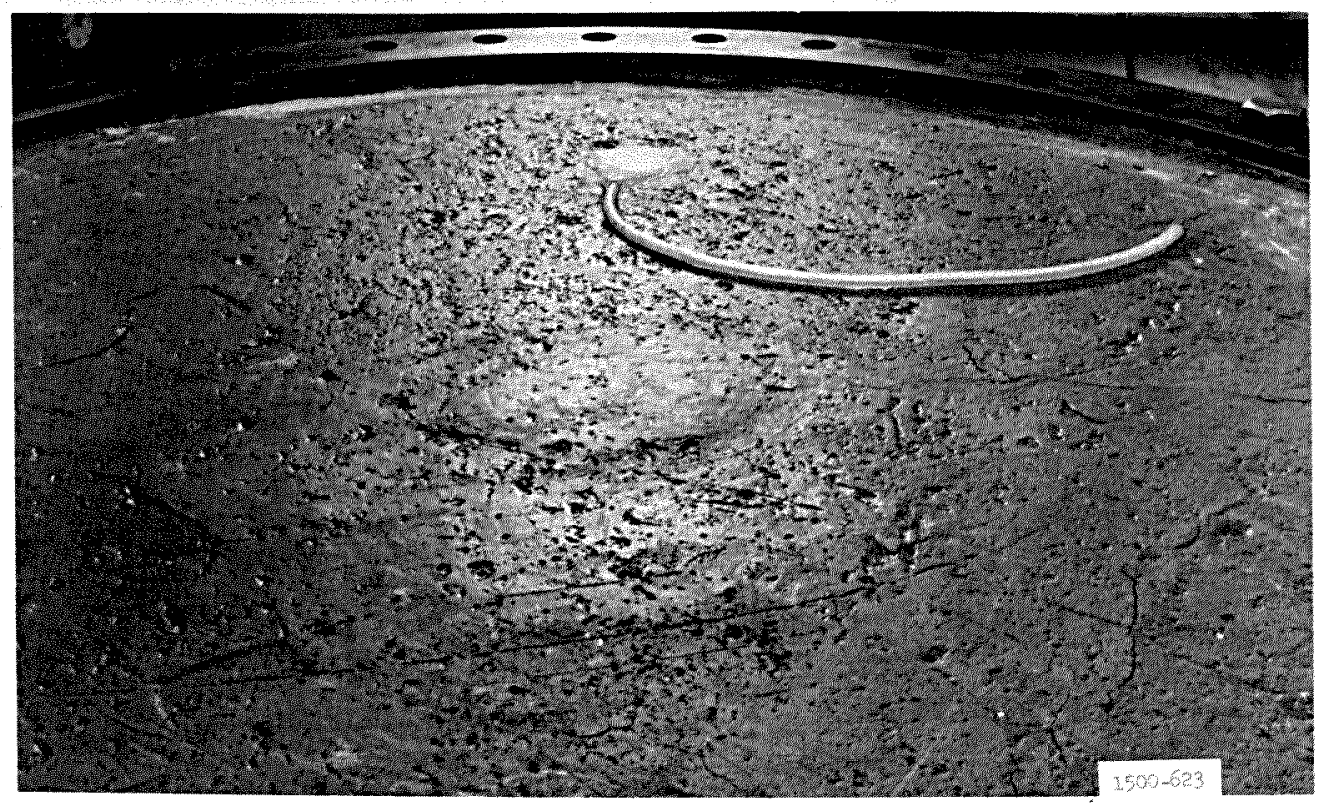

a. Oblique view of soil surface

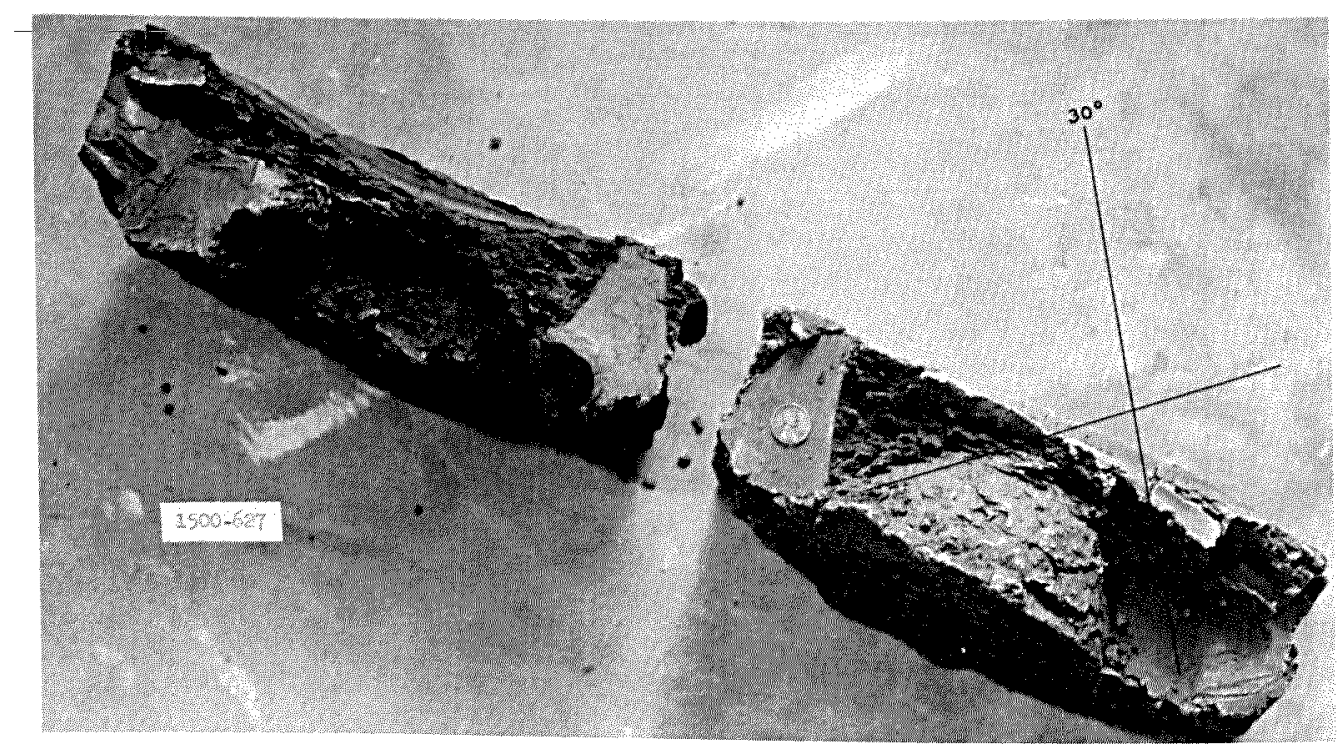

b. Cutaway view of 35- to 38-inch soil level

Fig. 48. Soil deformations, Test 5; H/B $=1, \mathrm{P}_{\mathrm{S}}=37.5 \mathrm{psi}$ 


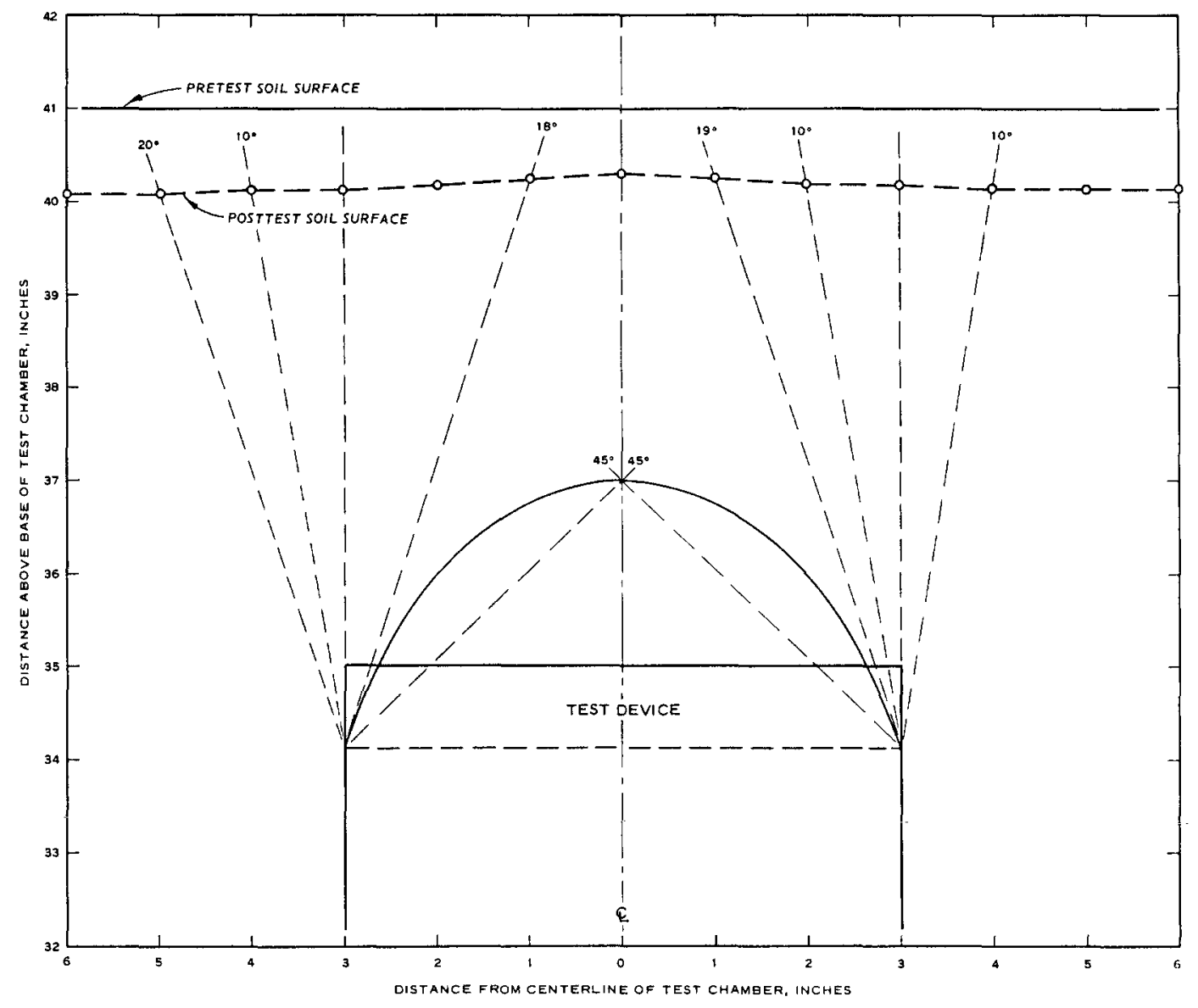

Fig. 49. Profile of soil deformations, Test 5; $\mathrm{H} / \mathrm{B}=1, \mathrm{P}_{\mathrm{S}}=37.5 \mathrm{psi}$ 


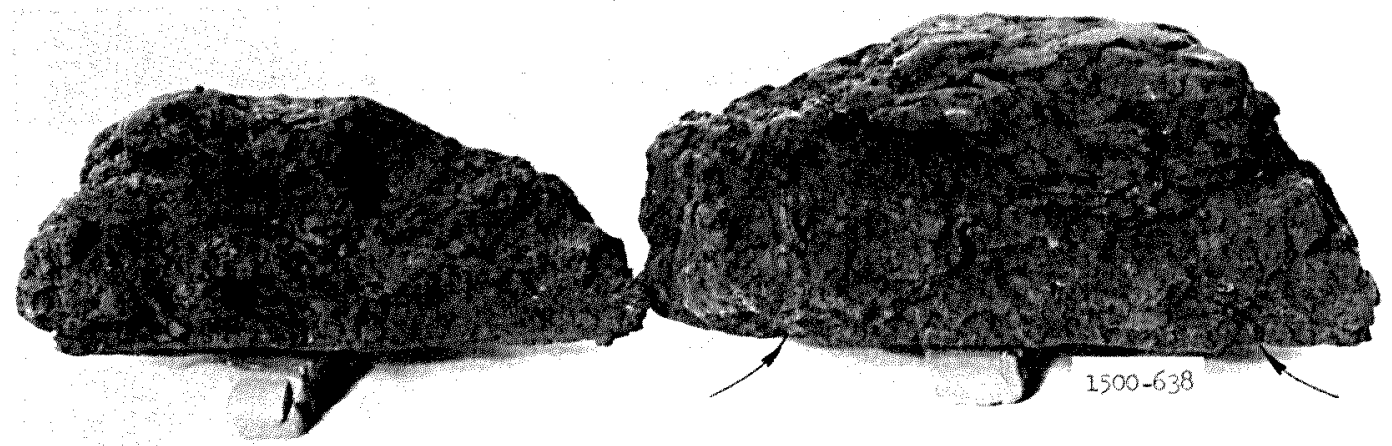

a. Soil directly above test device

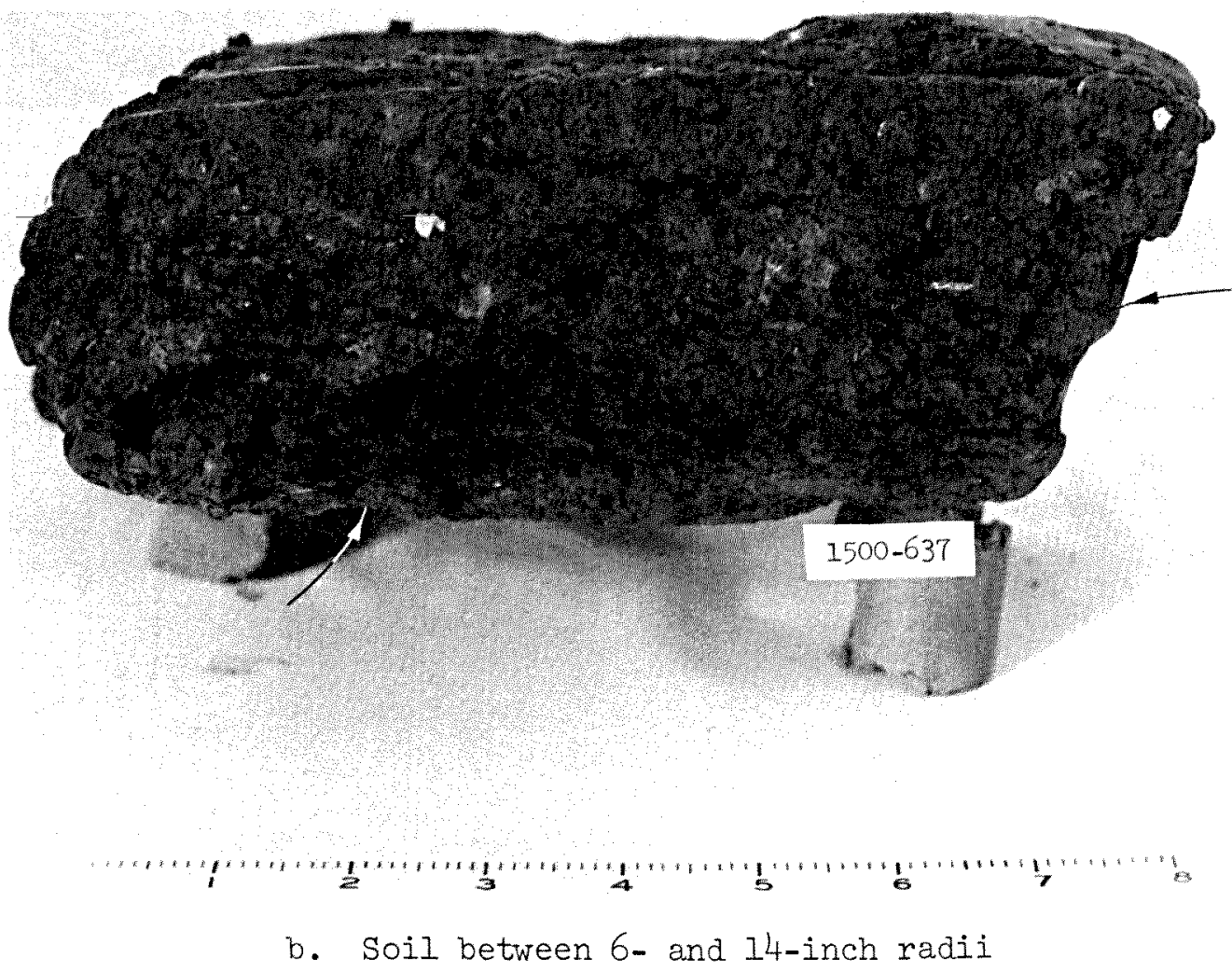

Fig. 50. Posttest soil samples removed from 35- to 38-inch layer, Test $6 ; \mathrm{H} / \mathrm{B}=1, \mathrm{P}_{\mathrm{S}}=37.5 \mathrm{psi}$ 

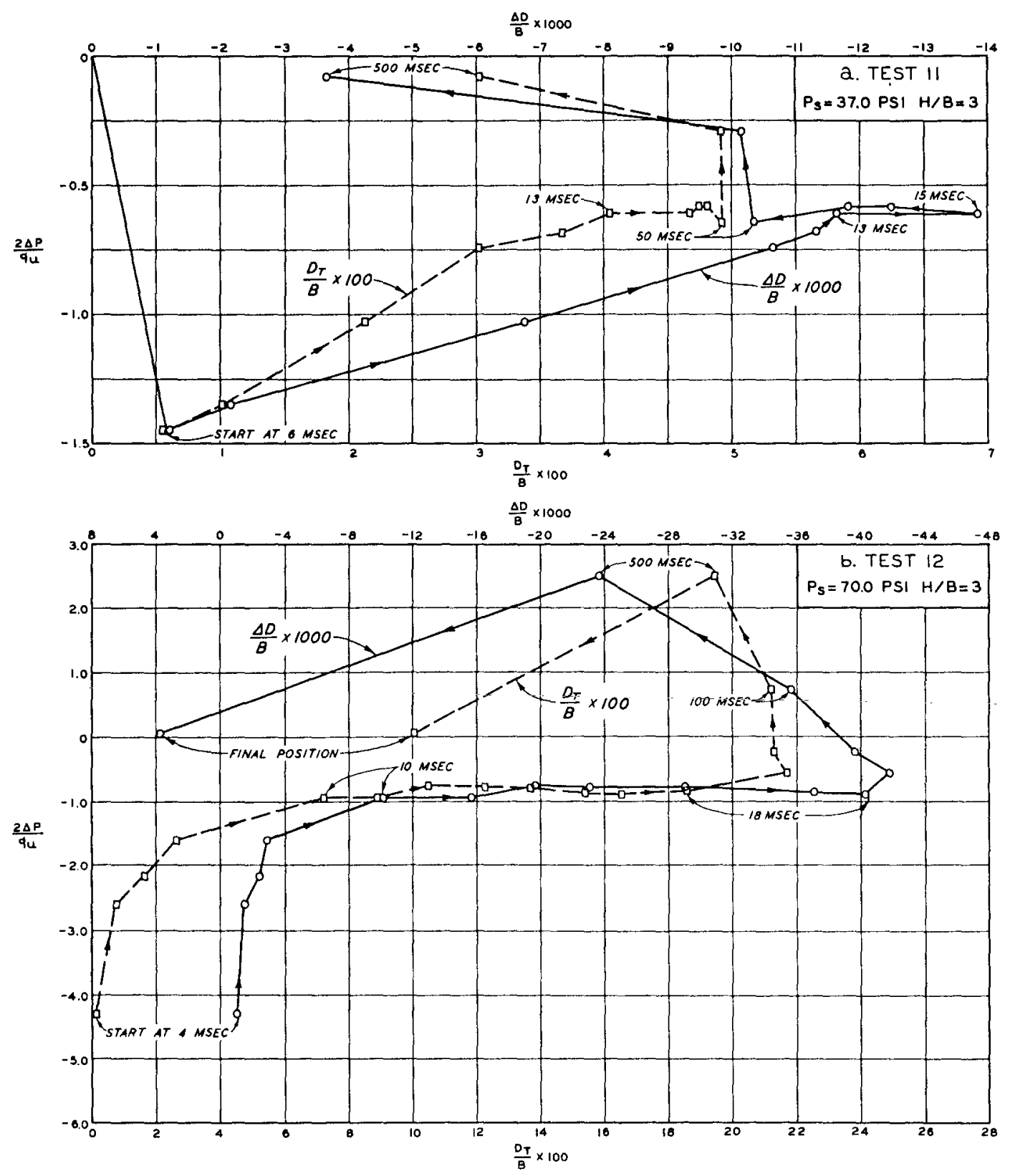

Fig. 51. Dimensionless plot of pressure versus deflection for dynamic Tests 11 and 12 

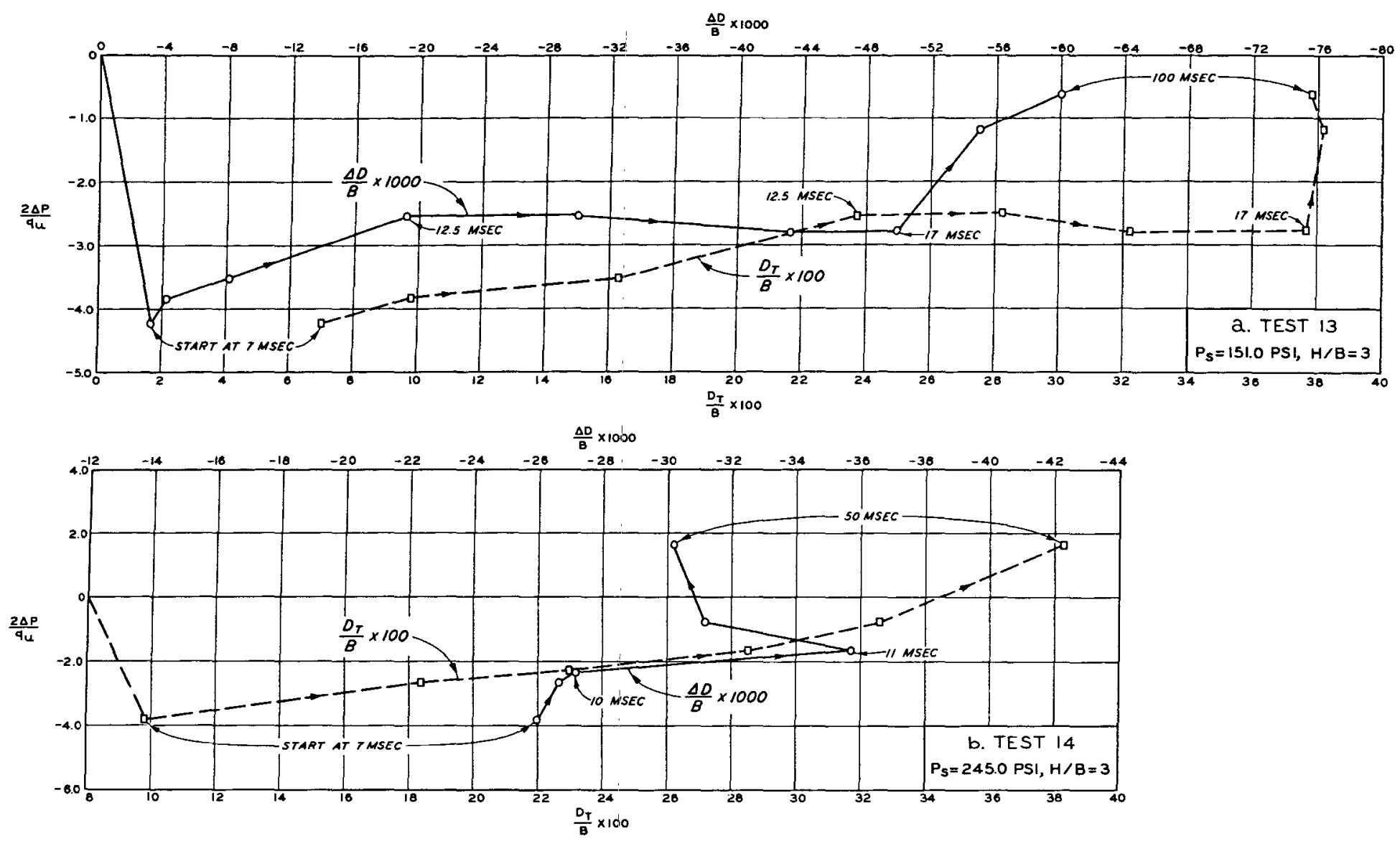

Fig. 52. Dimensionless plot of pressure versus deflection for dynamic Tests 13 and 14 

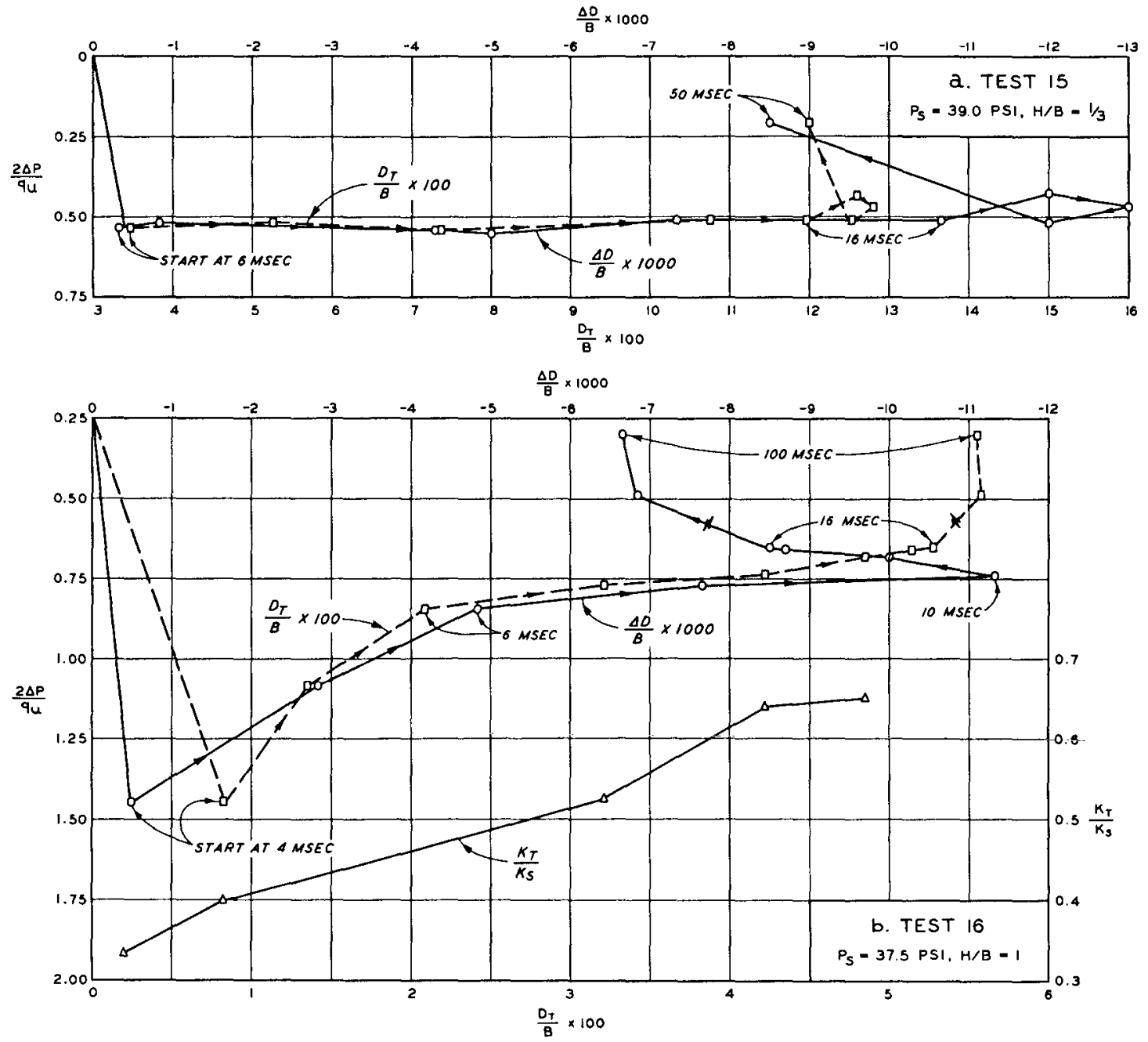

Fig. 53. Dimensionless plot of pressure versus deflection for dynamic Tests 15 and 16 

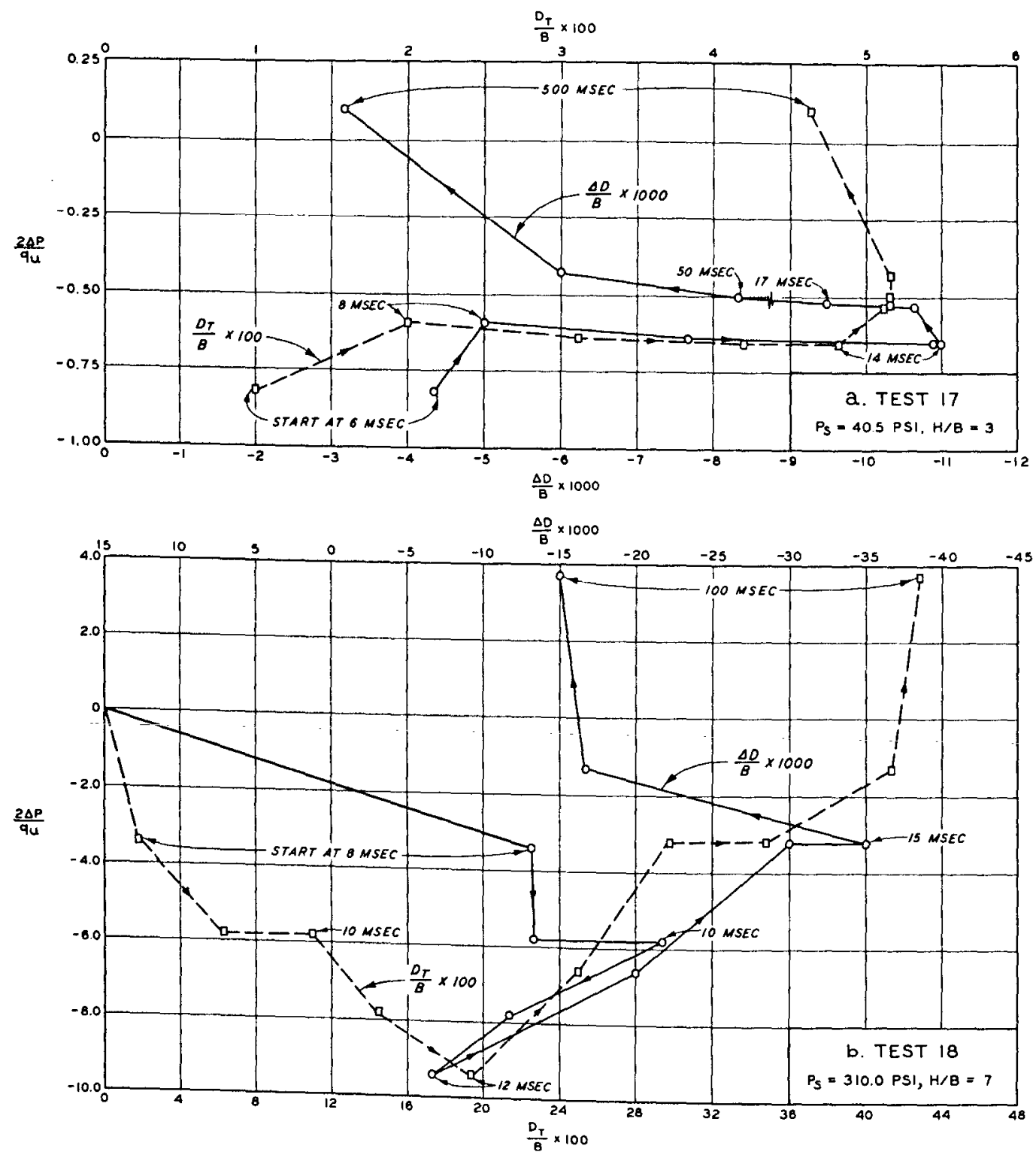

Fig. 54. Dimensionless plot of pressure versus deflection for dynamic Tests 17 and 18 

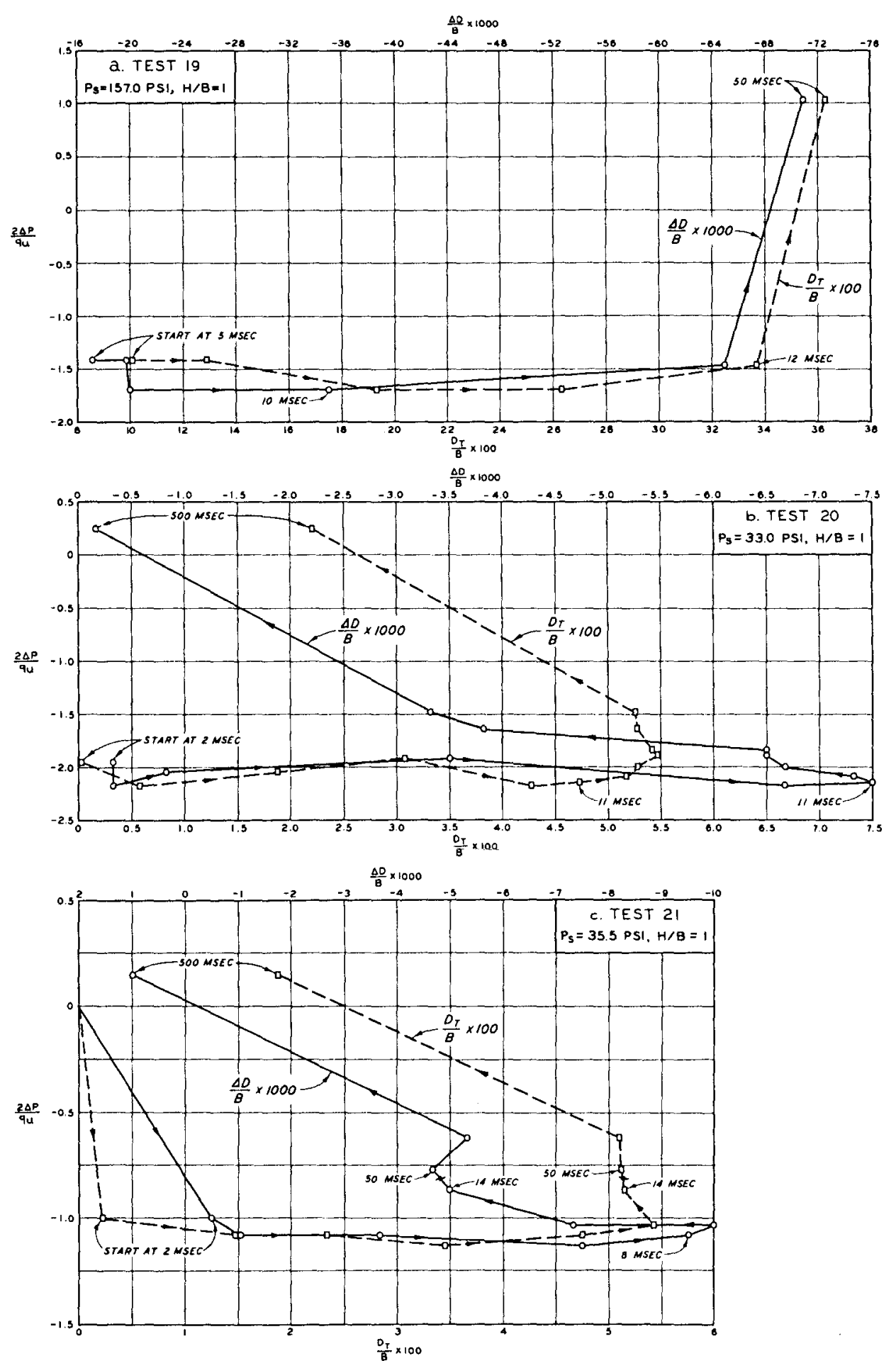

Fig. 55. Dimensionless plot of pressure versus deflection for dynamic Tests 19, 20, and 21 

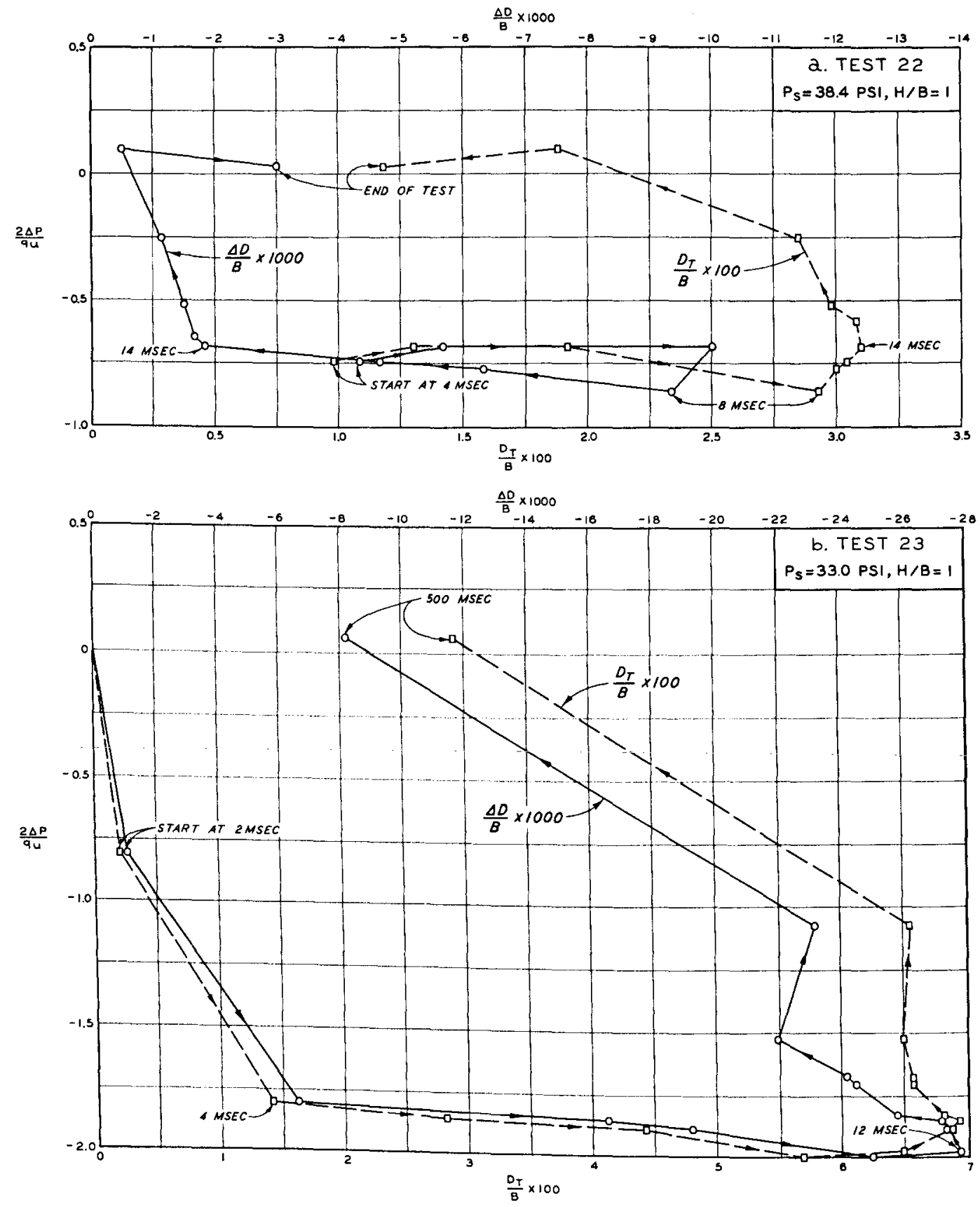

Fig. 56. Dimensionless plot of pressure versus deflection for dynamic Tests 22 and 23 


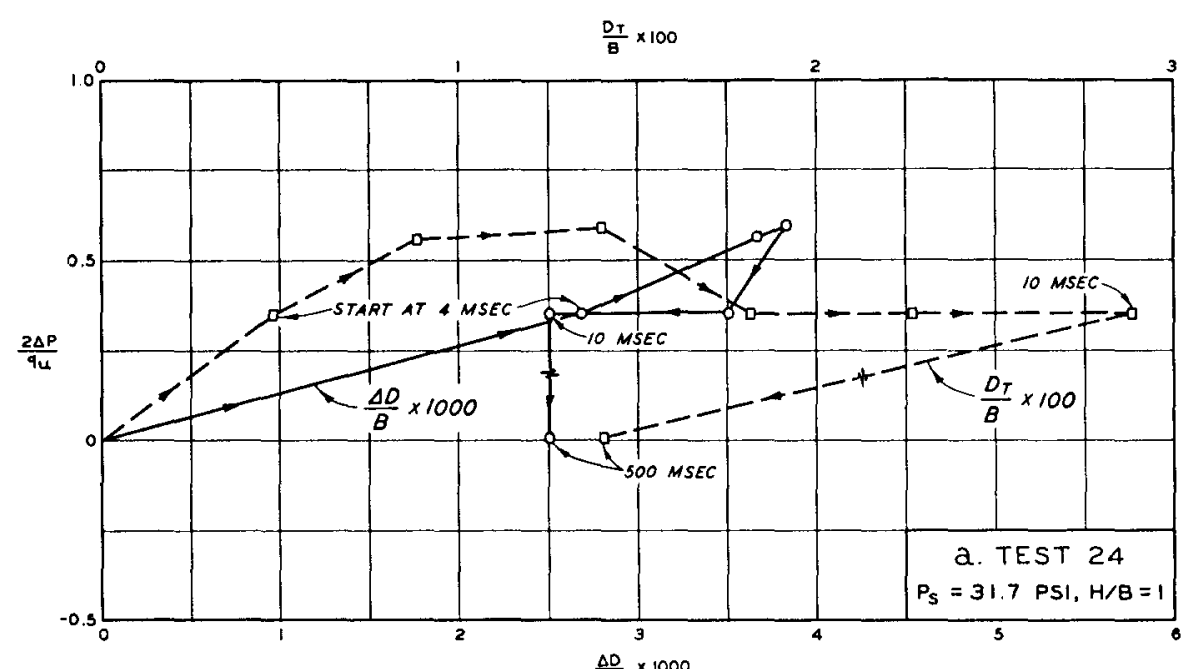

$\frac{\Delta \mathrm{D}}{\mathrm{B}} \times 1000$

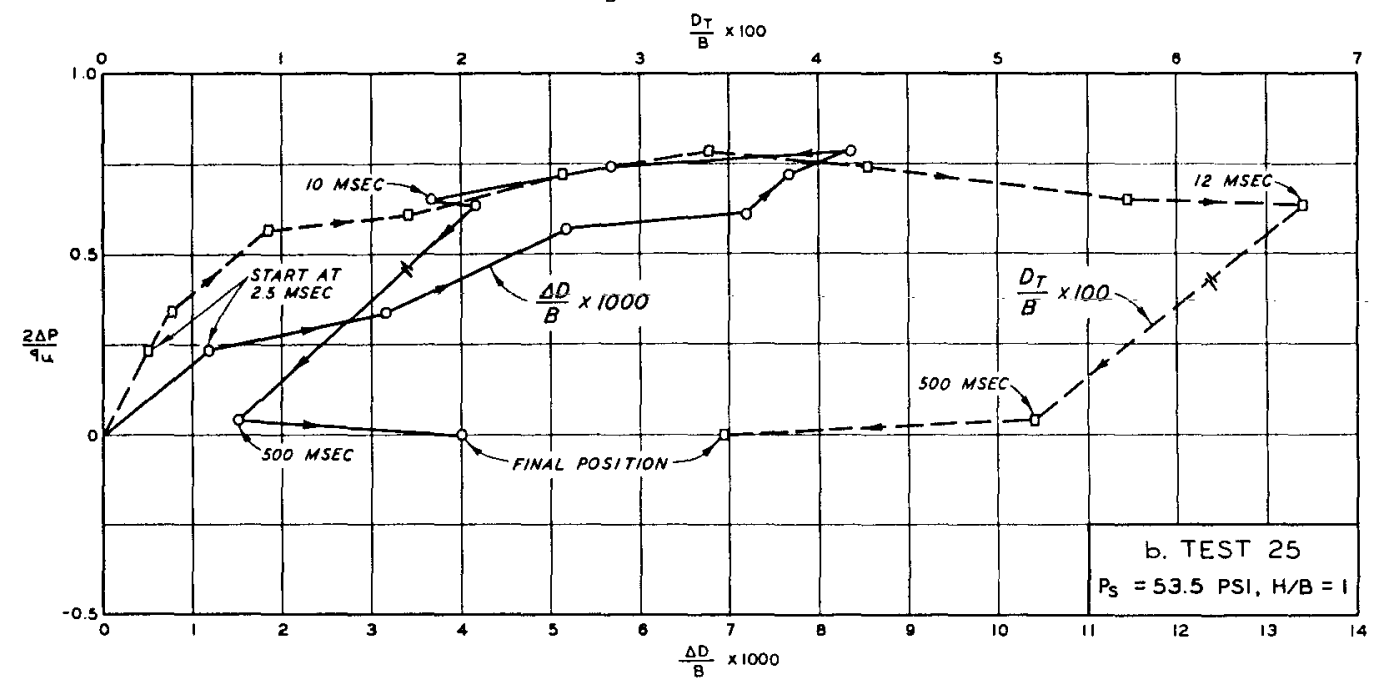

Fig. 57. Dimensionless plot of pressure versus deflection for dynamic Tests 24 and 25 

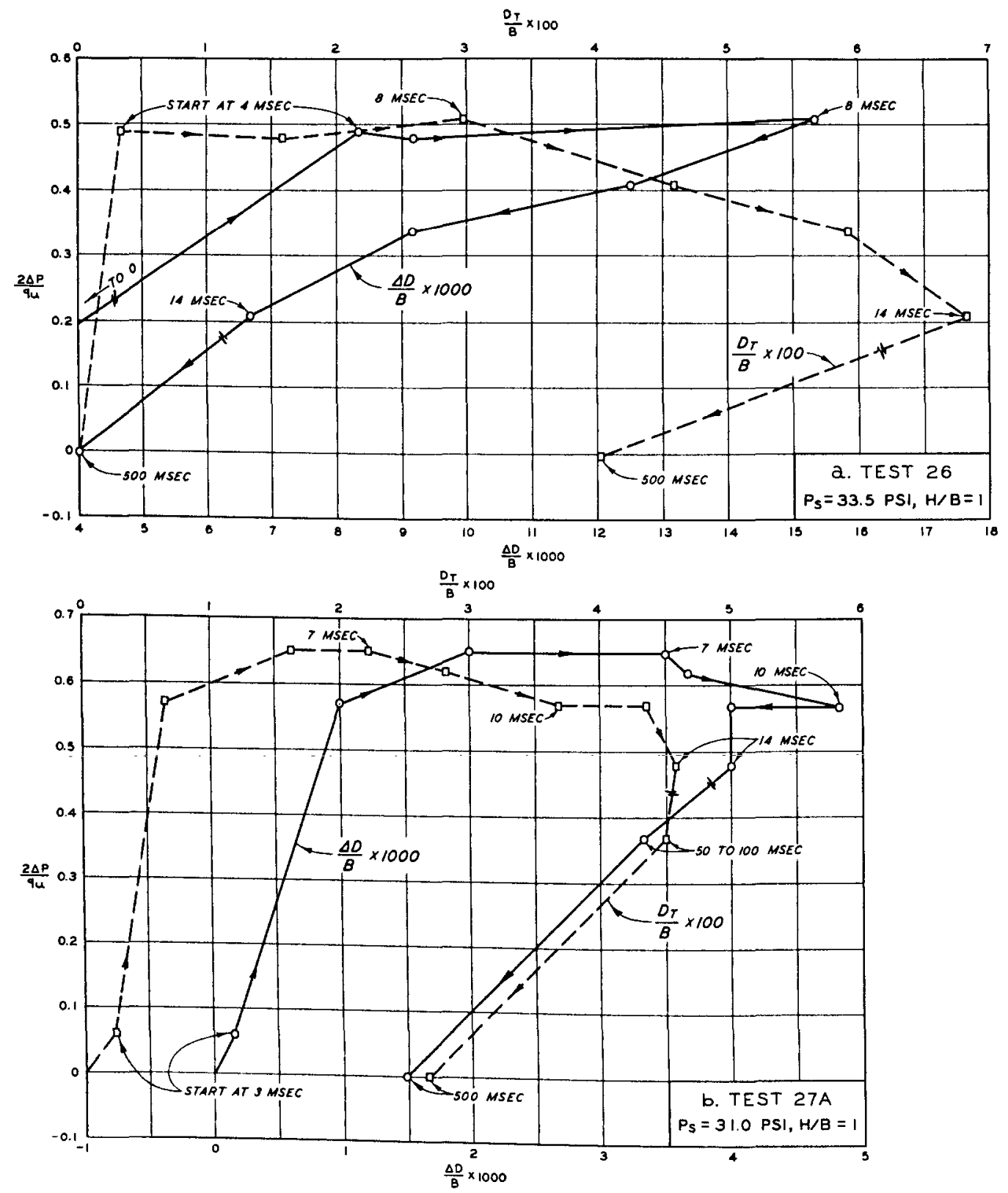

Fig. 58. Dimensionless plot of pressure versus deflection for dynamic Tests 26 and $27 \mathrm{~A}$ 

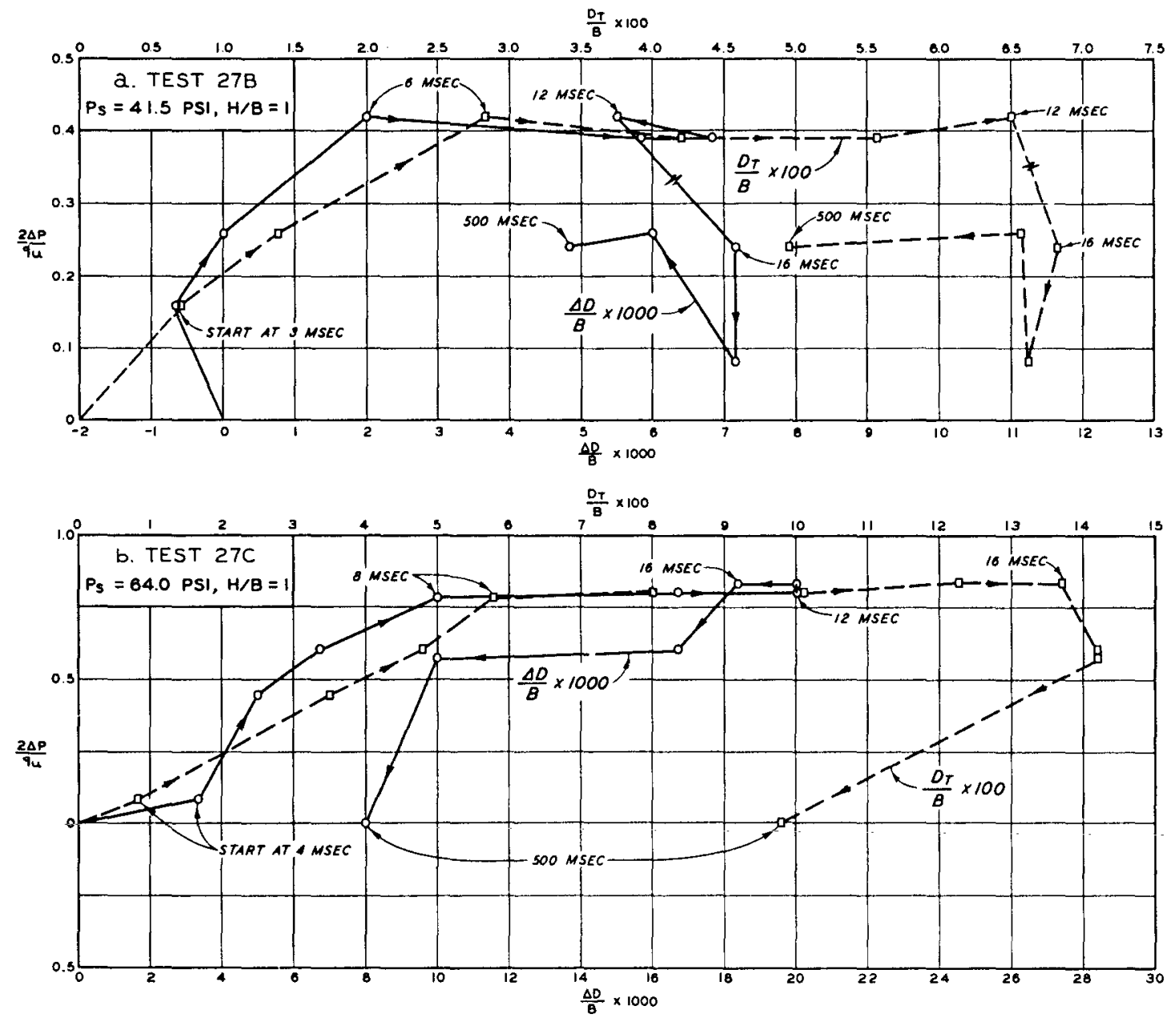

Fig. 59. Dimensionless plot of pressure versus deflection for dynamic Tests $27 \mathrm{~B}$ and $27 \mathrm{C}$ 
a. TEST $\|, H / B=3$

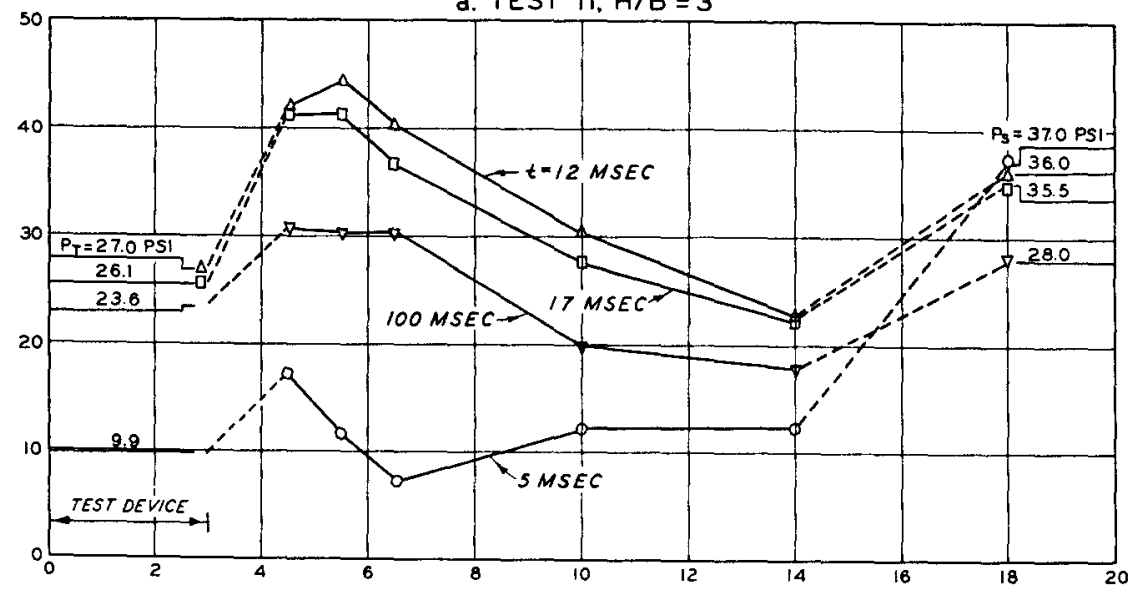

b. TEST $12, H / B=3$
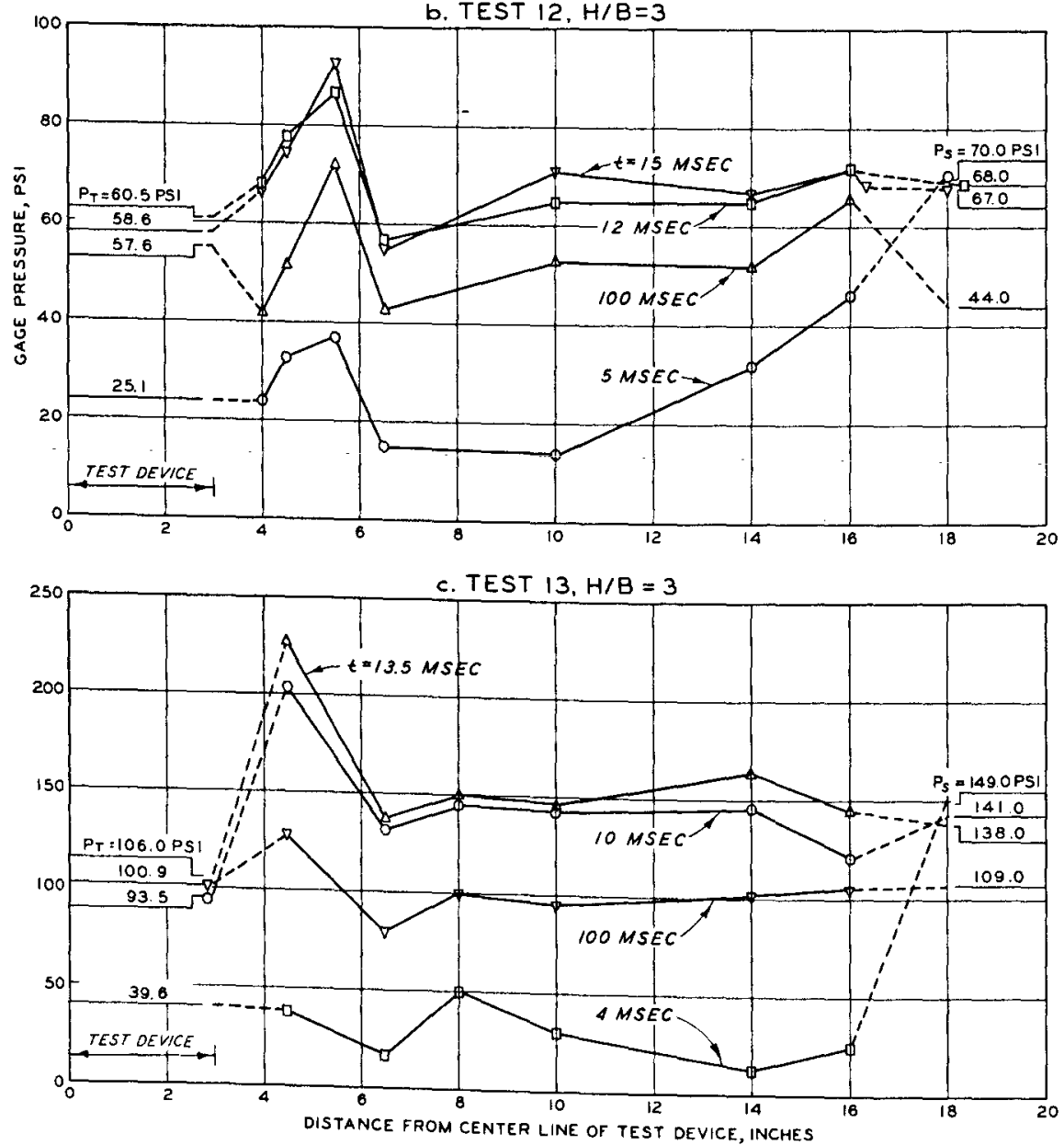

Fig. 60. Variation of vertical soil stress with time at the 35-inch level, Tests 11, 12, and 13 
a. TEST $15, H / B=1 / 3$

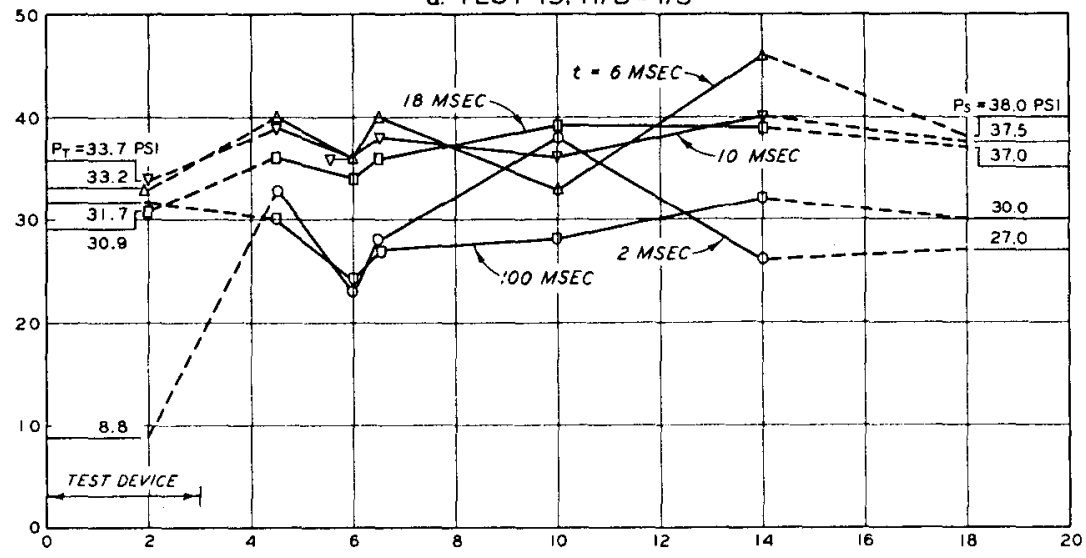

b. TEST $16, H / B=1$
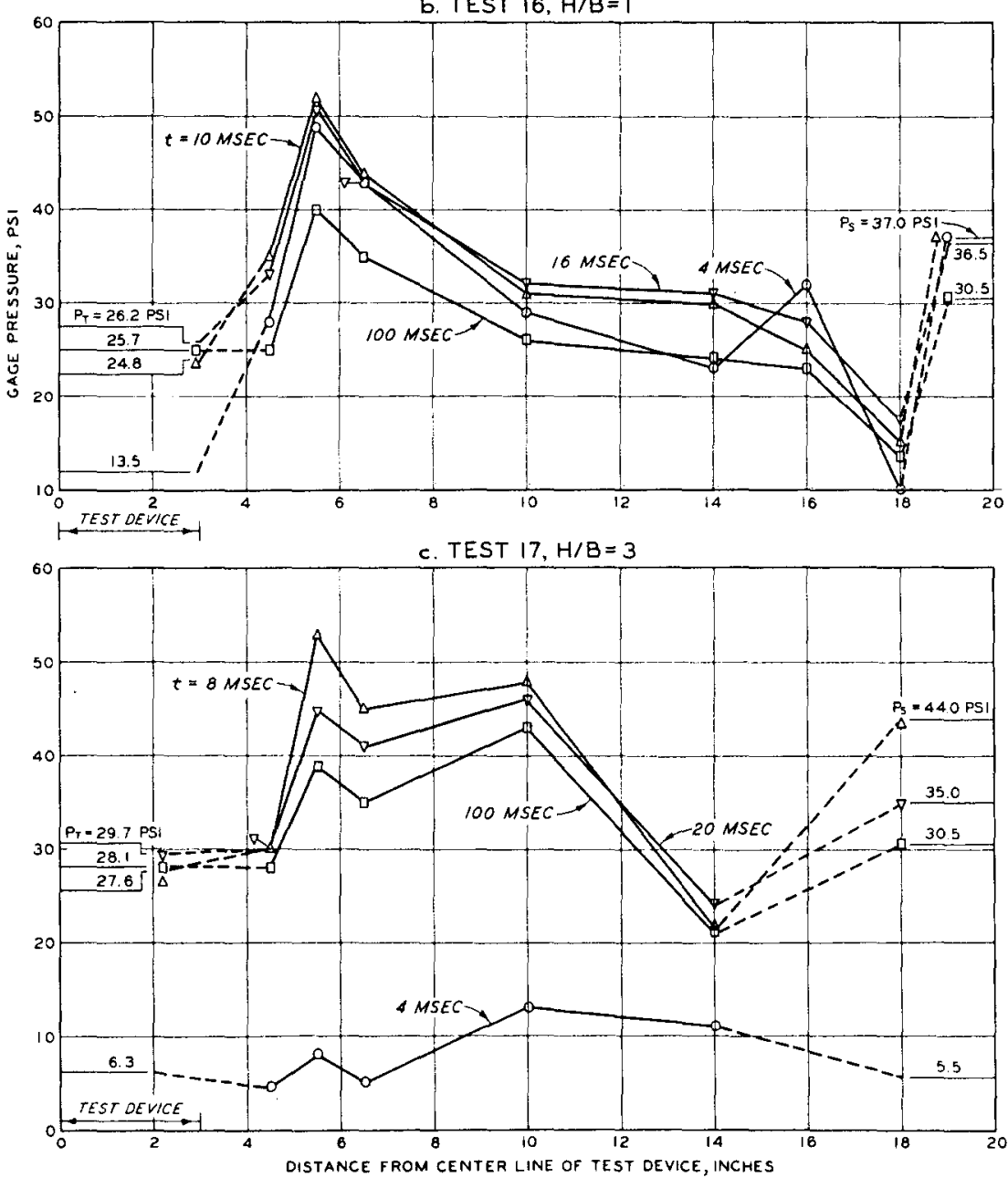

Fig. 61. Variation of vertical soil stress with time at the 35-inch level, Tests 15, 16, and 17 
a. TEST $20, H / B=1$

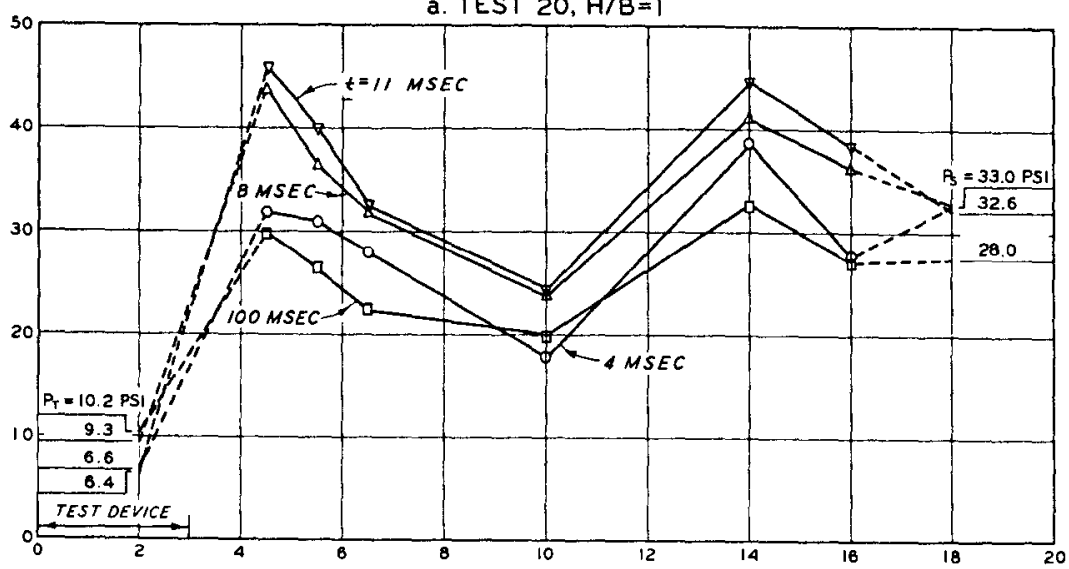

b. TEST 2I, $H / B=1$
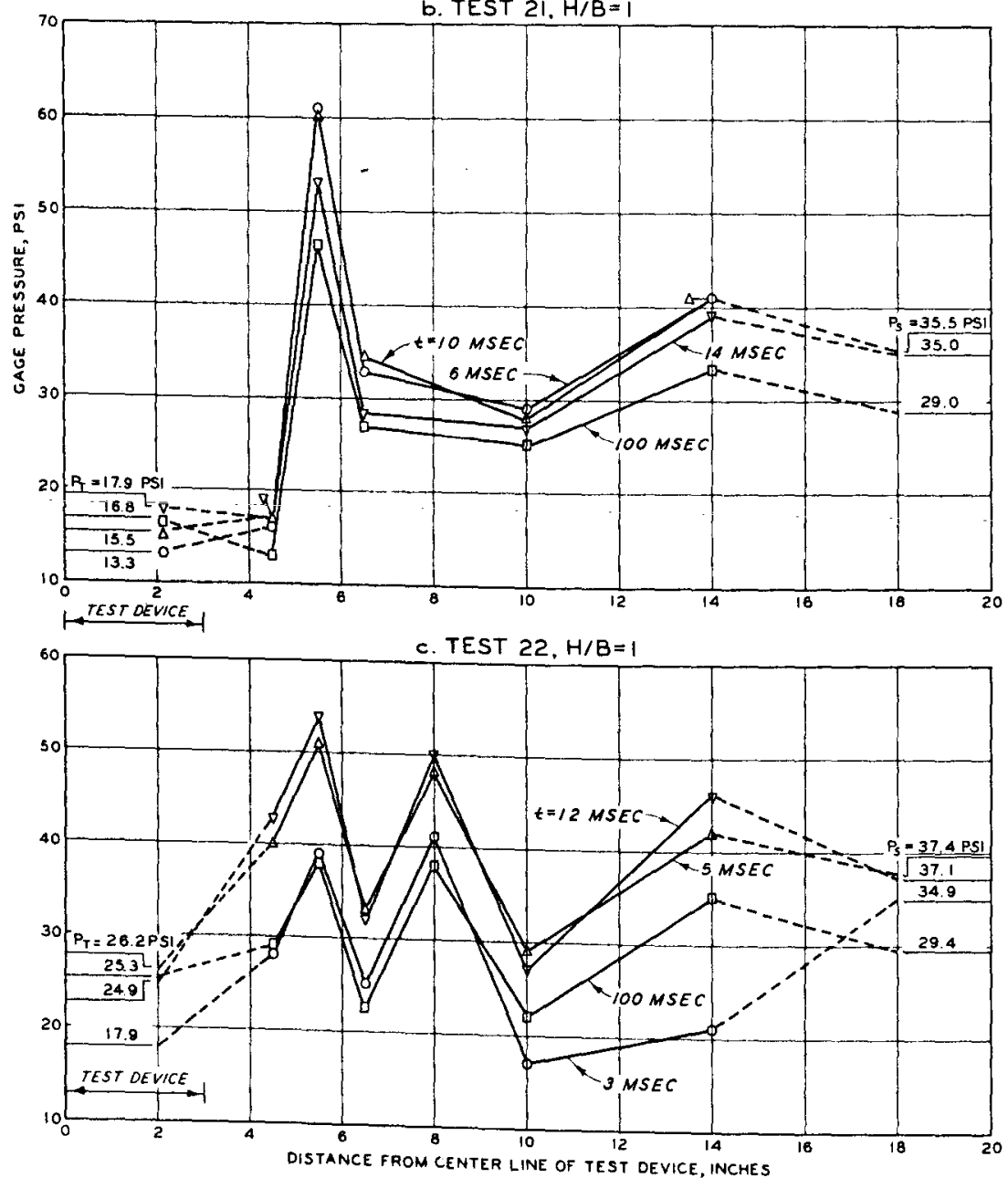

Fig. 62. Variation of vertical soil stress with time at the 35 -inch level, Tests 20,21 , and 22 
a. TEST $23, H / B=1$
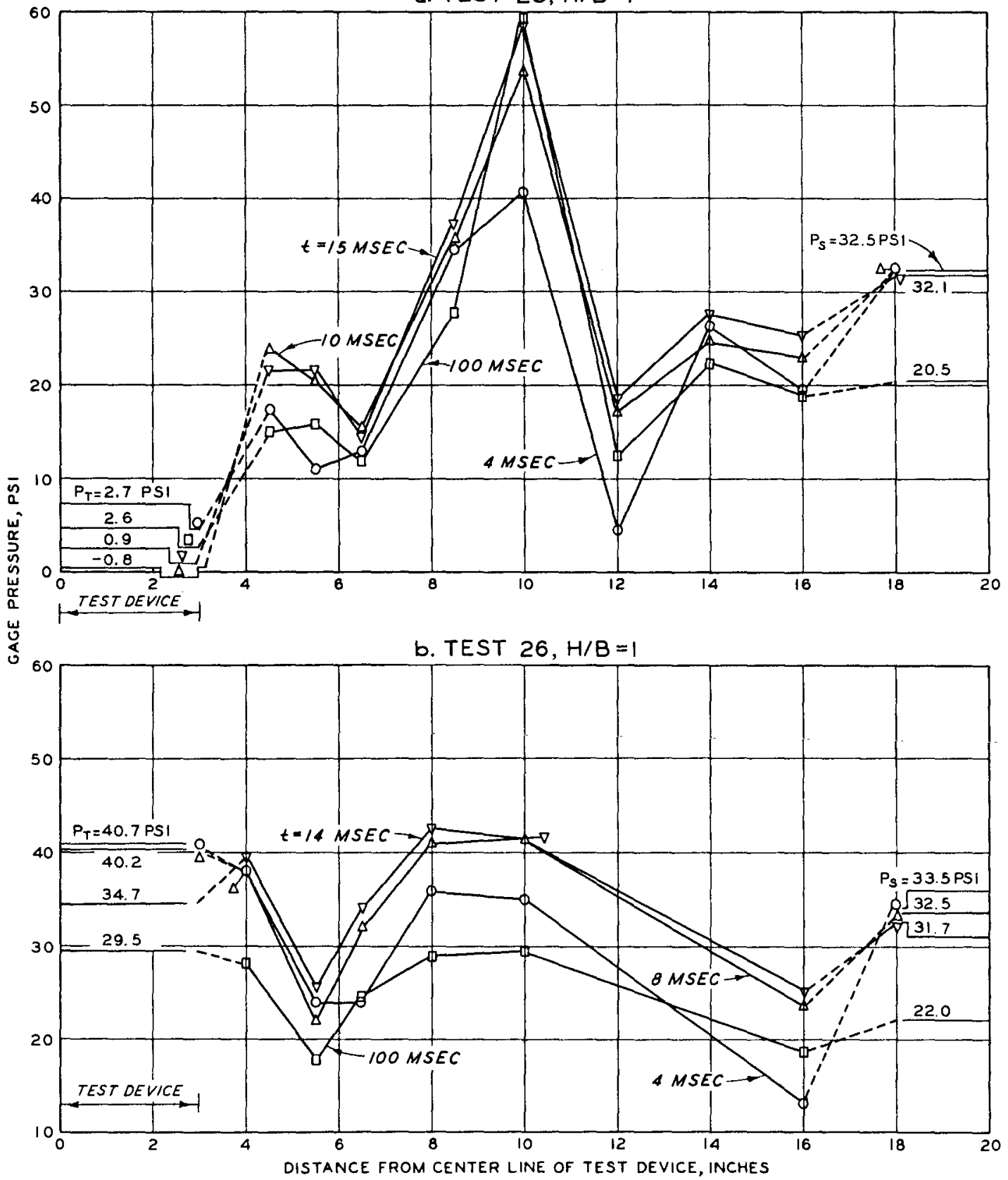

Fig. 63. Variation of vertical soil stress with time at the 35-inch level, Tests 23 and 26 
a. TEST $12, \mathrm{H} / \mathrm{B}=3$

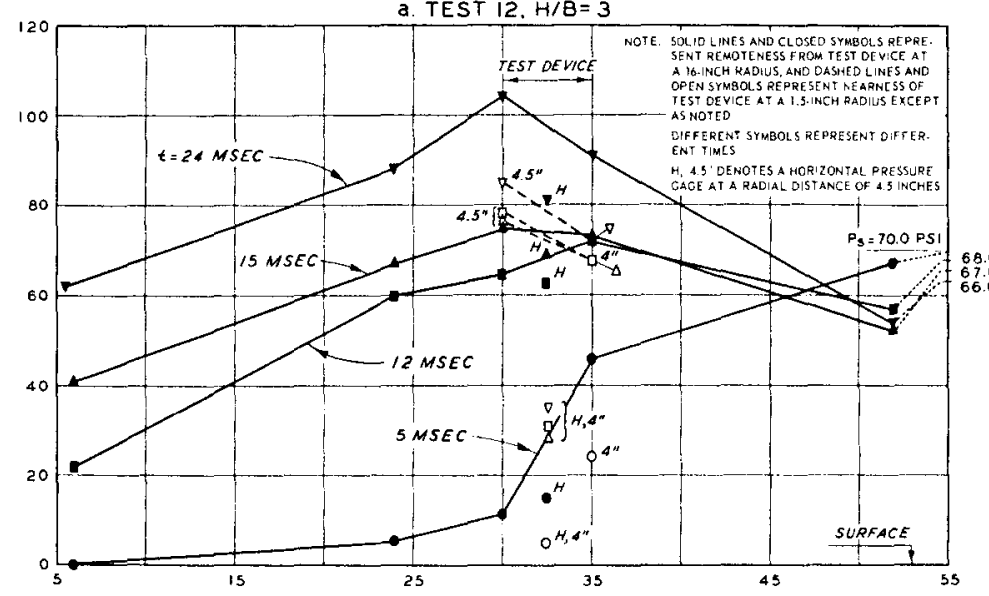

68.0
67.0
66.0
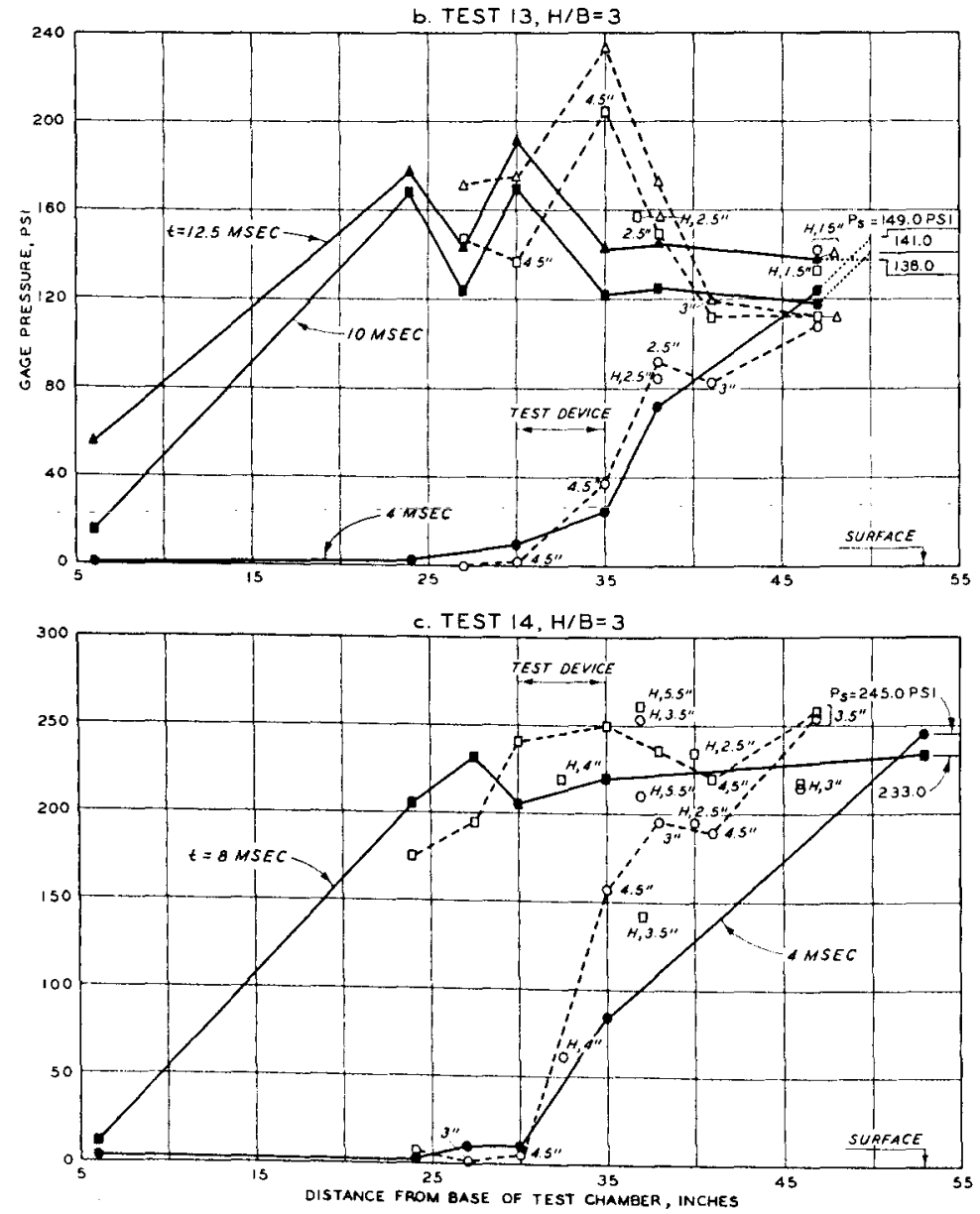

Fig. 64. Variation of soil stress with depth and time, Tests 12, 13, and 14 
a. TEST $18, H / B=7$

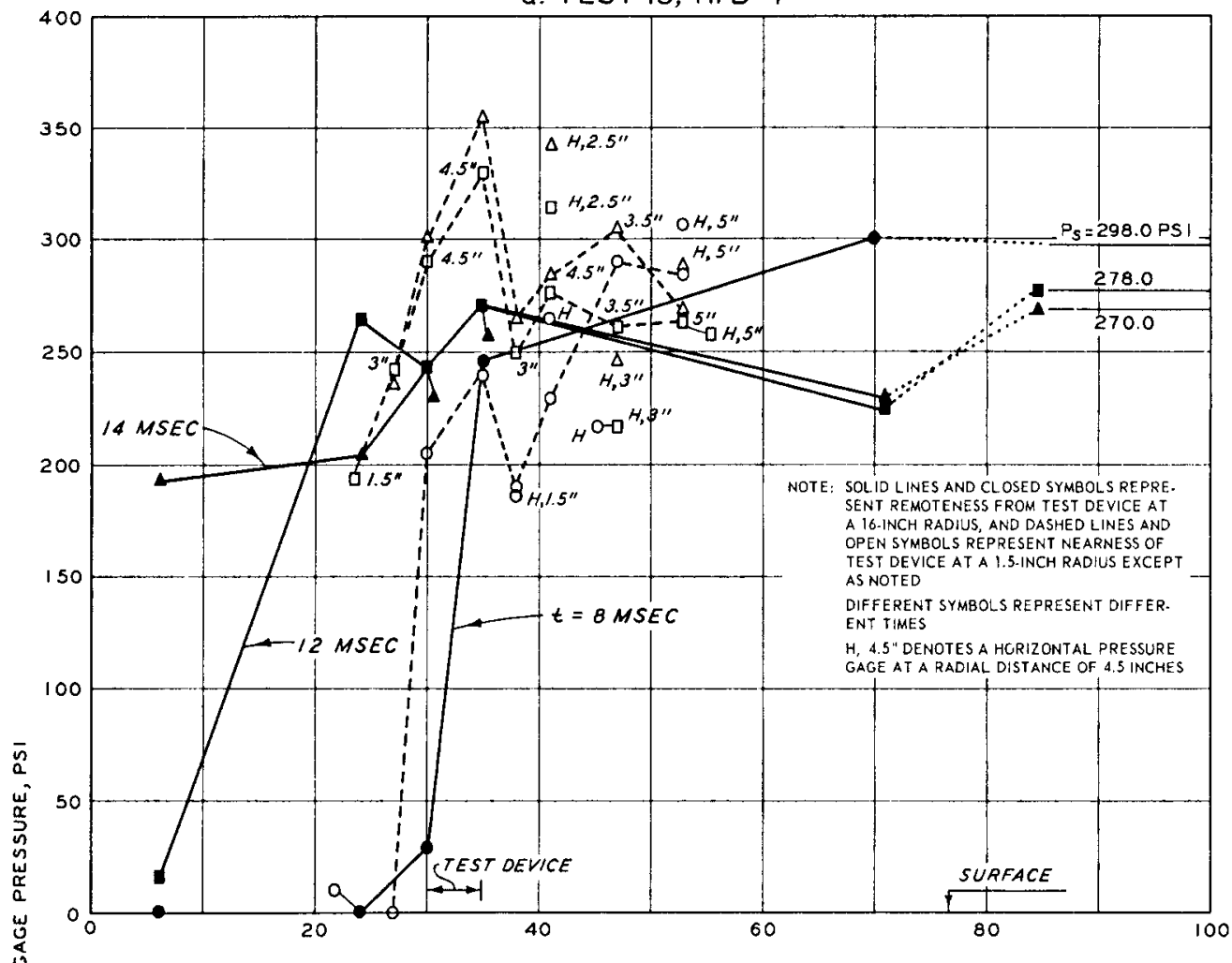

b. TEST $19, H / B=1$

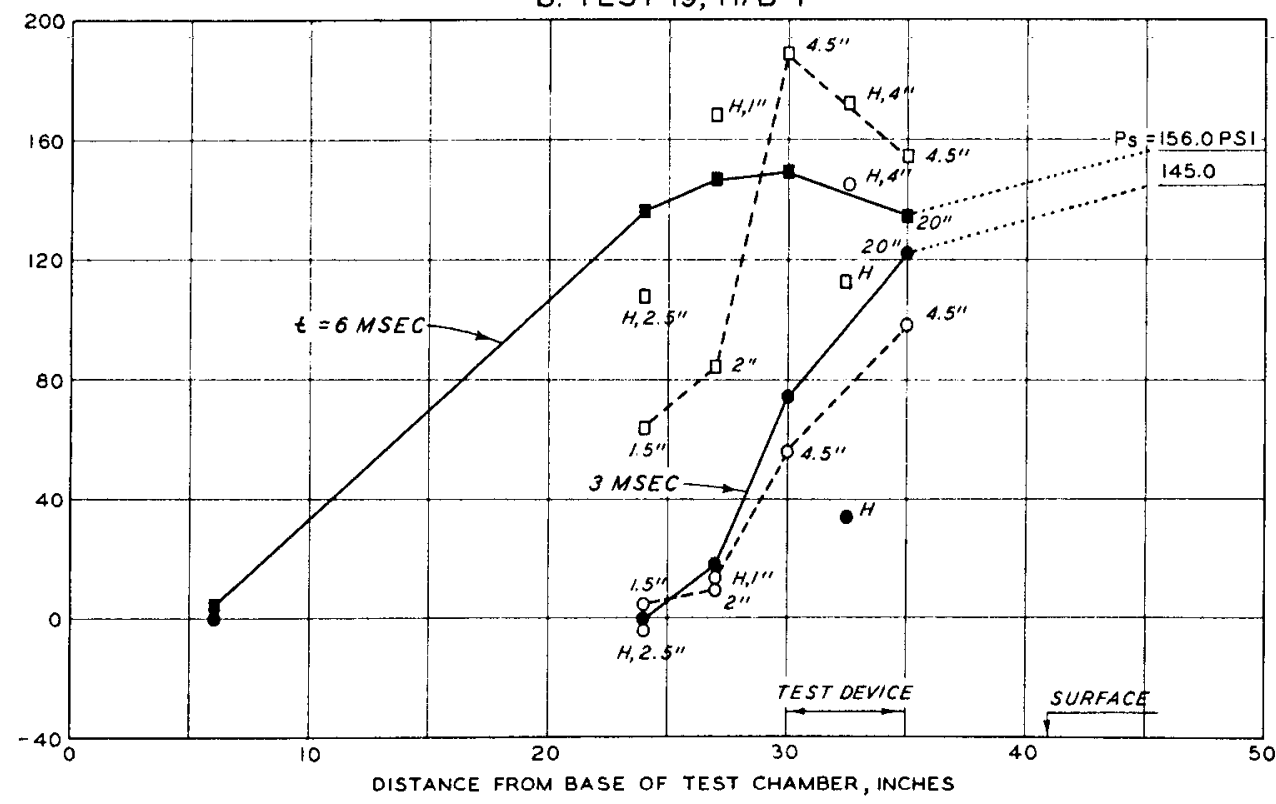

Fig. 65. Variation of soil stress with depth and time, Tests 18 and 19 
a. TEST 22, $H / B=1$

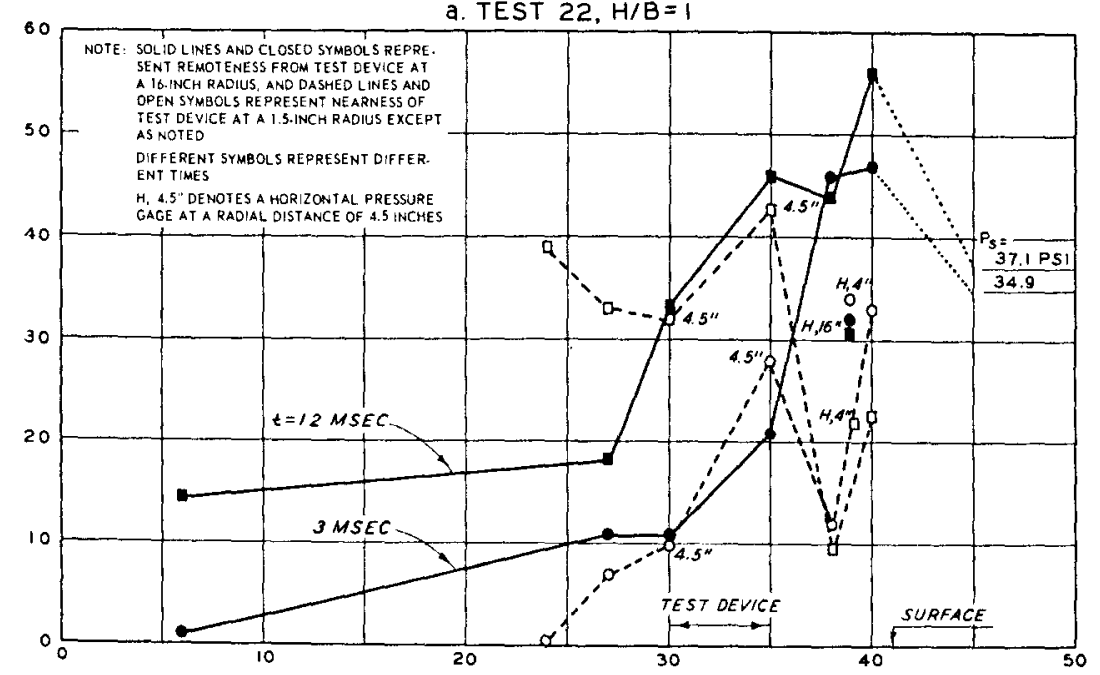

b. TEST $23, H / B=1$

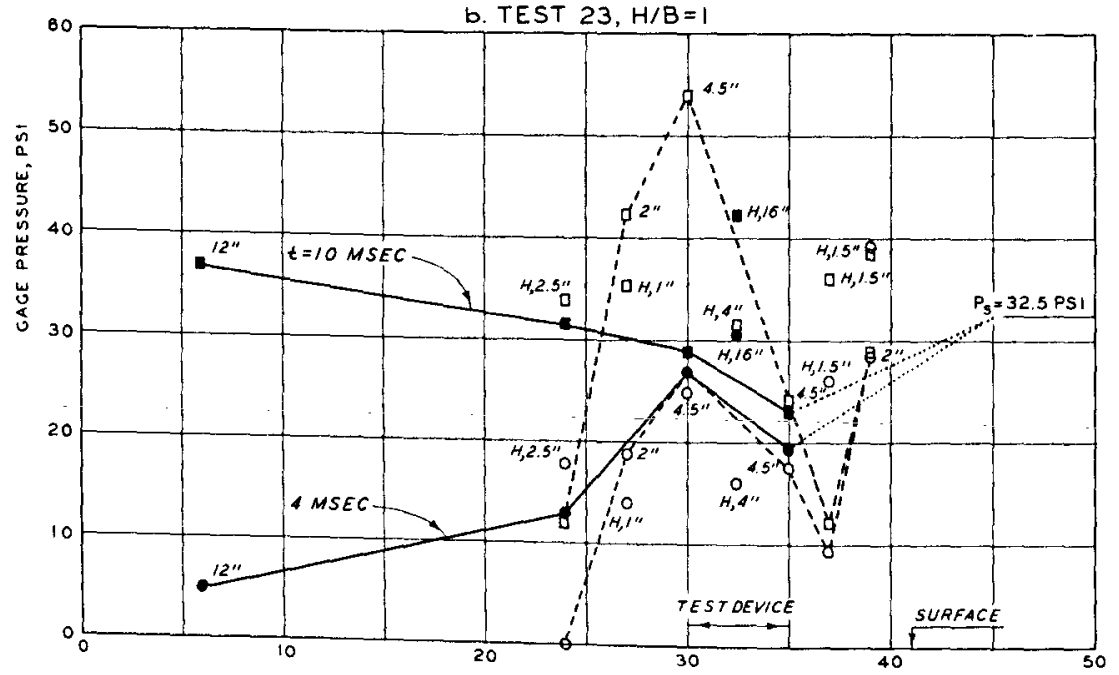

c. TEST $25, \mathrm{H} / \mathrm{B}=1$

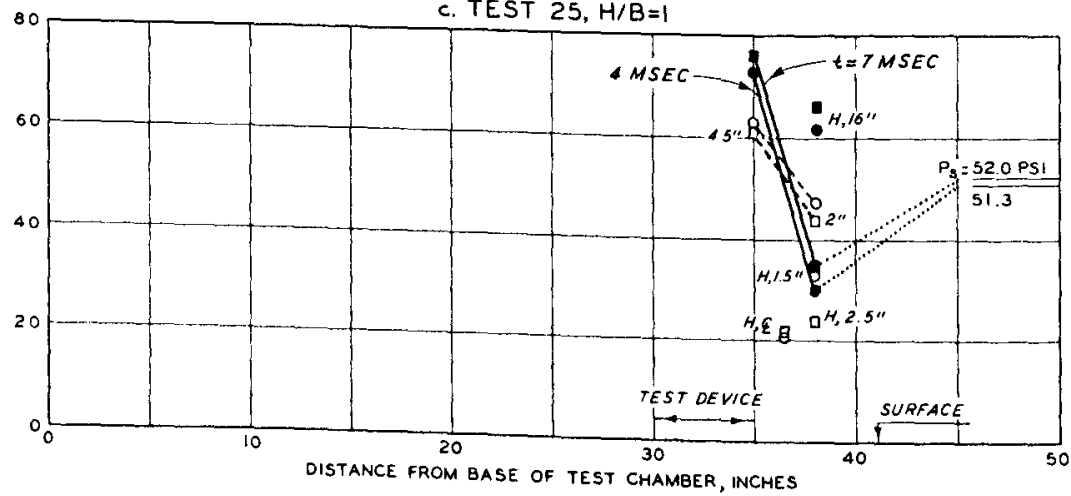

Fig. 66. Variation of soil stress with depth and time, Tests 22, 23, and 25 
a. TEST $C$

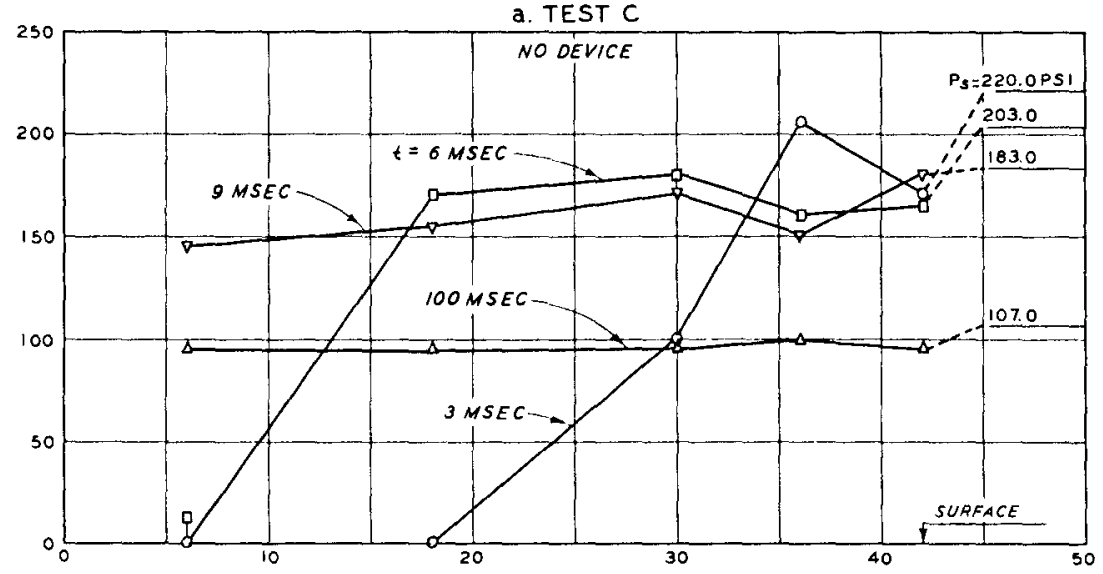

b. TEST E

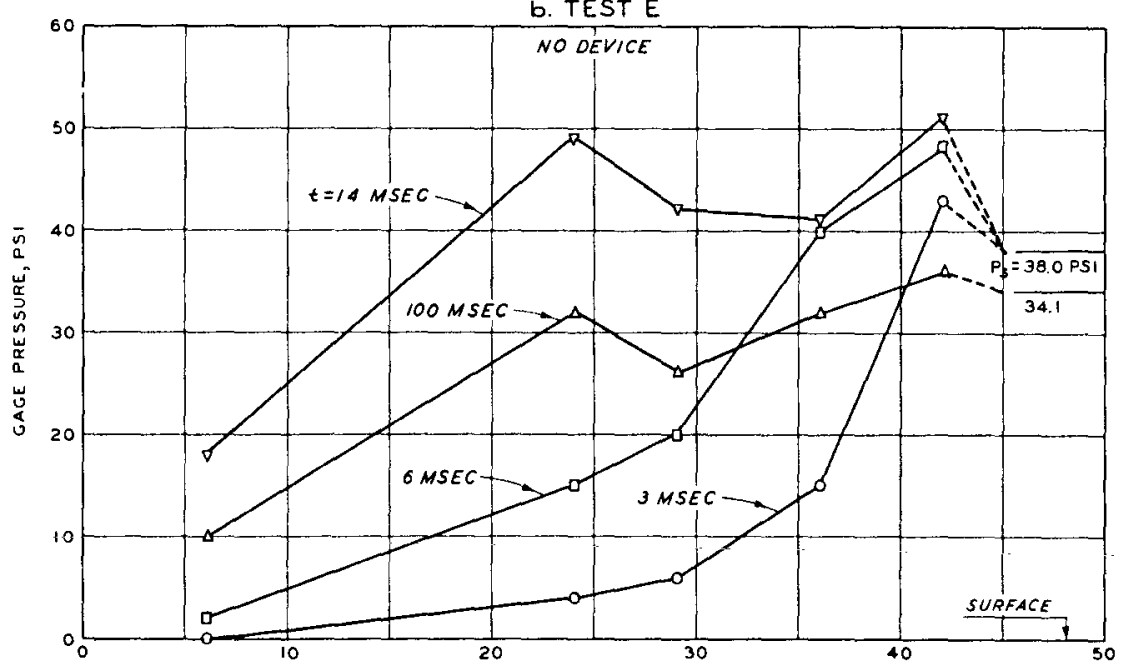

c. TEST F

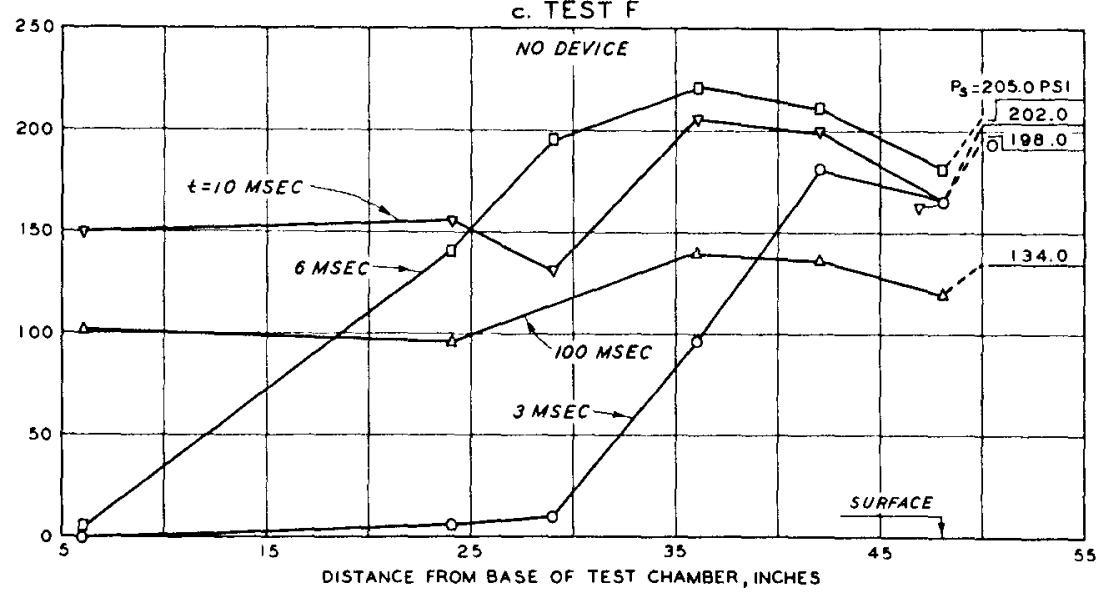

Fig. 67. Variation of soil stress with depth and time, preliminary Tests $\mathrm{C}, \mathrm{E}$, and $\mathrm{F}$ 


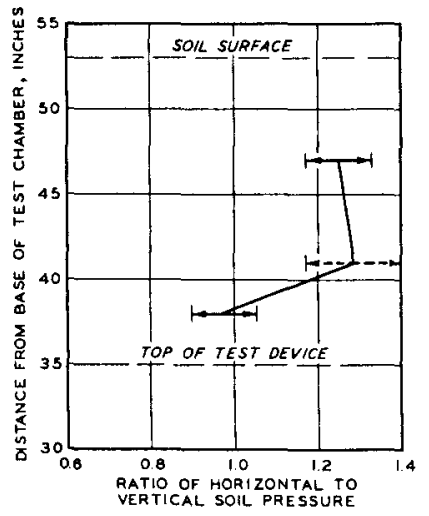

a. TEST 13

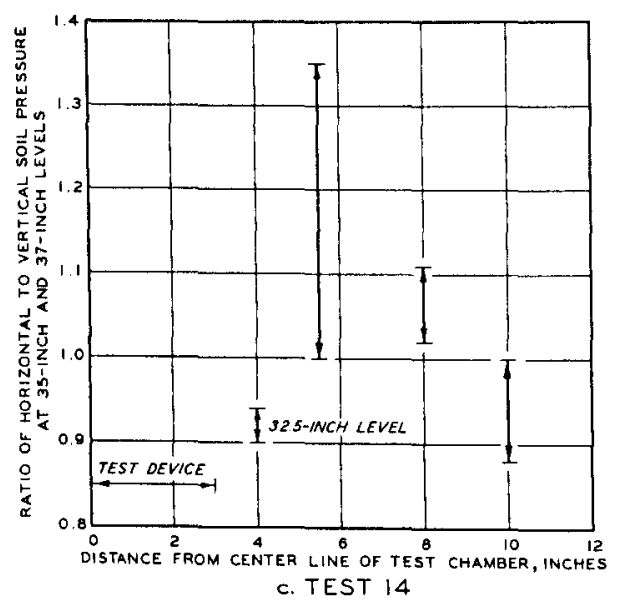

c. TEST 14

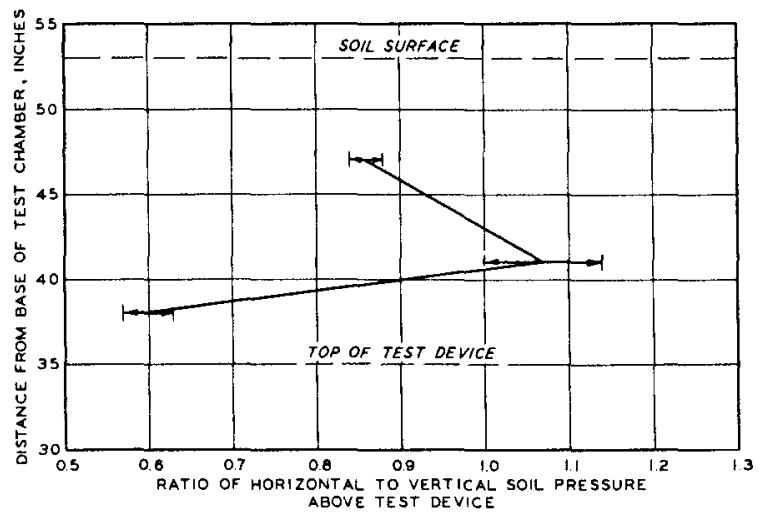

b. TEST 14

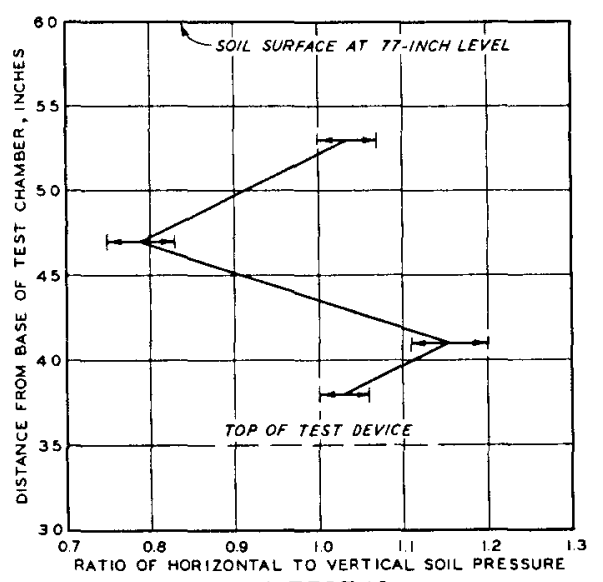

d. TEST 18

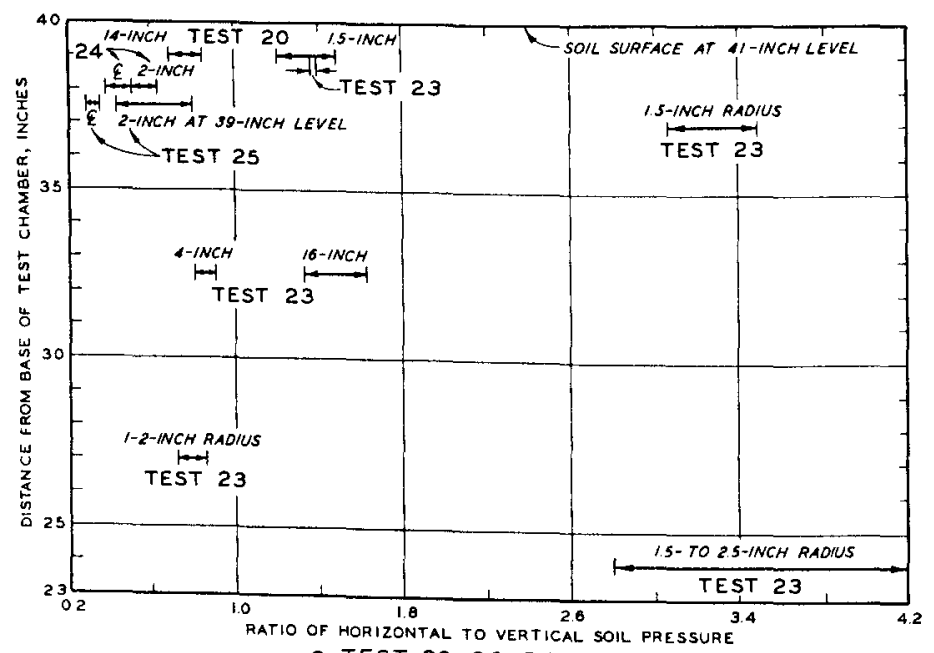

e. TEST 20, 23, 24, AND 25

Fig. 68. Variation of horizontal-to-vertical pressure ratio, Tests 13, 14, 18, 20, 23, 24, and 25 


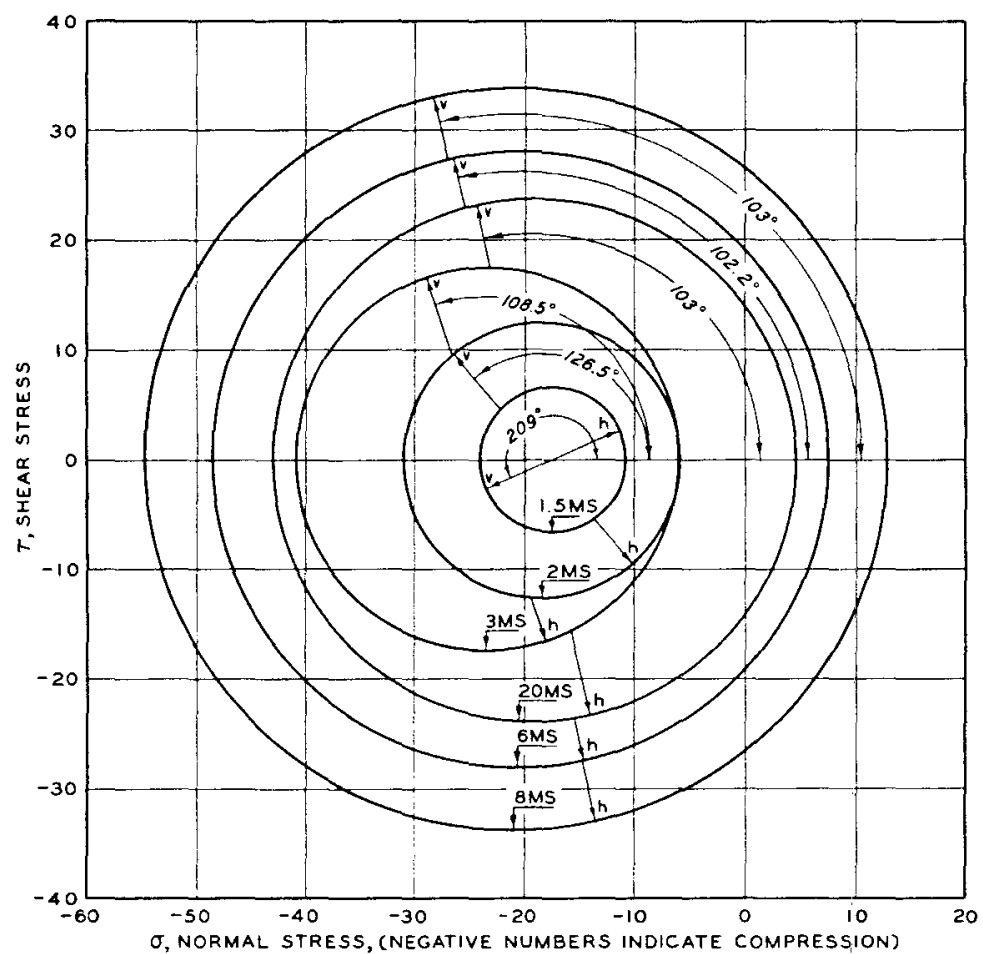

a. MOHR'S CIRCLES OF STRESS, GAGES PLACED AT 2-INCH RADIUS, 38-INCH LEVEL

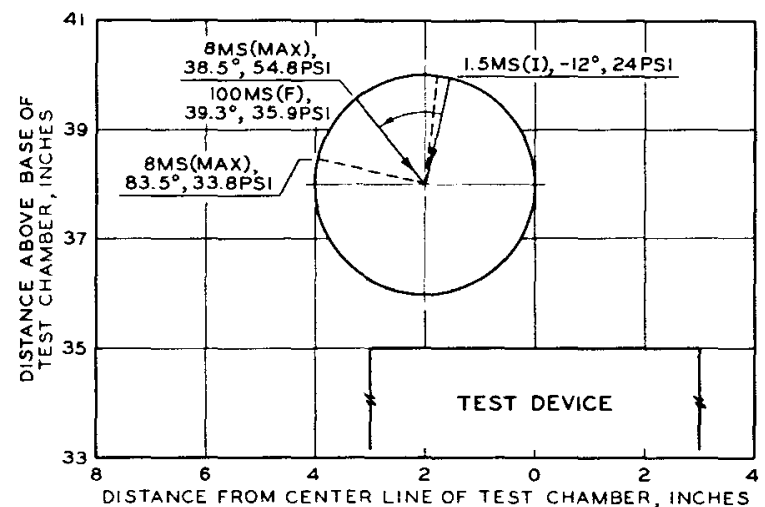

b. DIRECTION OF PRINCIPAL STRESS AND PLANES OF MAXIMUM SHEAR

$\underline{\text { KEY }}$

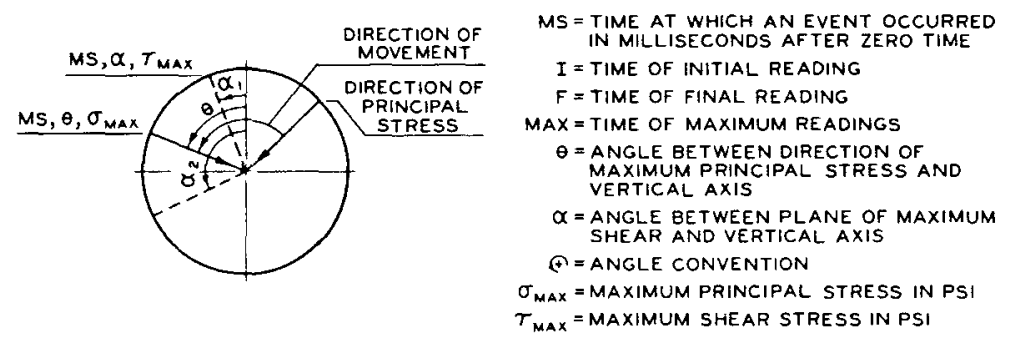

Fig. 69. Determination of direction and magnitude of principal stress, Test 24 

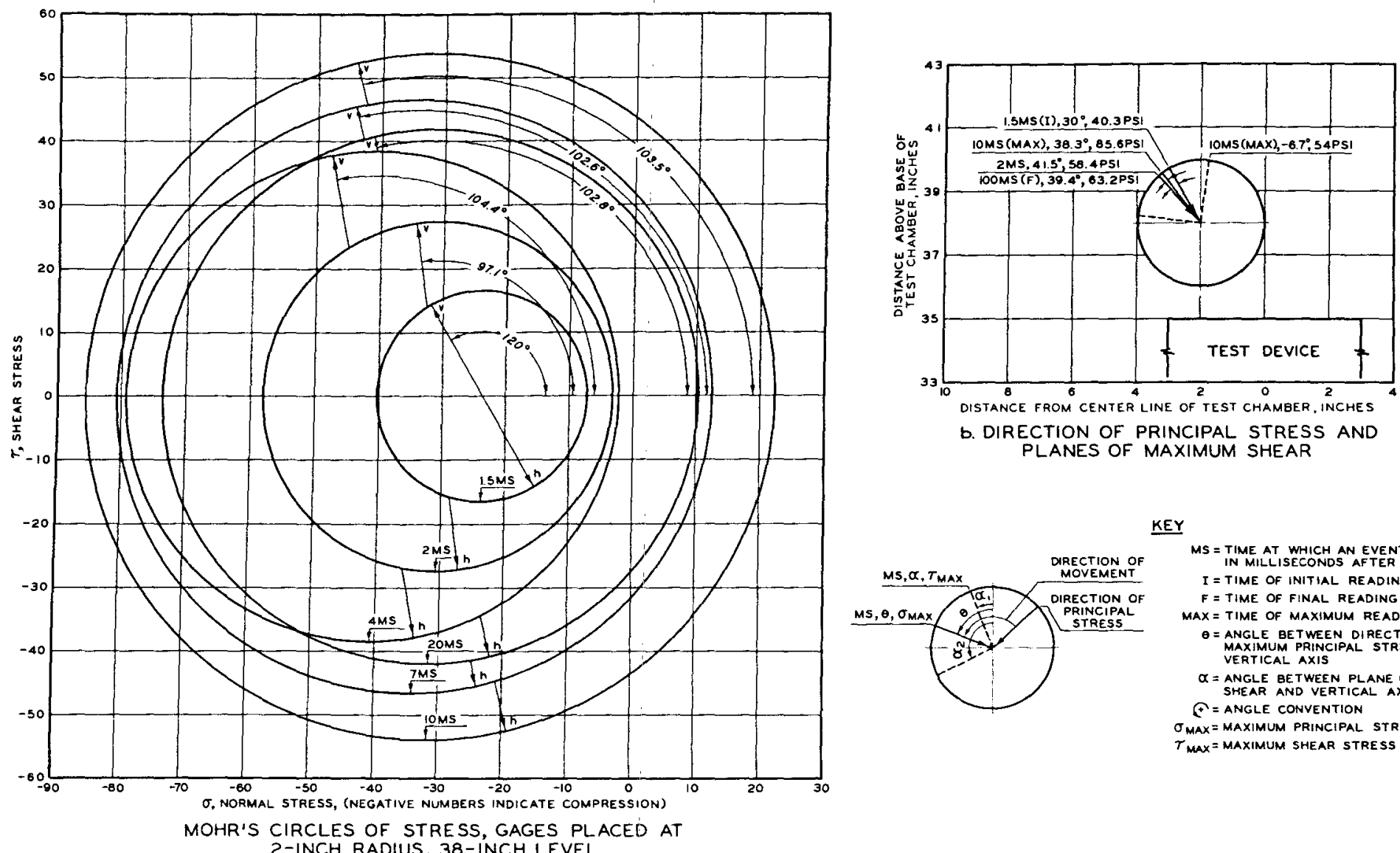

b. DIRECTION OF PRINCIPAL STRESS AND PLANES OF MAXIMUM SHEAR

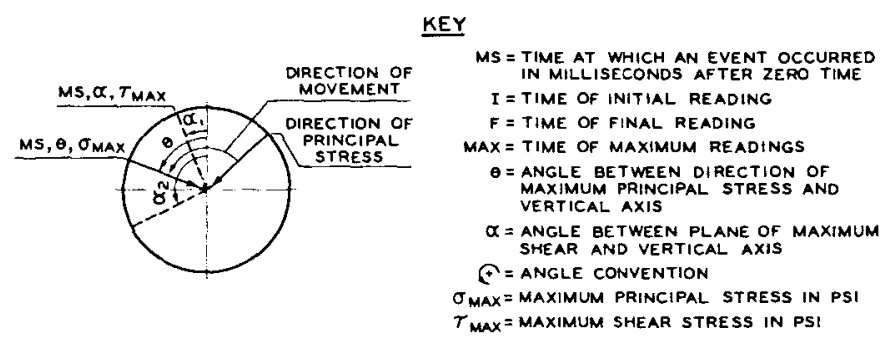

Fig. 70. Determination of direction and magnitude of principal stress, Test 25 
a. TEST $11: H / B=3, P_{S}=37 \mathrm{PSI}$

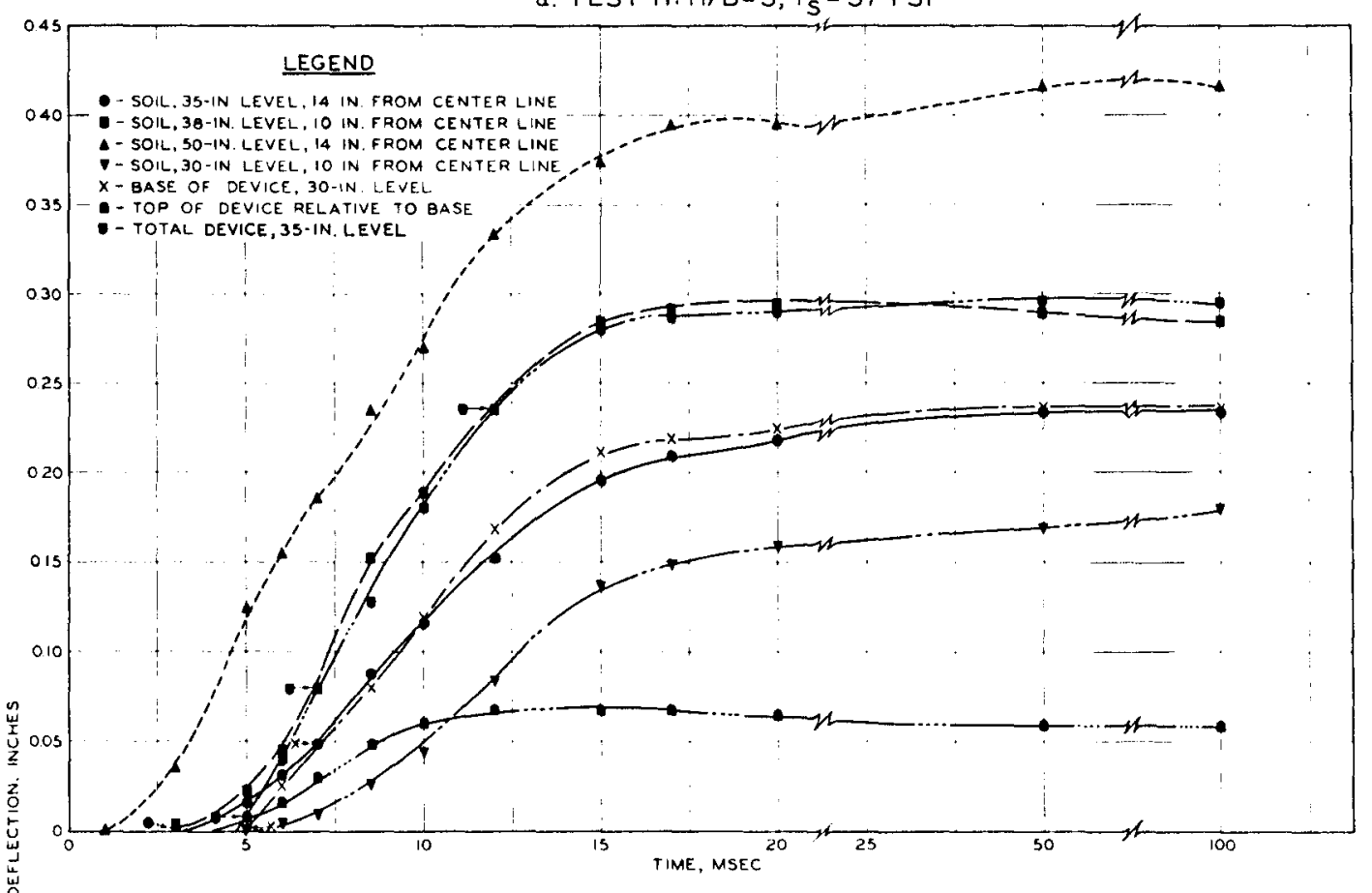

b. TEST $12: H / B=3, P_{S}=70$ PSI

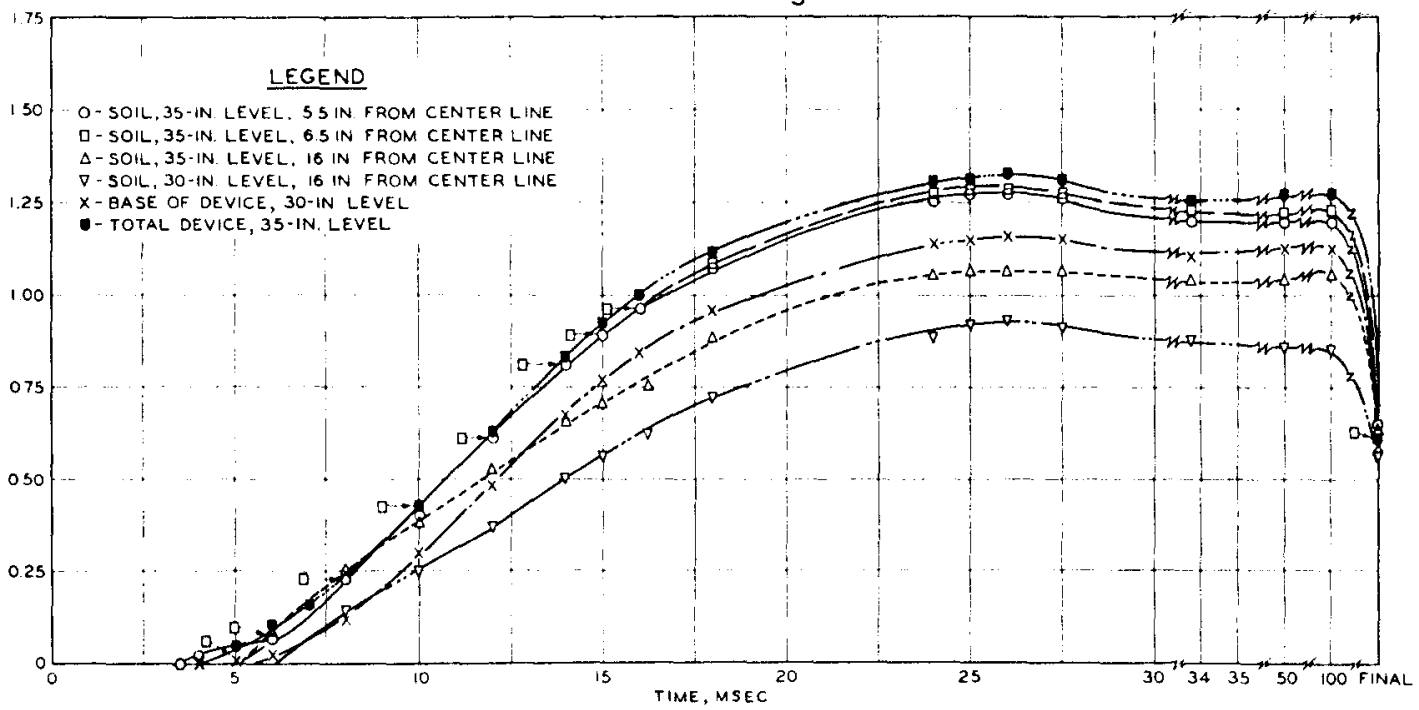

Fig. 71. Soil and structure deflection versus time, Tests 11 and 12 
a. TEST 15: $H / B=1 / 3, P_{S}=39$ PSI
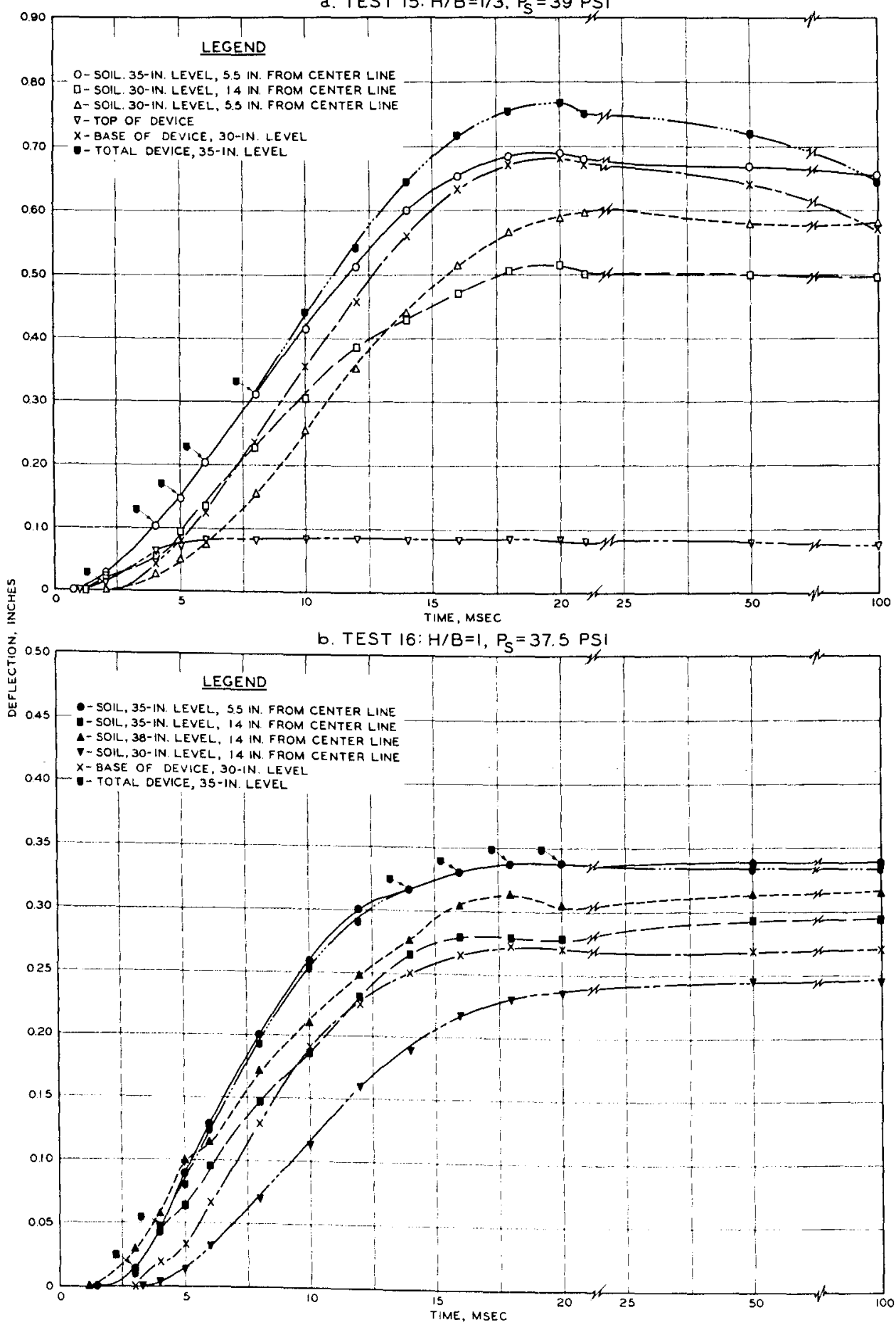

Fig. 72. Soil and structure deflection versus time, Tests 15 and 16 
a. TEST 18: $\mathrm{H} / \mathrm{B}=7, \mathrm{P}_{\mathrm{S}}=310 \mathrm{PSI}$
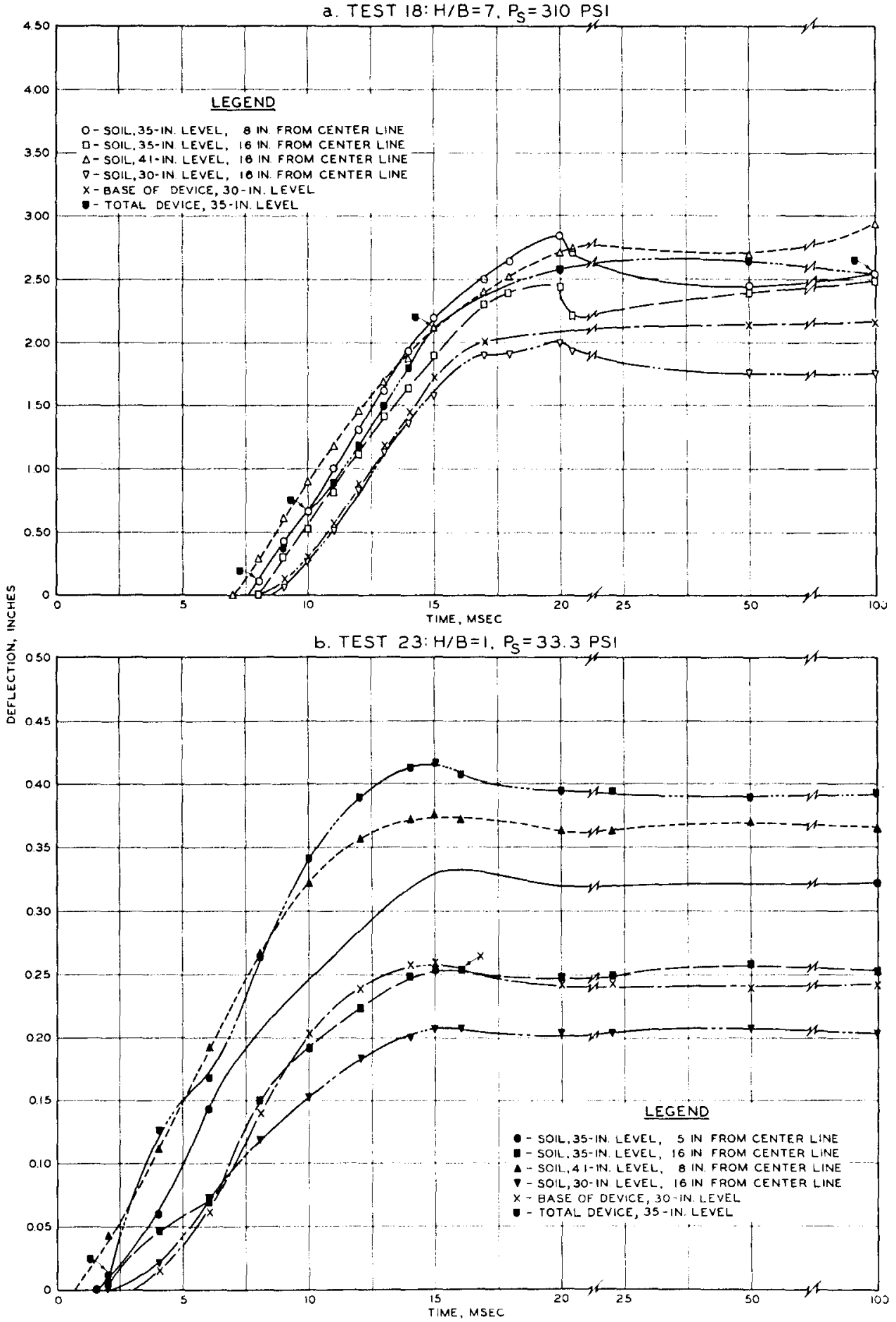

Fig. 73. Soil and structure deflection versus time, Tests 18 and 23 
a. TEST 24: $\mathrm{H} / \mathrm{B}=1, \mathrm{P}_{\mathrm{S}}=31.7 \mathrm{PSI}$
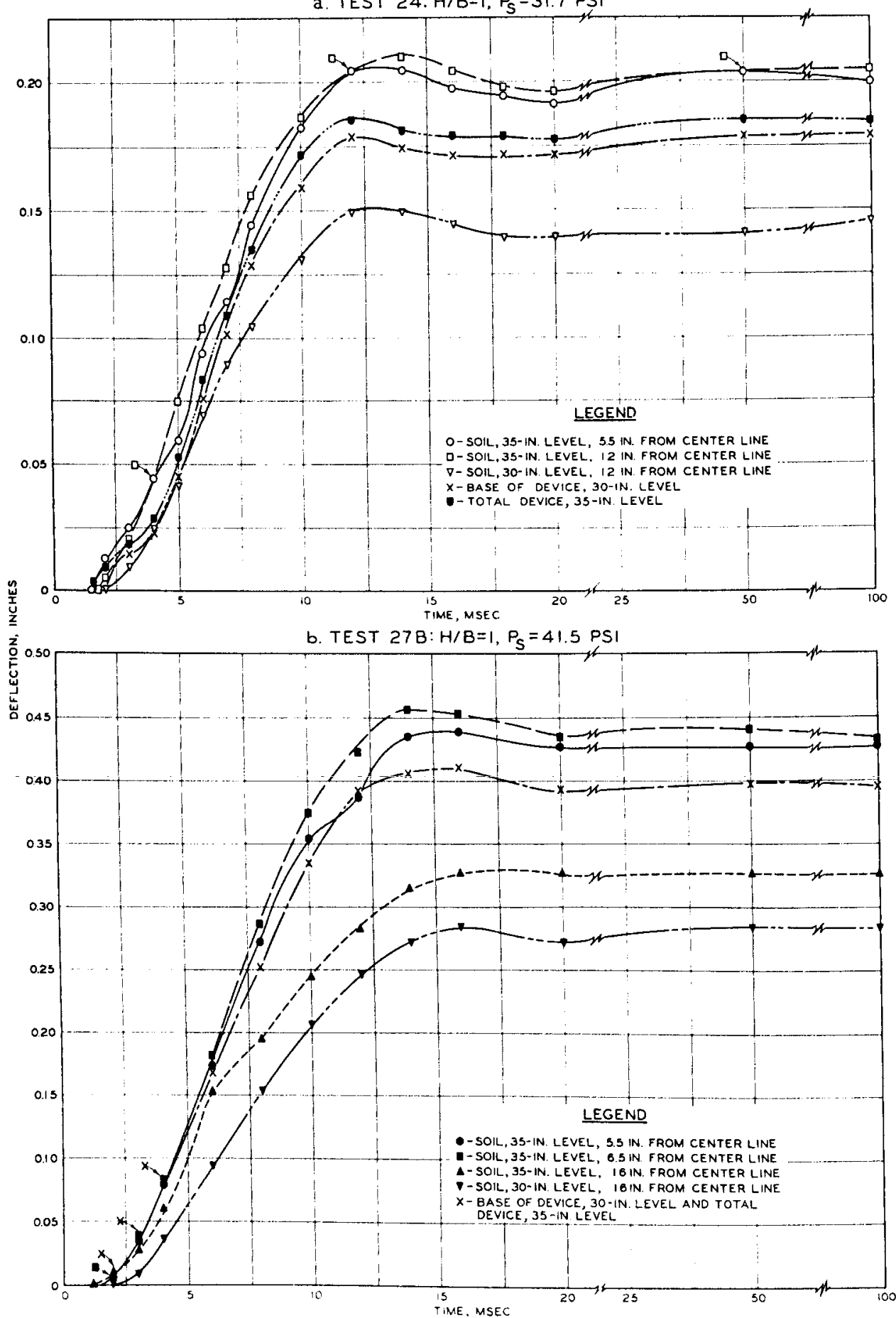

Fig. 74. Soil and structure deflection versus time, Tests 24 and 27B 
a. TEST E: $P_{S}=38$ PSI, TOTAL DEPTH $=48$ INCHES

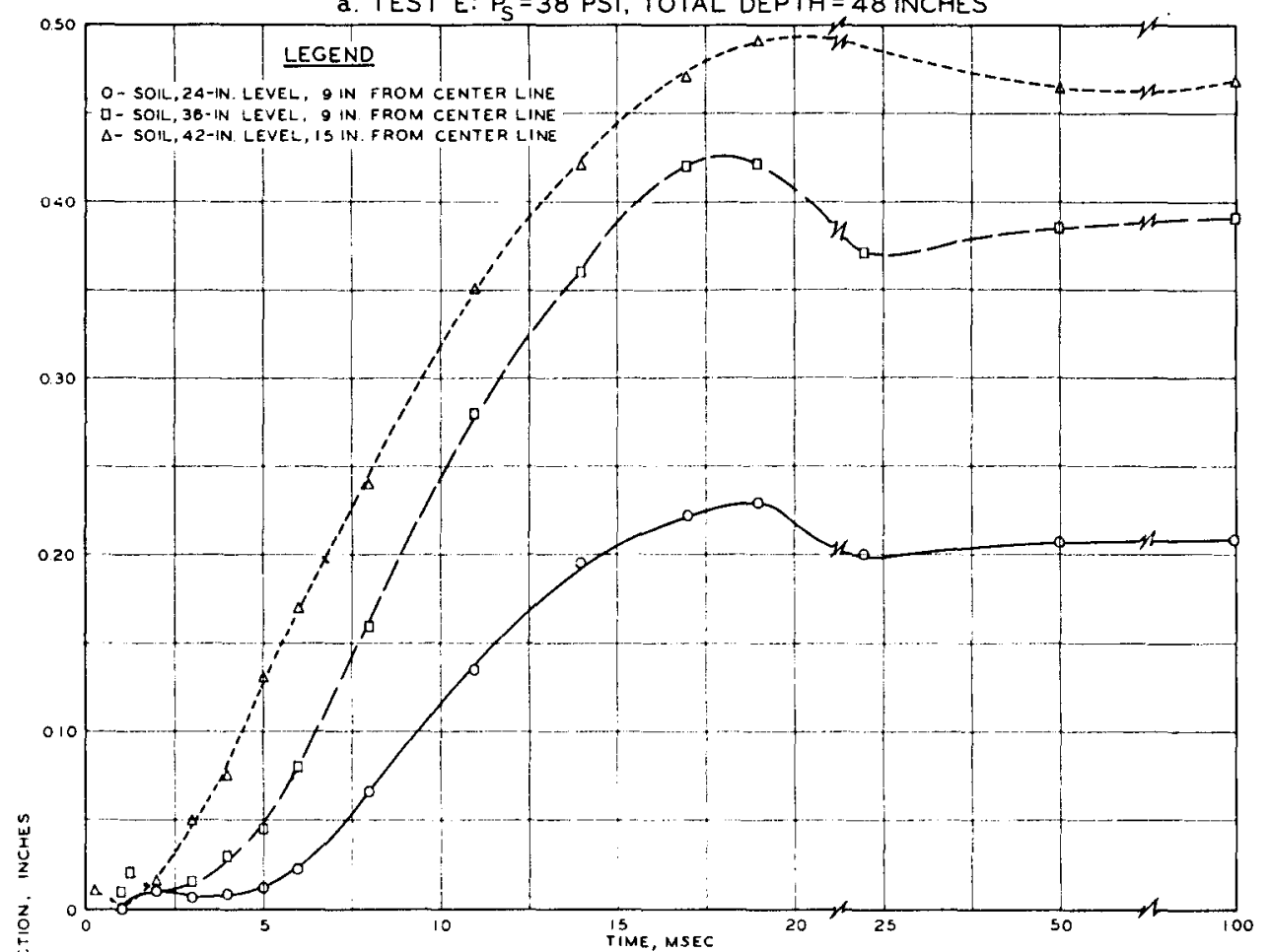

b. TEST $F: P_{S}=205$ PSI, TOTAL DEPTH $=48$ INCHES

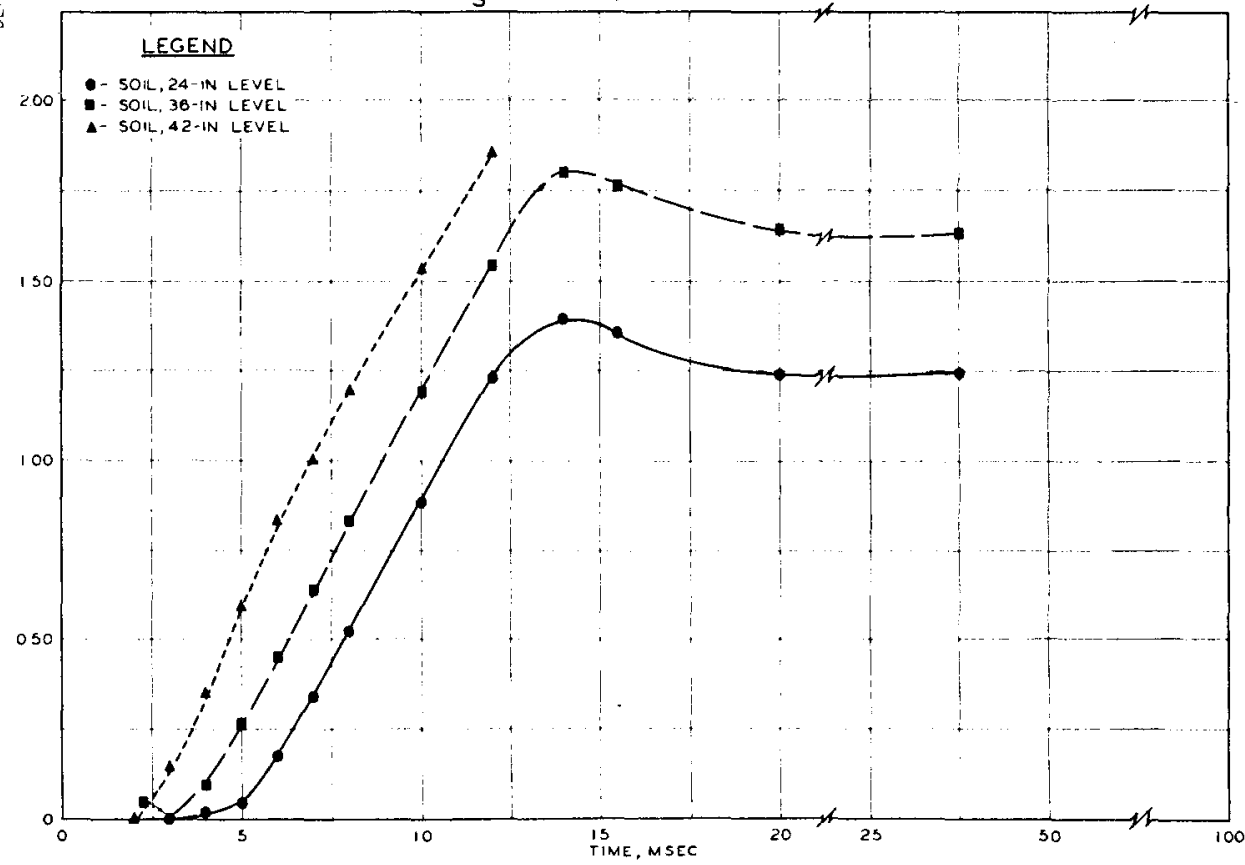

Fig. 75. Soil deflection versus time, preliminary Tests $E$ and $F$ 


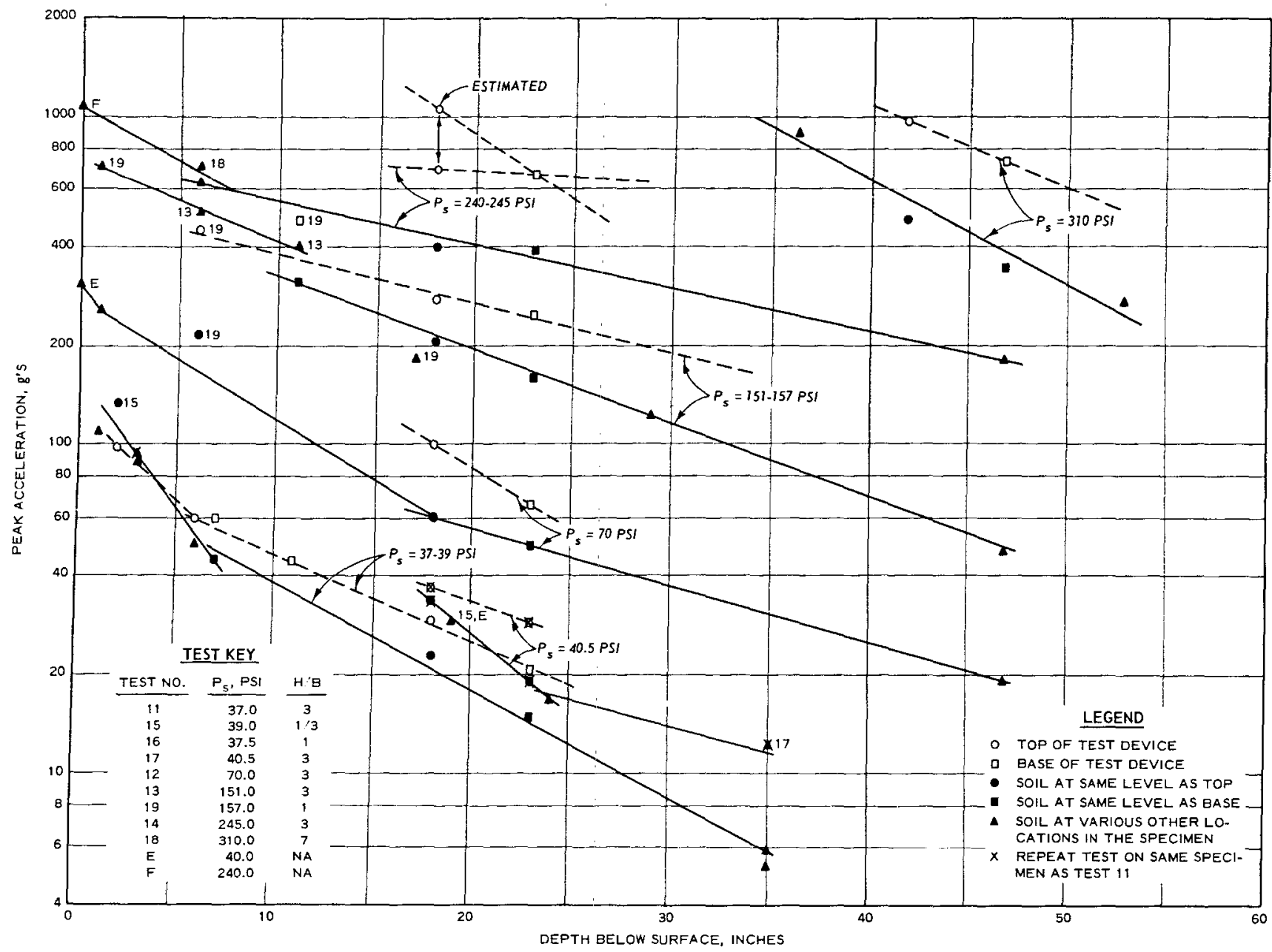

Fig. 76. Variation of soil and structure acceleration with changes in surface pressure and depth of burial at a relatively constant structural stiffness 


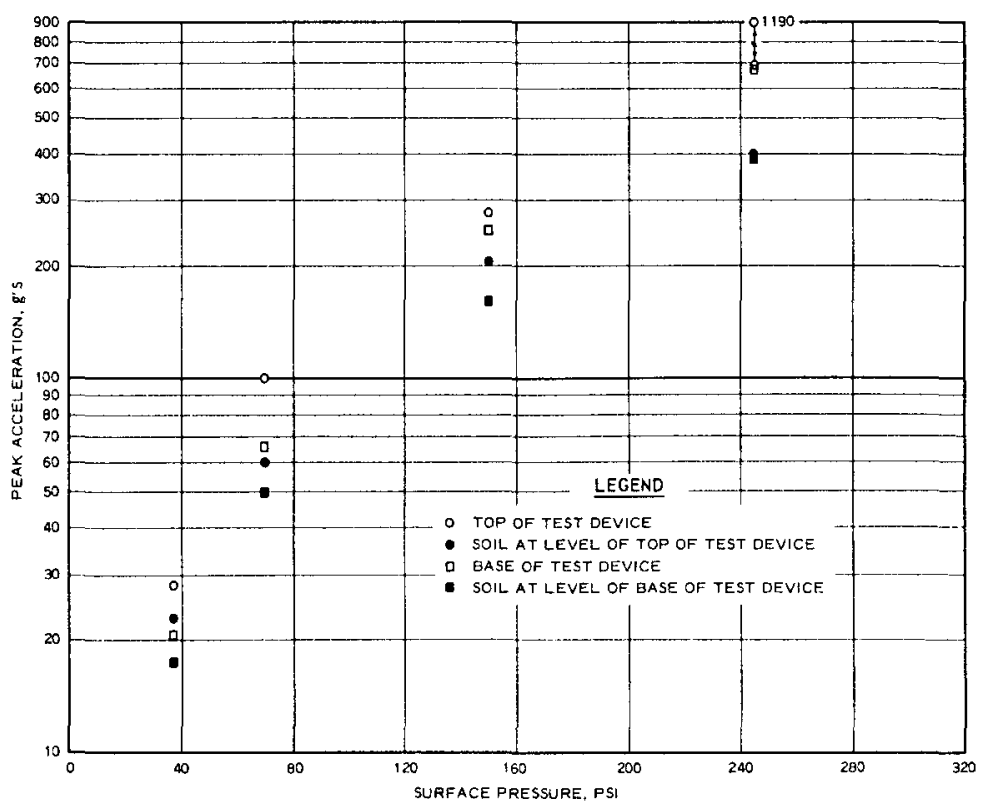

a. Variation with change in surface pressure at constant depth of burial; Tests 11, 12, 13, and 14; H/B = 3

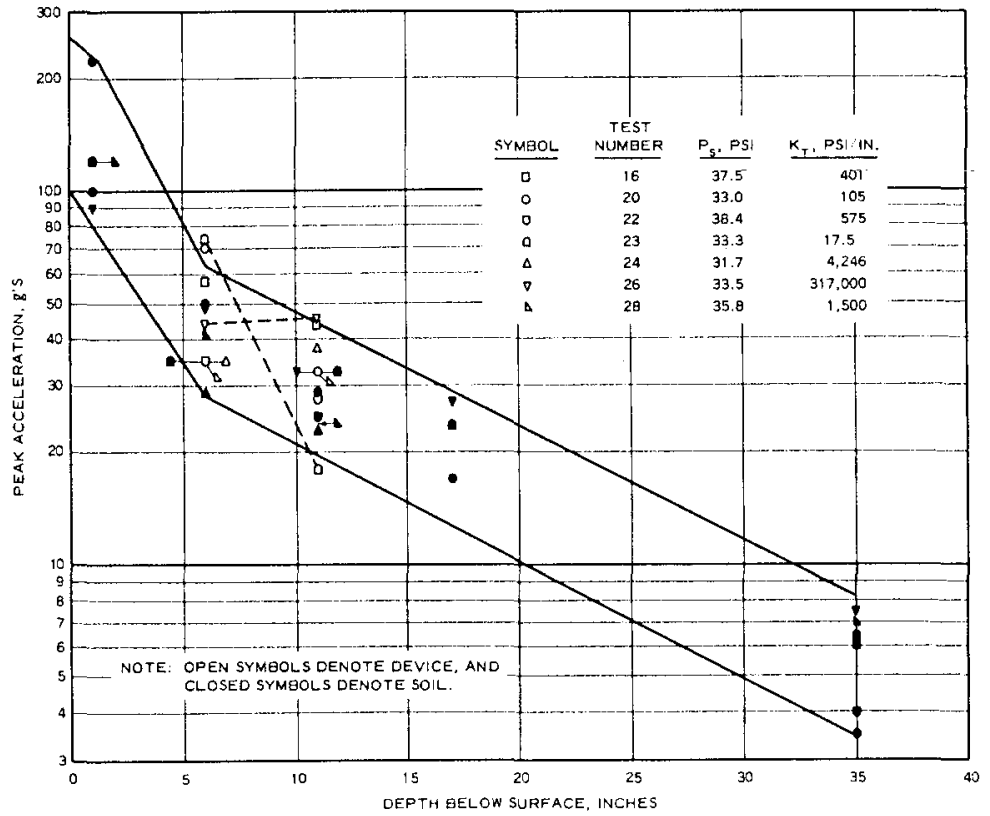

b. Variation with change in structure stiffness; $H / B=1$, $\mathrm{P}_{\mathrm{S}} \approx 32$ to $38 \mathrm{psi}$

Fig. 77. Variation of soil and structure acceleration with surface pressure and structure stiffness 


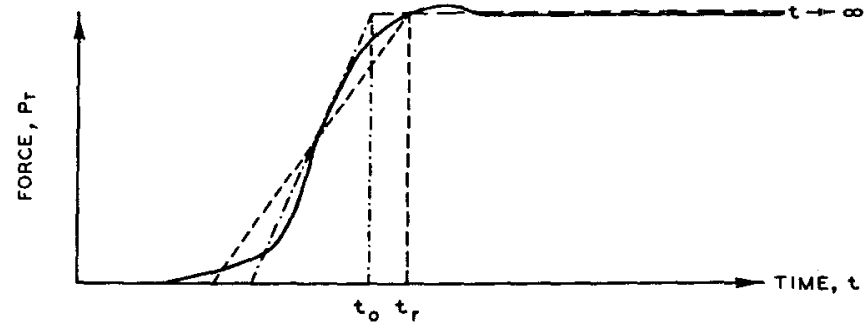

a. ACTUAL AND IDEALIZED FORCE-TIME RELATION

$\underset{\infty}{\leftarrow}$

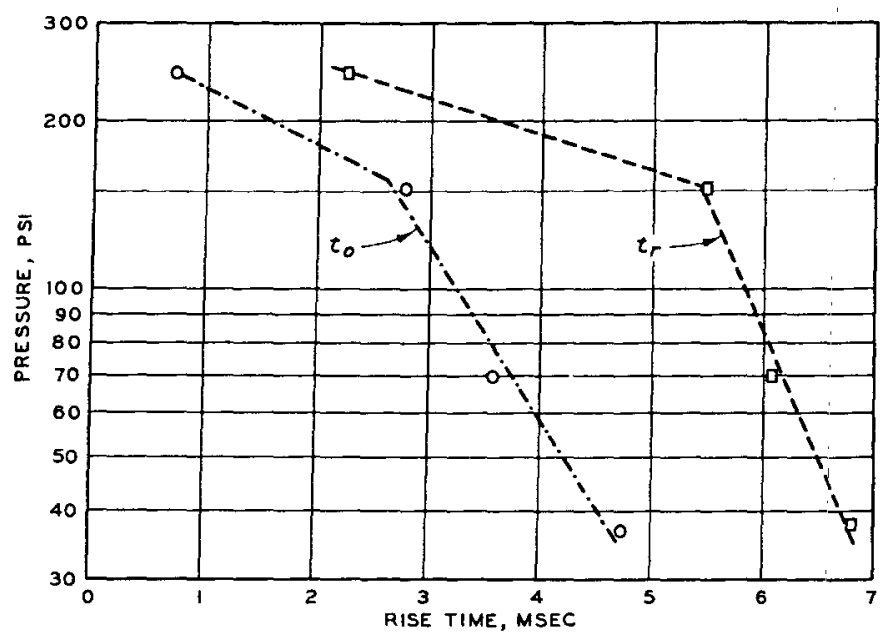

c. VARIATION OF SOIL PRESSURE RISE TIME WITH PEAK SURFACE PRESSURE, $H / B=3$

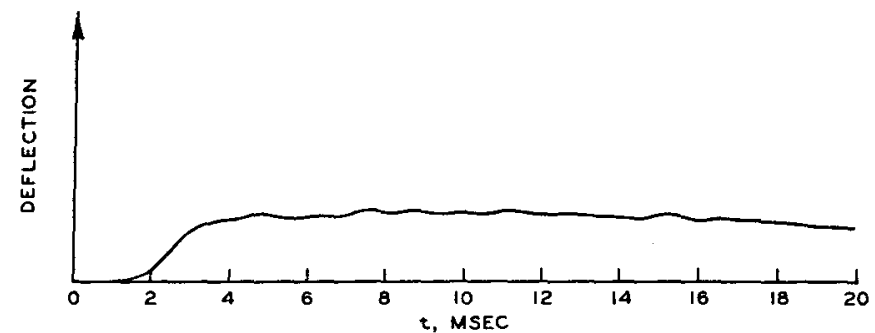

b. DEFLECTION OF TOP OF TEST DEVICE

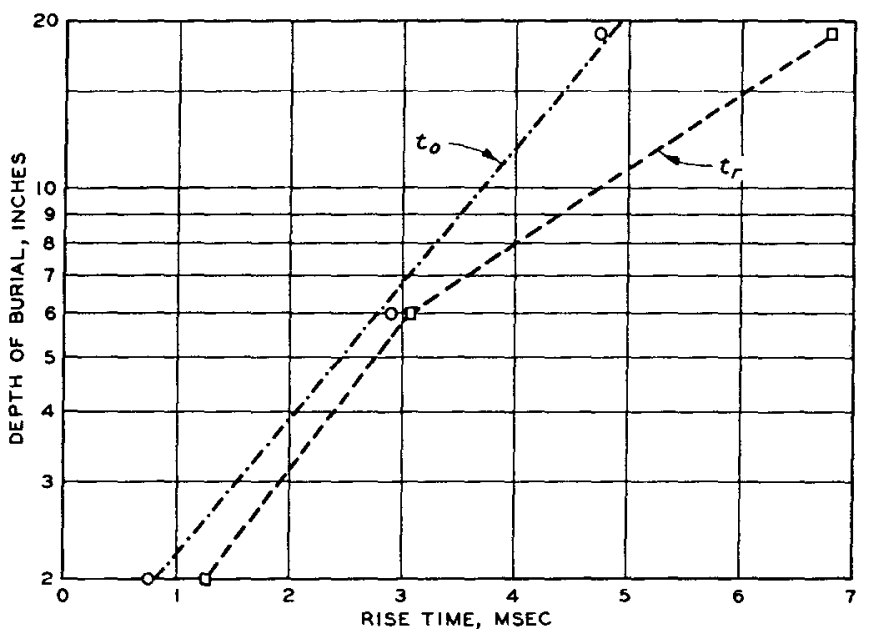

d. VARIATION OF SOIL PRESSURE RISE TIME WITH DEPTH, $P_{S} \approx 37$ PSI

Fig. 78. Rise time data 


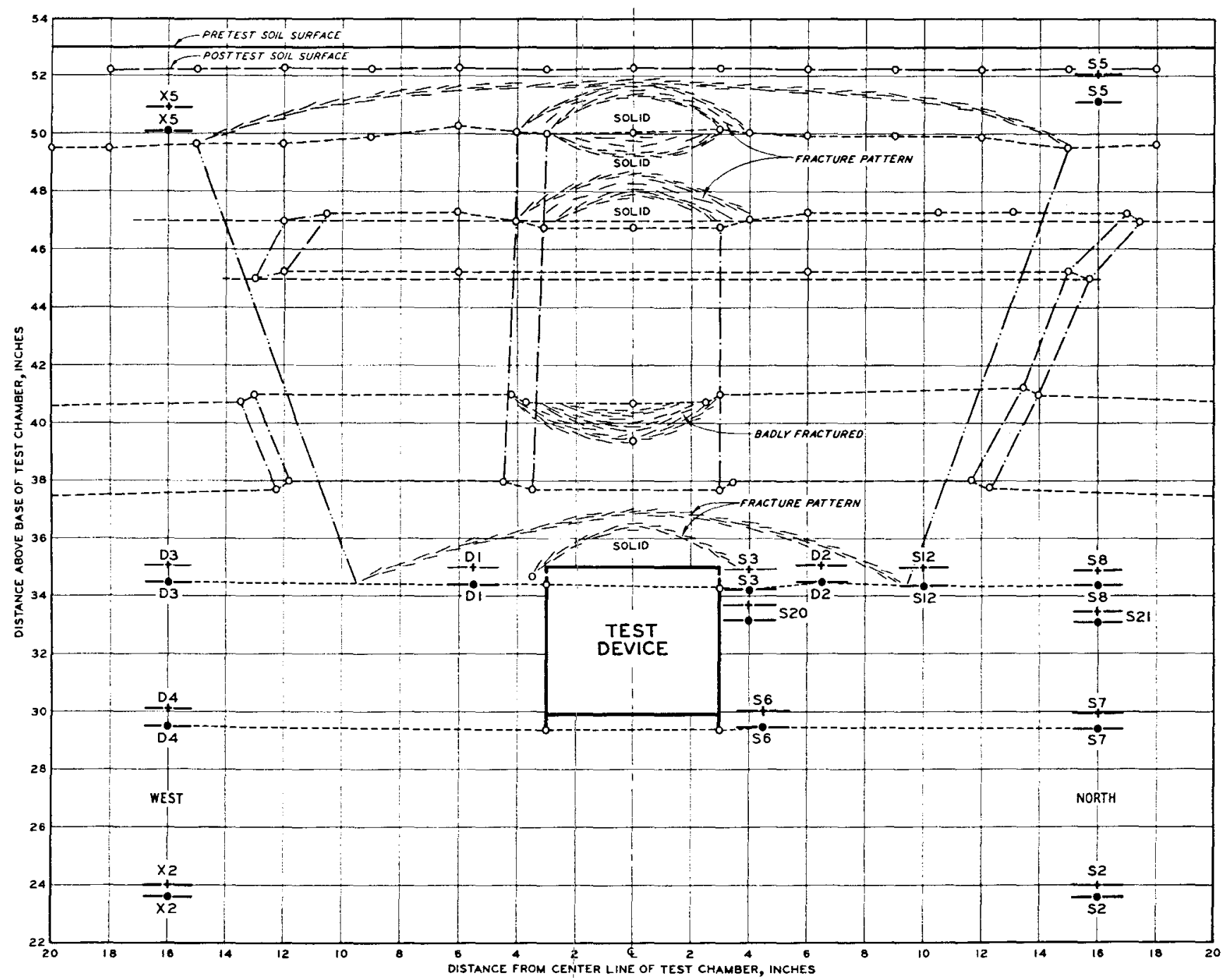

Fig. 79. Cross section of soil deformations, Test 12; H/B $=3, \mathrm{P}_{\mathrm{S}}=70$ psi 


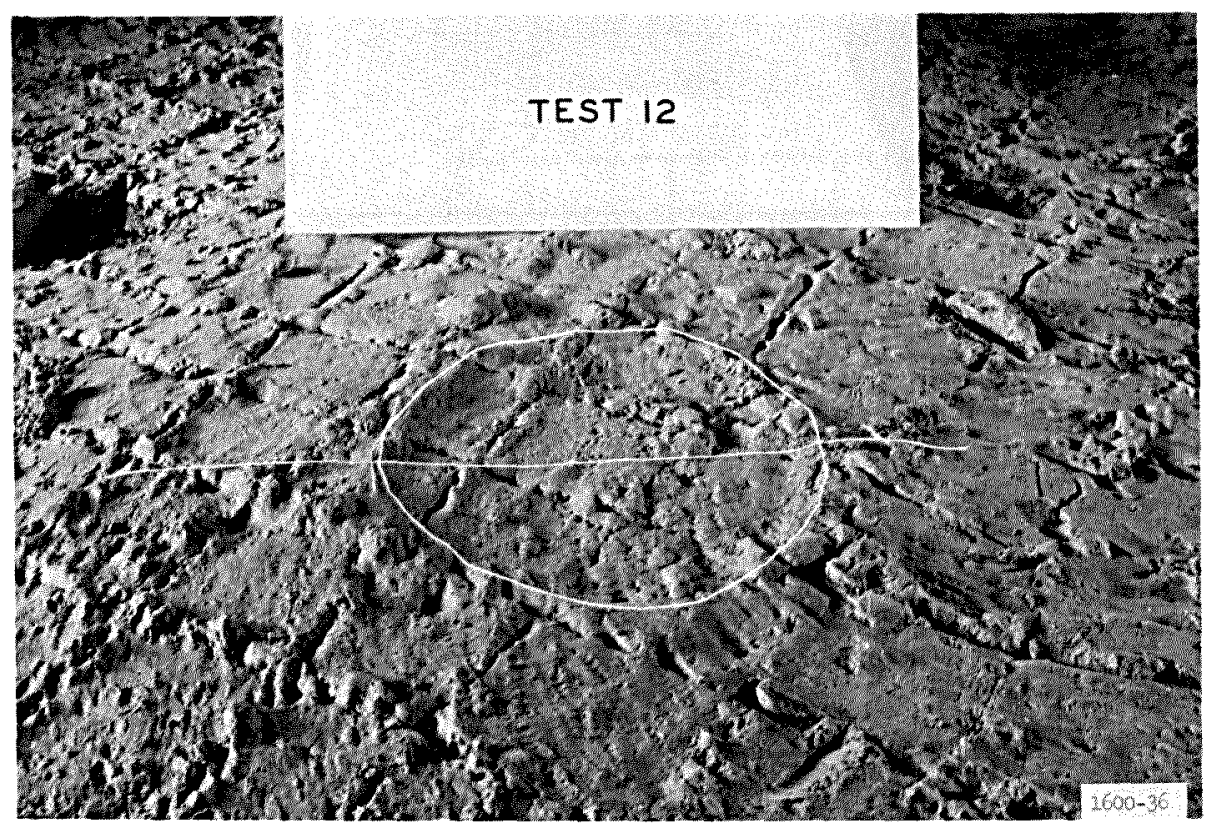

a. Oblique view of soil surface

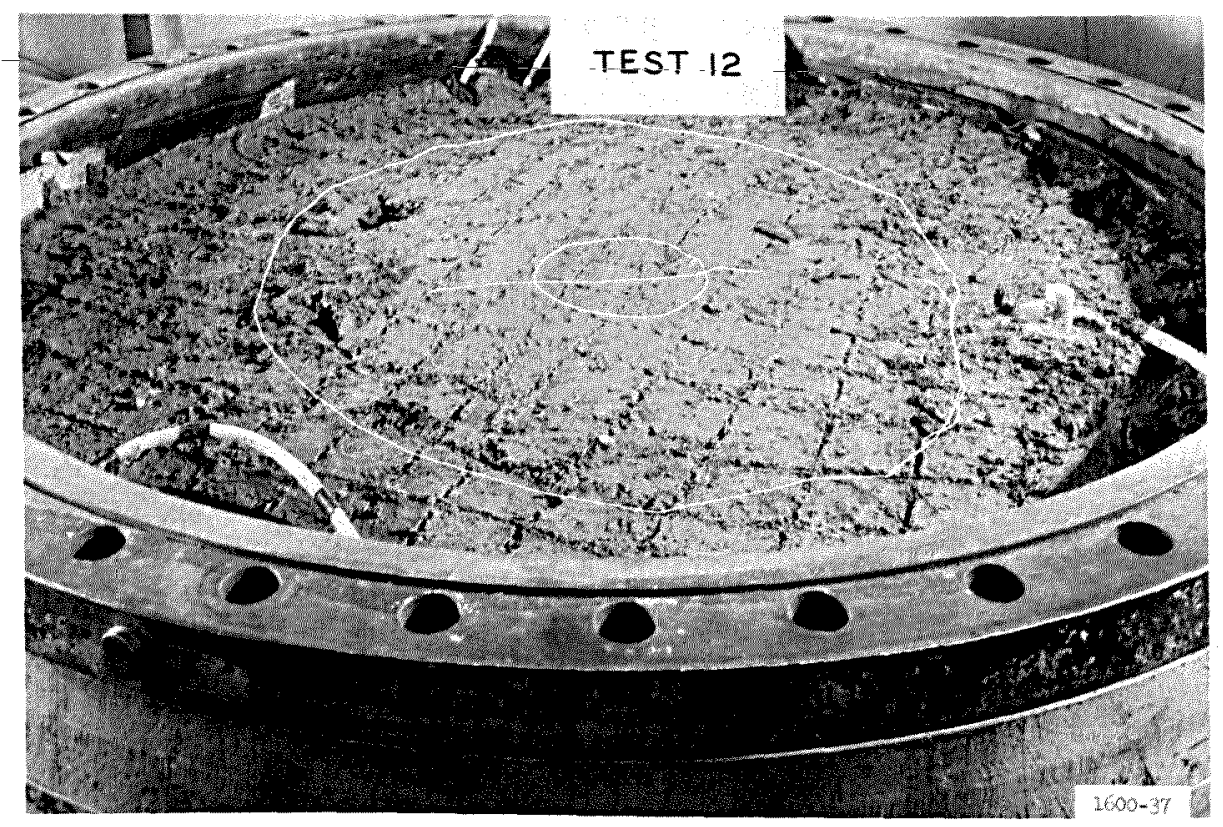

b. Oblique view of depression

Fig. 80. Hump and depression at 50-inch level of Test 12 


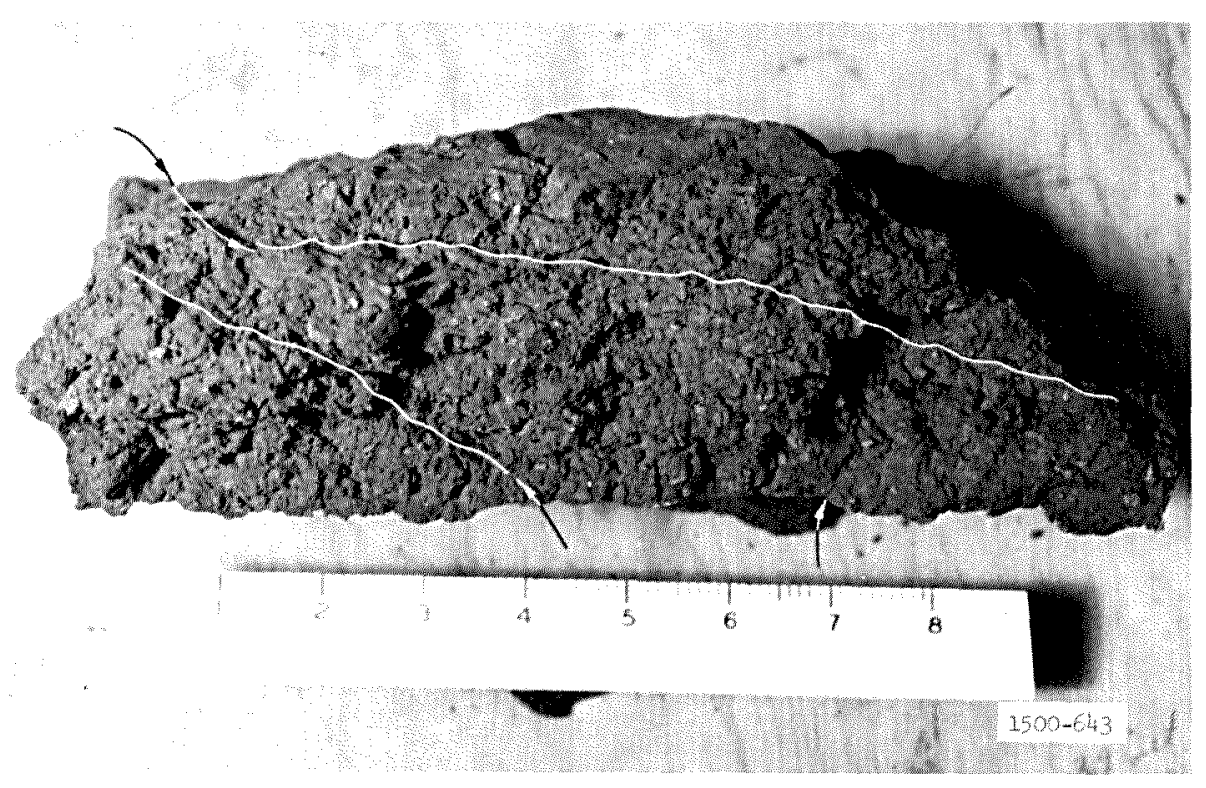

a. Cross-section view of broken soil layer

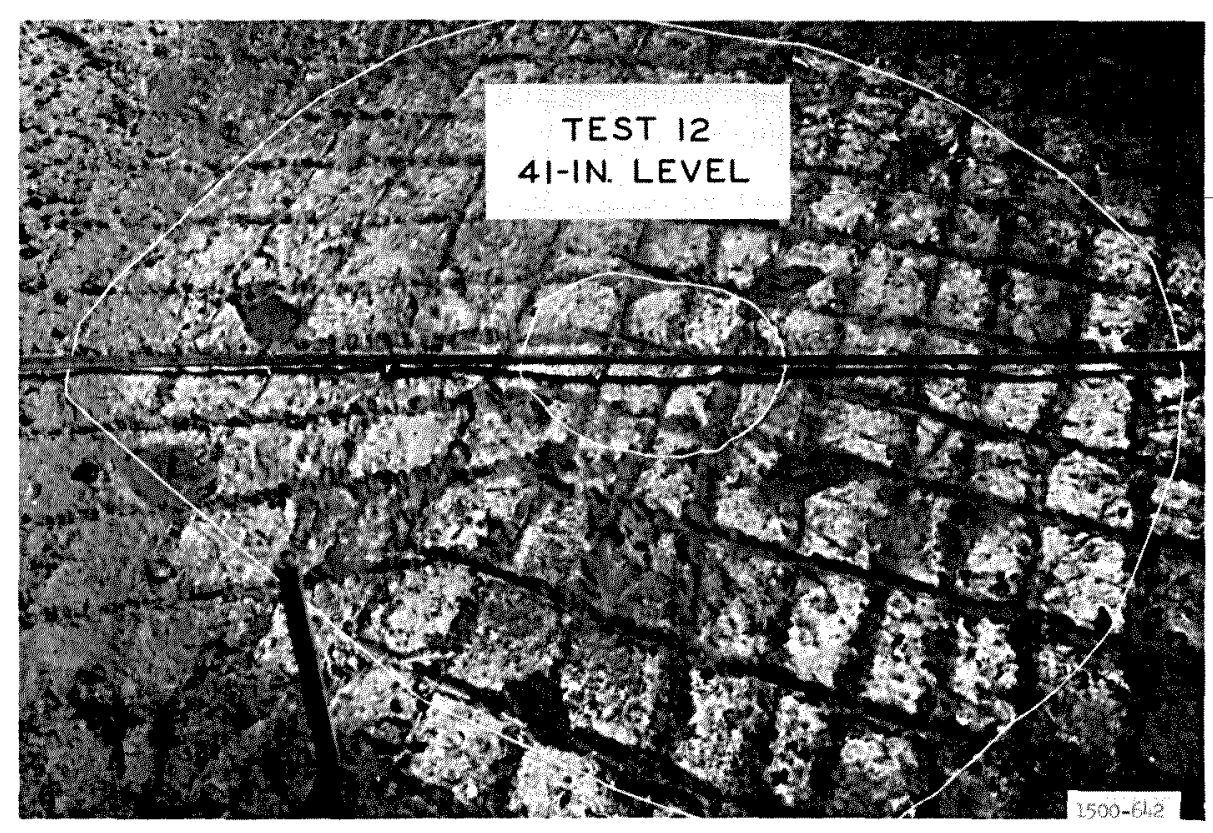

b. Oblique view of soil surface

Fig. 81. Views of a soil cross section and the soil surface at 41-inch level, Test 12 


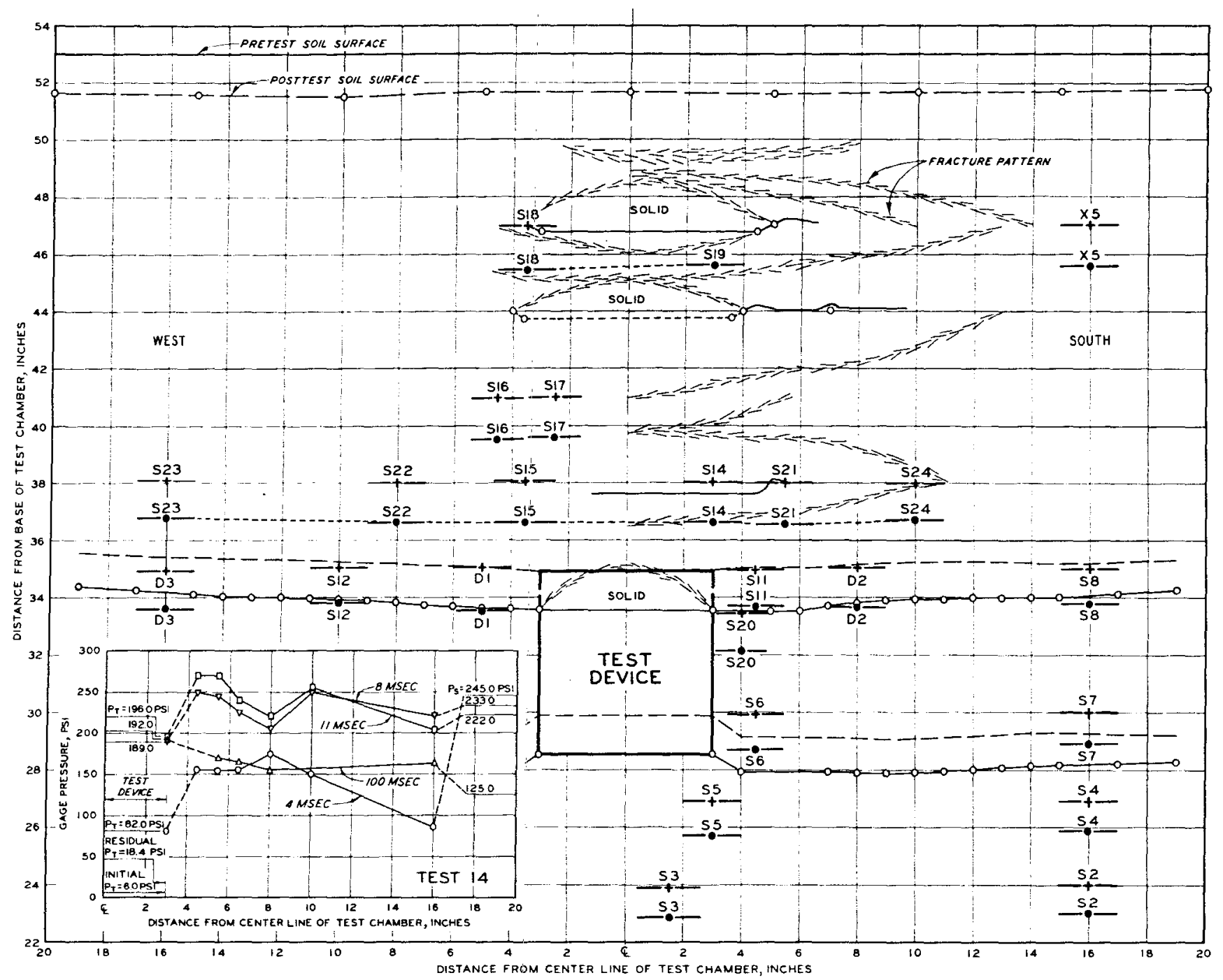

Fig. 82. Cross section of soil deformations, Test $14 ; \mathrm{H} / \mathrm{B}=3, \mathrm{P}_{\mathrm{S}}=245$ psi 


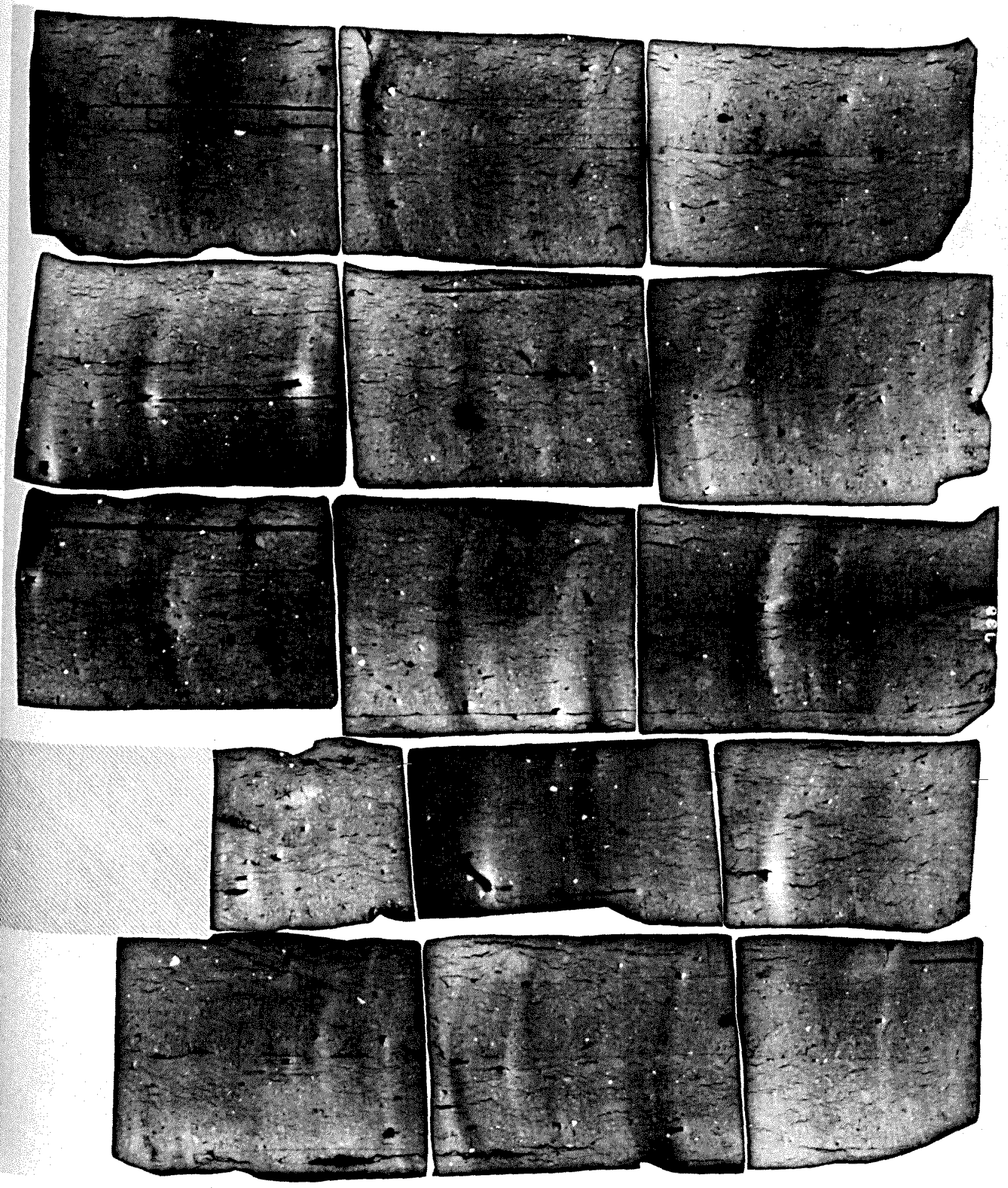

Fig. 83. Radiograph of soil deformation pattern, Test 14; $\mathrm{H} / \mathrm{B}=3, \mathrm{P}_{\mathrm{S}}=245 \mathrm{psi}$ 


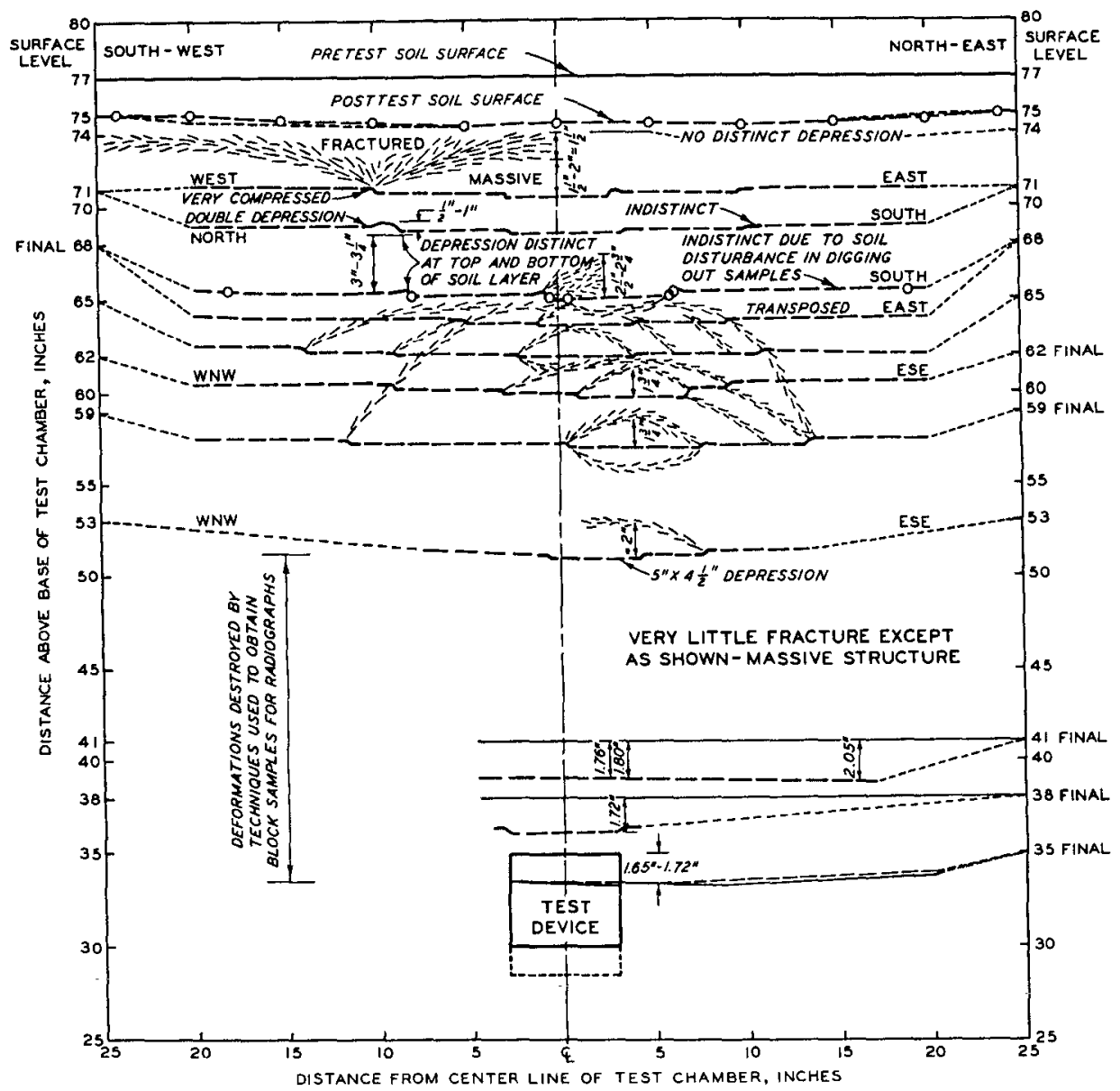

a. CROSS SECTION OF SOIL DEFORMATIONS

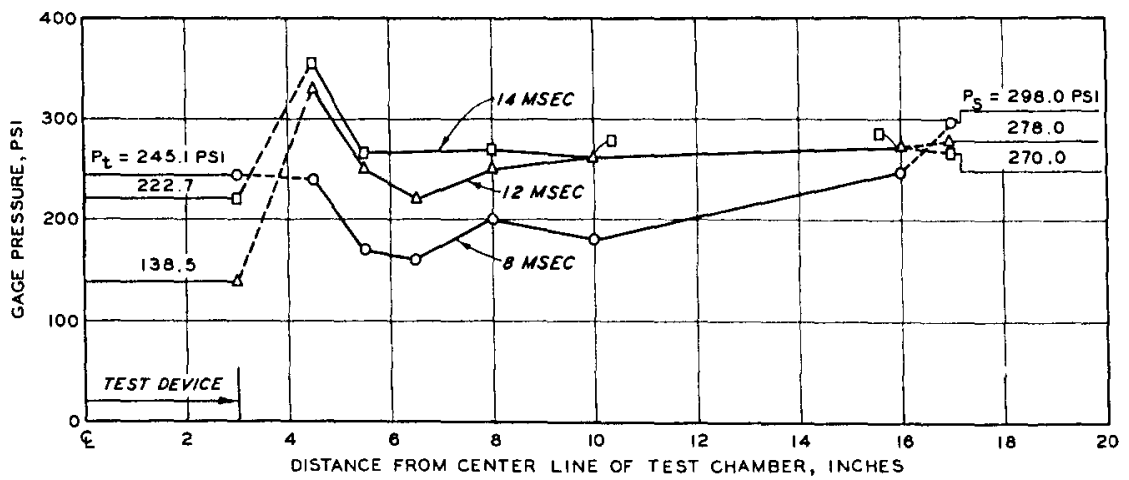

b. VARIATION OF SOIL STRESS WITH

TIME AT THE 35-INCH LEVEL

Fig. 84. Cross section of soil deformations, Test 18; $\mathrm{H} / \mathrm{B}=7, \mathrm{P}_{\mathrm{S}}=310 \mathrm{psi}$ 


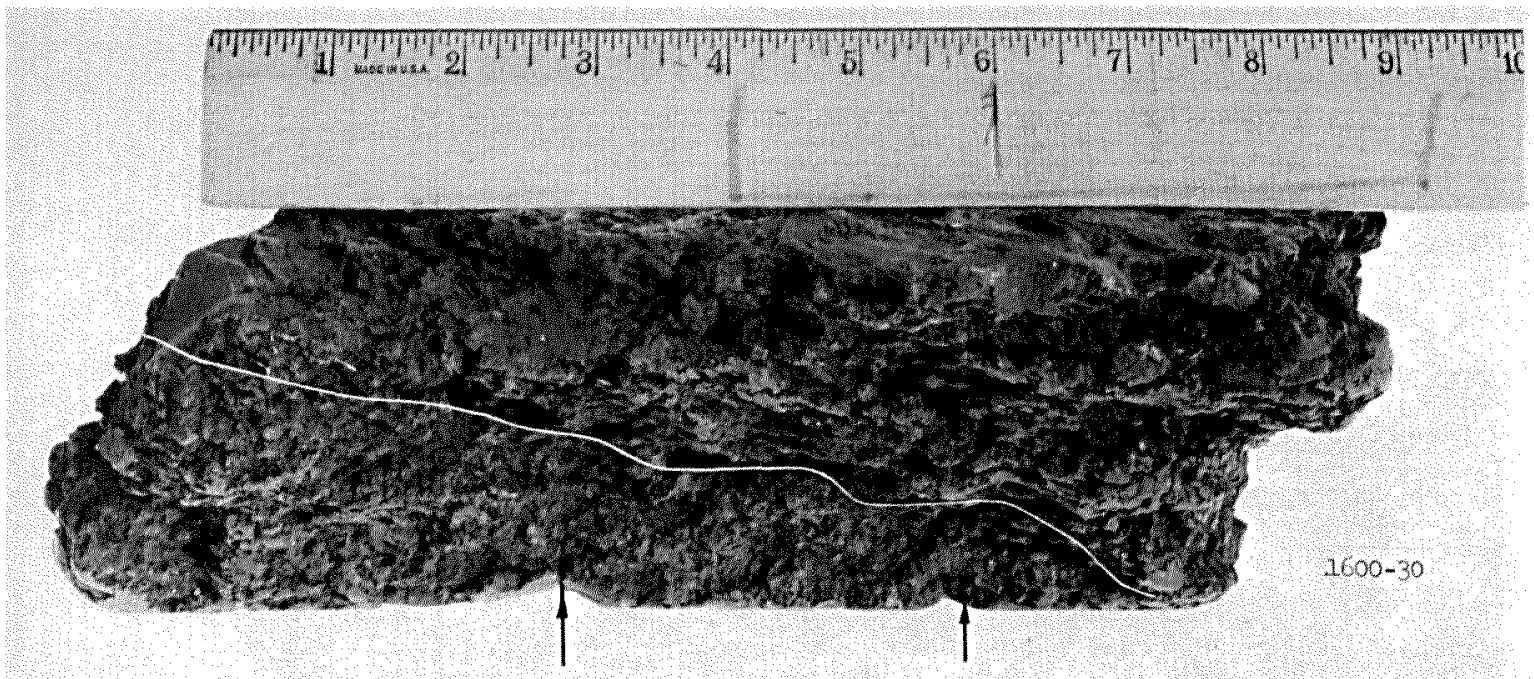

a. Side view of soil between 71 - and 74-inch levels
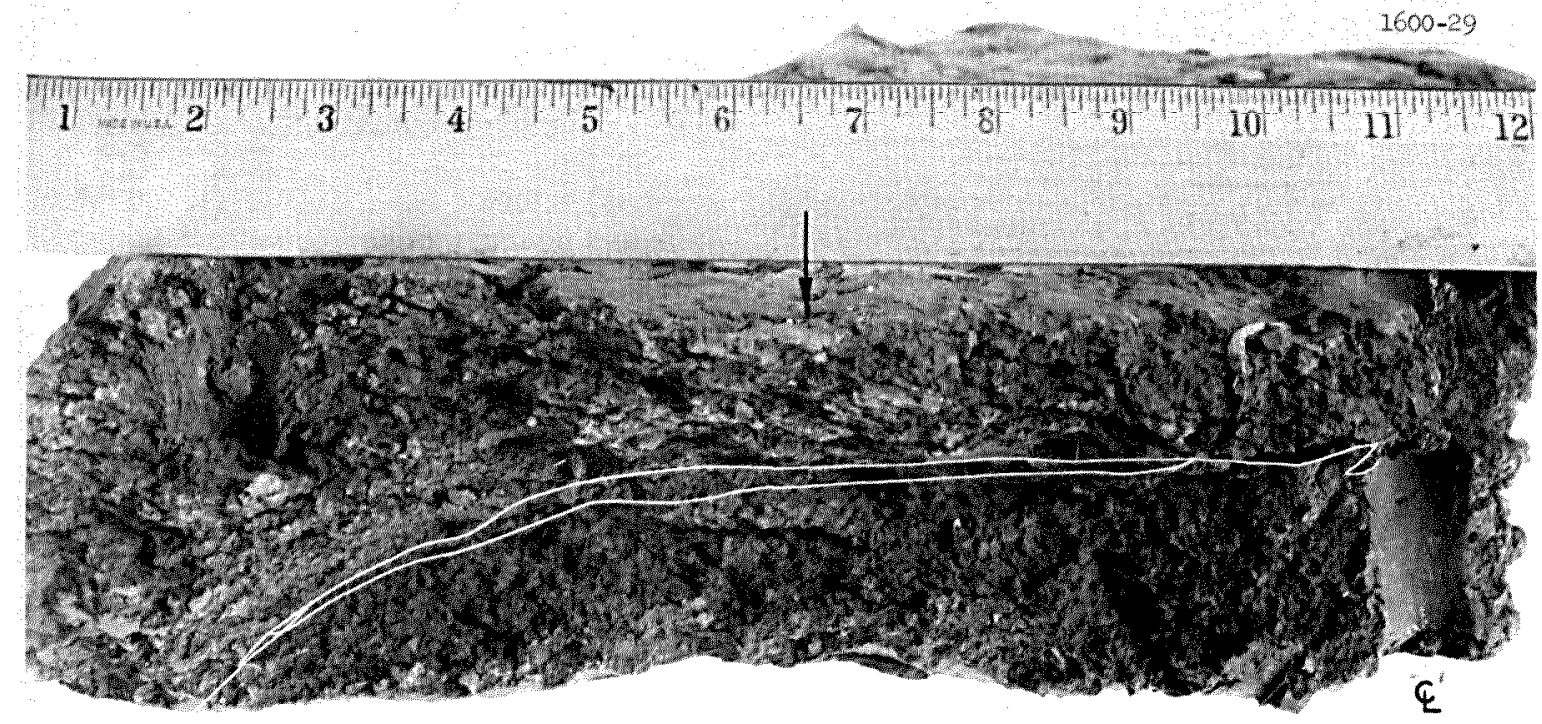

b. Side view of soil between 71 - and 74 -inch levels

Fig. 85. Soil deformations between 71 - and 74 -inch levels, Test 18 


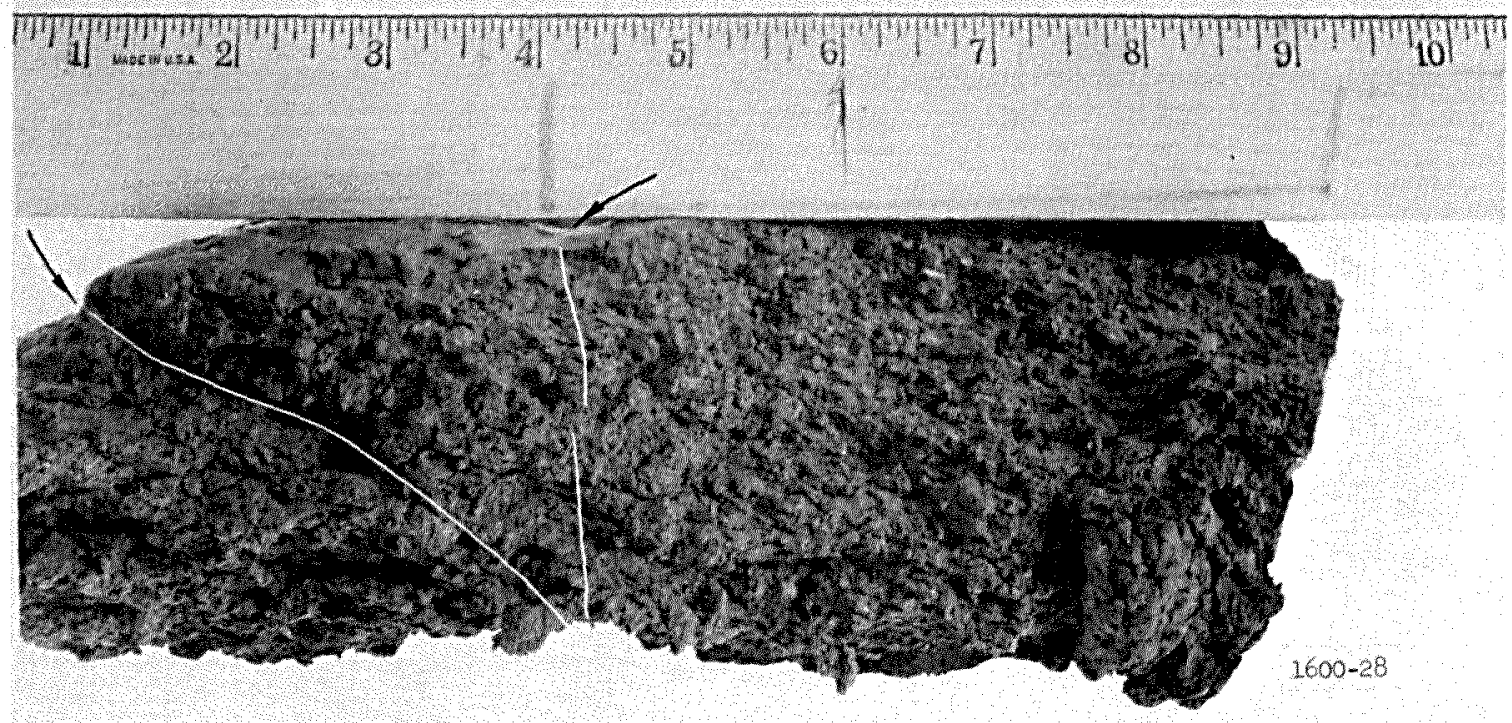

a. Side view of soil between 59- and 62-inch levels

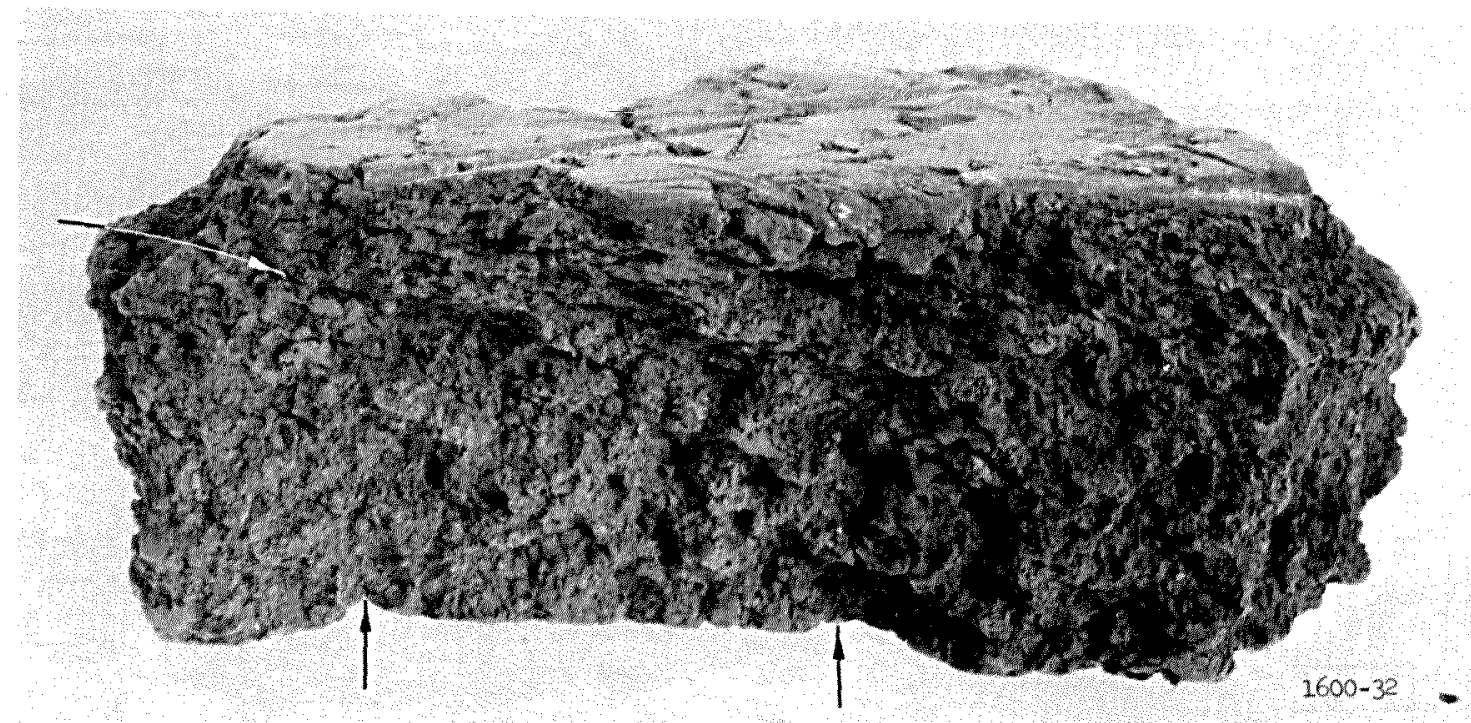

b. Side view of soil between 53 - and 56-inch levels

Fig. 86. Soil deformations between 53 - and 62 -inch levels, Test 18 


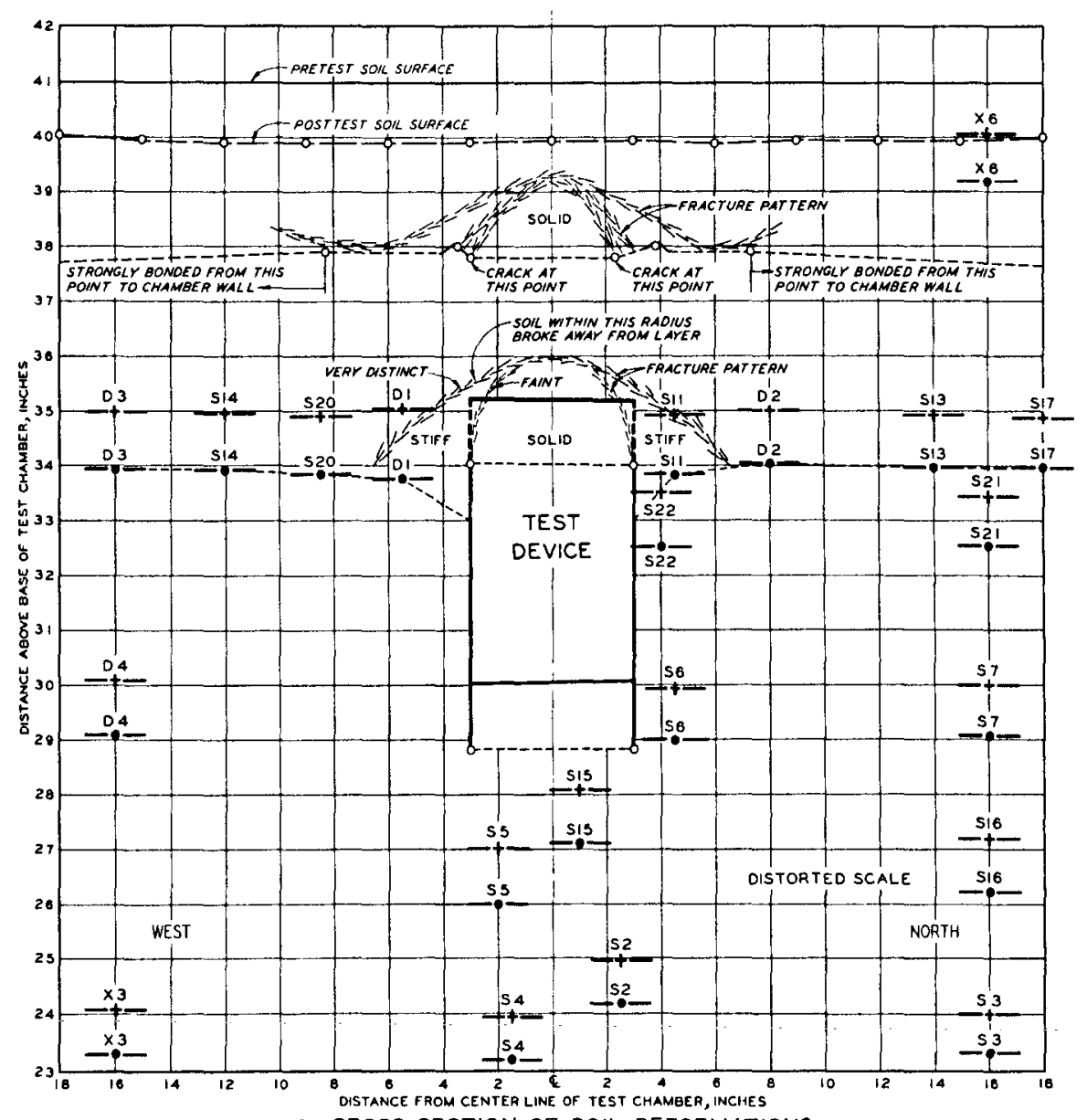

a. CROSS SECTION OF SOIL DEFORMATIONS

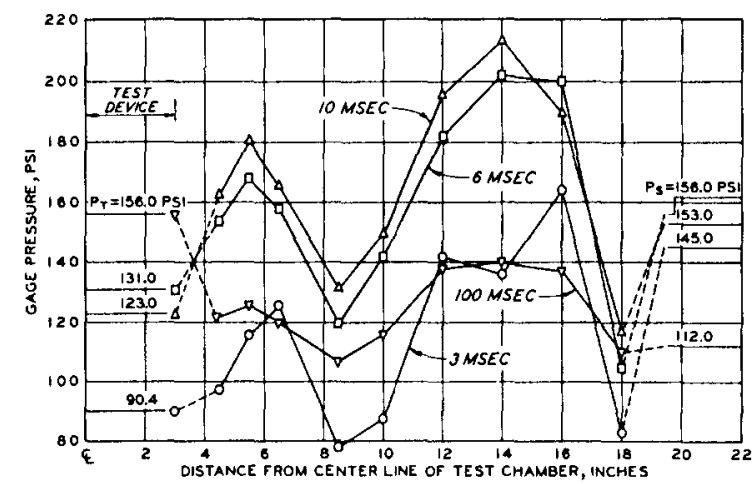

b. VARIATION OF SOIL STRESS WITH

TIME AT THE 35-INCH LEVEL

Fig. 87. Cross-section view of soil deformations, Test 19; $\mathrm{H} / \mathrm{B}=1, \mathrm{P}_{\mathrm{S}}=157 \mathrm{psi}$ 


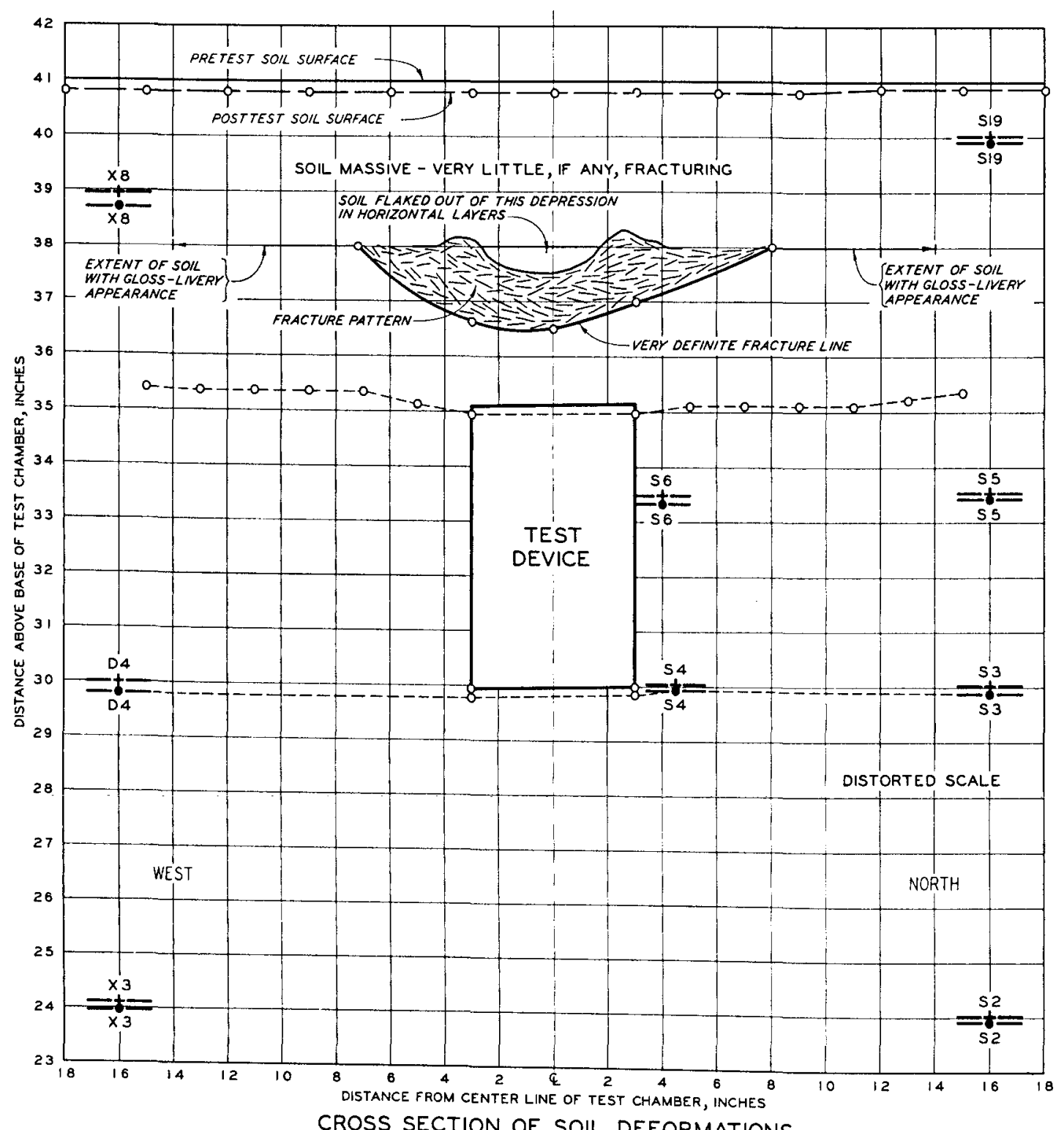

Fig. 88. Cross section of soil deformations, Test 26; $\mathrm{H} / \mathrm{B}=1, \mathrm{P}_{\mathrm{S}}=33.5 \mathrm{psi}$ 


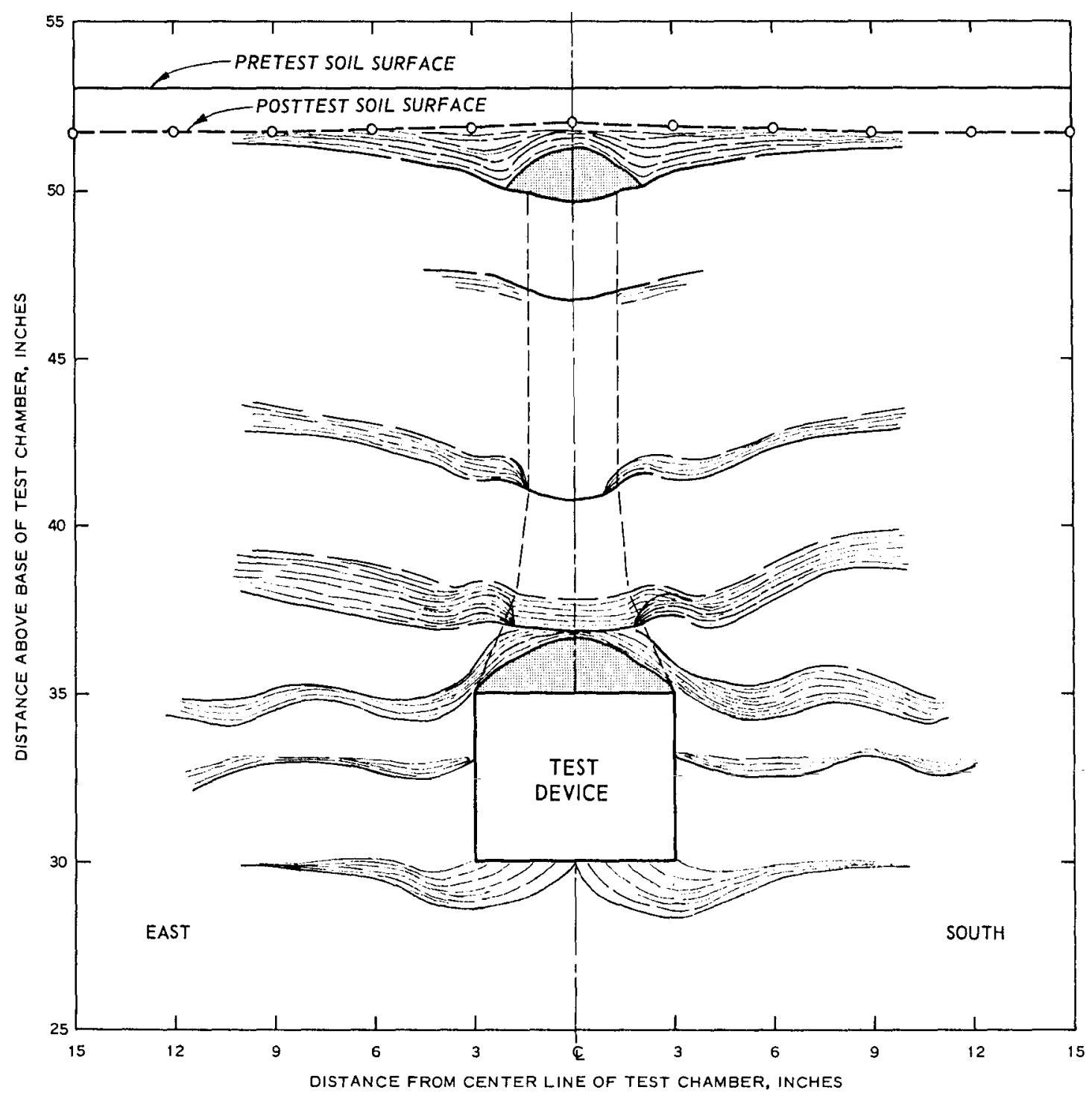

Fig. 89. Composite soil deformation profile under active arching conditions 


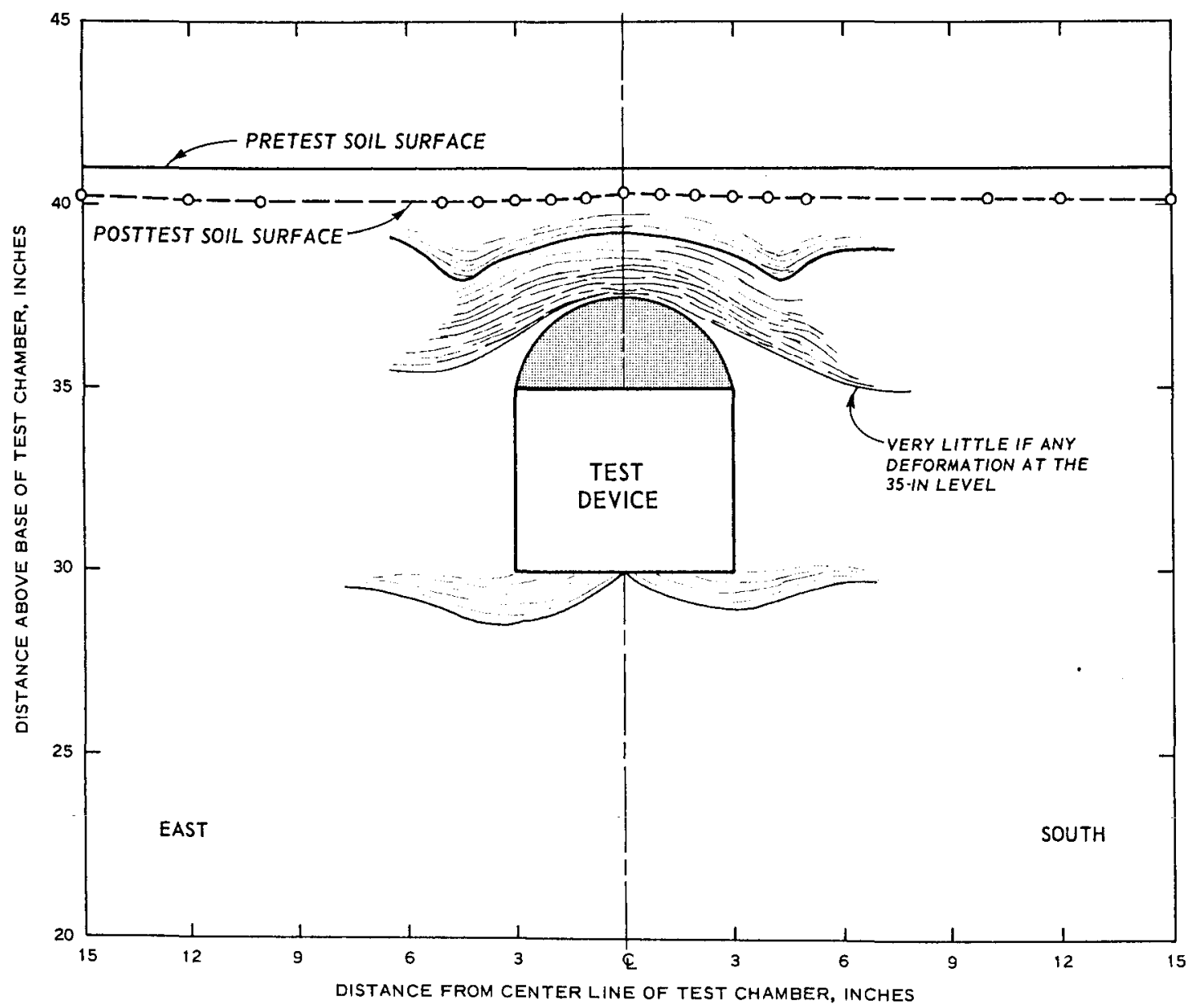

Fig. 90. Hypothetical soil deformations under passive arching conditions 


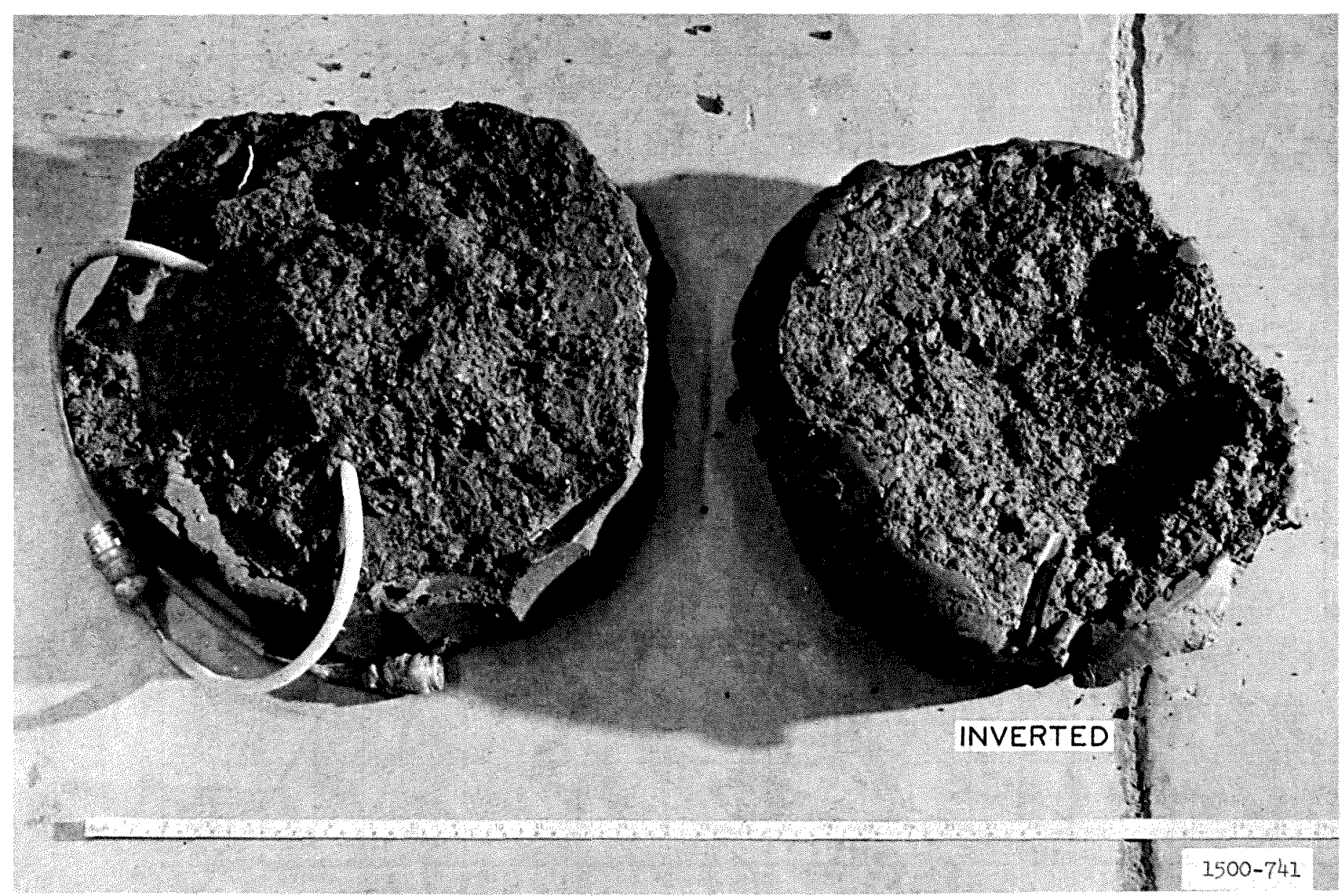

Fig. 91. Soil deformation pattern resulting from a 6-inch plate bearing test; $\mathrm{H} / \mathrm{B}=3$ 


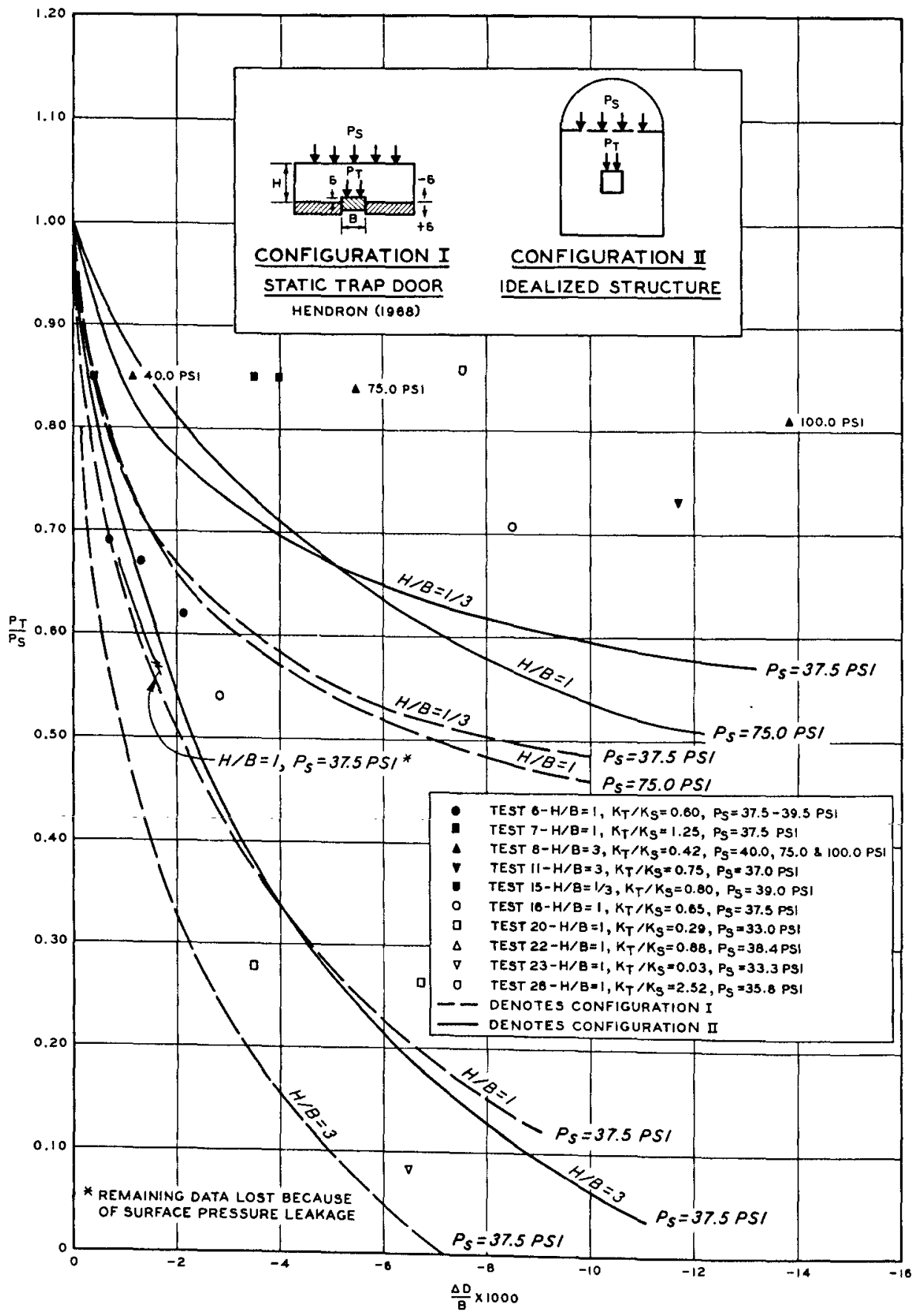

Fig. 92. Active arching curves, static surface pressure 


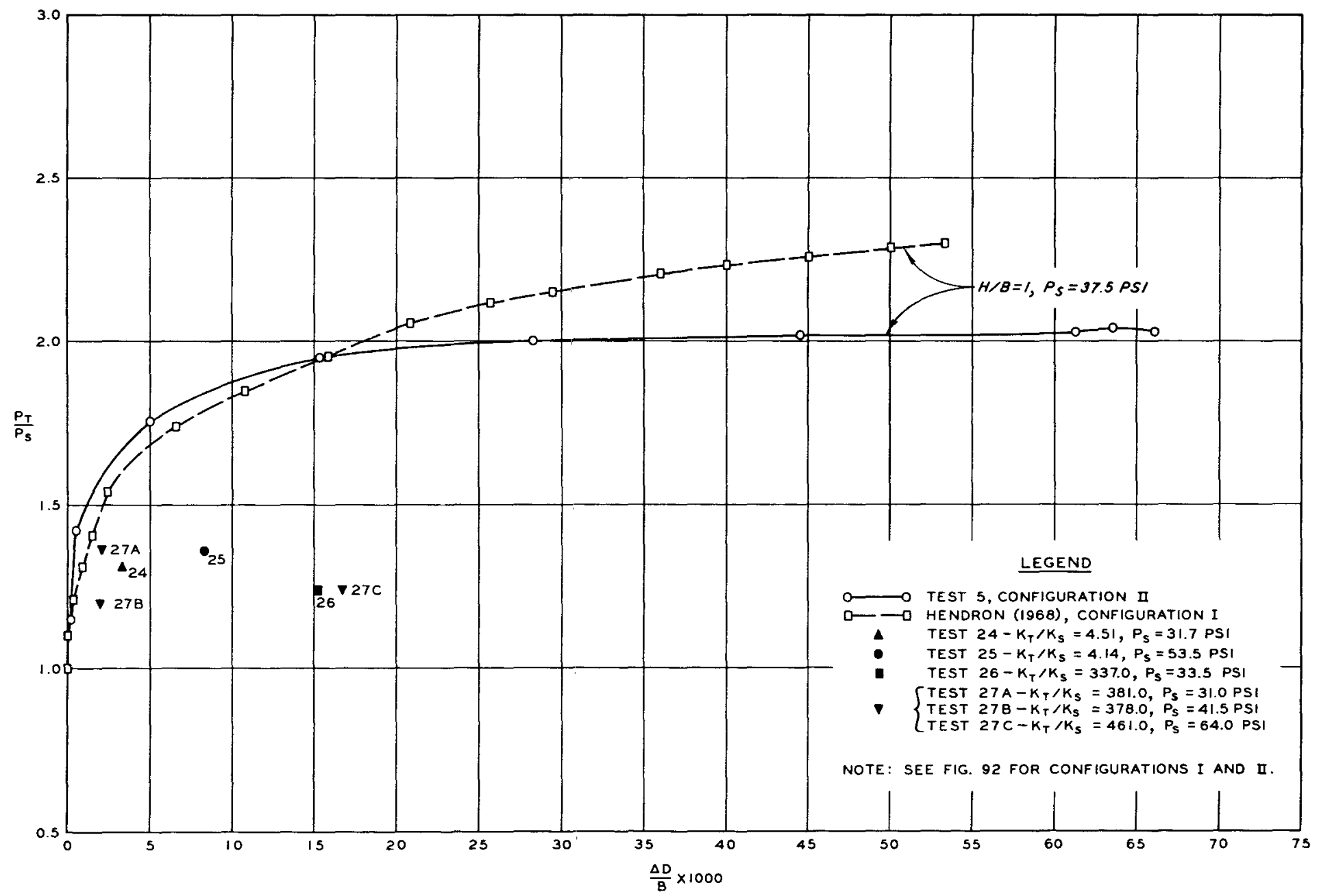

Fig. 93. Passive arching curves, static surface pressure 


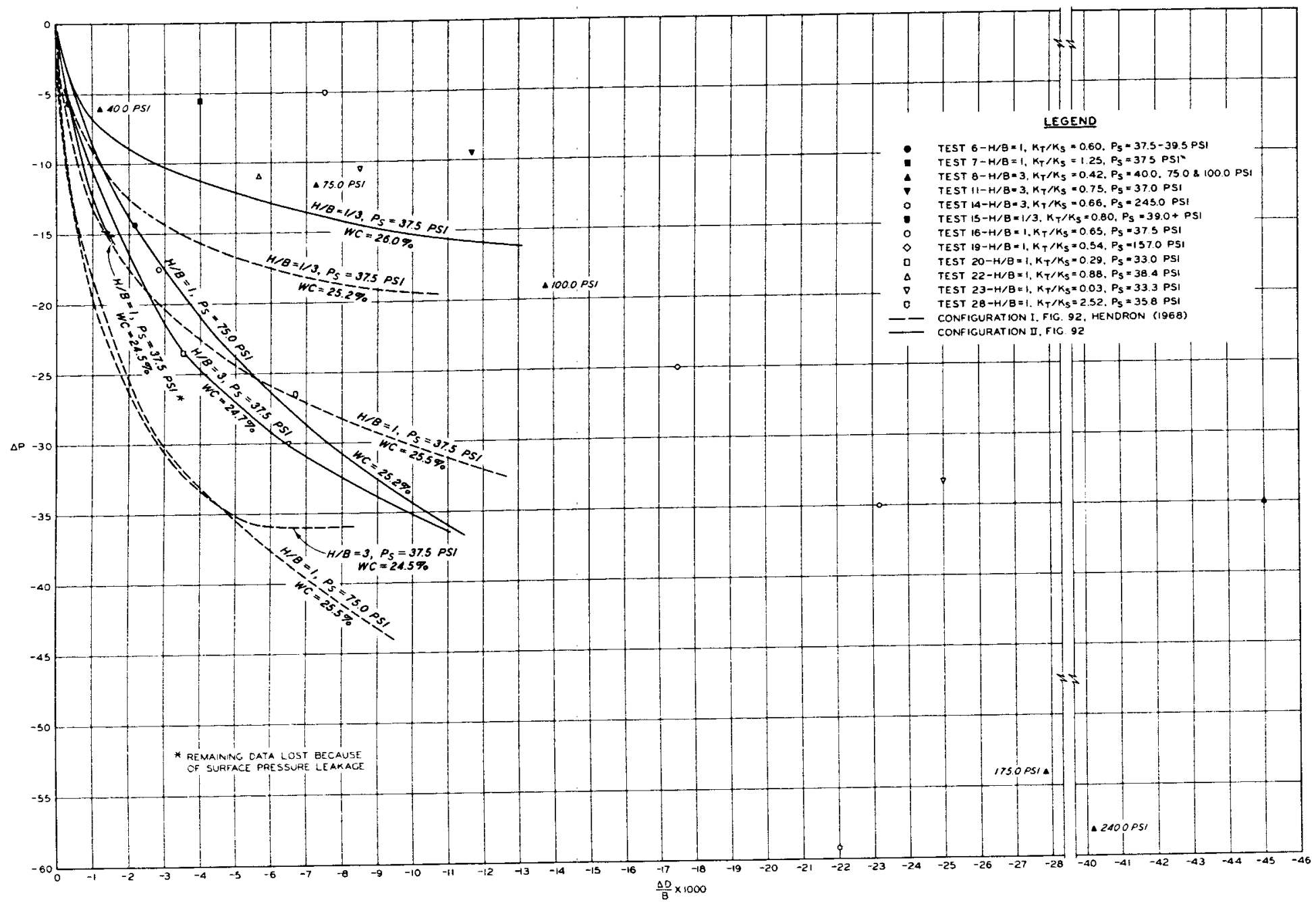

Fig. 94. Active arching, static and dynamic surface pressures, $\Delta P$ versus $\frac{\Delta D}{B} \times 1,000$ 


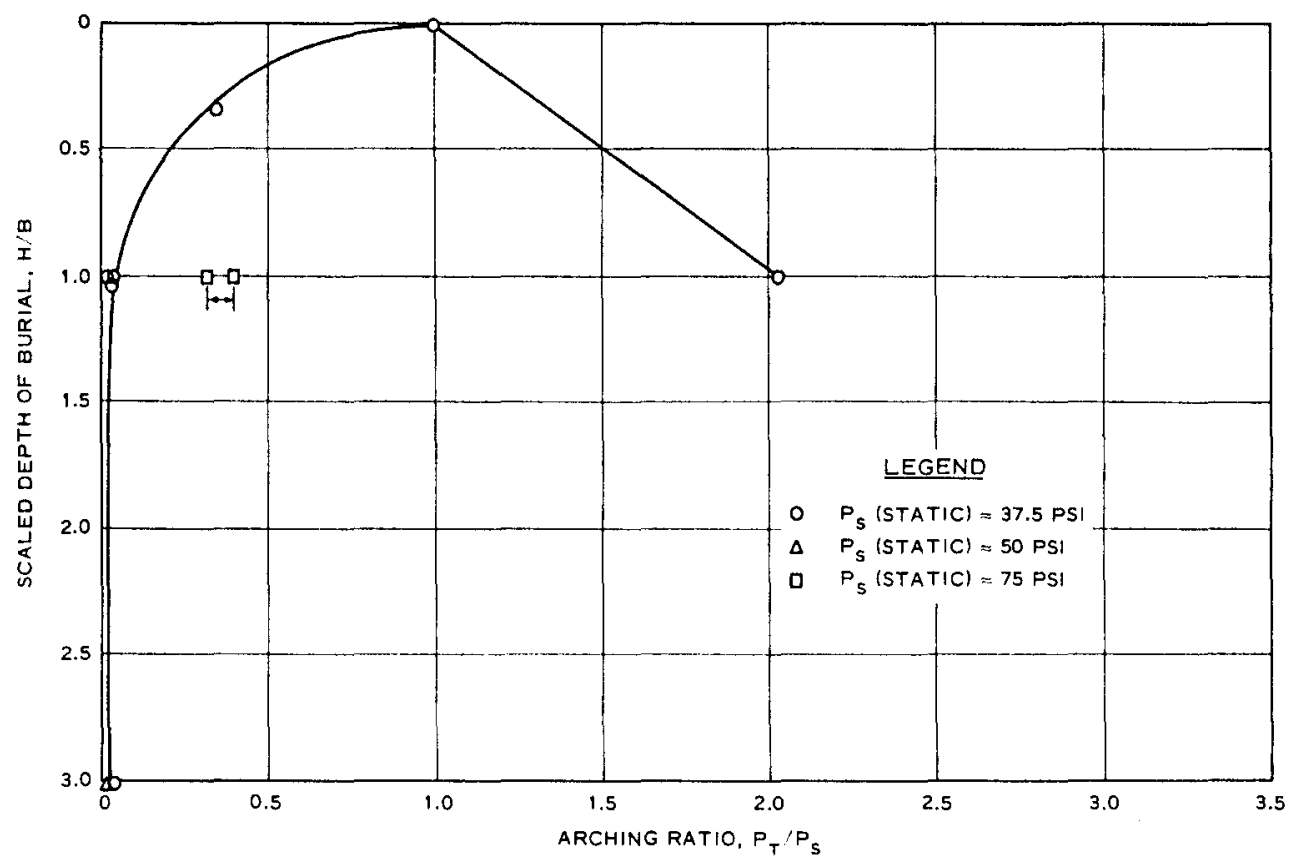

a. Variation of relative load on the structure

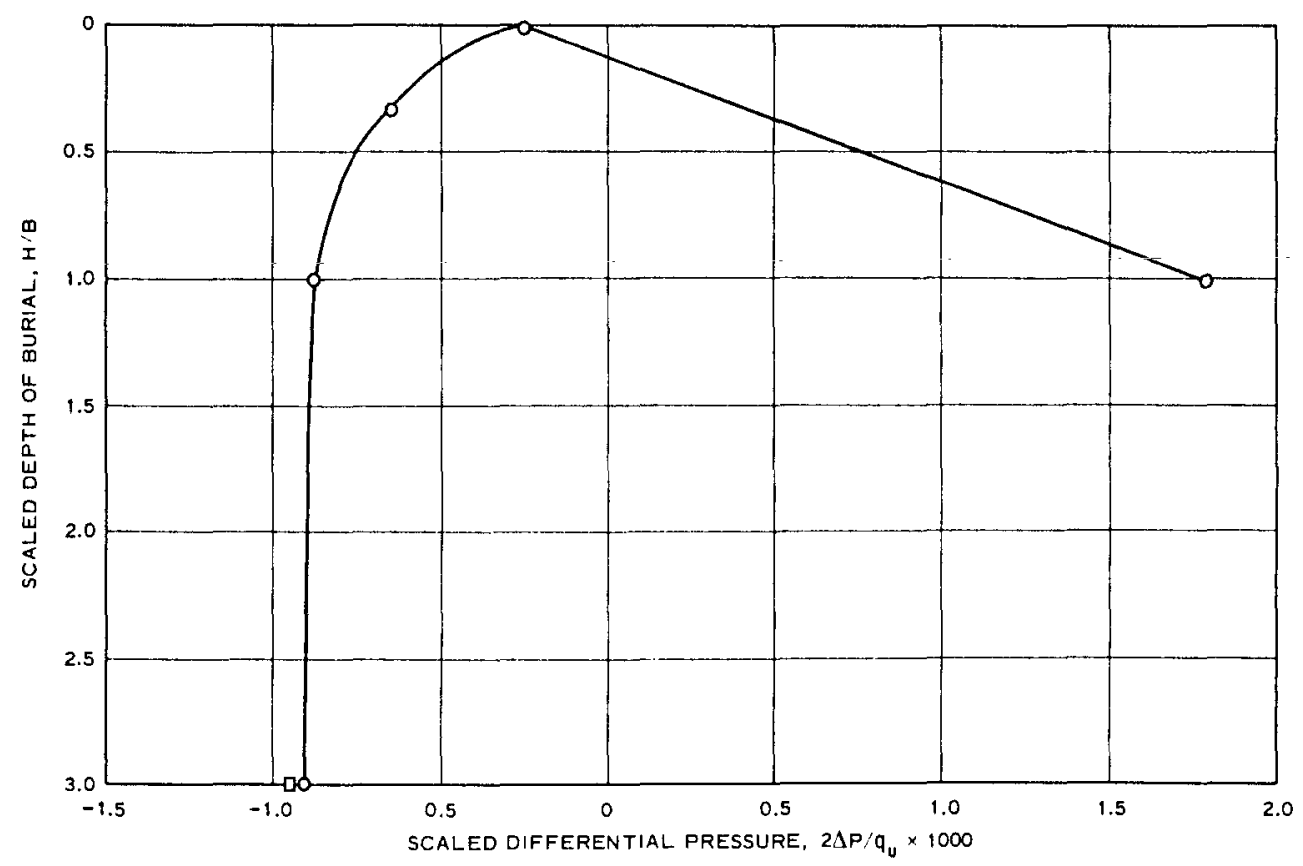

b. Variation of differential pressure at a scaled differential deflection of $\frac{\Delta D}{B}= \pm 2.5 \times 10^{-3}$

Fig. 95. Variations of relative load and differential pressure with depth of burial 


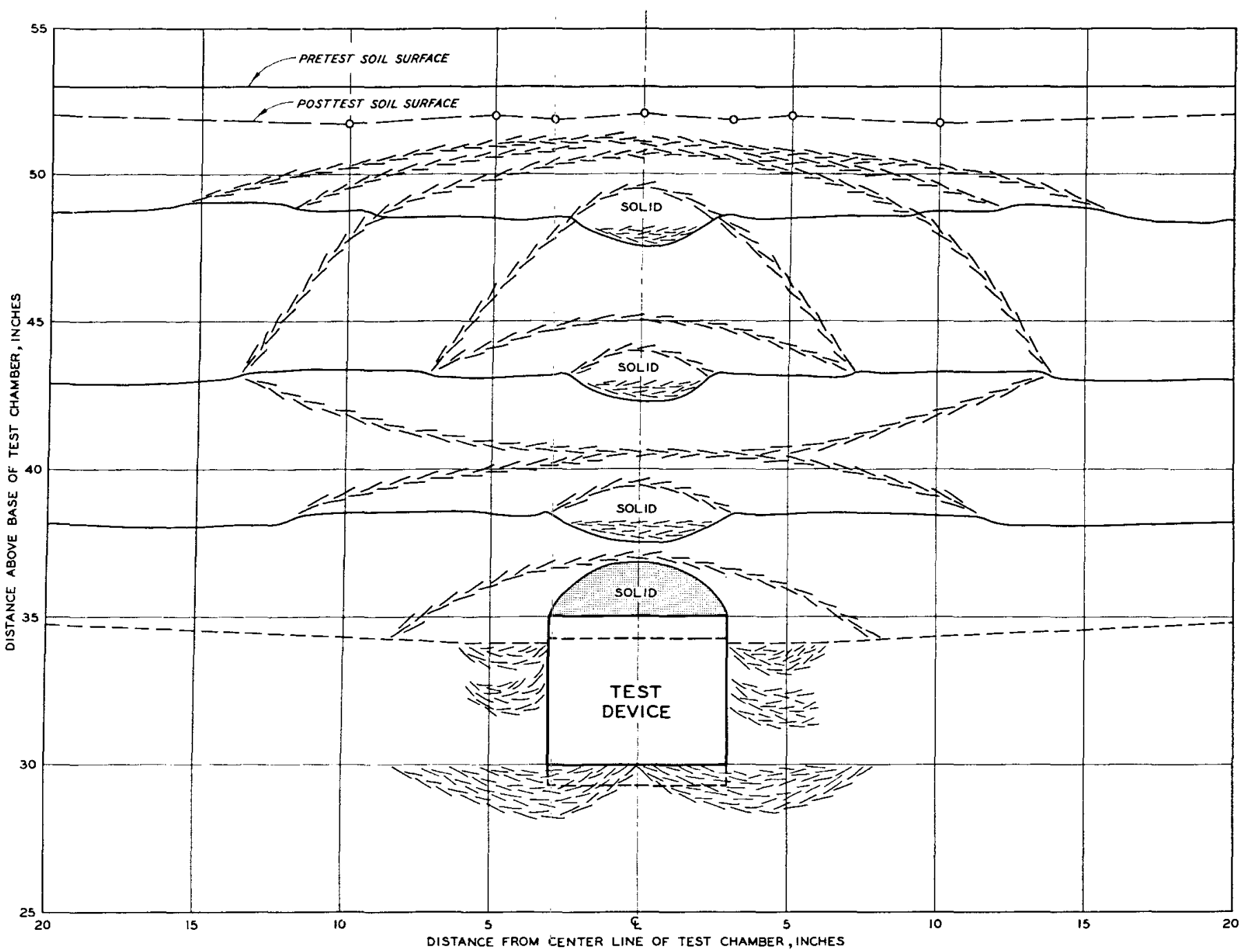

Fig. 96. Composite soil deformation profile under active arching conditions 


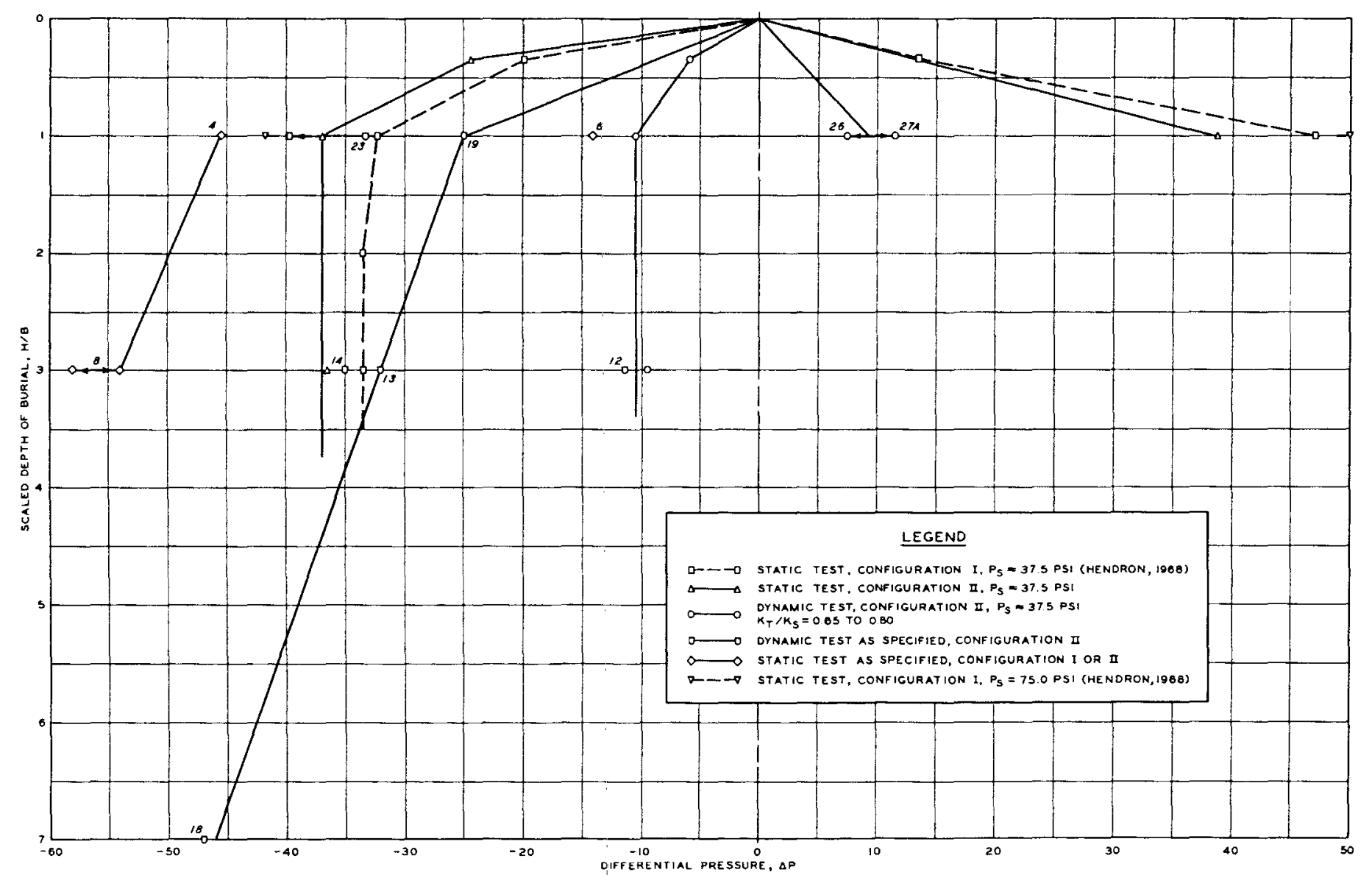

Fig. 97. Differential pressure versus scaled depth of burial, active and passive arching; $\mathrm{WC} \approx 26 \%, \mathrm{~B}=6$ inches 


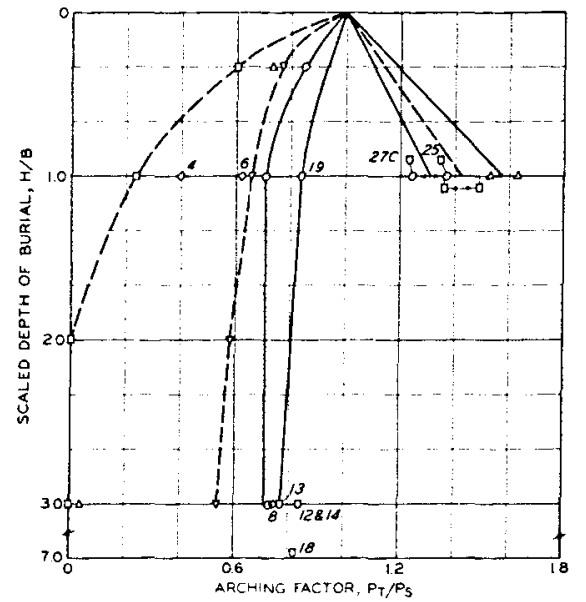

a. VARIATION OF RELATIVE LOAD ON THE STRUCTURE WITH DEPTH OF BURIAL; $W C \approx 26 \%$ AND $B=6$ INCHES

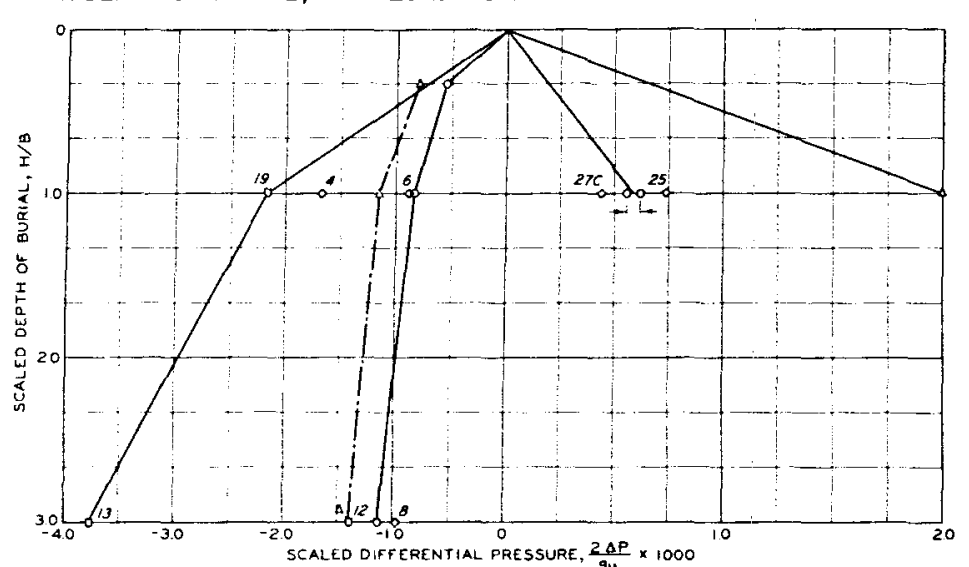

c. VARIATION OF SCALED DIFFERENTIAL PRESSURE WITH DEPTH OF BURIAL

AT SCALED DIFFERENTIAL DEFLECTIONS OF $\triangle D / B \times 1000=-50$ AND \pm 37

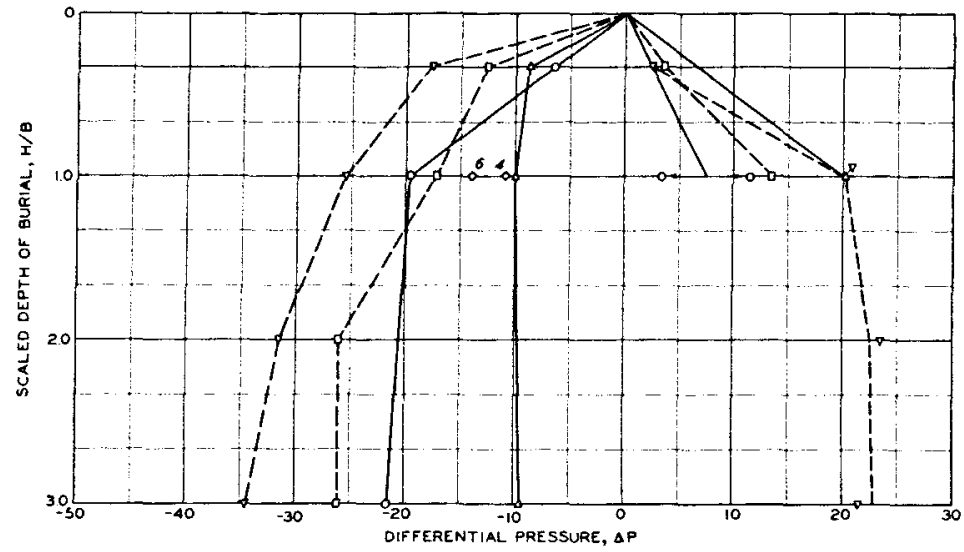

b. VARIATION OF DIFFERENTIAL PRESSURE WITH DEPTH OF BURIAL AT SCALED DIFFERENTIAL DEFLECTIONS OF $\triangle D / B \times 1000= \pm 2.0$

Fig. 98. Variations of relative load on structure and differential pressure with depth of burial

D-- 0 STATIC TEST, CONFIGURATION $I, P_{S}=37.5$ PSI (HENDRON, 1968) $\therefore$ A STATIC TEST, CONFIGURATION II, $P_{S}=375$ PSI

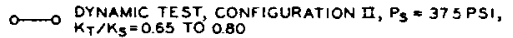

13 OrNAMic TEST AS SPEC

SPECIFIED, CONFIGURATION I

FIED, CONFICURATION I OR D

$\rightarrow$ STATIC TEST, CONFICURATION I, Ps $=75$ OPSI IHENDRON, 1968) 


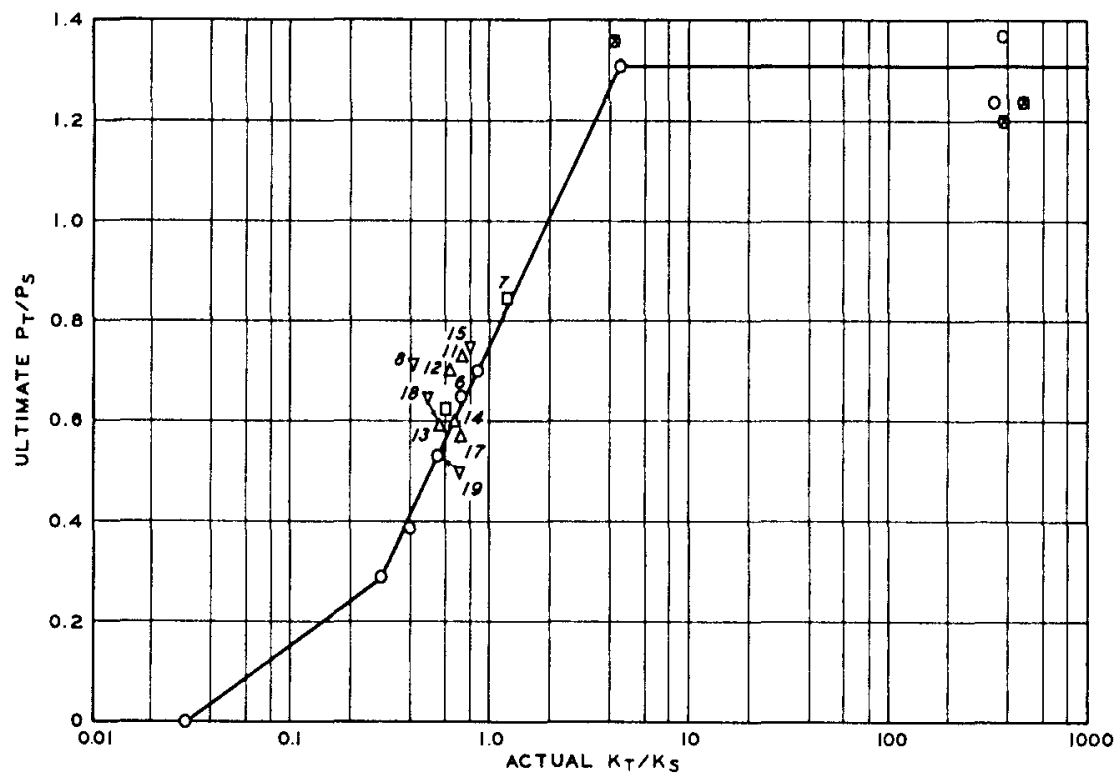

a. RELATION BETWEEN ARCHING FACTOR AND RELATIVE STIFFNESS

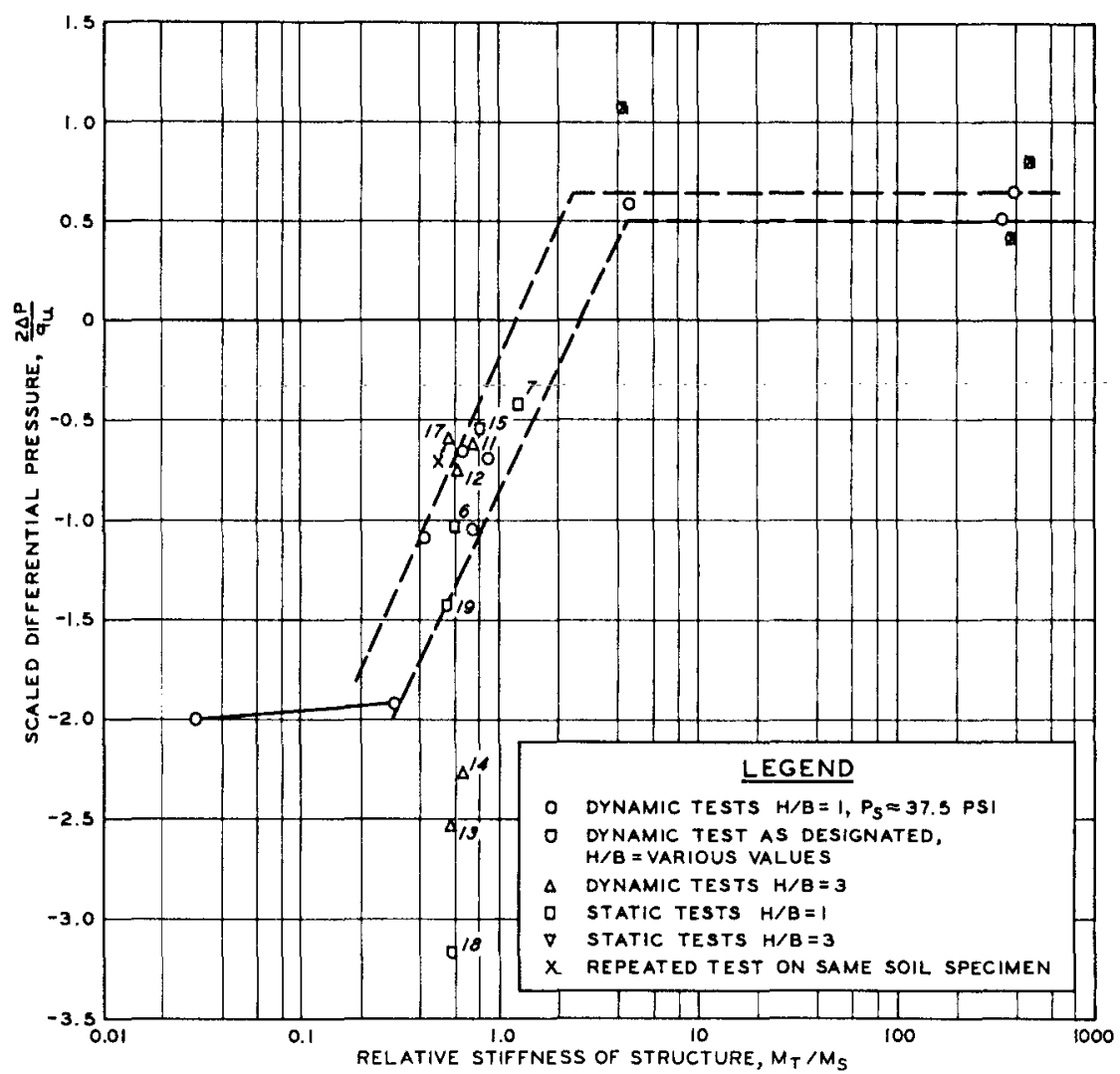

b. RELATION BETWEEN DIFFERENTIAL PRESSURE AND RELATIVE STIFFNESS

Fig. 99. Effect of structure stiffness on active and passive arching; $\mathrm{WC} \approx 24$ to $27 \%, \mathrm{~B}=6$ inches 
APPENDIX A

PROPERTIES OF COMPACTED BUCKSHOT CLAY; WALL FRICTION REDUCTION; SOII, GAGE, AND TEST DEVICE PLACEMENT

A.I Occurrence, Nature, and Index Properties of Clay. The soil used throughout this investigation was a highly plastic clay which is an alluvial material deposited in low-lying areas adjacent to the Mississippi River near Vicksburg, Mississippi, U. S. Army Engineer Waterways Experiment Station (1958). This material is generally referred to as "buckshot clay" because it forms small pellets in its dried condition, Jackson and Hadala.

The gradation curve and Atterberg limits for this material are shown in Figure A-1. The limits for all tests to date are generally parallel to the "A" line in the clay of high plasticity portion of the plasticity chart. The clay appears to have the same characteristics as that used by Jackson and Hadala in their study of the dynamic bearing capacity of clay. The material was derived from the same source as that used by both Carroll and Dorris and the properties appear to be very similar.

This material has been used for a considerable number of studies at the Massachusetts Institute of Technology (MIT) and other institutions. Several reports in MLT's study of The Response of Soils to Dynamic Loadings are based on investigations of buckshot or Vicksburg clay, synonymous terms. 
Buckshot clay classifies as a $\mathrm{CH}$ in the Unified Soil Classification System. The specific gravity of the soil particles is approximately 2.68. The relative consistency of the soil at 26 percent water content is medium with an average unconfined compressive strength of $14 \mathrm{psi}$.

A.2 Compaction Studies. Initially the test program included a study of the effects of soil saturation on arching. It was known that the constrained modulus of clay was sensitive to this parameter. The compaction study was undertaken to determine the compaction effort and water content required to produce degrees of saturation between 70 and 95 percent.

The soil was placed in the Small Blast Load Generator (SBLG) using the techniques described in section 3.5 of the main text. The thickness of the soil layers and the amount of compaction effort were varied. The saturation possible to attain within the workable range of water contents was approximately 80-90 percent, Figure A-2. Saturation increased with water content as anticipated. The numbers inserted beside the open data points are the number of passes of the mechanical compactor required.

The results of the tests indicated that it was possible to significantly increase saturation with increased compaction if the initial water content was lower than 20 percent. At water contents of above 25 percent, the effect of compaction on the degree of 
saturation was small. At water contents of 30 percent or above, the effects of compaction were negligible (Steen, 1966b). The compaction tests showed that the range of usable water contents fell within an area such that variation of saturation was not practical. The use of the degree of saturation as a test parameter was abandoned.

Based on these tests and previous work by Jackson and Hadala it was decided that a standard compaction technique would be adopted and water content would be used as the principal means of soil strength control. An attempt was made to hold water content constant at 26 percent throughout the main test series. It was only possible to limit water content to $26 \pm 2$ percent. Saturation varied over a small range between 86 and 92 percent.

In the previously cited work by Jackson and Hadala, a 4-pass compaction technique was developed and related to the standard Proctor compaction curve, Figure A-2. Using this relation, it was possible to prepare large laboratory samples for tests described later in this appendix.

After the test program started, it became evident that the clay was acting as if it were saturated in some tests, especially those involving high pressures and/or long periods of time. From a series of laboratory triaxial tests, the diagram shown in Figure A-3 was constructed. It was assumed that a saturated condition had been reached when the shear stress became constant. This figure was used 
as a guide in the planning and analysis of subsequent tests.

A.3 SBLG Wall Friction Reduction Studies. Just prior to and during the initial portions of the test program, a detailed study of wall friction in the SBLG was conducted by Hadala (1967b). Both unlined and lined (grease-polyethylene and grease-neoprene) specimens were tested. A summary of the test results is shown in Figure $\mathrm{A}-4$. This figure indicates that friction losses for unlined clay specimens was insignificant at static pressures of 250 psi or higher but that the losses could be significant for low pressure static and all dynamic tests. Figure A-4b shows that the friction losses for all lined specimens was less than 10 percent of the applied stress at a depth equal to one diameter.

Hadala also found that the grease-polyethylene liner was susceptible to more damage than the grease-neoprene liner during the construction of the clay specimen in the SBLG.

Based on Hadala's test results and considering the range of static and dynamic incident pressures planned, a double, 1/16-inch neoprene liner covered with a thick layer of G-403 automotive and artillery grease, constructed as explained in section 3.5 of the main text, was selected for use.

In the preliminary test program and the early static tests, two problems arose which caused a reexamination of the liner problem. Posttest examination of the soil specimen showed that grease was 
being squeezed from between the double liner and the friction losses during the static tests were higher than expected.

The horizontal expansion of the clay specimen would negate the one-dimensional compression conditions desired and thus affect the vertical stress wave velocity. Two methods of attacking this problem were tried. First, a neoprene-graphite combination was tested to determine its coefficient of friction. Tests showed that the friction coefficient for the neoprene-graphite combination was 0.13 as compared with a coefficient of 0.02 to 0.08 for the neoprene-thick grease combination. The graphite was abandoned and a second approach was tried. Instead of a thick coat of grease between the neoprene layers, the liners were wiped with a greasy rag. Checkout tests showed this procedure to be satisfactory; no apparent loss of frictionreducing capacity and no excess grease occurred.

The friction losses at depths below one diameter were attacked in another manner. As previously explained, the continuous neoprene liners were taped to the side of the test chamber during the construction of the soil specimen, Figure 1l. After the specimen was completed, the tape holding the inside liner was cut. In an attempt to improve the friction-reducing capabilities, it was decided that instead of stopping the inside liner a couple of inches short of the base as was the practice, it would be turned under the soil at the base. Secondly, the inside liner was segmented into 1- to 2-foot 
strips. This practice reduced the friction considerably. In all but the low pressure tests, the sidewall friction was practically insignificant. At the depths used for device burial, friction losses were very small even for the low pressure tests.

A.4 Soil Placement Techniques. The soil placement procedures explained in Section 3.5 of the main text were developed by Jackson and Hadala. The soil was generally uniform except between the layers. In spite of the scarifying technique, it was obvious that distinct layers existed and had some effect on the test results.

Although such a condition was undesirable, the time and funds necessary to develop a new soil placement technique were not available. Preliminary study showed that a penetrating device with a tapered head which could work on the sheepsfoot rolier principle probably would solve the problem. The device and technique presently are under study at WES. One major problem with this technique is the protection of instrumentation and cables buried in the soil.

A. 5 Gage Placement Techniques. The cut-and-cover gage placement technique explained in Section 3.3.2 was patterned after one of the procedures tested by Hadala (1967a) in his gage placement study. The mechanics of placing the gages, such as design of the cutter and the mounding technique, were perfected during this test program, Figure 16. During the dynamic tests, it was found that cable breaks were 
frequent. It was necessary to provide considerable slack in the cables, especially along the chamber wall and near the cable port. In addition, the cables were covered with 1/4-inch plastic tubing, Figure 16.

In the static test program, moisture migration was a problem. It was necessary to carefully wrap and waterproof the cable connectors and the connection between the cable and the gages. The cable had to be changed frequently since any break in the insulation allowed moisture to enter the circuit, especially at high pressures. The placement program conducted by Hadala (1967a) and Ingram (1965 and 1967) plus the preliminary tests performed in this program showed that soil pressures were normally accurate to less than \pm 10 percent. It had been planned to check each soil pressure gage in the soil. The -gages were to be installed in soil using the standard procedures and a series of registration tests were to be performed. However, the test device required to accomplish this task was not available soon enough. The soil gages were air calibrated several times during the test program.

A.6 Test Device Placement Techniques. It was necessary to develop a procedure for placing the test device which would minimize the possibility of damage during soil specimen construction and reduce the effects of sidewall friction. A wooden block with a diameter $1 / 8$ inch larger than the device was constructed, Figure A-5a. When the 30-inch 
soil level was attained, the block was placed in the position planned for the device. Then, the soil specimen construction was continued in two lifts to the 35-inch level. Extreme caution was required in the use of the mechanical tampers. When the level of the compacted soil reached the planned elevation, the block was removed and the device placed in the hole left by the block, Figures A-5b and 16. The next layer of soil was then placed directly on top of the device. The depth of soil in the next layer was sufficient to protect the top of the device.

To reduce the friction between the soil and the sides of the device and to prevent soil from obstructing movement of the device, it was necessary to cover the device. For this purpose a machined metal shield was used to cover the gap between the top and the main body of the static device. A single 0.015-inch layer of Teflon was wrapped around the entire device, Figure A-5b. For the dynamic device, only the Teflon was required. Preliminary tests to determine the coefficient of friction indicated that friction between the soil and the structure would be insignificant. Examination of the radiographs disclosed that sufficient friction existed to deform the soil in the vicinity of the device, especially at the 32- to 33-inch levels.

A.7 Laboratory Investigation During Test Program. A series of laboratory tests was performed on samples taken from each soil 
specimen to control and measure soil strength.

As the soil was being prepared in the pugmill and after delivery to the test site, its water content was checked. As the soil specimen was constructed, the dry density and water content were determined for every other layer. These data were determined from two Little Rock drive density samples removed from these layers. Initially, two Hvorslev unconfined compression tests were performed for every other layer. Because of the large number of tests required, this was later changed so that the Hvorslev tests were used at the 35-inch level and every layer above this level.

The procedures for performing the Hvorslev tests are explained by Hvorslev (1949). The sampler is shown in Figure A-6a and the unconfined compression machine in Figure A-6b. The Hvorslev tests proved to be a very rapid and accurate means of checking soil strength. Initially, it appeared that the Hvorslev data would always plot higher than the strength determined by the normal laboratory unconfined compression test. Later in the test program, when a large amount of data were available, this conclusion was found to be incorrect. Within the scatter of the data, it was not possible to distinguish such a trend, Figure A-7a.

In addition to the samples discussed above, two 4-inch undisturbed cube samples were taken from most soil specimens. These samples were used to perform unconfined compression, triaxial 
compression, and consolidation tests in the laboratory. After the correlation between the Hvorslev and laboratory test results was available, the number of undisturbed block samples was reduced, especially when more than one test was performed on the same batch of soil.

After most tests, Hvorslev samples were taken at the surface and at every other layer above and beside the test device. In addition, 4-inch undisturbed block samples were taken during the preliminary test program. Some of the posttest results are plotted in Figure A-7b.

A consolidation of all the density and unconfined compressive strength data is shown in Table 4. It is interesting to note the trends between the pre- and posttest data. In general soil strength increased and water content decreased, though this was not always the case with the water content. The amount of strength change appears to depend on the surface pressure and, for the sample taken directly over the test device, the flexibility of the test device. Stiff devices obviously compressed the soil more and thus raised the strength, while flexible devices had the opposite effect except at the high pressures used in Tests 13 and 14.

The only test in which the "all-samples" soil strength did not increase with testing was Test 25 . This is a repeat test on the same soil specimen used in Test 24 so the data are suspect.

The movement of the data upward and to the left, except for the 
one test at a water content above 30 percent, can be seen in Figure A-7b. The preponderance of the data increased in shear strength by approximately $3.5 \mathrm{psi}$ and decreased in water content by approximately 0.5 percent.

A range of water contents was used in the preliminary program so that a reasonable one could be selected. A water content that could be expected in the field but which would not mask the test results with effects predominated by water content was desired. Because only one water content was to be used, it was decided to select one wet of optimum if possible. A search of the literature concerning buckshot clay and previous arching studies furnished only a small portion of the information required to design the experiments and their instrumentation.

A.8 Pretest Studies. In order to design the main test program, it was necessary to study: dynamic and static properties of buckshot clay, the soil displacements and accelerations to be expected at various depths, the pressure wave characteristics, and particle velocities.

The constrained modulus was probably the most important soil property determined. It was the basis for establishing the range of relative compressibilities between the test device and soil.

In addition to the parameters used to design the test device, the preliminary test program established the ranges to be expected 
for the soil pressure, acceleration, and deflection gages. This was necessary so that proper size gages and gains could be used.

The preliminary test program consisted of two phases which were accomplished simultaneously. Instrumented soil specimens at various water contents were built and tested in the SBLG, Table A-1. Soil samples prepared from the same batch of soil were tested in an impact loading device.

A.8.1 Small Blast Load Generator Tests. A series of five dynamic tests at four water contents were conducted in the SBIG, Table A-1. The variation of soil pressure, displacement, and acceleration with height and water content of specimen were studied. The test layout is shown in Figure A-8. The number and location of the various gages varied somewhat during the tests.

The original Test $D$ was conducted on a soil specimen with 26 percent water content. The planned surface pressure was 40 psi. For some unknown reason, the explosive charge fizzled. The rise time to peak pressure was very long and the desired peak pressure was not attained. Results from this test are not included in this report.

As a result of this failure, a new soil specimen was prepared at a water content of approximately 25.5 percent. It was first exposed to a surface pressure of $41 \mathrm{psi}$. The results of this test are identified as Test $\mathrm{E}$ in Table A-l. After the surface deflection was measured and the gain on the instrumentation changed, the same 
specimen was subjected to a surface pressure of $238 \mathrm{psi}$. This test is identified as Test D.

Copies of the basic oscillograph records from which all time and magnitude measurements were made are contained in Appendix $C$. The type of instrumentation and its location are shown with each record. The SE soil pressure gage used in these tests was the same type described in the main body of the report. The accelerometers were the same piezoelectric gages explained in Chapter 3. The 2-inchdiameter soil displacement disks were constructed from a densitymatched epoxy. These disks were placed at various radii and levels and were used as a basis for measuring permanent soil displacements. In Tests $A, B$, and $C$, a water bag was used at the base of the soil specimen. This water bag was constructed of neoprene, and was 2 to $2-1 / 2$ inches high when evenly compressed. A Norwood and a piezogage were located in the water bag. With this test setup, it was possible to study the sidewall friction, base pressure, and reflected pressure waves.

In Tests $D$ and $E$, soil deflection rods were installed and instrumented for axial strain, Figure 15. The strain was measured as a precaution to ensure that the strain in the rod was small as compared with the movement of the soil disk. The results confirmed the fact that strain in the rods was very small at low pressure and still insignificant at high pressure. 
A.8.2 Confined Compression Tests. A series of static and dynamic confined compression tests was made using the gas-actuated impact hammer and instrumentation shown in Figure A-9. The soil was prepared by compacting it into "concrete" molds at the water content and density used in each of the preliminary SBLG tests, Figure A-10. Soils at each of the four water contents (23, 25, 27, and 32 percent) were subjected to static and dynamic pressures of $37.5,75,120$, and 240 psi.

To prepare the soil for testing, l-inch-high disks, Figure A-10, were cut from the prepared specimen by use of a piano wire. The edges of each specimen were wrapped in a layer of Teflon before the specimen was placed in the confining chamber. The confining chamber was placed under the impact machine and the head, which was the same diameter as the inside diameter of the confining chamber, was lowered onto the top of the soil disk.

When the trigger mechanism for the hammer was released, a timing device triggered in turn a camera which photographed the display of the stress and strain on the oscilloscope. Typical oscilloscope records are shown in Figure A-11. The stress and strain traces were not coupled to produce $X-Y$ plots for use as stress-strain curves. There is a danger of a phase shift with the viscous buckshot clay.

The test program explained in this section was used since the constrained modulus was so important to the test program and no other 
device was readily available. The one-dimensional compression device under design by Schindler was not ready, and the device at the University of Illinois, Kane, Davisson, Oleson, and Sinnamon, was not immediately available. After the test program had been completed, Schindler's device became available and was used to perform one static and one dynamic test on soil specimens prepared at a 26 percent water content. The test results are included in the following sections.

A.9 Static Properties. In order to study the properties of buckshot clay and relate them to the arching actions observed in the soil, a laboratory test program was designed and carried out both prior to and during the main arching test program. Each of the sections below will introduce and explain briefly the various soil tests and their results. Several investigators have shown that the strength and volume change associated with buckshot clay when subjected to a load is a function of the loading rate, Whitman et al. (1962a), Carrol, Kane et al., Perry. The change in shear strength appears to be related to the degree of drainage and some other phenomenon which has not been completely isolated. In spite of the low permeability of buckshot clay, it is important to specify the drainage conditions. In some of the rather long tests in the static program, drainage did occur. This was evidenced by the water seeping from the test chamber at the flanges. This was especially true in Test 8, one of the high pressure static tests. Although the soil was not 
saturated in its compacted state at the beginning of the test, it did become saturated during this test, Figures A-2 and A-3. Even in the dynamic tests, the very high pressures may have changed soil volume sufficiently to induce a saturated condition.

In those tests in which the clay became saturated or very nearly saturated and was subjected to strains without drainage, the clay behaved as a cohesive material with $\varnothing$, the friction angle, equal to zero.

From the preceding discussion and the test conditions explained in the main body of the report, it can be seen that no one laboratory test can depict the conditions which existed in each of the arching tests. In order to specify the strength of the material, it was necessary to establish the stress levels which existed, the drainage conditions, and the rise time and/or duration of the load.

The unconfined compression test does not allow control of drainage or confining conditions. For this reason it was initially planned that the unconfined test would be used only to estimate the shear strength. The closer the clay approached a saturated condition and its failure approximated an undrained condition, the more accurate the shear strength computed from the unconfined test became. Due to progressive failure, the average shearing resistance was taken as $\mathrm{q}_{\mathrm{u}} / 2$, Terzaghi and Peck. When clay is unsaturated, the confining pressure can have measurable effect on its shearing resistance and 
the triaxial test is more applicable. It is for these reasons that both unconfined and triaxial tests were used in this program.

Due to the significant nonlinearities in the stress-strain curves for buckshot clay, it was difficult to determine one modulus. The modulus actually was continually changing, depending upon the stress or strain level. Thus it was necessary to use tangent modulus values which were approximately constant over some range of stress or strain or to use a secant modulus which was dependent upon some selected stress or strain.

It would have been desirable to have the same stress and strain conditions in the laboratory test as those to which the soil was subjected in the arching test. Lateral strain appears to be minimized during underground arching; therefore, it was desirable to limit lateral strains. It was for this reason that one-dimensional compression tests were used in the preliminary test program. Time and funds did not allow an appropriate investigation of strain rate effects, and data from other investigations in this field have been used. A.9.I Unconfined Compression Tests. As previously explained, two types of unconfined tests were conducted, the normal laboratory test and the Hvorslev test. Figure A-7 compares the maximum strength determined by both type tests. Figure A-12 illustrates the stressstrain results from many of the laboratory tests. Inasmuch as the buckshot clay appeared to be strain rate sensitive, the tests were 
separated based on time to failure. In the tests which were conducted in more than 20 minutes, the soil displayed a plastic action. At a strain of approximately 7 percent, the soil became fully plastic and there was very little increase in the soil strength for an increase in strain, especially above 25 percent water content. The drier soil reached this point at approximately 10 percent strain. Obviously, the higher the water content the lower the strength.

The curves for specimens tested to failure at a rate faster than 20 minutes did not indicate as much scatter as those from the slower tests. They generally exhibited a higher strength than the curves from the slower tests with the exception of the soil at a water content of 24 percent. In the faster tests, the soil became fully plastic at lower strains, Figure A-12b.

The failures occurred in the test specimens by both splitting and bulging. In most cases, the failure appeared to be a combination of the two modes of failure. The soils were compacted wet of optimum water content, but were not saturated. Generally the saturation of the test samples varied from 86 to 92 percent, depending on the initial water content.

The unconfined tests were an excellent means of soil strength control. They also indicated relative strengths at various water contents. The test results appear to be applicable to the low pressure static tests which were conducted in a matter of hours rather 
than days, where confining pressure and drainage conditions may not have been significant.

A.9.2 Triaxial Tests. These were quick tests with confining pressures approximately equal to those used in the static test program.

Typical examples of the triaxial test results are plotted in Figure $\mathrm{A}-13$. These results indicate cohesion varying from 10 to 16.7 psi and friction angles varying from 1 to 6 degrees depending upon the water content. Thus, the buckshot clay acts almost like a $\phi=0$ material, especially at the desired water content of 26 percent.

Figure A-13 shows that confining pressures within the range tested had very little effect on the soil strength as compared with a slight change in the water content, except for the soil with a 23 percent water content. The variation was insignificant for the 26 percent water content material.

Time to failure during the triaxial compression tests varied from 50 to 60 minutes or a rate of strain of approximately 0.25 to 0.30 percent per minute.

A comparison of Figures A-12 and A-13 disclosed that at comparable rates of strain and with water contents approximating 26 percent, the average strength of the material as determined by both tests was virtually identical. At 8 percent strain the average 
strength as determined by the unconfined tests was 2 psi higher. The ultimate strengths were identical. The unconfined tests conducted in less than 20 minutes disclosed average strengths 8 psi larger than the triaxial results at both 4 and 8 percent strain. At ultimate strength the faster unconfined results were 7.5 psi larger. For 26 percent water content material it is evident that the effects of confining pressure within the range tested were insignificant as compared with the effects of rate of strain. At comparable strain rates the strengths as determined by unconfined compression tests are as valid as the triaxial test results. Carroll also found that the effects of confining pressure were insignificant as compared to water content and strain rate, Figure A-14.

During the triaxial tests, the samples failed by splitting and/or bulging.

A.9.3 Direct Shear Test. Consolidated-drained direct shear tests were not performed during this test program. Shear strength as determined by the normal shear box is very questionable and has fallen from use except for particular soil types. A large number of these tests were conducted by Jackson and Hadala, and Hadala (1965) during their investigation of the dynamic bearing capacity of buckshot clay. These were stress-controlled tests on 2.4- by 2.4- by 0.4-inch-high specimens. Although it is difficult to compare directly, the shear strength as determined by the direct 
shear test using specimens of equal water content was approximately 20 percent higher than comparable triaxial results.

\section{A.9.4 Consolidation Tests. Normal consolidation tests were} performed on samples removed from some of the test specimens. Normal e - $\log p$ curves were drawn and, in addition, the stress-strain curves shown in Figure A-15 were constructed. Until such time as better data were available, these curves were used (to obtain an estimate of the one-dimensional modulus of the material).

A.9.5 Stress-Strain Curves. The pretest stress-strain curves plotted in Figure A-16 were generated using confined soil samples prepared as explained in section A.8.2. The 1-inch-high by 6-inchdiameter specimens were loaded with the MIT constructed loader at the rate of 1,000 pounds per minute or 35.4 psi per minute, Figures A-9 and A-10. Three different specimens were loaded at three different pressures, 37.5, 75, and 240 psi.

The curves in Figure A-16 marked with test numbers were constructed by using data generated at the 30 - to 35 - or 30 - to 38-inch levels of the soil specimens placed in the SBLG. Paired soil deflection and pressure measuring gages were at the same level and radius. The surface pressure was applied at the rate shown in Figure A-16. A more uniform rate was desired but not always attainable. These curves depict the stress-strain relation within the soil and are not necessarily analogous to the one-dimensional compression test. 
The posttest curve in Figure A-16c was derived from data developed using Schindler's one-dimensional test device. The soil specimen was 1 inch high by 10 inches in diameter.

The low pressure curves in Figure A-16a indicate a plastic matexial which tends toward a fully plastic condition. Obviously, the loading rate even at this pressure level has a large effect on the total strain and the shape of the curves.

Figure A-16b contains the medium pressure curves. There is a point of inflection in both curves. This is an indication that some sort of locking action is taking place. In this case, it is probably due to the change in the degree of saturation and not to the type of loading action normally associated with sand.

The high pressure curves are shown in Figure A-16c. In the pre- and posttest curves, the locking action is marked. It is not as obvious in the curve derived from Test 8 . The small strains that resulted from the relatively slow rate of load application are difficult to explain. There appeared to be a malfunction in the deflection gage at the 35-inch level which could account for the discrepancies. The curves resulting from the two entirely different compression devices are remarkably similar.

In determining the constrained modulus from the pretest data, a secant modulus was used. In spite of the effects of strain rate, the data proved reliable enough to design the test device. The 
modulus calculated from the stress-strain curves in Figure A-14 also were used. They constitute one extreme of the effects of loading rate.

As can be seen from the stress-strain curves, the modulus of this material was continually changing during the course of the tests. This modulus was affected by the pressure, depth of soil, loading rate, and the saturation of the material. In studying the test results in Tables A-I and A-2 plus those in the main body of the report, this fact should be kept in mind.

A.9.6 Constrained Modulus. Table A-2 contains the range of values derived for the constrained modulus from all sources. In most cases, the values appear to be reasonable. In spite of the disparities in the strain rates, the modulus calculated from the onedimensional laboratory compression tests related closely to those calculated during the SBIG tests. The one major exception was Test 8 which was discussed in the first paragraph of Section A.9.

The modulus derived from the SBLG tests was calculated by measuring at a designated time the differential strain over a particular gage length, and the average pressure between the top and bottom of the gage length.

A.10 Dynamic Properties. In the study of the dynamic properties of buckshot clay, several properties were of particular interest. Of most importance were the stress-strain and modulus data. These 
were required to plan the various stiffnesses for the test device. The constrained modulus was calculated not only by using stressstrain, but also by using propagation velocity of the peak stress wave; thus, a study of velocities was conducted. In dynamic tests, the rise time of the pressure pulse can affect the loading a buried structure experiences; therefore, information on this subject was collected and used in the planning of the test program.

A.10.I Variation of Propagation Velocities. In the study of propagation velocities two times were of interest: the arrival of the initial disturbance, and the arrival of the peak pressure. The velocities between the soil surface and the level of the test device were of particular interest, i.e. the top 2 to 18 inches of material. The time for the pressure wave to reach the base of the test chamber also was important, since this was used to determine the amount of soil required below the test device and the amount of time available to study structural actions before the reflected waves interfered.

Figure A-17a is a plot of the propagation velocity of the initial disturbance. The data were taken from the records in Appendix C. There is considerable variation in the velocity except for the high water content material in Test C. This uniformity was probably a function of the high degree of saturation. The relation between the velocity and the water content did not appear to be reasonable, i.e. the soil with the lowest water content had the highest velocity and 
the soil with the highest water content had the lowest velocity. Further examination disclosed that if Test $\mathrm{E}$, the low pressure test, is omitted, the velocities are in an inverse relation to the water content.

The relation between the pressure and the velocity also appears to be out of order. The low pressure test, E, had a higher propagation velocity than the high pressure test on soil with the same water content. It was not until a point approximately 18 inches below the surface was reached that the position of the velocities appeared to be in proper relation to each other.

In Figure A-17D, the propagation velocity of the initial peak pressure, disregarding reflections, is plotted. These velocities were more uniform from surface to base. In addition, material with a higher water content had a higher velocity than the drier material. The higher pressure tests also developed higher propagation velocities than the low pressure test.

Based on the material shown in Figure A-17, the propagation velocity of the first stress peak was selected as one of the means for calculating the constrained modulus. Although these velocities are not exactly shock velocities, inspection of the pressure signatures in Figures $\mathrm{C}-73$ through $\mathrm{C}-87$ shows that they are close enough for the purposes desired.

Figure A-18 is a plot of the propagation velocities from several 
of the program tests. The water content varied from 26.5 to 25.1 percent, Table 4. This figure confirms the rather obvious fact that velocity is dependent upon the surface pressure initiating the stress wave. With the exceptions of Test 12 at 70 psi and Test 17 at 40.5 psi, the curves are in descending order of surface pressure. The reason for the transposition of Test 12 is not clear even though its water content was 26.4 percent and generally high in comparison with the other tests. The position of Test 17 is understandable since this was a repeat shot on the same soil specimen as that used in Test 11. Close examination discloses that the velocities in Test 17 exceeded those in Test 11 at all depths.

Figure A-19 shows the variation of peak stress velocity with surface pressure and soil water content. The pressure curves in Figure A-19a were not normalized for water content even though there was a 1.4 percent range. The water content curves in Figure A-19b were normalized to $200 \mathrm{psi}$. The 6-inch level was not plotted because of the scatter in the data. These curves indicate that peak pressure waves attained a relatively constant velocity at a water content of approximately 28 percent. This indicates that soil with this water content and above becomes saturated at 200 psi. This does not agree with the results shown in Figure A-3.

Based on Figures A-17b, A-18, and A-19, the calculated modulus should be fairly uniform in relation to depth, but should vary with 
pressure and water content. At higher pressures the water content does not appear to have a large effect until a point is reached where the material does not approach saturation at the test pressure of interest.

The velocity of the peak pressure does not appear to vary with depth as some soil model builders believe, at least not within the depths used for these tests. This wave velocity appears to be dependent primarily on the stiffness of the soil, the surface pressure, and, of course, the type material which was a constant for these tests. Thus, the velocity of the peak stress is directly related to the stress-strain characteristics of the soil. These are discussed in Section A.10.3.

The velocities plotted in Figures $A-17$ and $A-18$ were not calculated from the stress-strain curves, but were measured directly from the oscillograph records of the soil pressure gages.

A.10.2 Rise Times. Dynamic amplification effects can be very important to buried structures just as they are for surface structures. Figure A-20 is a plot of the variation of rise time of the stress wave with depth, water content, and pressure. The rise time was nondimensionalized by plotting the pressure at the depth of interest over its rise time divided by the surface pressure over the rise time at the surface.

The effects of high water content were dramatic. The effects of 
pressure were large at shallow depths. At a depth of 24 inches, the effects of water content below approximately 25.6 percent and of pressure within the range tested appeared to be minimal in the nondimensional form. Figure A-20 appears to confirm the findings reported in the main body of the report. At shallow depths of burial and high pressure levels with water contents around 26 percent, the rise time can affect the load which the test device experiences. Dynamic amplification was possible as the ratio of the rise times to the period of the structure and the test results showed.

A.10.3 Stress-Strain Curves. Stress-strain curves were generated at four water contents and at least four pressure levels using the impact loader previously described. The soil specimens were 6 inches in diameter and 1 inch high. Specimens 2-1/2 inches high were tried but the results were very erratic because of what appeared to be friction losses and the time necessary for the stress wave to traverse the specimen. Figures $\mathrm{A}-21$ and $\mathrm{A}-22$ show the results of these tests. Peak load was reached in 4 to $6 \mathrm{msec}$. In these curves the material displays a plastic behavior. It was only at high pressures in the wetter material that the shocking action caused by saturation became evident.

In addition to the laboratory stress-strain curves, similar curves were constructed using data developed during the SBLG tests. Data collected with the deflection and pressure gages located within 
the soil at the 30- to 35- or 30- to 38-inch levels were used. Figure A-23 contains these curves. The low pressure curves appear reasonable while the high pressure curves are erratic. The total strains and shapes of the curves are considerably different from those in Figure A-22.

Figure A-23b also contains a stress-strain curve generated by using Schindler's one-dimensional compression device. The sample was 1 inch high by 10 inches in diameter. This curve is not similar to all the previously described curves.

It is evident that the rate of strain has a large effect on the characteristics of the stress-strain curve. It almost masks the effect of water content. One-dimensional tests were not as good an approximation of the SBIG conditions as they were in the static tests. This was especially true at high pressures.

Carroll showed similar strain rate effects in his investigation of buckshot clay, Figure A-24.

The stress-strain curves resulting from the laboratory confined compression tests were used to determine the constants in the clay models suggested by Seaman. Using the parameters described in Seaman's report it was not found possible to duplicate his results or to predict the results experienced in the SBLG. A.10.4 Constrained Modulus. Constrained modulus was required to design the tests and the test devices. This modulus also was 
calculated after each test in order to determine the actual relative flexibility of the structure versus the soil.

Prior to the main test program, the one-dimensional laboratory tests discussed above, the preliminary tests in the SBLG, and a method of calculation suggested by Johnson were used to estimate the constrained modulus. The modulus was determined in two ways from the SBLG tests. First the actual stress-strain curves were constructed as previously explained from data generated during Tests $D$ and $E$. In addition, a modulus was calculated using the velocity of propagation of the peak stress. These were measured velocities and not calculated from the stress-strain curves. The stress waves shown in Appendix C were not considered shock waves, since their rise times, especially at depth, were normally measured in milliseconds and not microseconds. In spite of this "discrepancy," a modulus was calculated using the simple formula, $M_{z}=C^{2} p$, where $\rho$ is the wet density of the soil and $C$ is propagation velocity of the peak stress between any two points of interest. Table A-I contains the values of constrained modulus derived from all sources. The range of values shown for the SBLG tests was calculated using the velocity from the surface to the 30-inch level and from the surface to the base. The stress-strain data were derived from two different locations, between the 24- and 36-inch levels, and between the 24- and 42-inch levels. When a range of values is shown it signifies that several tests were performed at that 
water content and pressure level. The full range of values is given. The secant modulus and not the tangent modulus is used. Based on this, considerable difference was expected in the values of the modulus. The one-dimensional compression tests appear to agree more closely with the velocity data than the strain data which are larger in all cases. The values calculated using Johnson's approach also are shown. They agree with the one-dimensional tests rather well at the intermediate pressures, but appear to be high and low at the upper and lower extreme pressures respectively.

The soil moduli for the main test program are shown in Table 2 of the main text. Note that both velocity and strain data were used. In many cases the agreement was quite good; in others there was considerable difference. The modulus which appeared to be the mean of both ranges normally was used in the stiffness calculations. Just as in the static tests, the constrained modulus of the soil was changing throughout the tests. These changes were due to the pressure acting on and saturation of the soil. The modulus also was affected by whether the soil was loading or unloading at the particular time in question. Figure 53b contains a curve showing the change in the relative stiffness of the structure versus the soil as Test 16 progressed. The modulus selected and used for most comparisons was calculated at the time of minimum arching. In this manner, the stiffnesses compared should correspond fairly closely to the arching condition examined. 
Table A-I

Constrained Modulus of Soil Specimens Derived from All Sources, Preliminary Dynamic Tests

\begin{tabular}{|c|c|c|c|c|c|c|c|}
\hline \multirow[b]{2}{*}{$\begin{array}{r}\text { Test } \\
\text { No. }\end{array}$} & \multirow[b]{2}{*}{$\begin{array}{c}\text { Water } \\
\text { Content } \\
\% \\
\end{array}$} & & \multirow[b]{2}{*}{$\begin{array}{c}\text { Height } \\
\text { of } \\
\text { Specimen } \\
\text { in. } \\
\end{array}$} & \multicolumn{4}{|c|}{ Constrained Modulus } \\
\hline & & $\begin{array}{r}\mathrm{P}_{\mathrm{S}} \\
\mathrm{psi} \\
\end{array}$ & & $\begin{array}{c}\text { Velocity } \\
\text { SBLG } \\
\text { psi } \\
\end{array}$ & $\begin{array}{c}\text { Stress-strain } \\
\text { SBIG } \\
\text { psi } \\
\end{array}$ & $\begin{array}{c}\text { l-Dimensional } \\
\text { Lab } \\
\text { Compression } \\
\text { Tests, psi }\end{array}$ & $\begin{array}{c}\text { Calculated } \\
\text { psi } \\
\end{array}$ \\
\hline A & $23 \cdot 3$ & $\begin{array}{l}236.0 \\
107.0\end{array}$ & $\begin{array}{r}43.9 \\
1.0\end{array}$ & 2320 & & $\begin{array}{c}2190-2520 \\
2340\end{array}$ & \\
\hline B & 27.2 & $\begin{array}{r}212.0 \\
120.0 \\
103.0 \\
48.0\end{array}$ & $\begin{array}{r}42.1 \\
1.0 \\
1.0 \\
1.0\end{array}$ & $4180-4280$ & & $\begin{array}{c}2945-3210 \\
2120 \\
2180 \\
1990\end{array}$ & $\begin{array}{l}4300 \\
2600 \\
2200 \\
1200\end{array}$ \\
\hline $\mathrm{C}$ & 32.1 & $\begin{array}{r}205.0 \\
97.0 \\
89.0 \\
70.0 \\
43.0\end{array}$ & $\begin{array}{r}43.1 \\
1.0 \\
1.0 \\
1.0 \\
1.0\end{array}$ & $3280-5820$ & & $\begin{array}{l}4750 \\
2560 \\
2280 \\
1920 \\
1160\end{array}$ & $\begin{array}{l}4000 \\
2200 \\
1900 \\
1550 \\
1050\end{array}$ \\
\hline $\mathrm{D}$ & 25.4 & $\begin{array}{r}238.0 \\
192.0 \\
120.0 \\
81.0\end{array}$ & $\begin{array}{r}48.7 \\
1.0 \\
1.0 \\
1.0\end{array}$ & $35 ? 0-4430$ & $5710-6340$ & $\begin{array}{c}4230-4500 \\
2780 \\
2420-2720 \\
1875-2290\end{array}$ & $\begin{array}{l}5100 \\
4350 \\
2700 \\
1900\end{array}$ \\
\hline$E$ & 25.6 & $\begin{array}{l}41.0 \\
41.7 \\
60.4\end{array}$ & $\begin{array}{r}48.0 \\
1.0 \\
1.0\end{array}$ & $1100-1581$ & $1830-3360$ & $\begin{array}{c}1500-2100 \\
2340 \\
2560\end{array}$ & $\begin{array}{l}1120 \\
1150 \\
1500\end{array}$ \\
\hline
\end{tabular}


Table A-2

Range of Values of Constrained Modulus of Soil Specimens Derived from All Sources, Preliminary Dynamic Tests

\begin{tabular}{|c|c|c|c|c|}
\hline \multirow[b]{2}{*}{$\begin{array}{c}\text { Approx } \\
\text { Water } \\
\text { Content } \\
\% \\
\end{array}$} & \multirow[b]{2}{*}{$\begin{array}{c}\text { Test } \\
\text { Pressure } \\
\text { psi } \\
\end{array}$} & \multicolumn{3}{|c|}{ Constrained Modulus } \\
\hline & & $\begin{array}{c}\text { I-Dimensional } \\
\text { Lab } \\
\text { Compression } \\
\text { Tests, psi } \\
\end{array}$ & $\begin{array}{c}\text { SBLG } \\
\text { Tests } \\
\text { psi } \\
\end{array}$ & $\begin{array}{c}\text { Consolidation } \\
\text { Tests } \\
\text { psi }\end{array}$ \\
\hline \multirow{2}{*}{24.0} & 37.5 & $1875-2260$ & $1700-2040$ & 2230 \\
\hline & 240.0 & 3380 & & 6360 \\
\hline \multirow[t]{2}{*}{25.0} & 37.5 & & $1570-1870$ & \\
\hline & 75.0 & & $1650-1700$ & \\
\hline \multirow[t]{6}{*}{26.0} & 37.5 & $1920-2175$ & 1500 & \\
\hline & 50.0 & & 3830 & \\
\hline & 75.0 & $1850-1920$ & 1875 & \\
\hline & 100.0 & & 1545 & \\
\hline & 155.0 & 2980 & & \\
\hline & 240.0 & $3660-3840$ & & \\
\hline \multirow[t]{4}{*}{27.0} & 37.5 & $1500-2160$ & & 1910 \\
\hline & 75.0 & 2320 & & \\
\hline & 175.0 & & 6150 & \\
\hline & 240.0 & 4460 & $7450-7950$ & 7020 \\
\hline \multirow[t]{3}{*}{32.0} & 37.5 & 1040 & & 1420 \\
\hline & 75.0 & 1940 & & \\
\hline & 240.0 & & & 4660 \\
\hline
\end{tabular}




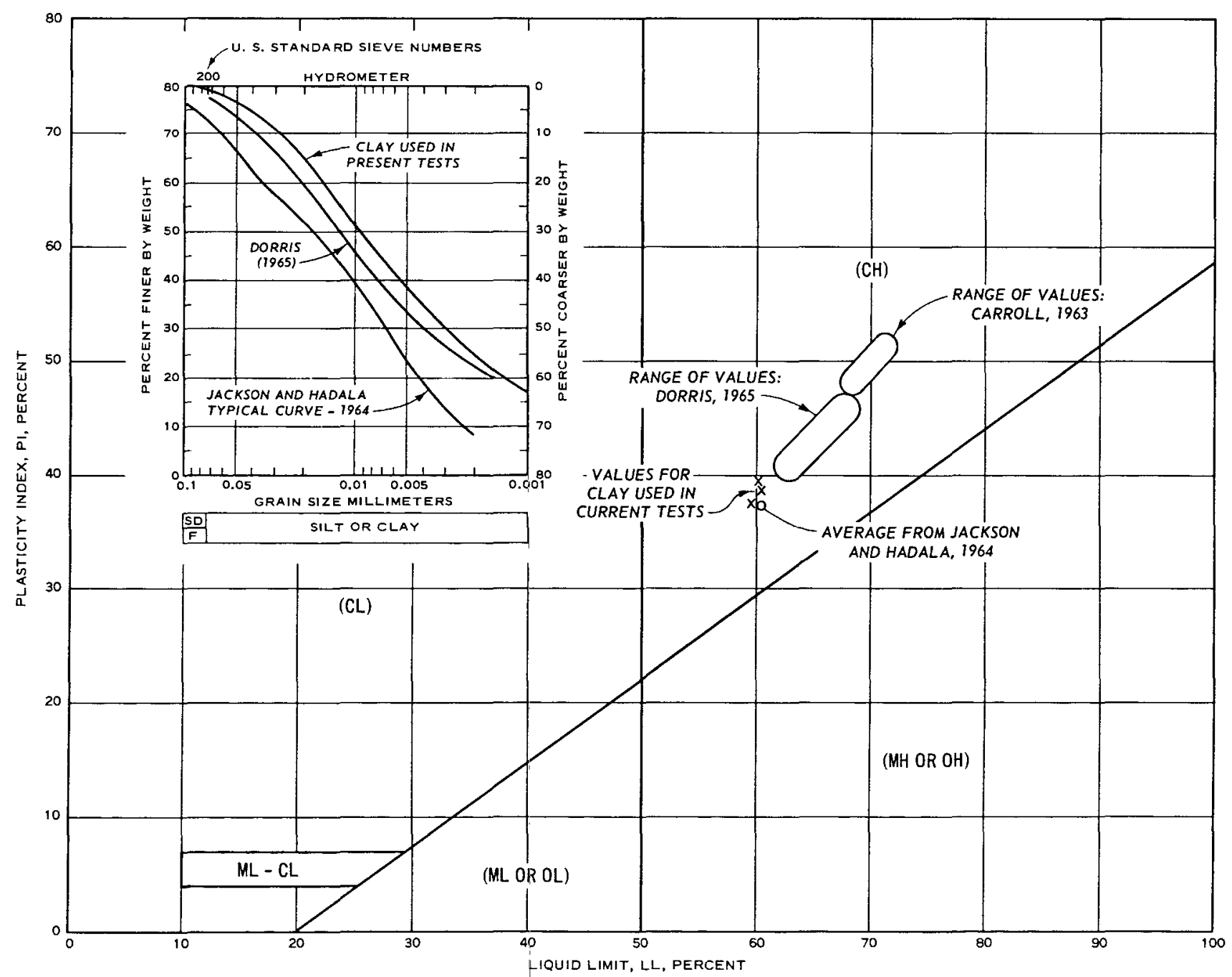

Fig. A-l. Gradation curve and Atterberg limits for buckshot clay 


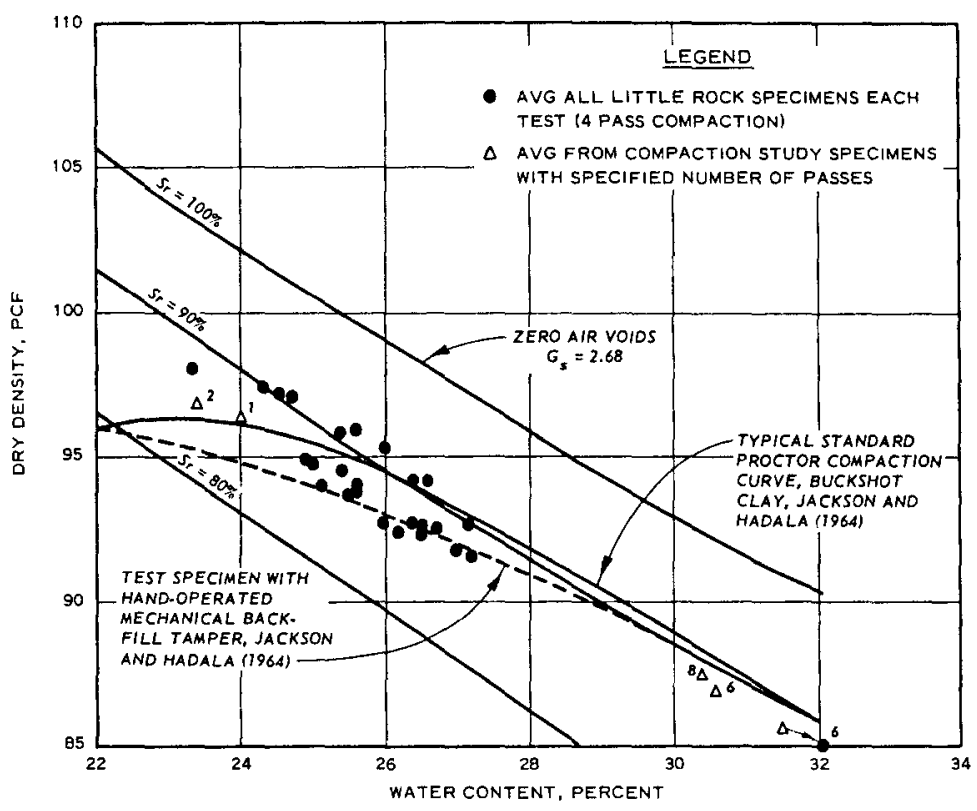

Fig. A-2. Water content-density relations for buckshot clay

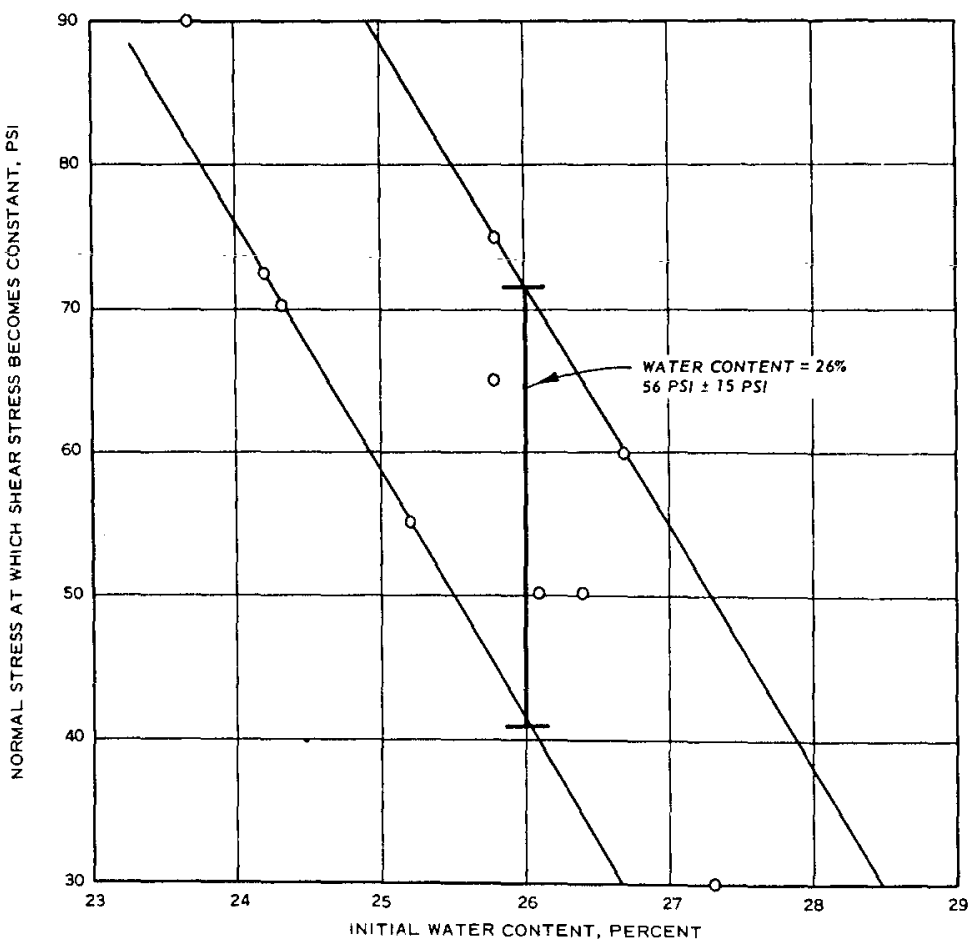

Fig. A-3. Approximate value of stress at which buckshot clay becomes saturated 


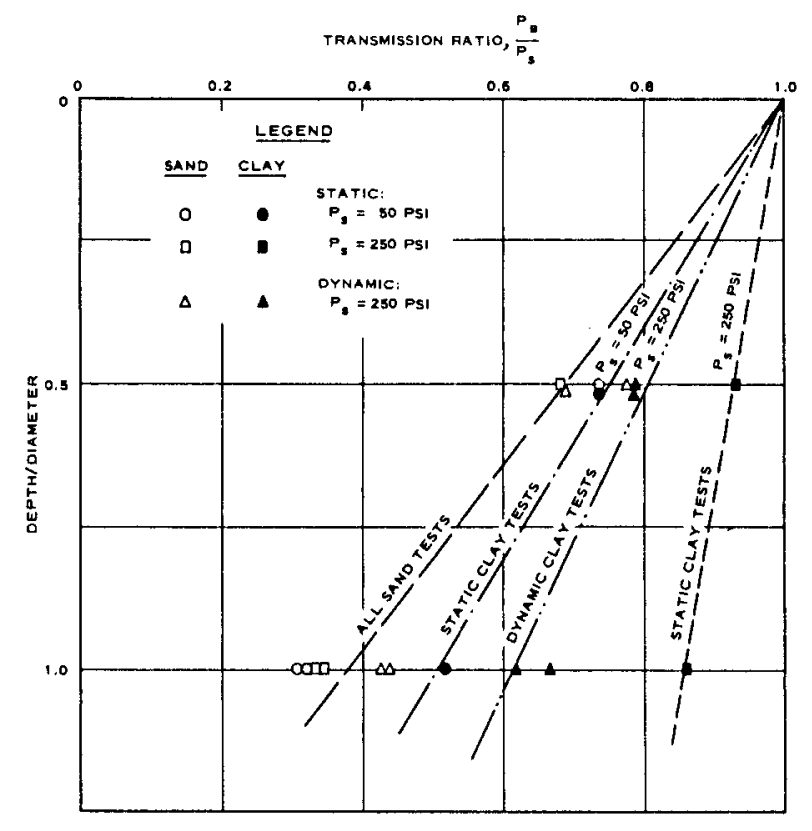

a. Unlined specimens

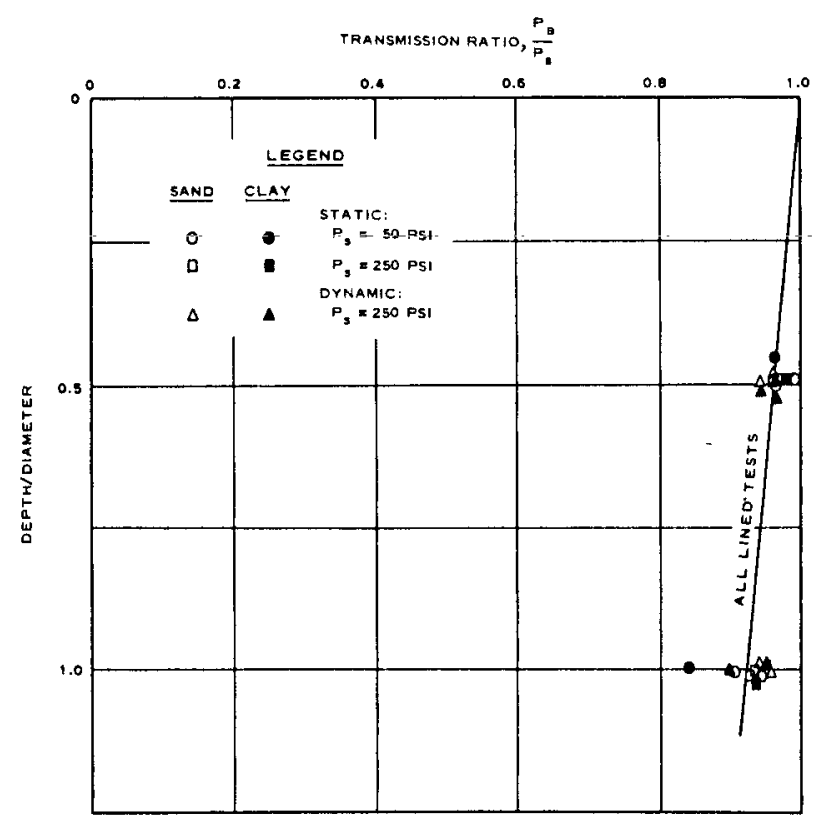

b. Iined specimens.

Fig. A-4. Results of wall-friction studies in the WES-SBLG by Hadala (19670) 


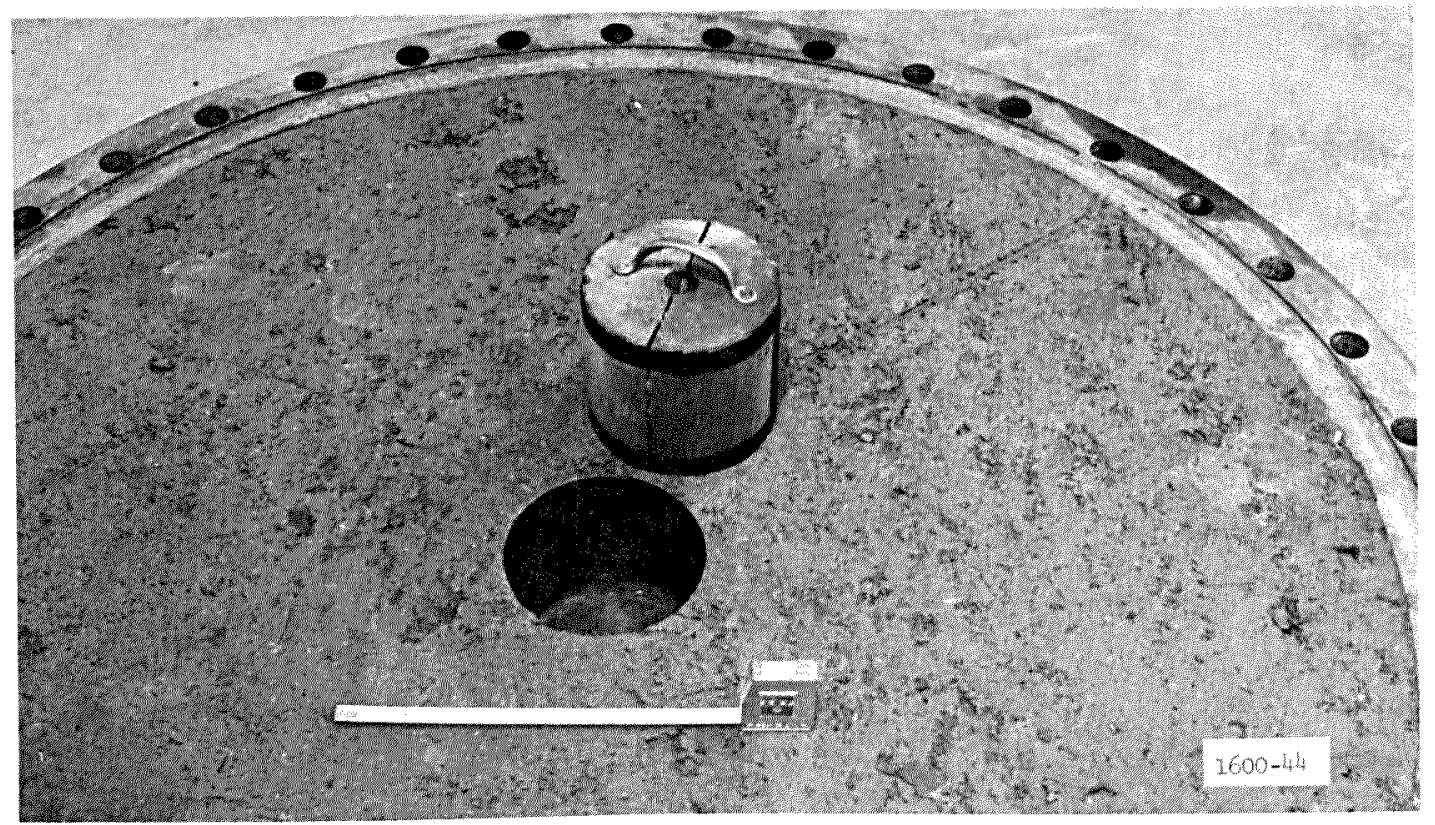

a. Form used for placement of test device

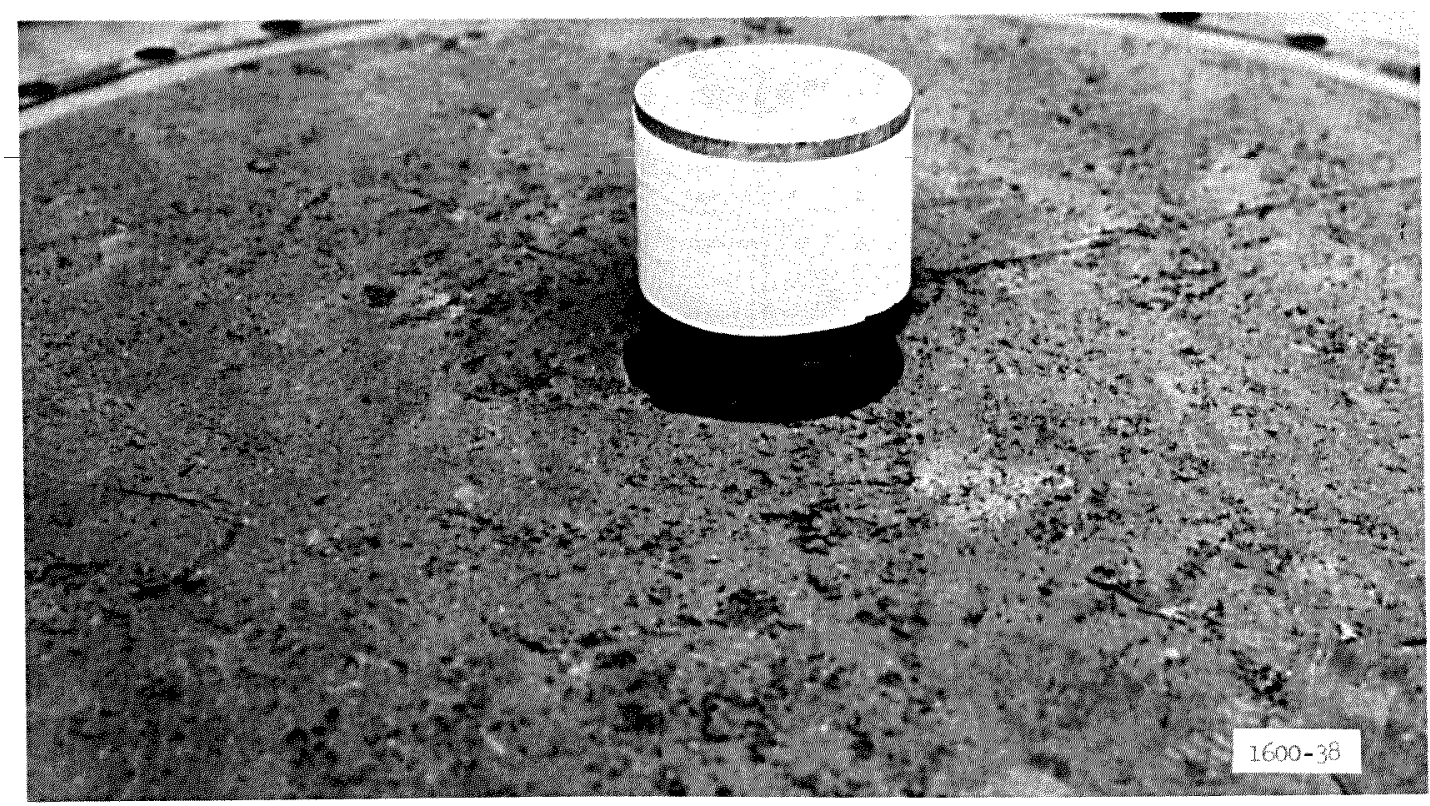

b. Test device with Teflon cover, ready for lowering in prepared hole

Fig. A-5. Placement of test device 


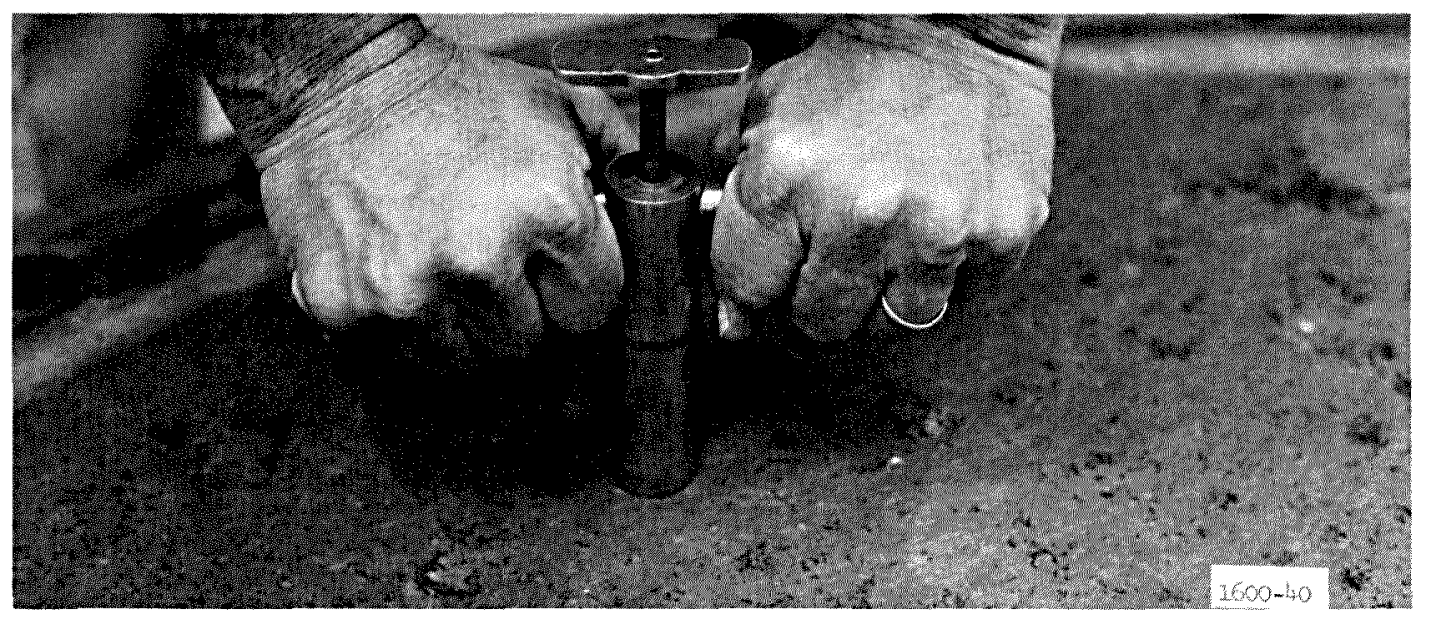

a. Sampler

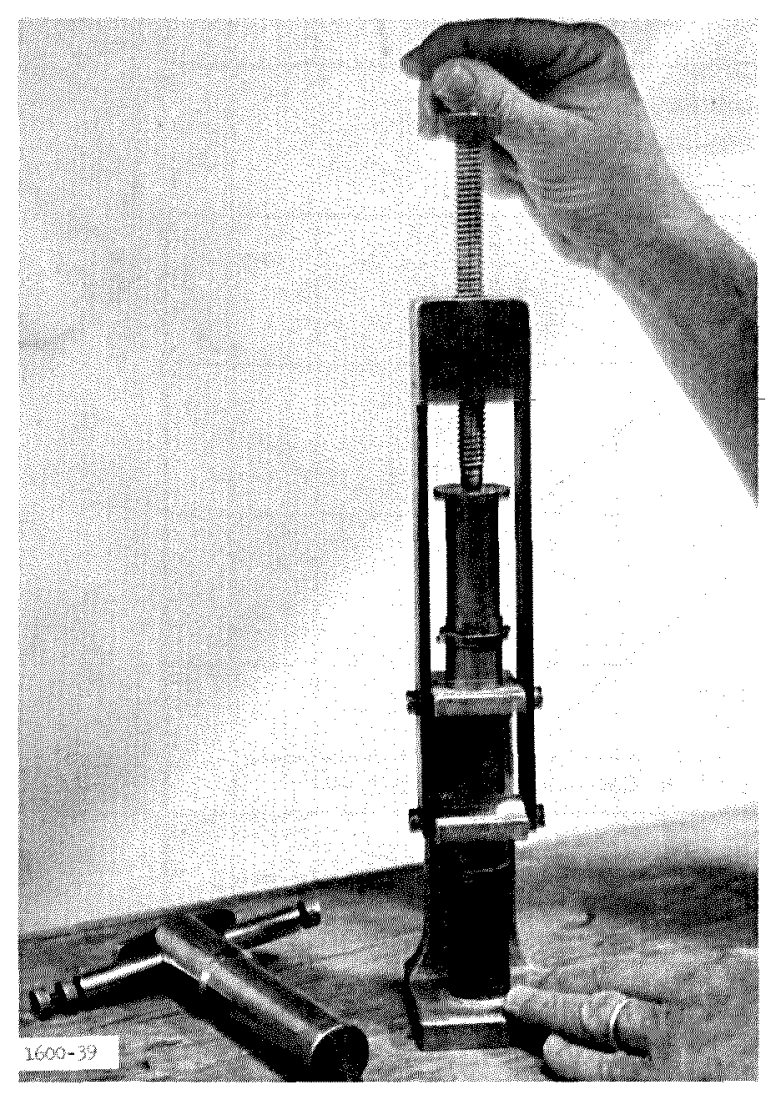

b. Unconfined compression device

Fig. A-6. Use of Hvorslev technique 


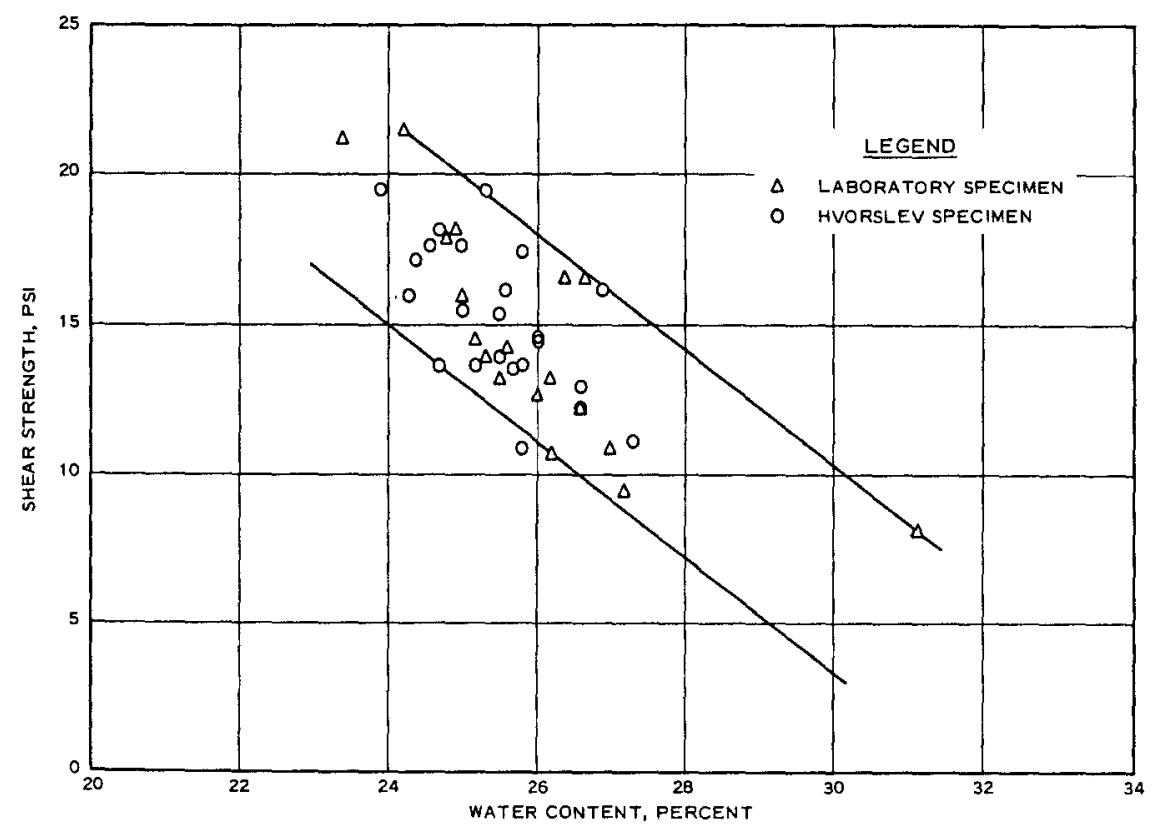

a. PRETEST UNCONFINED COMPRESSION

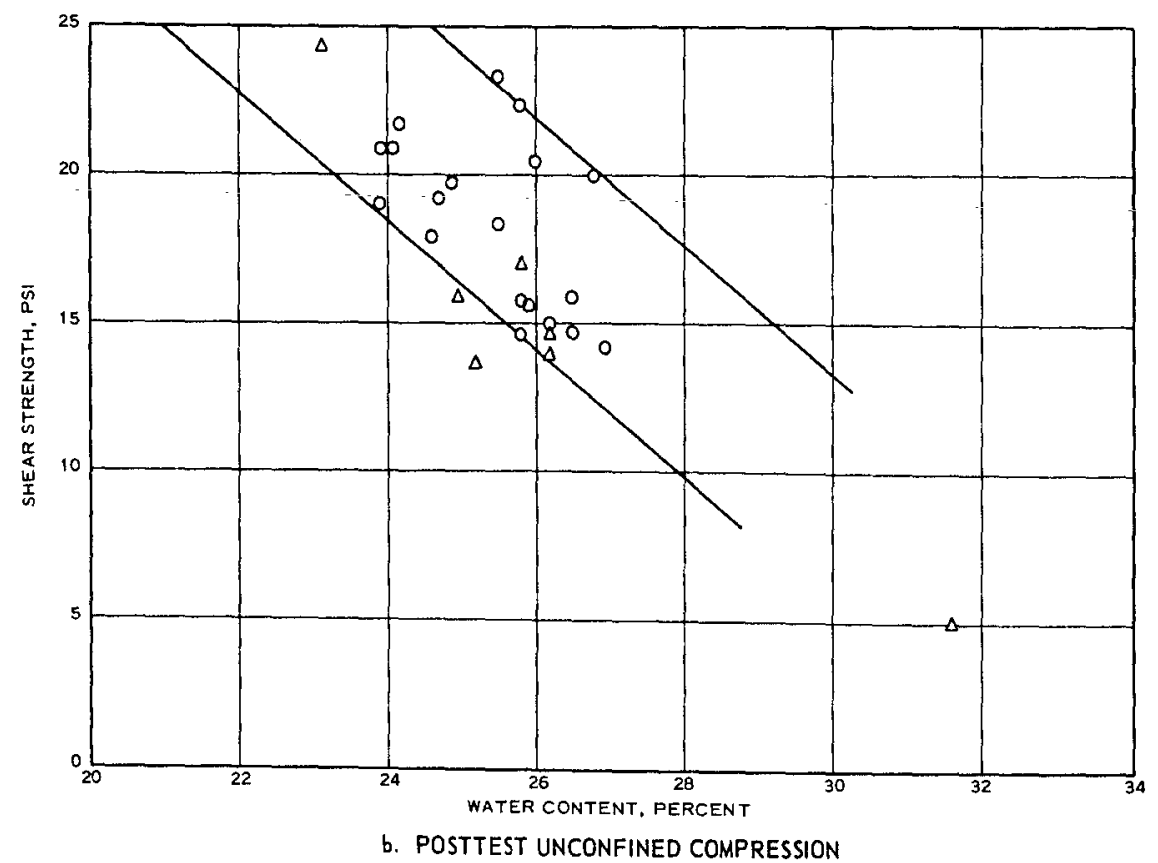

Fig. A-7. Comparison of unconfined compressive strength as determined by Horslev and laboratory techniques 


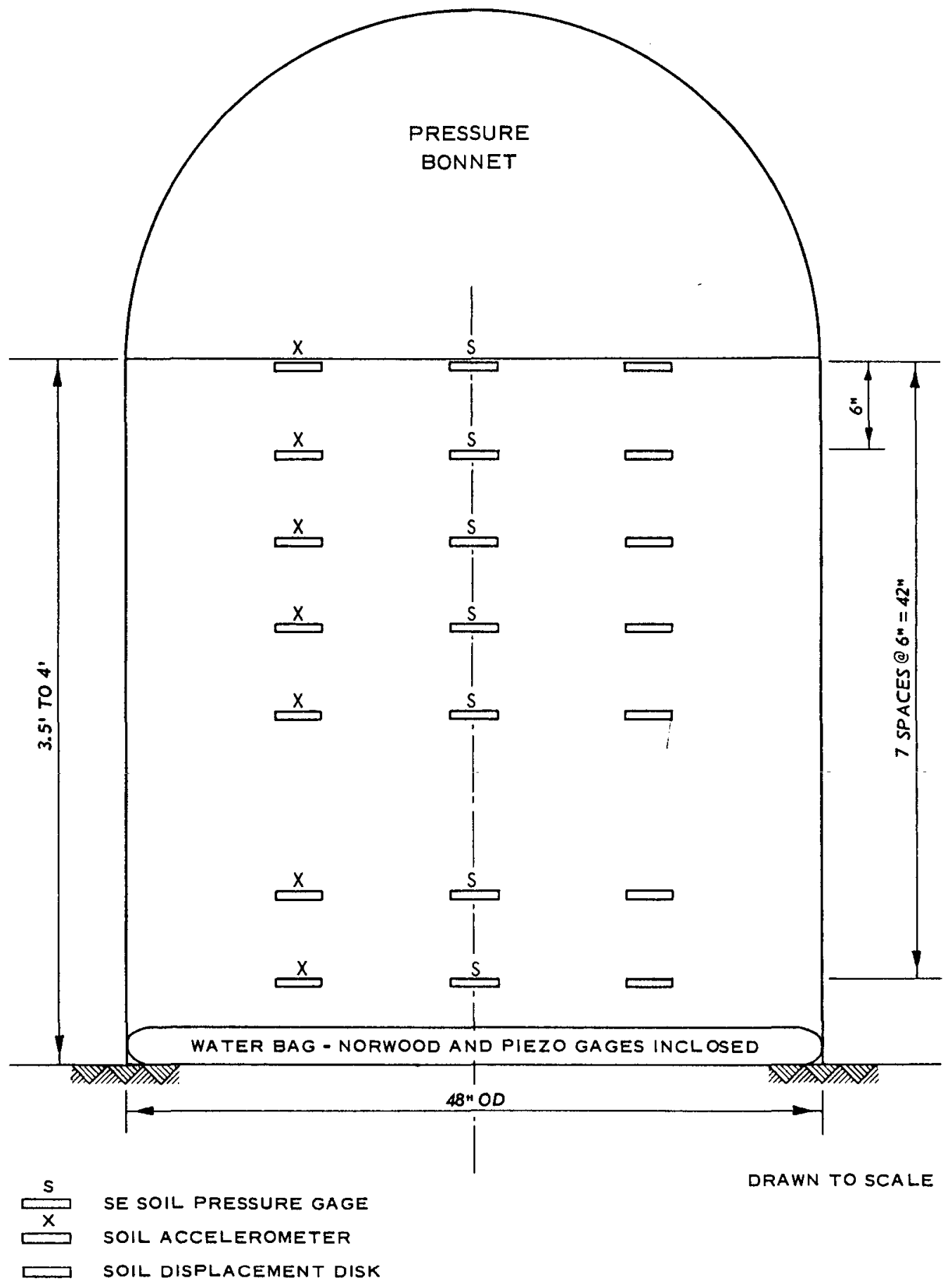

Fig. A-8. Test geometry for preliminary tests in SBLG 


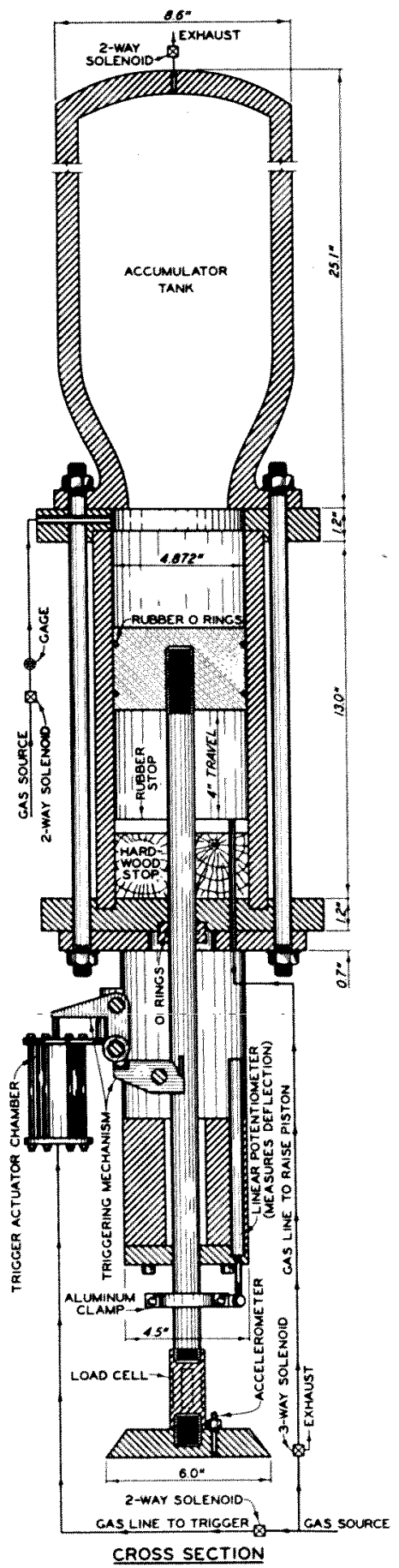

IMPACT AIR HAMMER APPARATUS

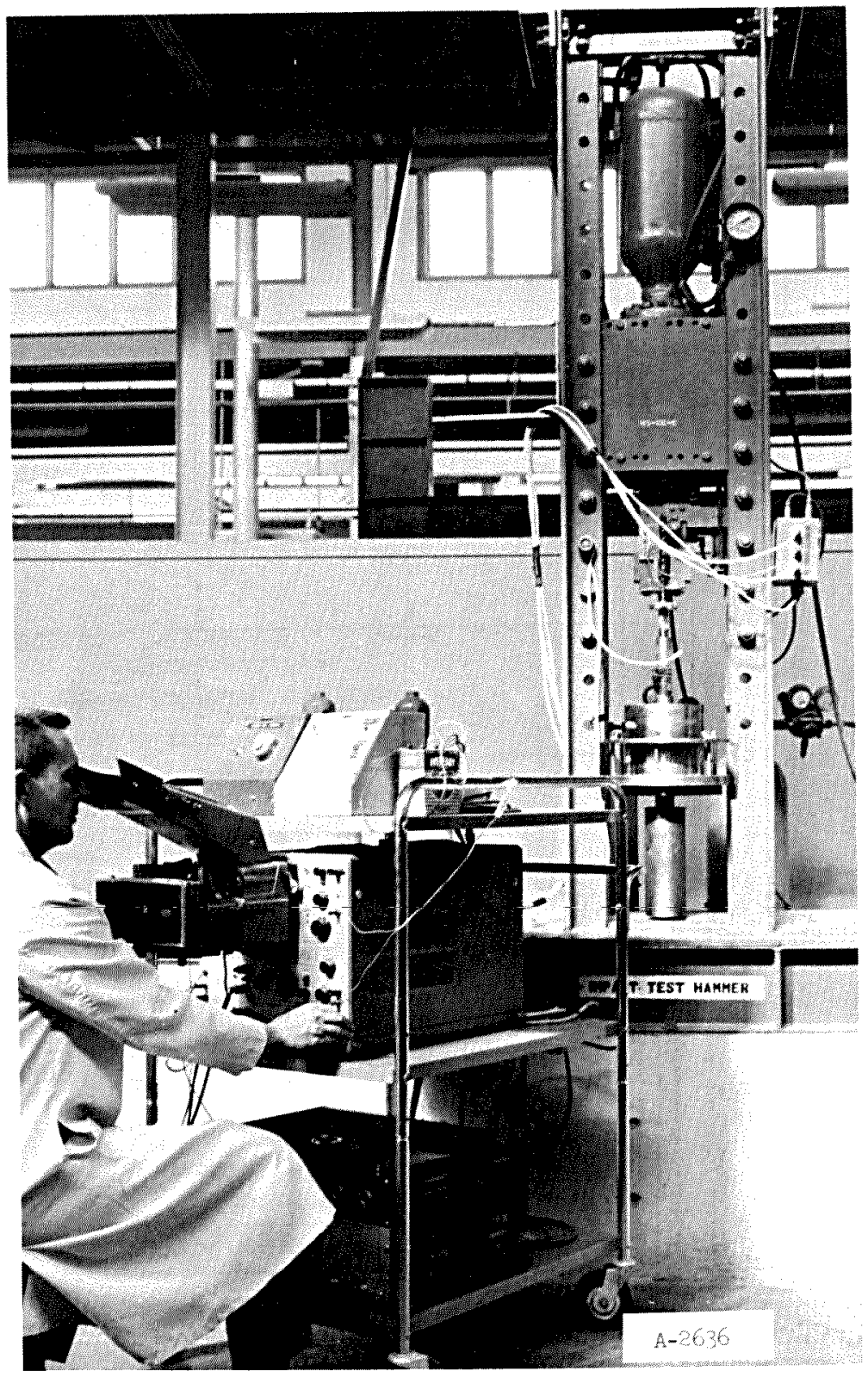

b. Impact hammer in test position with instrumentation

a. Cross section of impact hammer

Fig. A-9. Impact hammer used for confined compression tests 


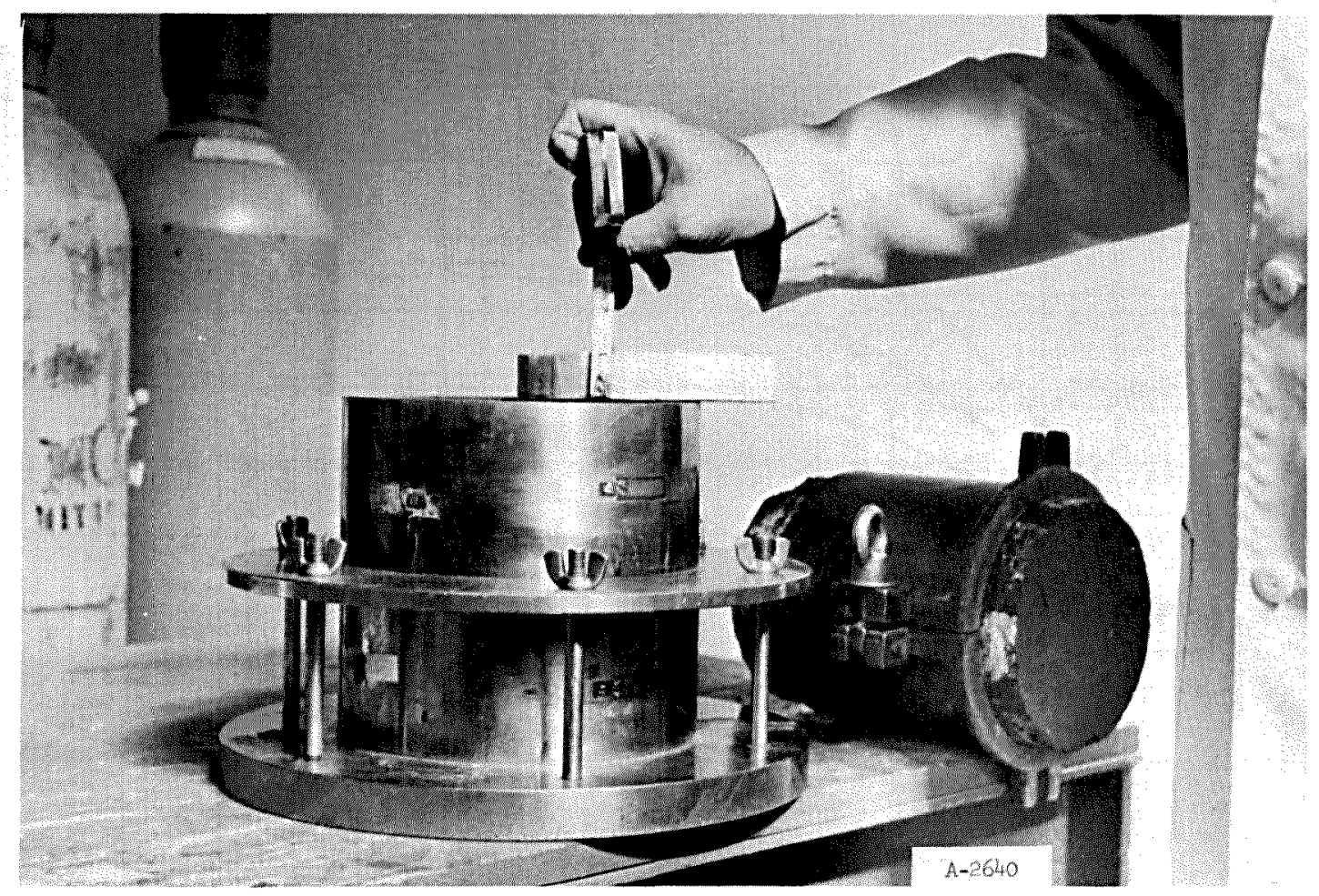

Fig. A-10. Mold, confining chamber, and soil disk used in confined compression tests
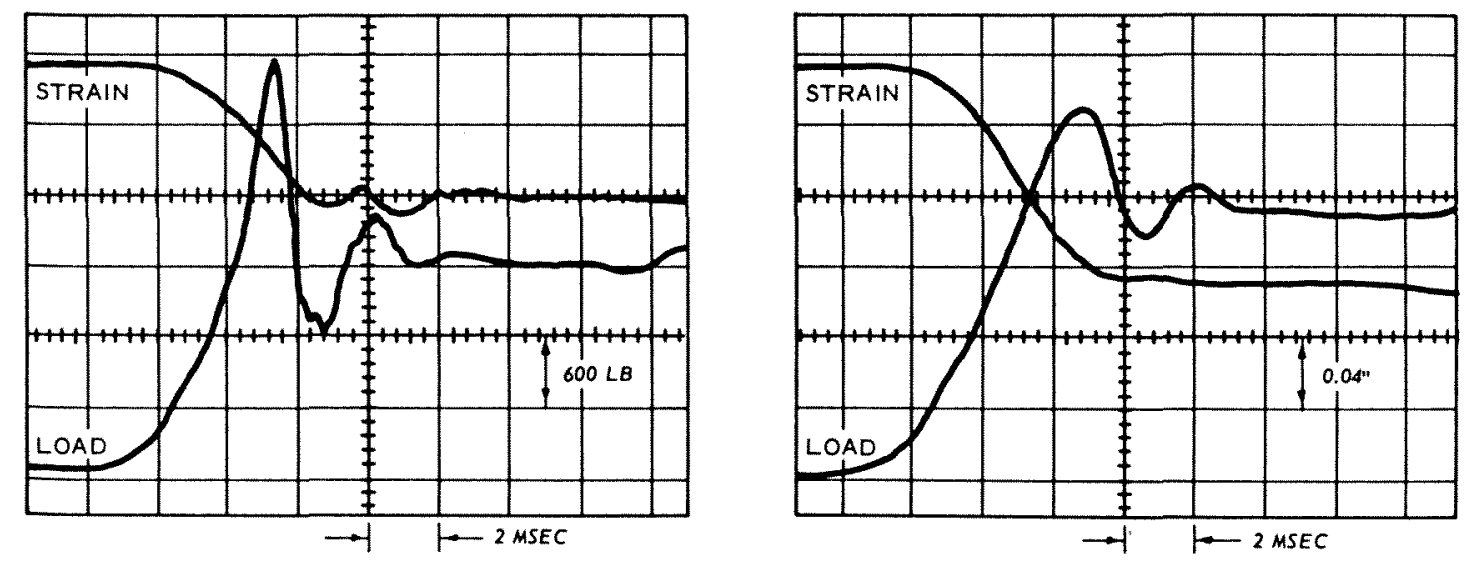

Fig. A-11. Typical oscilloscope records from confined compression tests 

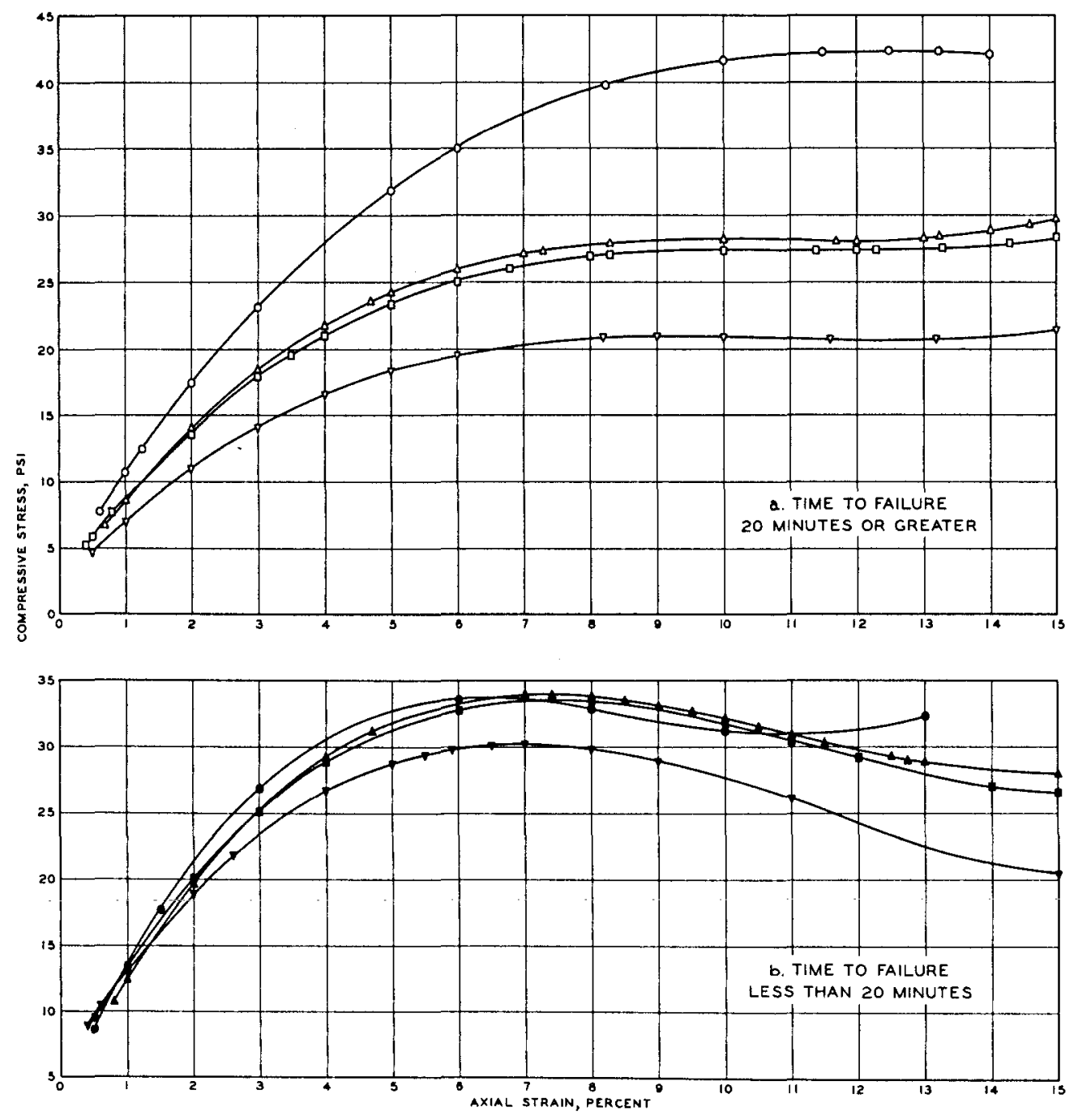

KEY-UNCONFINED COMPRESSION TESTS

\begin{tabular}{|c|c|c|c|c|c|c|c|c|c|}
\hline \multirow{2}{*}{ SYMBOL } & \multirow{2}{*}{$\begin{array}{l}\text { WATER CONTENT } \\
\text { RANGE } \\
\end{array}$} & \multirow{2}{*}{$\begin{array}{l}\text { NUMBER } \\
\text { OF TESTS } \\
\end{array}$} & \multirow{2}{*}{$\begin{array}{c}1 \\
1 \\
\text { RANGE } \\
\end{array}$} & \multirow{2}{*}{\multicolumn{2}{|c|}{$\begin{array}{l}\text { DRY DENSITY } \\
\text { RANGE }\end{array}$}} & \multicolumn{2}{|c|}{ E AT maXIMUM $\sigma$} & \multirow{2}{*}{\multicolumn{2}{|c|}{$\operatorname{MAXIMUM} \sigma$}} \\
\hline & & & & & & RANGE & MEAN & & MEAN \\
\hline & PERCENT & & MINUTES & \multicolumn{2}{|c|}{ LB PER F $F^{3}$} & \multicolumn{2}{|c|}{ PERCENT } & \multicolumn{2}{|c|}{ LB PER IN ${ }^{2}$} \\
\hline 0 & 24.1.24.4 & 5 & 22.78 & 96.5.98.7 & 97.4 & $8.3 \cdot 14.0$ & 11.0 & 33.5 .46 .5 & 40.8 \\
\hline$\Delta$ & 25.0 .25 .3 & 7 & $20-43$ & 94.7 .98 .0 & 96.5 & 9.5 .14 .5 & 11.8 & 25.8 .37 .1 & 30.6 \\
\hline D & 25.5 .26 .4 & 9 & 21.48 & 93.9 .97 .8 & 95.1 & 8.2 .15 .0 & 11.1 & $22.4 \cdot 36.7$ & 27.7 \\
\hline$\nabla$ & $26.5-27.3$ & 5 & 20.40 & 92.6 .93 .0 & 92.8 & 9.8 .13 .0 & 11.1 & 17.7 .28 .5 & 21.8 \\
\hline$\bullet$ & 24.4 & 1 & 14 & 96.7 & 96.7 & 9.0 & 9.0 & 33.1 & 33.1 \\
\hline$\Delta$ & 24.5 .25 .4 & 10 & 7.16 & 94.7.97.8 & 95.9 & $7.5 \cdot 10.5$ & 8.8 & 29.2 .40 .0 & 33.1 \\
\hline च & 25.5 .26 .4 & 18 & 7.19 & 91.2 .96 .4 & 94.5 & $3.0 \cdot 15.5$ & 8.6 & $23.4-52.1$ & 32.6 \\
\hline $\boldsymbol{V}$ & $26.6 \cdot 27.3$ & 9 & 6.18 & 88.5 .84 .1 & 92.3 & 3.3.-18.0 & 7.2 & 18.6.33.9 & 28.4 \\
\hline
\end{tabular}

Fig. A-12. Stress-strain relations for buckshot clay as determined by unconfined compression test 

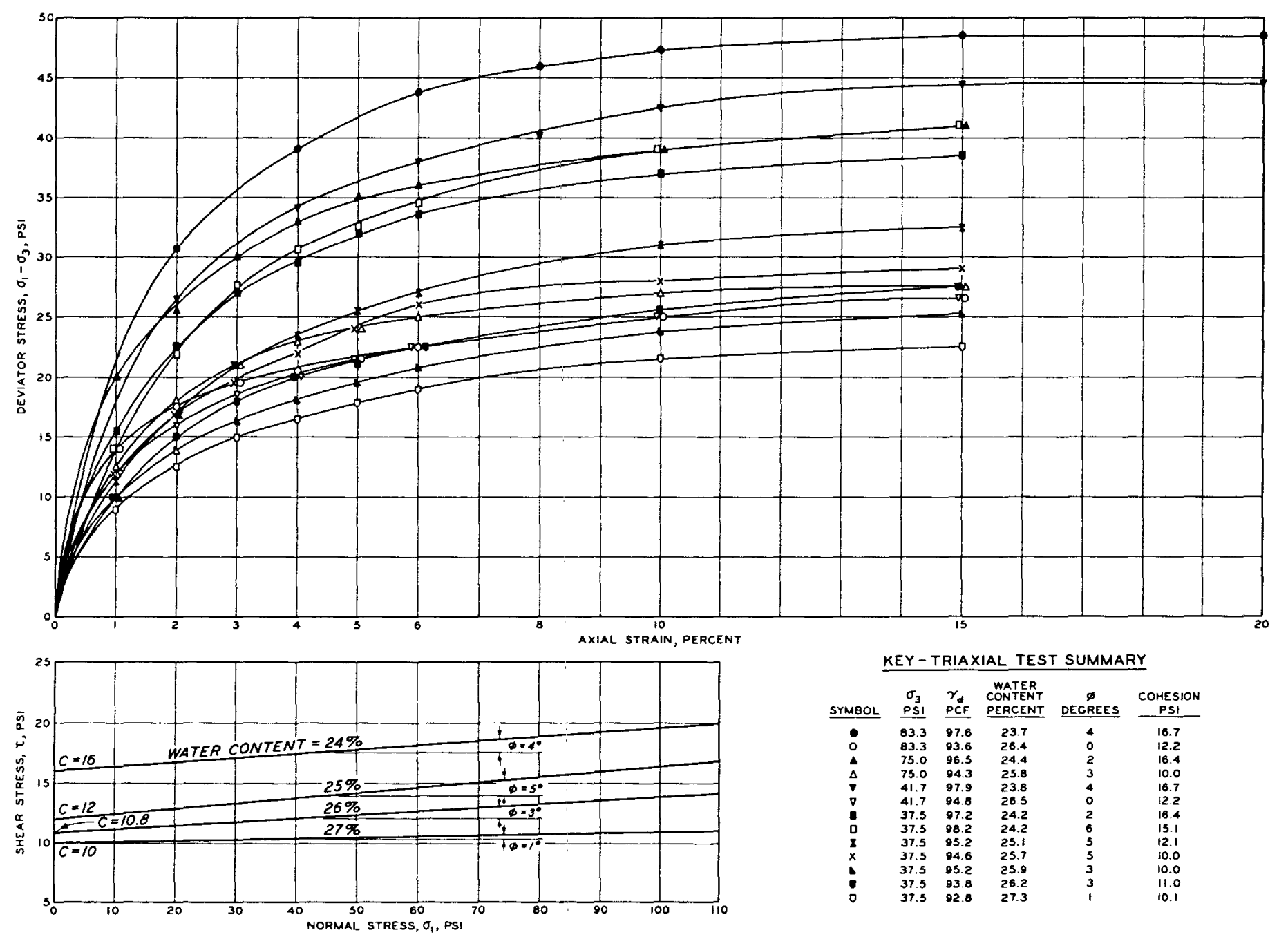

Fig. A-13. Stress-strain relations for buckshot clay as determined by triaxial compression tests 


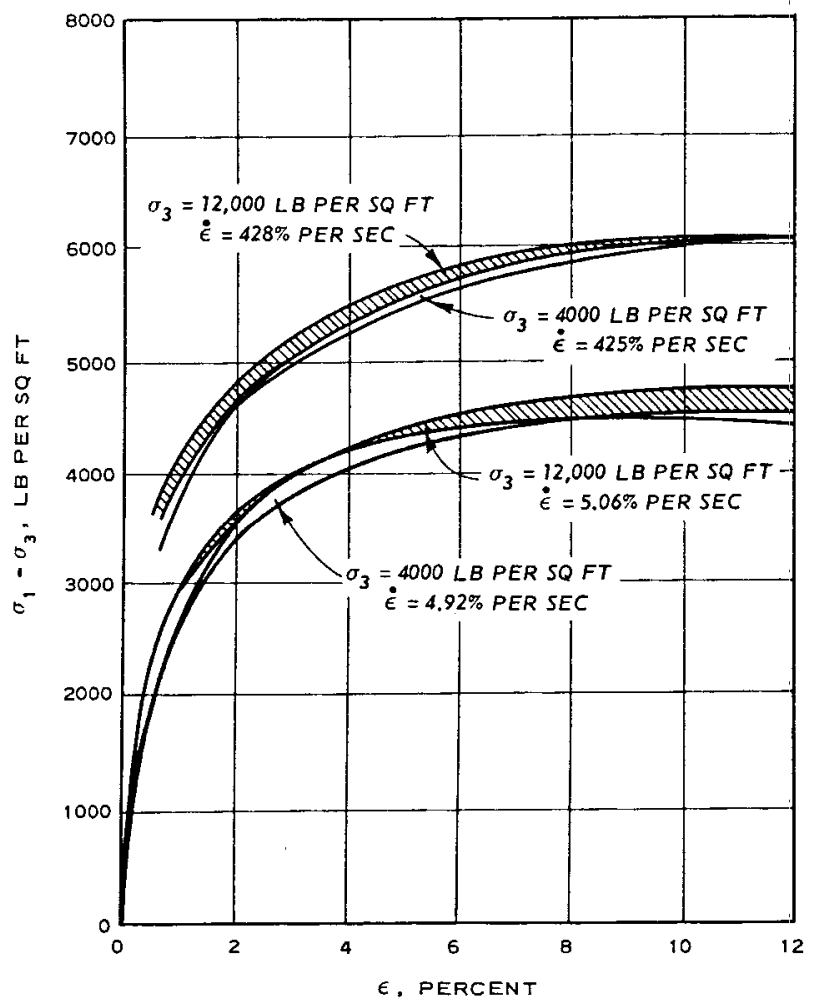

A. BUCKSHOT CLAY STRESS-STRAIN CURVES $W C=27.1 \pm 0.3 \%$

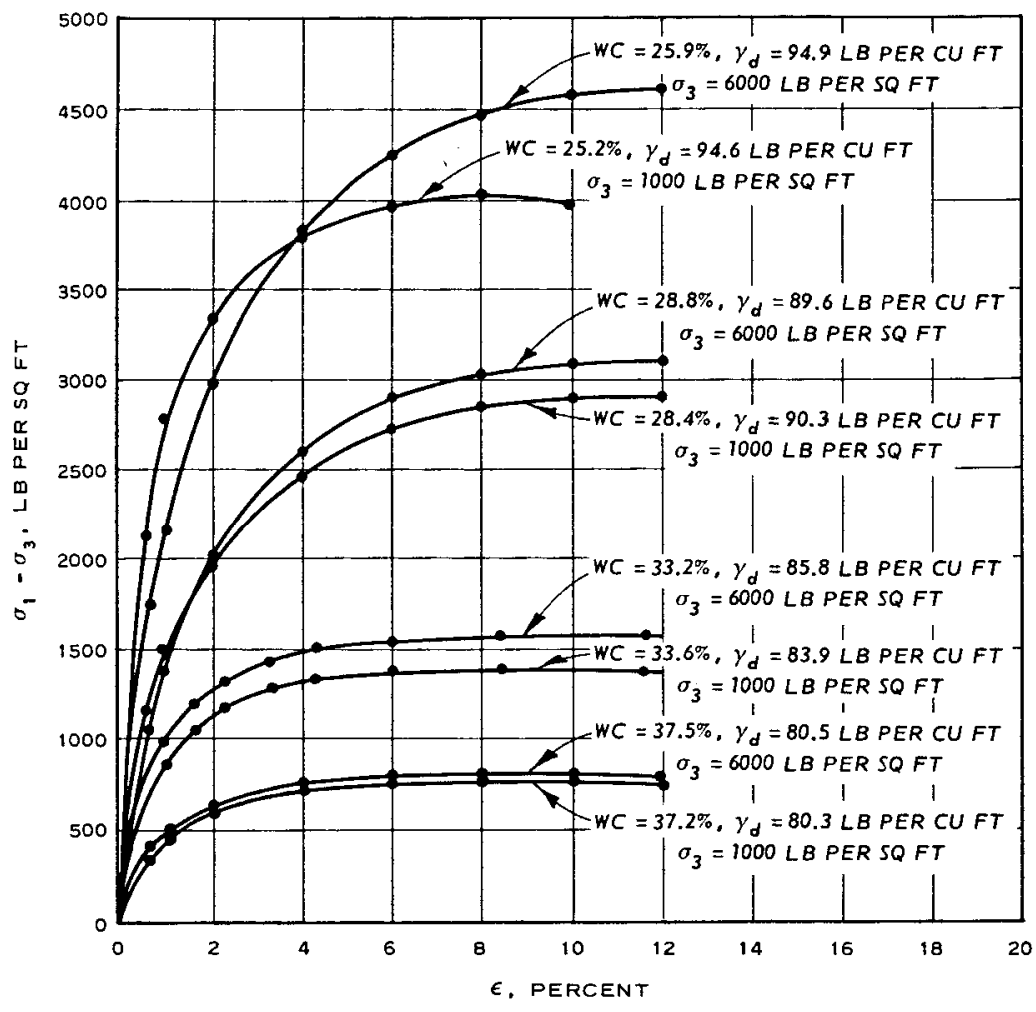

B. BUCKSHOT CLAY

UU STRESS-STRAIN CURVES

A-14. Effects of confining pressure, water content, and strain rate on the shear strength of buckshot clay (after Carroll) 


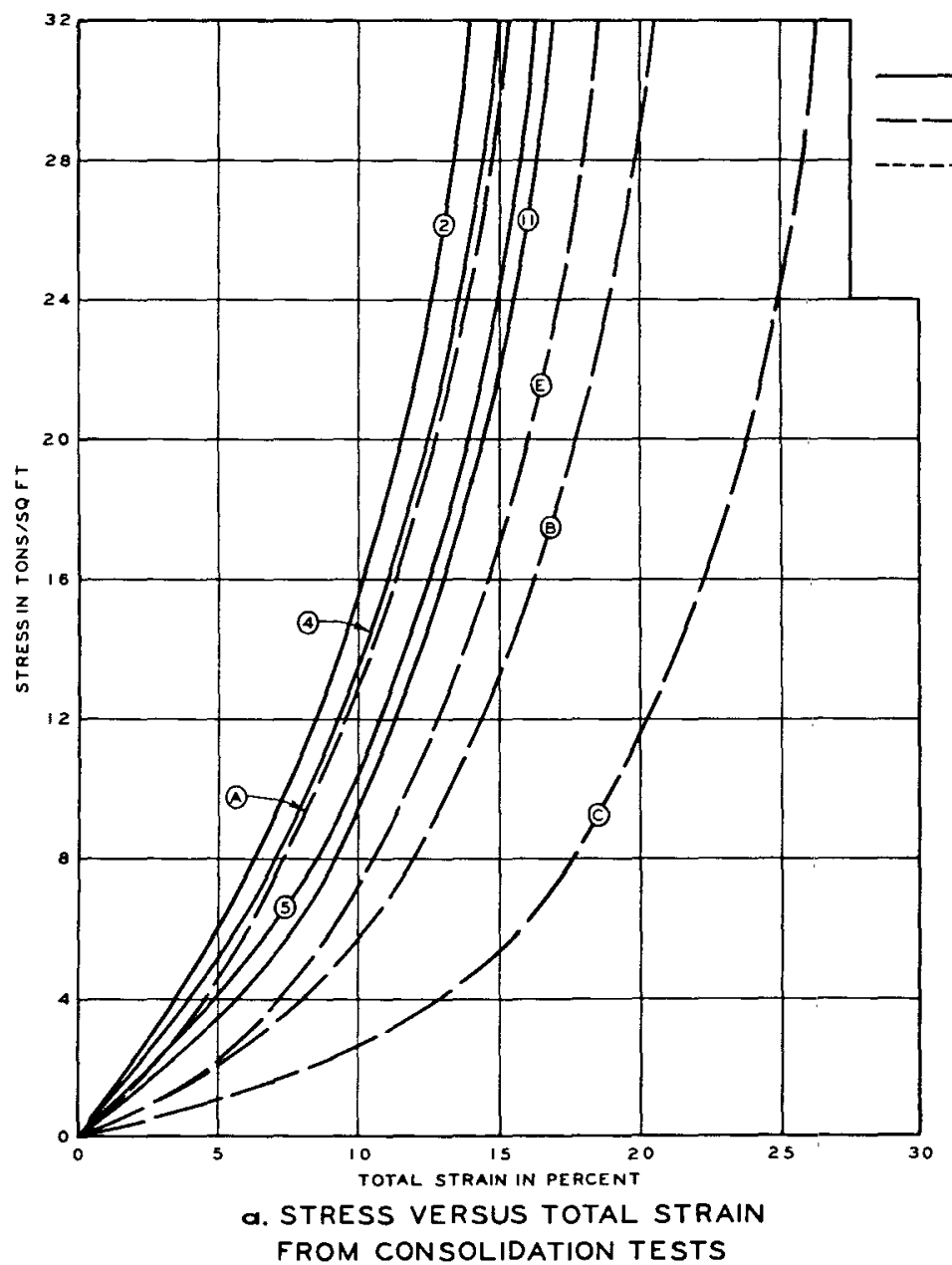

\section{LEGEND}

TESTS ARE CORRECTED FOR
COMPRESSIBILITY OF APPARATUS COMPRESSIBILITY OF APPARATUS CURVES ARE ERRATIC ON THESE
TESTS IN THE ZONE GELOW T TOS SOFT ZONE QLLOW GONSTSFT

FROM CONSOLIDATION TESTS

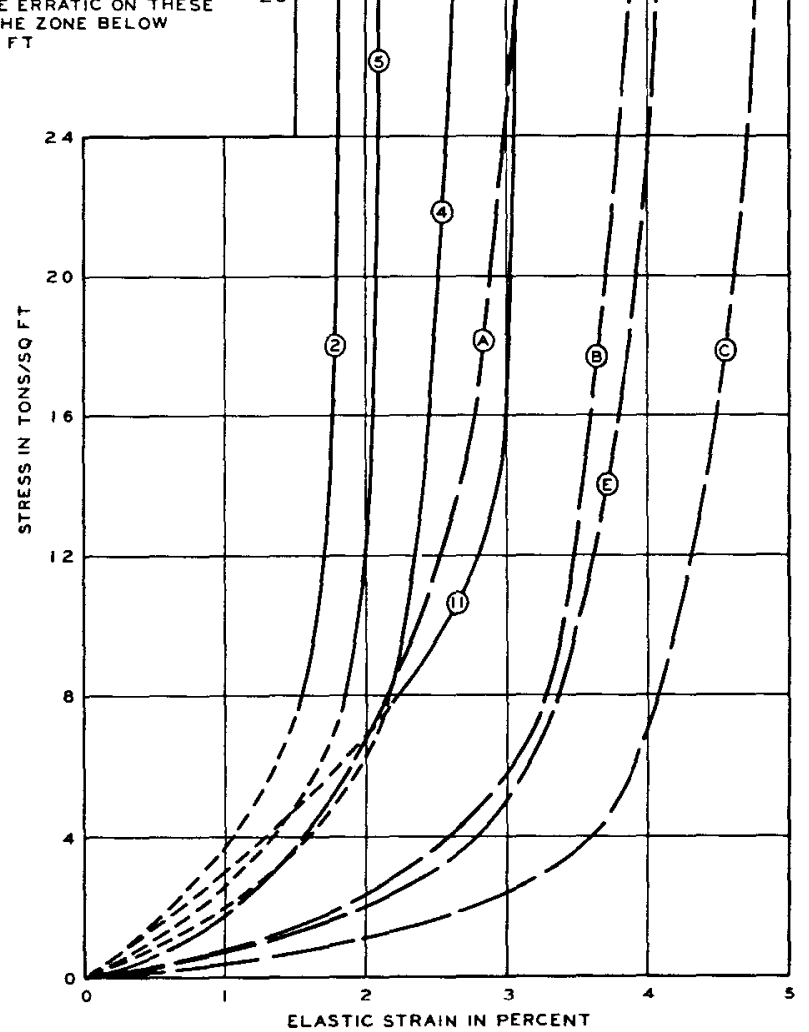

b. STRESS VERSUS ELASTIC STRAIN FROM CONSOLIDATION TESTS

Fig. A-15. Stress-strain curves constructed from consolidation test results 

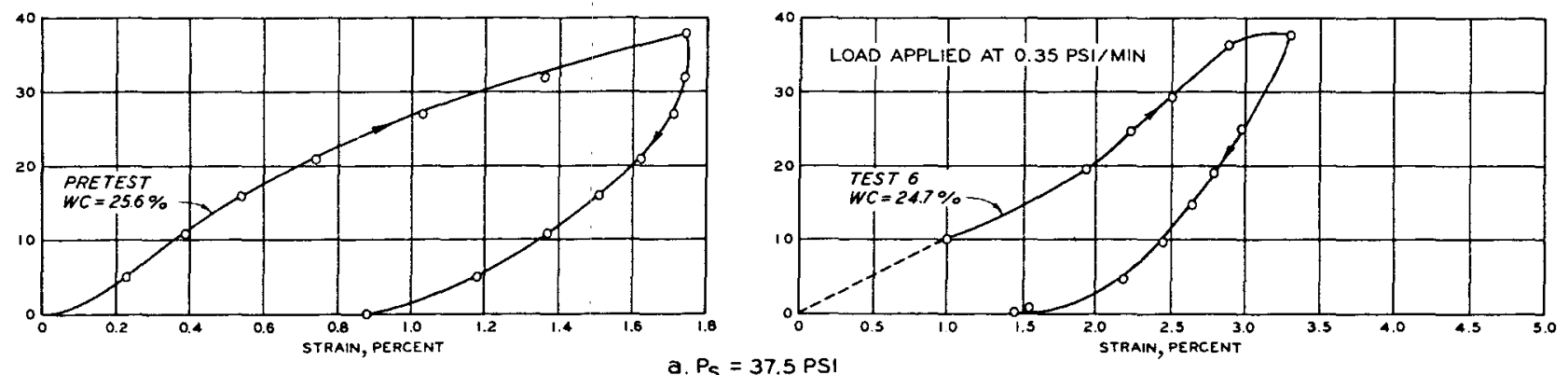

a. $P_{S}=37.5 P S$

요
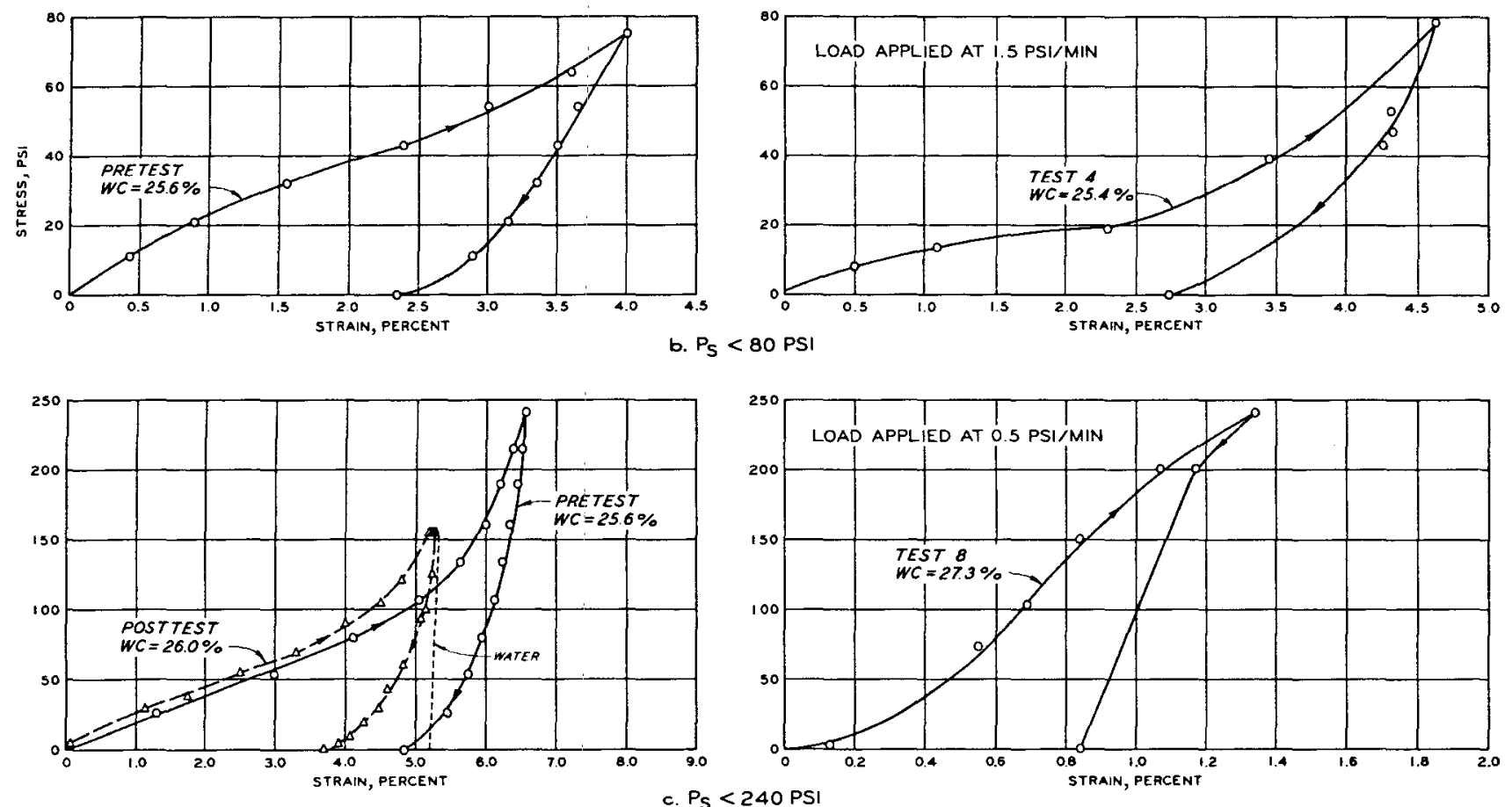

Fig. A-16. Stress-strain curves determined by static one-dimensional tests 


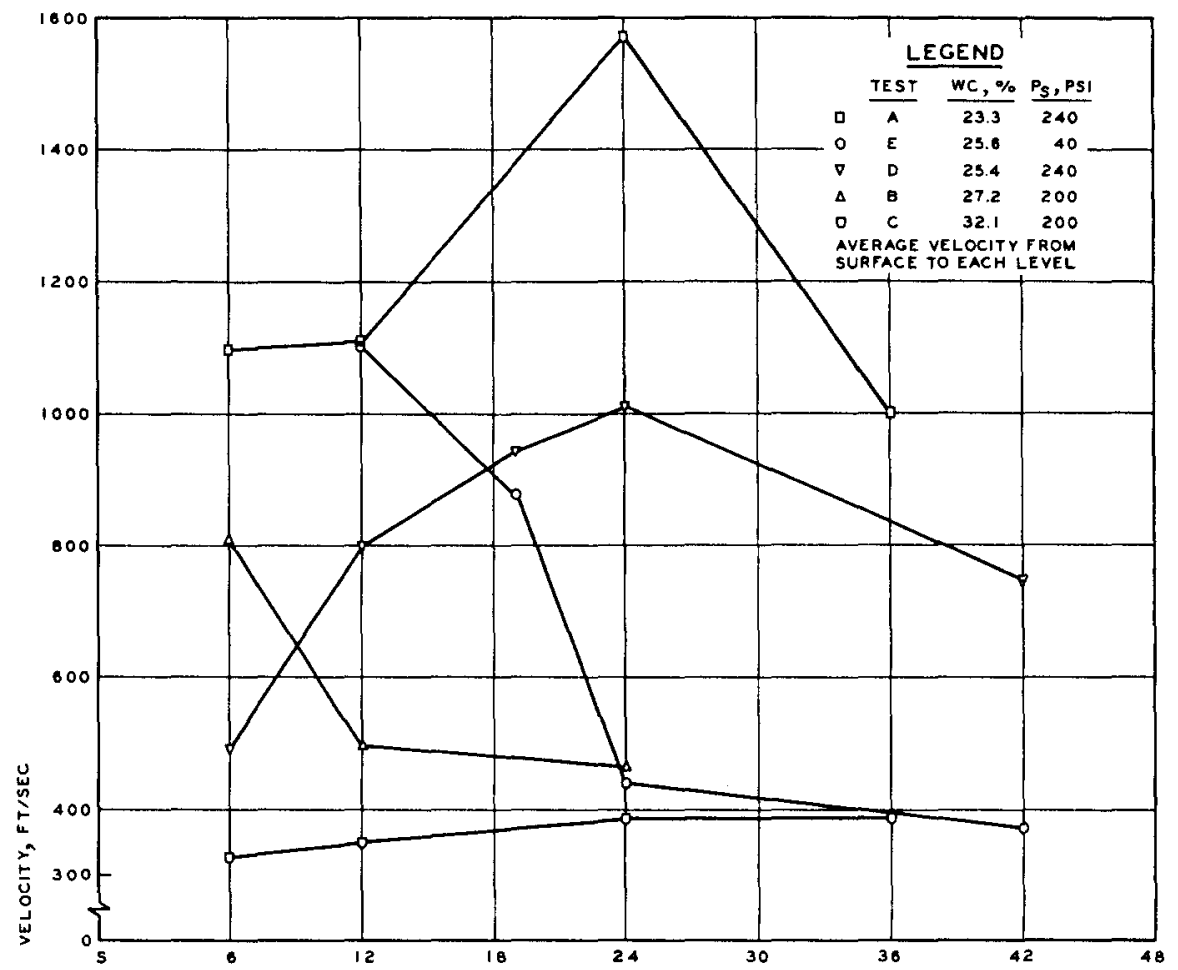

a. PROPAGATION OF INITIAL DISTURBANCE

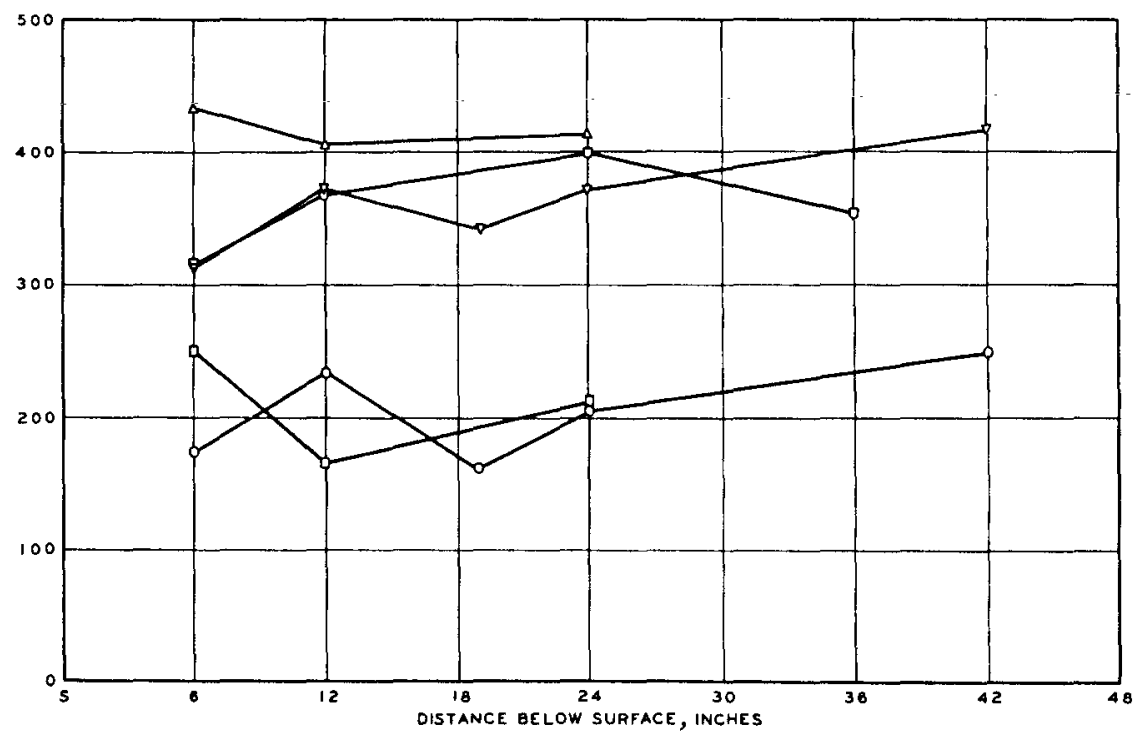

b. PROPAGATION OF FIRST STRESS PEAK

Fig. A-17. Variation of velocities with pressure and soil water content, preliminary SBIG tests 


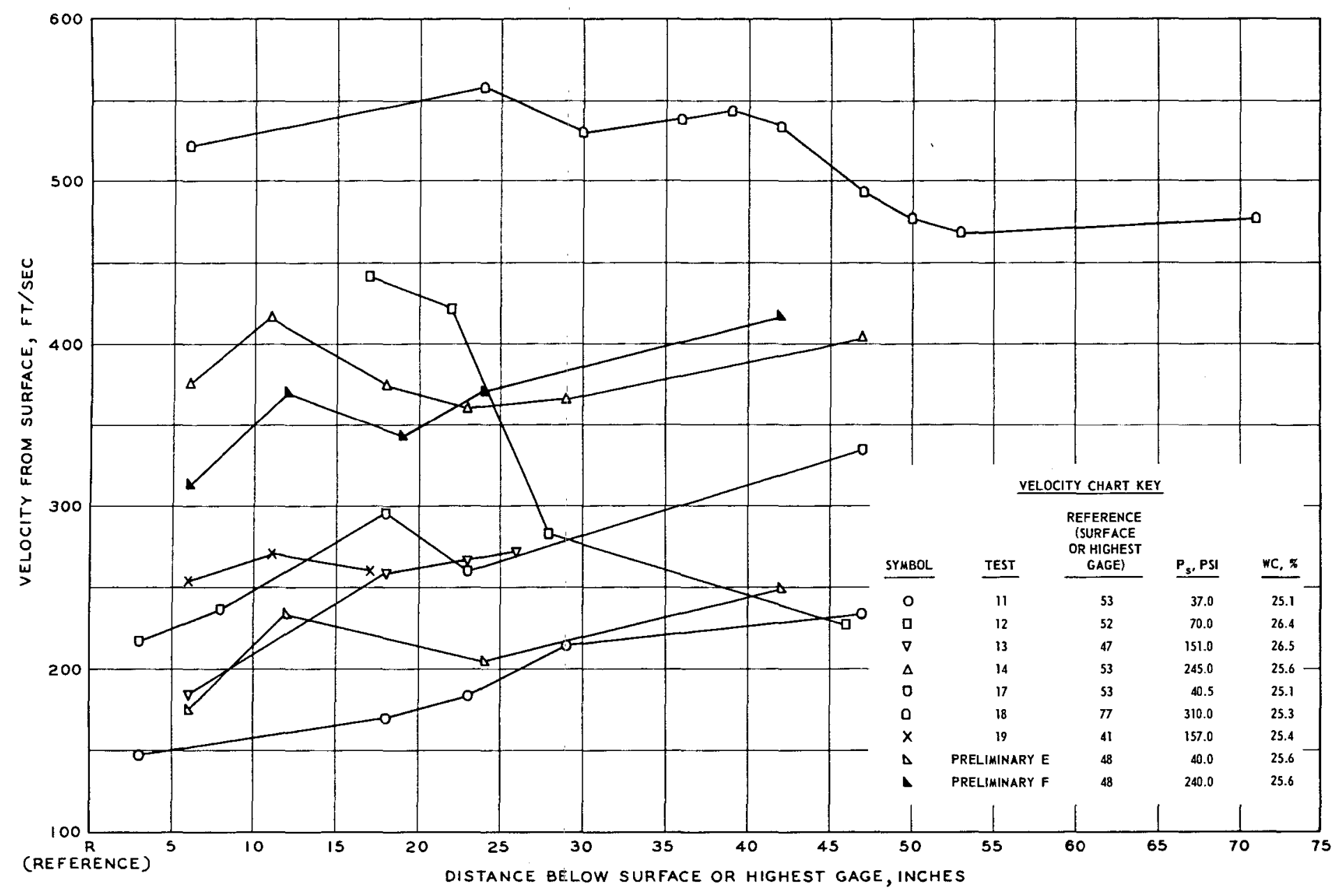

Fig. A-18. Variation of velocity of propagation of first stress peak with surface pressure 

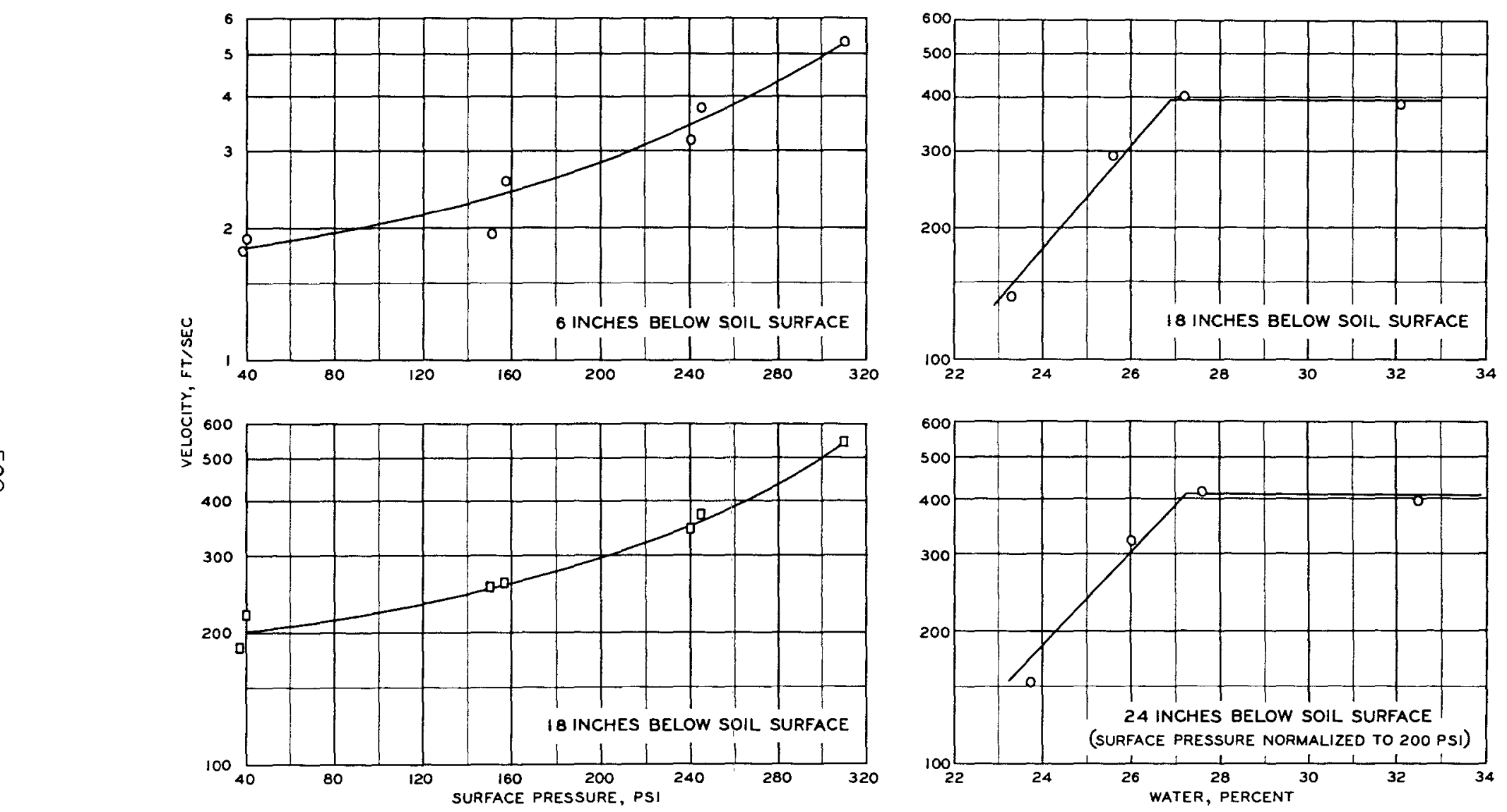

a. STRESS WAVE VELOCITY VERSUS SURFACE PRESSURE

b. PEAK STRESS WAVE VELOCITY VERSUS SOIL WATER CONTENT

Fig. A-19. Variation of peak stress velocity with initiating pressure and soil water content 


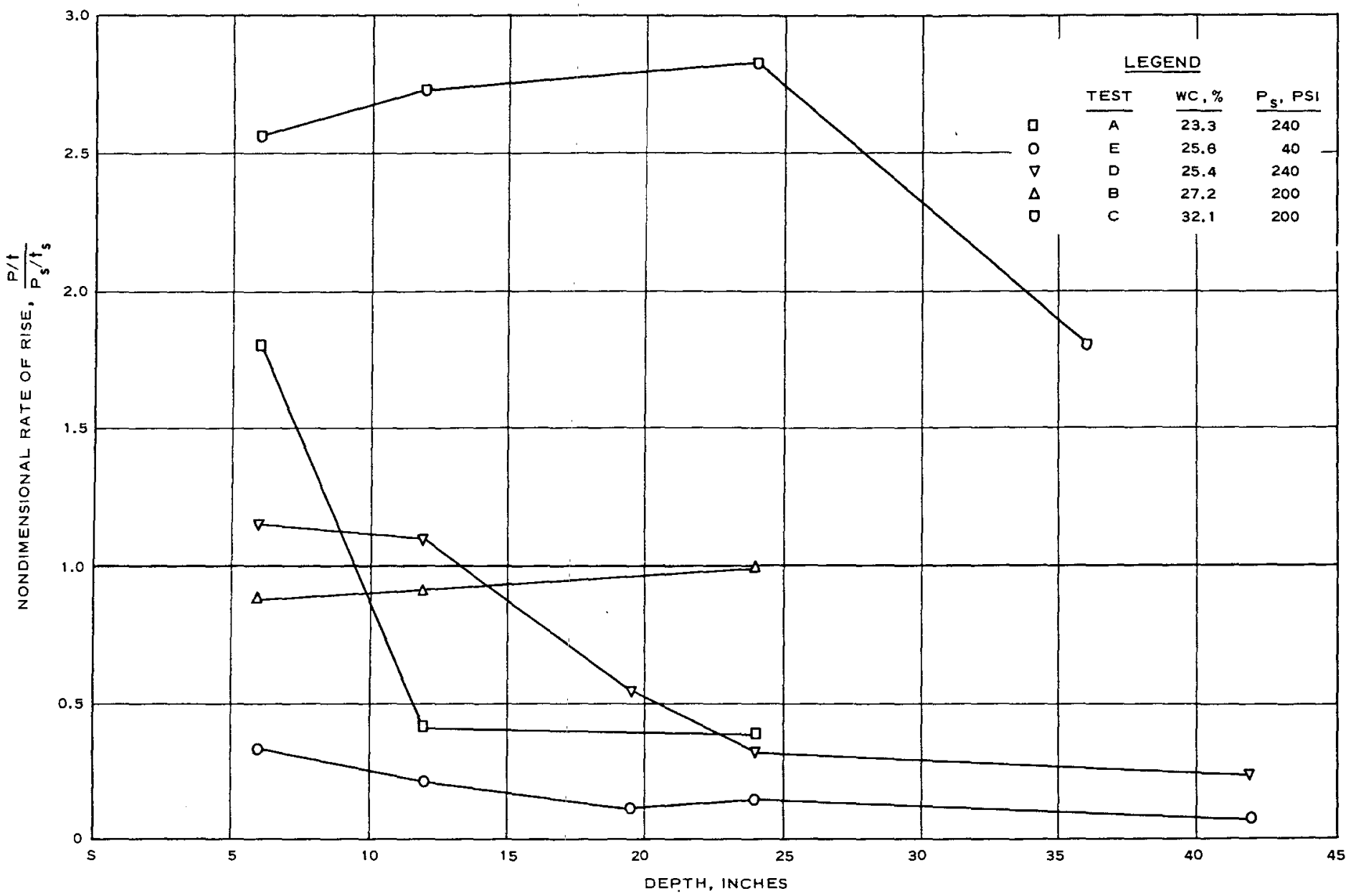

Fig. A-20. Nondimensional rate of rise variation with depth (average of north and south Norwood rates of rise set equal to 1.00) 


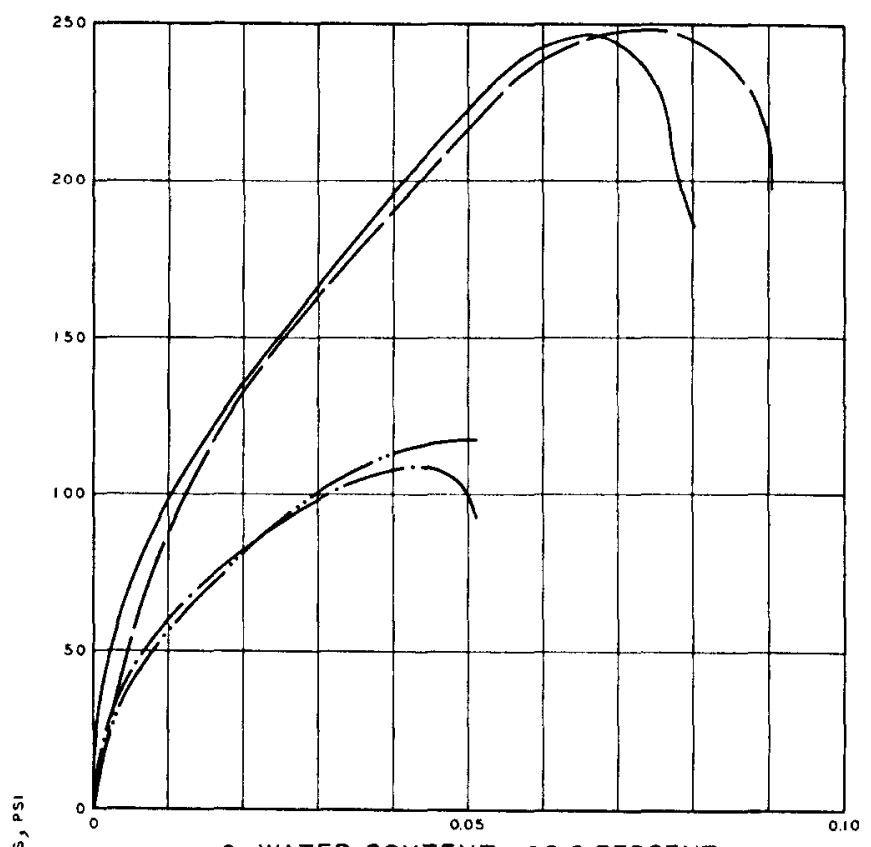

a. WATER CONTENT $=23.3$ PERCENT

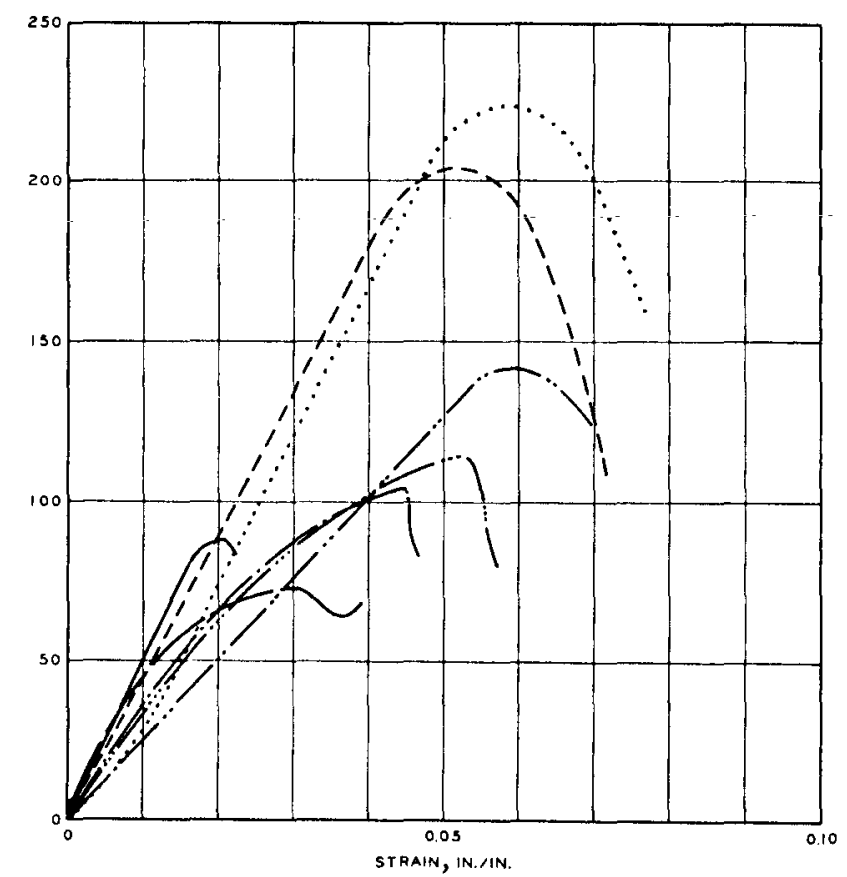

b. WATER CONTENT $=25.6$ PERCENT

Fig. A-2l. Stress-strain curves, buckshot clay, impact loader; water contents, 23.3 and 25.6 percent 


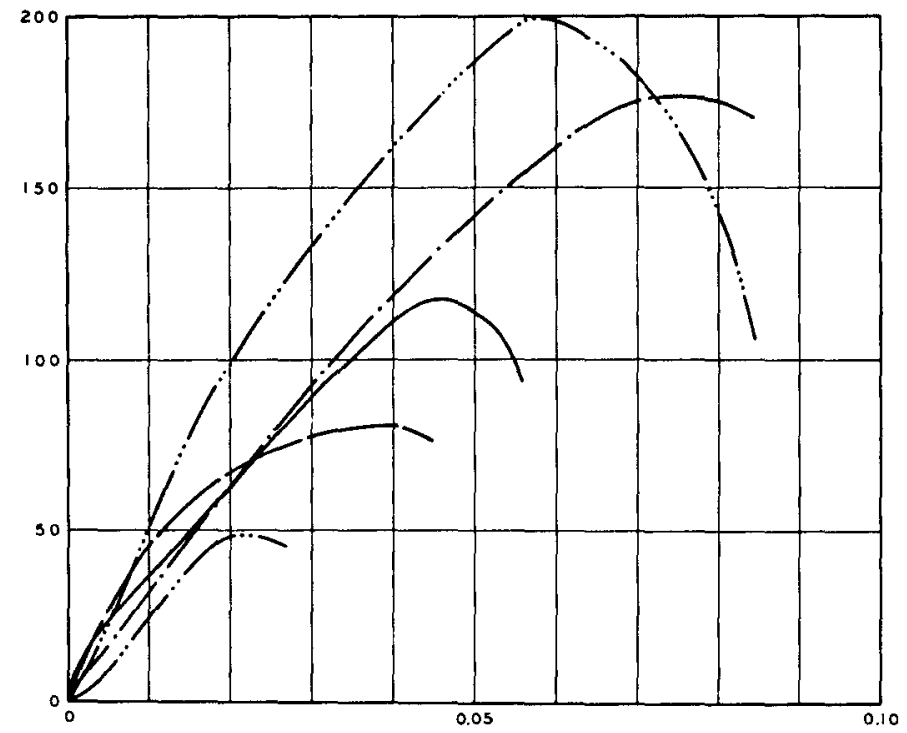

a. WATER CONTENT $=27.2$ PERCENT

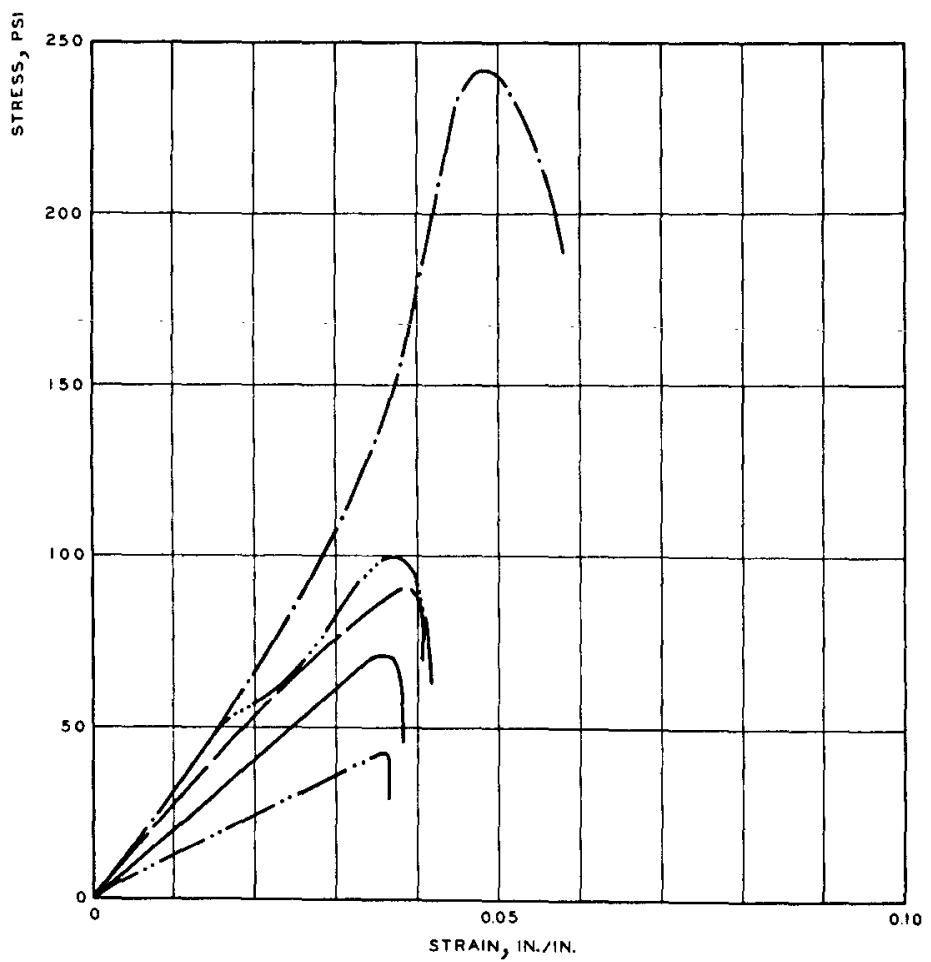

b. WATER CONTENT $=32.1$ PERCENT

Fig. A-22. Stress-strain curves, buckshot clay, impact loader; water contents, 27.2 and 32.1 percent 

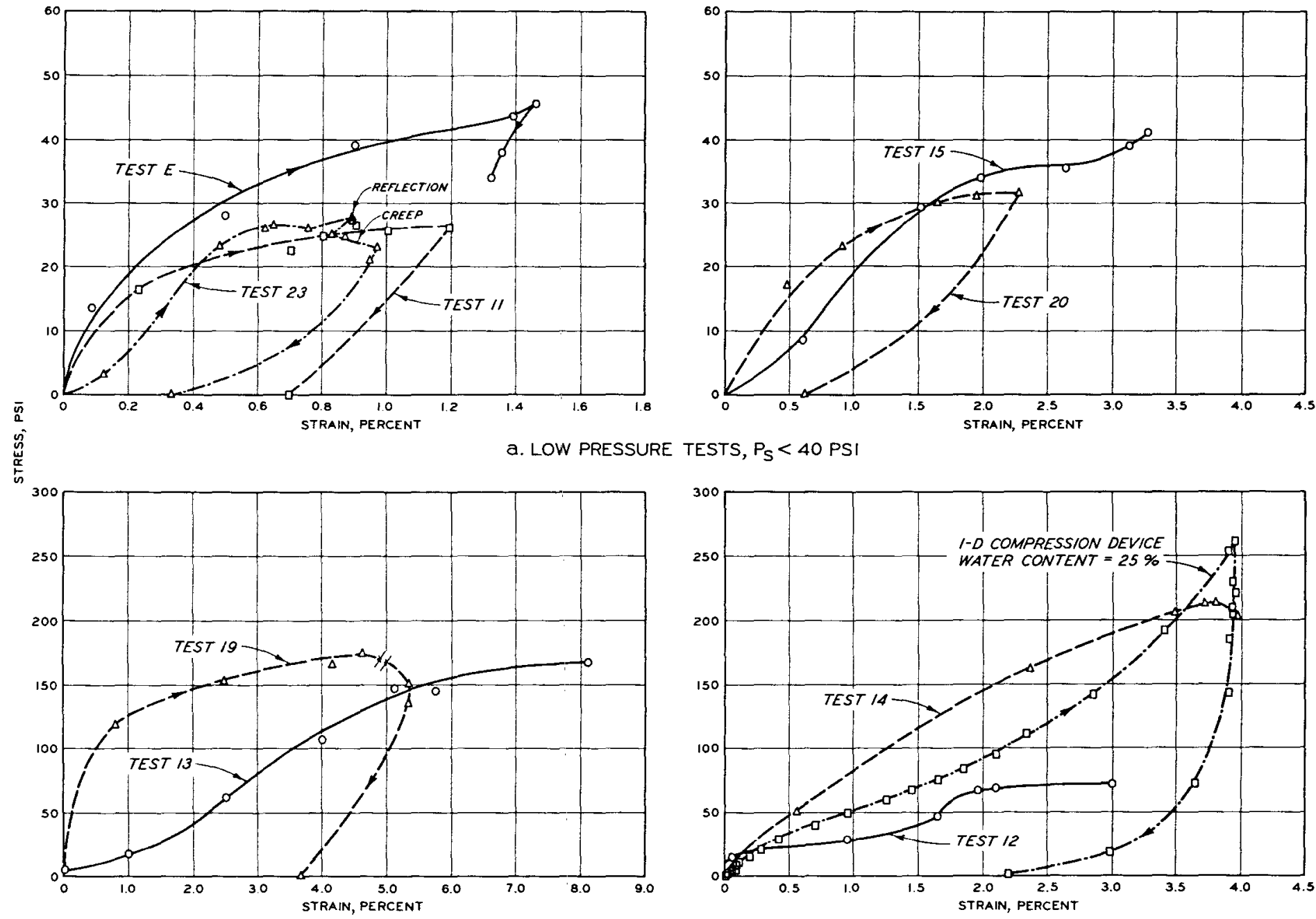

b. HIGH PRESSURE TESTS

Fig. A-23. Stress-strain curves, buckshot clay, SBLG tests 


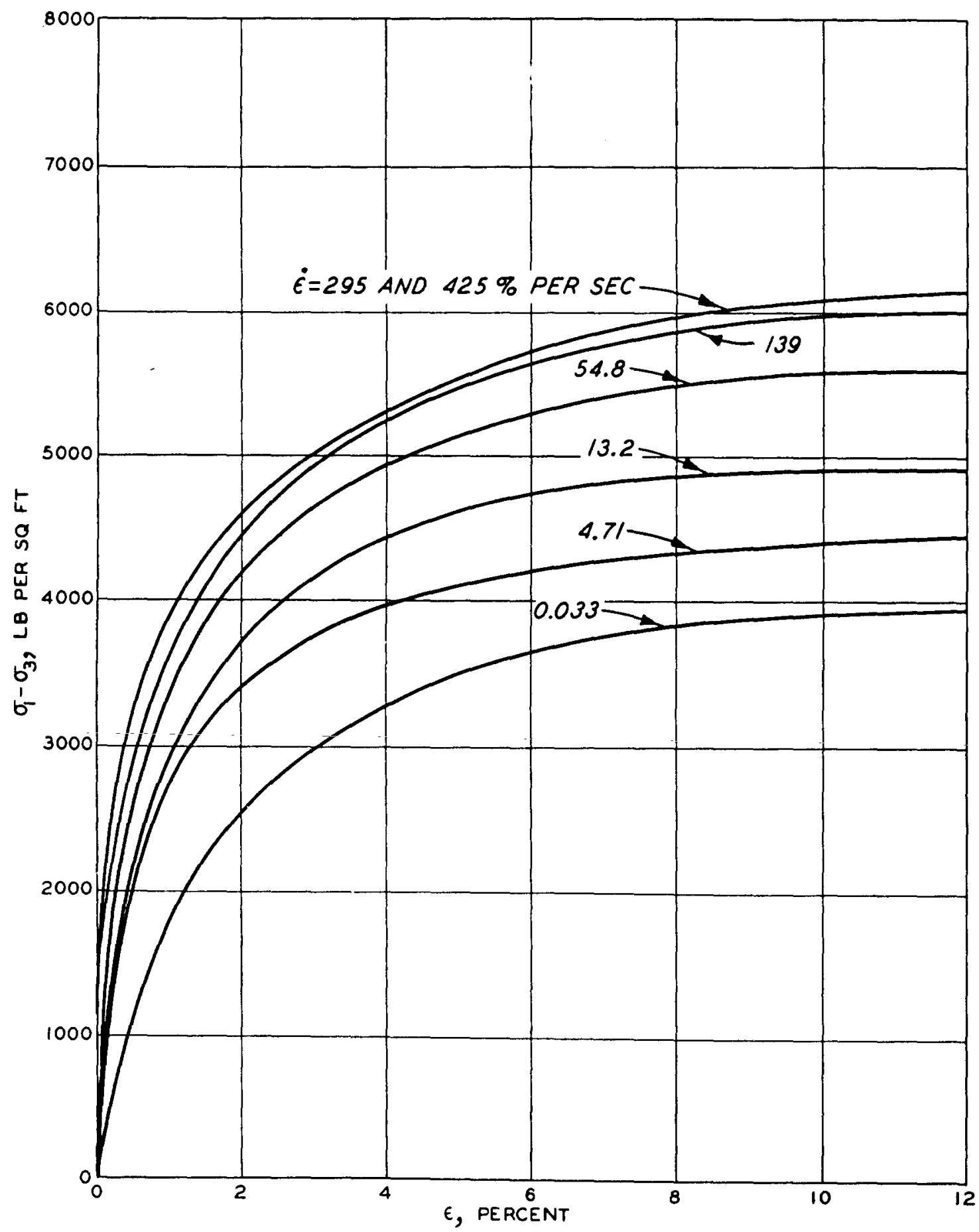

Fig. A-24. Stress-strain curves, buckshot clay (after Carroll) 


\section{APPENDTX B \\ DESIGN OF SPRING-RING TEST DEVICE}

The spring-ring test device used in the test program described in the main text is believed to be unique. In order to assist others with a similar design problem and to prevent repetition of similar mistakes, the step-by-step development of this device is outlined in this appendix.

The performance requirements of the flexible test device posed severe problems in the development and design of a structural member which could provide the prescribed elastic and deformation characteristics. The design of the experiments dictated that the size of the test device remain constant for all tests. Additional constraints were: (1) range of spring constants, (2) overall mass of test device, (3) radial stiffness of test device, (4) lateral strength and stiffness of test device, (5) space occupied by the elastic member, and (6) space requirements for displacement and acceleration gages.

Table 3 of the main text shows the range of spring constants desired: $495 \mathrm{Ib} / \mathrm{in}$. to $8,950,000 \mathrm{Ib} / \mathrm{in}$. These constants were selected based upon the stiffnesses of buckshot clay developed in the preliminary test program described in Appendix A. The main purpose of this program was an investigation of structure stiffness on 
soil arching; therefore, a large range of stiffnesses was desired. In addition to the stiffness requirements, sufficient displacement of the top of the device relative to the base was required to develop maximum arching at various depths of burial and surface pressure. These displacement requirements were estimated using the results from Hendron's (1968) trapdoor experiments with the same buckshot clay at similar water contents.

A number of approaches were considered in the initial attempts to design a suitable elastic structural member for the flexible device. In this phase of the design and development a broad range of spring types was surveyed. This survey is summarized in the following paragraphs.

The widely used helical coil spring was the first spring type studied extensively- structural configurations were evaluated for a single large helix, concentric large helices, and multiple small helix springs within the housing of the test device. Calculations showed that all the configurations tried for this spring type could not provide the required spring constants and displacement range. In addition this spring type had no inherent lateral stiffness and required that other structural members in the test device provide lateral strength. The design calculations using the helical spring were so discouraging that it was dropped from consideration early in the investigation. 
Another general spring type considered was the spring column approach attempted by Mason (1965). This approach utilized the differing moduli of elasticity of selected materials such as steel, aluminum, plastic, etc., to fabricate cylinders which gave the desired spring constants. This configuration provides structures with inherently high radial and lateral strength but is very limited in the range of physically realizable spring constants. Calculations using this approach quickly showed that it would be unsuitable for any aspects of the planned experiments except for the extremely rigid devices.

A closely related modification of Mason's approach was considered. Specially formulated plastics and grouts were studied and tested for use as spring cylinders. This approach had some possibilities, but the close control of spring constants and deflections required was not possible.

Other configurations considered were Bellville springs, leaf springs, metal bellows, diaphragms, proving rings, and tapered disk springs as described by Brecht and Wahl. The Bellville springs were eliminated from consideration when their inherently high hysteresis was discovered and also because of the limited displacement range obtainable with their use. Leaf springs could be devised for the lower spring constants only and were laterally weak. Commercially available bellows suffered from the same limitations 
as the leaf springs. Diaphragms did not permit the attainment of the necessary displacement range.

Tapered disk springs appeared to have good possibilities. A wide range of spring constants was available within the physical size constraints of the test device. The disk spring was inherently strong radially and was found to have fair lateral strength and stiffness. Several scale drawings were made of the test device utilizing this type of spring design. One of these designs could have been used for some of the desired spring constants. The tapered disk spring approach was finally discarded because of the development of still another spring design concept which had been maturing during the study and evaluation of the spring types described above.

The design approach which eventually lead to the adopted spring system is illustrated in Figure B-1. Initially, flattened proving rings were tried as the spring element (Figure B-la), but this configuration severely limited the allowable displacement. While the original approach was not suitable, it lead to the idea of using a machined outer cylinder as shown in Figure B-Ib. This configuration had a number of structural advantages over any of the other spring designs considered. First, the spring constant could be varied over a very wide range by selecting the machined dimensions of the individual spring elements. Second, the spring element was inherently 
rigid radially and fairly stiff laterally. Third, the efficient use of space was superior to any other configurations considered. Fourth, it permitted a greater vertical displacement for a given spring stiffness than any other type of spring studied. In addition, the multiple small slots made it much easier to fabricate a soil barrier around the wall of the test device.

In attempting to extend the slotted cylinder design of Figure B-Ib to provide greater vertical displacement, it became apparent that the machining difficulties would be severe for the low spring constant-high displacement spring cylinders. Additional development effort resulted in the configuration shown in Figure B-lc. In this case, the spring cylinder was fabricated from spring rings with spacers bonded between them. This concept made it possible to fabricate, from a relatively small number of spring rings and spacers, a spring cylinder of almost any desired spring constant. Figure B-2 shows some of the spring rings, spacers, and assembled spring cylinders.

Calculations based on nominal 1/16-inch-, 1/8-inch-, and 1/4inch-thick spring rings, all 6 inches outside diameter and 5 inches inside diameter, showed that these dimensions would satisfy all the spring cylinder stiffness requirements shown in Table 3 of the main text, except for the stiffest device. In order to maintain a constant spring cylinder height, and allow for the space occupied by 
the spacers, the final spring-ring thicknesses selected were 0.052 inch, 0.115 inch, and 0.242 inch.

In order to explain the method of fabricating a spring cylinder to a preselected spring constant, a brief review of the action of multiple springs in series and parallel is included. Each spring cylinder is made up of a stack of spring rings in series and the spring constant of the cylinder is the spring constant of the individual rings divided by the number of rings, where the spring rings are all uniform. This can be expressed as,

$$
K_{T}=\frac{K_{n}}{n}
$$

where

$$
\begin{aligned}
\mathrm{K}_{\mathrm{T}} & =\text { spring constant of cylinder } \\
\mathrm{K}_{\mathrm{n}} & =\text { spring constant of individual springs } \\
\mathrm{n} & =\text { number of springs }
\end{aligned}
$$

or more generally:

where

$$
\frac{1}{K_{T}}=\frac{1}{K_{1}}+\frac{1}{K_{2}}+\ldots+\frac{1}{K_{n}}
$$

$$
\begin{aligned}
& \mathrm{K}_{1}=\text { spring constant of ring } 1 \\
& \mathrm{~K}_{2}=\text { spring constant of ring } 2 \\
& \mathrm{~K}_{n}=\text { spring constant of ring } n
\end{aligned}
$$

Within the spring cylinder each individual spring ring also acts 
as a number of springs in parallel, the number being determined by the number of spacers. The spring constant of springs in parallel is given by the relation:

$$
K_{p}=K_{1}+K_{2}+K_{3}+\ldots+K_{n}
$$

where

$$
\begin{aligned}
& K_{p}=\text { ring spring constant } \\
& K_{I}=\text { segment spring constant } \\
& K_{n}=\text { segment spring constant }
\end{aligned}
$$

The example of springs in parallel could be applied to the spring rings only in a very general way, for the addition of more spacers was not just a summing of springs. When, for example, the number of spacers was increased from 4 to 5 , then each spring element was shortened, with a corresponding increase in stiffness. The resulting spring constant of the ring was therefore significantly greater than the ratio of 5 to 4 which would have been the case for summing springs of a constant $K$. Experimentally determined curves such as Figure B-3 were used for selecting the number of spacers to obtain a given spring constant. The curves of this figure give the spring constant per ring and this value had to be divided by the number of active rings to obtain the spring constant for the cylinder as explained above.

By the proper choice of spring-ring thickness, number of active 
rings, and number of spacers, the desired spring constant was fabricated. In all cases, it was desirable to utilize the largest number of active spring rings possible in order to achieve the largest allowable displacement without overstraining the spring rings. During fabrication, the spring rings and spacers were placed in a fixture which held the parts in place while the epoxy hardened. The fixture consisted of three smooth, round posts screwed into a flat, ground steel plate. The three posts were perpendicular to the base plate and positioned so that a 6-inch-diameter cylinder would fit snugly between them. The spring rings were placed in the assembly fixture one at a time and spacers, coated on both sides with epoxy, were positioned on each spring ring. The angular position of the spacers at each level of assembly was determined with a paper template. The epoxy adhesive used to bond the copper spacers to the steel spring rings was made by mixing three parts by volume of Epon 828 (Shell Oil Co.) with one part by volume of Versamid (Du Pont). The spacers were stamped from sheet copper to the desired sizes, $1 / 32$ by $1 / 8$ by $1 / 2$ inch to go with the $1 / 16$-inch rings and $1 / 16$ by $1 / 4$ by $1 / 2$ inch to go with the $1 / 8$ - and $1 / 4$-inch rings (Figure B-2).

Determination of the spring constant of the assembled spring cylinder was made by applying a known force and measuring the resulting deflection. This testing was performed in the apparatus shown 
in Figure B-4. In this machine a hydraulic cylinder was used to apply the load and, depending on the test, either a proving ring or a load cell was used to measure the load. Mechanical dial gages and the displacement transducers within the test device were used to monitor the deflection resulting from the load. A typical calibration curve derived from these measurements is shown in Figure 13 in the main text. 


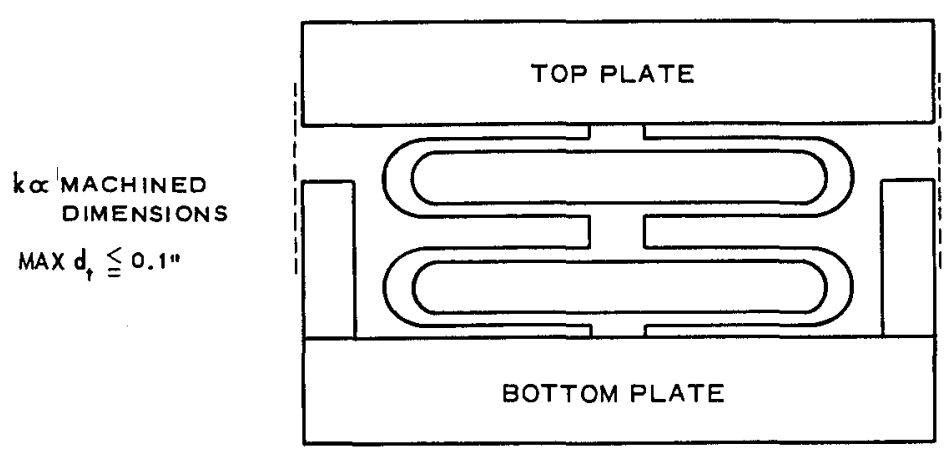

a. PROVING RINGS WITHIN TEST DEVICE HOUSING

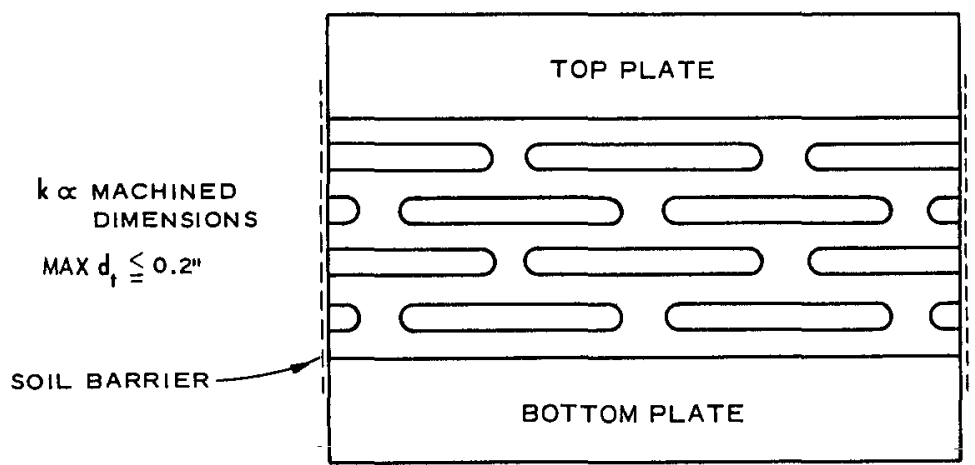

b. ONE-DIMENSIONALLY ELASTIC SURFACE CYLINDER MACHINED AS MULTIPLE PROVING RINGS JOINED TO FORM CYLINDER
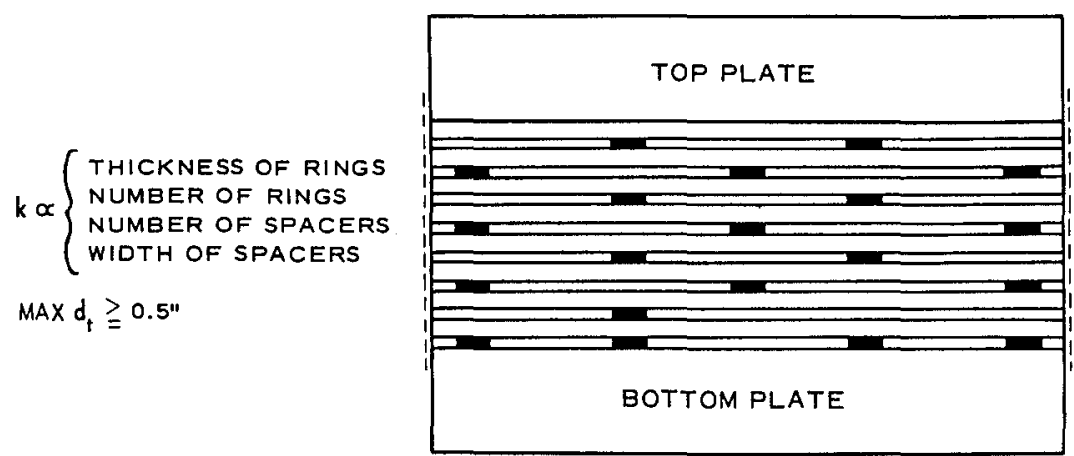

c. ONE-DIMENSIONALLY ELASTIC SURFACE CYLINDER FABRICATED FROM SPRING RINGS AND SPACER SHIMS

Fig. B-1. Stages in the development of the spring-ring concept 


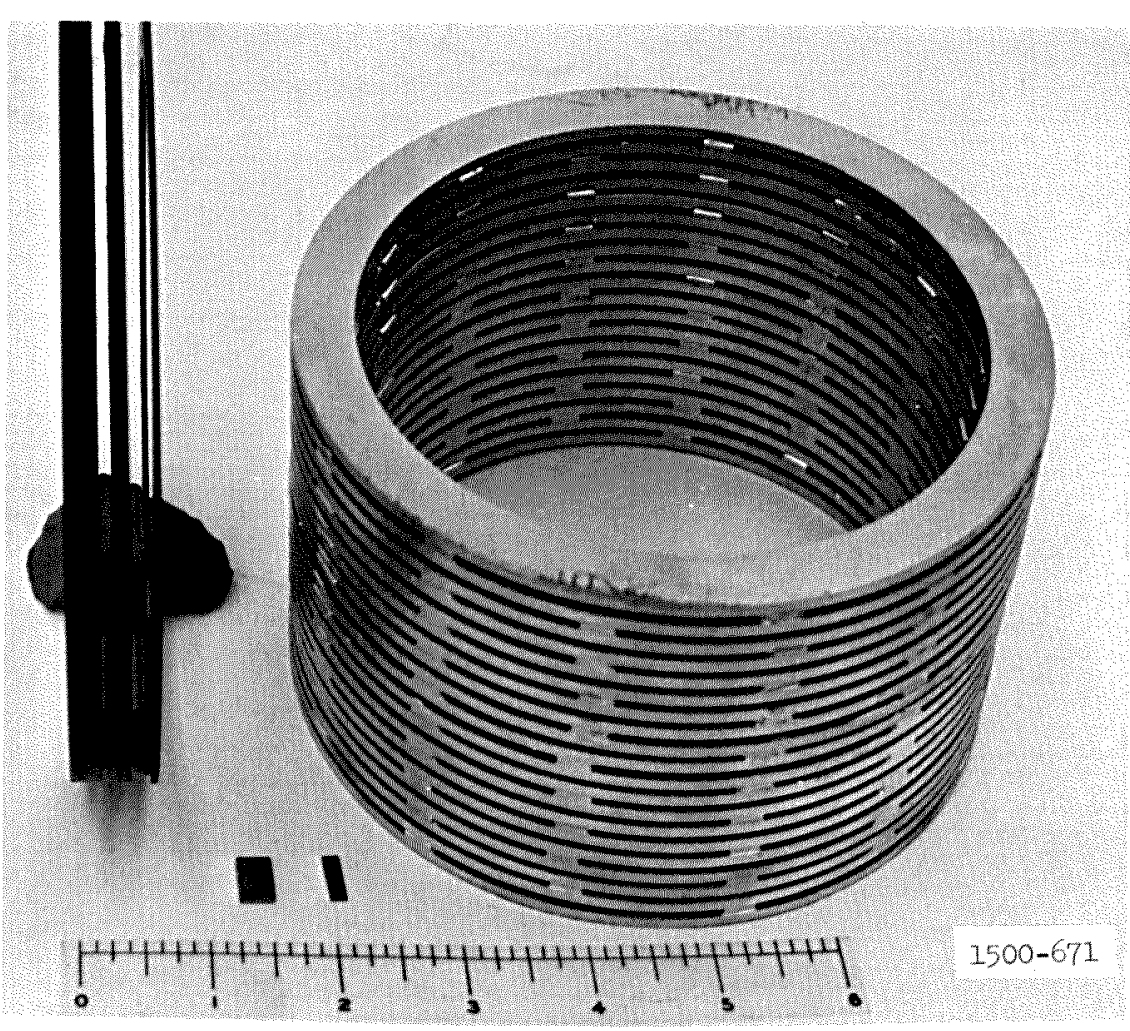

a. Springs, spacers, and assembled spring cylinder
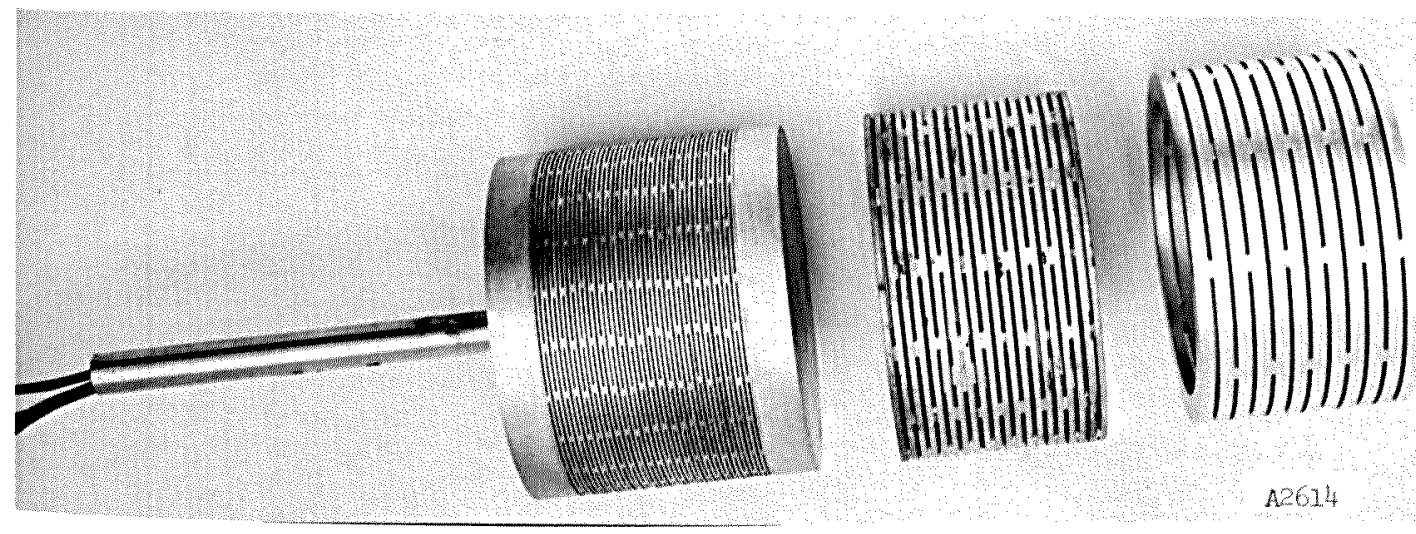

A261.4

b. Spring cylinders fabricated of nominal 1/16-inch, 1/8-inch, and 1/4-inch rings

Fig. B-2. Spring cylinders for spring-ring test device 


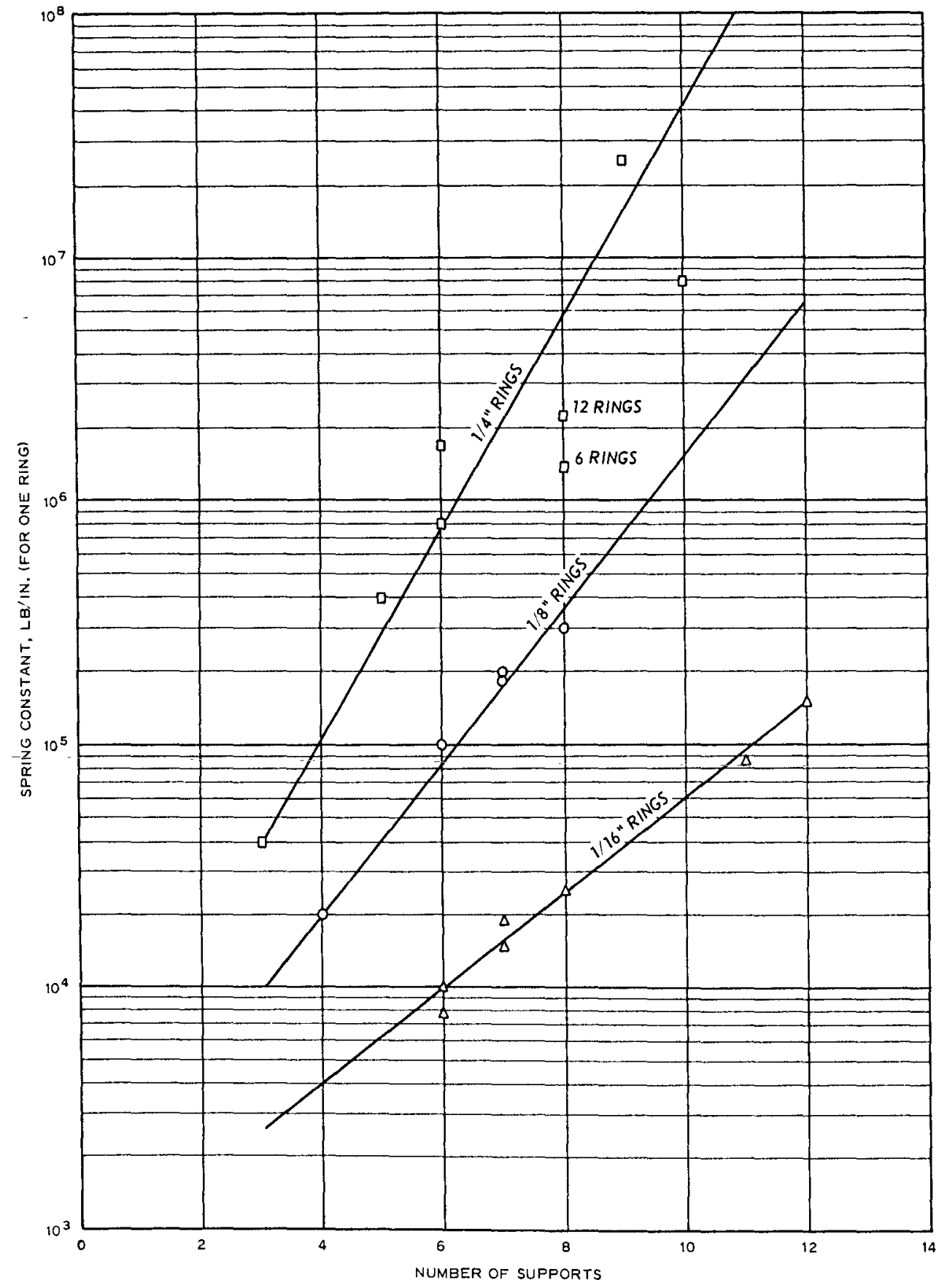

Fig. B-3. Spring constants of individual spring ring 


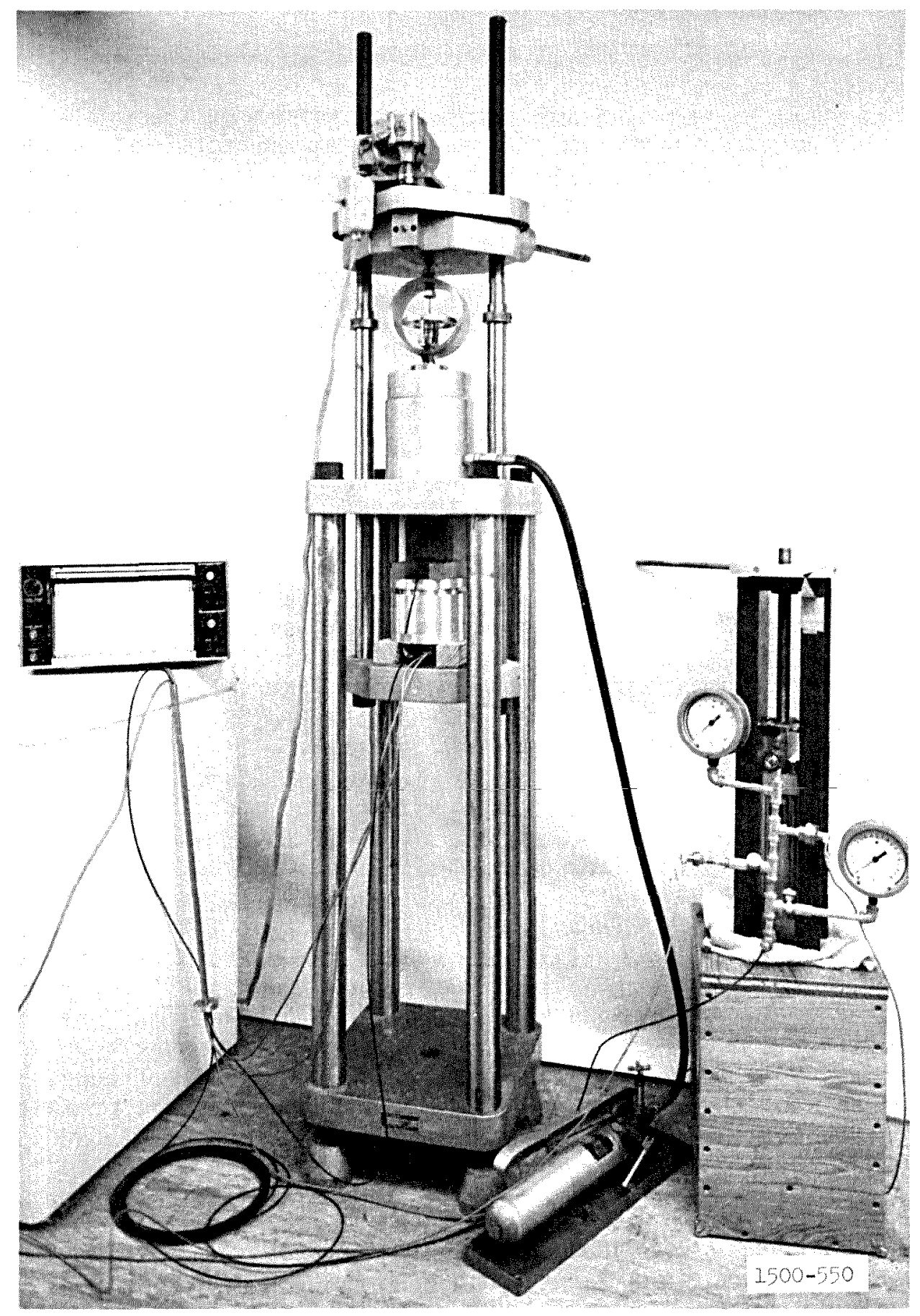

Fig. B-4. Test device calibration apparatus 


\section{APPENDIX C \\ OSCILIOGRAPH RECORDS FROM THE DYNAMIC TESTS}

This appendix contains photographic reproductions of many of the oscillograph records from the dynamic test program. These figures are referred to frequently throughout the main text of the report. These records should be of particular value to both investigators and designers in the field of dynamic design.

With the exception of Figure $\mathrm{C}-1$, the records were produced by gages installed in the soil or on the test device. Figure C-l is a typical record from the tests used to determine the frequency and damping properties of the test devices. Figures C-2 through C-75 were produced during Tests 11 through 28. Figures C-76 through C-87 originated from the preliminary test program.

Normally the first 10 to $20 \mathrm{msec}$ were the most important part of the records. After this time, the records showed considerable disturbance. Therefore only the first $50 \mathrm{msec}$ of the records are shown in continuous form. The $100-\mathrm{msec}$ and final portions, approximately after $1 \mathrm{sec}$ unless specified otherwise, also have been included to show the instrument recovery after the surface pressure had decreased.

The zero timing line during the main test program was produced by using the explosive cap to break a wire placed in the bonnet. In 
preliminary Tests $A$ and $B$, a time of arrival (TOA) gage was placed on the soil surface and compared with the zero time produced by the wire technique. The initiation of the TOA gage lagged the wire break by less than $0.5 \mathrm{msec}$ and corresponded very closely with the initiation of the Norwood pressure gages in the bonnet, Figures C-76, $\mathrm{C}-77$, and $\mathrm{C}-78$. The wire break normally preceded the initiation of the bonnet pressure gages by less than $0.5 \mathrm{msec}$, Figures C-80, C-83, and $\mathrm{C}-86$. In some of the records, especially those from any type of strain gage, excursions can be seen at zero time. Exploratory tests showed that these disturbances were caused by the E. M. F. generated in the explosion. The pressure gages in the bonnet appeared to initiate at the same time as those placed on the soil surface, Figure $\mathrm{C}-3$.

The format preceding each record identifies the instrumentation channel, the level of the top of the gage measured with reference to the test chamber base, the radial distance from the centerline of the chamber, the radial angle measured clockwise from north, and the final level of the top of the gage. In addition, the calibration for each channel is shown in the last column. As the records have been reduced considerably from their original size, a reference scale is shown on each record. A reference trace also has been placed on each record.

The channel number in the first column identifies the type of 
measurement being made; $S$ or SE identifies soil pressure; PN, PS, $P E$, or FW identifies the bonnet pressure in the north, south, east, or west quadrant; $\mathrm{X}$ or ACC signifies an acceleration measurement; $D$ designates deflection; $\epsilon$ is a strain measurement; IP designates pressure measurement inside the test device; and $F$ is a measurement of force on the top of the test device produced by a strain gage-bridge arrangement.

When possible, distorted traces have been labeled. Questionable traces also have been labeled.

Timing lines have been placed at the bottom of each record. In addition, important timing lines measured with reference to zero time have been placed on many of the records. 


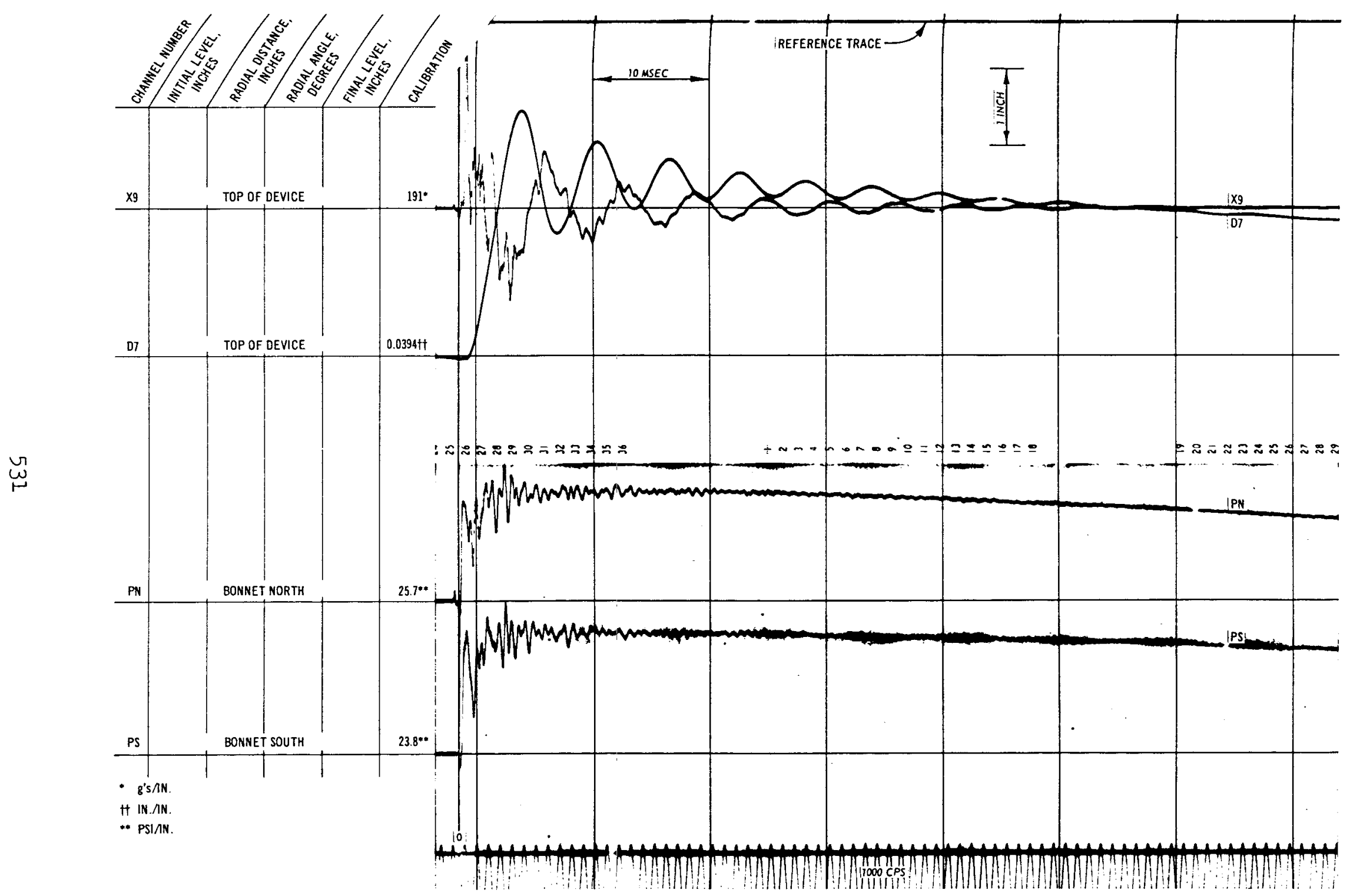

Fig. C-1. Typical dynamic airblast test record for spring-ring test device 


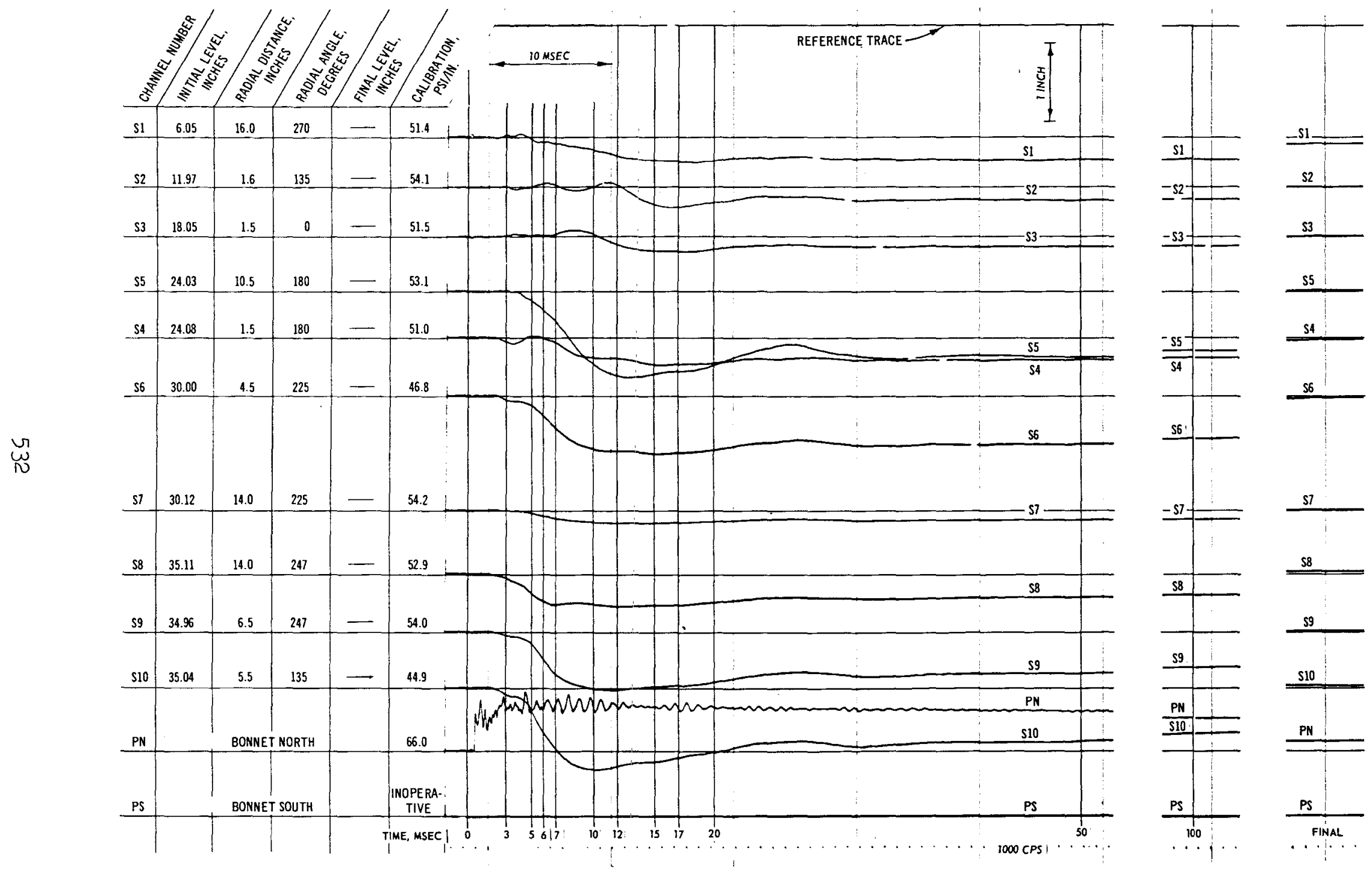

Fig. C-2. Test 11, pressure record (channels S1-S10) 

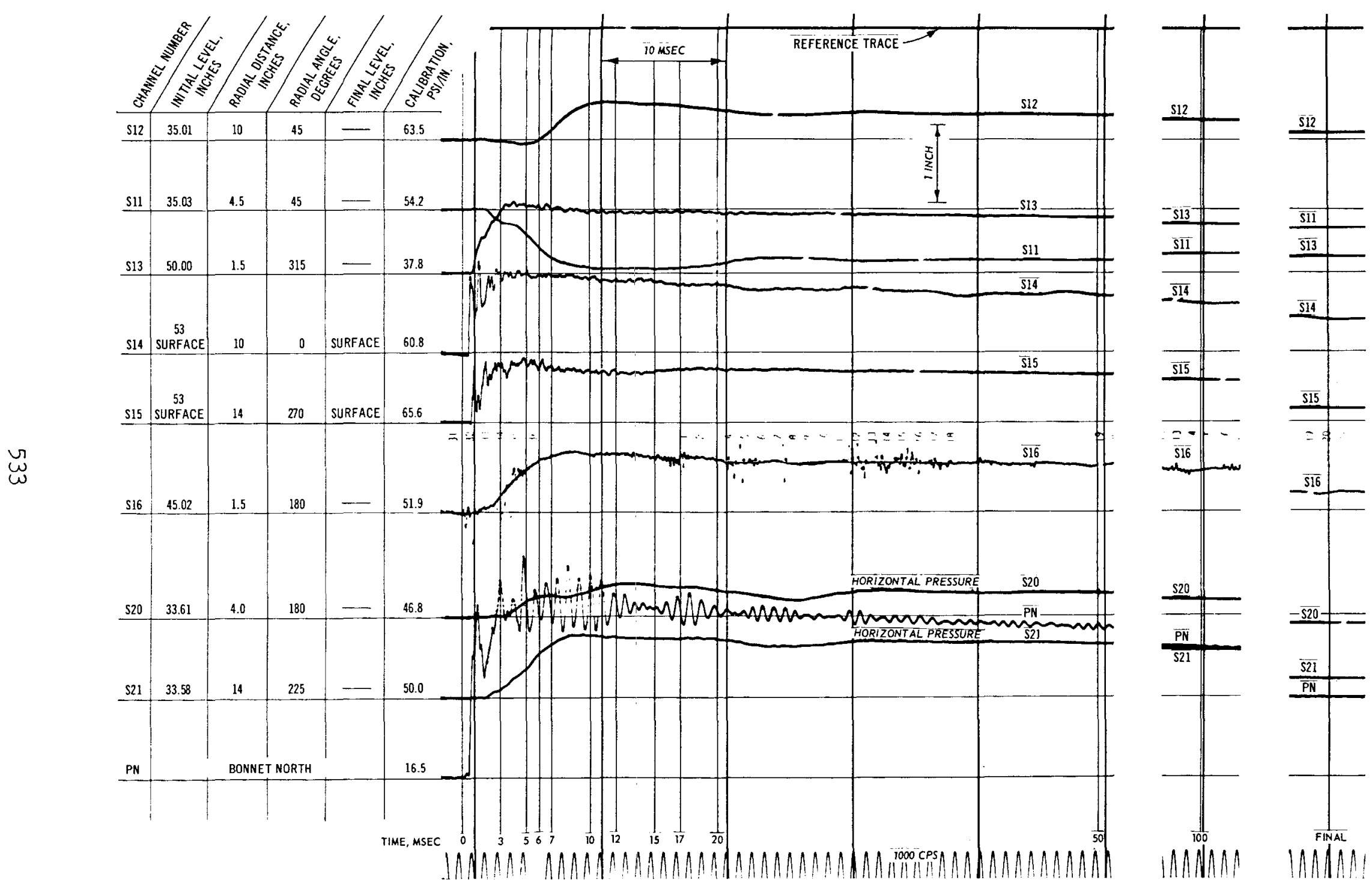

Fig. C-3. Test 1I, pressure record (channels SII-S2I) 


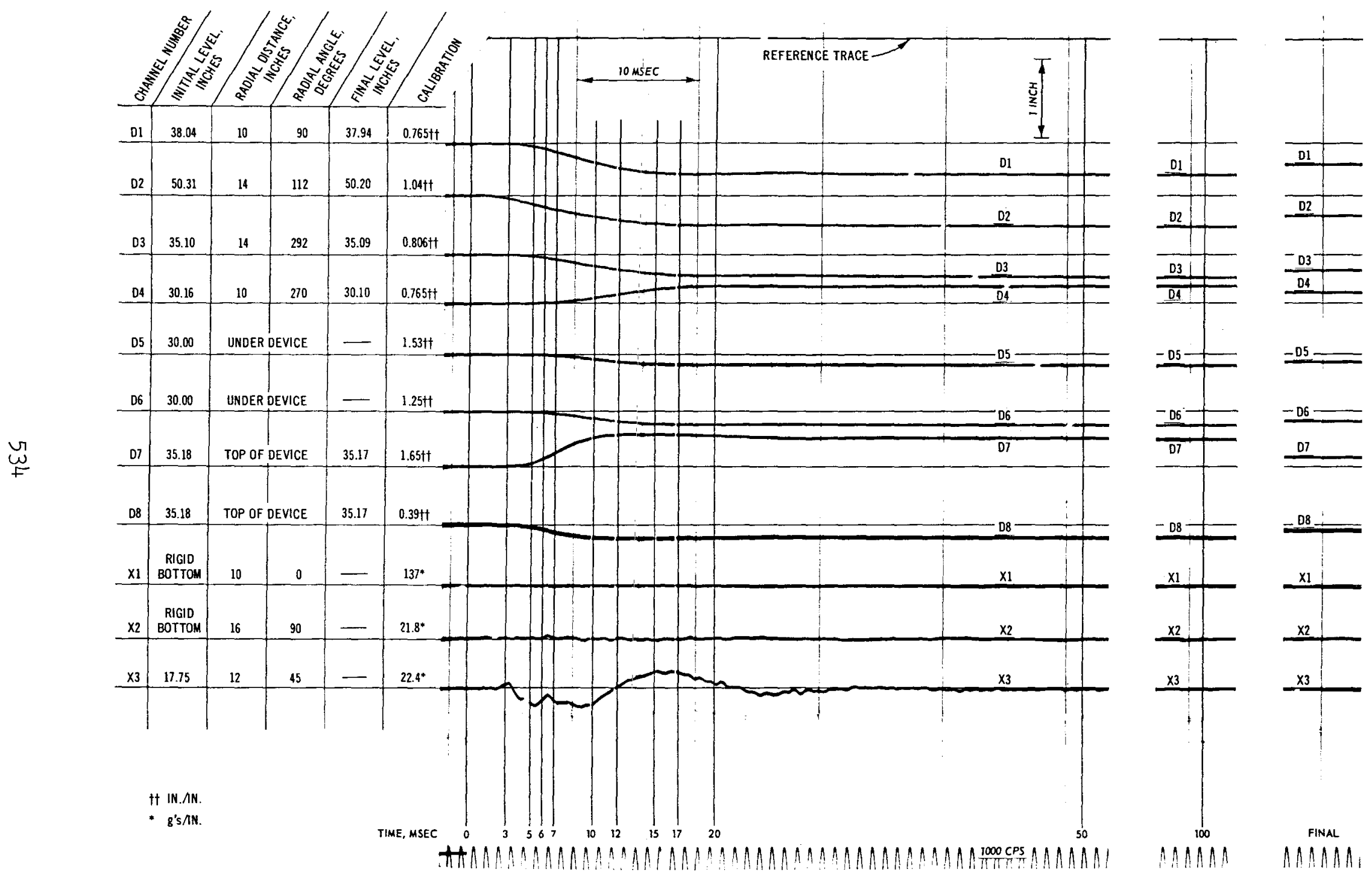

Fig. C-4. Test 11 , deflection and acceleration (X1-X3) record 

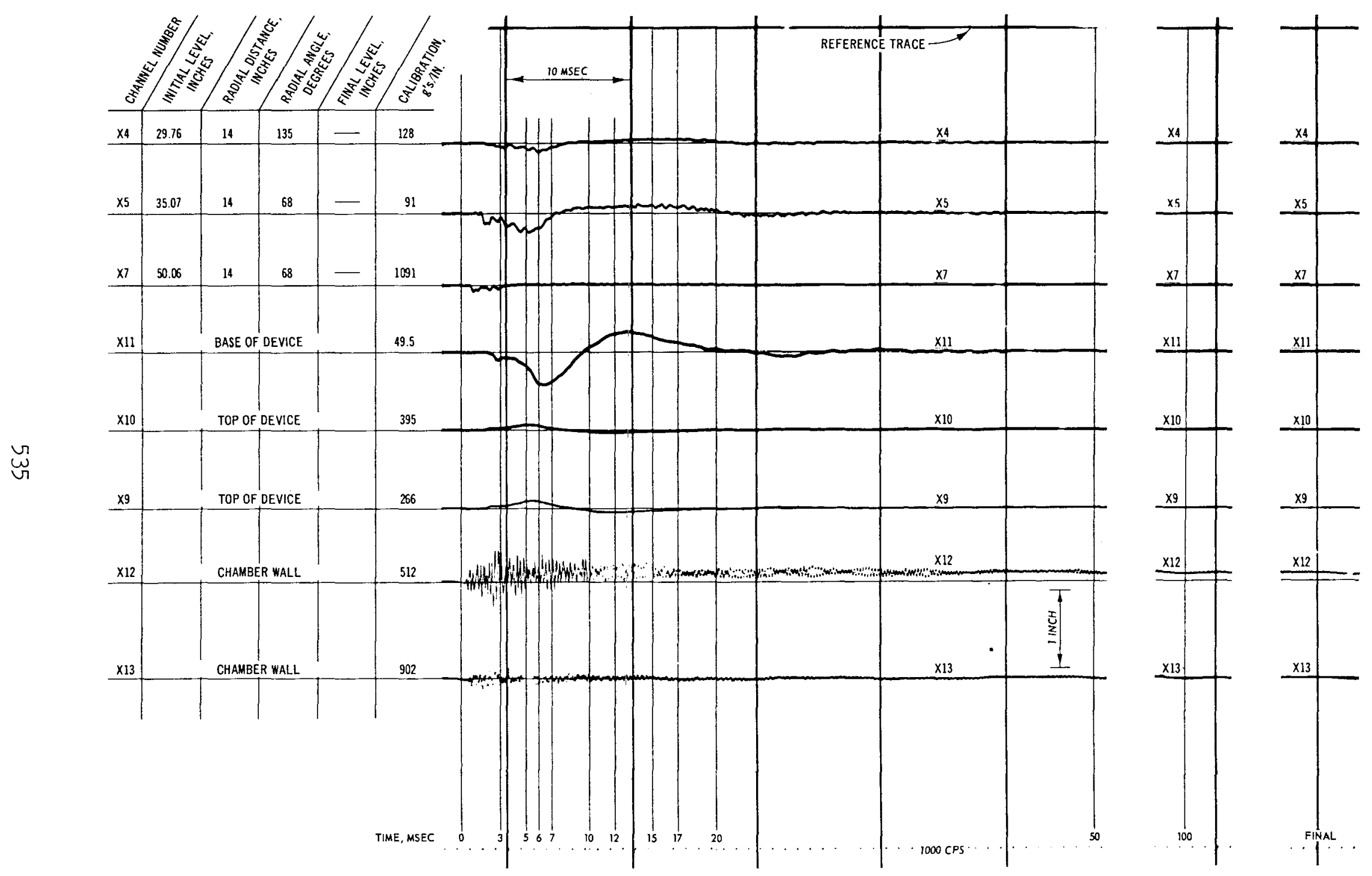

Fig. C-5. Test II, acceleration record (X4-XI3) 


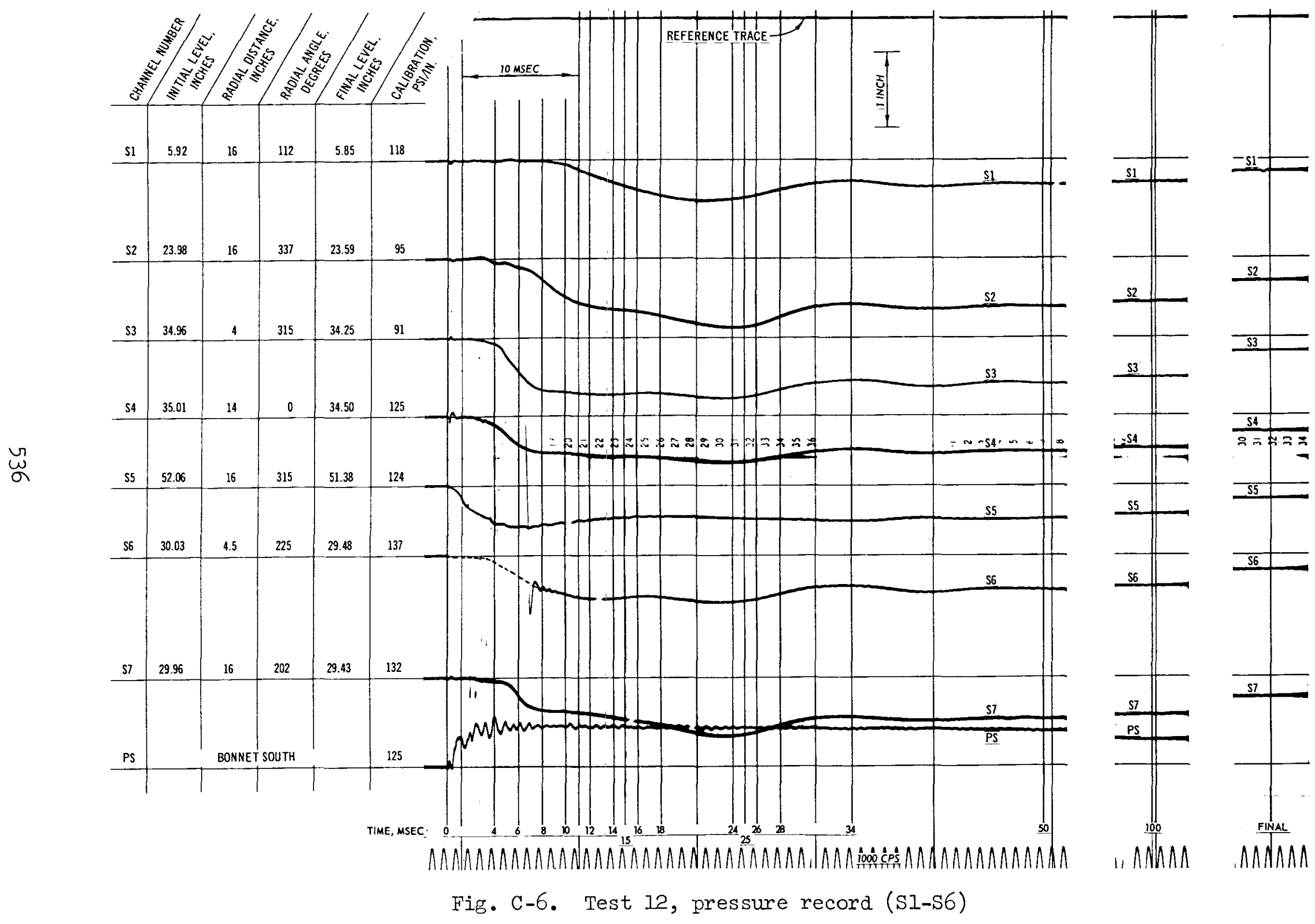



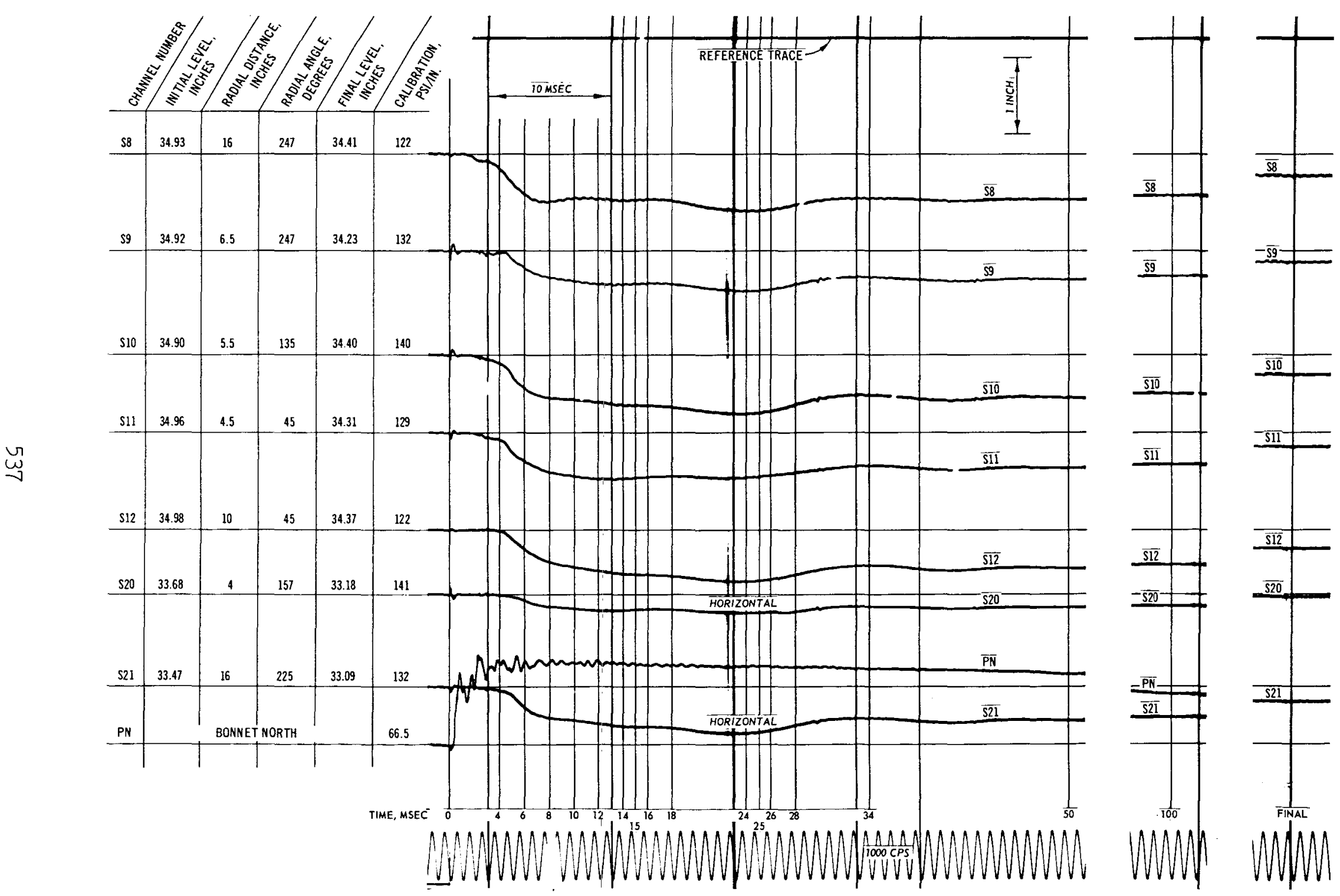

Fig. C-7. Test 12, pressure record (S8-S21) 

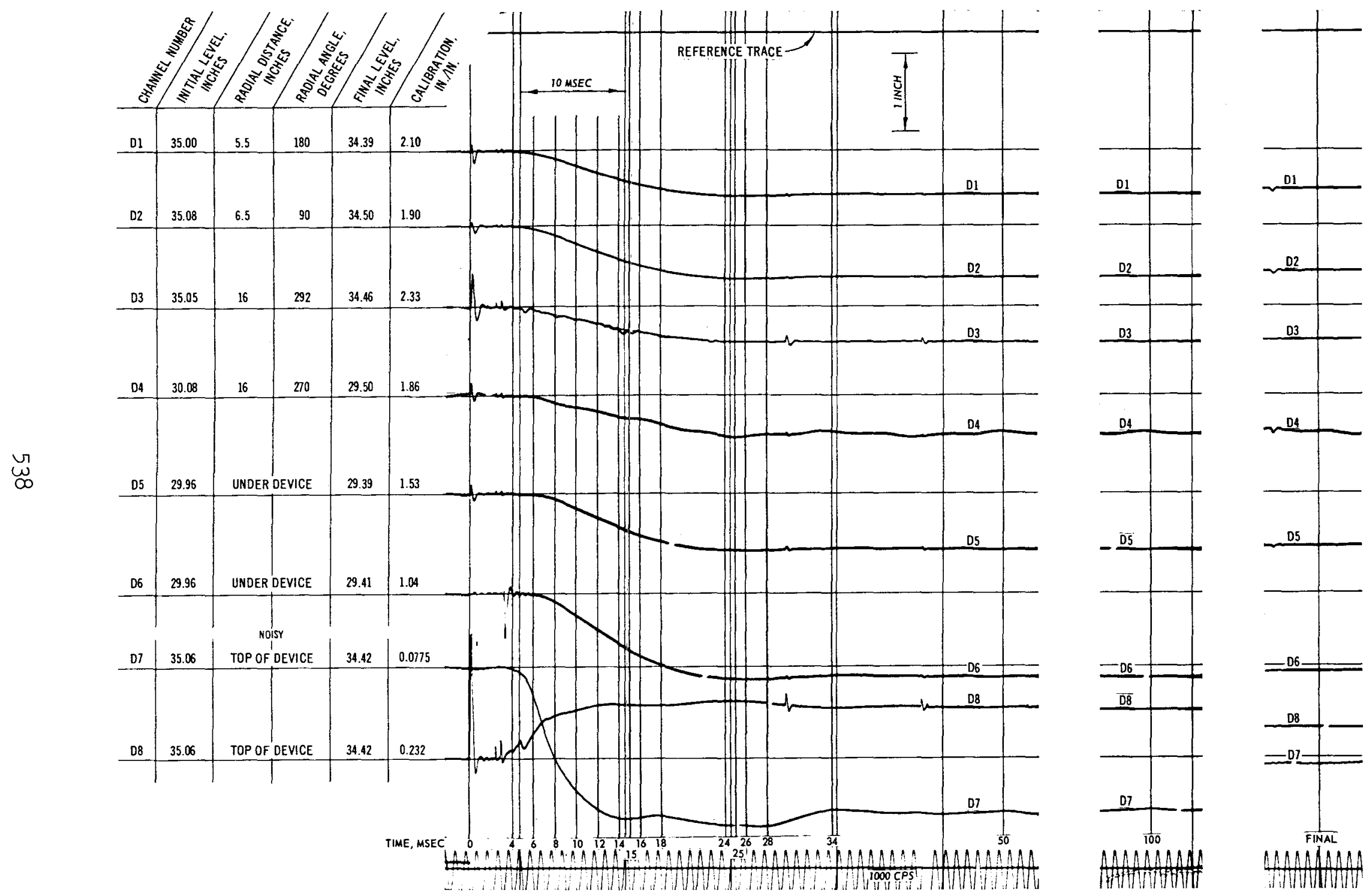

Fig. C-8. Test 12, deflection record 

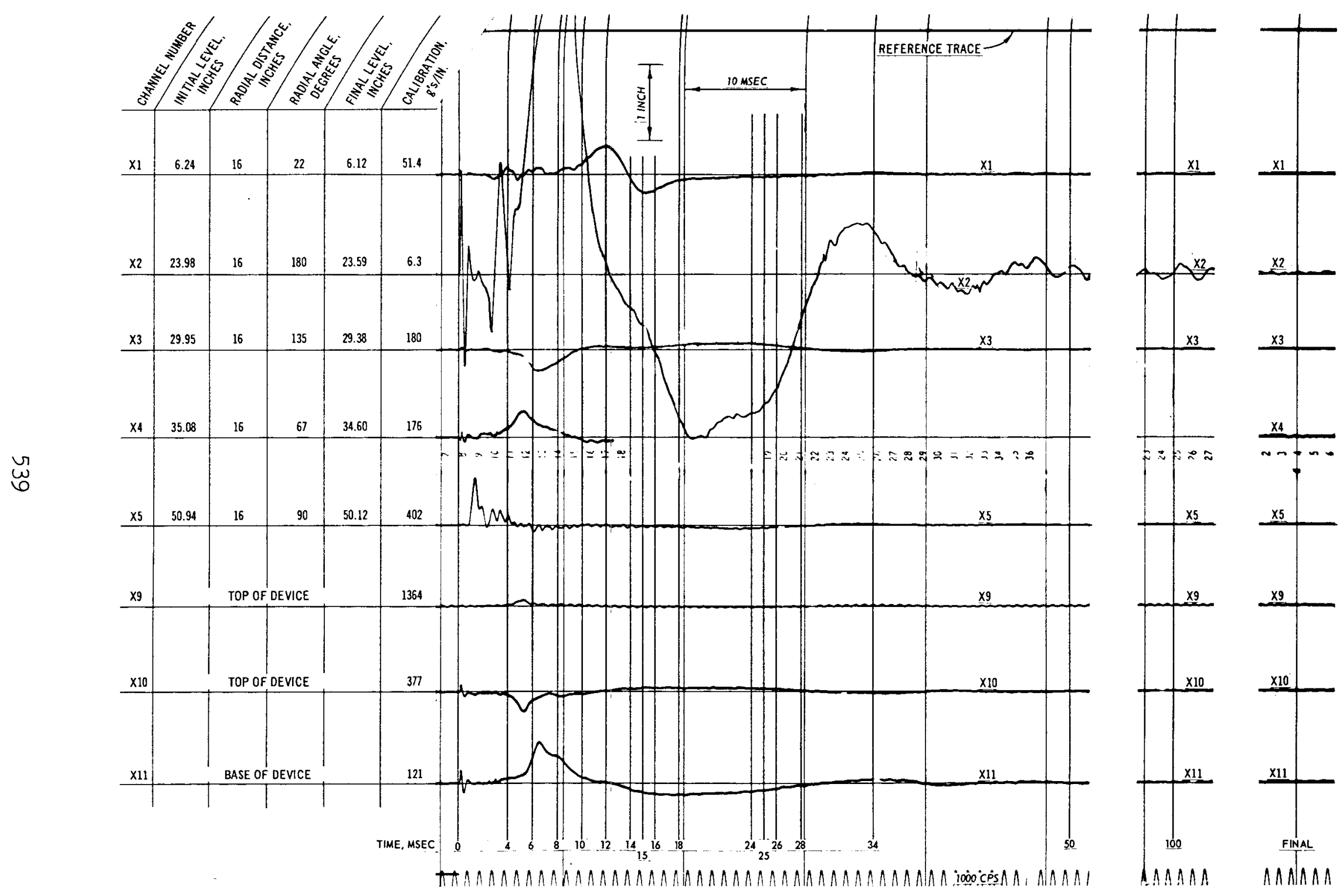

Fig. C-9. Test 12, acceleration record 

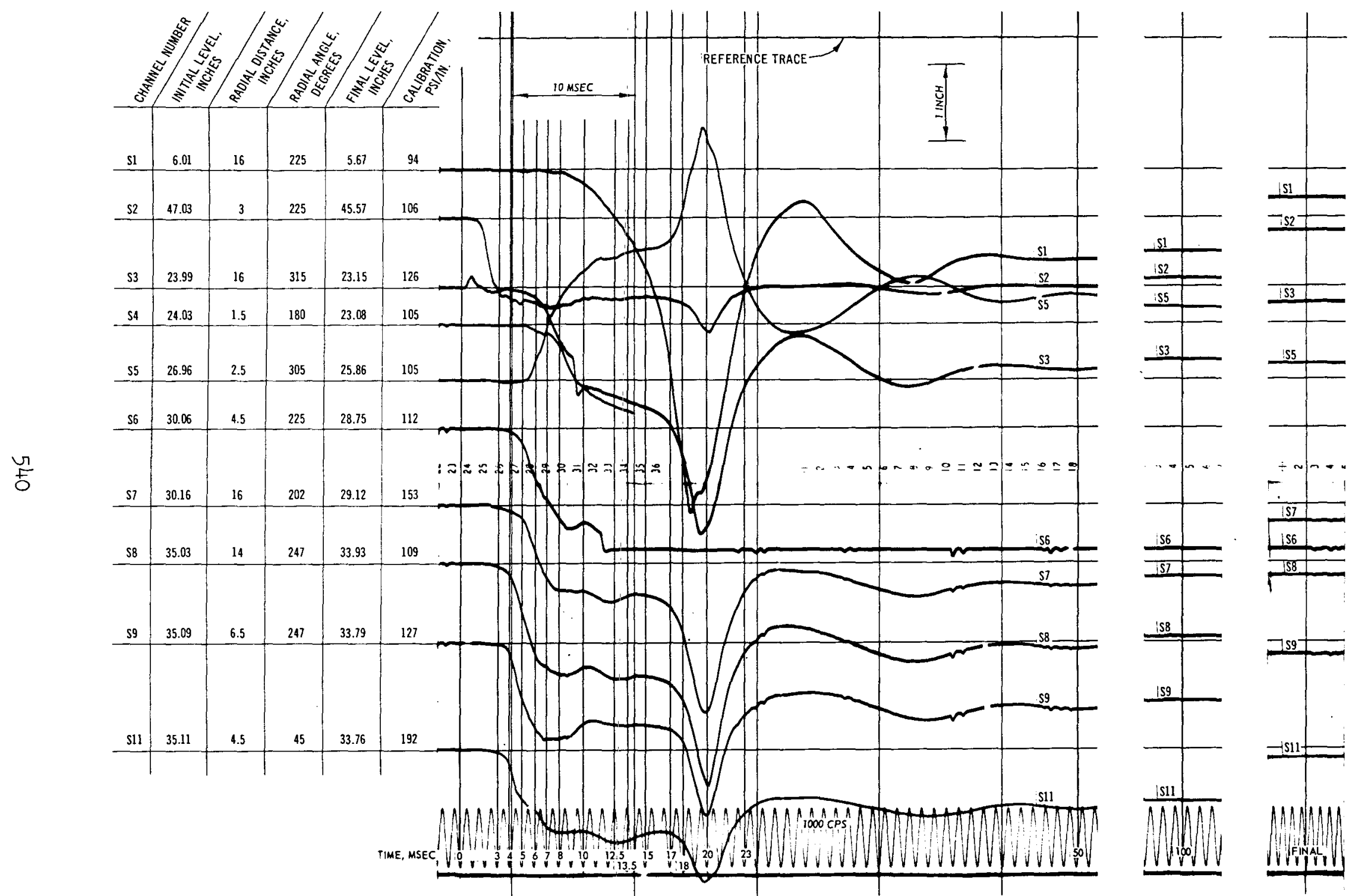

Fig. C-10. Test 13, pressure record (SI-SII) 


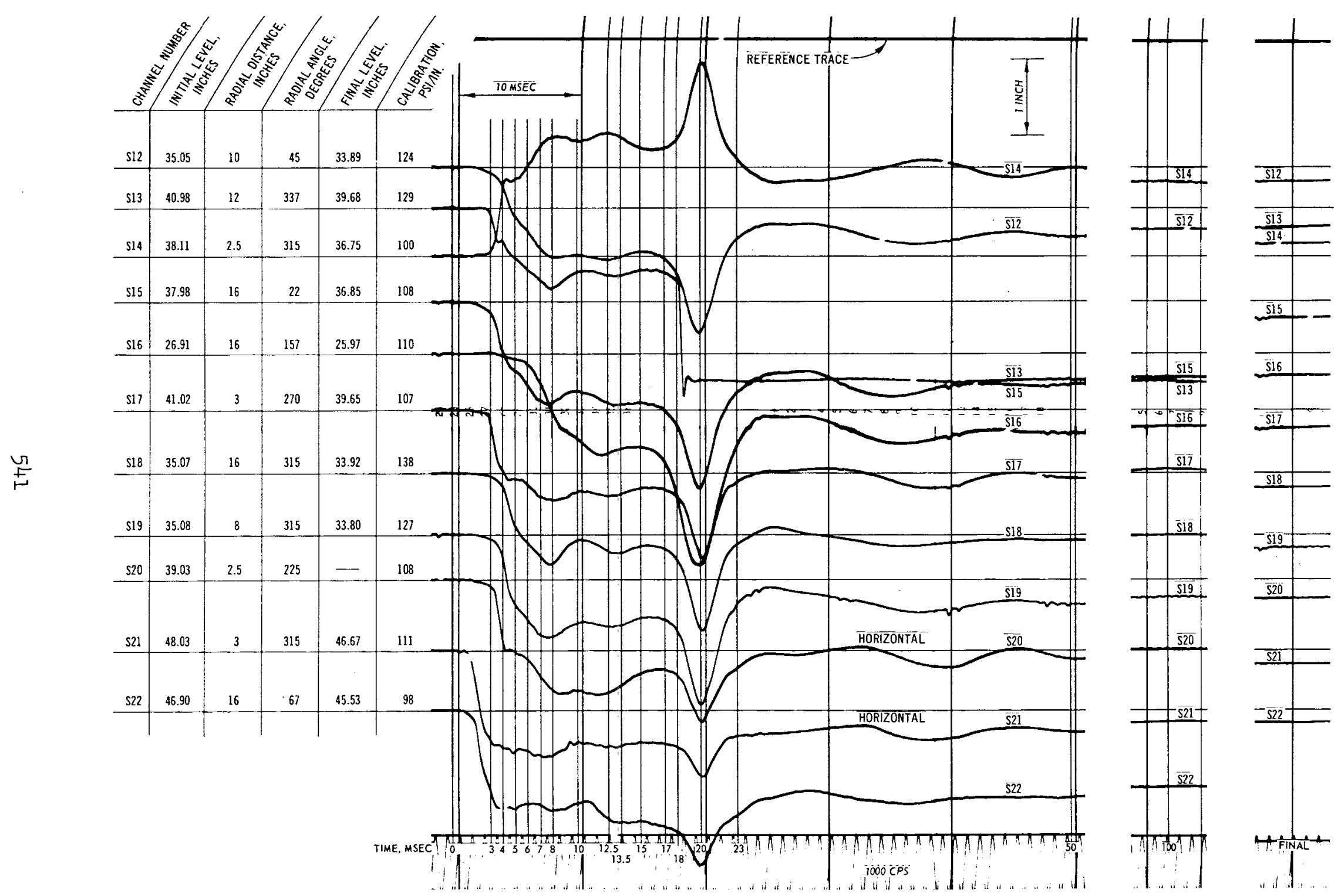

Fig. C-11. Test 13, pressure record (S12-S22) 


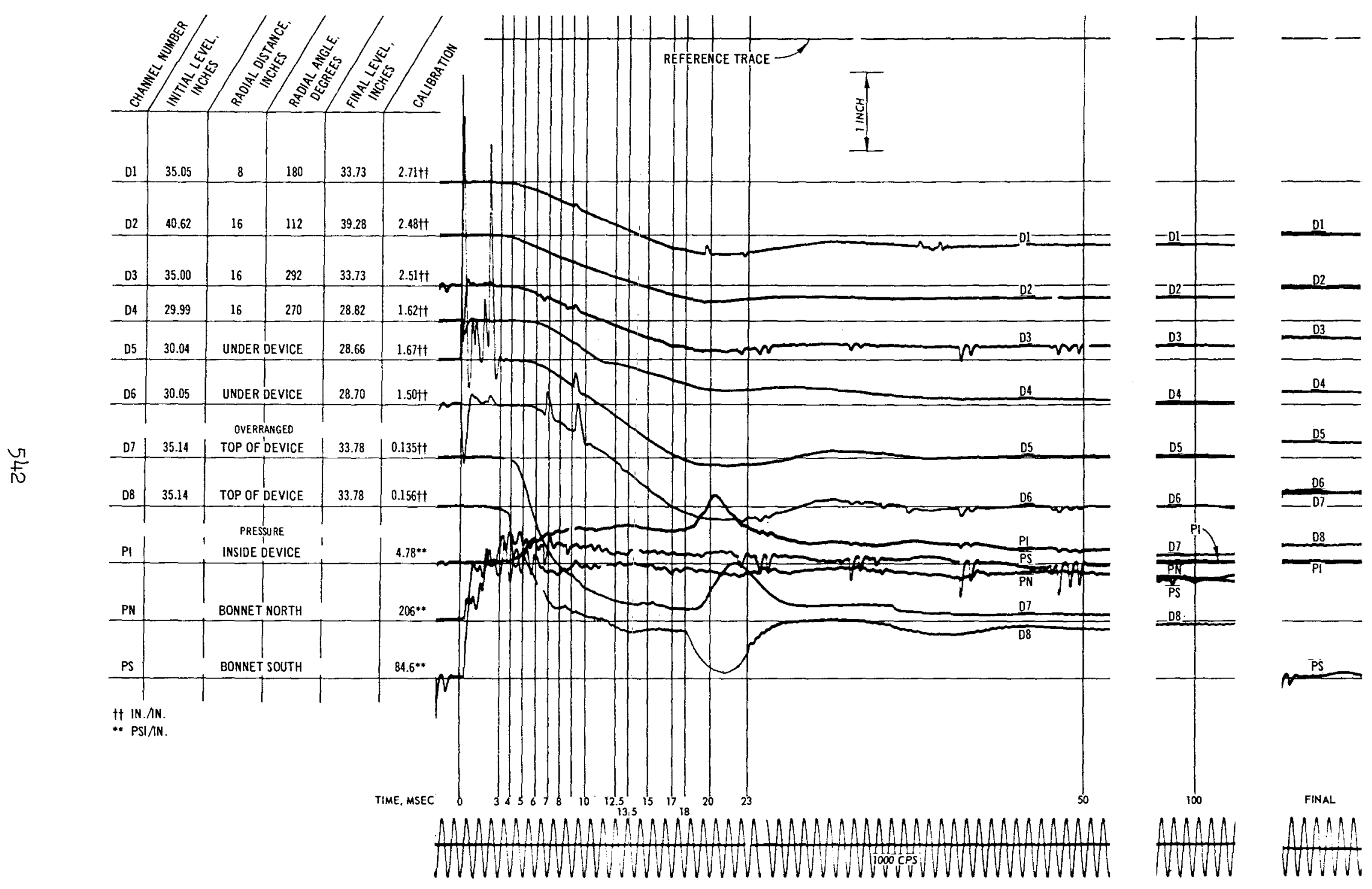

Fig. C-12. Test 13, deflection and pressure record 


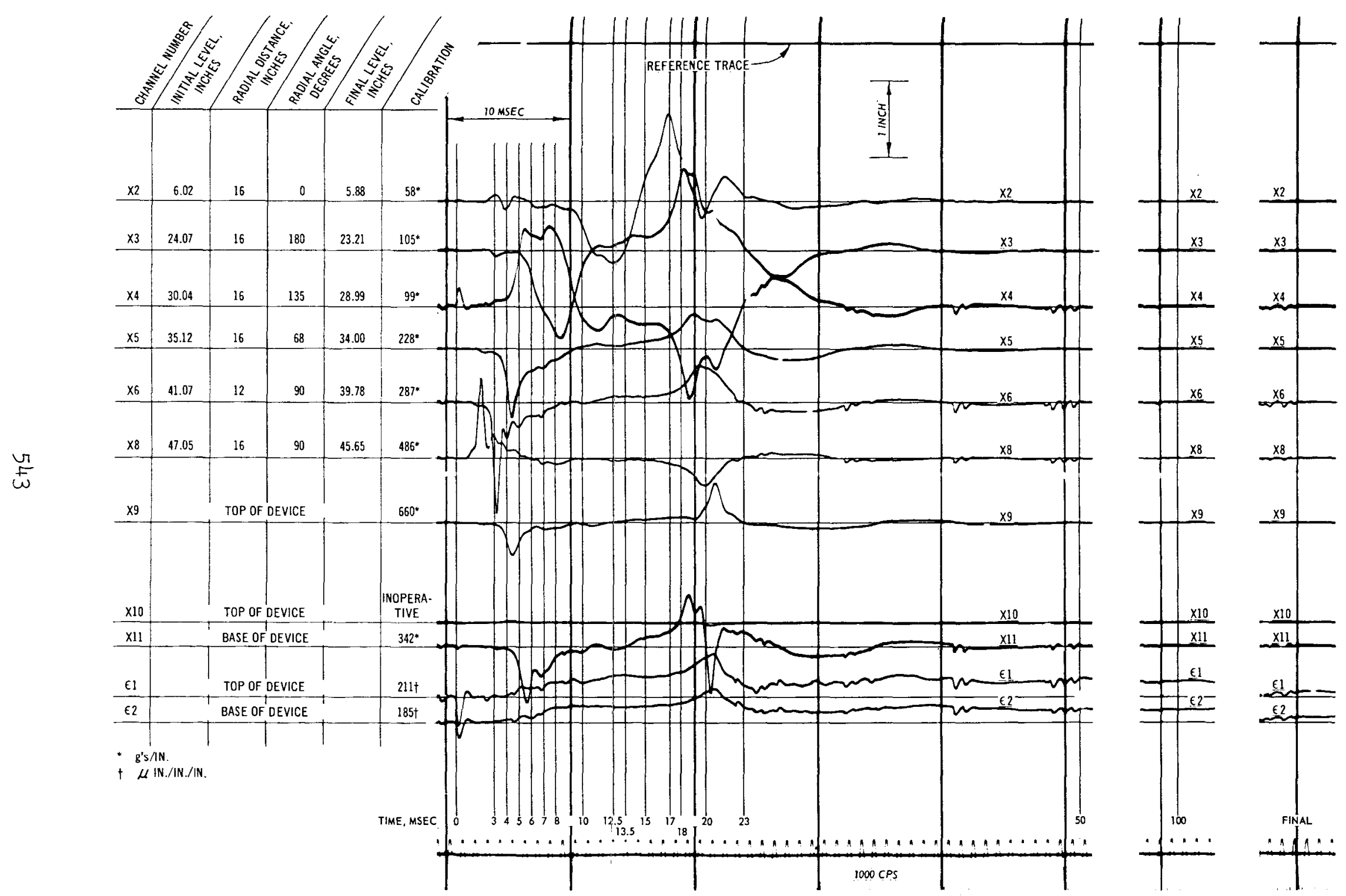

Fig. C-13. Test 13, acceleration and strain record 

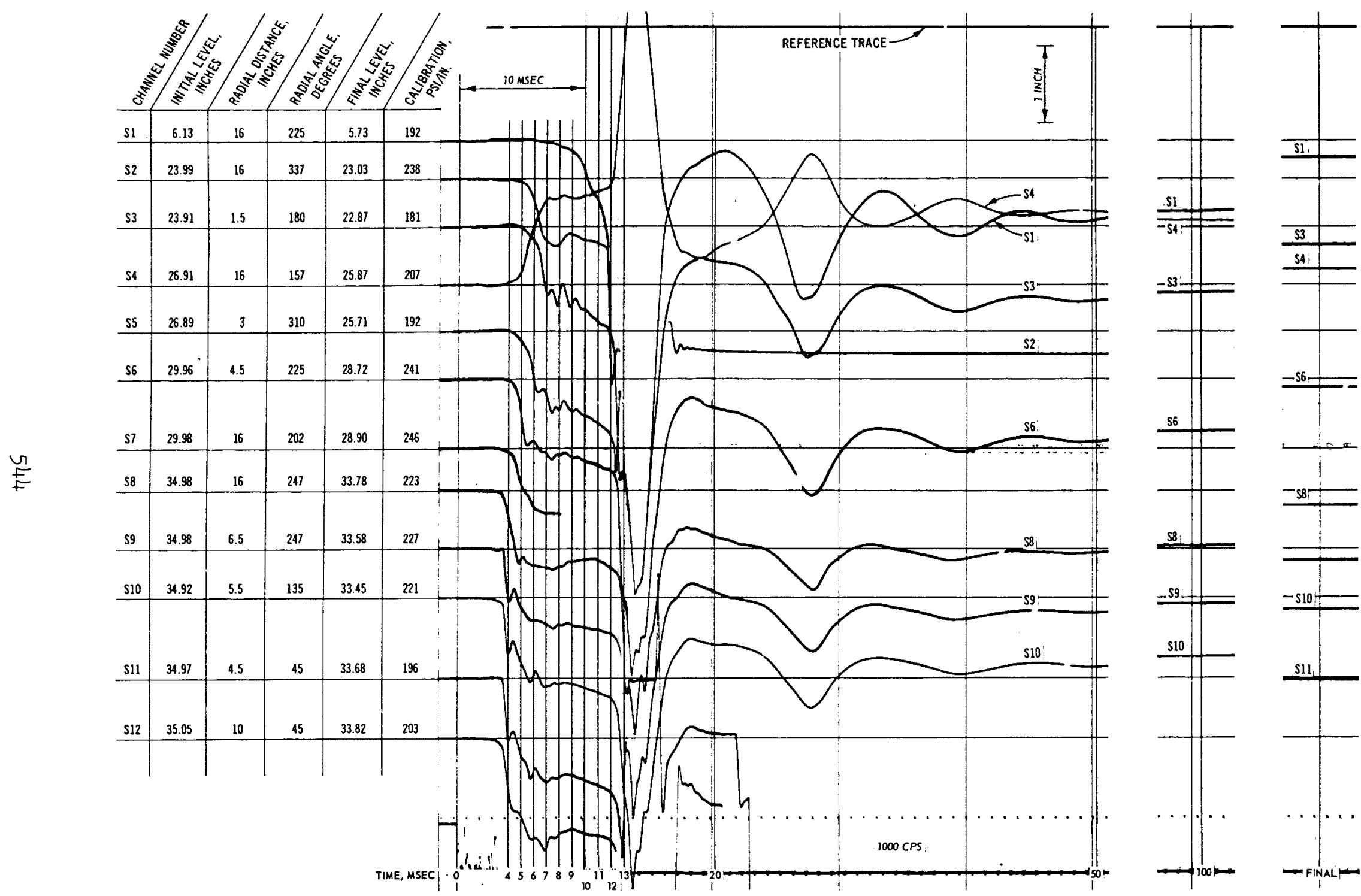

Fig. C-14. Test 14, pressure record (SI-S12) 


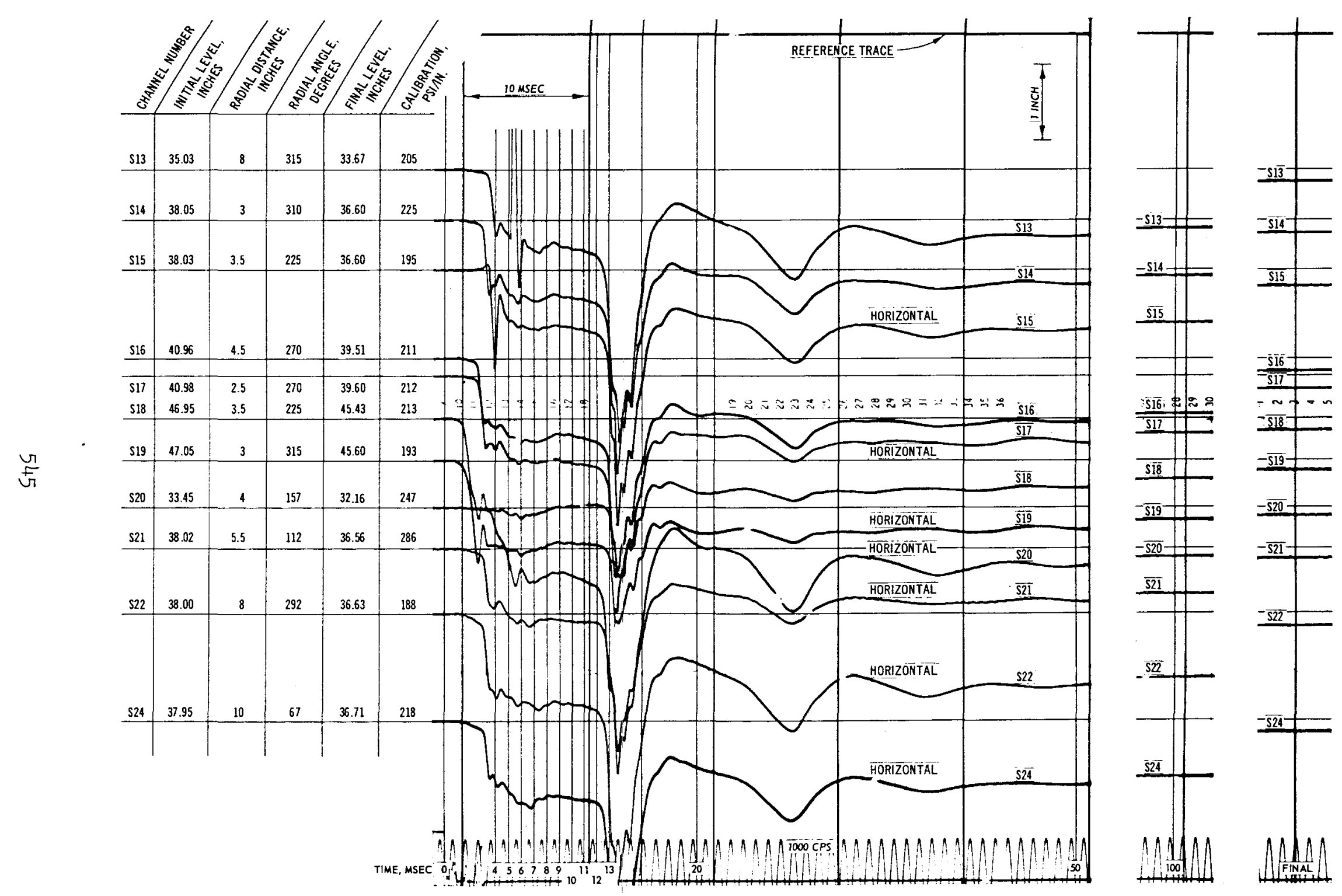

Fig. C-15. Test 14, pressure record (S13-S24) 

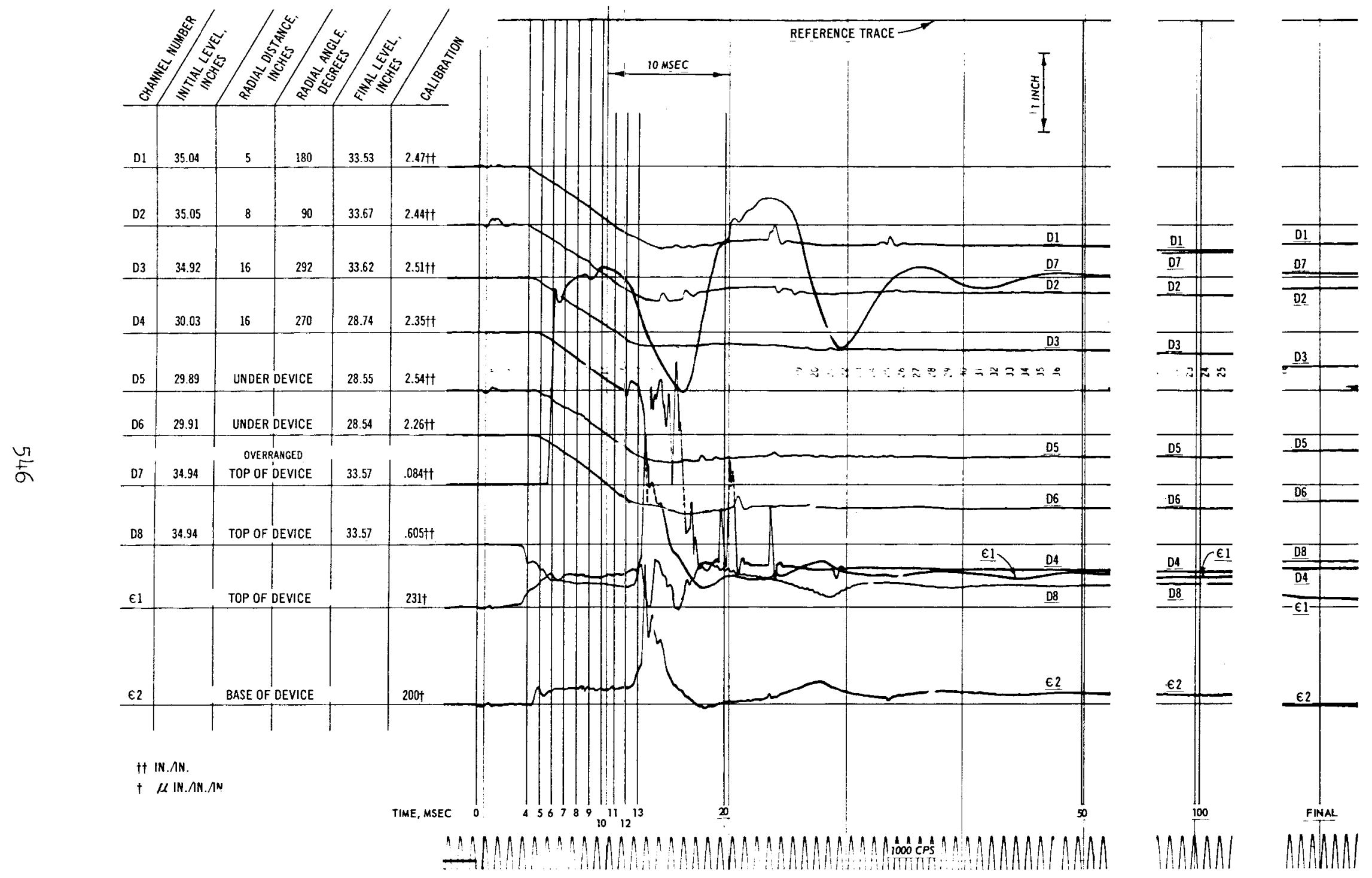

Fig. C-16. Test 14, deflection and strain record 


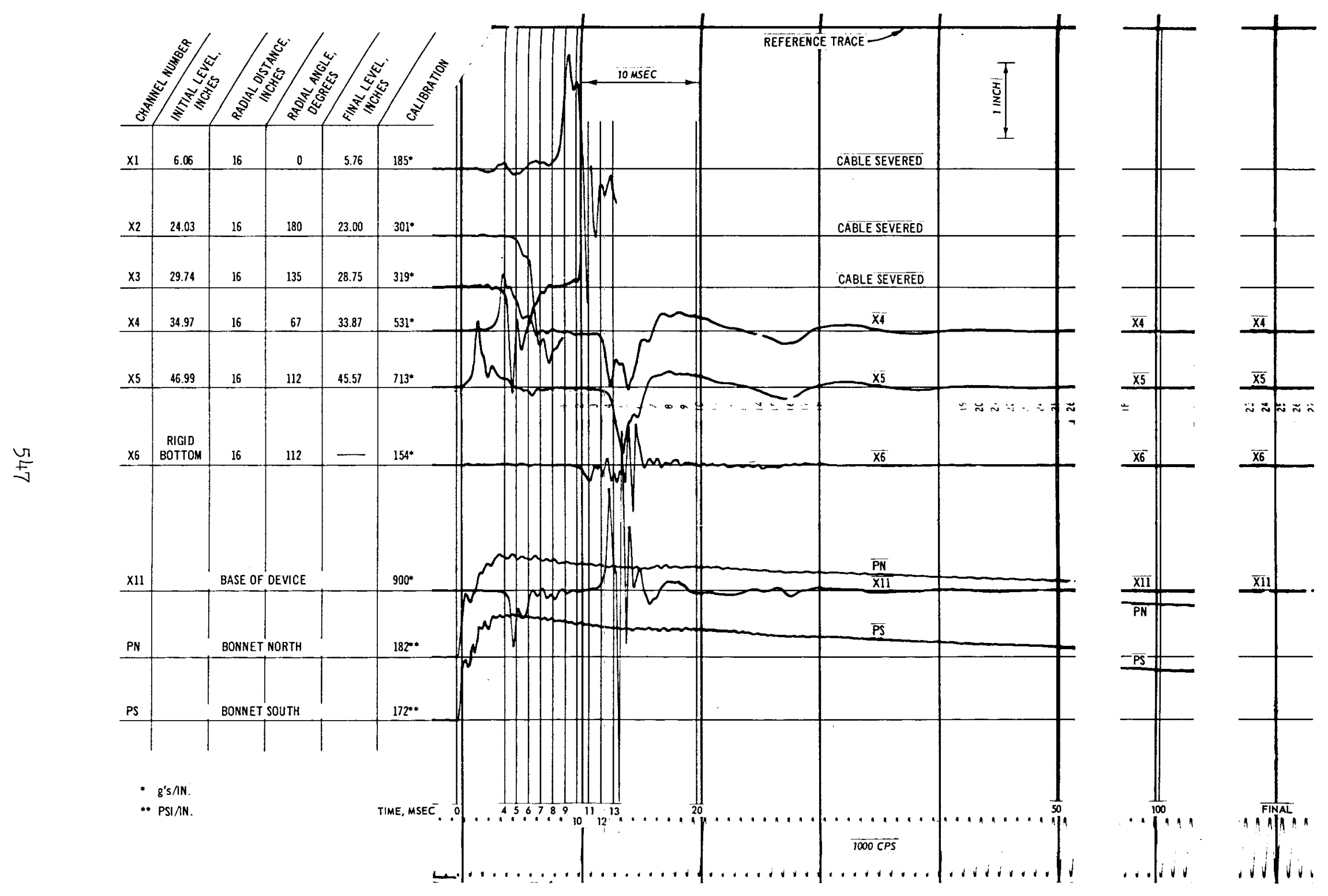

Fig. C-17. Test 14, acceleration and pressure record 

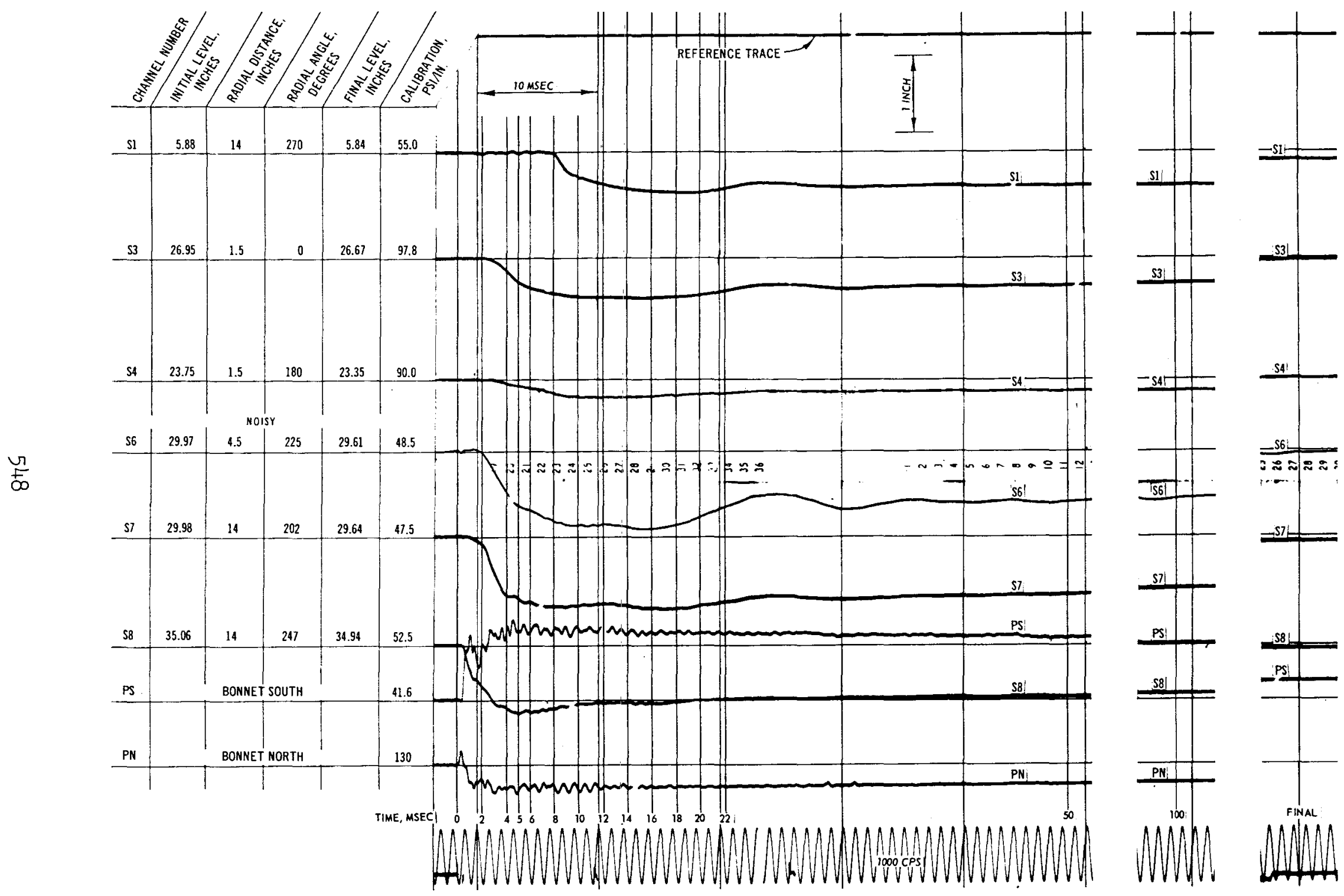

Fig. C-18. Test 15, pressure record (SI-S8) 


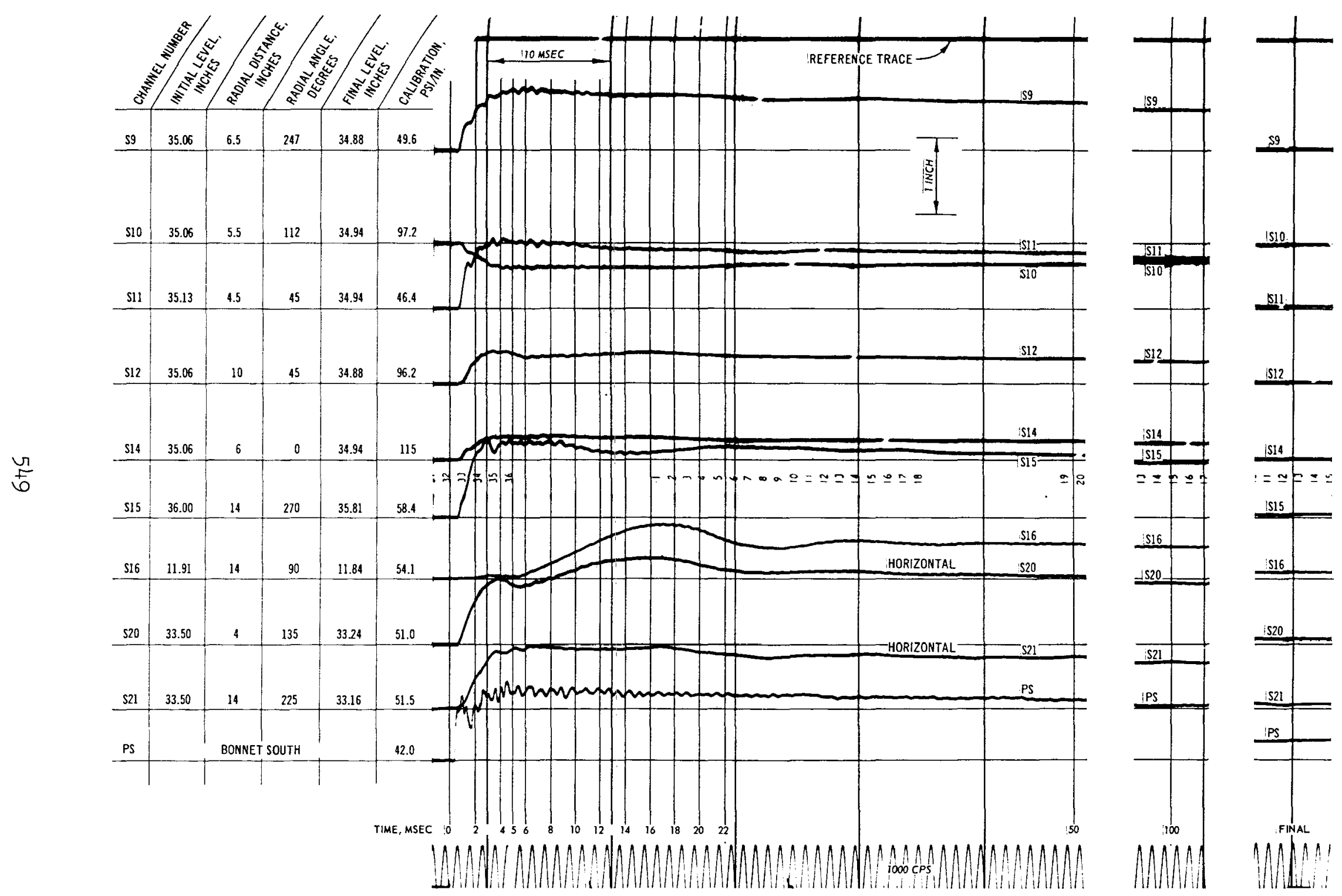

Fig. C-19. Test 15, pressure record (S9-S21) 

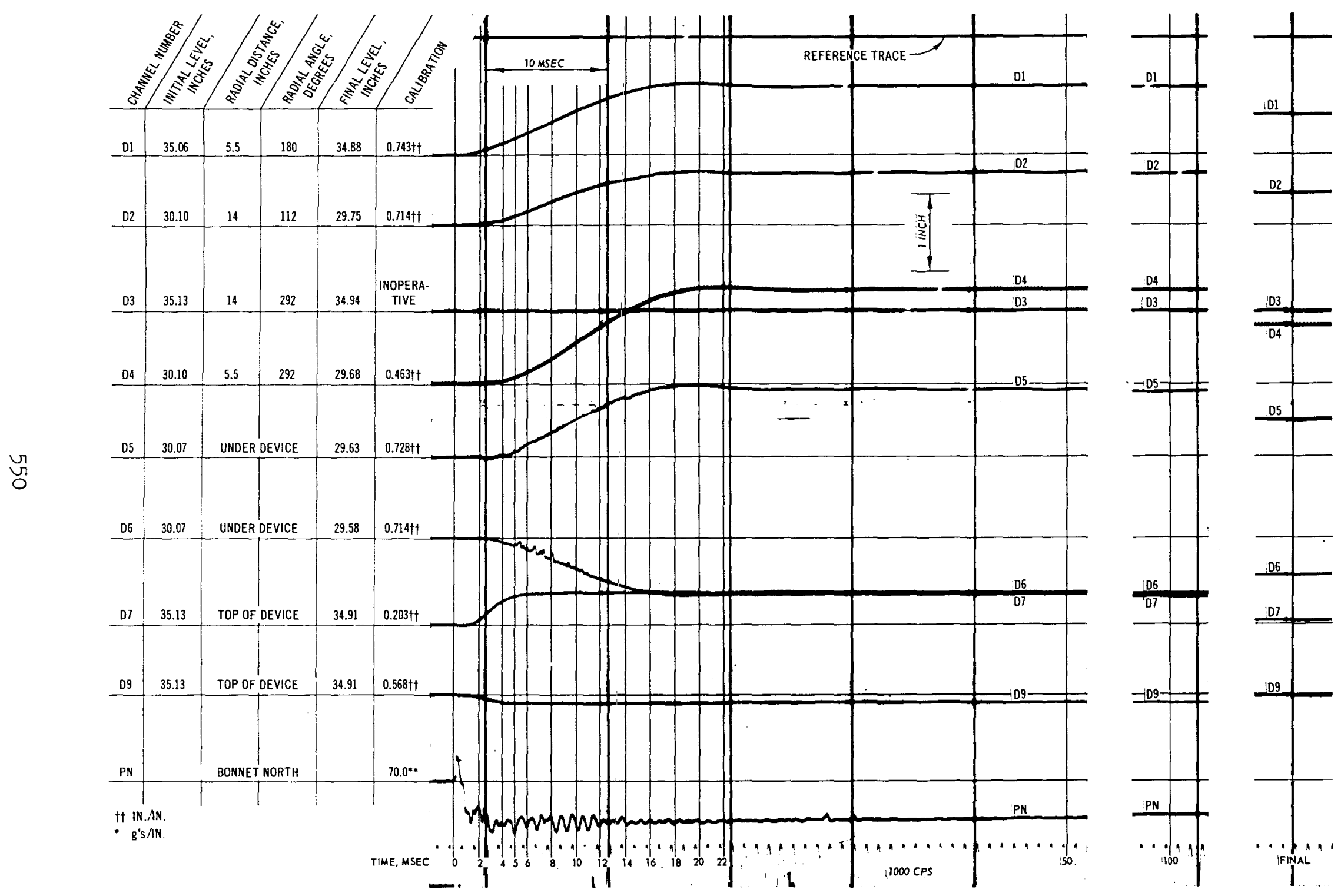

Fig. C-20. Test 15, deflection and pressure record 


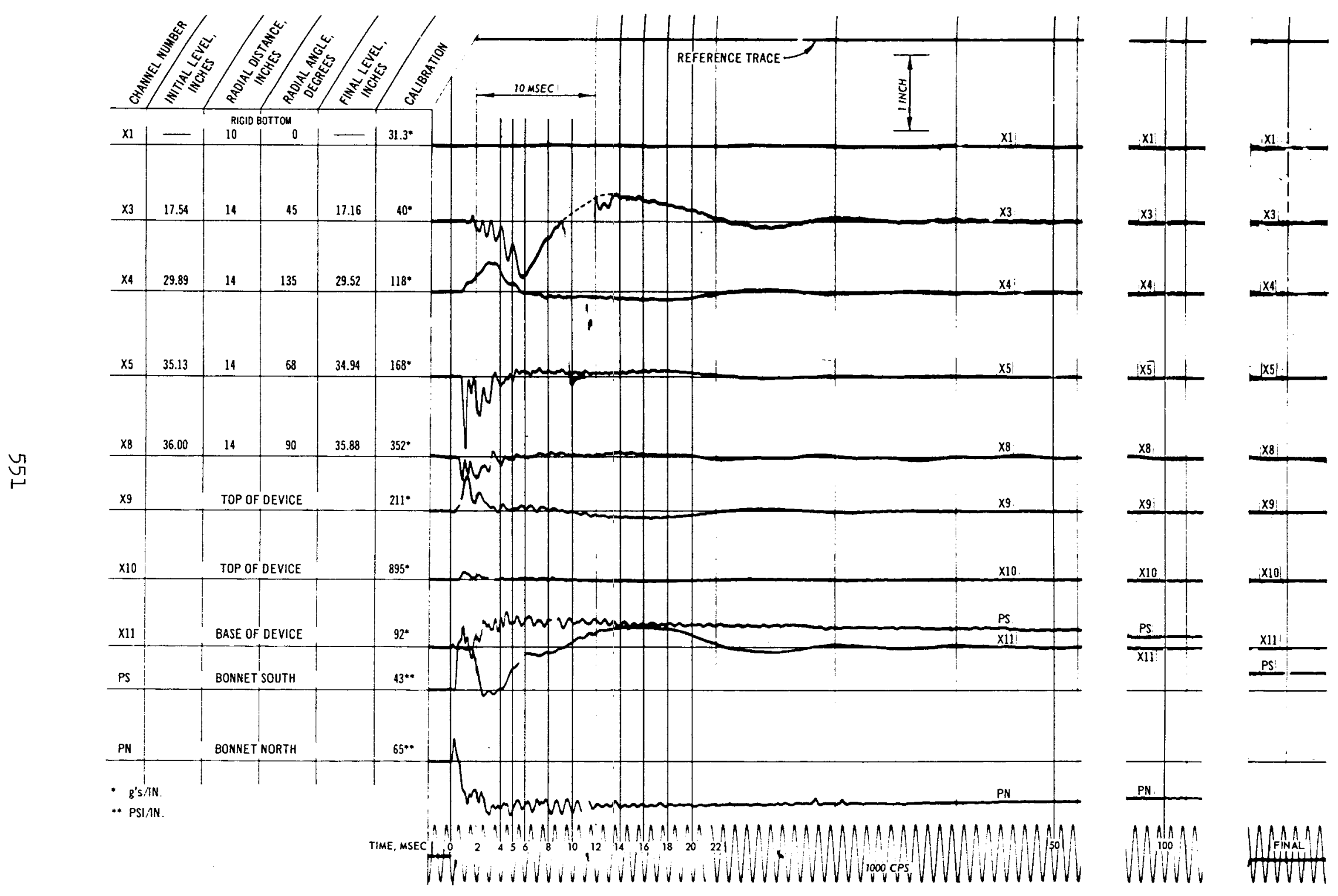

Fig. C-21. Test 15, acceleration and pressure record 

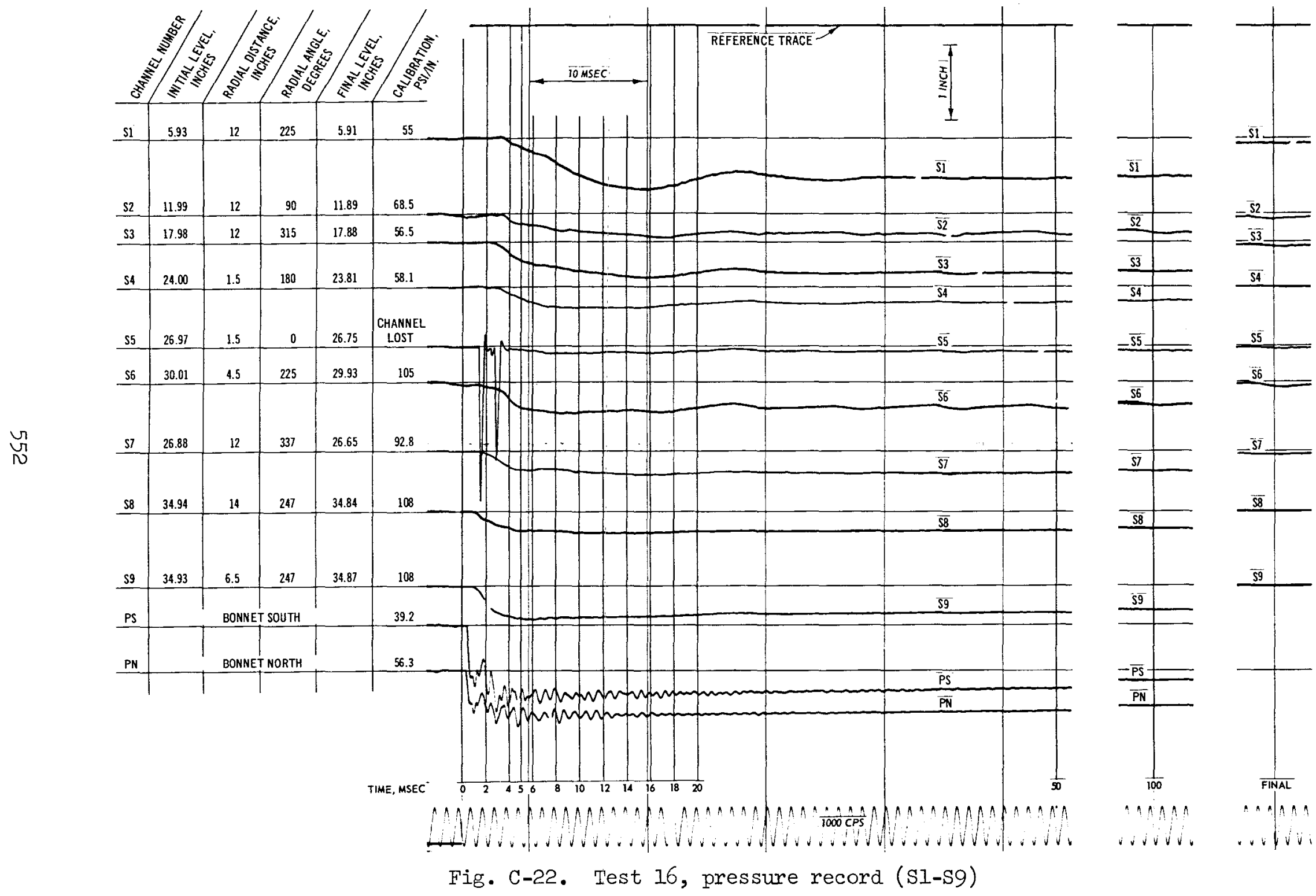

Fig. C-22. Test 16, pressure record (S1-S9) 


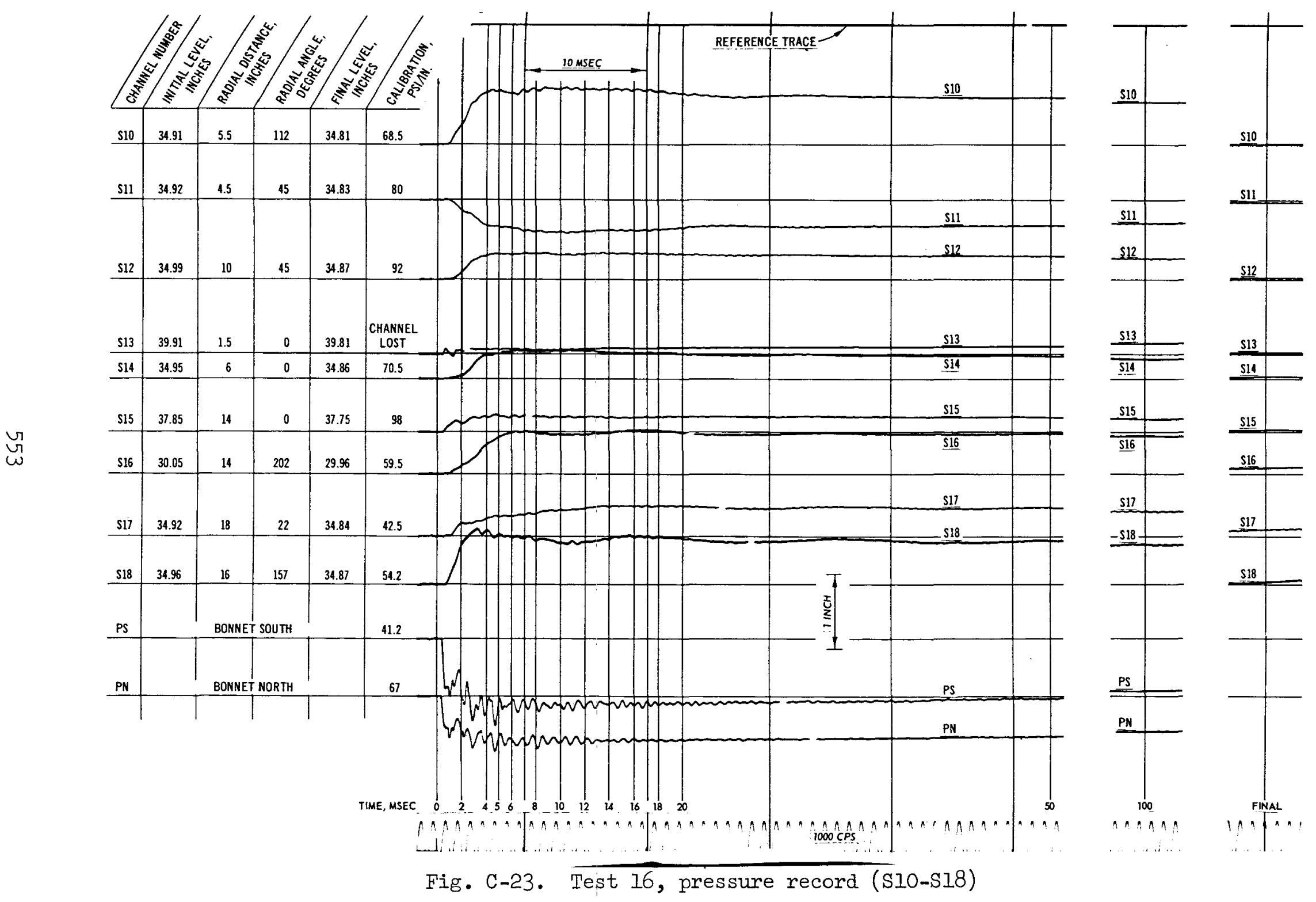



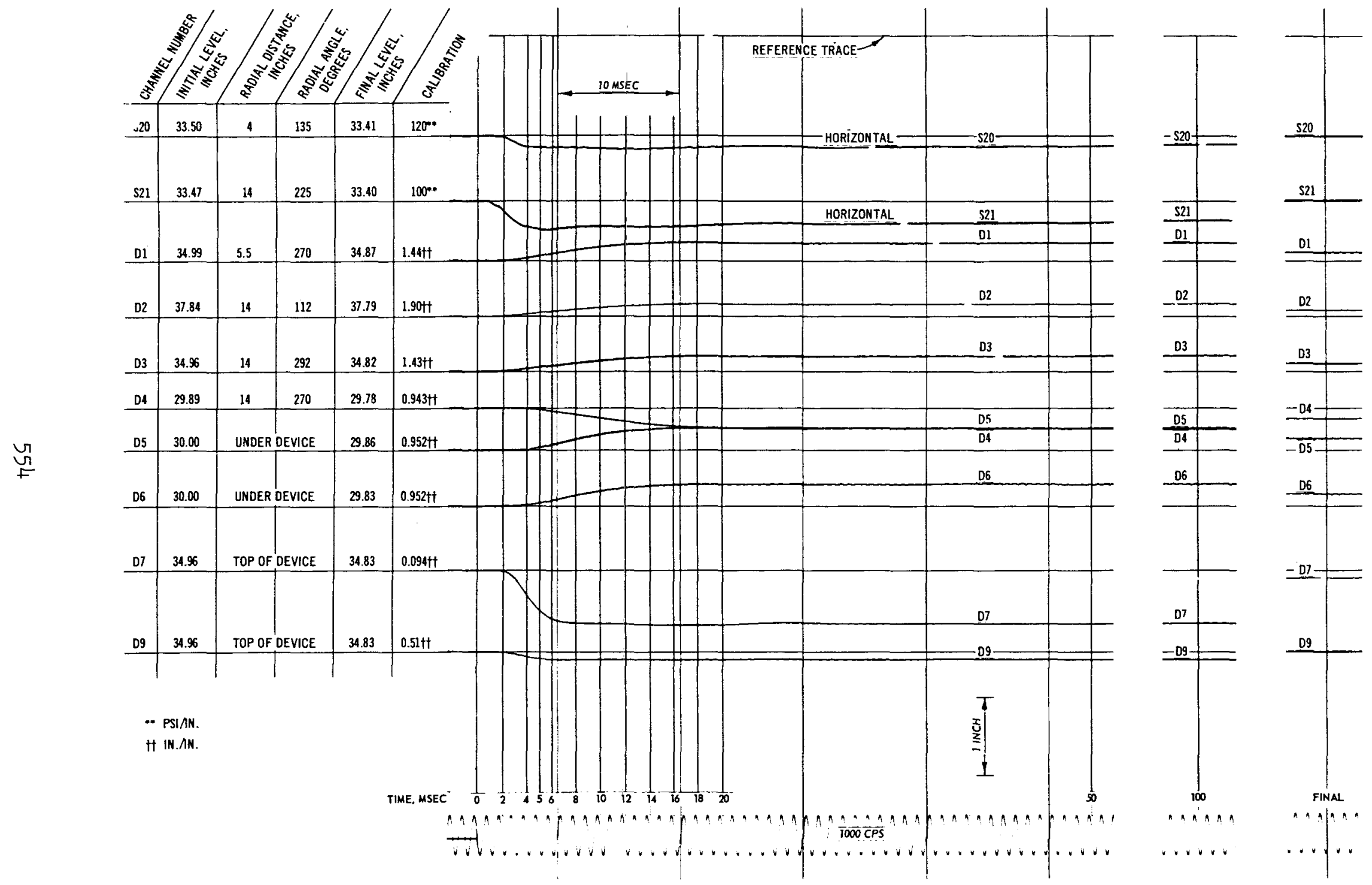

Fig. C-24. Test 16, pressure and deflection record 

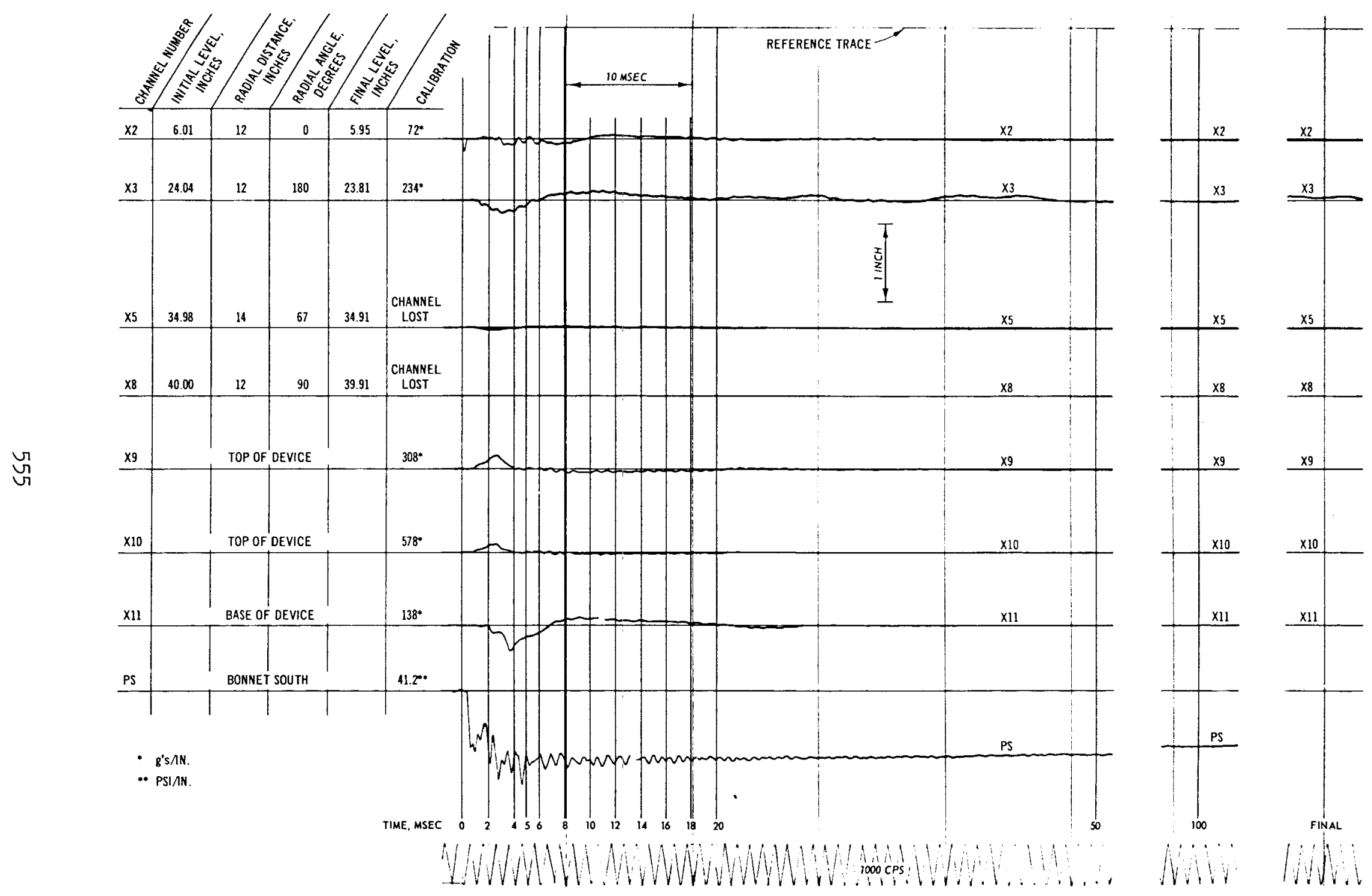

Fig. C-25. Test 16, acceleration and pressure record 

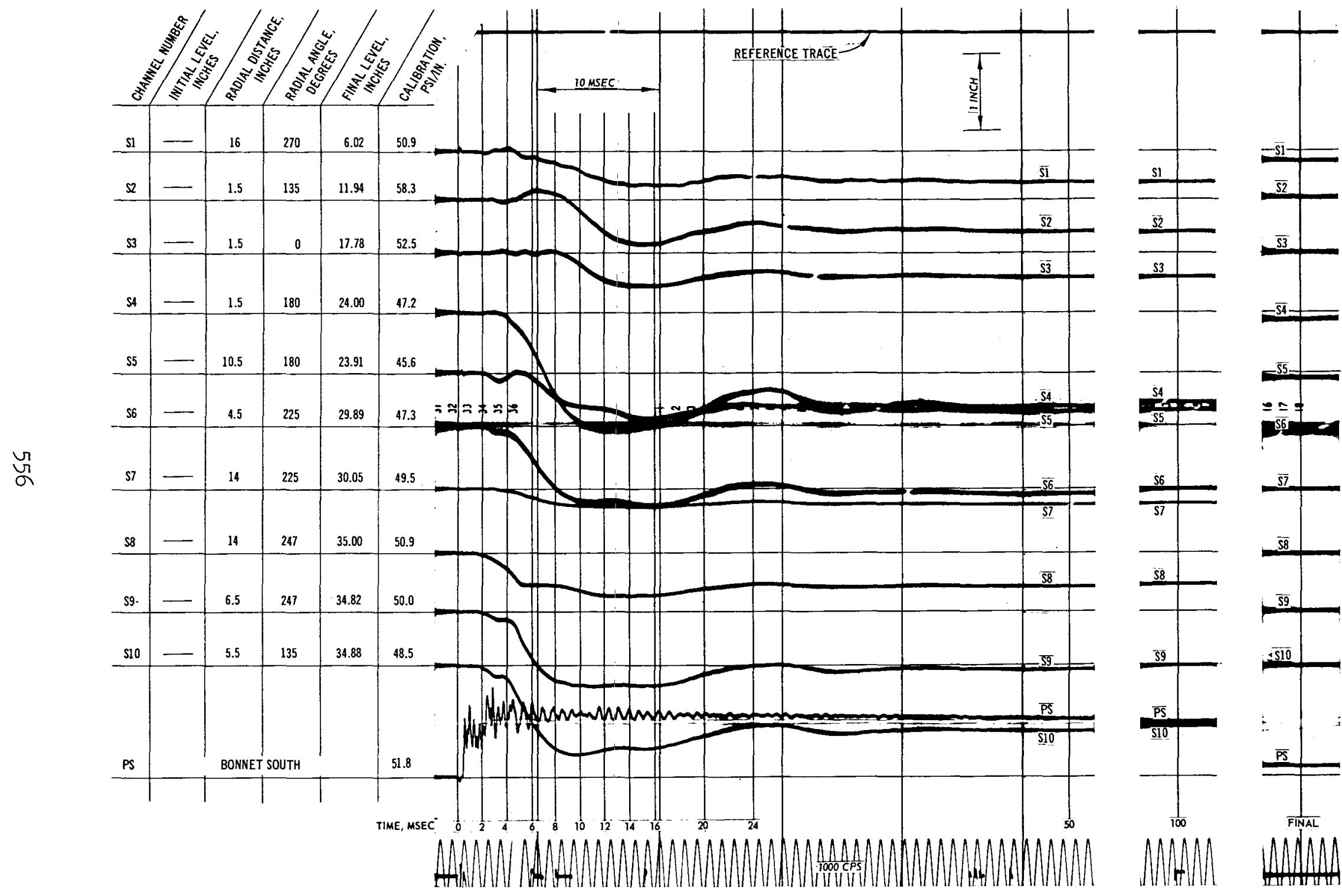

Fig. C-26. Test 17, pressure record (SI-S10) 

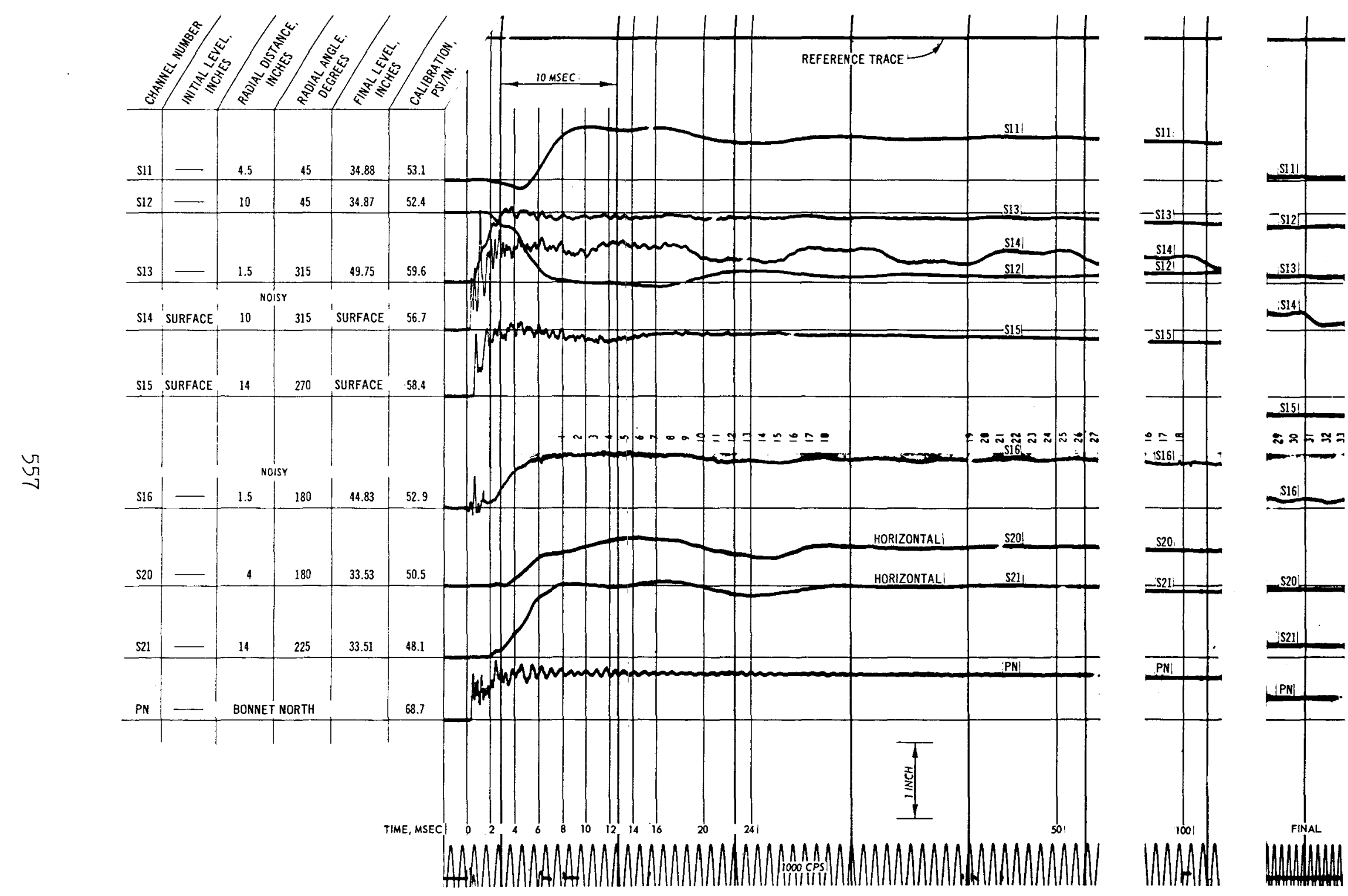

Fig. C-27. Test 17, pressure record (S1工-S21) 

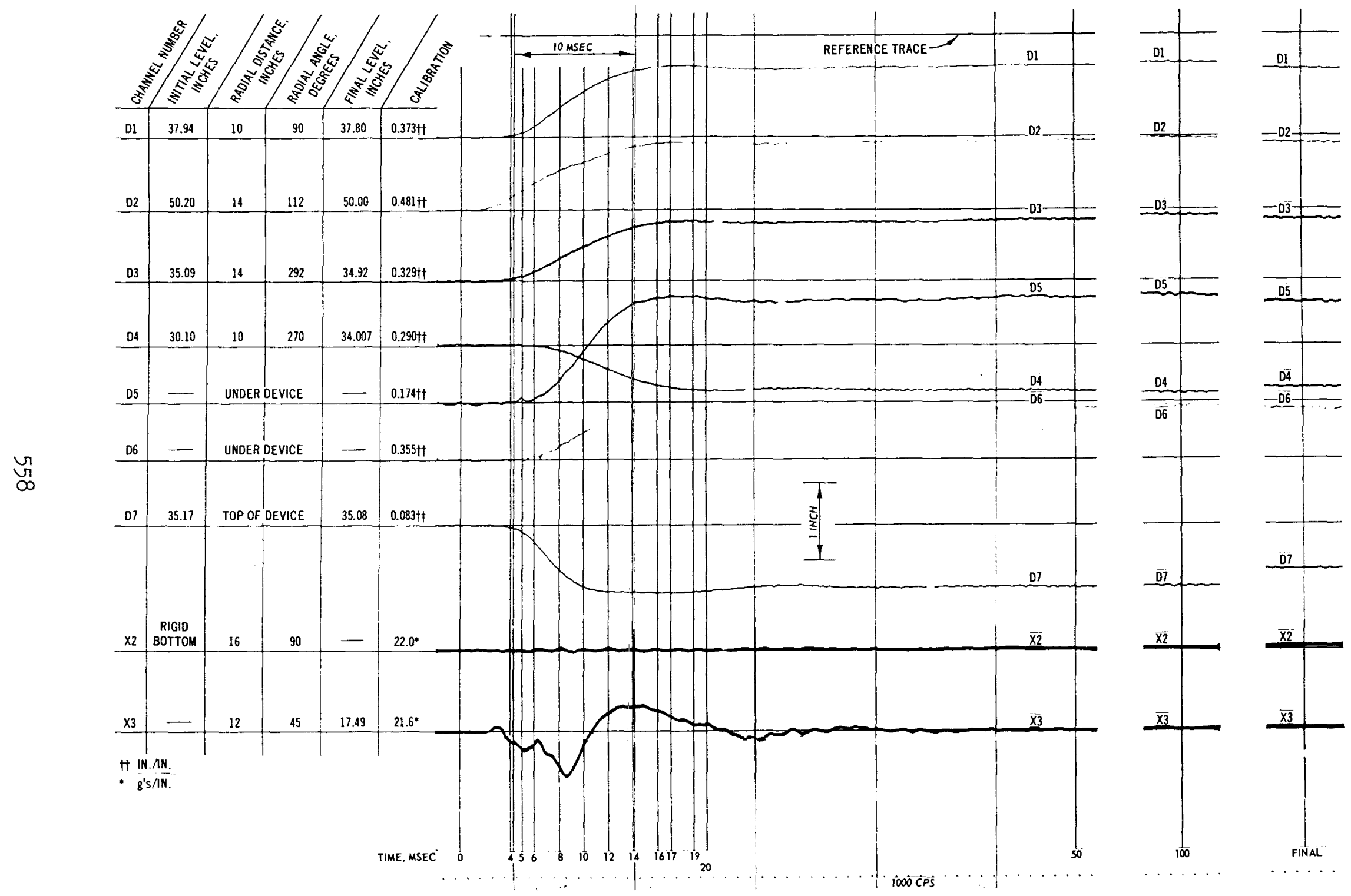

Fig. C-28. Test 17, deflection and acceleration record 


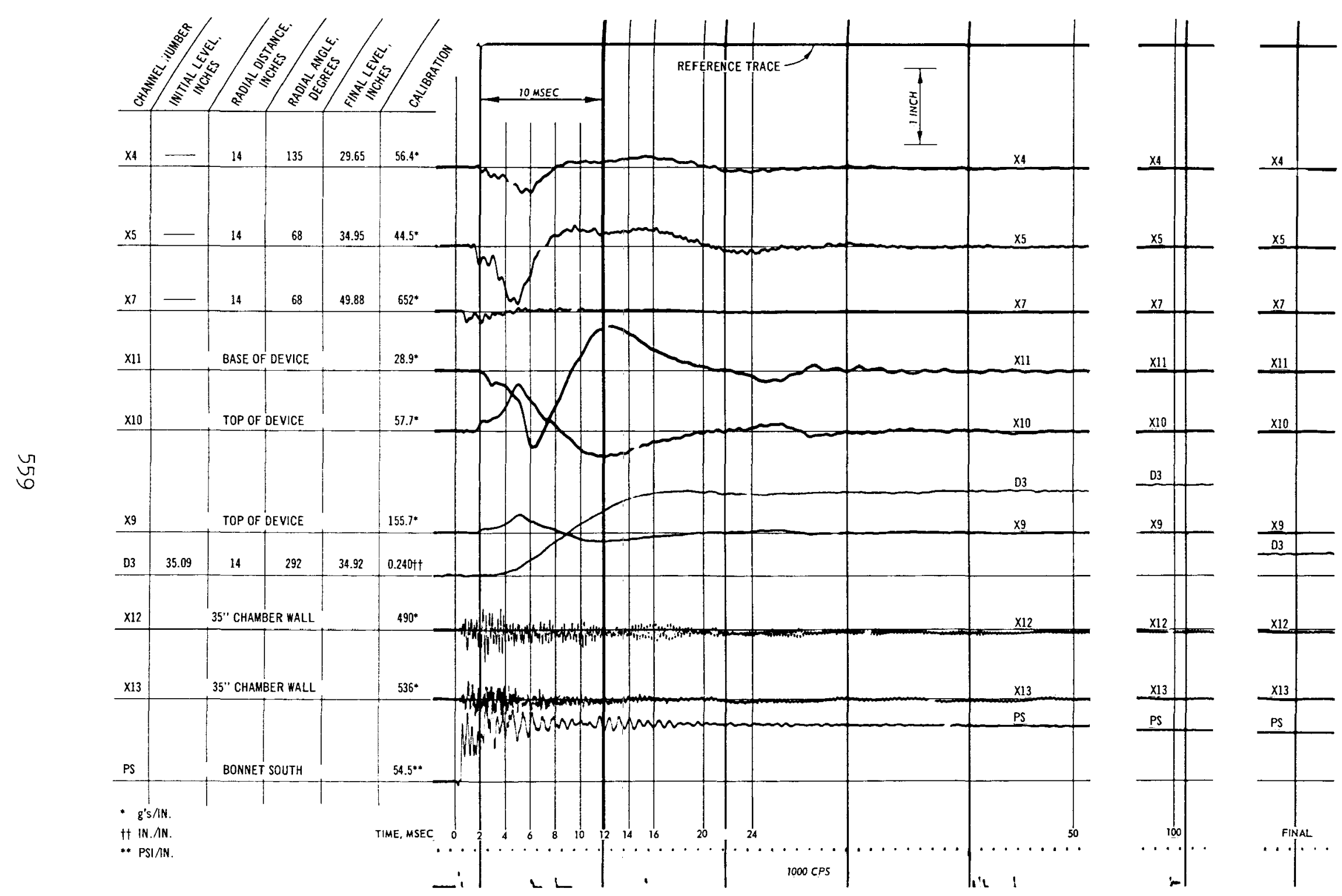

Fig. C-29. Test 17, acceleration, deflection, and pressure record 

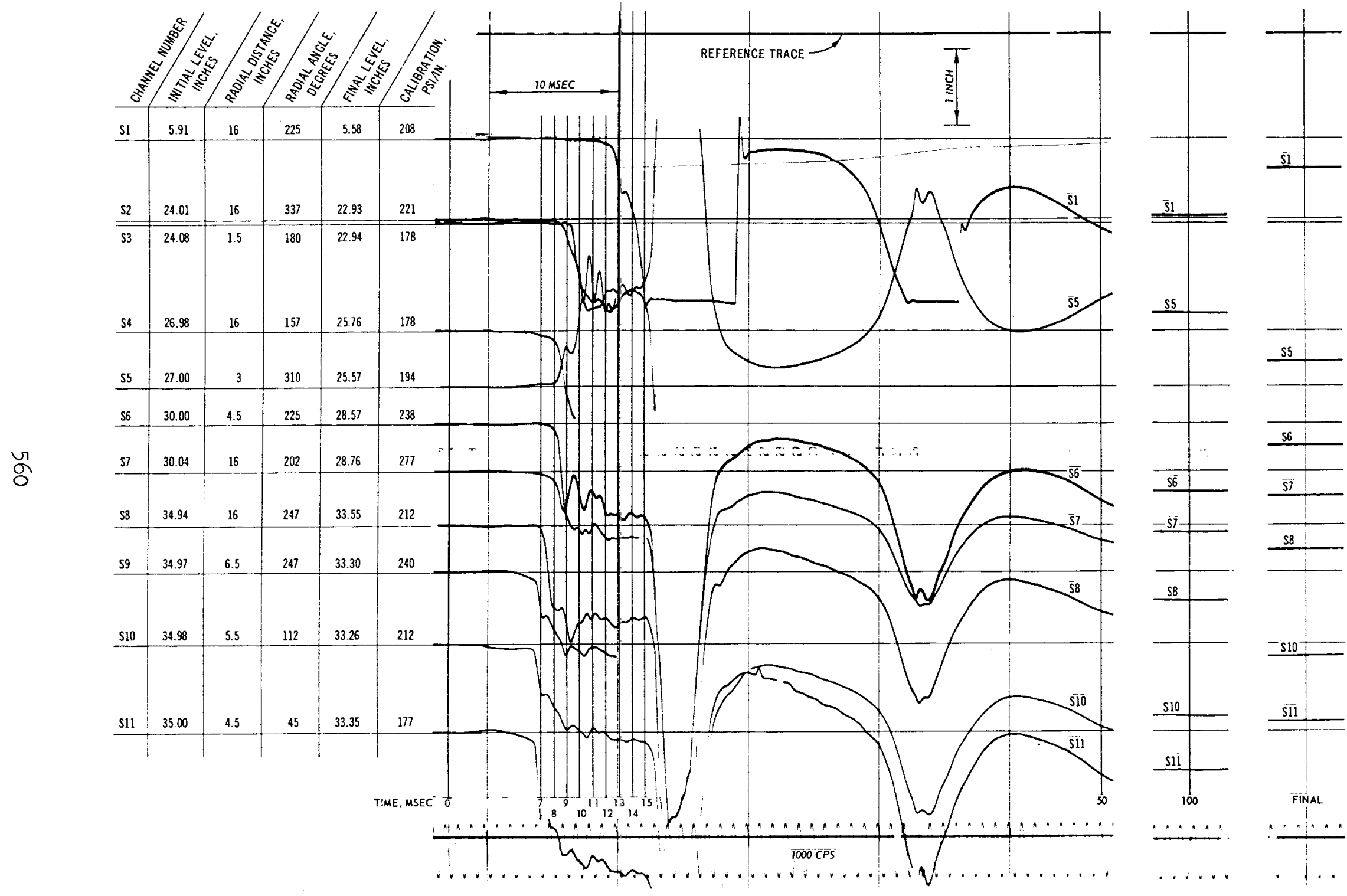

Fig. C-30. Test 18, pressure record (SI-SII) 


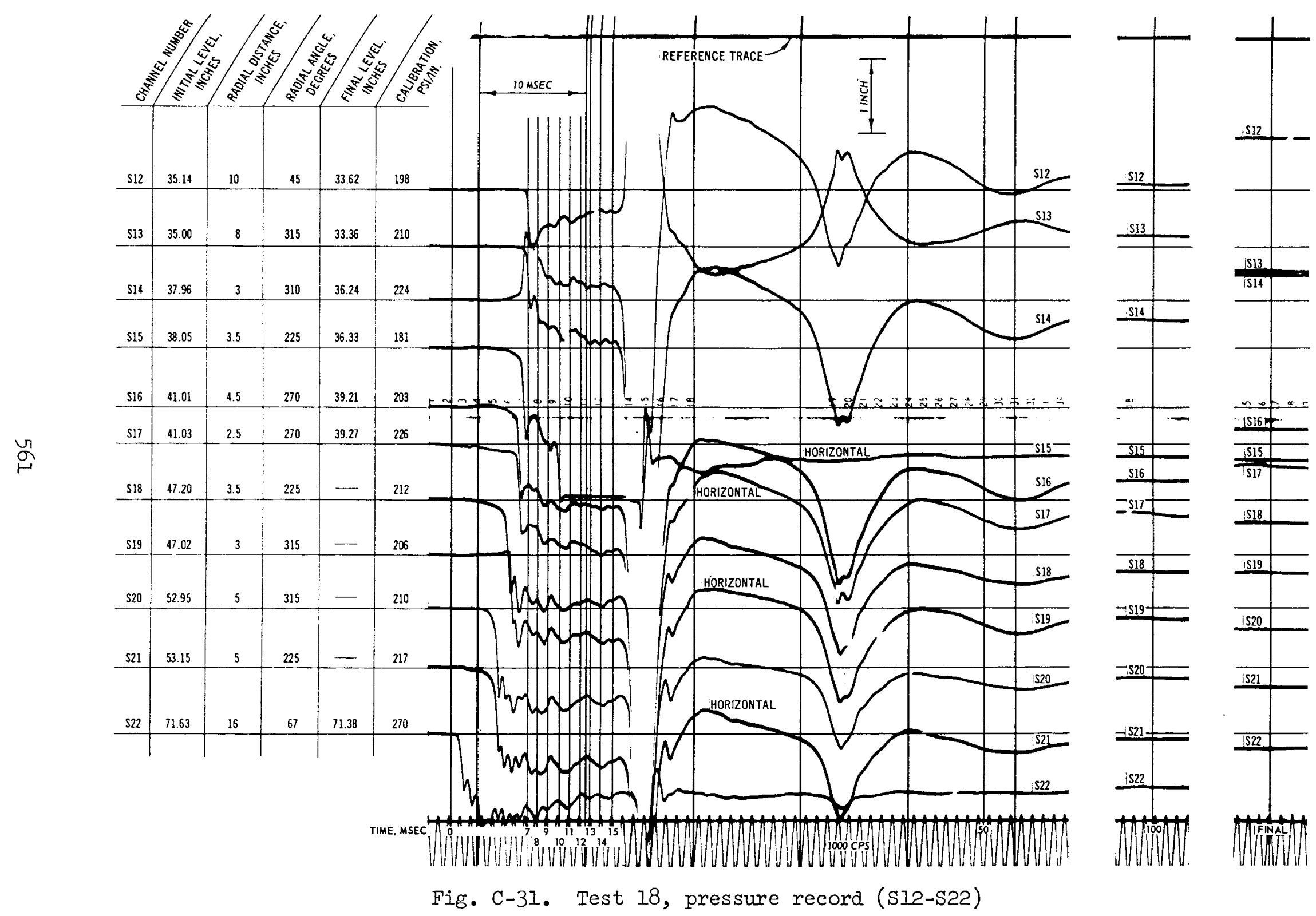




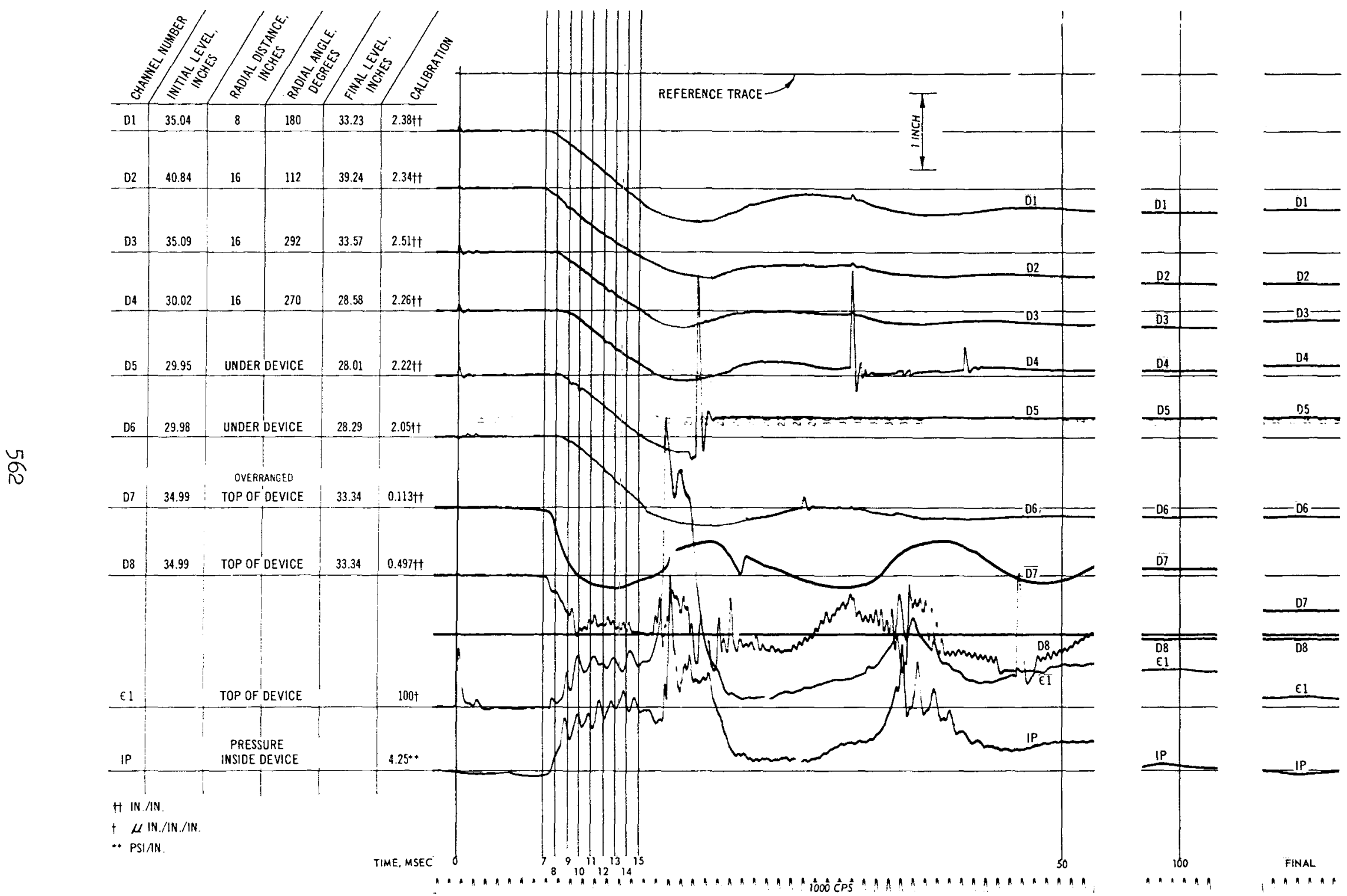

Fig. C-32. Test 18, deflection, strain, and pressure record 


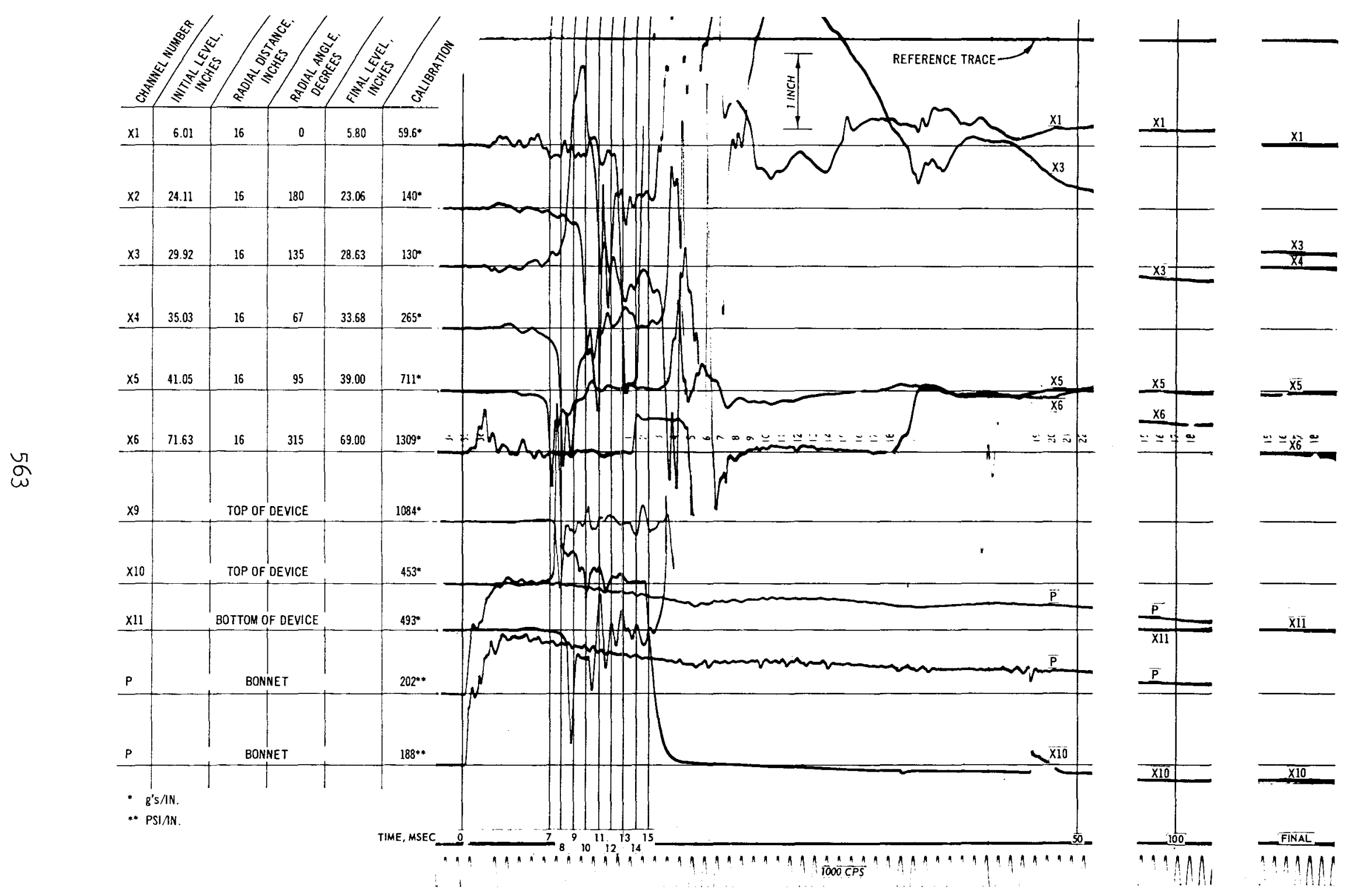

Fig. C-33. Test 18, acceleration and pressure record 

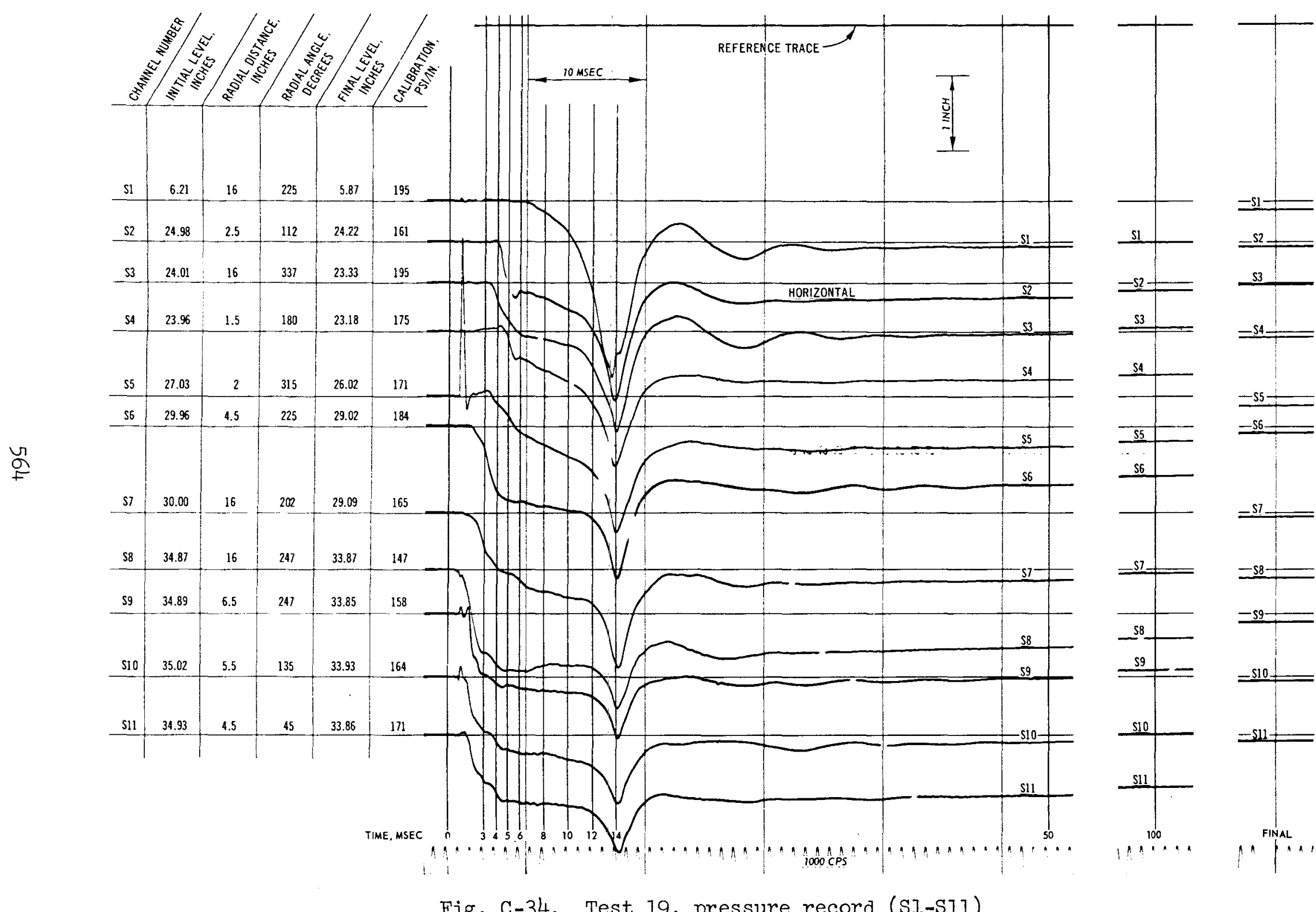

Fig. C-34. Test 19, pressure record (SI-SII) 

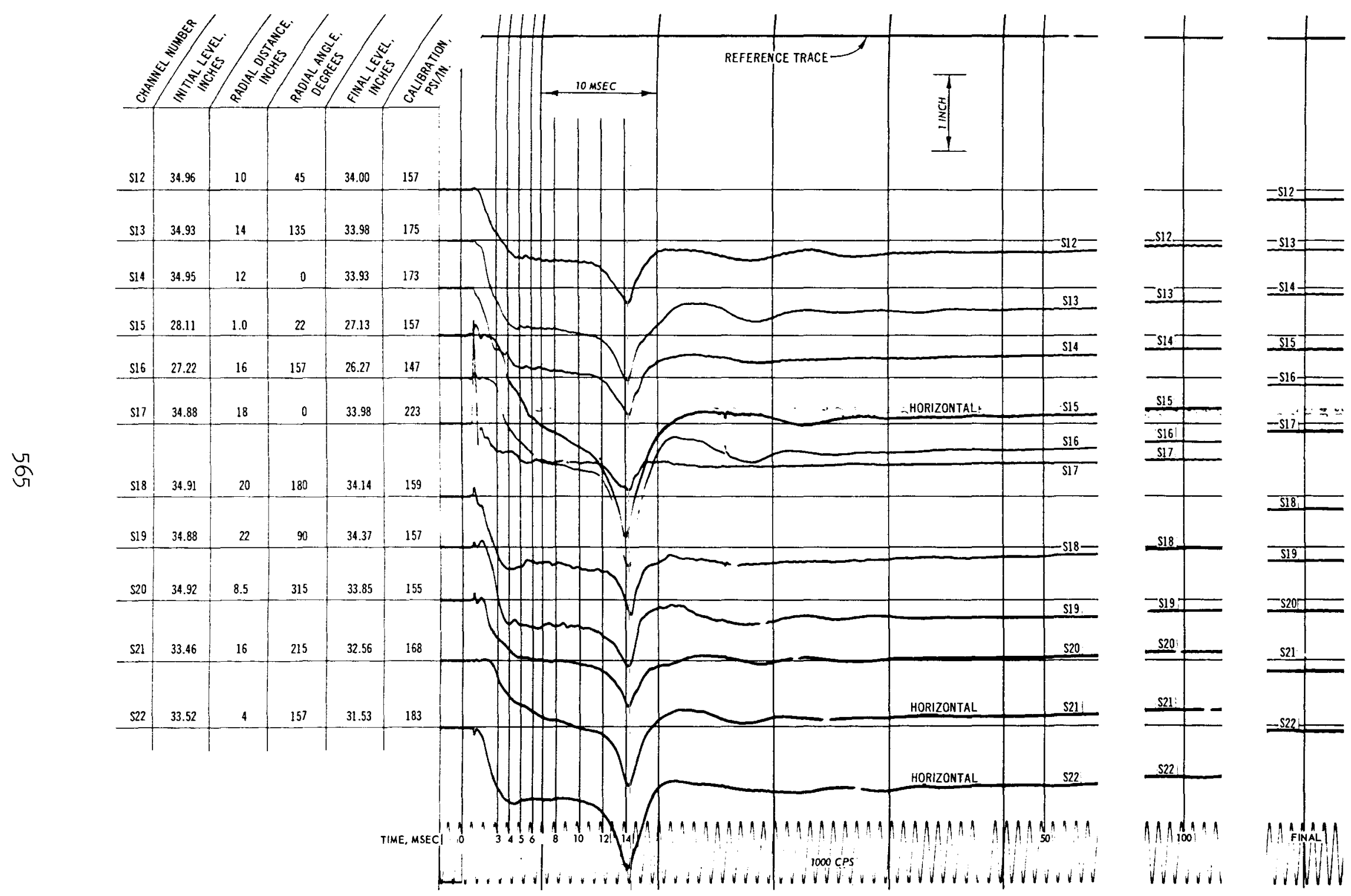

Fig. C-35. Test 19, pressure record (S12-S22) 

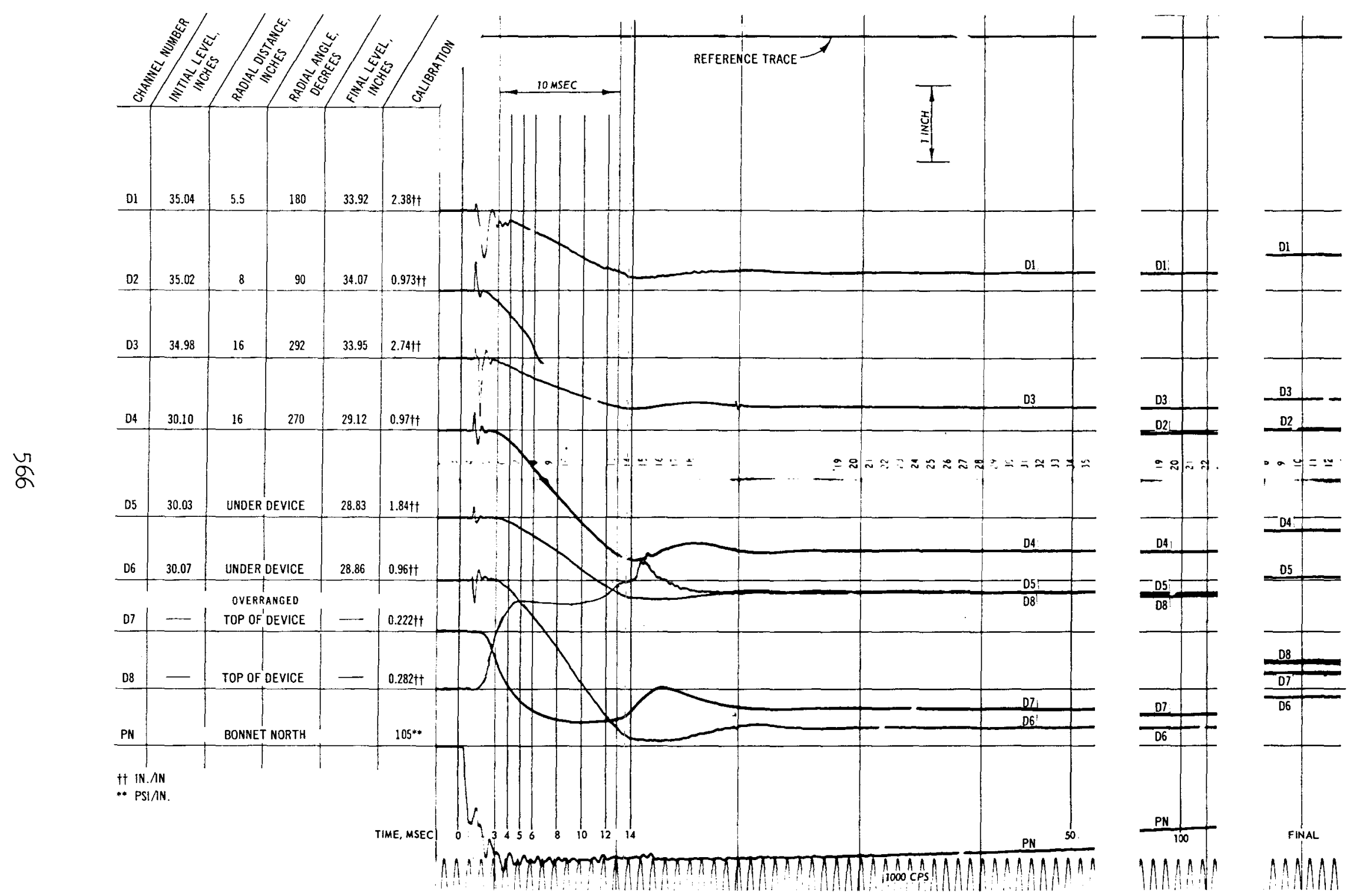

Fig. C-36. Test 19, deflection and pressure record 

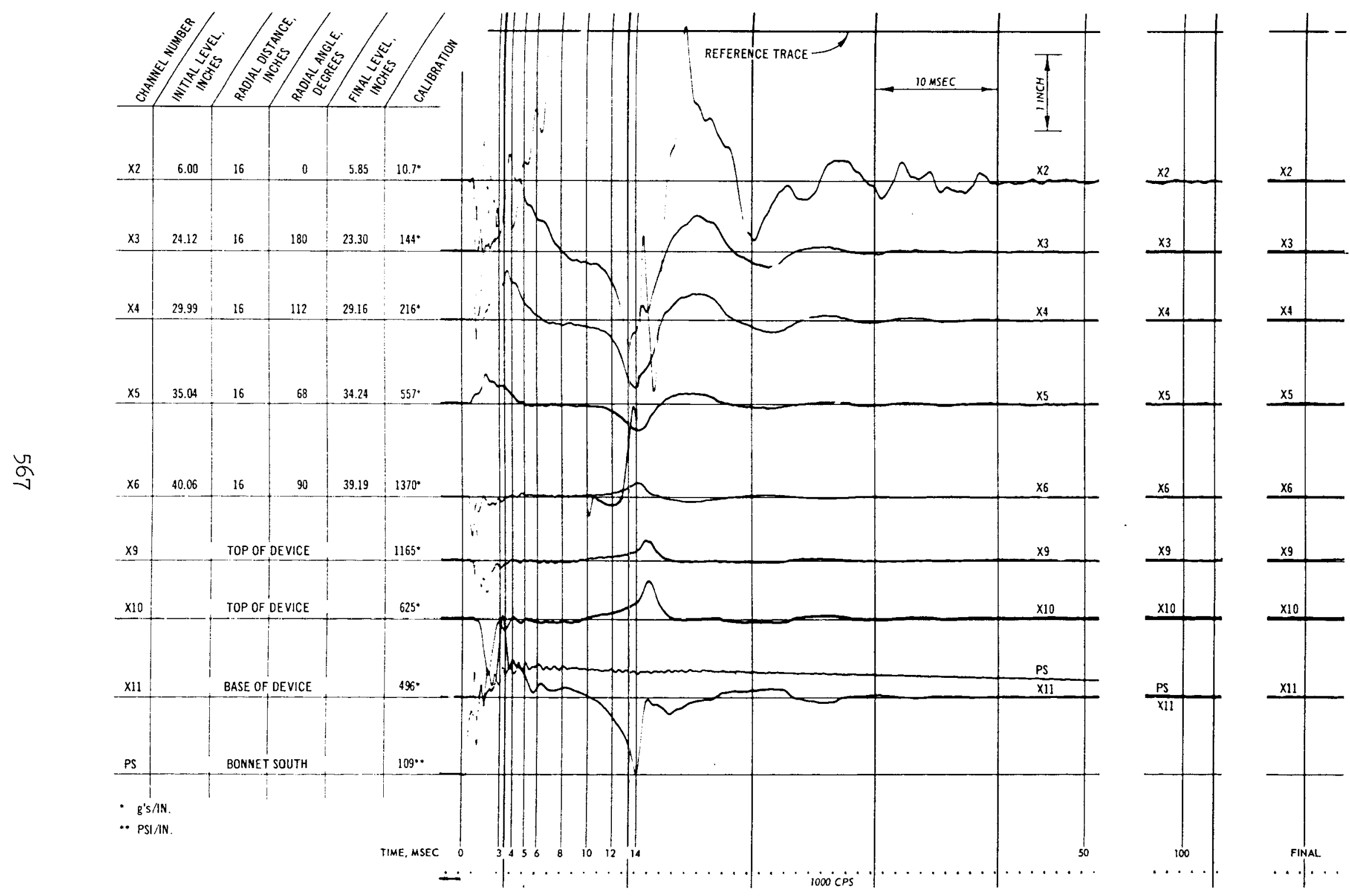

Fig. C-37. Test 19, acceleration and pressure record 


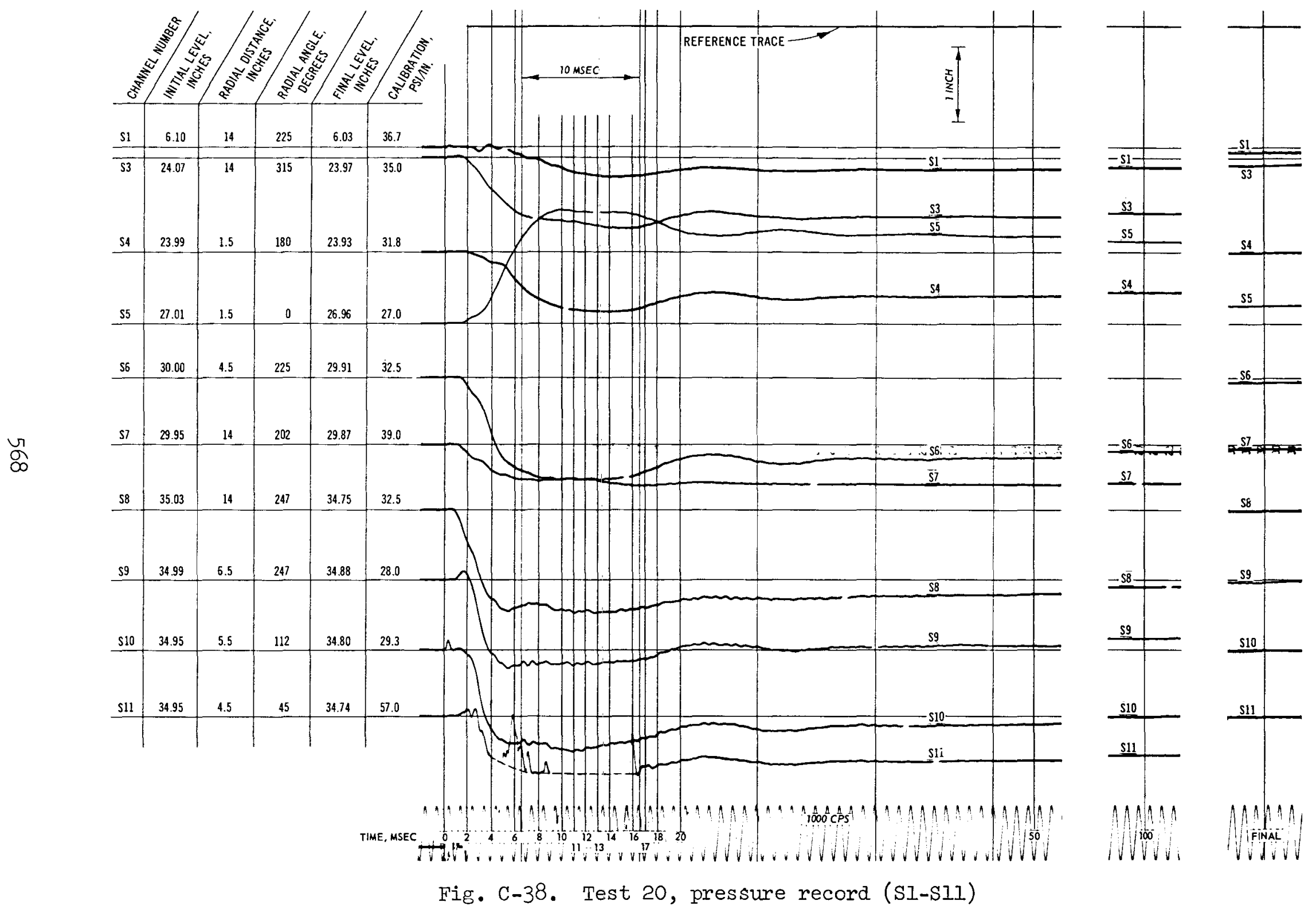




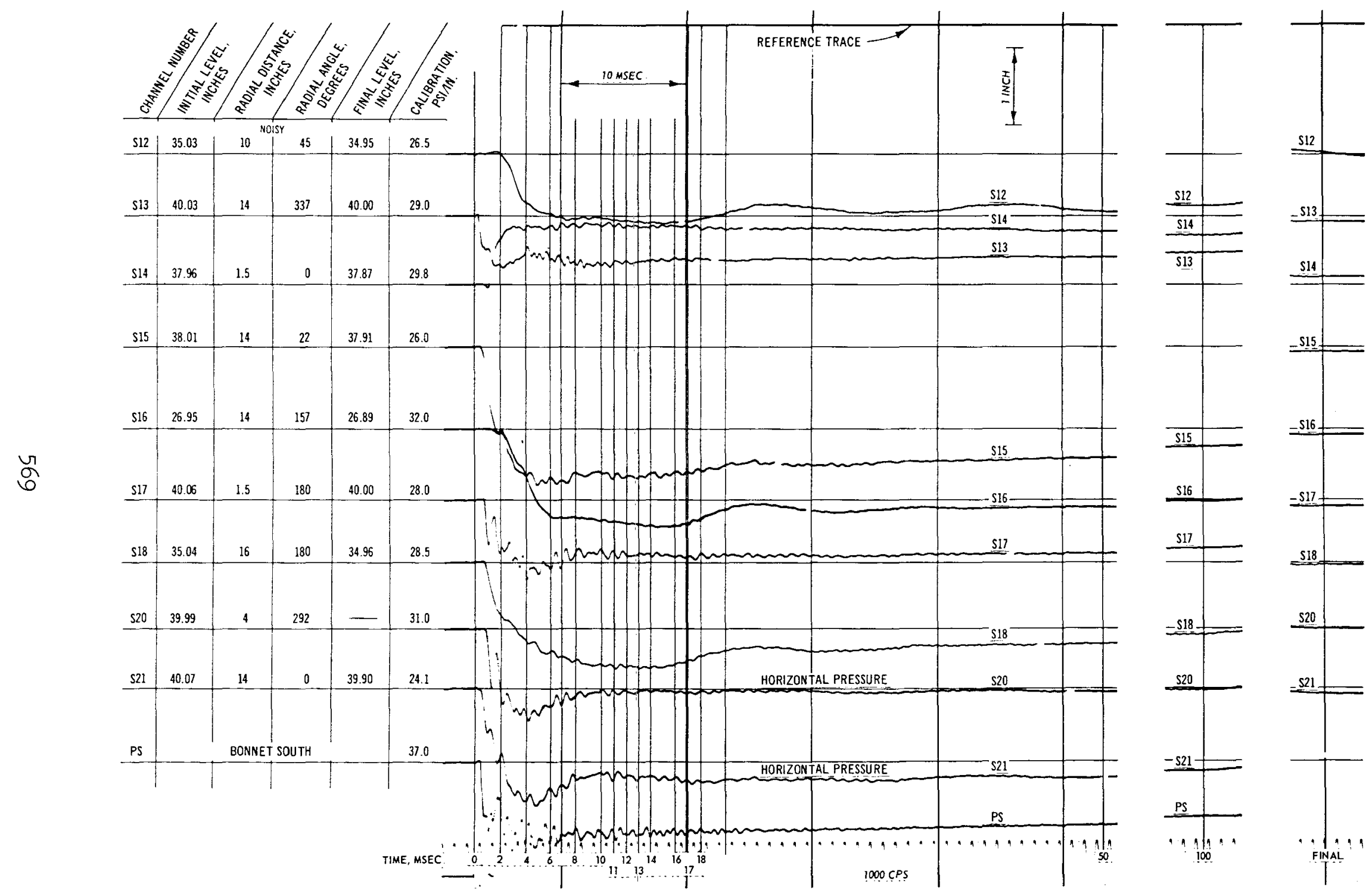

Fig. C-39. Test 20, pressure record (S12-S21) 

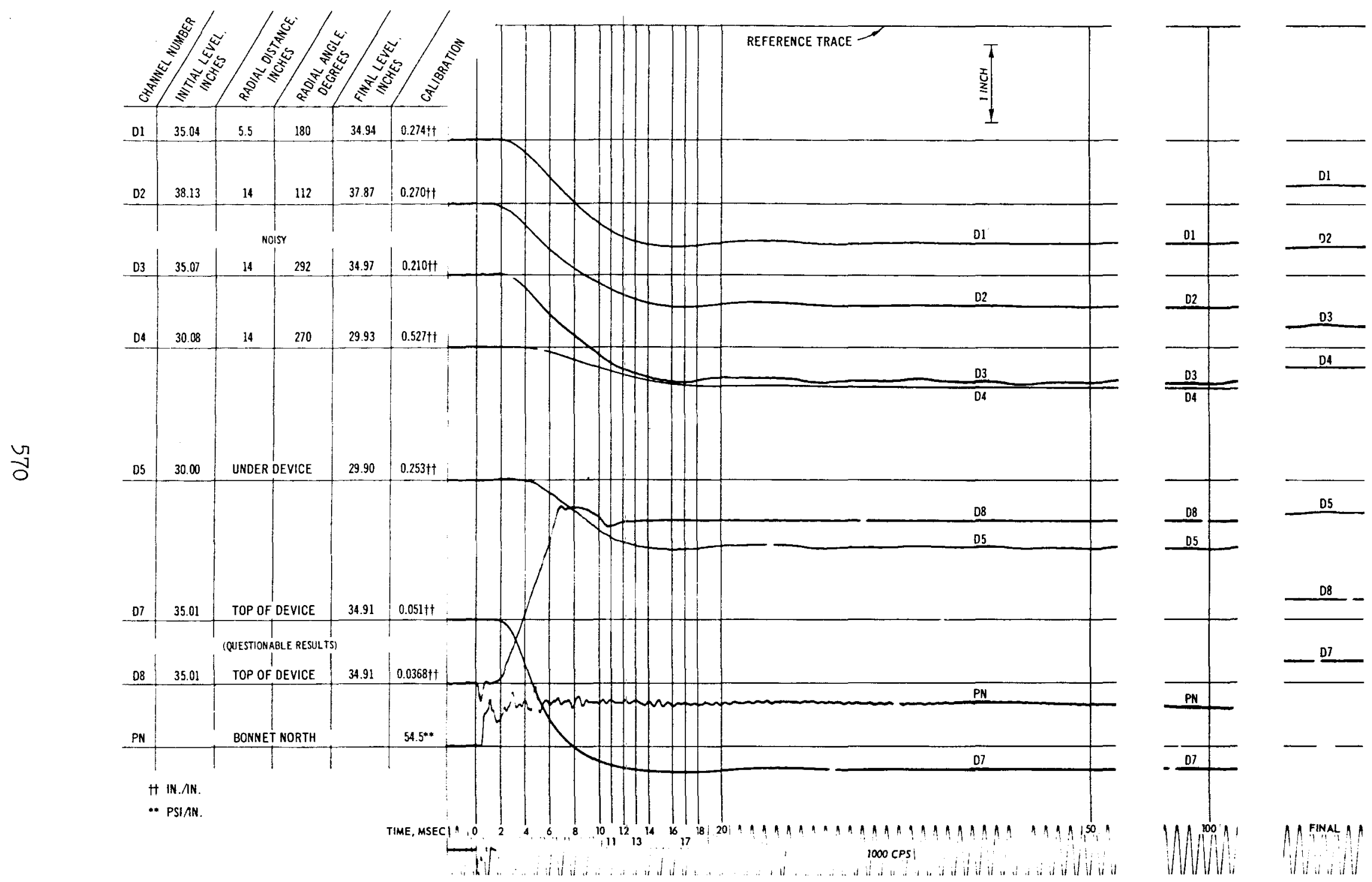

Fig. C-40. Test 20, deflection and pressure record 


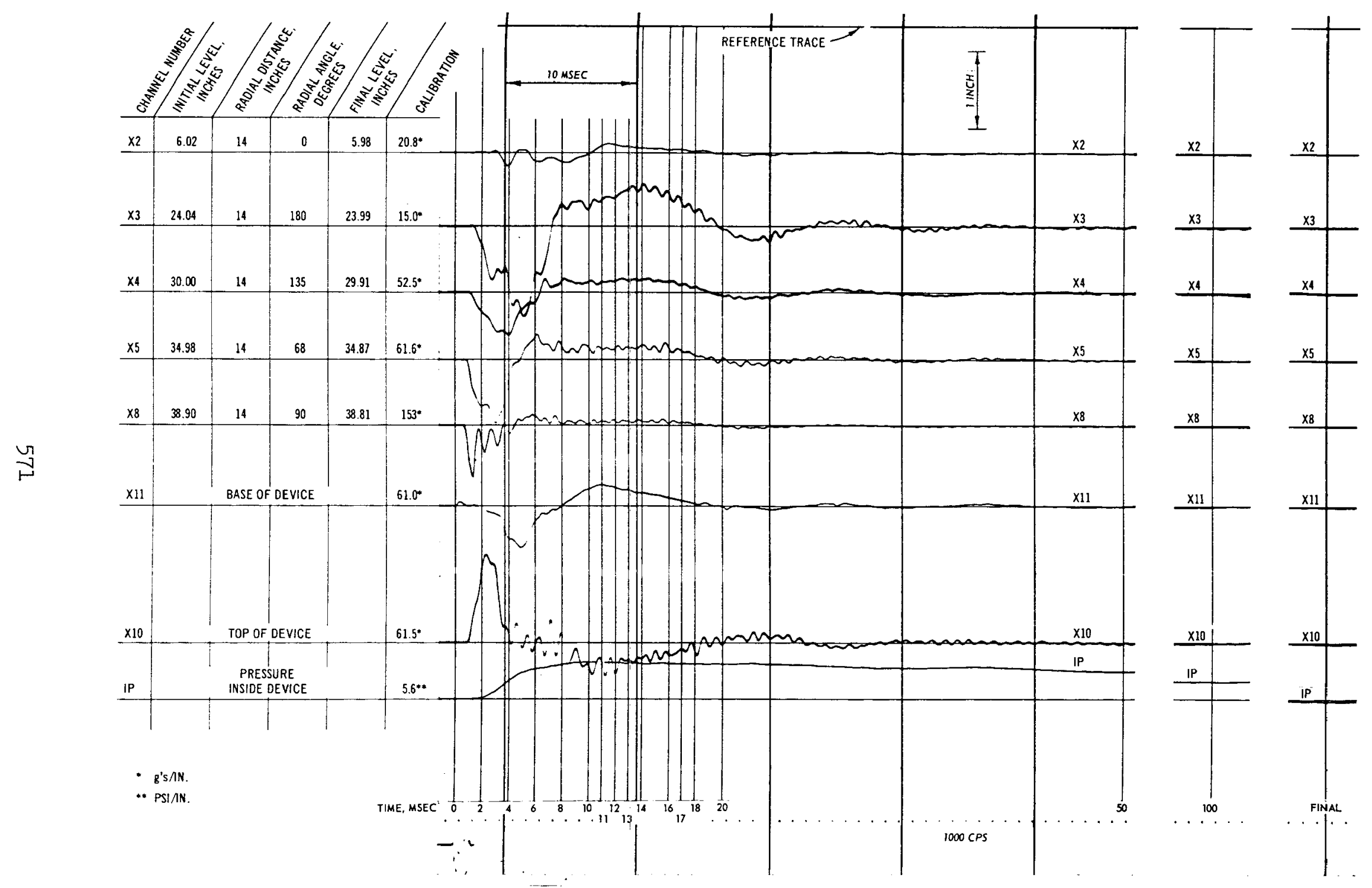

Fig. C-41. Test 20, acceleration and pressure record 

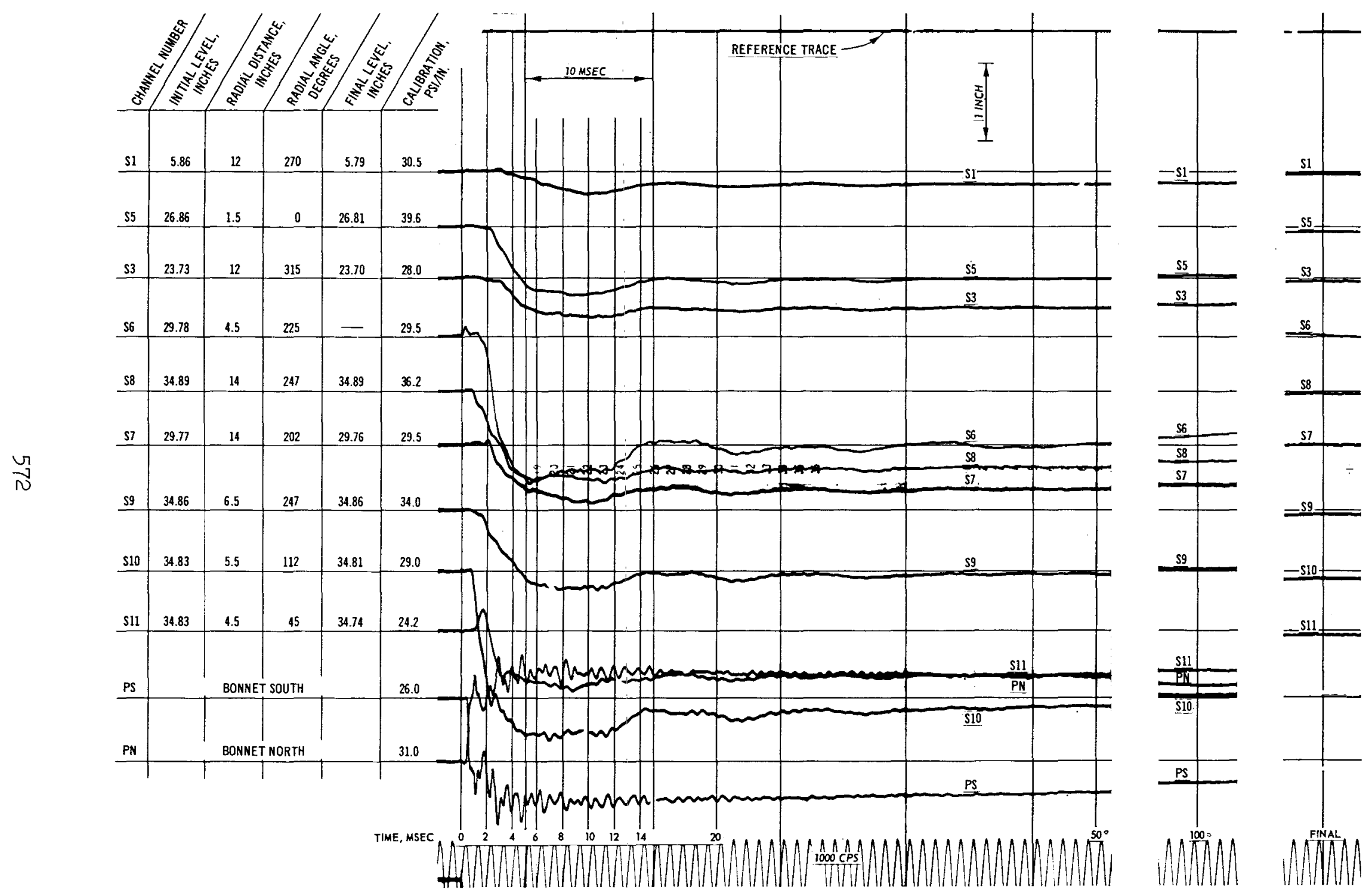

Fig. C-42. Test 2I, pressure record 


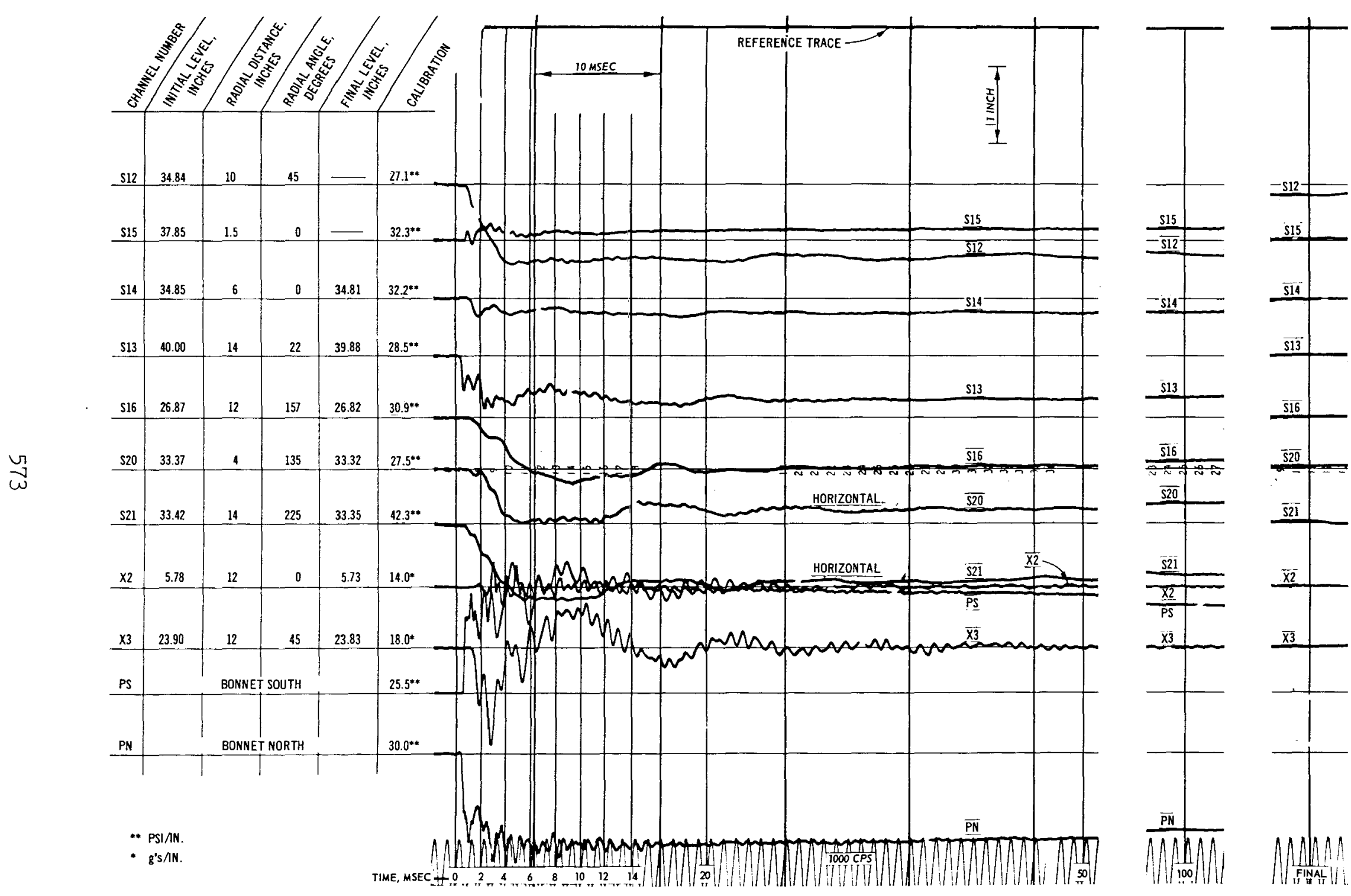

Fig. C-43. Test 2I, pressure and acceleration record 

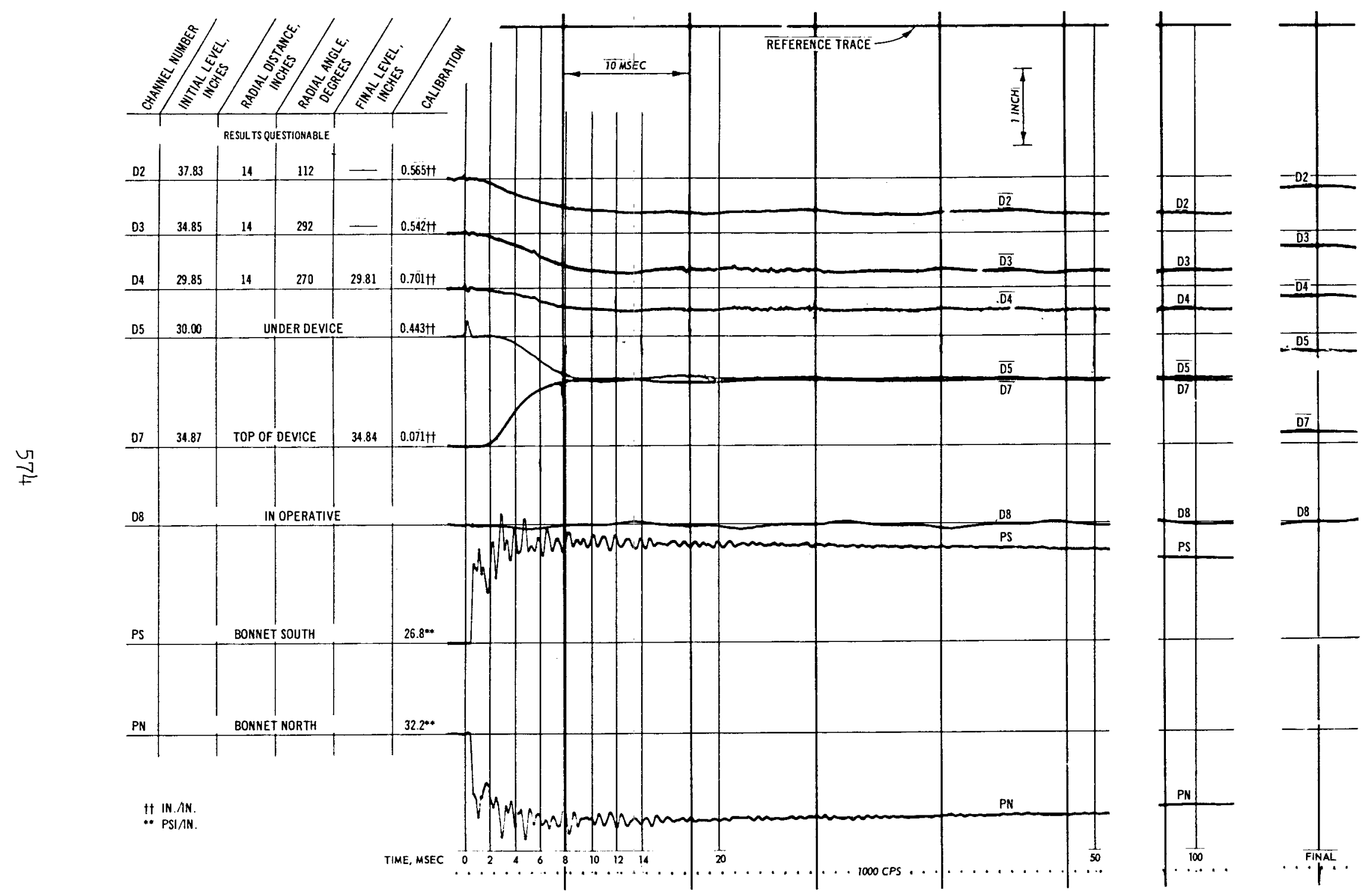

Fig. C-44. Test 21, deflection and pressure record 


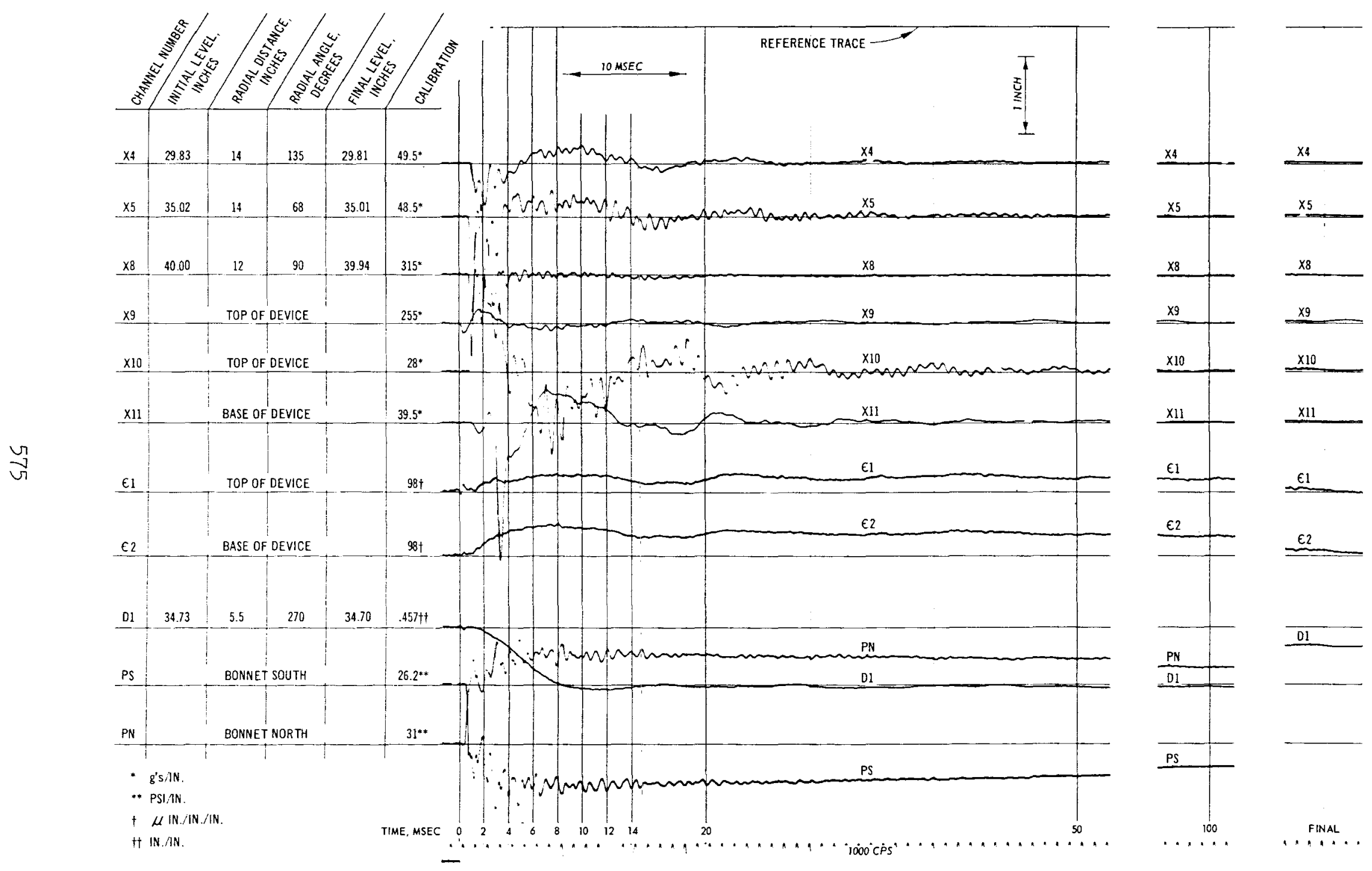

Fig. C-45. Test 21, pressure, acceleration, deflection, and strain record 

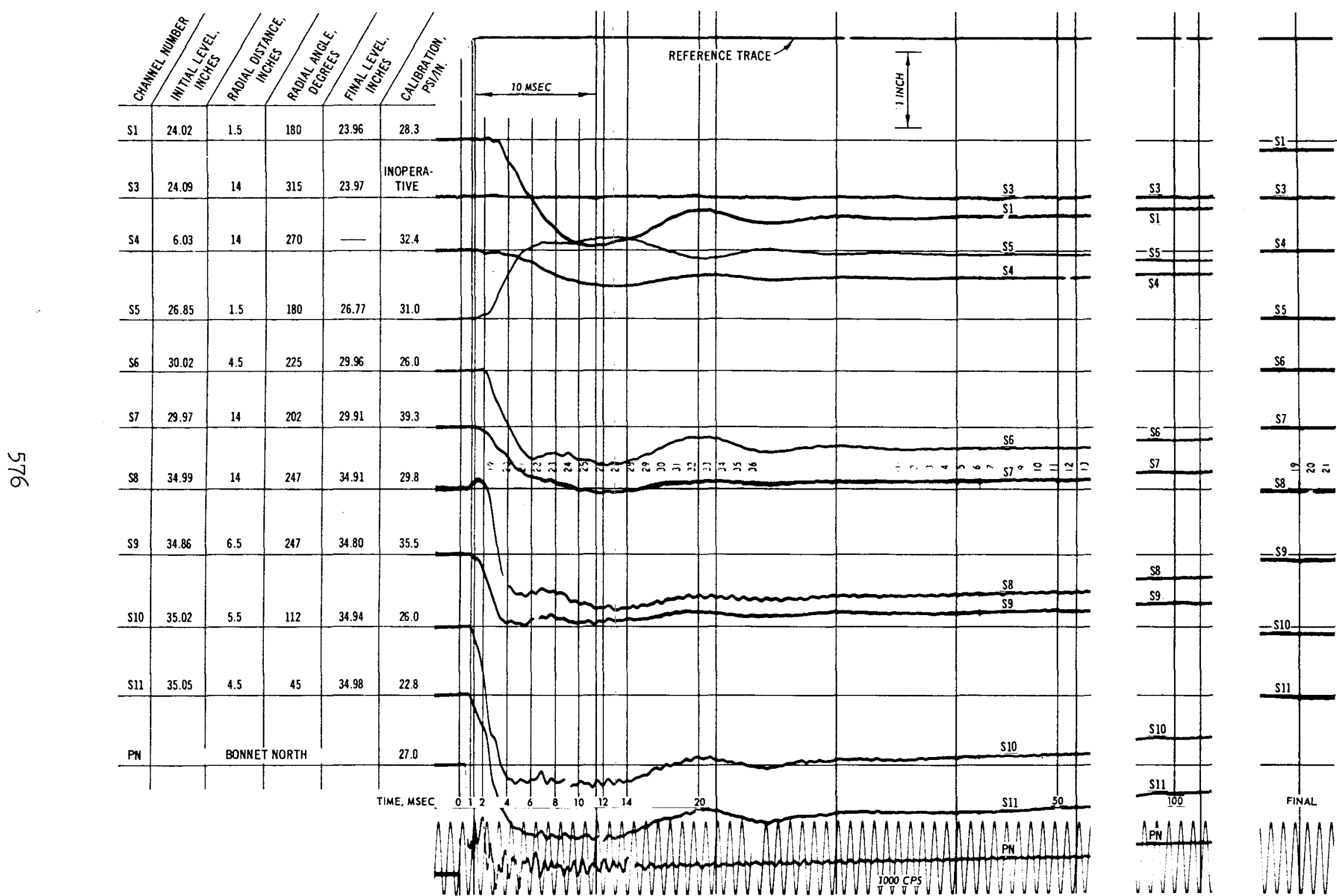

Fig. C-46. Test 22, pressure record (S1-Sll) 

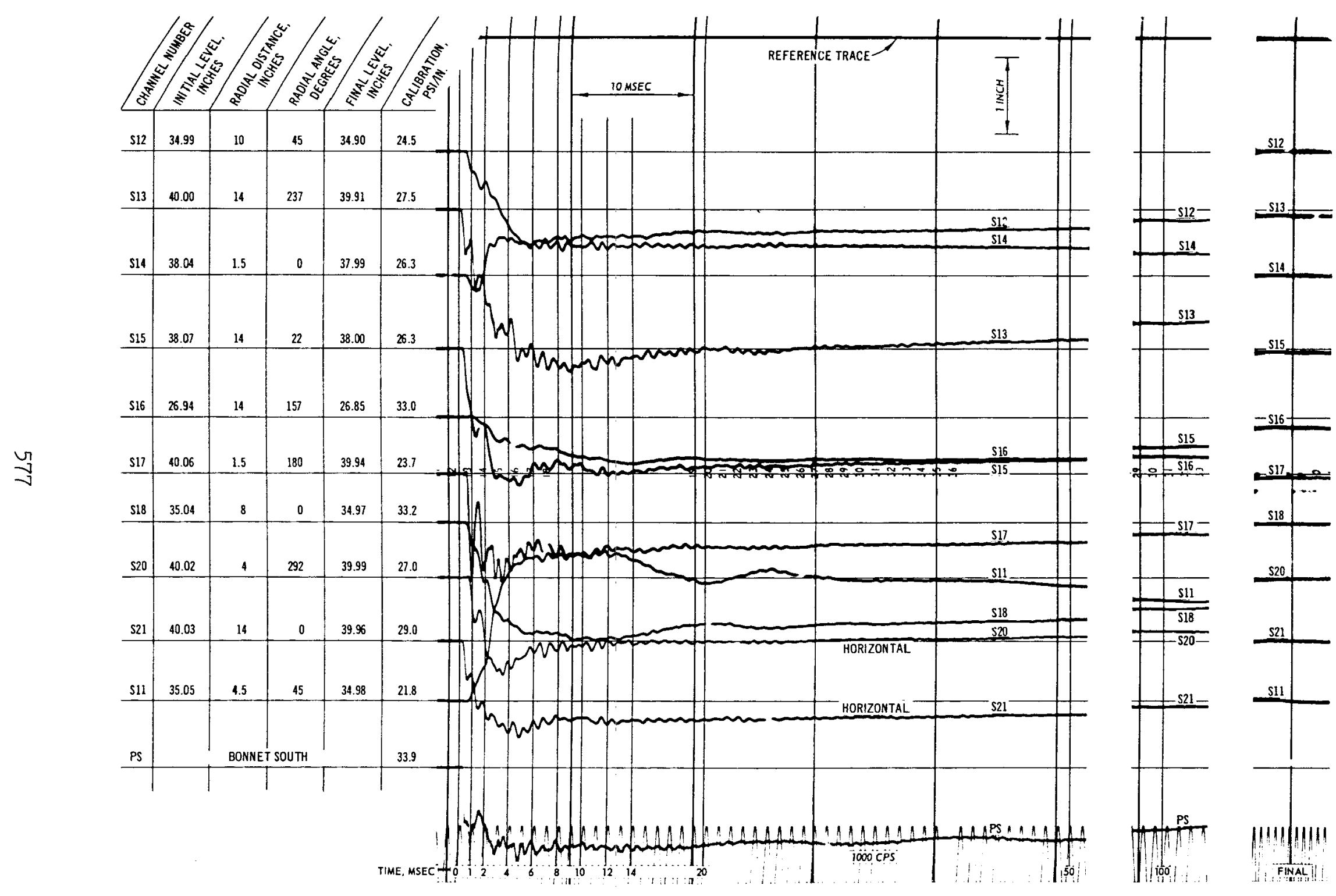

Fig. C-47. Test 22, pressure record (S12-S21, S11) 

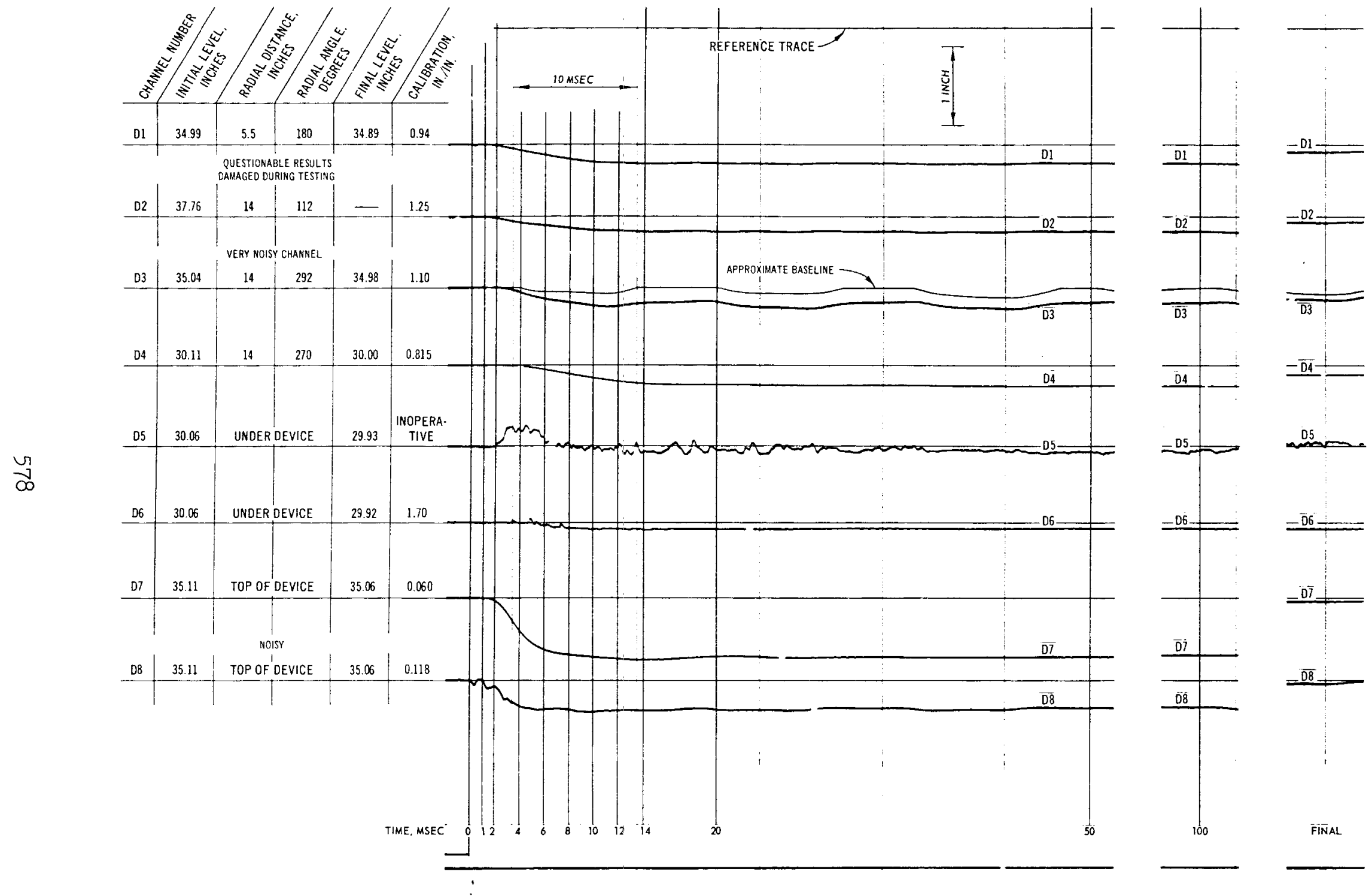

Fig. C-48. Test 22, deflection record 


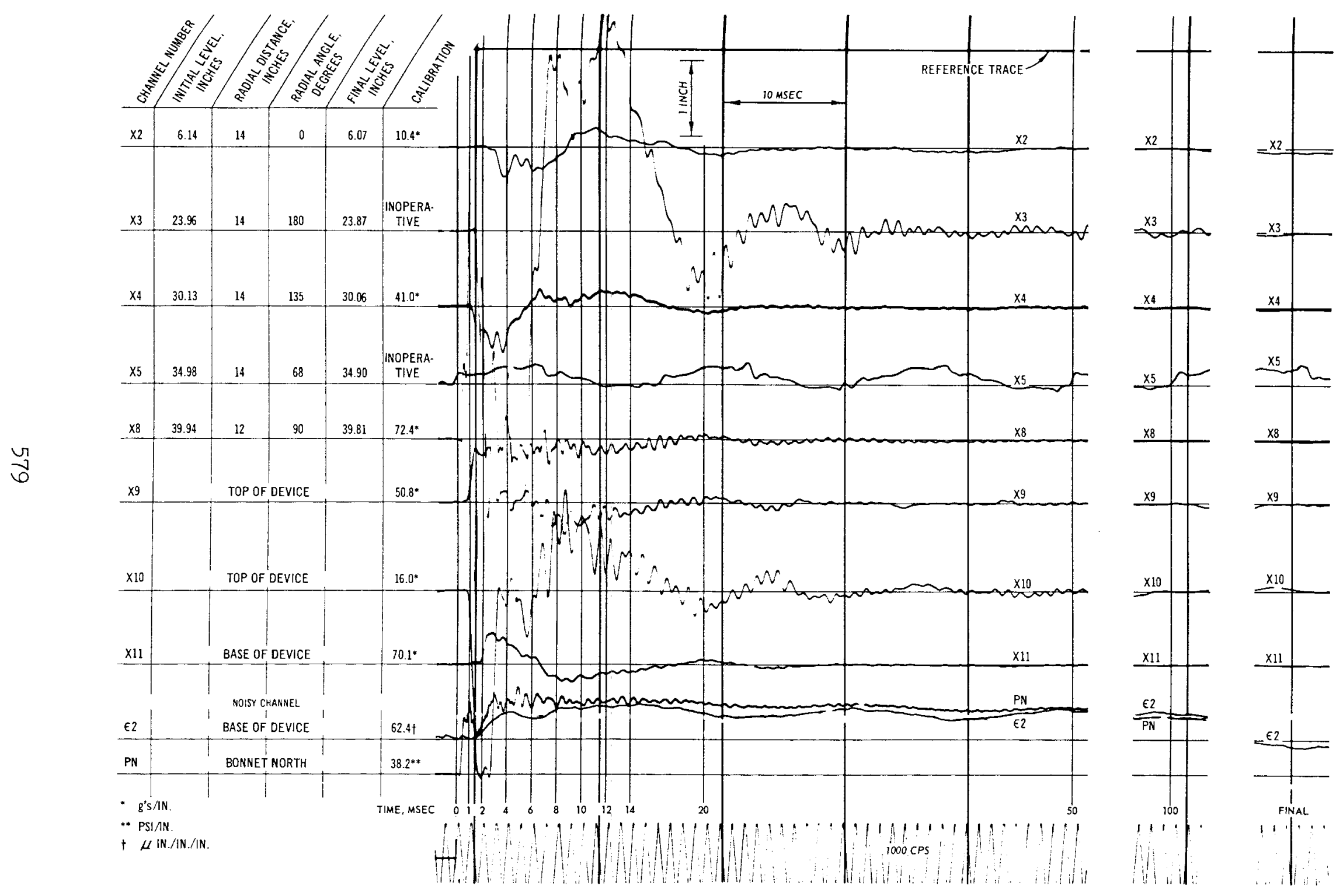

Fig. C-49. Test 22, acceleration, strain, and pressure record 

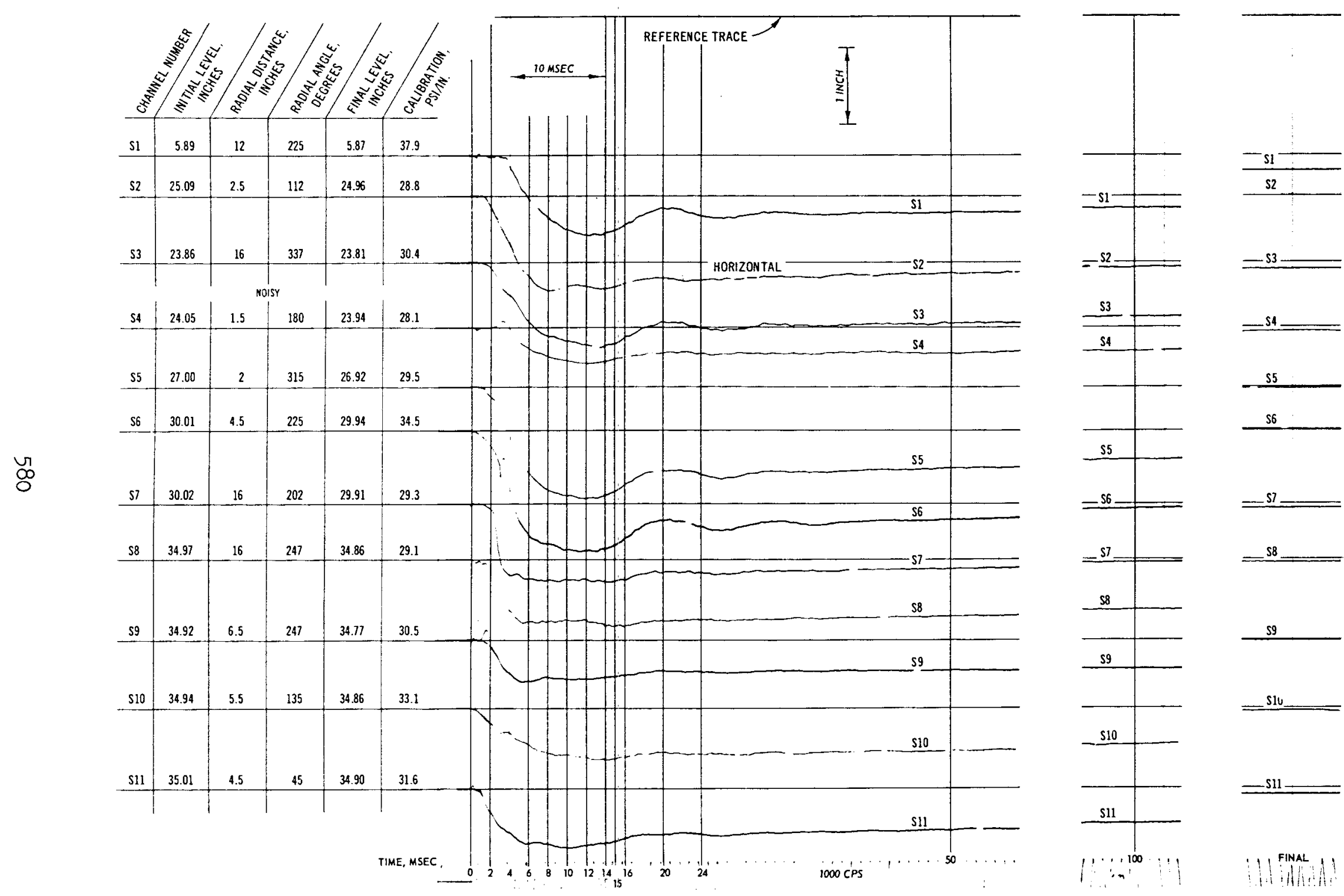

Fig. C-50. Test 23, pressure record (SI-SII) 


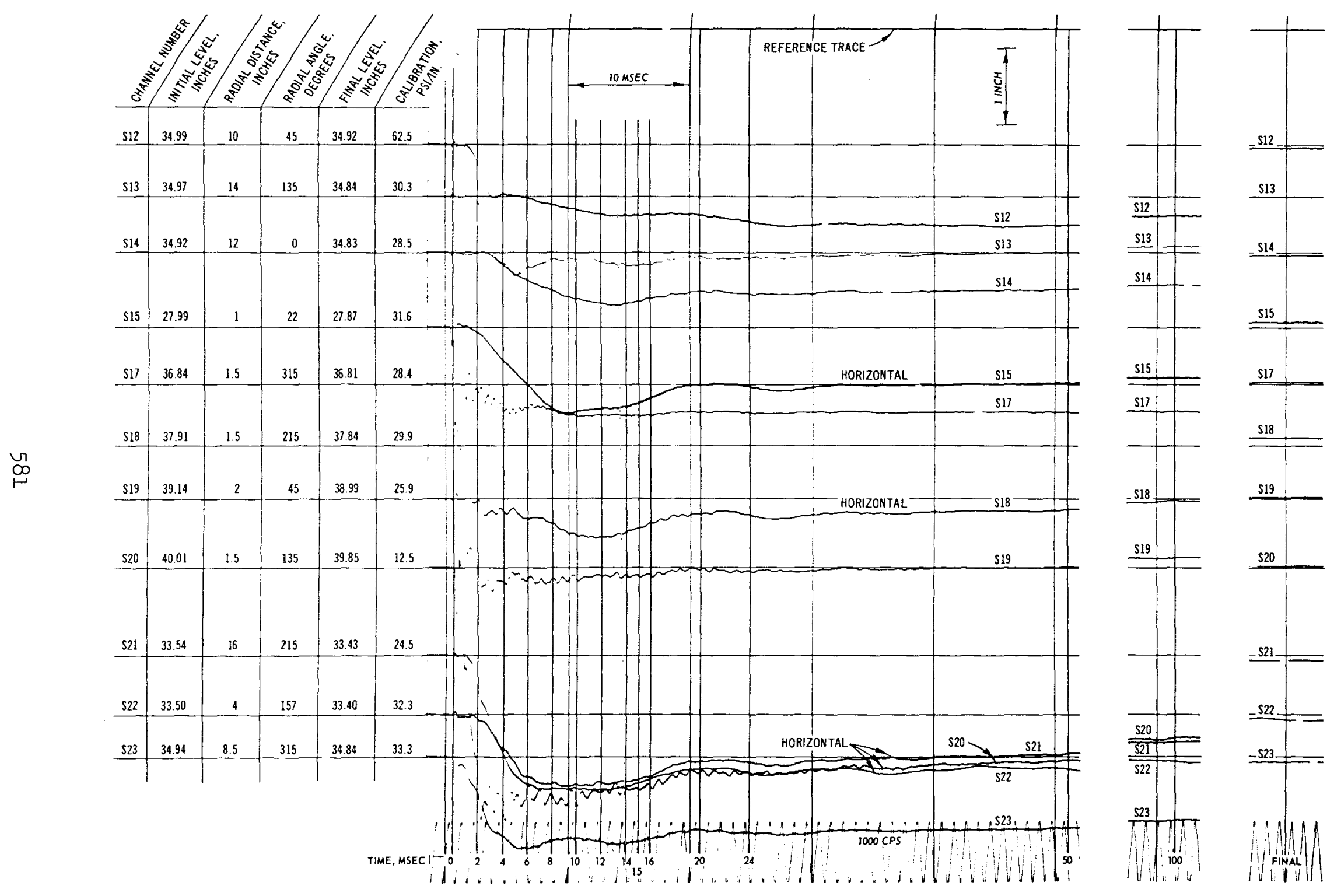

Fig. C-51. Test 23, pressure record (S12-S23) 

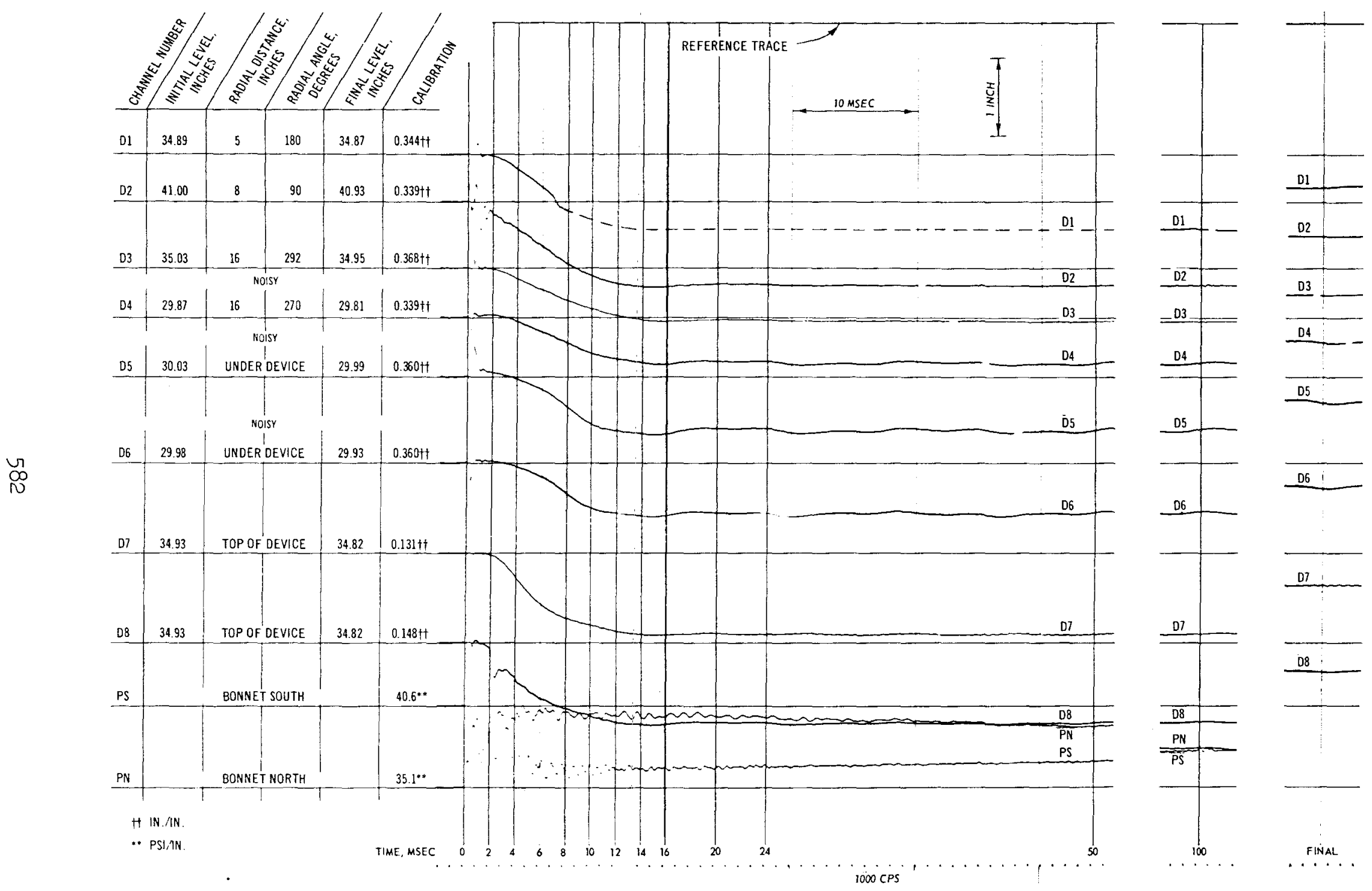

Fig. C-52. Test 23, deflection and pressure record 


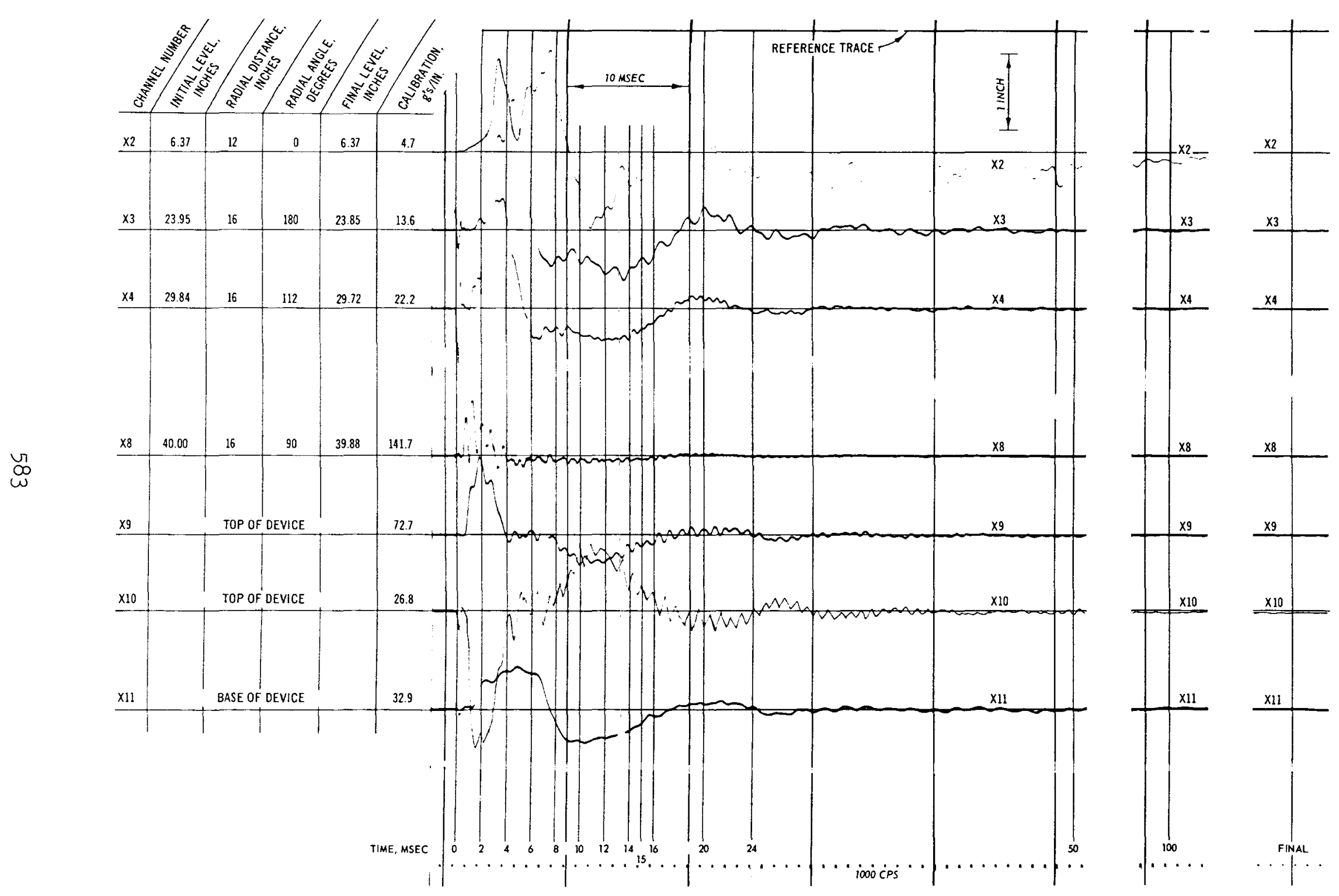

Fig. C-53. Test 23, acceleration record 


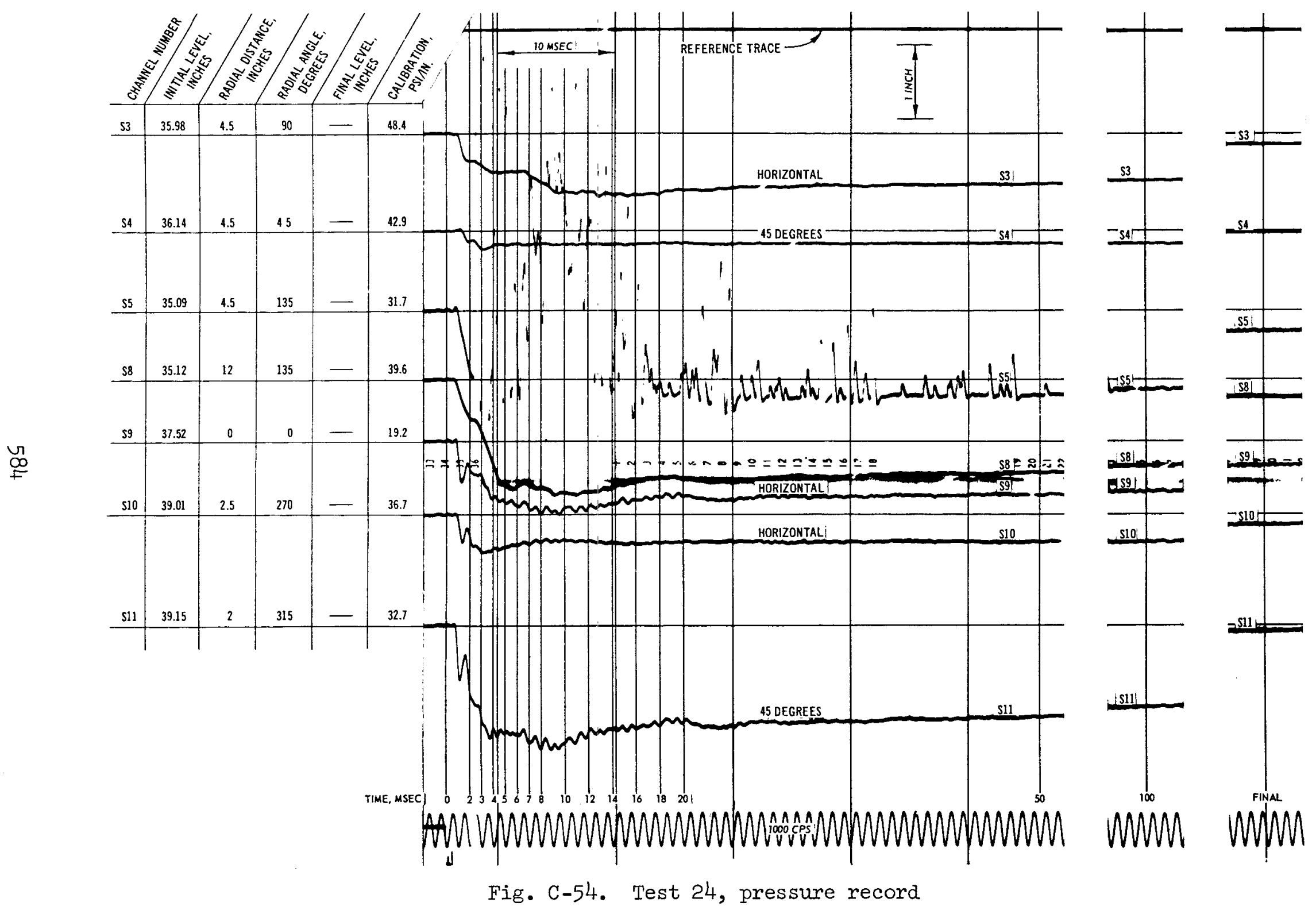




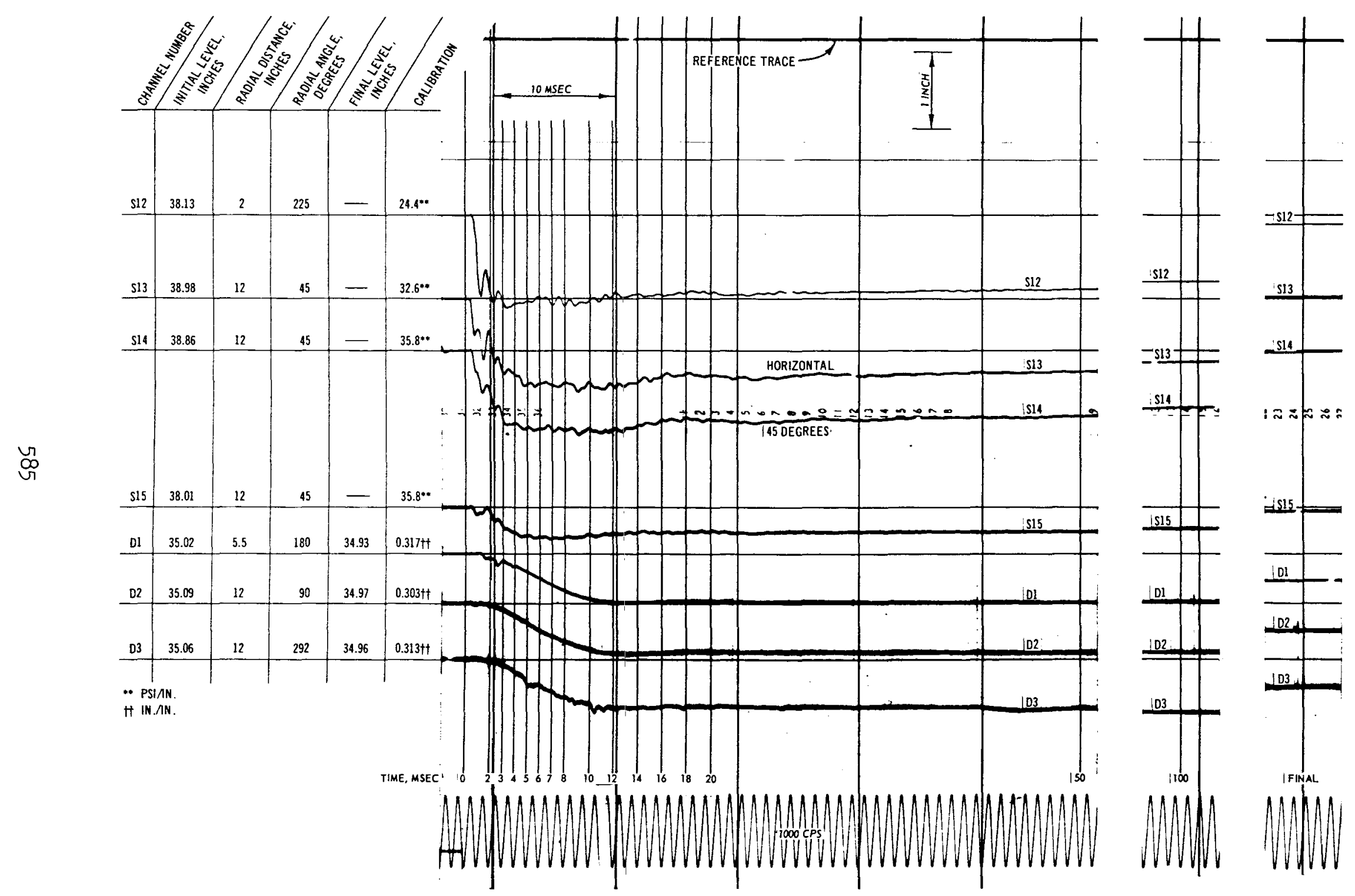

Fig. C-55. Test 24, pressure and deflection record 

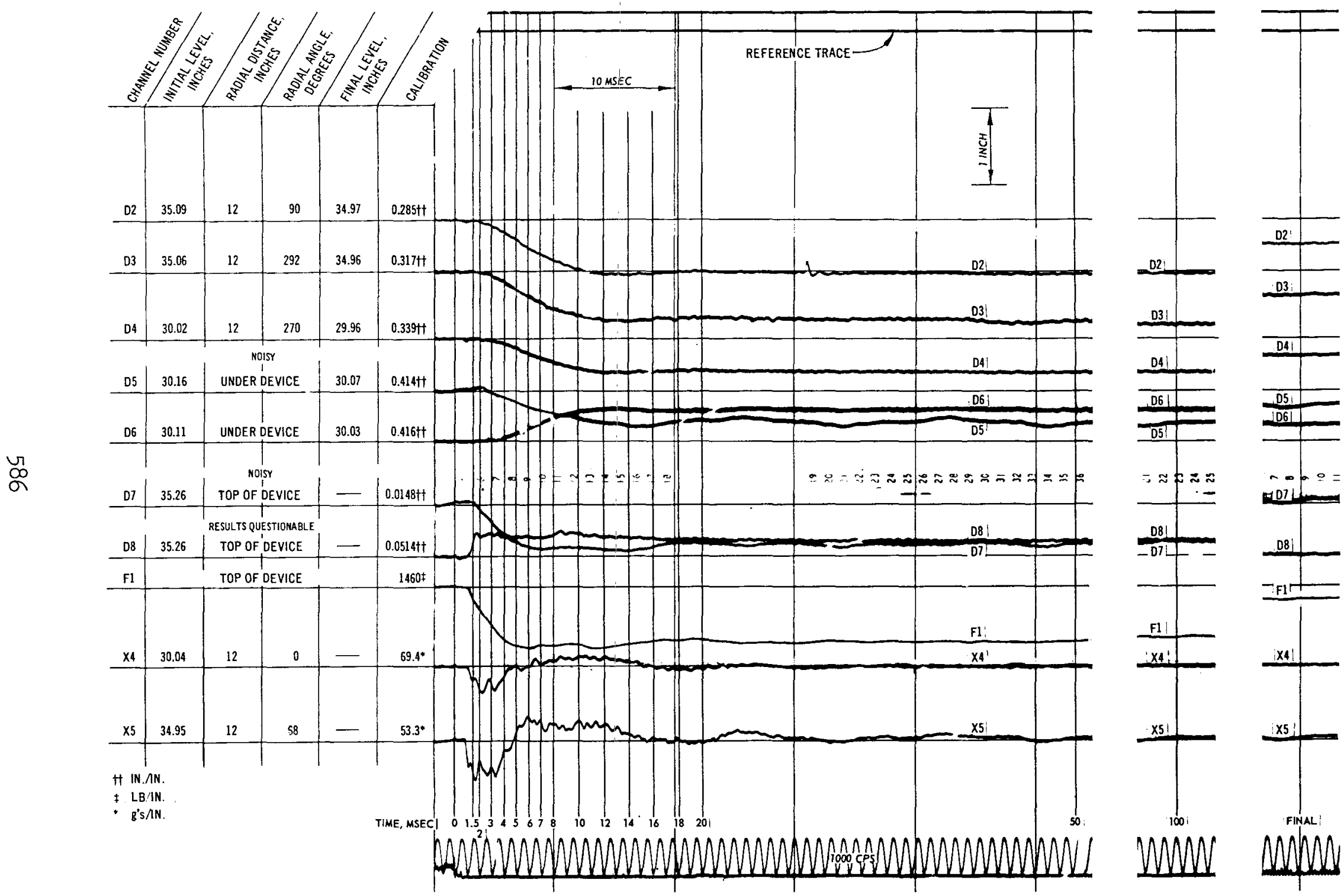

Fig. C-56. Test 24, deflection, force, and acceleration record 


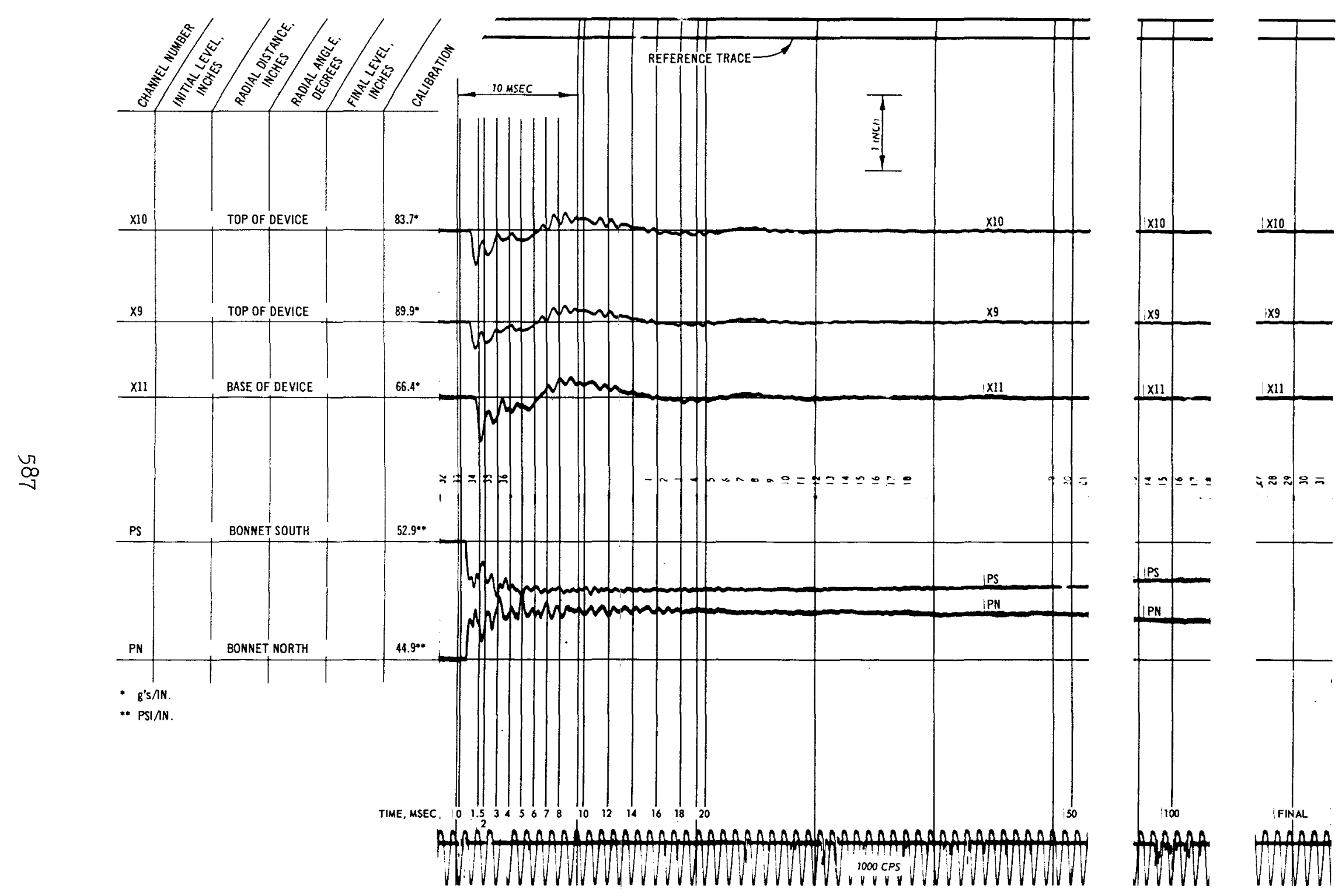

Fig. C-57. Test 24, acceleration and pressure record 


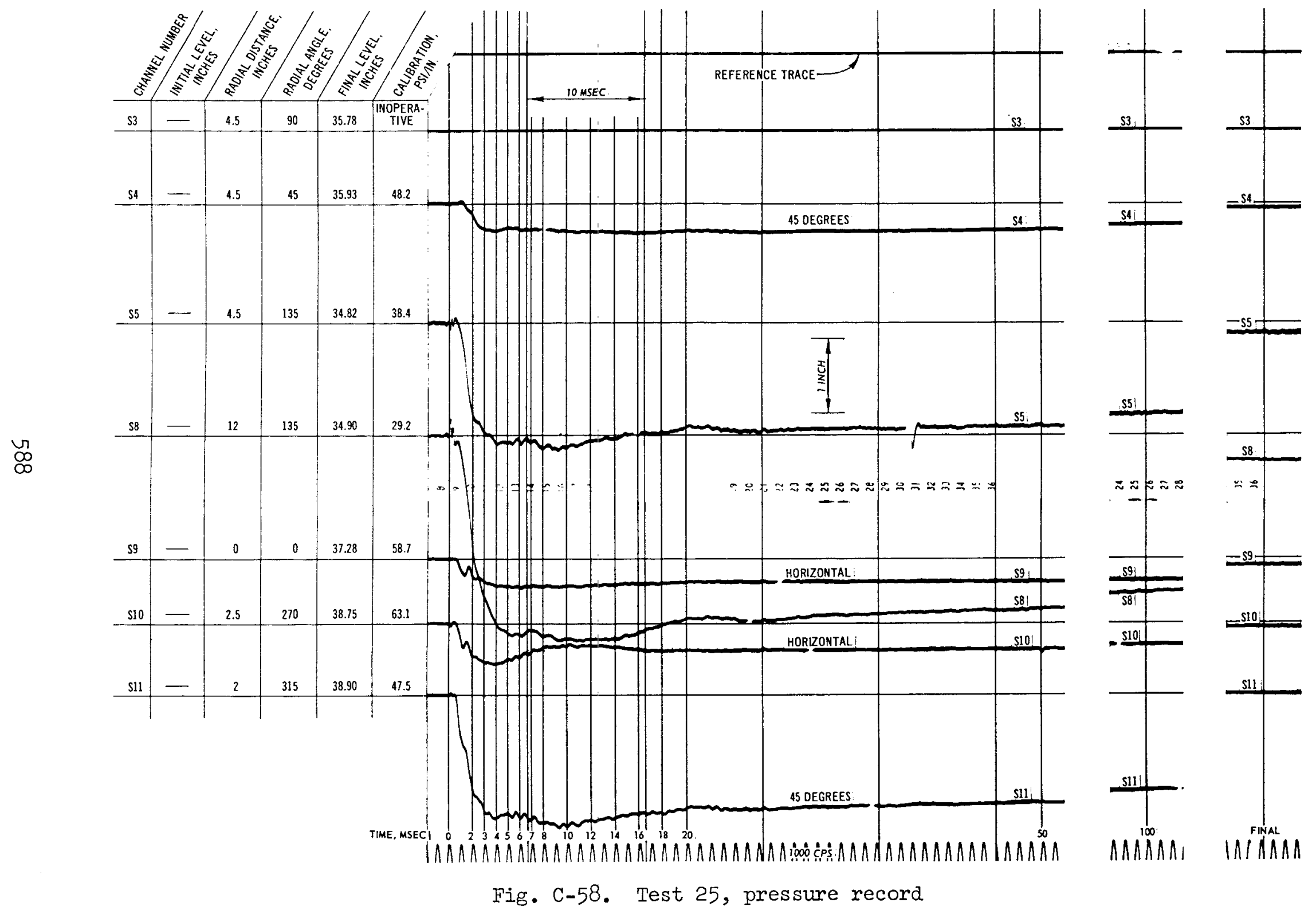




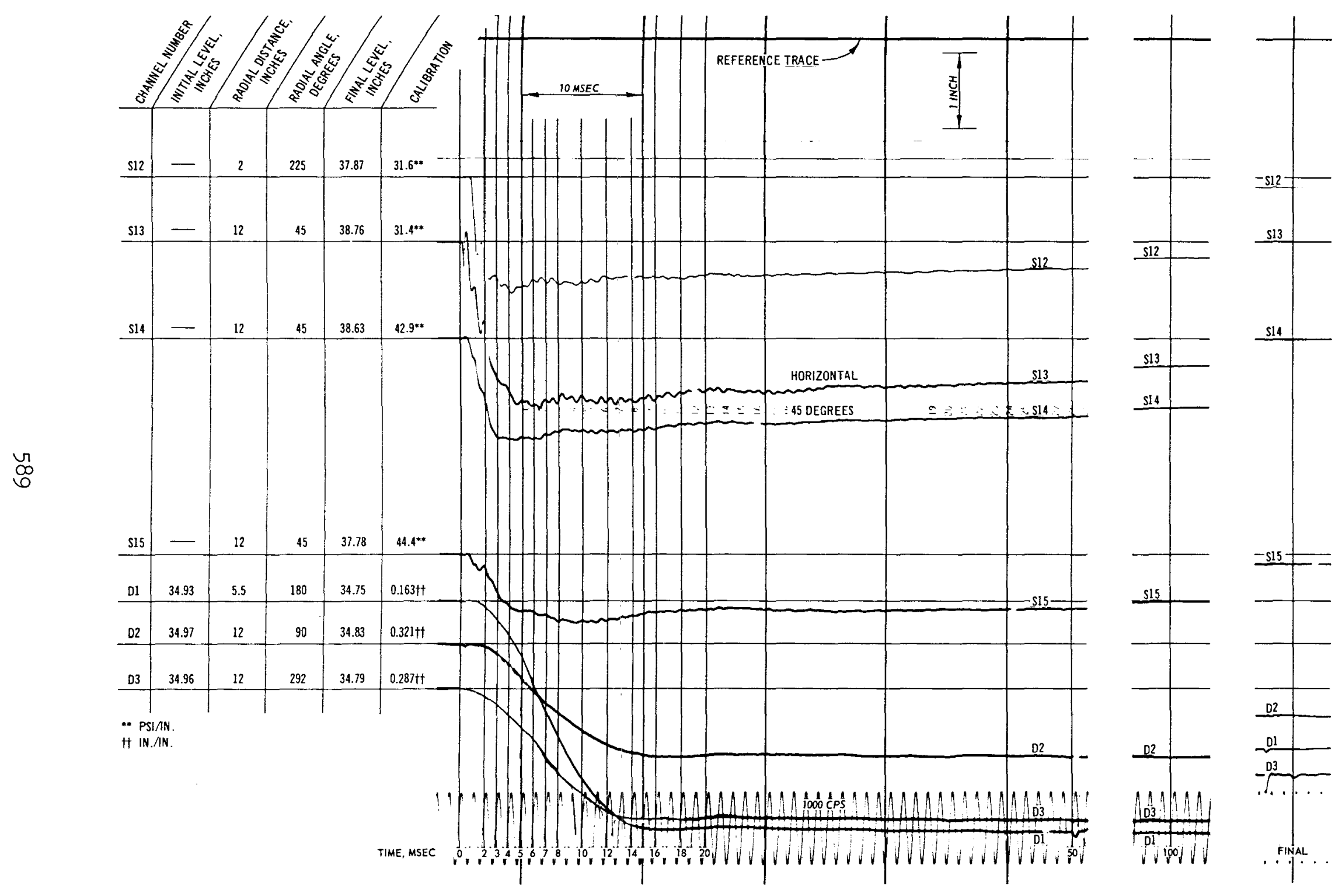

Fig. C-59. Test 25, pressure and deflection record 

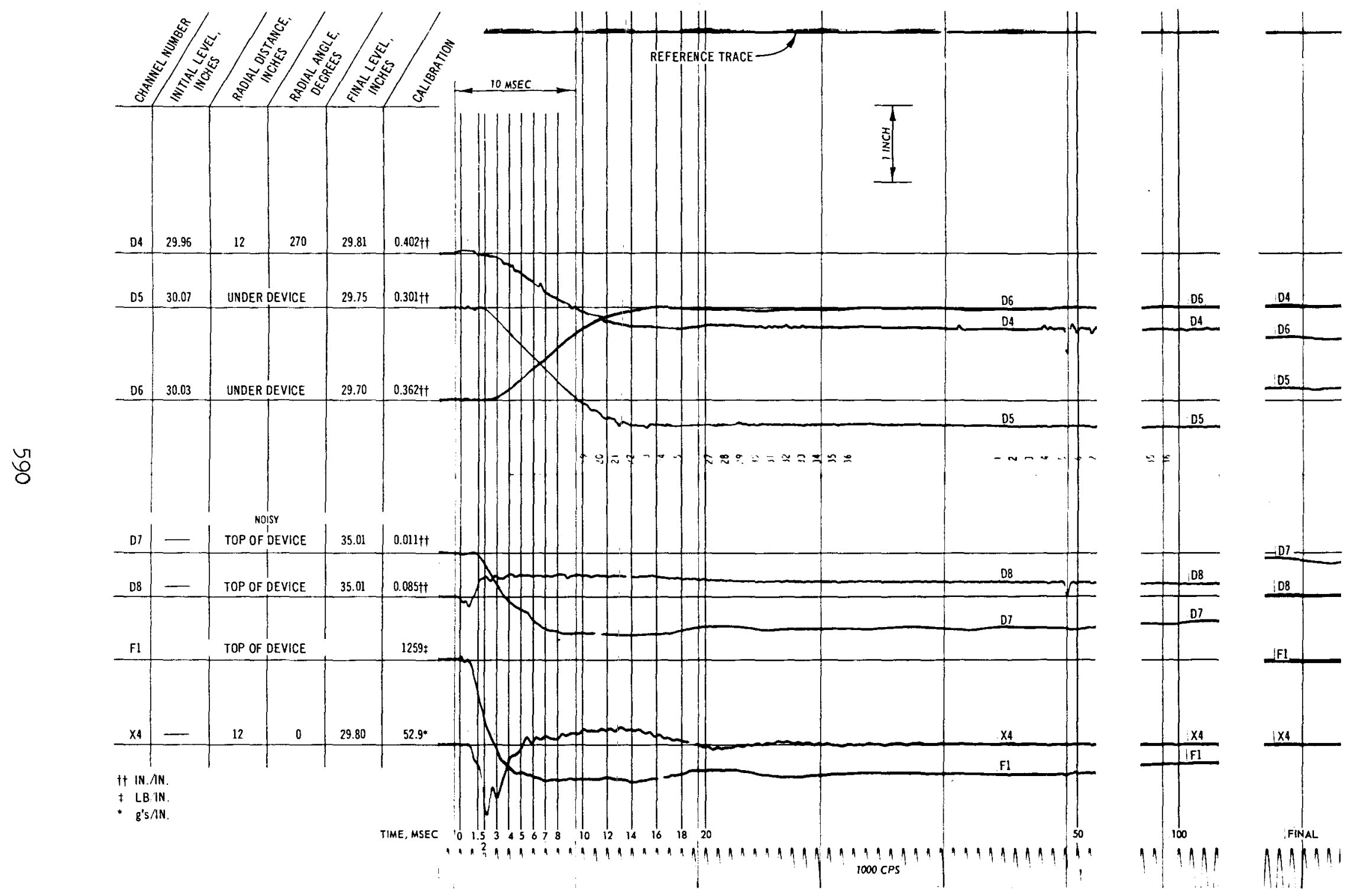

Fig. C-60. Test 25, deflection, force, and acceleration record 


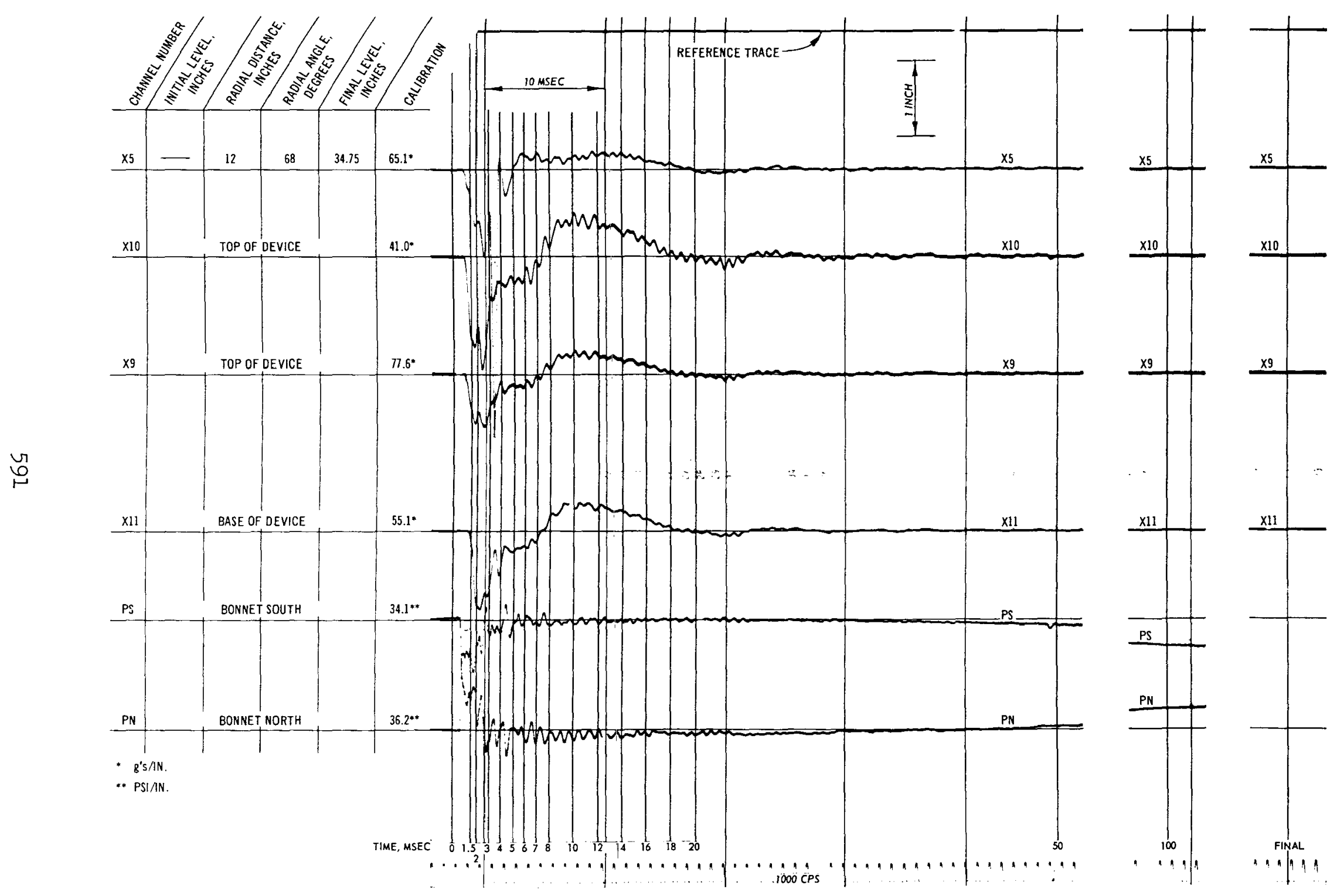

Fig. C-6I. Test 25, acceleration and pressure record 

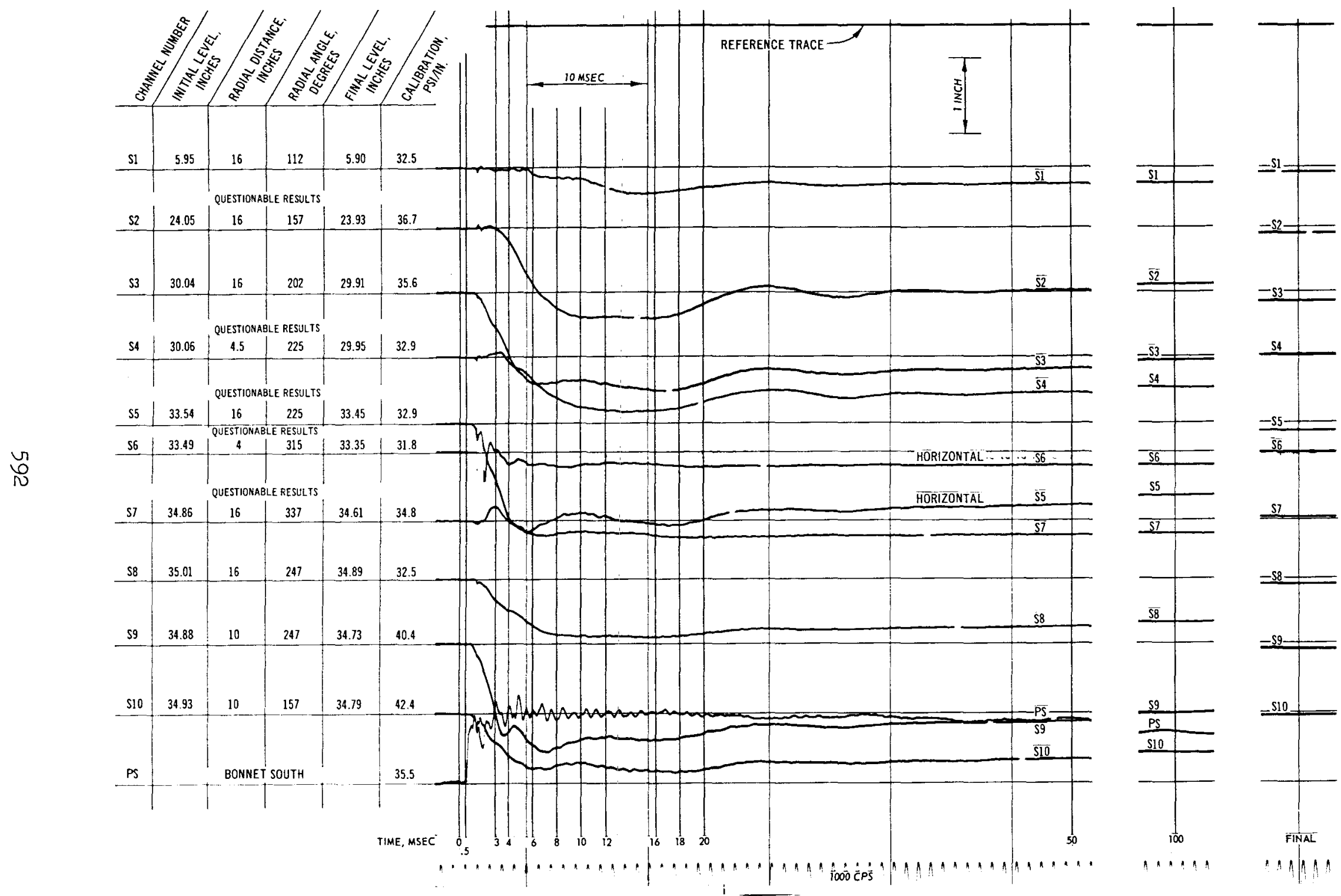

Fig. C-62. Test 26, pressure record (S1-S10) 

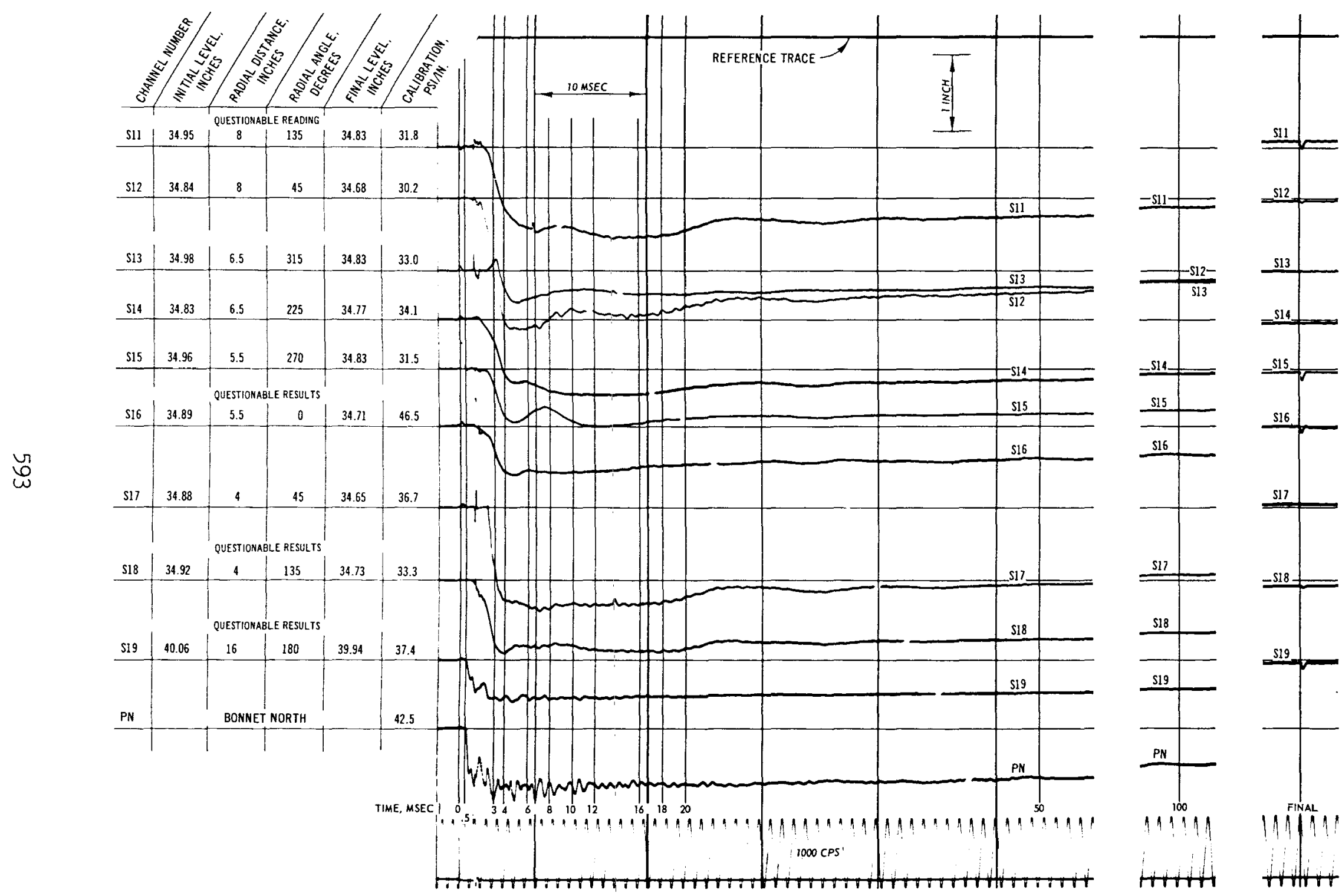

Fig. C-63. Test 26, pressure record (S11-S19) 

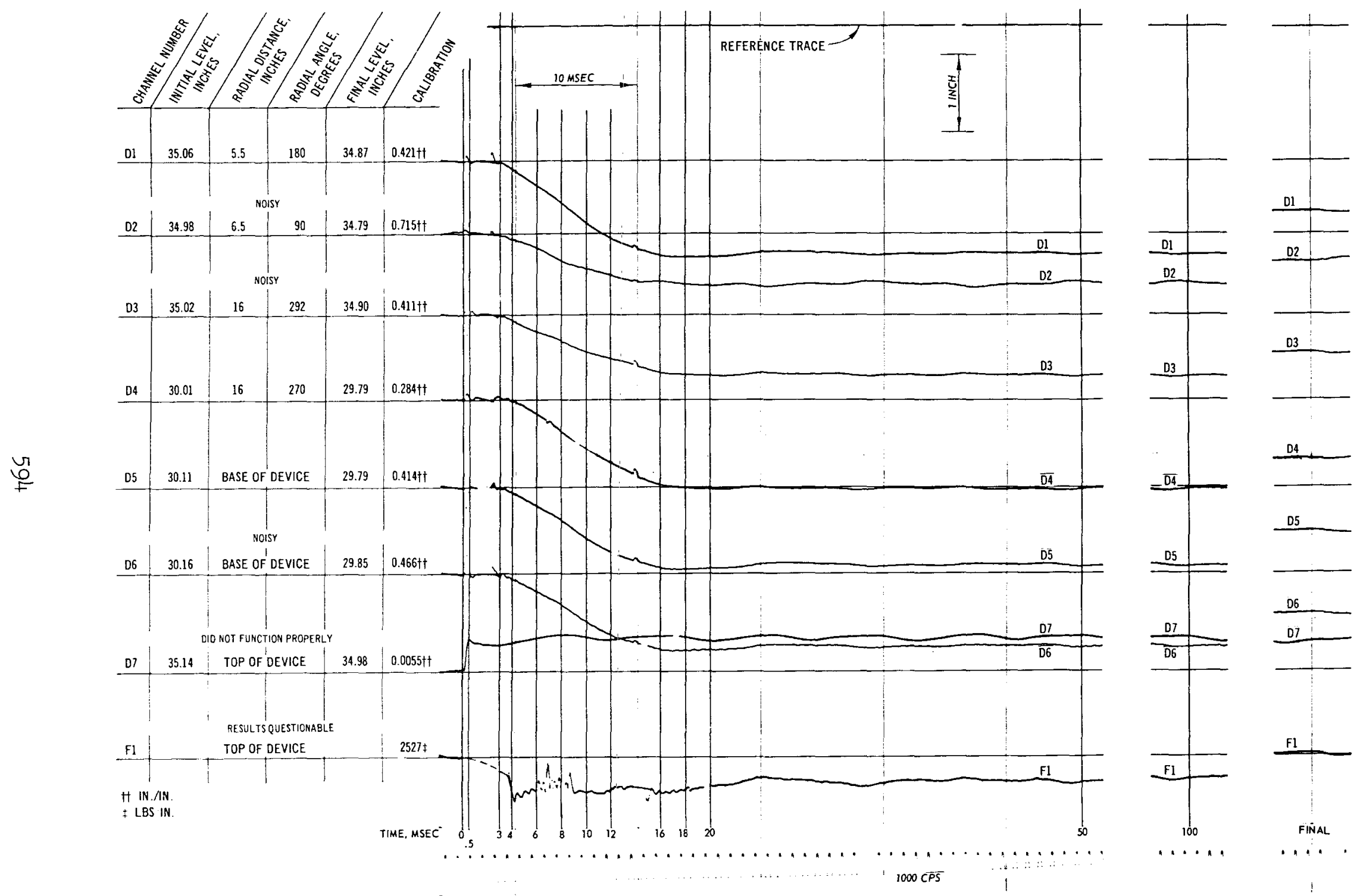

Fig. C-64. Test 26, deflection and force record 


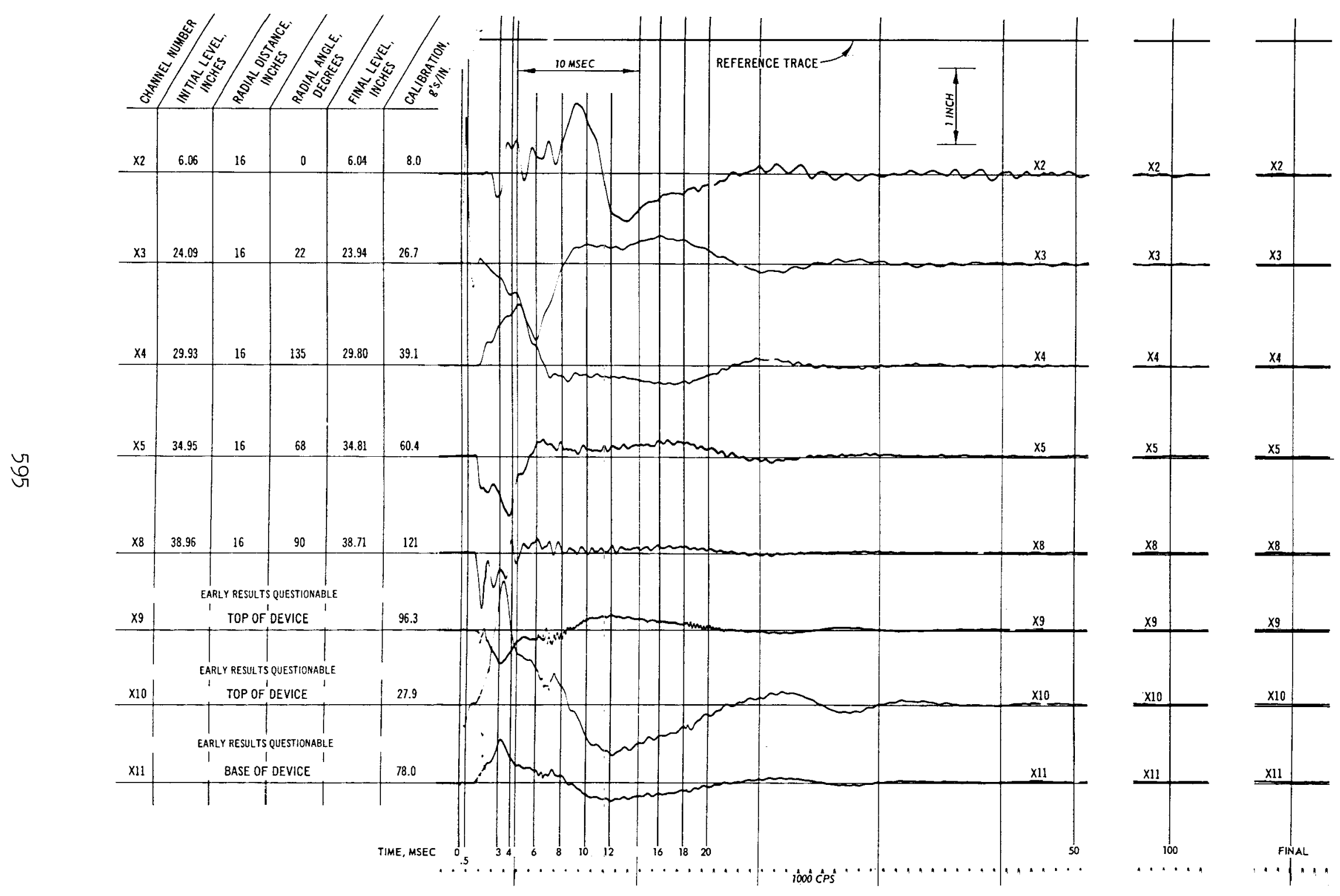

Fig. C-65. Test 26, acceleration record 


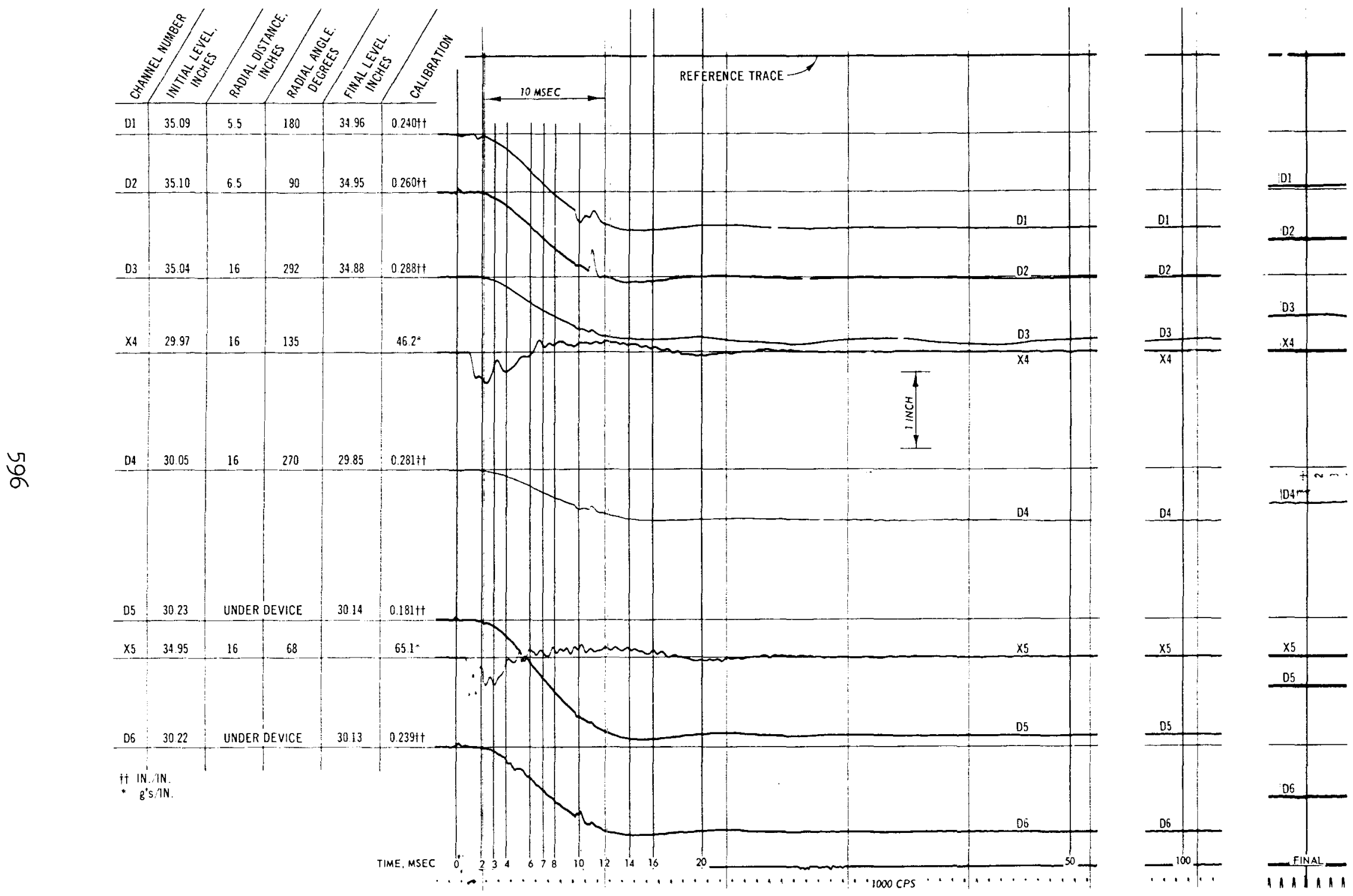

Fig. C-66. Test 27A, deflection and acceleration record 


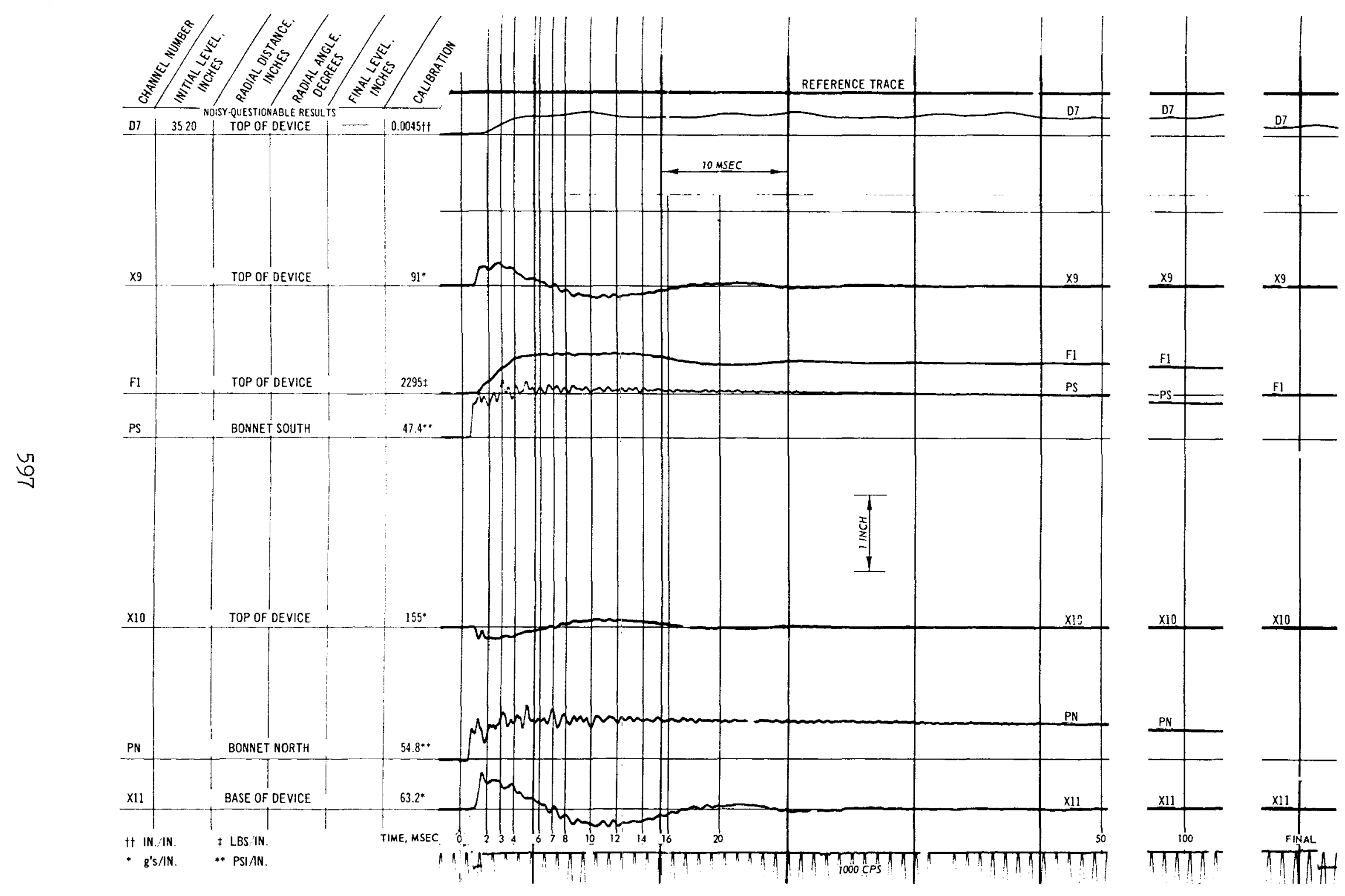

Fig. C-67. Test 27A, deflection, pressure, acceleration, and force record 


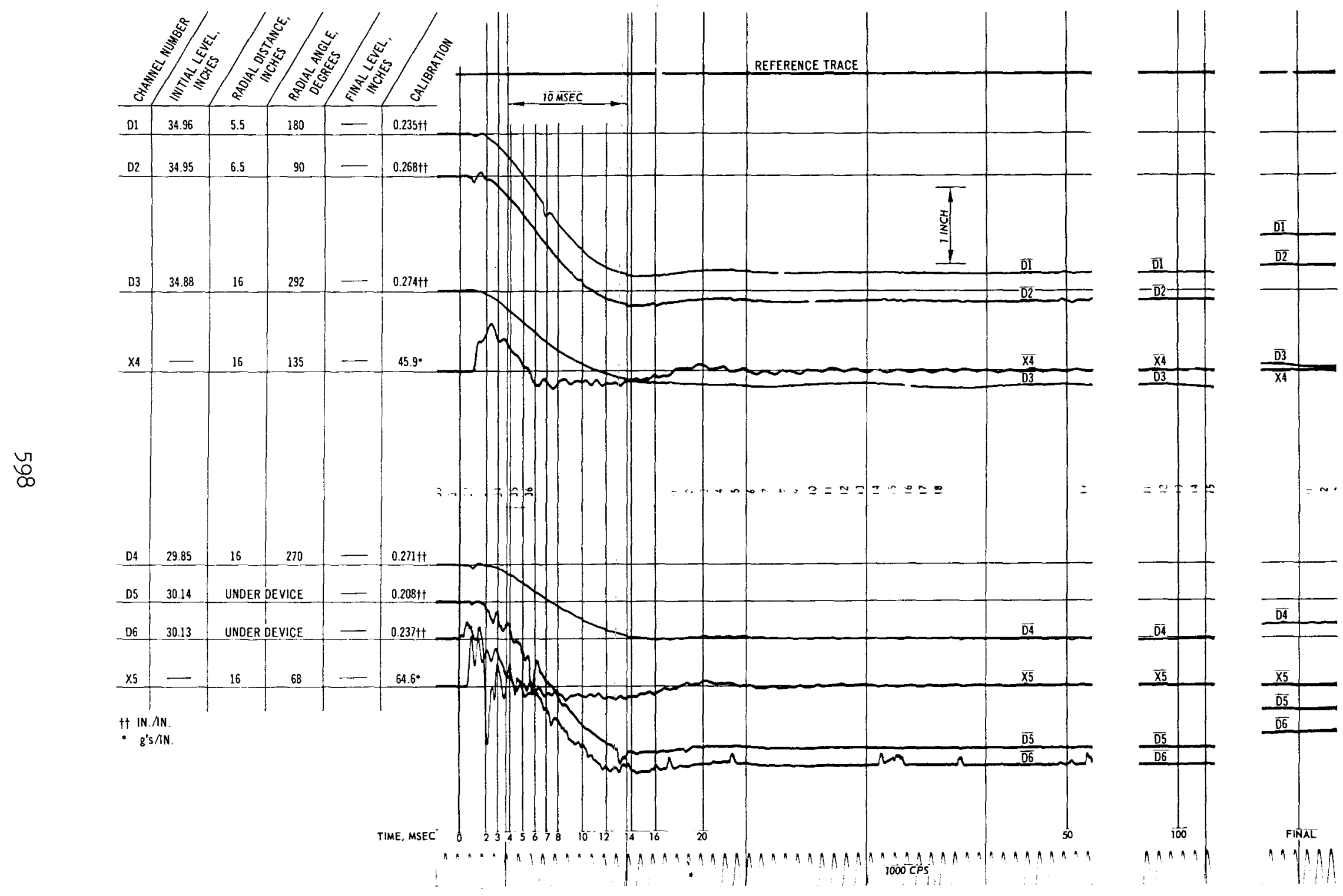

Fig. C-68. Test 27B, deflection and acceleration record 


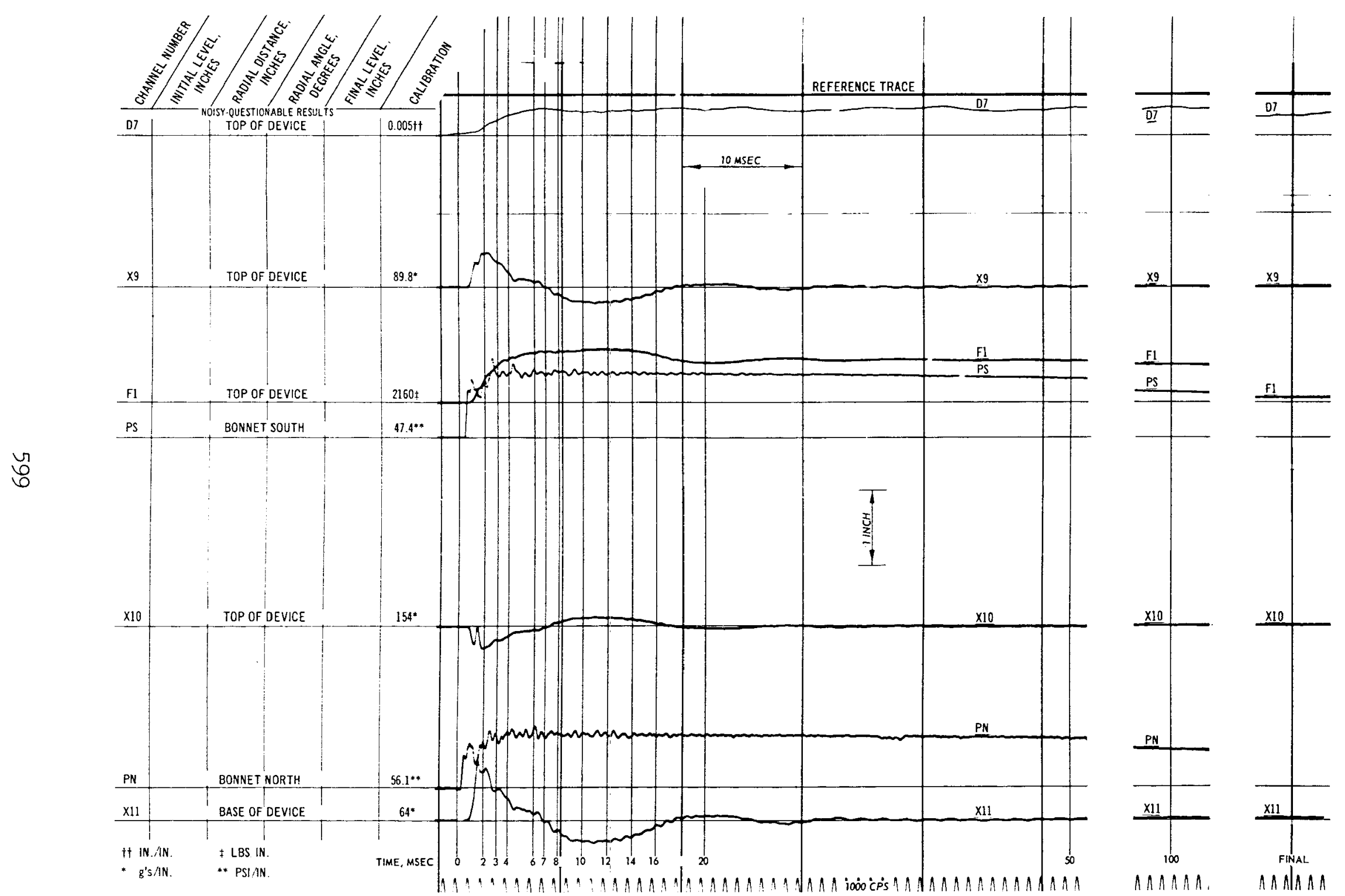

Fig. C-69. Test 27B, deflection, acceleration, pressure, and force record 

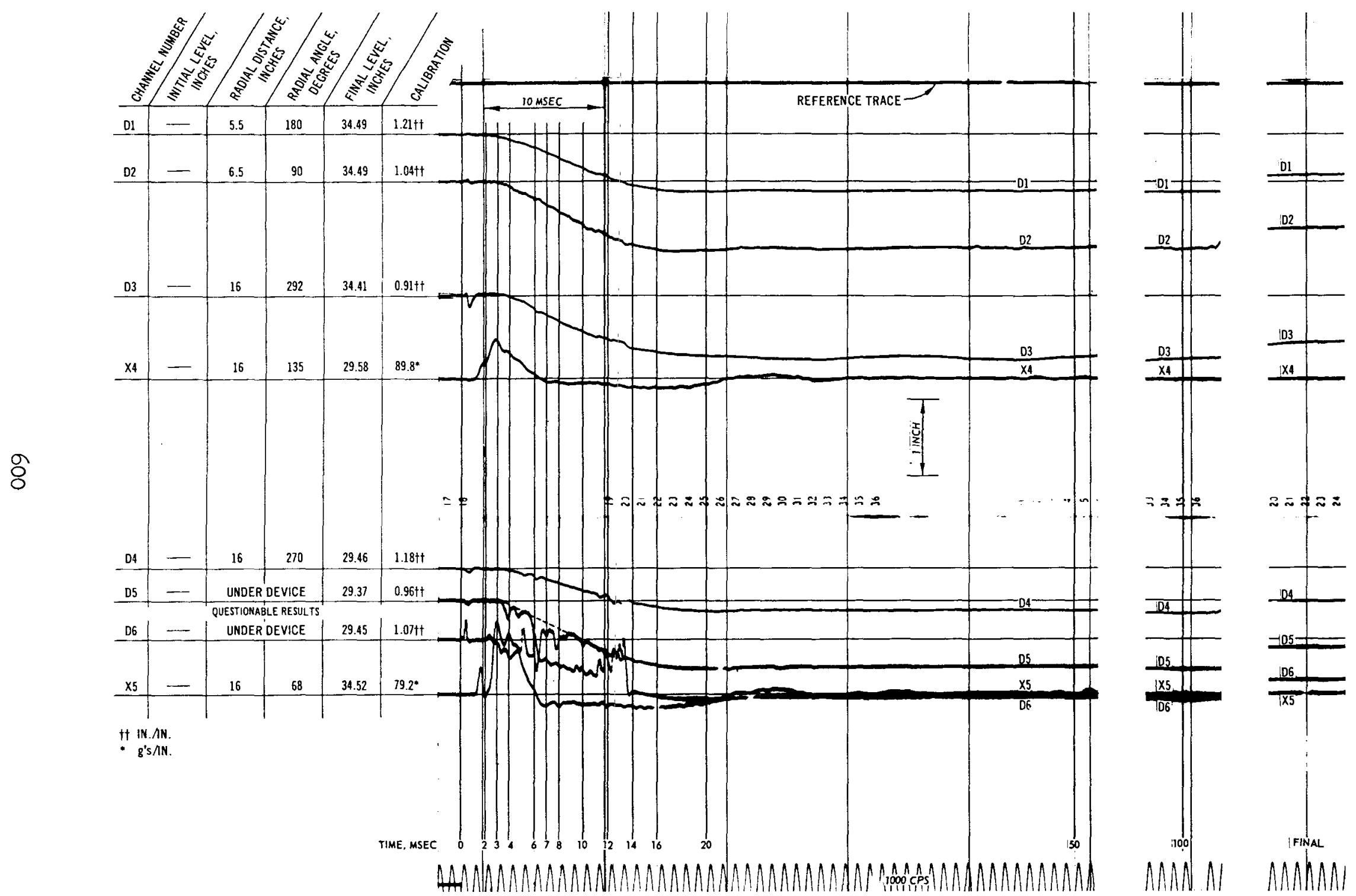

Fig. C-70. Test 27C, deflection and acceleration record 


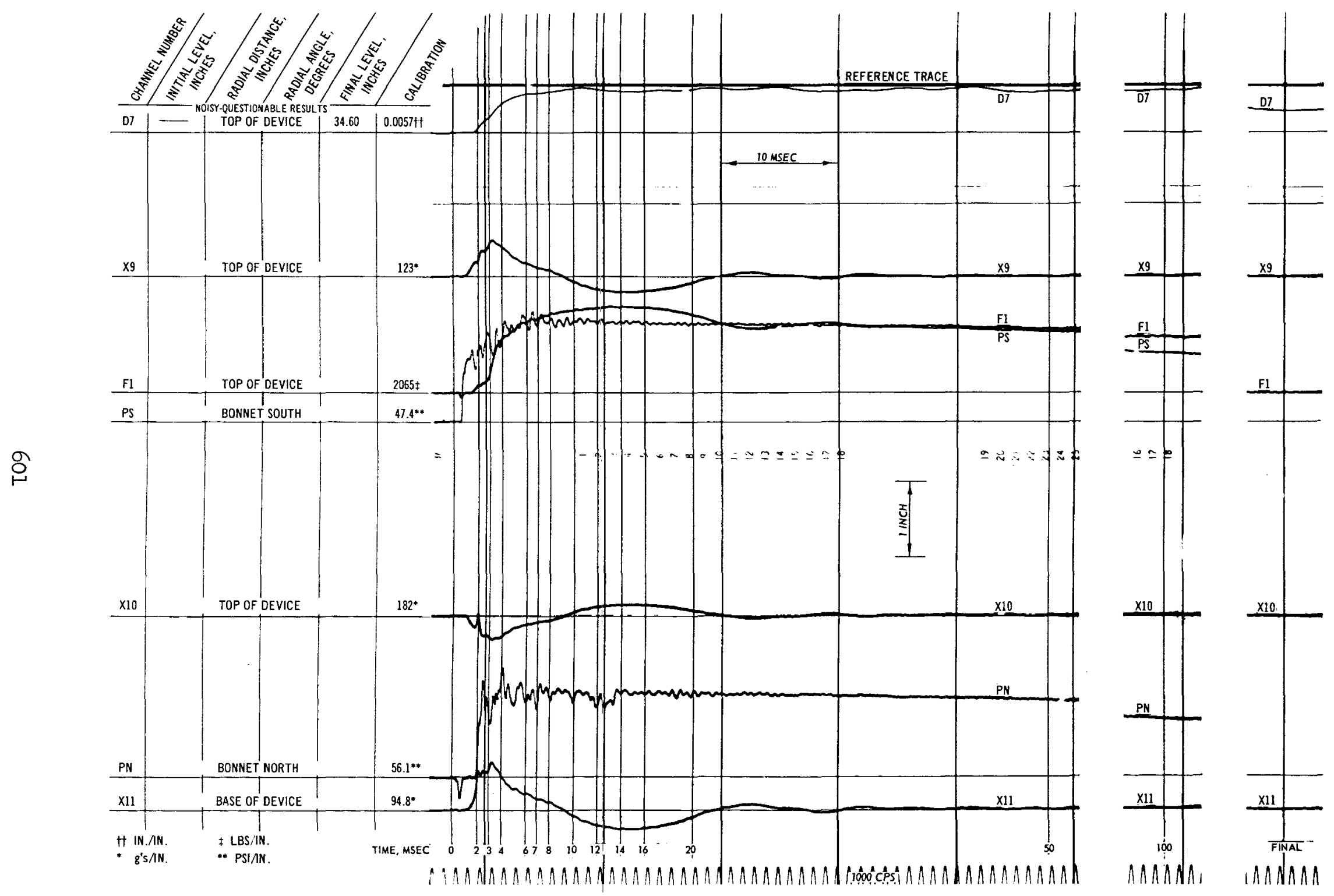

Fig. C-71. Test 27C, deflection, pressure, acceleration, and force record 

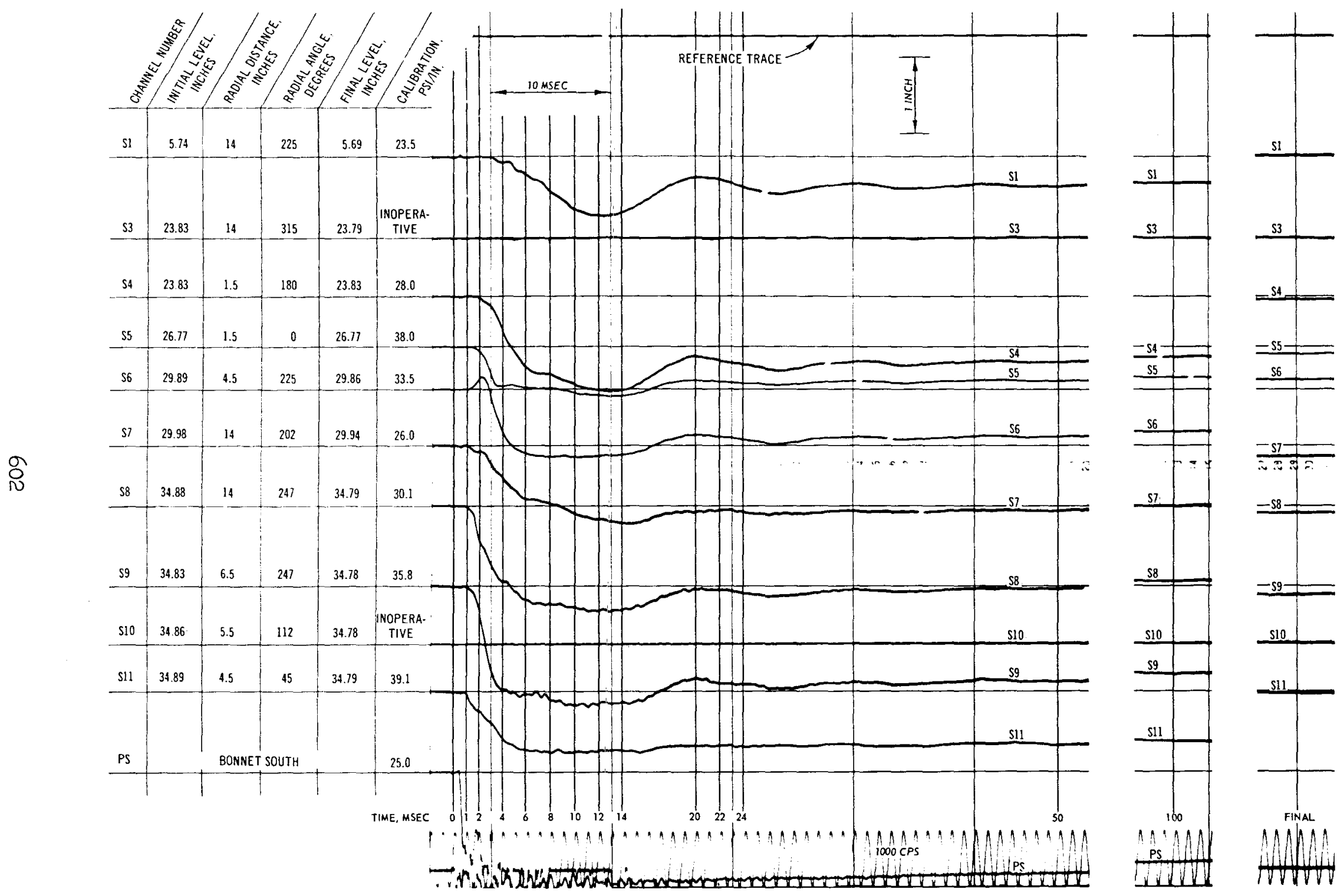

Fig. C-72. Test 28, pressure record (SI-SII) 


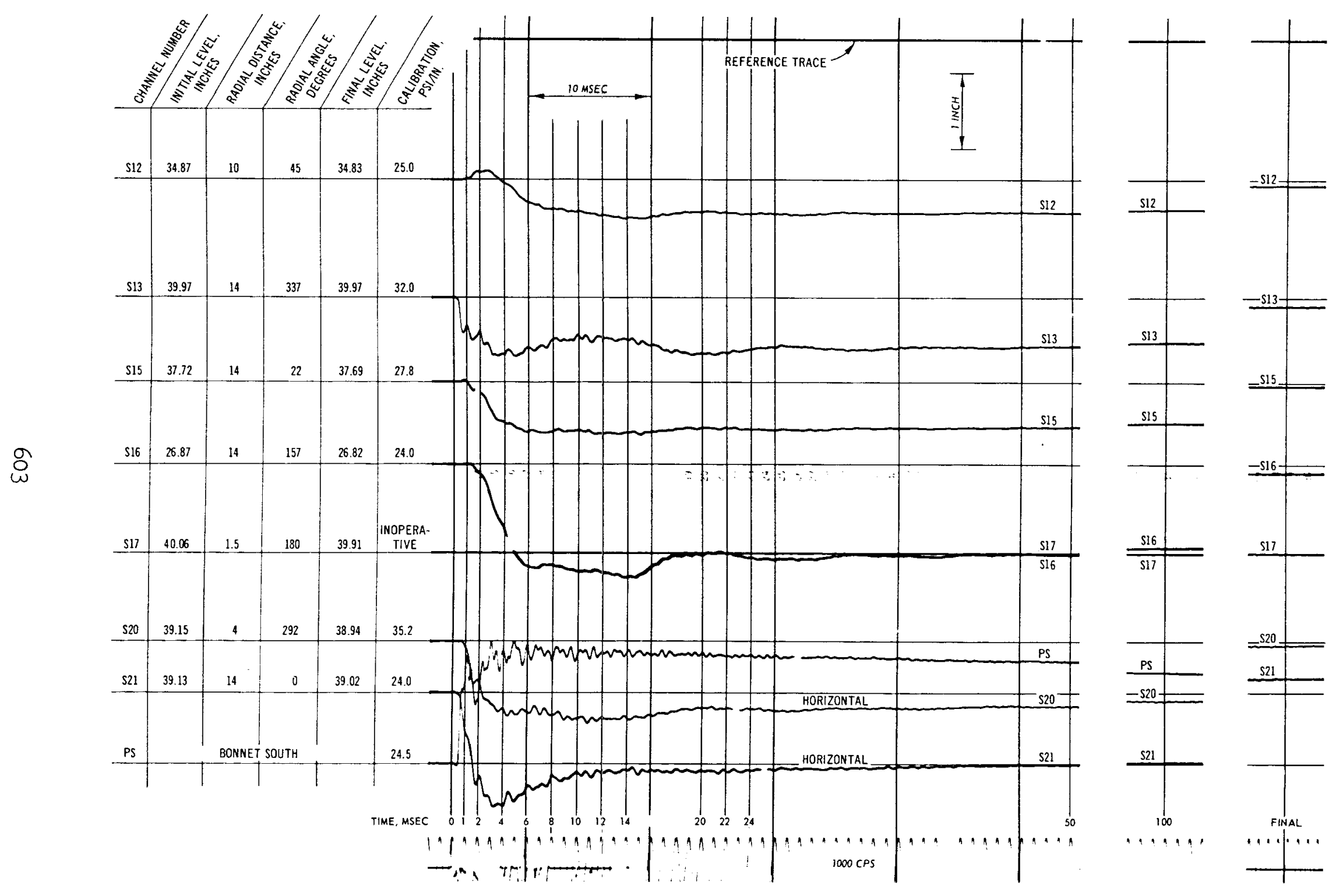

Fig. C-73. Test 28, pressure record (S12-S21) 


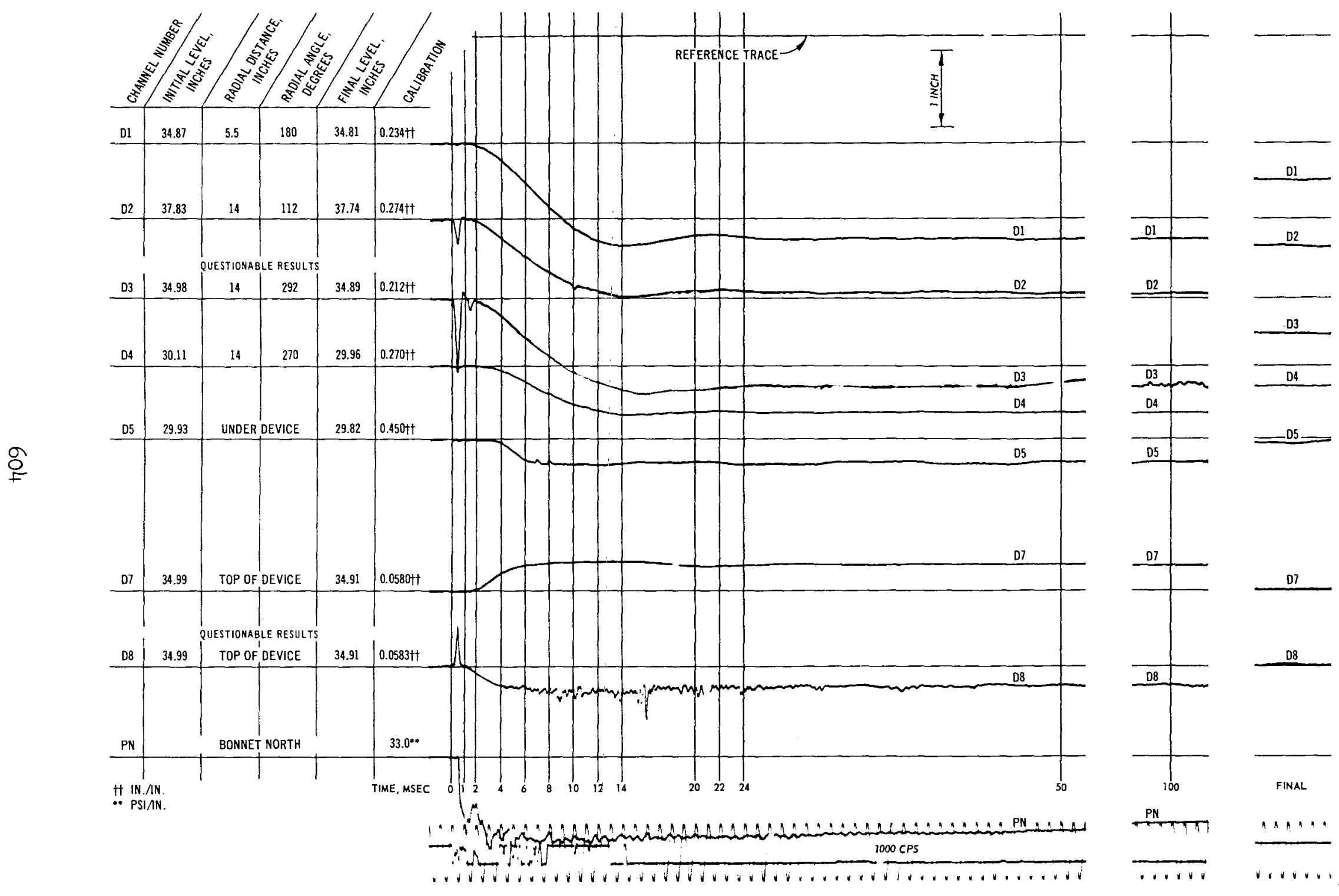

Fig. C-74. Test 28, deflection and pressure record 


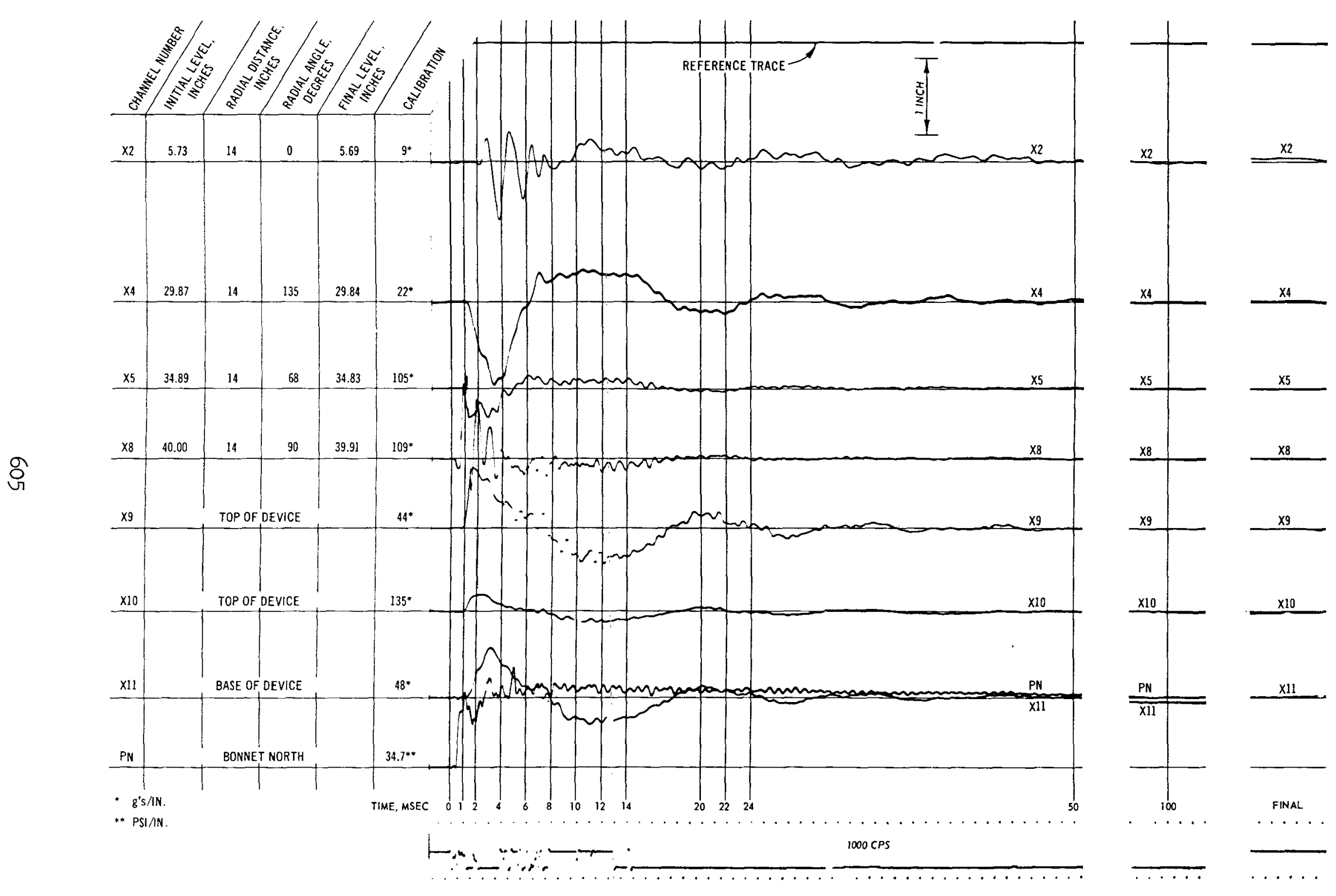

Fig. C-75. Test 28, acceleration and pressure record 


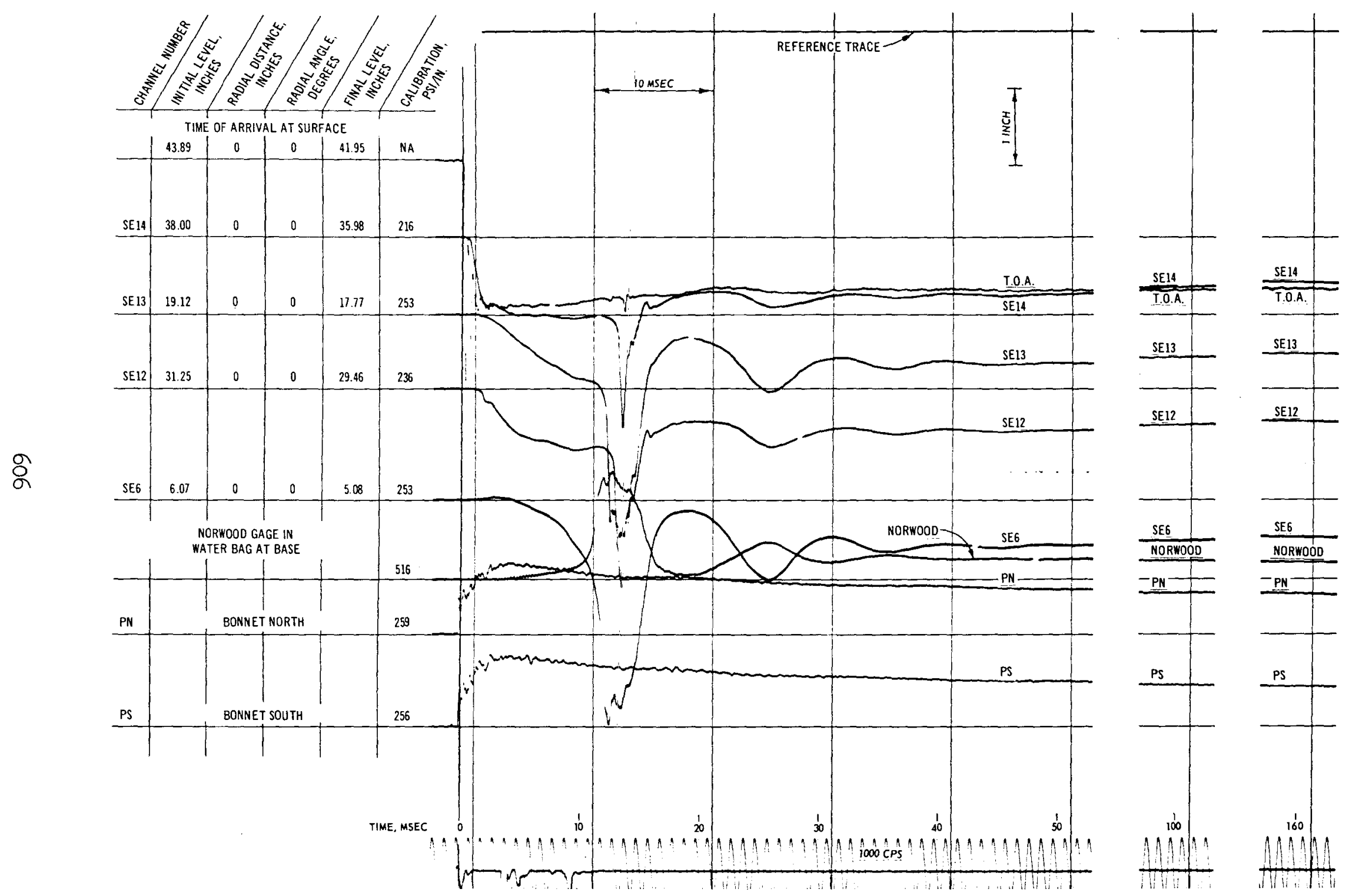

Fig. C-76. Test A, pressure record 


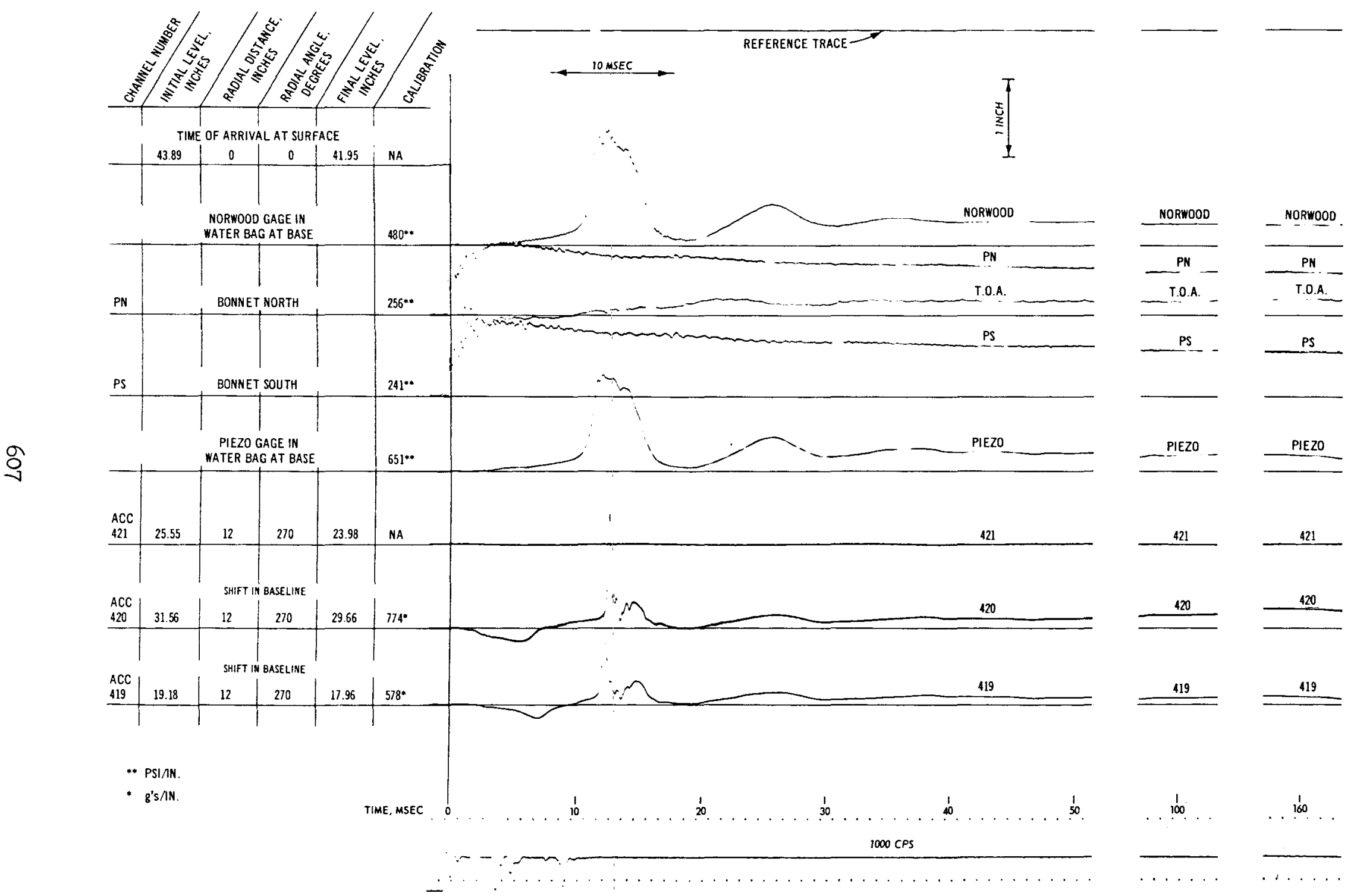

Fig. C-77. Test A, pressure and acceleration record 


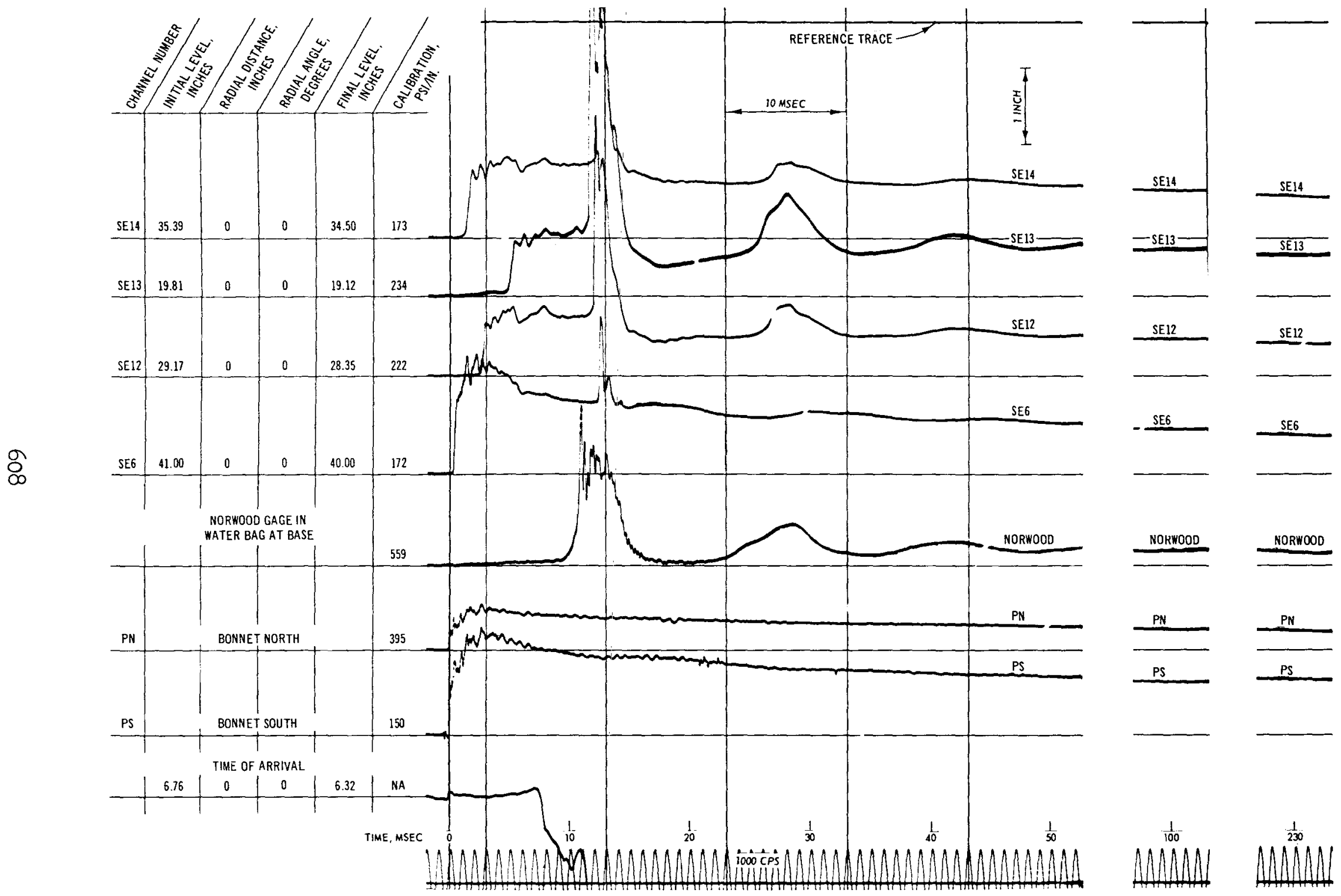

Fig. C-78. Test B, pressure record 


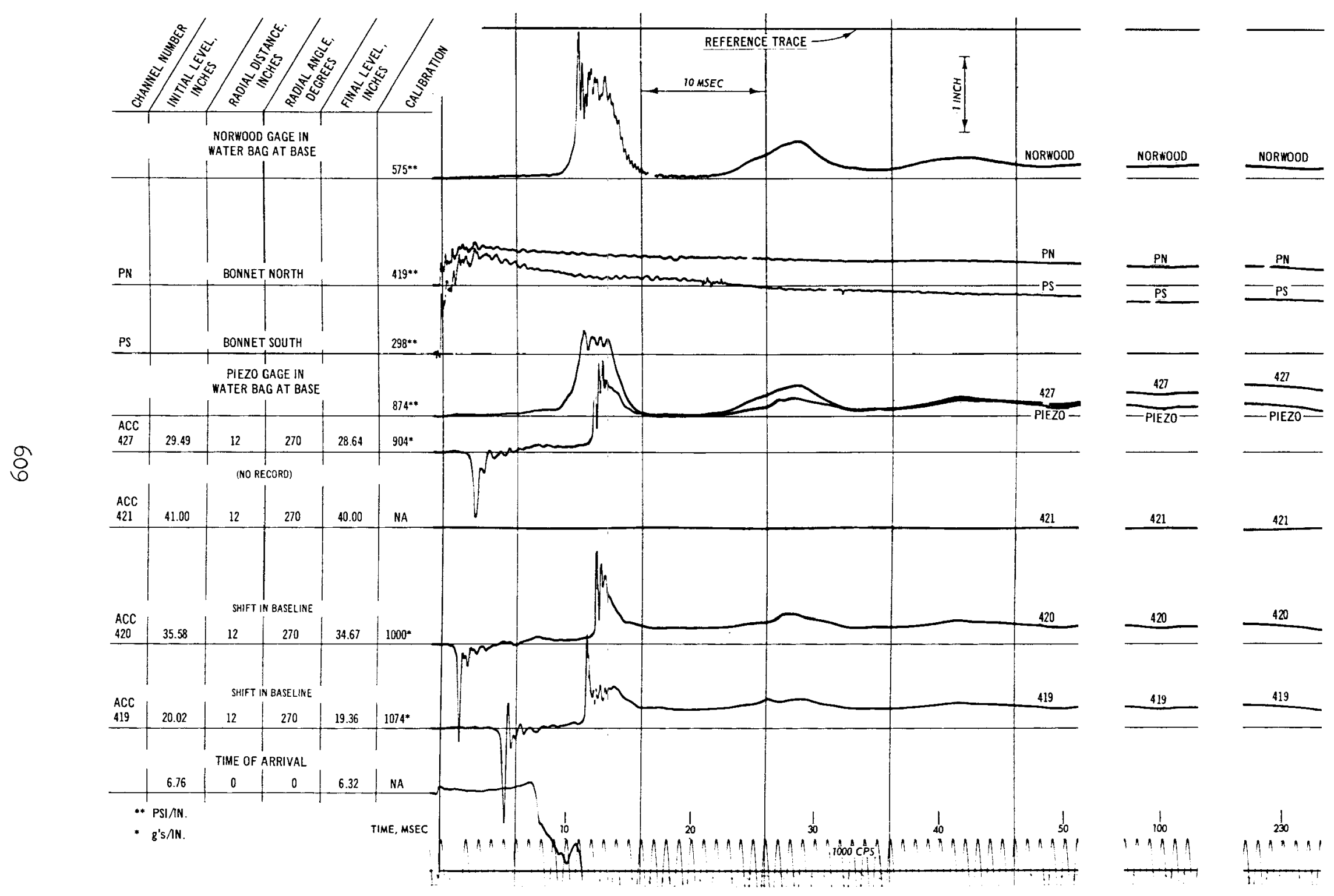

Fig. C-79. Test B, pressure and acceleration record 

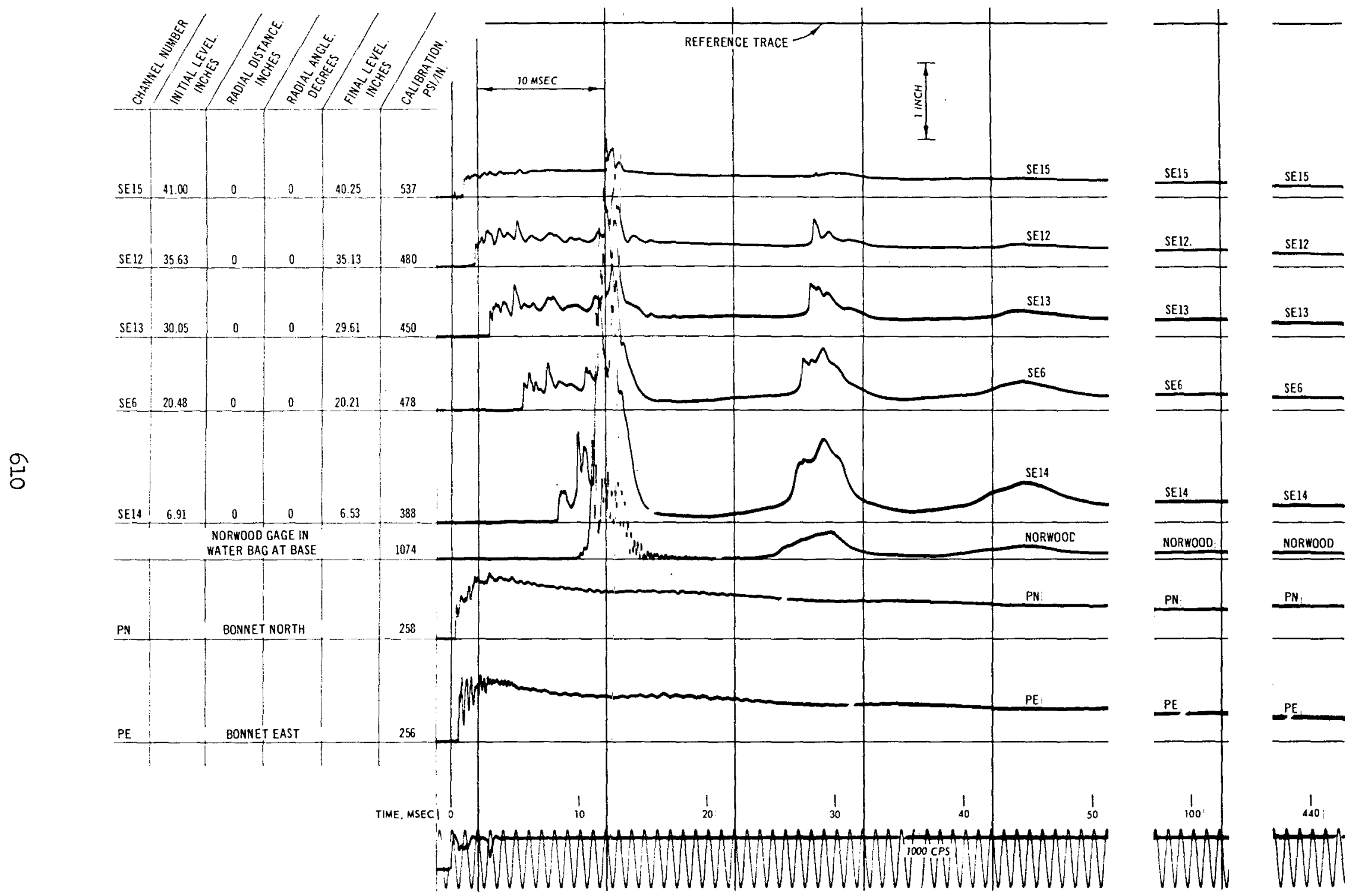

Fig. C-80. Test C, pressure record 

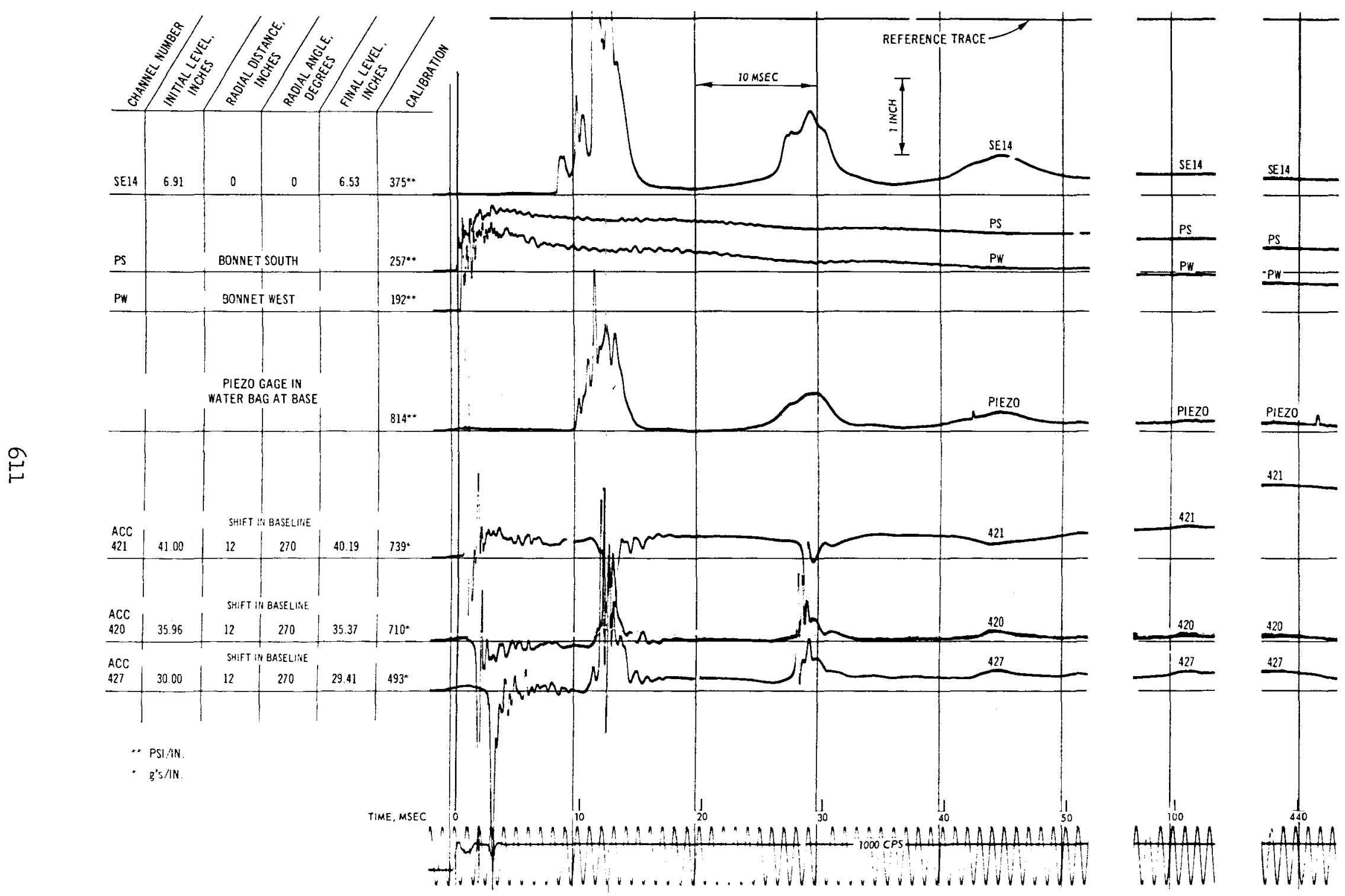

Fig. C-81. Test C, pressure and acceleration record 

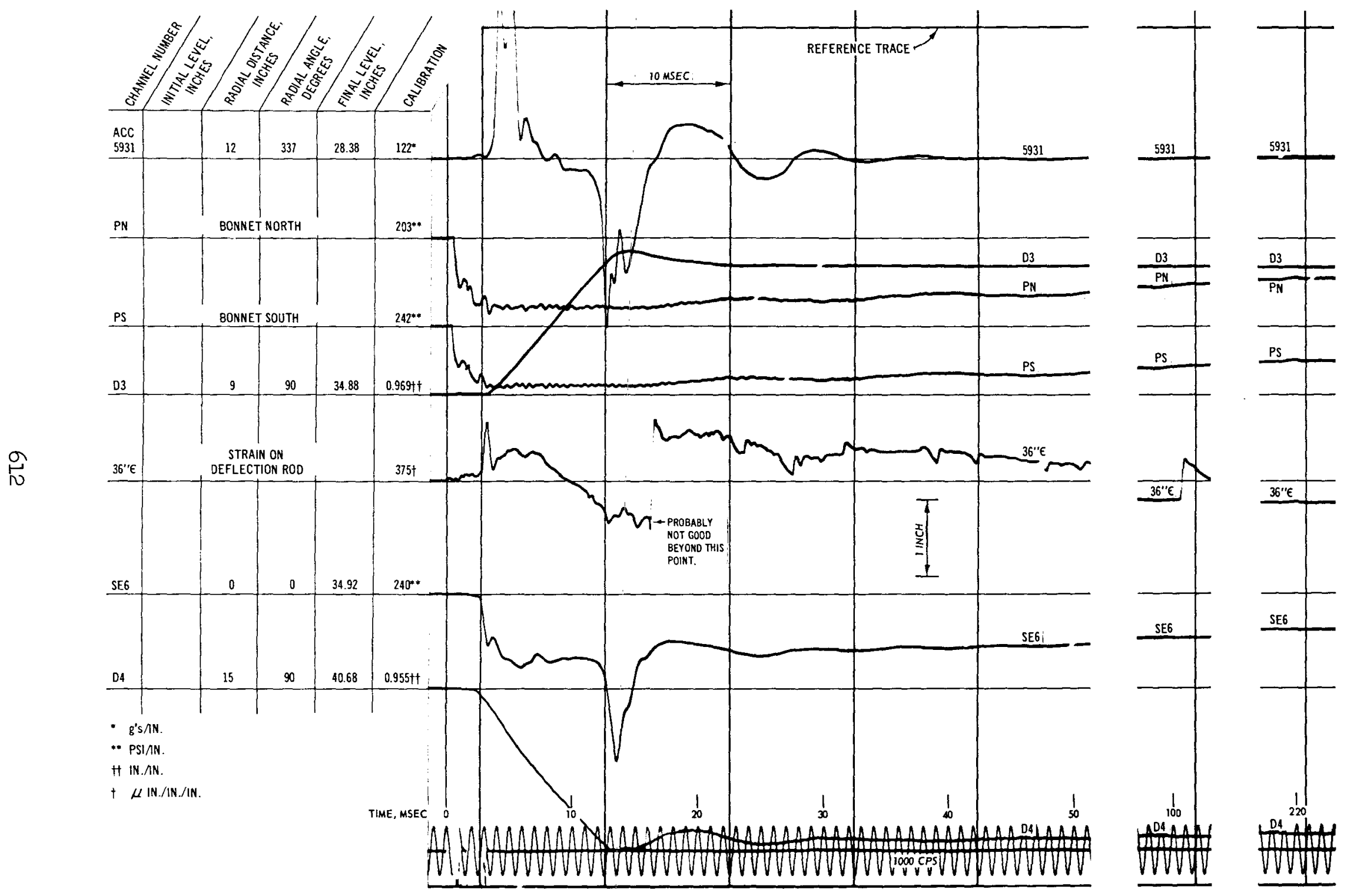

Fig. C-82. Test D, pressure, acceleration, deflection, and strain record 


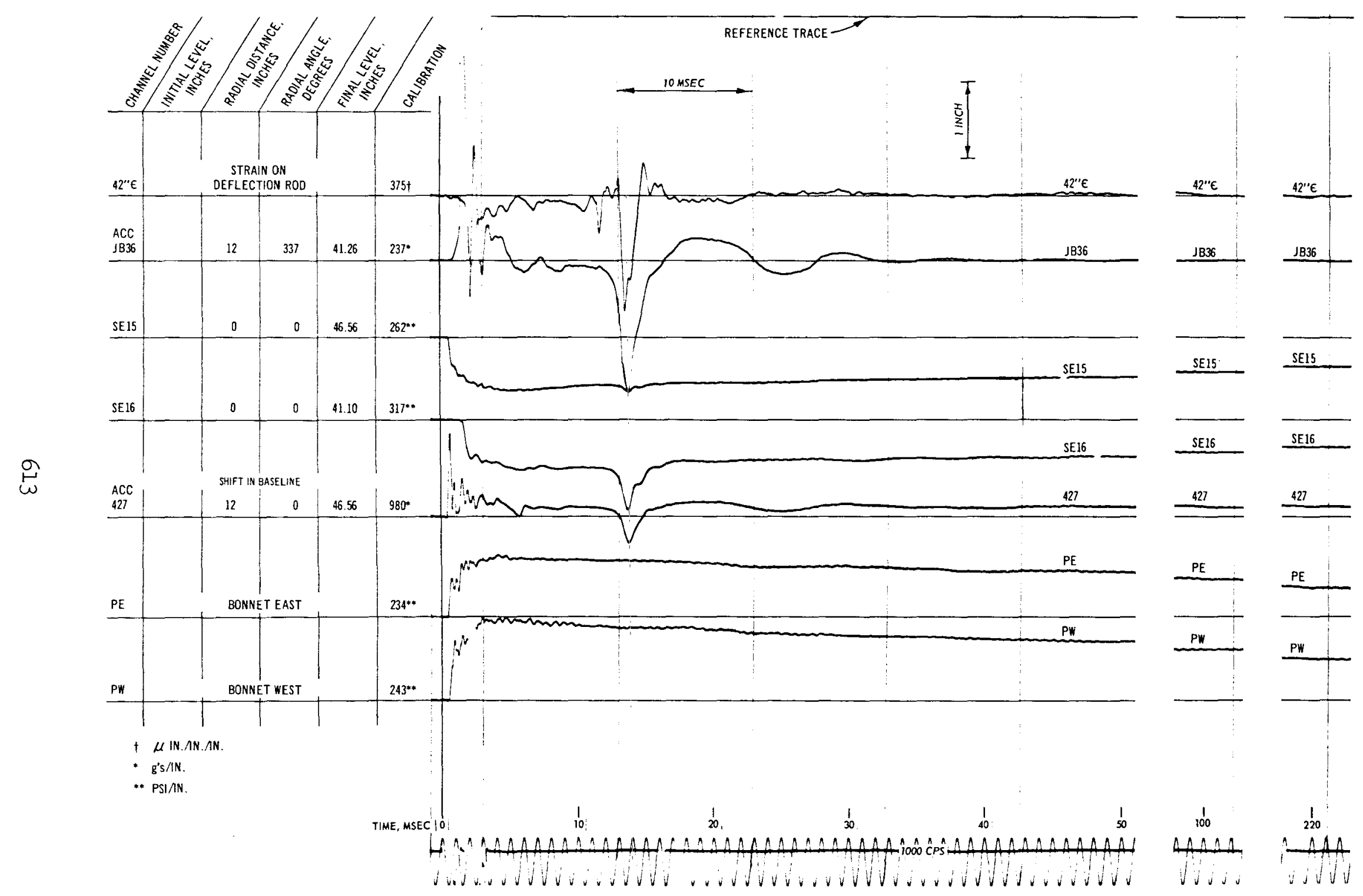

Fig. C-83. Test D, pressure, acceleration, and strain record 


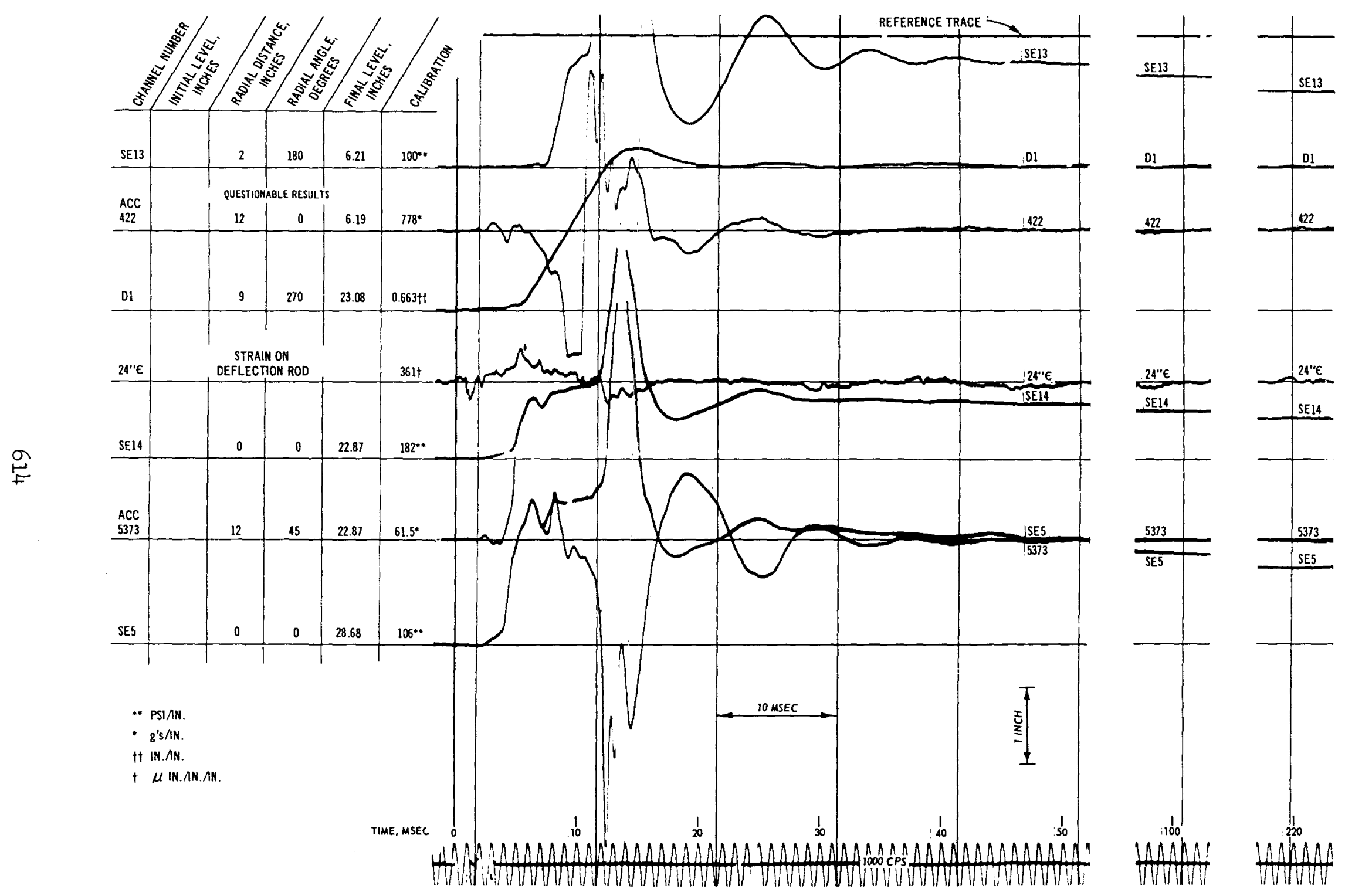

Fig. C-84. Test D, pressure, acceleration, deflection, and strain record 


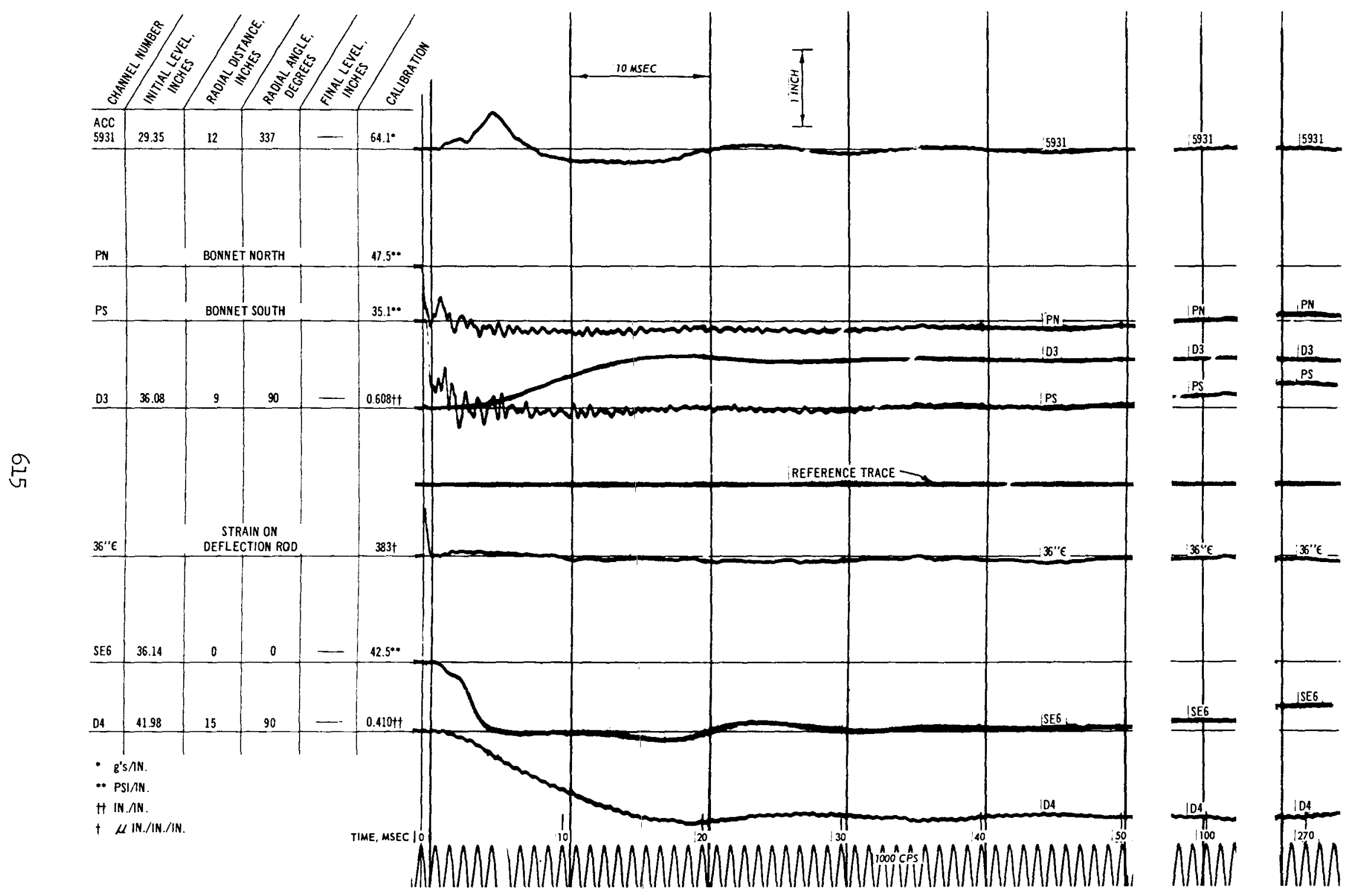

Fig. C-85. Test E, pressure, acceleration, deflection, and strain record 


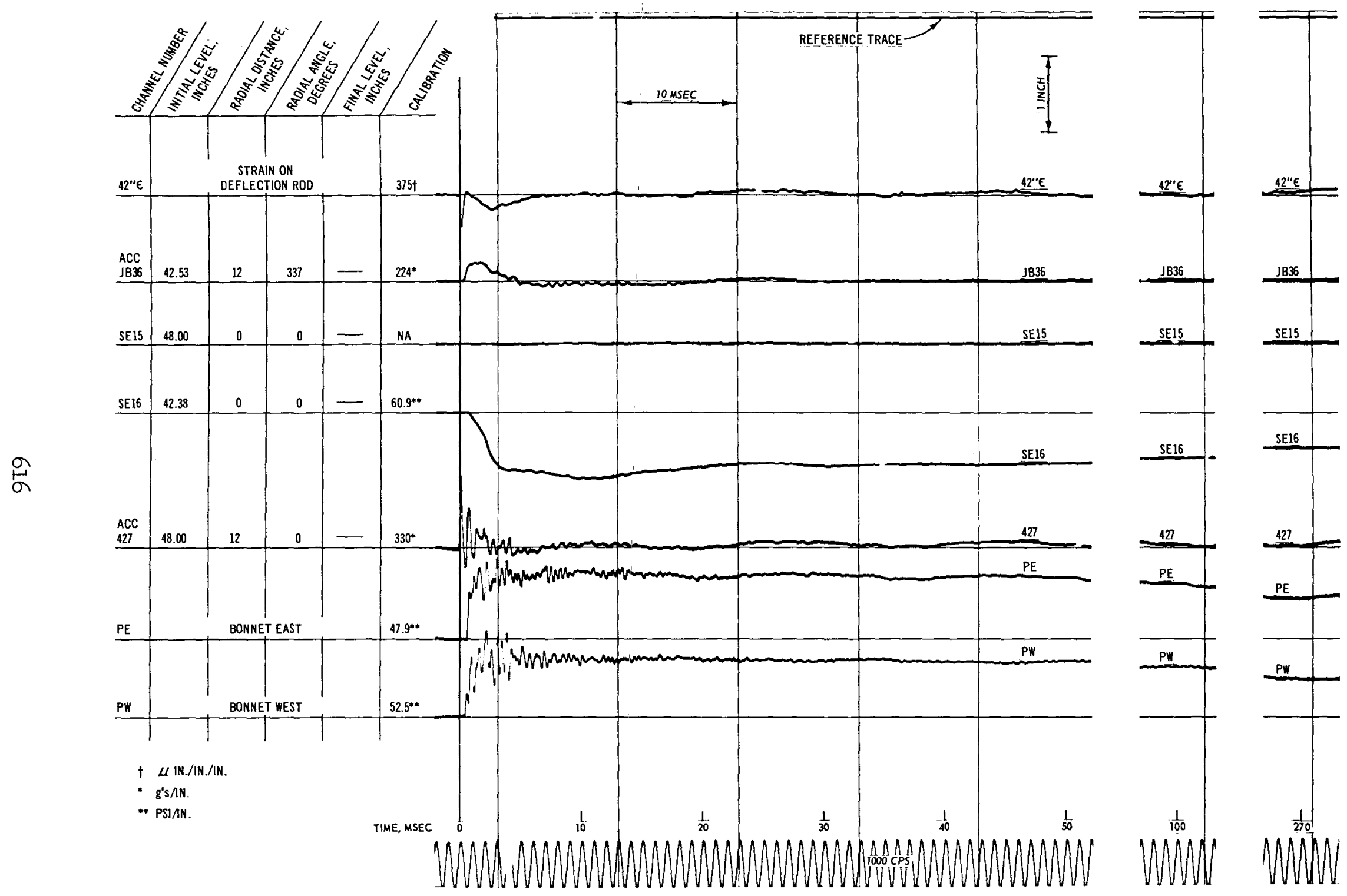

Fig. C-86. Test E, pressure, acceleration, and strain record 


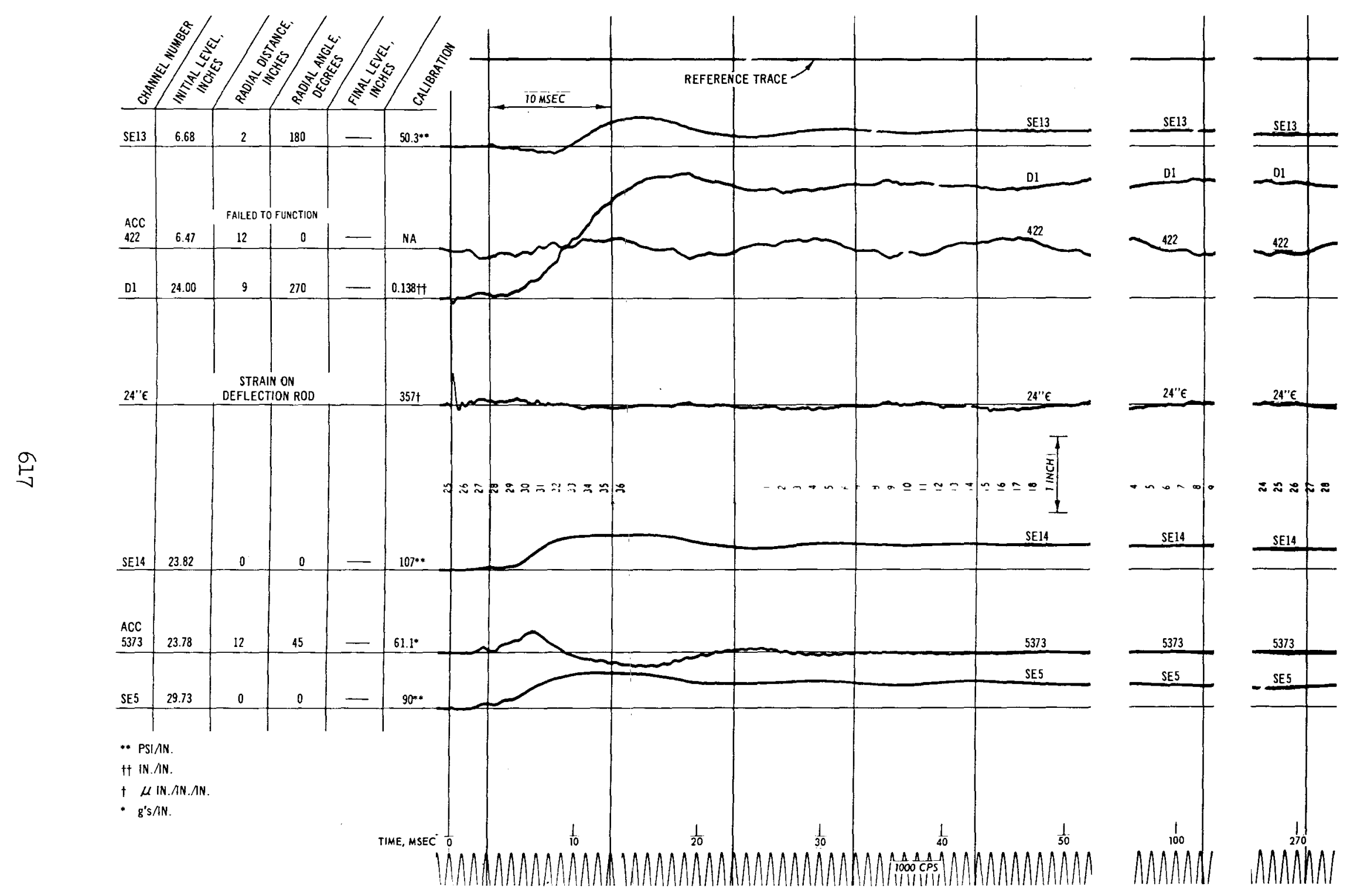

Fig. C-87. Test E, pressure, acceleration, derlection, and strain record 
Army

Chief of Engineers, Department of the Army, Washington, D. C. 20314

ATTN: ENGME-S

ENGME

ENGCW-E

ENGCW-Z

ENGMC-E

ENGMC-EM

ENGMC-DE

ENGAS-I

ENGAS-I, Library

ENGNA

Chief of Research and Development, Headquarters, Department of the Army, Washington,

D. C. 20310

ATTN: Director of Army Technical Information

Chief of Research and Development, Department of the Army, Washington, D. C. 20310

ATTN: Atomic Office

CRDES

Division Engineers, U. S. Army Engineer Divisions, Continental United States

Cy to ea

Commandant, U. S. Army Air Defense School, Fort Bliss, Tex. 79906

Commandant, U. S. Army Command \& General Staff College, Fort Leavenworth, Kans. 66027

ATTN: Archives

Commandant, Army War College, Carlisle Barracks, Pa. 17013

ATTN: Library

Commanding General, Aberdeen Proving Ground, Aberdeen, Md. 21005

ATTN: Director, Ballistic Research Laboratories

Commanding General, The Engineer Center, Fort Belvoir, Va. 22060

1

ATTN: Assistant Commandant, Engineer School

Commanding General, U. S. A. Electronics Command, Fort Monmouth, N. J. 07703

ATTN: AMSEL-GG-DD

Commanding General, USA Missile Command, Huntsville, Ala. 35809

Commanding General, USA Munition Command, Dover, N. J. 07801

Commanding General, U. S. Continental Army Command, Fort Monroe, Va. 23351 
NWED Reports Distribution List

ATTN: Technical Documents Center, Building 315

Commanding Officer, U. S. Army Nuclear Defense Laboratory, Edgewood Arsenal Edgewood, Md. 21040

ATTN: Technical Library

Department of the Army, CE Ballistic Missile Construction Office, P. O. Box 4187 Norton AFB, Calif. 92409

Director of Civil Defense, Office of the Secretary of the Army, Washington, D. C. 20310 ATTN: Mr. George Sisson (RE-ED)

Director, Nuclear Cratering Group, U. S. Army Corps of Engineers, Lawrence Radiation Laboratory, P. O. Box 808, Livermore, Calif. 94550

Director, U. S. Army Corps of Engineers, Coastal Engineering Research Center Washington, D. C. 20016

ATTN: Mr. T. Saville, Jr.

Director, U. S. Army Corps of Engineers, Ohio River Division Laboratories, 5851 Mariemont Avenue, Cincinnati, Ohio 45227

Technical Library

USAMERDC, Bldg 314

Fort Belvoir, Va. 22060

Director, U. S. Army CRREL, P. O. Box 282, Hanover, N. H. 03755

ATTN: Mr. K. Boyd

Director, U. S. Army Construction Engineering Research Laboratory, P. O. Box 4005, Champaign, Ill. 61820

ATTN: Library 
District Engineer, U. S. Army Engineer District, Omaha, 6012 U. S. Post Office and Court House 215 N. 17th Street, Omaha, Nebr. 68101

ATTN: MROGS-B

President, U. S. Army Air Defense Board, Fort Bliss, Tex. 79906

U. S. Army Engineer Division, Missouri River, P. O. Box 103, Downtown Station Omaha, Nebr. 68101

ATTN: Mr. Ken Lane

\section{Navy}

Commander-in-Chief, Pacific, FPO, San Francisco 94129

Commander-in-Chief, U. S. Atlantic Fleet, U. S. Naval Base, Norfolk, Va. 23511

Chief of Naval Operations, Navy Department, Washington, D. C. 20350

ATTN: OP-75

$$
\text { OP-03EG }
$$

Chief of Naval Research, Navy Department, Washington, D. C. 20390

ATTN: Code 811

Commandant of the-Marine- Corps, Nary-Department, Washington, D. C. 20380

ATTN: Code A04E

Commander, Naval Facilities Engineering Command, Navy Department, Washington, D. C. 20370

ATTN: Code 04

Code 03

Commander, Naval Ordnance Systems Command, Washington, D. C. 20360

Commander, Naval Ship Engineering Center, Washington, D. C. 20360

ATTN: Code 6115

Commanding Officer, Nuclear Weapons Training Center, Atlantic Naval Base, Norfolk, Va. 23511 ATTN: Nuclear Warfare Department

Commanding Officer, Nuclear Weapons Training Center, Pacific, Naval Station, North Island San Diego, Calif. 92136

Commanding Officer \& Director, Naval Electronics Laboratory, San Diego, Calif. 92152 
Commanding Officer \& Director, U. S. Naval Civil Engineering Laboratory

ATTN: Code L3I

Commanding Officer, U. S. Naval Civil Engineer Corps Officer School, U. S. Naval Construction Battalion Center, Port Hueneme, Calif. 93041

Commanding Officer, U. S. Naval Damage Control Training Center, Naval Base

ATTN: ABC Defense Course

Commanding Officer, U. S. Naval Weapons Evaluation Facility, Kirtland Air Force Base

Albuquerque, N. Mex. 87117

ATTN: Code WEVS

Commanding Officer, U. S. Naval Weapons Laboratory, Dahlgren, Va. 22448

ATTN: TE

Commander, U. S. Naval Oceanographic Office, Suitland, Md. 20023

Commander, U. S. Naval Ordnance Laboratory, Silver Spring, Md. 20910

ATTN: EA

EU

$E$

Commander, U. S. Naval Ordnance Test Station, China Lake, Calif. 93555

Director, U. S. Naval Research Laboratory, Washington, D. C. 20390

President, U. S. Naval War College, Newport, R. I. 02840

Special Projects, Navy Department, Washington, D. C. 20360

\section{Air Force}

Air Force Flight Dynamics Laboratory, Wright-Patterson AFB, Dayton, Ohio 45433 ATTN: Mr. Frank Janik, Jr.

Air Force Institute of Technology, AFIT-L, Building 640, Wright-Patterson AFB, Ohio 45433 


\section{Air Force (Continued)}

Air Force Technical Applications Center, Department of the Air Force, Washington, D. C. 20333

Air Force Weapons Laboratory, Kirtland AFB, N. Mex. 87117

ATTW: Library

WLDC

WLDC/R. W. Henny

Director, Air University Library, Maxwell AFB, Ala. 36112

Commander, Strategic Air Command, Offutt AFB, Nebr. 68113

ATTN: OAWS

Commander, Tactical Air Command Langley AFB, Va. 23365

ATTN: Document Security Branch

Space and Missile Systems Organization, Norton AFB, Calif. 92409

ATTN: SAMSO (SMQNM)

Headquarters, USAF, Washington, D. C. 20330

Director, Air Research and Development Command Headquarters, USAF

ATTN: Combat Components Division

Director of Civil Engineering, Headquarters, USAF, Washington, D. C. 20330

ATTN: AFOCE

Director, U. S. Air Force Project RAND, Via: U. S. Air Force Liaison Office, The RAND

Corporation, 1700 Main Street, Santa Monica, Calif. 90406

ATTN: Library

Dr. Harold L. Brode

Dr. Olen A. Nance

\section{Other DOD Agencies}

Administrator, National Aeronautics \& Space Administration, 400 Maryland Avenue, S. W. Washington, D. C. 20546

Assistant to the Secretary of Defense (Atomic Energy), Washington, D. C. 20301

Commandant, Armed Forces Staff College, Norfolk, Va. 23511

Commandant, National War College, Washington, D. C. 20310

ATTN: Class Rec. Library 
Director, Advanced Research Projects Agency, Washington, D. C. 20301

Director, Defense Intelligence Agency, Washington, D. C. 20301

ATTN: DIAAP-IK2

DIA-AP8B-1

Director, Weapons Systems Evaluation Group, Washington, D. C. 20305

Langley Research Center, NASA, Langley Field, Hampton, Va. 23365

ATTN: Mr. Philip Donely

Manager, Albuquerque Operations Office, USAEC, P. O. Box 5400, Albuquerque, N. Mex. 87115

Manager, Nevada Operations Office, USAEC, P. O. Box 1676, Las Vegas, Nev. 89101

National Aeronautics \& Space Admínistration, Man-Spacecraft Center, Space Technology 1

Division, Box 1537, Houston, Tex. 77001

National Military Command System Support Center, Pentagon BE 685, Washington, D. C. $20301 \quad 1$ ATTN: Technical Library

U. S. Atomic Energy Commission, Washington, D. C. 20545

ATTN: Chief, Classified Tech Lib, Tech Information Service

U. S. Documents Officer, Office of the United States National Military Representative-SHAPE APO New York 09055

\section{Other Agencies}

Aerospace Corporation, 1111 E. Mill Street, San Bernardino, Calif. 92408 


\section{Other Agencies (Continued)}

Applied Theory, Inc., 1728 Olympic Blvd, Santa Monica, Calif. 90404

ATTN: Dr. John G. Trulio

AVCO Corporation, Research and Advanced Development Division, 201 Lowell Street

Wilmington, Mass. 01887

ATTN: Mr. R. E. Cooper

Battelle Memorial Institute, 505 King Avenue, Columbus, Ohio 43201

ATTN: Dr. P. N. Lamori

Bell Telephone Laboratories, Inc., Whippany Road, Whippany, N. J. 07981

ATTN: Mr. R. W. Mayo

The Boeing Company, P. O. Box 3707, Seattle, Wash. 98124

ATTN: Technical Library

Corrugated Metal Pipe Institute, Crestview Plaza, Port Credit, Ontario, Canada

ATTN: Mr. W. A. Porter

Defence Research Establishment, Suffield, Ralston, Alberta, Canada

General Research Corporation, P. O. Box 3587, Santa Barbara, Calif. 93105

ATTN: Mr. Benjamin Alexander

Denver Mining Research Center, Building 20, Denver Federal Center, Denver, Colo. 80225

ATTN: Dr. Leonard A. Obert

Dynamic Science Corporation, ${ }^{-1900}$ Wälker Âvenue, Monrovia,-Calif. 91016

ATTN: Dr. J. C. Peck

Edgerton, Germeshausen \& Grier, Inc., 95 Brookline Avenue, Boston, Mass. 02129

ATTN: D. F. Hansen

Engineering Physics Company, 12721 Twinbrook Parkway, Rockville, Md. 20852

ATTN: Dr. Vincent J. Cushing

Mr. W. Danek

General American Transportation Corporation, General American Research Division 7449 North Natchez Avenue, Niles, nl. 60648

ATTN: Dr. G. L. Neidhardt

General Electric Company, Missile and Space Vehicle Department, Valley Forge Space Technology Center, Goddard Boulevard, King of Prussia, Pa. 19406

General Electric Company, TEMPO, 816 State Street, Santa Barbara, Calif. 93101 ATTN: Mr. Warren Chan (DASIAC) 
Ehysics International Company, 2700 Merced Street, San Leandro, Calif. 94577

ATTN: Dr. Charles Godfrey

Mr. Fred M. Sauer

Research Analysis Corporation, Document Control Supervisor, McLean, Va. 22101.

Dr. John S. Rinehart, Senior Research Fellow (R.2), IER/ESSA, Boulder, Colo. 80302

TRW Space Technology Laboratories, One Space Park, Redondo Beach, Calif. 90278

ATTN: Dr. Millard Barton Mr. M. V. Anthony Mr. J. L. Merritt

ATTN: Mr. Harold Mason

U. S. Department of the Interior, Geological Survey, Geologic Division, Branch of Engineering Geology, 345 Middlefield Road, Menlo Park, Calif. 94025

ATTN: Harold W. Olsen

Paul Weidlinger, Consulting Engineer, 110 East 59th Street, New York, N. Y. 10022 ATTN: Dr. M. L. Baron 


\section{College and Universities}

University of Arizona, Tucson, Ariz. 85721

ATTN: Dr. Donald A. DaDeppo, Department of Civil Engineering Professor Bruce G. Johnston, Dept of Civil Engineering Dr. George Howard, College of Engineering

University of Illinois, Urbana Campus, Department of Civil Engineering, Urbana, III. 61801

ATTN: Professor N. M. Newmark

Professor S. L. Paul

Professor M. T. Davisson

Professor $\bar{G} . \bar{K}$. Sinnamon

Professor W. J. Hall

Professor A. J. Hendron, Jr.

Professor M. A. Sozen

Iowa State University of Science and Technology, Ames, Iowa 50010

ATTN: Professor Glen Murphy

Lehigh University, Bethlehem, Pa. 18015

ATTN: Dr. J. F. Libsch, Materials Research Center

Dr. D. A. Van Horn, Department of Clvil Engineering

University of Massachusetts, Department of Civil Engineering, Amherst, Mass. 01002

Massachusetts Institute of Technology, Division of Sponsored Research, 77 Massachusetts

Avenue, Cambridge, Mass. 02139

ATTN: Dr. Robert J. Hansen

Dr. Robert V. Whitman

University of Michigan, Civil Engineering Department, Ann Arbor, Mich. 48104

ATTN: Professor Frank E. Richart, Jr., Consultant 


\section{College and Universities (Continued)}

Dr. George B. Clark, Director, Rock Mechanics Research Group, University of Missouri at Rolla, Rolla, Mo. 65401

University of New Mexico, Eric H. Wang Civil Engineer Research Facility, Albuquerque,

N. Mex. 87106

ATTN: Dr. Eugene Zwoyer

University of New Mexico, Eric H. Wang Civil Engineering Research Facility, P. O. Box 188

University Station, Albuquerque, N. Mex. 87106

Nova Scotia Technical College, School of Graduate Studies, Halifax, Nova Scotia, Canada

ATTN: Dr. G. G. Meyerhof

Pennsylvania State University, University Park, Pa. 16802

ATTN: Professor G. Albright, Dept of Architectural Engineering

Professor Richard Kummer, 101 Eng. A

1

Purdue University, School of Civil Engineering, Civil Engineering Building,

Lafayette, Ind, 47907

ATTN: Professor M. B. Scott

Rensselaer Polytechnic Institute, Troy, N. Y. 12180

ATTN: Dr. Clayton Oliver Dohrenwend, Security Officer, Mason House

Rice University, Department of Civil Engineering, Houston, Tex. 77001

ATTN: Professor A. S. Veletsos

San Jose State College, Department of Civil Engineering, San Jose, Calif. 95114

ATTN: Dr. Franklin J. Agardy

University of Texas, Balcones Research Center, Austin, Tex. 78712

ATTN: Dr. J. Neils Thompson

Utah State University, Department of Mechanical Engineering, Logan, Utah 84321

ATTN: Professor R. K. Watkins

University of Washington, Seattle, Wash. 98105

ATTN: C. H. Norris, Department of Civil Engineering

Dr. A. B. Arons, Department of Physics

Professor William Miller, Department of Civil Engineering, 307 More Hall

1

1

1

The George Washington University, Nuclear Defense Design Center, School of Engineering and Applied Science, Washington, D. C. 20006

Worcester Polytechnic Institute, Department of Civil Engineering, Worcester, Mass, 01609 ATTN: Dr. Carl Koontz

Northern Arizona University, Box 5753, Flagstaff, Arizona 86001

ATTN: Professor Sandor Popovics

1

1

2

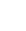


Unclassified

Security Claseification

\begin{tabular}{|c|c|c|}
\hline \\
\hline \multicolumn{2}{|l|}{ 1. ORIGINATING ACTIVITY (Corporato Quthor) } & $\begin{array}{l}\text { 2a. REPORT BECURITY CLABSIFICATION } \\
\text { UnClaSSified }\end{array}$ \\
\hline $\begin{array}{l}\text { U. S. Army Engineer Waterways Experiment Station } \\
\text { Vicksburg, Mississippi }\end{array}$ & Experiment Station & Unclassified \\
\hline \multirow{2}{*}{\multicolumn{3}{|c|}{$\begin{array}{l}\text { AN EXPERIMENTAL INVESTIGATION OF SOIL-STRUCIURE INTERACTION IN A COHESIVE SOIL; } \\
\text { VOLUMES I AND II }\end{array}$}} \\
\hline & & \\
\hline \multicolumn{3}{|l|}{$\begin{array}{l}\text { 4. OESCRIPTIVE NOTES (TYpe ol report and inclualve detes) } \\
\text { Final report (in two volumes) }\end{array}$} \\
\hline \multicolumn{3}{|l|}{ 5. AUTHOR(S) (Flrel name, midef Inltial, leot neme) } \\
\hline $\begin{array}{l}\text { 6. REPORT DATE } \\
\text { March } 1970\end{array}$ & \multicolumn{2}{|c|}{\begin{tabular}{|c|c|} 
7. TOTAL NO. OF PAGES & 7b. NO. OF REFS \\
666 & 133 \\
\end{tabular}} \\
\hline OO. CONTRACT OR GRANT NO. & \multicolumn{2}{|c|}{ Technical Report $\mathrm{N}-70-7$; Volumes I and II } \\
\hline c. NWER Subtask SC210 & \multicolumn{2}{|c|}{$\begin{array}{l}\text { Db. OTHER REPOAT NO(S) (AnY Other numbere that anay be aceloned } \\
\text { Qhit roport) }\end{array}$} \\
\hline \multicolumn{3}{|c|}{$\begin{array}{l}\text { 10. DISTRIDUTION STATEMENT } \\
\text { This document has been approved for public release and sale; its distribution is } \\
\text { unlimited. }\end{array}$} \\
\hline $\begin{array}{l}\text { 11. SUPPLEMENTAAY NOTE Report was also sub- } \\
\text { mitted to University of Illinois, Urbana, } \\
\text { Illinois, as thesis for degree of Doctor } \\
\text { of Philosophy in Civil Engineering }\end{array}$ & \multicolumn{2}{|c|}{$\begin{array}{l}\text { 12. SPONSORING MILITARY ACTIVITY } \\
\text { Defense Atomic Support Agency } \\
\text { Washington, D. C. }\end{array}$} \\
\hline \multicolumn{3}{|c|}{ 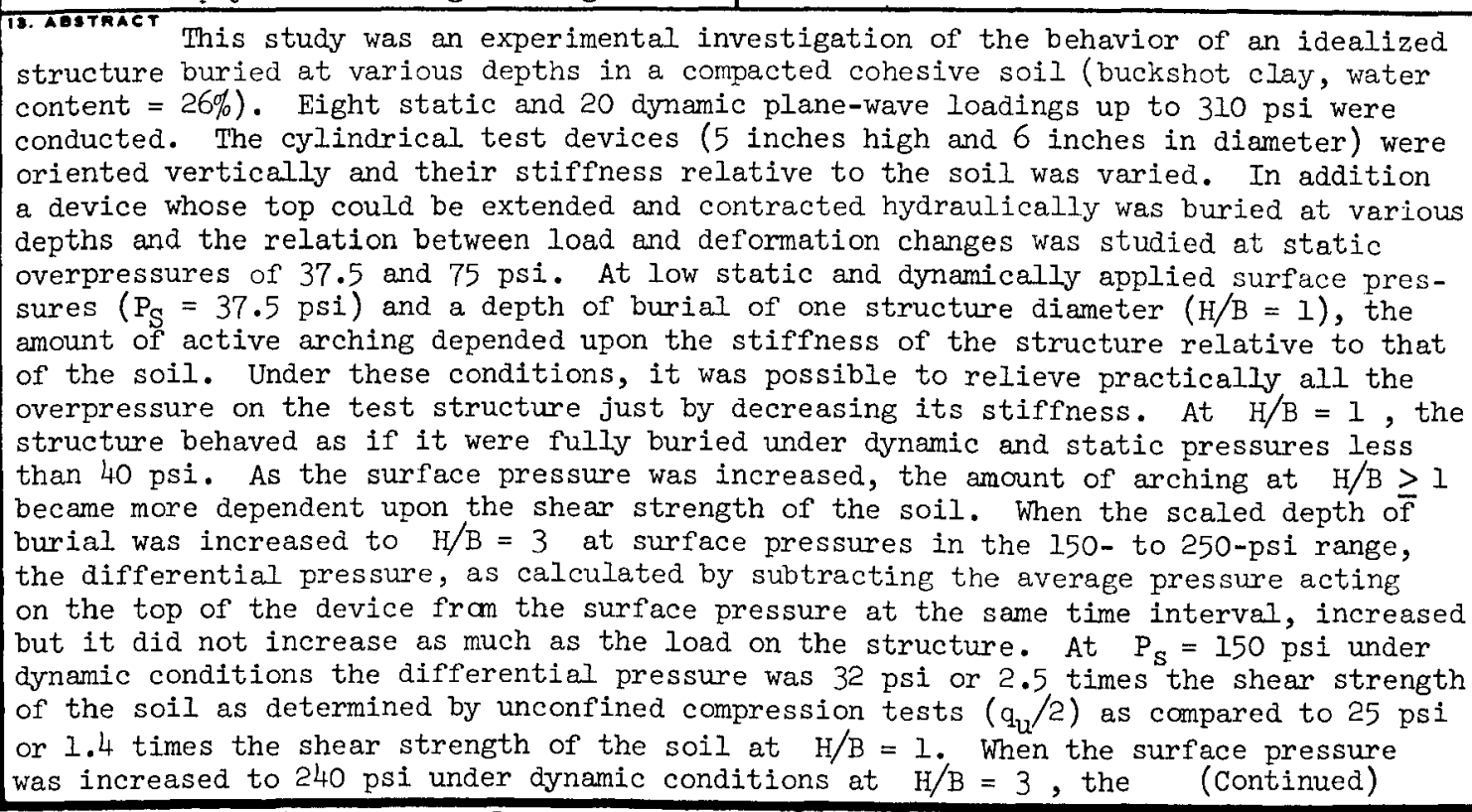 } \\
\hline
\end{tabular}

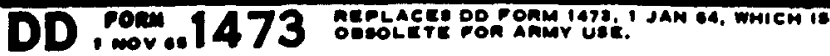

Unclassified 
Unclassified

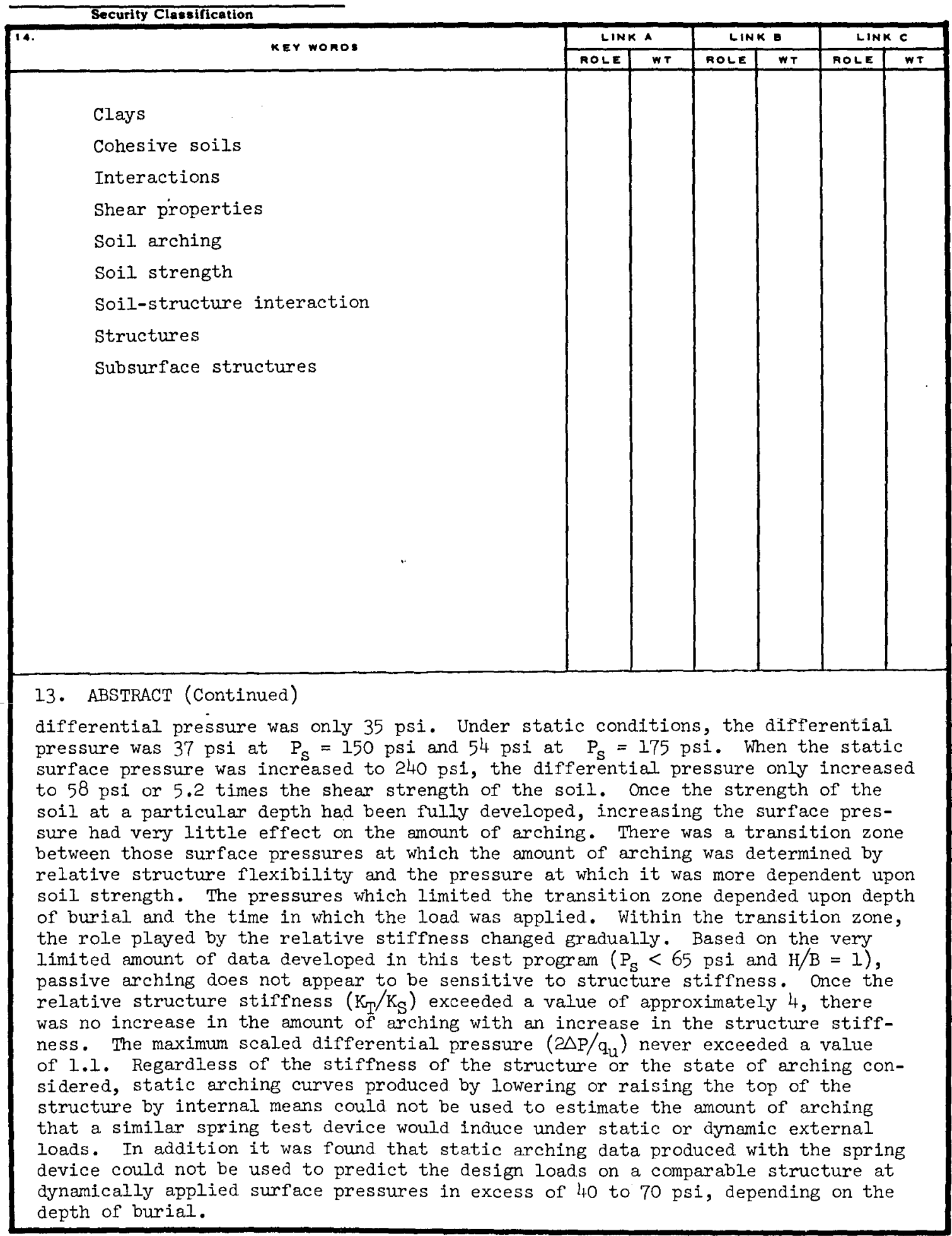

